\title{
TIRYPANOSOMES
}

\section{AND \\ TRYY PANOSOMIASES}

2A KURAN B MESNIZ

D. NABARRO 


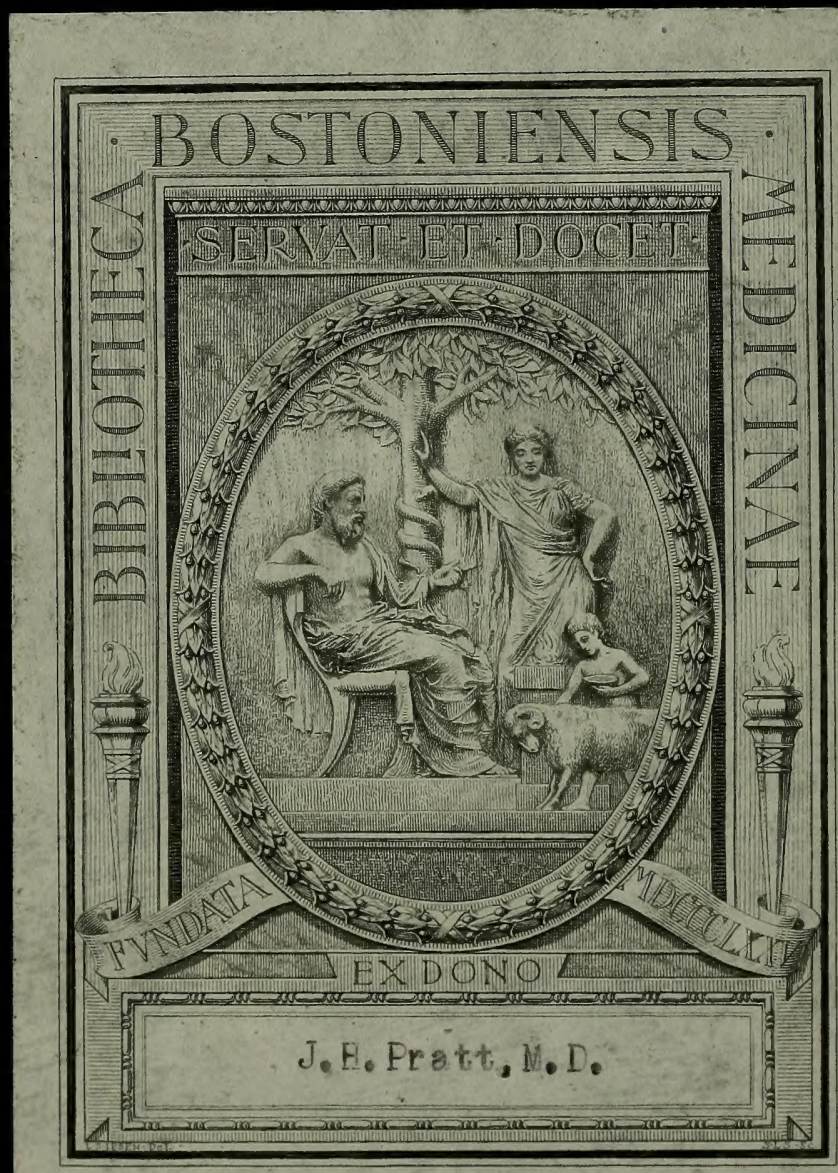


10. B. 5 . 


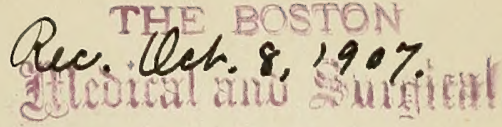

JOURNAL:

TRYPANOSOMES AND TRYPANOSOMIASES 



\section{TR Y PA NOSOMES}

AND

\section{TRYPANOSOMIASES}

BY

A. LAVERAN

Membre de l'Institut, et de l'Académie de Médecine, Paris:

AND

F. MESNIL

Chef de Laboratoire a l'Institut Pasteur

TRANSLATED AND MUCH ENLARGED BY

DAVID NABARRO, M.D., B.Sc., D.P.H., Lond.

Member of the Royal College of Physicians, London; Assistant-Professor of Pathology and Bacteriology, University College, London; Scientific Assistant in Pathology at the University of London; Royal Society Commissioner for the Study of Sleeping Sickness in Uganda, Igo3

WITH COLOURED PLATE AND EIGHTY-ONE FIGURES IN THE TEXT

\section{CHICAGO}

W. T. KEENER \& CO.

90 WABASH AVENUE 


\section{3}

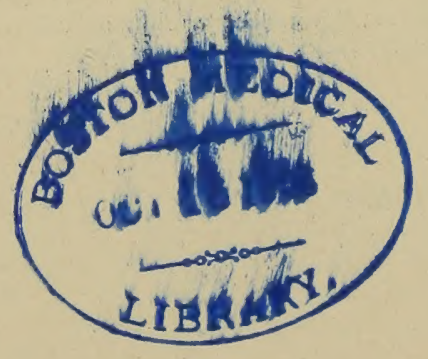




\section{PREFACE TO THE ENGLISH EDITION}

Three years have elapsed since the publication of Professors Laveran and Mesnil's 'Trypanosomes et Trypanosomiases.' When the work appeared it contained practically all that was known about the subject at the time; consequently, it has been an indispensable book of reference to all subsequent investigators in this particular branch of pathology. During the past three years a great deal of work has been done in Europe, Asia, Africa, and America upon the life-history and development of the trypanosomes, as well as upon the trypanosome diseases of man and the lower animals. The great activity in this direction is, no doubt, largely due to the stimulus given by the discovery (made in I903) that sleeping sickness is a stage of human trypanosomiasis - in fact, that it is a human tsetsefly disease.

This large output of original work-over 200 new references to recent papers are given in this edition-has made it necessary to do more than merely publish a translation of the original book. The work has been brought thoroughly up to date, considerable additions being made to practically every chapter. All such additions are enclosed within square brackets [], and for the added matter I alone am responsible. Among the most important additions may be mentioned: The spirochætes; the Leishman-Donovan body; several new trypanosomes of small mammals, birds, Batrachians, and fishes; recent observations on the occurrence of flagellates in tsetse-flies, mosquitoes, leeches, etc., and the possible relation of these parasites to the sexual forms of the trypanosomes of vertebrates; the recent observations upon the prevalence of human and animal trypanosomiases in various parts of Africa and in Asia; many facts in conrection with human trypanosomiasis; and the microscopic changes found in the central nervous system in sleeping sickness and in dourine. A new chapter has been written on the "Treatment of the 'Trypanosomiases.'

The result of incorporating all the latest information has been to increase the work by more than one half the original size; but by the use of a larger page, and the rather free use of closer type for a considerable portion of the added matter, the book contains only roo pages more than the original. The labour involved in carrying out the work has been very great-far greater, indeed, than I anticipated 
when I began the translation. The excellent abstracts by Mesnil in the Bulletin de l'Institut Pasteur of practically all papers dealing with trypanosomes have been of considerable assistance to me, and have rendered my task somewhat lighter than it would otherwise have been. I am greatly indebted to Professor Mesnil for permission to quote from these abstracts, and I have done this in several instances, due acknowledgment being made in the text.

I wish to acknowledge my indebtedness to many English and foreign periodicals, more particularly to the various reports of the Sleeping Sickness Commission of the Royal Society; the Comptes Rendus des Séances de l'Académie des Sciences, and of the Société de Biologie; the Annales and the Bulletin de l'Institut Pasteur; the Journal of Infectious Diseases; the Thompson Yates and Johnston Laboratory Reports; the Lancet and the British Medical Journal; the Arbeiten aus dem kaiserlichen Gesundheitsamte; and the Centralblatt für Bakteriologie.

My thanks are due to all who have kindly helped me during the progress of the work, especially to the authors for sending me copies of their own papers, as well as of those of their pupils, and for the interest they have taken throughout; to Dr. A. C. Stevenson for drawing many of the new illustrations ; to Professors E. A. Minchin and F. G. Novy and Drs. Mott and G. Martin for sending me copies of their publications; to Professor L. Brandin, Dr. G. C. Chubb, and Messrs. E. E. Austen and W. G. Hartog for constant help; and, lastly, to Mr. Wilfred Trotter and Dr. H. M. Woodcock for much valuable criticism and help in reading the proofs. My thanks are also due to the publishers for the patience they have shown during the delayed production of the work, and for their invariable courtesy.

In conclusion, I can only hope that this amplified English edition may be as favourably received as was the original, and that it may prove as useful to subsequent investigators; I shall then not have laboured in vain.

DAVID NABARRO.

UNIVERSITy College, LoNdon, July, I907. 


\section{CONTENTS}

PAGE

AUTHORS' INTRODUCTION - $\quad$ - $\quad$ - $\quad$ - $\quad-\cdots \quad-\quad$ - $x v$

CHAPTER I.

Historical : Geographical Distribution of the Trypanosomiases - I

\section{CHAPTER II.}

Technique for the Study of the Trypanosomes.

Section 1.-Examination in the Living State - - - - - - $\quad 7$

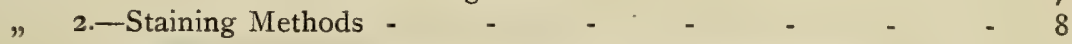

" 3.-Preservation in vitro and Cultivation of Trypanosomes - _ 13

"4.-Inoculation Experiments - _ _ _ - _ $\quad$ - 15

\section{CHAPTER III.}

\section{Comparative StUdy of the TRYPanosomes.}

Section I.-General and Comparative Morphology - $\quad$ - _ - $\quad$ I6

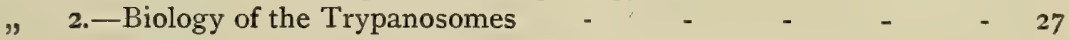

" 3.- Historical Survey of the Genera of Trypanosomes - - - 31

" 4.-Position of the Trypanosomes amongst the Flagellates - - 35

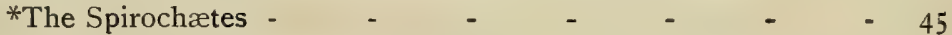

*The Leishman-Donovan Body - $\quad$ - $\quad$ - $\quad$ - $\quad$ - 48

" 5.-Significance of the Basal Corpuscule of Trypanosomes; its

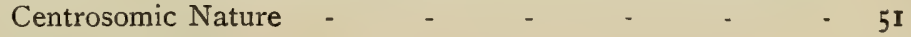

CHAPTER IV.

TRYPANOSOMA LEWIST-PARAsite Peculiar to Rats.

Section I.-Historical Review and Geographical Distribution - $\quad$ - 58

" 2.-The Course of Experimental Infection - - - - - 6I

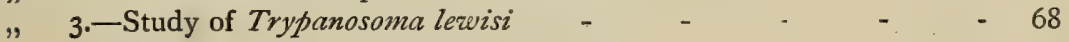

" 4.-Agglomeration of Trypanosoma lewisi - $\quad$ - $\quad$ - $\quad$ - 80

" 5.-Natural Modes of Infection in Rats - $\quad$ - $\quad$ - $\quad$ - 88

" 6.-Active Immunity : its Mode of Production - - - 90

" 7.-Passive Immunity: Attempts at Treatment _ _ - . 93

\section{CHAPTER V.}

\section{Different Trypanosomes of Small Mammals.}

Section I.- * Trypanosome of the Mouse ( $T$. duttoni) -

"2.-Trypanosome of the Bandicoot $(T$. bandicotti $)$ - - - 102

" 3.-Trypanosome of the Rabbit (T. cuniculi) - - - - - - 103

* The Sections marked with an asterisk have been added. 
Section 4.-Trypanosome of the Guinea-pig - _ _ _ - - 105

" 5.-Trypanosome of the Hamster (T. rabinowitschi) - _ - _

" 6.-Trypanosome of the Spermophile - _ - - - - 106

"7.-Trypanosome of the Indian Squirrel (T. indicum) - - 106

" 8.- Trypanosome of the Dormouse (T. myoxi, T. blanchardi) - 107

" 9.-Trypanosome of Bats (T. vespertilionis) - _ - - $\quad$ - 107

" Io.-*Trypanosome of the Mole (T. talpa, n. sp.) - - - I I0

"II._* Trypanosome of the Badger (T.pestanai) - - - - IIO

\section{CHAPTER VI.}

\section{Nagana and Allied African Diseases.}

Historical Review and Geographical Distribution

\section{PART I. $-N A G A N A$.}

Section 1.-Animals Susceptible to Nagana; Refractory Animals ; Symptoms and Course of the Disease in Different Animals - - I2I

9)

9)

9)

29

2)

99
2.-Pathological Anatomy

*Changes in the Blood and Internal Organs -

3.--Résumé of Symptoms and Pathological Anatomy

4.-Morphology of Trypanosoma brucei; Action of Heat and Cold ; Cultivation Experiments; Agglomeration; Involution Forms 5.-The Etiology of Nagana; the Rôle of the Tsetse-Fly and of the Big Game

6.-Treatment -

7.--Prophylaxis
I 2 I

143

146

150

I64 169 178

\section{PART II.-DISEASES ALLIED TO NAGANA.}

Section I._* General Considerations ; Classification of the Trypanosomiases; Methods of Differentiation and Identifying the Pathogenic Trypanosomes - $\quad-\quad-\quad-\quad-\quad-\quad-185$

" 2.-Trypanosomiasis of German East Africa - _ _ _ _ $\quad$ - 190

" 3.-Trypanosomiasis of Togoland - - - _ - 193

" 4.-* Trypanosomiases of Cameroon - . _ _ _ _ $\quad$ - 199

" 5.-*Trypanosomiasis of Somaliland - - - - - - 201

" 6.- - Trypanosomiases of Abyssinia and Erythrea - - - 202

" 7.- *Trypanosomiases of the Anglo-Egyptian Sudan _. - 203

, 8. - * Trypanosomiases of Uganda and Adjacent Parts of East Africa 204

" 9.-Trypanosomiases other than Dourine, found in Algeria - 210

" I0.-Trypanosomiases of the French Sudan - - - - 219

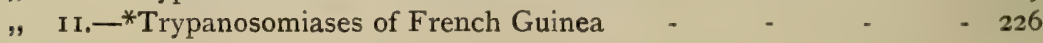

\section{CHAPTER VII.}

TRYPANOSOMIASIS OF HORSES IN GAMBIA.

Section 1.- * Geographical Distribution 


\section{CHAPTER VIII.}

SURRA.

Section I.-Historical: Geographical Distribution of the Disease - _ $\quad 246$

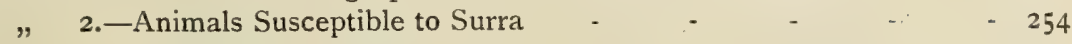

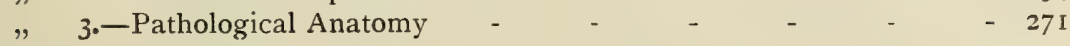

" 4.-Trypanosoma evansi - _ - - - $\quad-\quad 272$

" 5.-Surra is a Distinct Disease - _ _ - - - - 277

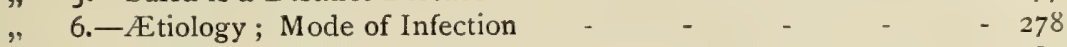

"7.-Treatment; Prophylaxis - _ _ _ _ _ _ _ $\quad 282$

Appendix A.-Lingard's Giant Trypanosome of Cattle _ _ _ - - $\quad 285$

" B. - *'Trypanosomiasis of Horses in Annam _ _ - $\quad 286$

\section{CHAPTER IX.}

Mal de Caderas.

Section I.-Historical ; Geographical Distribution

\begin{tabular}{|c|c|c|c|c|c|}
\hline & 2.-Animals Susceptible to Caderas & - & - & - & - \\
\hline & 3.-Pathological Anatomy & - & - & . & - \\
\hline & 4.-Trypanosoma equinum & . & - & - & - \\
\hline & 5.-Caderas is a Distinct Disease & - & - & - & - \\
\hline & 6.--Mode of Propagation & - & - & - & - \\
\hline & 7.-Treatment; Prophylaxis . & - & - & - & - \\
\hline
\end{tabular}

\section{CHAPTER X.}

\section{DOURINE.}

Section I.-Historical Survey and Geographical Distribution - _ _ _ - 312

" 2.-Dourine in the Equidæ - - - _ - - - 314

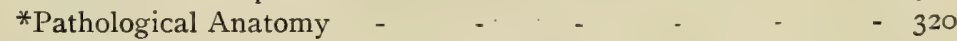

" 3.-Experimental Dourine in Susceptible Animals - - $\quad$ - 322

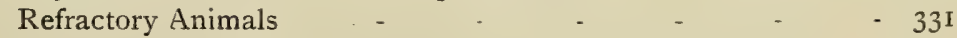

"4.-Trypanosoma equiperdum - _ _ _ _ - $\quad$ - 332

" 5.-The Individuality of Dourine - _ _ - _ - $\quad$ - 335

" 6. -Mode of Propagation _ _ _ _ _ _ _ _ $\quad$ - 337

"7.-Treatment ; Immunity; Prophylaxis - - - - 338

Appendix._-'Maladie de Soemedang,' Java _ _ _ _ _ _ _ $34 \mathrm{I}$

\section{CHAPTER XI.}

GALZIEKTE (GALl-Sickness).

Section I.-Historical ; Geographical Distribution - $\quad$. $\quad$ - $\quad 343$

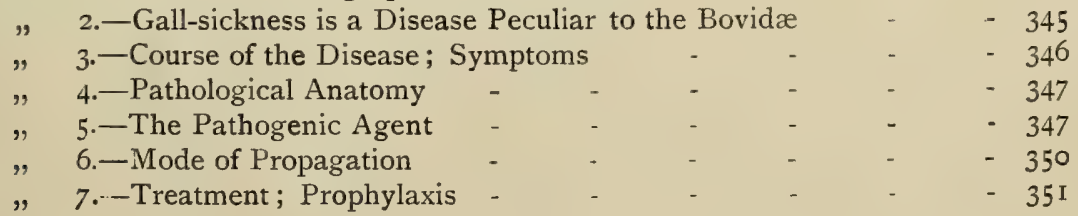

CHAPTER XII.

Human Trypanosomiasis, including Sleeping Sickness.

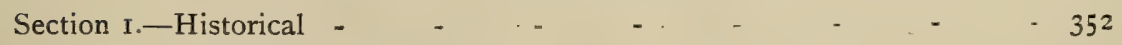

"2.-Geographical Distribution - $\quad$ - $\quad$ - $\quad$ - 359 
Section 3.-Predisposing Causes; The Influence of Age, Sex, Occupation,

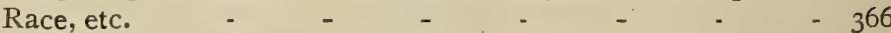

" 4.-Description of the Disease $\quad$ - $\quad$ - $\quad$ - $\quad$ - $\quad 369$

" 5.-The Pathogenicity of Trypanosoma gambiense for Different

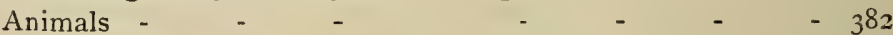

"6.-Pathological Anatomy - - - - - - - 391

*The Changes in the Brain and other Organs in Sleeping Sickness -

: Trypanosoma gambiense

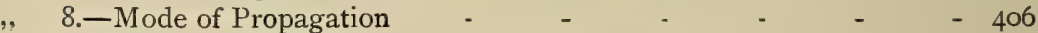

", 9.-Diagnosis - $\quad-\quad$ - $\quad$ - $\quad$ -

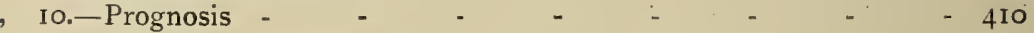

" II.-Treatment; Prophylaxis - $\quad$ - $\quad$ - $\quad$ - $\quad$ - $\quad$ - 4 II

\section{CHAPTER XIII.}

*The Treatment of the TRypanosomiases.

Section 1.- ${ }^{*}$ Historical Review of the Treatment of the Trypanosomiases in General -

"2. - Experiments on Treatment with Agents other than Chemical Substances: Serums and Spleen Extracts; Cultures of Organisms; X Rays and other Rays; Light - - -

" 3. - Recent Experiments on Treatment with Arsenic Compounds, including Atoxyl, and with Trypanred - $\quad-\quad-\quad-420$

" 4. - * Treatment by Means of Benzidine Dyes r _ - - - - 426

" 5.-*Treatment of Human Trypanosomiasis and of Experimental Infections with Trypanosoma gambiense in Animals

\section{CHAPTER XIV.}

TRYPANOSOMES OF BIRDS.

Section I.-Historical Review and Geographical Distribution _ - $\quad 439$

" 2.-Morphology and Cultivation of the Trypanosomes of Birds - 443 *Novy and McNeal's Observations on the Trypanosomes of Birds 45I

\section{CHAPTER XV.}

TRYPANOSOMES OF REPTILES.

Trypanosoma damonice of the Tortoise -

* Trypanosoma boueti of the Lizard

\section{CHAPTER XVI.}

TRYPANOSOMES OF BATRACHIANS.

Section I.-Historical and Geographical Distribution -

"2.-Trypanosoma rotatorium -

* Trypanosoma borreli

* Cultivation of Trypanosoma rotatorium .

3. - Trypanosoma inopinatum -

* Development of Frog Trypanosomes in Leeches

" 4.- - Other Trypanosomes of Batrachians

* Trypanosoma nelspruitense of Frogs in the Transvaal

* Trypanosoma belli, n. sp. of Frogs in Hong-Kong

* Trypanosoma somalense of the Somaliland Toad

* Trypanosome of the Newt

"5-Modes of Infection -

460 465 469 470 473 


\section{CHAPTER XVII.}

TRYPANOSOMES OF Fishes.

Section I.-Historical; Species known to be Infected - _ _ _ _ $\quad$ - 479

"2.-Technique ; Preservation of the Trypanosomes of Fishes

" 3.-Description of the Trypanosomes of Fishes belonging to the

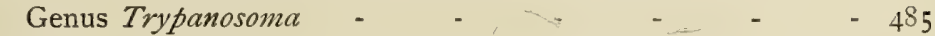

*The recently described Species of this Genus - $\quad$ - $\quad-493$

" 4.-Description of the Trypanosomes of Fishes belonging to the

Genus Trypanoplasma - $\quad$ - $\quad$ - $\quad$ - $\quad$ - $\quad .496$

*Recent Observations on the Trypanoplasms of Fishes - - $50 \mathrm{I}$

" 5.-Mode of Multiplication of the Trypanosomes of Fishes - $\quad 502$

"6.-Modes of Infection - - - - - 502

*Development of Trypanosomes and Trypanoplasms in Leeches 504

\section{CHAP'TER XVIII.}

The TSETSE-Flies AND THEIR TRYPanosomes.

Part I._*The Trypanosomes of Tsetse-Flies - _ _ - - $\quad 508$

,2.-The Tsetse-Flies

INDEX -

\section{TABLES.}

I. Table showing the Results obtained in Various Animals infected with

Nagana by Different Investigators - - - - - -

2. Table showing the Results obtained by Schilling and by Martini with the

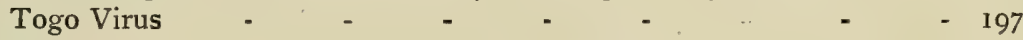

3. Table giving the Dimensions of the Bird Trypanosomes - . - - 447

4. Dichotomous Table of the Species of the Genus Glossina - - - $52 \mathrm{I}$

\section{MAPS.}

I. Map showing the Geographical Distribution of the Animal Trypanosomiases

2. Map showing the Distribution of Surra in India and Indo-China - $\quad 250$

3. Map of Equatorial Africa, showing the Distribution of Human Trypanosomiasis 



\section{LIST OF ILLUSTRATIONS}

FIG.

I. Map showing the Geographical Distribution of the Animal Trypanosomiases

2. Trypanosomes of Mammals and Batrachia-Trichomonas - .

3. Trypanosomes of Fishes - - - - - - - - 18

4. Flagellates. Spermatozoa. Flagellated Spore of Noctiluca - . 39

5. Transformation of an Ookinete of an Intracorpuscular Parasite into a

Trypanosome - $\quad-\quad$ - $\quad$ - $\quad$ - $\quad$ - $\quad$ - $\quad-42$

6. Transition of Trypanosomes into Spirochætes - - - - $\quad$ - 43

*7. Treponema pallidum and various Spirochætes - - - - $\quad 46$

*8. The Development of the Leishman Bodies in Cultures - _ _ 49

*9. Stages in the Development of the Flagellum in Cultures of the Leishman

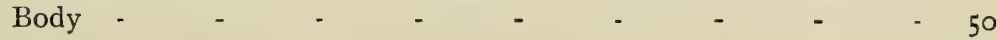

Io. Multiplication Forms of Trypanosoma lewisi - $\quad$ - $\quad$ - $\quad$ - 71

II. Trypanosoma lewisi in the Guinea-pig. Involution Forms - - $\quad$ - 73

12. Culture Forms of Trypanosoma lewisi - $\quad$ - $\quad$ - $\quad$ - $\quad$ - 79

13. Agglutination Forms of Trypanosoma lervisi - - - - - $\quad$ - 83

*I4. Sexual and Conjugation Forms of Trypanosoma lewisi - _ $\quad$ - 89

I5. Engulfment of a Trypanosome by a Leucocyte - - - - $\quad 92$

I6. Trypanosomes of Mus rattus, of the Rabbit (Petrie), and of the Indian Squirrel (Donovan) - $\quad$ - $\quad$ - $\quad$ - $\quad$ - $\quad$ - _ - I03

*I7. Trypanosomes of the Mole (T.talpce) and Bat (T. vespertilionis) - Iog

I8. Temperature Charts of Three Dogs with Nagana - - - $\quad$ I27

19. Temperature Charts of a Donkey and Horse with Nagana - - I33

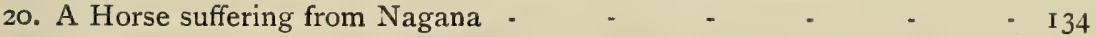

2I. Chart showing the Temperature and Number of Parasites in a Cow with

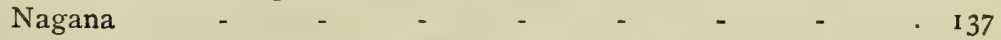

22. Multiplication of Trypanosoma brucei - _ _ - _ $\quad$ - $\quad$ - I53

*23. Details in the Division of Nucleus and Centrosome of Trypanosoma brucei I 55

24. Involution and Agglomeration Forms of Trypanosoma brucei - $\quad$ - 163

25. Temperature Charts of Two Horses suffering from the Gambian Trypanosomiasis - $\quad$ - $\quad$ - $\quad$ - $\quad$ - $\quad$ - $\quad$ - $\quad$ - 232

26. Temperature Chart of a Dog infected with Trypanosoma dimorphon - 237

27. Temperature Chart of a Goat infected with Trypanosoma dimorphon - 239

28. Trypanosoma dimorphon - $\quad-\quad$ - $\quad-\quad-\quad$ - $\quad$ : 242

29. Map showing the Distribution of Surra in India and Indo-China - 250

30, 3I. Temperature Charts of Horses suffering from Surra - $\quad$ - 254, 256

32. Temperature Chart of a Calf infected with Surra - - - - 260

33, 34. Temperature Charts of Two Dogs infected with Surra _ - 262, 263

35. Temperature Chart of a Sheep infected with Surra - - - 270

36. Trypanosomes of Surra, of Nagana, and of Caderas _ - - $\quad 273$

*37. Culture forms of the Philippine Surra Trypanosome - - - 275

38. The Giant Trypanosome of Lingard - - - _ - - $\quad$ - 286

39. Temperature Chart of a Horse with Caderas - - - - $\quad$ - 294

40. Temperature Chart of a Dog with Caderas - - - - $\quad 298$

* The figures marked with an asterisk have been added, and are not given in the original. 
FIG

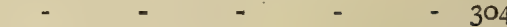

42. Photograph of a Horse with Dourine - - - - - $\quad$ - 315

43. Temperature Charts of a Horse and Donkey with Dourine - $\quad$ - 3I

44. Trypanosoma equiperdum - - $\quad$ - $\quad$ - $\quad$ - $\quad$ - $\quad$ - 333

45. Trypanosoma theileri and Trypanosoma transvaaliense - $\quad-\quad-347$

46. Hippobosca rufipes - $\quad$ - $\quad$ - $\quad$ - $\quad$ - $\quad$ - $\quad$ - $\quad 350$

47. Map of Equatorial Africa showing the Distribution of Human Trypano-

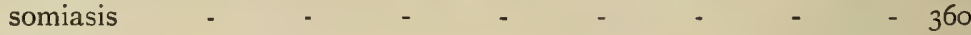

48. Temperature Chart of a Patient in the Early Stage of Human Trypano-

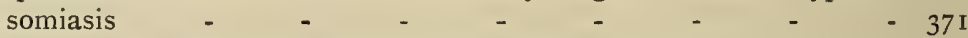

49. Temperature Chart of a Sleeping Sickness Patient - - - $\quad 373$

*50. Photograph of an Advanced Case of Sleeping Sickness in a Native of

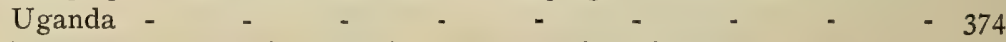

*51. Photograph of a Persian suffering from Sleeping Sickness $\quad-\quad$ - 375

52. Temperature Chart of a Dog inoculated with Trypanosoma gambiense - 386

* 53, 54, 55. Changes seen in the Brain in Sleeping Sickness - - 394, 395

*56. Section of a Vessel in a very Chronic Case of Sleeping Sickness, showing

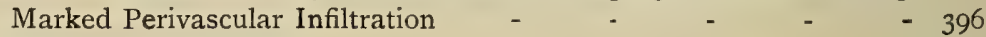

57. Trypanosoma gambiense - _ - $\quad$ - $\quad$ - $\quad$ - $\quad . \quad-402$

*58. Sexual and Indifferent Forms of Trypanosoma gambiense - - - 403

59. Trypanosomes of Birds (after Danilewsky) - $\quad$ - $\quad-\quad-\quad-443$

6o. Trypanosoma avium of the Owl - $\quad$ - $\quad$ - $\quad$ - $\quad$ - $\quad$ - 445

6r. Trypanosomes of Different Birds - _ - - _ $\quad$ - $\quad$ - $\quad$ - 446

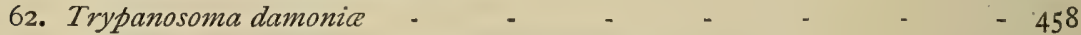

63. Trypanosoma rotatorium of Rana esculenta $\quad-\quad$ - $\quad$ - $\quad$ - 467

64. Trypanosoma mega and Trypanosoma karyozeukton - - $\quad$ - $\quad .468$

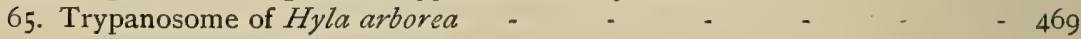

*66. Culture forms of Trypanosoma rotatorium - _ - _ - $\quad$ - $\quad$ - 472

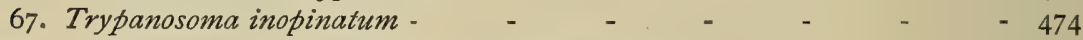

*68. Trypanosoma belli from a Frog in Hong-Kong .. . - . . - - 476

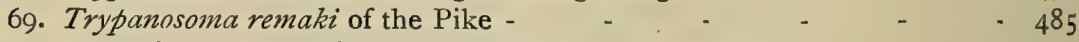

70. Stages in the Multiplication of Trypanosoma remaki - $\quad$ - $\quad$ - 487

71. Trypanosomes of the Carp and Tench - _ - _ $\quad-\quad-\quad 488$

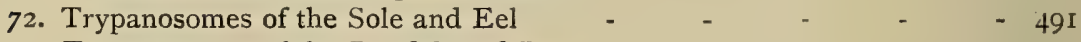

73. Trypanosomes of the Dogfish and Ray - _ - _ $\quad$ - $\quad-492$

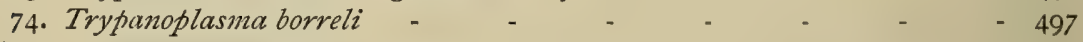

*75. Trypanosomes of the Tsetse-Fly $(T$. grayi $) \quad-\quad$ - $\quad-\quad$ - 509

*76. Trypanosomes of the Tsetse-Fly $(T$. tullochi $) \quad-\quad-\quad-\quad$ - 5 Io

77. Glossina morsitans - - - - - - - $\quad$ - $\quad$ - 512

78. Glossina morsitans, Before and After Feeding - _ _ _ _ - 513

79. Pupa of the Zululand Tsetse-Fly - - _ - - _ - $\quad$ - 515

8o. External Mouth Parts and Pharynx of Glossina morsitans - $\quad$ - 517

81. Glossina palpalis - - . - - - - - .

Coloured Plate at End of Volume. 


\section{AUTHORS' INTRODUCTION}

IN I892 one of us was able within the compass of a short article in a medical journal ${ }^{1}$ to give a résumé of our knowledge of the Trypanosomes. To-day it requires a whole volume to relate all that is known about these hæmatozoa and the diseases to which they give rise.

The subject of the Trypanosomes and the Trypanosomiases has grown very considerably during the past twelve years. In 1892 the existence of trypanosomes had been demonstrated in several of the lower animals, but the morphology of the parasites was little known, and only one pathogenic species had been described-namely, that which produces, especially in the Equidæ, the disease known as surra in India.

In recent years numerous investigators have devoted themselves to the study of the trypanosomes. Methods of staining, preserving, cultivating, and inoculating the parasites into a number of different animal species have made immense progress; questions of morphology have been elucidated; and, finally, successive discoveries of the greatest interest and importance have shown that trypanosomes play, in veterinary and in human pathology, a part the importance of which was hitherto unsuspected.

In addition to surra, it has been found necessary to include successively amongst the animal trypanosomiases the following diseases: nagana, or tsetse-fly disease; mal de caderas; dourine; galziekte (gall-sickness); a disease amongst horses in the Gambia Colony; and several other African trypanosome affections, of which the study is still incomplete. The importance of surra itself has increased, on account of serious epidemics of the disease in Mauritius, Java, and the Philippines outside its endemic area.

The discovery of a human trypanosomiasis in Africa-a trypanosomiasis to which must undoubtedly be attributed the symptoms known for more than a century under the name of sleeping sickness-has shown that trypanosomes must henceforth take their place amongst the pathogenic agencies, whose study is as indispensable to the physician as to the veterinary surgeon.

In I892 trypanosomes had little interest for practitioners outside certain regions of India where surra was prevalent. This is no

1 A. Laveran, Arch. méa. expérim., March I, I 892. 
longer the case. It can now be said that trypanosomiases are amongst the most widely distributed of diseases. The importance of the trypanosomiases cannot be too much impressed upon veterinary surgeons. All practitioners, as well as all veterinary surgeons, may be called upon to diagnose cases of trypanosomiases, even away from their endemic areas.

The extension of the colonial empires of European nations in Africa, the means of rapid transport, and the growing facilities for ocean travel, will undoubtedly favour the spread of human trypanosomiasis, of which a certain number of cases have already been observed among Europeans.

On the Congo and in Uganda the invincible progress of sleeping sickness has been evident for several years.

As to the animal trypanosomiases, the danger of their spread from the endemic areas is proved by a number of facts. This danger is increased by the development of commercial intercourse and the transportation of animals by sea, and also by the fact that the trypanosomiases of cattle and horses often run a slow and insidious course.

The serious outbreak of surra which has recently afflicted Mauritius is a notable instance of the ravages which certain trypanosomiases can cause, when prompt and energetic measures are not taken to prevent their spread.

In two years (1902-I903) the people of Mauritius lost nearly all their draught animals (horses and mules) and a large number of their cattle. The nature of the disease having been unrecognised at the outset, it is easy to see how the animals, scattered over the whole island, rapidly caused the spread of the infection. In Java more energetic prophylactic measures succeeded in limiting the extent of a similar outbreak. [Animal trypanosomiases also occur in the Philippines, and quite recently have been recognised in Hong Kong.] So far as the French possessions are concerned, these diseases have been already noted in Algeria, Tonkin, Cochin China, French Guinea, and the Sudan. Moreover, the fact cannot be ignored that Réunion and Madagascar are seriously threatened.

[Most of the British possessions in Africa, as well as India and Burmah, are similarly smitten by these animal trypanosomiasesparts of Cape Colony, Natal, Orange River Colony, and Transvaal; British Central Africa, Uganda, and the East African Protectorates; the River Gambia Colony, Sierra Leone, and Nigeria.]

For several years we have been studying the trypanosomes. It appeared to us that the time had come to collect and to condense the numerous publications on the subject, in order to lessen the labour of investigators, who must find it increasingly difficult to procure the monographs written in all languages, or the articles scattered throughout a large number of journals. Moreover, it is 
impossible to get these references in the small stations in our colonies, where the study of the human and animal trypanosomiases is so essential at the present day.

It might have been wiser perhaps to wait some years before writing this book. Our knowledge of the trypanosomes is still incomplete, and we are justified in believing that the rich harvest of discoveries concerning these hæmatozoa made during the last few years is not nearly completed. Science, especially natural and medical science, is always undergoing evolution, and one can never hope to have said the last word upon any branch of it. The only aim in writing a book such as this, is to gather together facts known at the present time about a special subject, and to facilitate the study of that subject by future investigators.

We have been particularly fortunate during our researches upon the trypanosomes. We have succeeded in getting together at the Pasteur Institute in Paris an almost complete collection of trypanosomes, and we have been able to study them, not only in stained preparations, but also in the living state, which has enabled us to follow the evolution of the trypanosomiases in a large number of species of lower animals, to compare the trypanosomes in them, and to differentiate several species which had hitherto been confused. The trypanosome of the rat, which is found almost everywhere, is an excellent one to study. We used it in our earliest researches upon the morphology and biology of trypanosomes. We have succeeded in procuring successively the trypanosomes of nagana, dourine, surra, mal de caderas, a disease amongst horses in the Gambia Colony, mbori, and human trypanosomiasis of the Gambia Colony and Uganda.

Further, we have been able to study the trypanosomes of birds, Batrachia, Chelonia, and fishes. We have described several new species of trypanosomes, notably amongst salt-water fishes, and we have found a trypanosome in a fresh-water fish-the red-eye-which has enabled us to establish the existence of a new genus, the genus Trypanoplasma.

The study of the trypanosomes, or at least of some of them, presents considerable difficulties. Several mammalian trypanosomes resemble one another so closely that it is impossible to distinguish them by their morphological characters alone. This applies to the trypanosomes of nagana, surra, dourine, and sleeping sickness. Moreover, trypanosomes belonging to distinct species may present close analogies from the point of view of their pathogenic action on certain animals. On the other hand, the virulence of a trypanosome is influenced by various factors. It varies specially with the origin and race of the animals inoculated. A trypanosome only slightly virulent for a species of animal from the Sudan, for example, may be very virulent for the corresponding European species. Also the same trypanosome may present slightly different 
morphological characters in the blood of different species of animals. It follows, therefore, that it may often be difficult to identify a trypanosome, and on that account several of these hæmatozoa still lack identification.

Some observers have maintained that the names surra and nagana referred to the same disease, while others have sought to identify mal de caderas with surra. On the other hand, it was believed for some time that the trypanosome found in the blood of man in Gambia was different from that found in Uganda in the cerebrospinal fluid of sleeping sickness patients. At the present day there is a little uncertainty about the nature of several African trypanosomes -notably that of the epizootic disease amongst dromedaries in Timbuctoo, which has been described under the name mbori. ${ }^{1}$

To identify a trypanosomiasis one must take into consideration: (I) the morphological and biological characters of the trypanosomes; (2) the symptoms of the disease as it occurs naturally; (3) the pathogenic action on different mammals; and (4) the action of the particular trypanosome upon animals immune against allied species of trypanosome. In this way we have succeeded in showing that nagana, surra, and mal de caderas are three distinct morbid entities; that the trypanosomiasis of horses in Gambia has nothing in common with the human disease; and, lastly, that the Trypanosoma gambiense and Trypanosoma ugandense should be regarded as identical.

Nocard and Lignières have shown in the same way that dourine is distinct from nagana and from mal de caderas.

In certain trypanosomiases and amongst certain species of animals the parasites are very scanty in the blood and body fluids, a fact which greatly increases the difficulties of investigation. In animals with dourine it is rare to find trypanosomes in the blood, whereas they are met with in large numbers in the odema fluids. In sleeping sickness it is often necessary, in order to demonstrate the presence of trypanosomes, to centrifuge the blood or the cerebrospinal fluid obtained by lumbar puncture.

The paucity of the parasites in the blood does not preclude their pathogenic action. In addition to dourine and human trypanosomiasis, animals may succumb to other trypanosomiases even when the parasites are very scanty in their blood. In this respect there are great differences amongst mammals. The trypanosomes of surra, nagana, and mal de caderas swarm in the blood of infected rats, mice, and dogs, whilst they are scanty, or very scanty, in the blood of infected rabbits, goats, sheep, and cattle. Although the trypanosomes appear to multiply with difficulty in these animals, the infection is none the less serious. Surra, nagana, and mal de caderas always cause death in rabbits.

${ }^{1}$ [It has recently been shown by Laveran that mbori is merely a variety of surra. See Chapter VI.] 
We know how the majority of the trypanosomiases are spread. Tsetse-flies convey nagana and human trypanosomiasis. Other biting flies convey surra and gall-sickness, whilst dourine is transmitted by coitus.

Prophylactic measures based on these ætiological data can render, and already have rendered, great service.

Our knowledge as to the treatment of trypanosome diseases is less advanced. It is especially to that aspect of the subject that future investigators should direct their efforts and attention.

Professors Nocard and Vallée have been good enough to communicate to us the results of experiments upon the different trypanosomiases carried out at the Veterinary College at Alfort, parallel with those which we carried out at the Pasteur Institute, and with the same parasites. These communications have been a great help to us. We should much have liked to express our sincere thanks to Nocard, but unfortunately we can only deplore the untimely death of this indefatigable worker-our devoted friend.

Professor Vallée is kindly continuing the valuable co-operation which we have been accustomed to find in the college at Alfort. We ask him to accept our best thanks.

PARIS, June I, I904. 



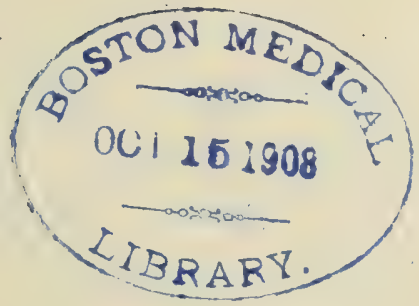

\title{
TRYPANOSOMES \\ AND THE TRYPANOSOMIASES
}

\author{
CHAPTER I
}

\section{HISTORICAL-GEOGRAPHICAI, DISTRIBUTION OF THE TRYPANOSOMIASES}

UNDER the general name Trypanosomes are included organisms belonging to the class Flagellata, of the phylum Protozoa, characterized by the possession of a fusiform body, more or less elongated; with, at its anterior extremity, a flagellum, which is continued along the body of the parasite as the thickened edge of an undulating membrane. In some cases there is a flagellum at both ends. All the known species of typical trypanosomes have their normal habitat in the blood of vertebrates.

We shall designate under the name of the Trypanosomiases ${ }^{1}$ those human and animal diseases produced by certain of these trypanosomes. Everyone agrees in ascribing to Valentin of Berne the discovery of the first trypanosome, observed by him in the blood of the trout (Salmo fario) in I84I. In I 842 and I843 appeared papers by Gluge of Brussels, Mayer of Bonn, and Gruby of Paris, upon the trypanosome of frogs. It was for these parasites of the frog that Gruby introduced the name Trypanosoma (from tpútavov, trupanon, a wimble or borer; and $\sigma \hat{\omega} \mu a$, soma, body).

Between I843 and I880 our knowledge of the trypanosomes made but little progress. They were rediscovered and studied anew at varying intervals in the blood of Batrachia (Wedl, I850 ; Chaussat, I850; Ray Lankester, I87I; Rättig, I875) and of various fishes (Remak, I842; Gros, I845; Berg, I845 ; Chaussat, I850). It is possible that Gros and Wedl had seen them in birds, but this is doubtful. Gros, in I845, found trypanosomes in the blood of the field-mouse and mole; Chaussat, in I 850 , found them in the black rat. But attention was only effectually drawn to the trypanosomes of mammals by the work of Lewis (I878) on the parasites of the

${ }^{1}$ [Brumpt has suggested the name 'trypanosomosis' for the disease due to a trypanosome, which agrees with the nomenclature of other protozoan diseasese.g., piroplasmosis, coccidiosis, etc.] 
blood of rats in India. The reason is that Lewis's discovery preceded by a short time that of the first truly pathogenic trypanosome.

It is a fact which at first sight may seem remarkable that the first pathogenic trypanosome was only discovered in I88o, forty years after Valentin's discovery. But one must remember that trypanosome diseases are essentially tropical in distribution, and that the study of them was taken up only during the last quarter of the last century. From I880, the date of the discovery by Evans ${ }^{1}$ of the trypanosome of surra amongst horses and camels in India, down to I894, the date of the discovery by Bruce of the trypanosome of nagana amongst horses and cattle in Zululand, apart from some experiments upon the artificial reproduction of surra, investigations were confined to the non-pathogenic trypanosomes.

Our knowledge of these different parasites was enriched by the researches of Lewis (I884), Crookshank (I886), Danilewsky and Chalachnikov (I888), upon the trypanosomes of rats; of Danilewsky (I888) on those of birds; of Danilewsky (I885), Chalachnikov, Mitrophanov (I883), on those of Batrachia and fishes. The most important amongst those researches are certainly those of Danilewsky, who studied at the same time the intracorpuscular hæmatozoa of reptiles, and especially those of birds, which are so closely allied to that other pathogenic Protozoon, the malarial parasite. But the knowledge of the trypanosomes acquired was limited to the different aspects of the parasite in the living state, and, in the case of some of them, to certain details concerning their mode of equal or unequal longitudinal division.

A résumé of the state of our knowledge towards the end of that period will be found in an article written by one of us in March, $1892,{ }^{2}$ with the object of bringing into prominence the interest of those researches upon the trypanosomes. In the writer's opinion, these researches ought to be undertaken side by side with those of the endoglobular hæmatozoa of the type of the hæmatozoon of malaria.

During the last twelve years our knowledge of the trypanosomes has increased by leaps and bounds. Two notable investigations should be mentioned as the starting-point of this forward movement:

First, the remarkable paper by Bruce upon nagana in $Z$ ululand, which appeared in extenso in 1897 , and proved conclusively the rôle played by a trypanosome in this disease, and at the same time cleared up the rtiology (the rôle of the tsetse-flies; the big game as reservoirs of the virus). Moreover, it was through Bruce that the first pathogenic trypanosome was introduced into the laboratories of Europe, where it has given rise to innumerable investigations which have materially increased our knowledge.

1 It is interesting to note that the publication of this discovery, made on December 3, I880, followed by about a week that of the malarial parasite (November 23, 1880).

${ }^{2}$ A. Laveran, Arch. méd. expér., March 1, 1892. 
Next should be mentioned the work of Rabinowitsch and Kempner, published in 1899 , on the trypanosome of rats. Here for the first time the cytological study of a trypanosome was made. These authors showed that satisfactory results are obtained only by employing those methods which had just been shown to succeed so well with the intracorpuscular hæmatozoa-namely, a suitably combined mixture of eosin and methylene blue. ${ }^{I}$. It is true Rabinowitsch and Kempner from their earlier work did not arrive at unassailable conclusions; and the researches carried out in Igoo, first by Wasielewski and Senn and later by ourselves, have introduced corrections into the morphological work of Rabinowitsch and Kempner. But to these investigators undoubtedly must be given the credit of having inaugurated the cytological study of the trypanosomes.

We believe we were the first to give a precise cytological account of the pathogenic trypanosomes, and to describe in detail their mode of longitudinal division into equal parts, with figures illustrating these changes. Our results have all been confirmed and extended by our own further researches and by the work of others on the pathogenic trypanosomes, as well as upon the non-pathogenic trypanosomes of the Batrachia, fishes, reptiles, and birds. During the progress of our researches we discovered in the blood of a freshwater fish - the rudd or red-eye-a trypanosome having, amongst other peculiarities, a flagellum at each extremity of its body. In Chapter III. we shall dwell more fully upon its morphological interest. We may add that closely-allied organisms have recently been found in the blood of the carp and minnow, ${ }^{2}$ and that they appear to be pathogenic for these fishes. Finally, in connection with the trypanosomes themselves, we have the interesting discovery made in 1903 by Novy and McNeal, who obtained pure cultures in a blood-agar medium, first of the rat trypanosome and later of the nagana parasite.

But it is especially in the discovery of the trypanosome diseases in man and the lower animals that the last decade was so prolific. We shall enumerate in the chronological order of the discovery of their causal agent all the trypanosomiases known at present, with a general survey of their geographical distribution (for the Animal Trypanosomiases, see Fig. I).

Surra, the trypanosome of which was discovered in I 880 by Evans, is very prevalent among the Equidæ and Camelidæ of the provinces of Northern India, Bombay, Burmah, Southern China, and various parts of French Indo-China. It has also been described in Sumatra, Java, and the Philippines. [Trypanosomiases identical with, or closely allied to, surra have been found to occur in various parts of Africa.]

\footnotetext{
1 Ziemann, since 1898 , had thus stained the two chromatic masses in the trypanosome of the frog.

2 [In Chapter XVII. will be found the names of other fishes in which similar parasites occur.]
} 
In India cattle are but slightly affected. On the other hand, during the past four years surra has caused a heavy mortality amongst the cattle in Central Java, and for three years amongst those in Mauritius.

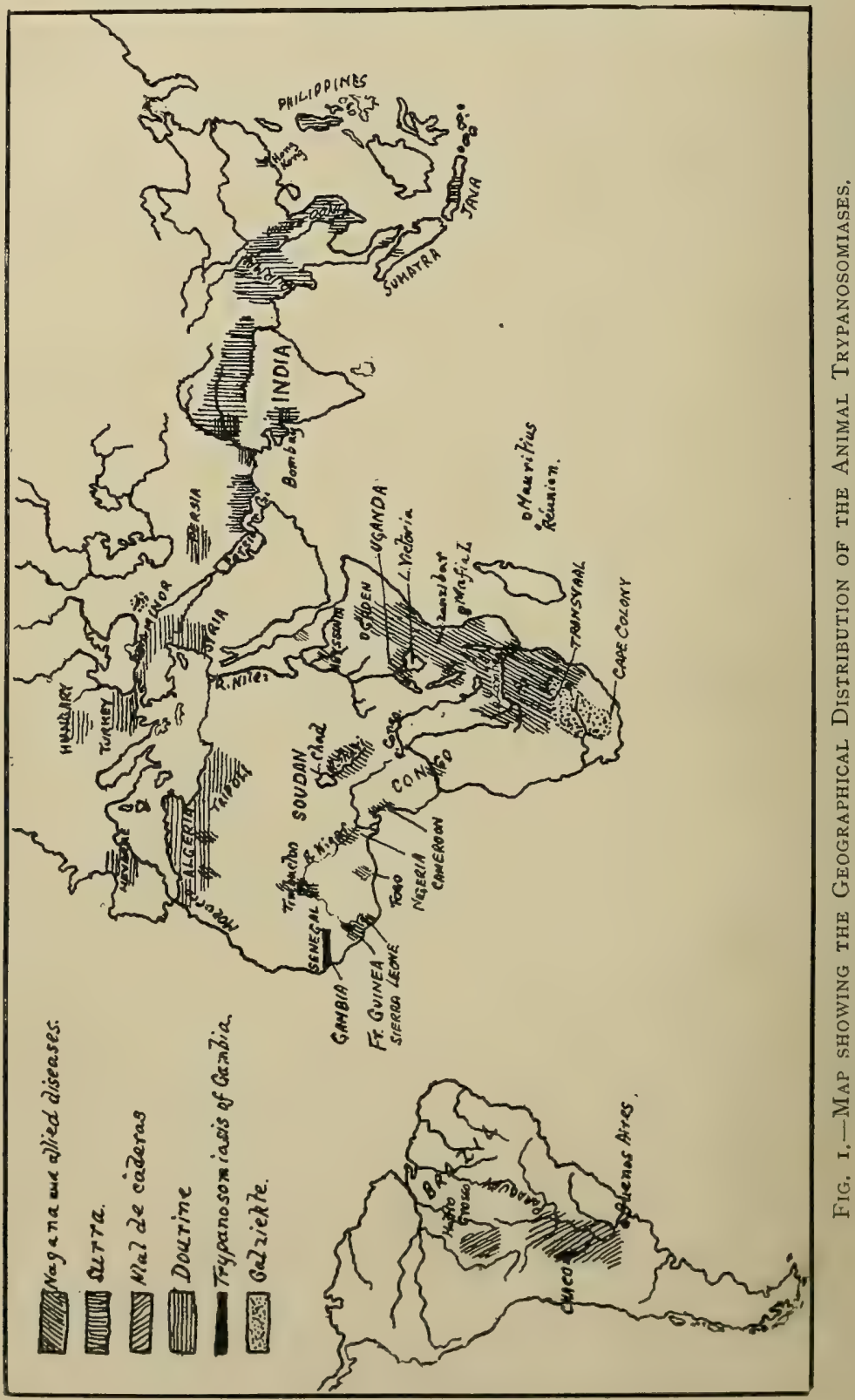

Nagana, or a disease closely resembling it, appears to be associated with the presence of one or more species of tsetse-fly. Since Bruce's discovery the trypanosome has been found in many parts of Africa 
where fly diseases are prevalent: in Northern Transvaal; amongst cattle in German East Africa; cattle and horses in British East Africa; dromedaries in Ogaden; cattle, sheep, and donkeys in the Belgian Congo territory; various mammals in the Cameroons; horses and cattle in Togoland; horses in Nigeria, French Guinea, and the valley of the River Shari ; and amongst the dromedaries and cattle in Timbuctoo.

Perhaps there are several distinct diseases in the areas just enumerated.

In the Gambia Colony there is also a trypanosome disease affecting horses, discovered in 1903 by Dutton and Todd, which is undoubtedly distinct from the nagana of $Z$ ululand.

In I894 Rouget found a trypanosome in the blood of a horse suffering from dourine in Constantine, in Algeria. This discovery was confirmed in I899 by Schneider and Buffard, who proved conclusively the causal relation of the trypanosome to the disease. This trypanosomiasis, known also by the name of mal du coüt (for so far as is known it is conveyed only by coitus), attacks only stallions and brood mares. It occurs along the African and Asiatic shores of the Mediterranean, in Persia, and in Turkey; $;^{1}$ cases are still occasionally met with in Hungary and the North of Spain. It occurs also in the United States, in the region of the Illinois, and possibly, too, in Java.

In Algeria dourine is apparently not the only trypanosome disease which attacks animals. Quite recently trypanosomiases amongst horses and dromedaries have been described, differing from dourine both in their mode of propagation, which is not by coitus, and by the characters of their respective trypanosomes.

In I90 I Elmassian, director of the Bacteriological Institute at Asuncion, Paraguay, discovered a trypanosome in the blood of horses with mal de caderas (disease of the hind-quarters). This disease is prevalent over the whole of the Gran Chaco, the hunting and cattle-rearing district, and over the adjacent parts of the Argentine, Uruguay, Paraguay, and Bolivia. Mal de caderas has also been recognised further north, in the Brazilian province of Matto-Grosso.

In 1902 Theiler discovered in the Transvaal a disease peculiar to cattle-galziekte, or gall-sickness, due to a trypanosome which Bruce and Laveran have described, at the same time drawing attention to its unusually large size. [This disease has since been described in other parts of Africa and in Transcaucasia.]

Until 1982 it was thought that the trypanosome diseases were confined to the lower animals, and that man was immune. In support of this hypothesis, nagana and other allied fly diseases of horses and cattle were frequently cited as having never attacked man, although he had often been bitten by the fly.

${ }^{1}$ [Dourine occurs also in India, where it has recently been studied by Lingard and others. (See Chapter X.)] 
The researches of the past five years have clearly shown the part played by trypanosomes in human pathology. Towards the end of I90I Dutton discovered a new trypanosome in the blood of a European resident in the Gambia Colony who was suffering from irregular pyrexia and enlarged spleen. ${ }^{1} \quad$ This discovery of a trypanosome pathogenic for man was soon confirmed in the Gambia Colony by Dutton and Todd, and elsewhere (Congo, Uganda) by others.

In May, Ig03 was published the important discovery by Castellani in Uganda of a trypanosome in the cerebro-spinal fluid of negroes suffering from sleeping sickness. This discovery was speedily confirmed by Bruce and Nabarro, who clearly demonstrated the intimate association between this trypanosome and sleeping sickness. It has been recognised, moreover, that this trypanosome is identical with that discovered in Igor by Dutton [and Forde]. Sleeping sickness is merely the final stage of a blood infection with trypanosomes.

This human trypanosome is conveyed by a particular species of tsetse-fly. Human trypanosomiasis has the same geographical distribution as sleeping sickness-the valleys of West Africa from the Senegal down to St. Paul de Loanda, and the northern shores of the Victoria Nyanza. (See special map, Chapter XII.)

This rapid résumé shows clearly the importance of the trypanosome diseases. Their elucidation is, therefore, one of the important problems of colonial expansion. The experimental study of the various trypanosomiases has already led to very important results, which will be found in detail in this book.

Unfortunately, we have progressed very little so far as prevention and treatment are concerned. It is not possible to inoculate against these diseases. Graduated treatment with arsenic leads to an improvement in the condition of animals or human beings suffering from trypanosomiasis, but a cure has never been effected. 'Trypanrot' of Ehrlich and Shiga has hitherto cured only mice inoculated with the trypanosome of mal de caderas. Serum-therapy has furnished a result interesting from a theoretical point of view, but not of practical value-namely, the action of human serum upon the animal trypanosomes.

[During the past few years some progress has been made in the preventive inoculation of animals, and the recent investigations of Laveran upon the action of arsenic and trypanred combined, of Mesnil and Nicolle and of Thomas upon the action of various dyes and of an arsenic compound (atoxyl), mark a distinct advance in the prevention and treatment of the trypanosomiases. (See Chapter XIII. on ' Treatment.').]

1 [It would seem that Forde first discorered the parasite in blood-films, but that Dutton recognised its character. (See chapter on Human Trypanosomiasis.)] 


\section{TECHNIQUE FOR THE STUDY OF THE TRYPANOSOMES}

\section{Section 1.-Examination in the Living State.}

To study the trypanosomes in fresh blood, a drop of blood from an infected animal is necessary. In the case of a mammal this is obtained from a prick or small incision in the ear, or, in the case of rats and mice, from the end of the tail. In birds prick one of the big veins on the internal surface of the wing; in the case of reptiles take the blood from the extremity of the tail or from a digit; in Batrachia cut a digit of one of the limbs, preferably the thumb of an anterior limb; finally, in fishes cut one or two rays of the caudal fin, or make a small cut in the gills.

The drop of blood is taken on a slide and covered with a coverslip. To study the structure of the parasites the blood should be in a thin layer, otherwise the trypanosomes may be entirely hidden by the red corpuscles. The movement which the trypanosomes impart to the red corpuscles enables one to recognise the presence of the parasites with very slight magnification (I0o diameters) when they are numerous in the blood, and it facilitates finding them, with greater magnification (300 or 400 diameters), when they are scanty. In the latter case, if one wishes merely to ascertain the presence of the parasite in the blood, it is better to take a relatively thick film of blood, so that the corpuscles form an apparently uniform layer by being pressed one against the other. With a little practice the presence of trypanosomes can be recognised by the rotary movements imparted to the adjacent corpuscles. In this way one has the advantage of examining a relatively big drop of blood.

In doubtful cases, especially if a microbiological diagnosis of a trypanosome is required, it may often be necessary to examine successively several blood preparations, and even to repeat the observation several days afterwards, so long as the examination is negative (e.g., in the case of ruminants suffering from nagana or surra; men and various mammals infected with the human trypanosome).

To facilitate the finding of trypanosomes in the blood, Kanthack, Durham, and Blandford advised centrifuging the blood rendered 
uncoagulable (as by the addition of sodium citrate solution). The trypanosomes are found in the middle or leucocytic layer. ${ }^{1}$

In the case of dourine, we shall see that it is the blood-stained œdema fluid which should be examined. In sleeping sickness the blood and the cerebro-spinal fluid obtained by lumbar puncture should be examined for trypanosomes after centrifuging.

When it is desired to watch the parasites for a considerable time, it is necessary to make hanging-drop preparations ringed with vaseline or paraffin. Such preparations are very useful, especially for studying the phenomena of agglutination. The blood is diluted with physiological salt solution and then defibrinated, or with citrated salt solution to prevent coagulation, or with serum from another animal. Francis, ${ }^{2}$ for Trypanosoma lewisi, advises letting the blood coagulate: the trypanosomes pass out into the serum, where they can be studied apart from the red corpuscles. Generally at the end of an hour in an ordinary slide and coverslip preparation, or a little longer in the case of a hanging drop, the trypanosomes become less active, and one is then able to study their shape and the movements of their various parts more in detail.

In order to reduce or arrest this movement of the trypanosomes, Plimmer and Bradford ${ }^{3}$ recommend adding a drop of a I per cent. solution of gelatine [or of a weak solution of cherry-gum] to the blood.

\section{Section 2.-Staining Methods.}

In the fresh state the general form of trypanosomes, their movements, and the action of physical and chemical agents upon them, can be studied. In order to obtain, however, a clear idea of their intimate structure, it is essential to study preparations stained by a special method-namely, a mixture of eosin and methylene blue in definite proportions. This mixture was first used by Romanowsky for staining hæmatozoa (the malarial parasite). It was applied to the staining of trypanosomes in 1898 by $Z$ iemann, ${ }^{4}$ who thus stained the nucleus and centrosome in the trypanosome of the frog. The following year Rabinowitsch and Kempner ${ }^{5}$ stained $T$. lewerisi by this method; but the most beautiful results were obtained in 1900 by Wasielewski and Senn, ${ }^{6}$ also with T. lewisi. They used Nocht's modification of Romanowsky's method.

The following method ${ }^{7}$ has given us excellent results:

1 We found this method useful in collecting together above the layer of red corpuscles the trypanosomes from a rat or dog with nagana when the parasites were more numerous than the red corpuscles.

${ }^{2}$ Francis, Bull. No. II, Hyg. Laby, U.S. Pub. Health and Mar. Hosp. Serv. Washington, 1903 .

3 Plimmer and Bradford, Centralbl. f. Bakter, I, v. 26, I899, p. 440 ; [also Quart. Journ. Micr. Sc., v. 45, I90 I, p. 45i.]

${ }^{4}$ Ziemann, Centralbl. f. Bukter., I, v. 24, 1898.

5 Rabinowitsch and Kempner, Zeitschr. f. Hyg., v. 30, I899, p. 25 I.

6 Wasielewski and Senn, Zeitschr. f. Hyg., v. 33, 1900, p. 444.

7 A. Laveran, Compt. Rend. Soc. Biol., June 9, 1900. 
The blood is spread in a thin layer on a glass slide with the edge of a visiting-card [or of another microscope slide], dried very rapidly, and fixed in absolute alcohol for five to ten minutes. The following solutions should be prepared beforehand:

I. Methylene blue with silver oxide or Borrel blue. Take 50 or fo c.c. distilled water in a flask of about I5O c.c. capacity, and add some crystals of silver nitrate. When these are dissolved, fill the flask with a concentrated solution of caustic soda and shake. A precipitate of silver oxide is formed. This is washed several times with distilled water, so as to get rid of any silver nitrate or excess of soda. A saturated watery solution of methylene blue (' medicinal methylene blue,' Hoechst) is then poured on to the silver oxide and allowed to remain for a fortnight, the flask being shaken from time to time.

2. Watery solution of eosin, I per I,0oo ('water-soluble' eosin, Hoechst).

3. Solution of tannin, 5 per cent., or, better, a solution of tanninorange, which can be obtained ready made up.

The staining mixture is prepared at the time of using, according to the following formula :

$\begin{array}{lccccc}\text { Solution of eosin }(\mathrm{I} \text { per } \mathrm{I}, \mathrm{OOO}) & \ldots & \ldots & 4 \text { c.c. } \\ \text { Borrel blue } \ldots & \ldots & \ldots & \ldots & \ldots & \text { I c.c. } \\ \text { Distilled water } & \ldots & \ldots & \ldots & \ldots & 6 \text { c.c. }\end{array}$

The mixture is at once poured into a flat dish, such as a Petri dish, or, better, a square or oblong dish with sloping bottom specially made for the purpose. ${ }^{1}$ The slide on which the blood-film has been spread and fixed is placed film downwards in the staining solution (a piece of glass rod or a projection from the bottom of the dish preventing the slide from touching the bottom, where a precipitate nearly always forms), and left there for five to twenty minutes. Five to ten minutes' staining is long enough for most trypanosomes, especially for the mammalian parasites; twenty minutes' staining is necessary for certain species-e.g., T. lewisi, particularly when undergoing reproduction. After removal from the staining solution the slide is well washed in a large quantity of water [or by blowing a strong stream of water on the film with a wash-bottle], then treated with the solution of tannin for several minutes, again washed in excess of water, and finally in distilled water and dried. If a precipitate is formed, which would render microscopical examination difficult, wash the film with oil of cloves, then with xylol, and gently rub the surface of the preparation with a piece of soft linen soaked in xylol.

${ }^{1}$ [Such dishes, made of vulcanite, each to take one slide $3^{\prime \prime} \times \mathrm{I}^{\prime \prime}$, have been made at the suggestion of Dr. A. C. Stevenson by Messrs. Townson and Mercer, of 89 , Bishopsgate Street, E.C. We have found these dishes extremely useful, especially for the prolonged staining of parasites, such as the trypanosomes and the Treponema pallidum (Spirochata pallida) of syphilis.] 
The preparations keep better uncovered than if covered with balsam or cedar-wood oil, in which case they rapidly lose their stain. When the staining has been successful, the protoplasm of the parasite is pale blue; the nucleus, flagellum, and edge of the undulating membrane are purplish; the centrosome is deep violeta little different from the nucleus; the undulating membrane is unstained, or stained a very pale blue (see coloured plate). The red corpuscles are pink, the nuclei of the leucocytes dark purple.

Equally good results are obtained with a mixture of azure blue II and eosin. This mixture has been strongly recommended by Giemsa, ${ }^{1}$ and Grübler supplies the blue prepared according to Giemsa's directions. We have modified the method as follows : ${ }^{2}$ Dry and fix the film in absolute alcohol, then stain for ten minutes in-

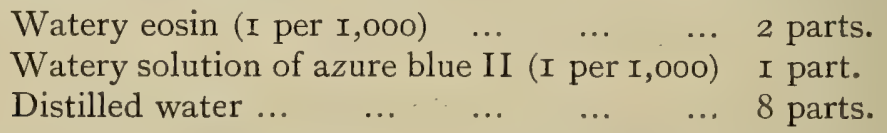

Wash in water; treat with 5 per cent. tannin for two to three minutes; wash again and dry. By this method we have obtained good specimens of the trypanosomes of nagana and caderas.

Finally, we have used the powders prepared by Jenner, Leishman, J. H. Wright, etc., by mixing together solutions of eosin and methylene blue, collecting the precipitate formed, and, after washing thoroughly in distilled water, drying and pulverizing. The powder is dissolved in absolute methyl alcohol, and is then ready for use. In staining blood-films by this method, preliminary fixation of the film is unnecessary.

[Leishman's stain answers admirably for trypanosomes, as well as for the malarial parasite, piroplasms, the 'Leishman body' of kala-azar and tropical splenomegaly (see Chapter III.), and other chromatin-containing parasites. It is best used as follows: Having obtained a blood-film or a smear of an organ or tissue in the ordinary way, draw two lines with a wax pencil (preferably blue, as with the other colours the wax tends to float off) across the whole width of the slide, one on either side of the bloodsmear. This prevents the stain from running all over the slide, so that

1 Giemsa, Centralbl.f. Bakter., I, Orig., v. 37, Iço2, p. 308.

[Giemsa's solution is prepared as follows: Take azure II-eosin, 3 grammes, and azure II, 0.8 gramme ; dry and powder. Dissolve at $60^{\circ} \mathrm{C}$. in 250 grammes chemically-pure glycerine, shaking the mixture to hasten solution. Add 250 grammes methyl alcohol, previously warmed to $60^{\circ} \mathrm{C}$.; shake, leave standing for twenty-four hours at room temperature, and filter.

To use, fix air-dried films in ethyl alcohol, or, more quickly (two to three minutes), in methyl alcohol; dry with blotting-paper. Dilute the stain with water in a wide graduated vessel, shaking the mixture. Use I drop of stain (dropped from a drop-bottle) to about I c.c. distilled water, preferably warmed to $30^{\circ}$ or $40^{\circ} \mathrm{C}$. When properly mixed, pour the freshly diluted stain on to the film, and leave on for ten to fifteen minutes (or the staining may be done in the small dishes mentioned on p. 9). Then wash in a stream of water, blot, dry, and mount in balsam. Giemsa states that it is not advisable to use his solution in the way that Leishman's stain is used.

If an alkaline solution of the stain is required, add to Io c.c. distilled water (before diluting the stain) I or 2 drops of a $\mathrm{I}$ per cent. solution of potassium carbonate.]

${ }^{2}$ A. Laveran, C. R. Soc. Biol., March 7, I903, p. 304. 
less stain is necessary, and a cleaner preparation is ultimately obtained. Without previous fixation, run a few drops of stain on to the film from a fine pipette ( 5 to Io drops, according to the size of the film), and after thirty seconds run on double the number of drops of distilled water-again from a pipette--taking care that the water does not overstep the wax boundary lines. Tilt the slide carefully to mix the stain and the water, and allow the diluted stain to act for five to fifteen minutes. The stain is then poured away, the film is washed for ten or fifteen seconds in distilled water in a beaker, or blown on from a wash-bottle, and allowed to dry in the air. The slide may be preserved uncovered, or it may be covered in the ordinary way with a coverslip and xylol balsam, the latter, however, causing the stain to fade after a time.]

[Marino's ${ }^{1}$ stain also gives very good results with trypanosomes. The alcoholic solution is obtained by dissolving in pure methyl alcohol the precipitate formed by adding an aqueous solution of eosin to an alkaline solution of methylene blue and azure blue in certain proportions. ${ }^{2}$ Four to 8 or ro drops of the alcoholic stain are run on to the film (the number of drops varying with the size of the film), and left on for three minutes. Then double the number of drops of an aqueous solution of eosin (0.05 gramme to I,000 c.c. water) are added, and left on for two minutes, after which wash, dry, and mount. Some trypanosomes, such as those of birds and fishes, require rather longer to stain well-four to ten minutes with the blue, and eight to twenty minutes with the eosin.]

If it be desired to stain a film rapidly, or if these special stains be not at hand, an alcoholic solution of fuchsin, or, better, a watery solution of magenta, or, better still, a solution of carbol thionin, may be used. Staining takes place rapidly, often in less than a minute. The nucleus, flagellum, and centrosome are stained more deeply than the protoplasm, and in this way sufficiently good results can be obtained when one wishes merely to ascertain the presence of the trypanosomes and their relative numbers.

By Heidenhain's method (hæmatoxylin and iron alum) the nucleus, centrosome, and flagellum are stained darker than the protoplasm, but of course are less distinctive than when stained by our method or that of Romanowsky [or Leishman, Giemsa, etc.], in which they assume a tint different from that of the protoplasm.

The methods of fixation and staining just described for blood will answer for other liquids which may contain trypanosomes, such as the blood-stained œdema fluids, cerebro-spinal fluid, culture media, anæmic blood, or blood diluted with salt solution or sodium citrate. In these cases, however, fixation is never so perfect as with pure blood; often the trypanosomes in these liquids appear vacuo-

i [Marino, Ann. Inst. Pasteur, v. 18, 1904, pp. 76I-766.]

2 [The directions for preparing the stain are as follows: Take an aqueous solution of methylene blue and azure blue (methylene blue, 0.5 gramme; azure blue, 0.5 gramme; water, I0o c.c.) and mix with an aqueous solution of sodium carbonate ( 0.5 per cent.). Keep the mixture in the $37^{\circ} \mathrm{C}$. incubator for one to two days, or, better, 'in the thermostat at a higher temperature ; then add an aqueous solution of eosin to the blue mixture. The best strength of eosin solution varies with the quality of the blue, and must be ascertained by actually trying the effect of different strengths $\left(0^{*} \mathrm{I}\right.$ to $0^{\circ} 3$ per cent.). The eosin-blue mixture is then filtered, and the precipitate obtained is soluble in water and in methyl alcohol. The alcoholic solution mentioned in the text contains 0.04 gramme of the dried precipitate dis. solved in 20 c.c. pure methyl alcohol.] 
lated. This is particularly the case with the trypanosomes in the cerebro-spinal fluid of sleeping sickness patients, so much so that at first we looked upon this as a characteristic feature of the sleeping sickness trypanosome. The examination of the same trypanosome in the blood, however, soon showed that such was not the case.

When the trypanosomes are very scanty in the blood, it is best to make thick smears, from which the hæmoglobin is subsequently dissolved out. ${ }^{1}$ In Ross's method ${ }^{2}$ the thick blood-film is dried and stained by one of the methods mentioned above without previous fixation. The watery solutions of the stains dissolve out the hæmoglobin, at the same time staining the leucocytes and the parasites. This method is specially to be recommended for mammalian blood, which often contains very few parasites. By this method, however, the blood-film is often washed away, and, moreover, the trypanosomes are very deformed and often difficult to recognise.

Ruge $^{3}$ has advised fixing the blood before staining by means of a 2 per cent. solution of formalin, to which is added acetic acid to the extent of $0^{\circ} 5$ to I per cent. Fixing by this method does not prevent the subsequent solution of the hæmoglobin.

The following method has yielded good results in our hands: fixing in absolute alcohol, then dissolving out the hæmoglobin with I per cent. acetic acid.

[Bradford and Plimmer ${ }^{4}$ got the best results by fixing with osmic and acetic acid vapours (osmic acid, 2 per cent., and glacial acetic acid, equal parts), and staining with a mixture of methylene blue and erythrosin.

In the case of cerebro-spinal fluid, lymphatic gland juice, and intestinal contents of flies containing trypanosomes, it is often difficult to stain the chromatin. Gray and Tulloch ${ }^{5}$ overcame this difficulty by fixing the films while still wet in osmic acid vapour, then adding fresh blood-serum, as recommended by Leishman for sections (see below). After washing off the serum, the films were stained as usual by Leishman's stain.]

[Staining Trypanosomes in Sections.-For staining trypanosomes and other chromatin-containing parasites in sections, Leishman ${ }^{6}$ recommends the following method: Paraffin sections (stuck on slides or coverslips), after treating with xylol and spirit, are washed in water and carefully blotted ; fresh blood-serum is added and allowed to remain on for five minutes; the excess is then removed by blotting and the rest allowed to dry on. The stain is mixed-2 parts 'Leishman' to 3 of distilled water-and poured on the section, which is then covered and left for one to one and a half hours. It is advisable to put on $f_{i}$ esh stain once or twice during that time. The stain is poured off and the section washed in distilled water. With a low power, the cell nuclei should be almost black. Decolourize and differentiate, using first a I in I, 500 solution of acetic acid, then a $I$ in 7,000 solution of caustic soda. The acid removes excess of blue, the alkali excess of eosin, and the solutions are used alternately until

\footnotetext{
1 The method, which consists in dissolving out the hæmoglobin with water or water acidulated with acetic acid, in order to show up any parasites which may be in the blood, is an old one. It has been recently reintroduced by Ronald Ross.

2 Ronald Ross, Lancet, January Io, 1903, p. 86.

3 Rugre, Diutsche med. Wochensitir., March I9, 1903, p. 205.

* [Bradford and P'limmer, Quart. Journ. Micr. Sc., v. 45, pp. 451, 452.]

"[Gray and Tulloch, Sleeping Sickness Comm. Reports, No. 6, p. 286.]

6 [Leishman, Journ. Hyg., v. 4, 1904, pp. 434-436.]
} 
the desired contrast stain is got, as seen under a low power. When the cell nuclei of the tissue are a deep Romanowsky-red colour, and the rest of the tissue a very pale pink or light blue, wash well in distilled water, drain and blot off excess of water, dehydrate rapidly with absolute alcohol, clear with xylol, and mount in balsam.]

[Giemsa's stain used as in Leishman's method, safranin and indigo carmine (Neporojny and Jakimoff), and polychrome methylene blue (Marchand and Ledingham, Mott, Halberstaedter), also stain the trypanosomes well in sections.]

[Halberstaedter ${ }^{1}$ gives the following details of the method used by him: Small pieces of tissue are fixed immediately after death in sublimateacetic mixture (concentrated watery solution $\mathrm{HgCl}_{2}$, I 50 parts; distilled water, I50 parts; glacial acetic acid, 4 parts) for twelve to twenty-four hours; then washed in running water for twenty-four hours, and, after passing through alcohol, etc., embedded in paraffin. Stain thin sections for ten minutes in polychrome methylene blue, wash in water, treat for a few seconds in absolute alcohol (not too long), clear with xylol, and mount in balsam.]

\section{Section 3.--Preservation in vitro and Cultivation of Trypanosomes.}

Until quite recently no method of cultivating trypanosomes was known, but they could be kept alive in vitro for a variable time, either in hanging-drop or in sterile tubes. The essential condition is to obtain the blood aseptically from the heart or carotid artery in the case of small animals, from the jugular vein in larger animals. It is at once mixed with physiological saline, and defibrinated in sterile flasks containing glass beads, or, better still, it may be mixed with citrated salt solution $(\mathrm{NaCl} 5$ grammes, sodium citrate 5 grammes, water I,000 c.c.) to prevent coagulation.

Even under these conditions most parasites can be kept alive only a few days, a notable exception being $T$. leweisi, which will live for a considerable time if kept in the ice-chest. The addition of different serums keeps the trypanosomes alive a little longer.

$\mathrm{McNeal}$ and $\mathrm{Novy}^{2}$ in 1903 succeeded in cultivating trypanosomes-first T. lewisi, and later T. brucei. We have also been able by using McNeal and Novy's method to cultivate $T$. lewisi and T. evansi. ${ }^{3}$

The culture medium employed is comparatively simple. It consists of nutrient agar containing I to 3 per cent. of peptone, to which defibrinated blood is added. From certain observations of $\mathrm{McNeal}$ and Novy it appears that the hæmoglobin plays an important part in this medium, for if the hæmoglobin undergo any change, the medium is useless and growth in it will cease.

Agar prepared in the ordinary way is cooled down to $59^{\circ} \mathrm{C}$,

1 [Halberstaedter, Centralbl.f. Bakter., v. 38, I905, pp. 525-532.]

$2 \mathrm{McNeal}$ and Novy, 'Contributions to Medical Research,' dedicated to V. C. Vaughan, June, I903; Journ. Infec. Dis., v. I, January 2, 1904.

3 [Recently Novy and McNeal and Thiroux have succeeded in cultivating bird trypanosomes, Thiroux that of the mouse, Petri that of the rabbit, and Bouet that of the frog.] 
and two or three times ${ }^{1}$ its volume of defibrinated blood taken aseptically from a rat, guinea-pig, or, more commonly, a rabbit, is added to it. The culture tubes are sloped in the usual way, and the medium allowed to solidify. As soon as this has occurred, the tubes are placed upright, so as to ensure the rapid formation of a large quantity of water of condensation, which collects at the bottom of the tubes. This water of condensation is inoculated with a loopful of blood from an infected animal or of a previous culture.

As the culture tubes are often kept for months, precautions are necessary to prevent the evaporation of this water of condensation. If the cultures are made at $20^{\circ}$ to $25^{\circ} \mathrm{C} ., \mathrm{McNeal}$ and Novy at first recommended sealing the tubes with wax; now they use thick rubber caps for the tubes, which we have also found to answer well. If incubated at $34^{\circ}$ to $37^{\circ} \mathrm{C}$., McNeal and Novy put the tubes in a large desiccator or apparatus such as is used for anaerobic cultures containing some cotton-wool well soaked in perchloride of mercury solution.

In the water of condensation in blood-agar tubes, the trypanosomes grow either at the room temperature or in the incubator at $34^{\circ}$ to $37^{\circ} \mathrm{C}$. In this way pure cultures can be obtained. Bacterial contamination usually causes rapid death of the trypanosomes; nevertheless we have succeeded in keeping $T$. leroisi alive and virulent for more than a fortnight in a tube in which many organisms had developed. [Novy and Knapp? also found that exceptionally some bacteria are not unfavourable to trypanosomes, and that mixed cultures may be kept alive for six months or more.]

[For the isolation of trypanosomes from accompanying bacteria they recommend the following method: With a small glass spatula take a little of the mixed growth, and spread it in a series of streaks on solidified blood-agar in six Petri dishes, using dishes which can be effectually sealed by means of a wide rubber band. At the end of ten to twelve days at room temperature the last plate or two of the series will show isolated colonies of the trypanosomes, which can then be subcultivated in the ordinary way in test-tubes.]

[Mathis ${ }^{3}$ has recently improved upon Novy and McNeal's original medium by heating it to $75^{\circ}$ to $100^{\circ} \mathrm{C}$. for half to one and a half hours one or more times. The advantages claimed for this modification of the original medium are-(I) that it sterilizes the medium, which therefore need not be prepared so carefully; (2) that it is fixed or more uniform in composition, and can therefore be prepared long in advance; and (3) that it alters the blood of various animals, such as the goose, rabbit, dog, and goat, so that they are all equally serviceable.

1 T. lewisi, according to McNeal and Novy, grows in media containing $\mathrm{I}$ part of blood to 5 or even Io parts of agar; but it prefers media rich in bliod, the optimum being apparently 2 parts of blood to 1 of agar. T. brucei will only grow in media containing at least as much blood as agar, the optimum mixture contain. ing 2 to 3 parts of blood to $x$ of agar. Even under these conditions cultures are difficult to obtain. (See special chapter.)

2 [Novy and Knapp, Juzurn. Hyg., v. 6, 1906, p. III.]

3 [C. Mathis, C. R. Soc. Biol., v. 6r, 1906, pp. 550-552.] 
This modified medium was found most useful for the nonpathogenic trypanosomes, less so for the pathogenic. Thus T. rotatorium in two days showed numerous trypanosomes, and in seven days they were very abundant. T. lewisi in two days showed fairly numerous characteristic rosettes; in one month the culture was very rich in trypanosomes, and intraperitoneal injection infected rats in less than three days.]

\section{Section 4.-Inoculation Experiments.}

Nothing very special need be said upon this point. Blood (pure, defibrinated, or citrated), blood-stained œdema fluid, or cerebrospinal fluid containing trypanosomes, may be injected with an ordinary hypodermic syringe or pipette. In the case of the pathogenic trypanosomes the inoculation may be made subcutaneously (or even to the freshly injured surface of an abrasion), intraperitoneally, or intravenously, with hardly any difference as to the final result. The mode of inoculation merely affects the period of incubation, parasites appearing in the blood sooner after an intraperitoneal than after a subcutaneous injection.

In the case of the non-pathogenic trypanosomes ( $T$. lewisi, trypanosomes of fishes), intraperitoneal inoculations appear to be the most reliable.

Certain trypanosomes (T. equiperdum of dourine) can apparently be inoculated upon healthy non-excoriated (?) mucous surfaces.

Inoculation through the mouth or stomach does not occur with any trypanosome, except when the buccal mucous membrane presents recent abrasions.

Trypanosomes appear to be virulent in the minutest doses, a single trypanosome sufficing to bring about an infection. It is merely a question of the duration of the incubation period. For example, in the case of nagana, the incubation period may be less than twenty-four hours after the intraperitoneal inoculation of many parasites; on the other hand, it may be as long as ten days when few are present in the material injected. The difference is still more marked in the case of other trypanosomes.

Before inoculation it is as well to make sure, by microscopic examination, of the condition of the trypanosomes to be injected. The incubation period and, in the case of trypanosomes that have been kept, the success of the inoculation depend on the condition of the parasites injected.

Finally, the inoculation of a large quantity (up to ro c.c.) of blood or other fluid is always indicated when the trypanosomes have not been visible on microscopic examination (as in the case of ruminants and pigs infected with or suspected of nagana, and horses suspected of dourine). The inoculation should be made into a susceptible animal-rat, mouse, or dog in the case of nagana; dog in the case of dourine. If rats or mice are used, several must be inoculated. 


\section{CHAPTER III}

\section{COMPARATIVE STUDY OF THE TRYPANOSOMES}

\section{Section 1.-General and Comparative Morphology.}

WE shall begin by giving a general account of the structure of trypanosomes, such as appears to us to embody the results of recent observations. Figures 2 and 3 show the principal variations in the form of trypanosomes, and at the same time bring out clearly the features by which these parasites may be recognised, at least in the form we shall call adult. There is a spindle-shaped, more or less elongated, protoplasmic body containing two chromatic masses-one small mass, which we call the centrosome, generally placed at the posterior end; the other, nearly always mesially situated, which is larger and is called the nucleus. A folded membrane, which is known as the undulating membrane, starts from the centrosome, and runs along the protoplasmic body. Its thickened border is generally prolonged anteriorly into a free part, constituting the flagellum.

In the case of certain parasites found in the blood of fishes, for which we have created a special genus, Trypanoplasma, several peculiarities are to be noted: first, the undulating membrane, which extends from one end of the body to the other, is prolonged posteriorly into a free flagellum, while anteriorly it bends round and reaches the end of a large elongated mass of chromatin, from which the anterior flagellum also starts; secondly, the protoplasmic body does not contain a large and a small chromatic mass, but two masses of almost the same size and about as large as the nucleus of the true trypanosomes.

We shall now study more in detail the various parts of the trypanosomes (using this term in its wider sense), pointing out at the same time the variations found in different members of the group.

The body proper is, as a rule, spindle-shaped. In certain parasites found in the Batrachia this spindle is almost as broad as it is long ${ }^{1}$ ( $40 \mu$ by $60 \mu$ ), and the ends are rounded off, leading

1 In measuring trypanosomes, it is advisable to measure the width in the region of the nucleus, taking into consideration the undulating membrane, and the length including the flagellum. At the same time the length of the body and of the free flagellum should be measured separately; but these measurements are 
eventually to flattened forms with an ellipsoidal outline, which would be a true geometrical figure were it not for a slight prolongation anteriorly which accompanies the flagellum. We find the opposite extreme in forms such as those seen in Fig. 2, $I$, where the body is only $\mathrm{I} \cdot 5 \mu$ wide, or, better still, in certain parasites of fishes, such as those of the eel, which are $80 \mu$ long by only $2.5 \mu$ broad. Between these two extremes there are many intermediate forms, some of which are characteristic of certain species parasitic in reptiles or birds, others not possessing any specific value. Thus, for example, the trypanosomes of Batrachia may assume various forms-some relatively thin (see Fig. 63, Chapter XVI.), which we give in Fig. 2 ( 3 and 4 ), as types of 'stumpy' forms; also the broad forms of T. lewisi (our 'thin' type, Fig. 2, $I$ ) when reproduction is about to take place.

The trypanoplasms are of medium length, the body being in the form of a ribbon, which is constantly changing its shape.
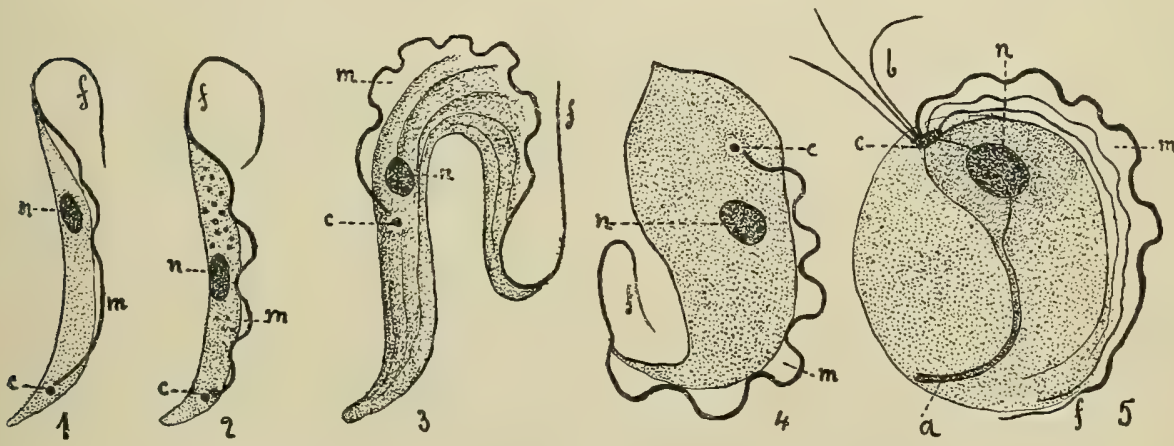

Fig. 2.-Trypanosomes of Mammals and Batrachia-Trichomonas.

I. $T$. lewisi : $n$, nucleus ; $c$, centrosome ; $m$, undulating membrane. These letters have the same significance in the other figures. $2 . T$. brucei. 3 and $4 . T$, rotatorium of Rana esculenta (frog): striated form (3) and flat form (4). 5. Trichomonas intestinalis, from the intestine of the guinea-pig : $a$, internal rod; $b$, anterior Hagella. (Magnification about $\mathrm{r}, 800$ diameters.)

The shape and size of trypanosomes afford very good means of diagnosing species. ${ }^{1}$ It must be remembered, however, in the case of trypanosomes pathogenic for different species of animals, that

often difficult, for it is sometimes not easy to determine where the free flagellum commences, so that there is in such a case a liability to err, which is not present when the whole parasite (flagellum included) is measured. It is for this reason that we lay most stress upon that dimension (namely, total length) in differentiating species.

[Lingard (Journ. Trop. Vet. Science, v. 1., 1906) suggests making the following measurements : (I) Post-centrosomic part; (2) from the centrosome to the posterior end of nucleus; (3) length of nucleus; (4) distance from the anterior end of nucleus to anterior end of body; (5) free flagellum. The sum of these five measurements gives the total length. He also measures the maximum width. As pointed out in the first part of this note, however, it is often difficult, if not impossible, to say where the body ends anteriorly and the free flagellum begins.]

1 [The recently described - probably sexual - morphological differences in various mammalian trypanosomes are referred to later on in this chapter.] 
these trypanosomes show certain variations in length, and even in shape, according to the species of animal infected. Thus, T. brucei is longer in Equidæ than in experimental rodents; it is more stumpy in rats and mice than in dogs. It follows, therefore, that if one wishes to compare two allied species of trypanosomes-for example, $T$. brucei and T. evansi-one should examine the parasites in the blood of the same species of animal.

The anterior extremity is generally elongated; it extends some way along the flagellum, sometimes (as in the case of $T$. dimorphon of horses in Gambia and of $T$. johnstoni of birds) even to its tip.

The posterior extremity varies very much in shape, not only in different species of trypanosomes, but also in the same species, and even in the blood of the same animal. Evidently this part of the body has a certain amount of plasticity or power of amœboid move-
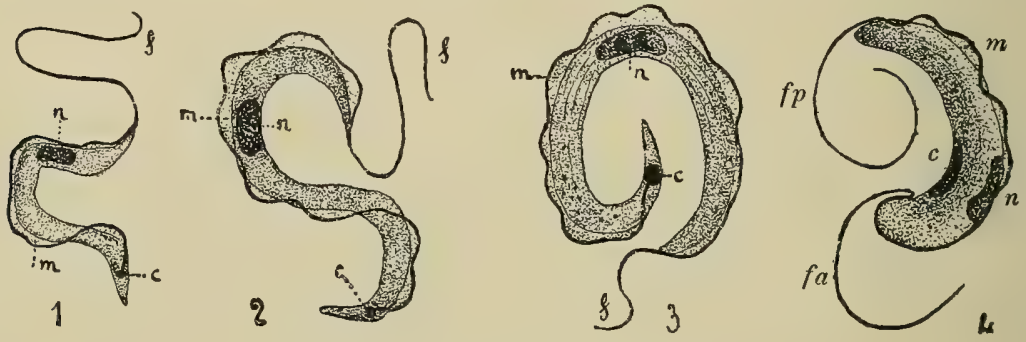

Fig. 3.-Trypanosomes of Fishes.

I. T. remaki, var. parva: $n$, nucleus ; $c$, centrosome; $m$, undulating membrane. These letters have the same significance in the other figures. 2. T. remaki, var. magna. 3. T. solea. 4. Trypanoplasma borreli: $f a$, anterior flagellum; $f p$, posterior flagellum. The anterior end is the lower one in the figure.

ment, by virtue of which it can elongate and shorten. None the less it presents, in a given species, certain peculiarities which are really specific, but we must always be very careful in interpreting such peculiarities.

Thus, $T$. lewisi (Fig. 2, $I$ ) is characterized by a long pointed posterior extremity, like an acute-angled cone. In some cases this part is almost as long as the rest of the body, whilst in others it is relatively short. In the trypanosomes of the type brucei (Fig. 2, 2) the posterior extremity is less slender and generally short; it is rarely. pointed, but nearly always in the form of a blunt cone. In the other pathogenic trypanosomes of mammals, the posterior end is usually rounded off in the form of a hemisphere, and the centrosome is very near the end; but even in these species many of the parasites have a bluntly conical end. It is, however, in the trypanosomes of the Batrachia that the greatest variation in shape is seen. Some have a posterior extremity as long as the rest of the body, and at times it even appears to be prolonged into a flagellum, so much does it taper off; in others, as has been mentioned before, the end is rounded off in the form of an ellipse, and one often observes 
under the microscope the conical posterior extremity of a parasite becoming rounded off.

The protoplasm stains more or less deep blue by our eosinmethylene blue mixture. ${ }^{I}$ This is due to the presence of a great number of barely visible very fine granules. The blue colour is deeper with some trypanosomes than with others; for example, the pathogenic trypanosomes of mammals and those of fishes stain more deeply than $T$. lewisi or other members of the same group. The blue colour is never very uniform, and there are always paler areas to be seen ; but we would draw attention to the fact that these clear areas always appear to be irregularly distributed, and we have never found, in the case of any particular trypanosome, anything characteristic in their form or distribution.

The clear oval vacuole which authors have described in the posterior part of certain trypanosomes, and which they identify with the contractile vacuole of the non-parasitic Flagellata, has appeared to us of variable form when present. Moreover, it is found especially in preparations made from liquids other than the blood (e.g., bloodstained œedema fluid, cerebro-spinal fluid in cases of sleeping sickness), and the presence of this vacuole is doubtless due, as we have said above, to the fact that we do not know how to fix these liquids properly. These vacuolated forms are not found in the blood. One cannot, therefore, attribute any specific importance to the presence of these vacuoles, to their shape, or to their relation to the centrosome. ${ }^{2}$

The protoplasm often contains chromatic granules stained deep violet, very variable in number, shape, and size. They are generally a little irregular in shape, but more or less rounded, and do not exceed I $\mu$ in diameter. Scme species of trypanosomes do not possess them; in others they are scanty, and often localized to the immediate neighbourhood of the nucleus; in yet others they are numerous and scattered throughout the body of the parasite. They are absent from $T$. lewisi, are very scanty in $T$. dimorphon and $T$. evansi, less rare and larger in $T$. brucei and $T$. equimum. In the latter species sometimes one or two of the granules are more than I $\mu$ in diameter and quite circular, in which case they may be mistaken for the centrosome. Up to a certain point these granules are of value in determining species, but too much weight must not be attached to their presence, as this depends to a certain extent upon the condition of health of the animal under investigation. When the death of an infected animal is approaching, the trypanosomes are usually less vigorous, and their protoplasm contains more granules than in the case of normal parasites. The same thing is seen in the

1 Throughout this description we shall refer solely to preparations stained by this method (see p. 9 et seq.).

2 [I do not agree with the authors that 'vacuolated' forms are not normally found in the blood; see footnote in Chapter XII. under the morphology of T. gambiense; also article by Woodcock, 'The Hæmoflagellates,' in Quart. Journ. Micr. Sc., v. 50, part i., April, 1906, pp. 211, 212.] 
trypanosomes from animals undergoing treatment with human serum or arsenious acid.

These violet granules are almost the only ones seen in trypanosomes. In addition, we may mention the non-staining granules seen in $T$. rotatorium of the frog and the refractile non-staining granule seen in $T$. lewisi in the blood of guinea-pigs. The refractile granules seen in culture forms of $T$. bruce $i$ are undoubtedly of the same nature.

In some trypanosomes one can distinguish fine longitudinal striæ; more or less marked, generaily characterized by a linear arrangement of the microsomic granules. These striæ are apparently quite superficial. They probably represent the broad, well-marked striæ seen in some forms of $T$. rotatorium. In this trypanosome the striæ are arranged in the form of a helix, the axis of the helix being almost transverse and the striæ arranged in a longitudinal direction.

The body of the parasite appears to have no definite investing membrane. We have been unable to make out around the body a special layer of protoplasm, the so-called ectoplasm or periplast. ${ }^{1}$ It is only along the ridge formed by the undulating membrane that the surface of the body presents any peculiarities, and these will be referred to later on.

The nucleus [macronucleus, trophonucleus] - that is to say, the larger chromatic corpuscule of trypanosomes-round or oval in shape, is generally situated about the middle of the body. Rarely, as in $T$. lewisi, it is situated in the anterior part of the body, which is a diagnostic feature of that species of trypanosome. [In $T$. longocaudense (Lingard) of the rat, $T$. duttoni (Thiroux) of the mouse, and in the bat trypanosome, the nucleus is also anteriorly placed. In some forms of $T$. theileri the nucleus is situated behind the middle of the body.] The size of the nucleus appears to be independent of the size of the parasite; for example, the nucleus of the large trypanosomes of frogs is scarcely bigger than that of the thin trypanosomes of mammals.

The nucleus appears as a collection of chromatic granules, staining purple by the ordinary methods. This collection of granules is relatively compact, the granules being of various sizes and shapes, and joined together by a feebly staining cement substance. No network or nuclear membrane can be distinguished. It is a relatively simple type of nucleus. Prowazek, ${ }^{2}$ who distinguishes four types of nucleus among the Flagellata, considers this nucleus to be of the simplest type.

\footnotetext{
1 [It is pointed out later on (pp. 24 and 69) that Prowazek and others describe the undoubted occurrence of an ectoplasmic layer in some cases.]

2 [Prowazek, Arch. f. Protistenkunde, v. 3, 1903, p. 195. But, according to Prowazek's recent investigations, the nucleus of 1 . lcavisi and $T$. brucei is very complex, and resembles the complex nucleus originally described by Schaudinn in $T$. noctuce. The nuclei of other mammalian trypanosomes are probably also more complex in structure than has hitherto been supposed. (See the accounts given later of $T$. lewisi and $T$. brucei.)]
} 
Usually the granules are scattered uniformly throughout the nuclear mass. There are few exceptions: $T$. remaki of the pike, which has in the centre of the nucleus a clear space containing a single large granule, the smaller granules forming a ring around the central vacuole. Perhaps this is also the case in $T$. solea, but we have seen too few specimens of it to be sure.

The nucleus always divides amitotically. ${ }^{1}$ Sometimes it elongates hardly at all before dividing; at others (e.g., T. brucei, Fig. 22, 2) the nucleus elongates considerably and becomes rod-shaped, and, finally, the intervening part where no chromatic granules are present becomes constricted and obliterated. In such cases there is a suggestion of mitotic division, but much less than in the micronuclei of the ciliated Infusoria, for example.

The small chromatic body which we call the centrosome ${ }^{2}$ is situated quite near the posterior extremity in many species of trypanosome. But that position is not absolutely constant. At first, in certain species (e.g., T. lewisi of the rat), when the parasite has just divided, the centrosome is close to the nucleus. In other species-at least, in certain varieties-it occupies normally, and without any reference to fission-this central position near the nucleus. This is seen in some forms of $T$. rotatorium of the frog (Fig. 2, 3) and in T. transvaaliense (Fig. 45, Chapter XI.). In these cases the centrosome is situated deep down in the interior of the protoplasm.

The centrosome of true trypanosomes occurs as a small compact and homogeneous mass of chromatin, staining a deep violet, slightly different from the colour of the nucleus. It is generally round, rarely elliptical, though it is frequently so in $T$. lewisi. In size it is usually $0^{\circ} 5 \mu \mathrm{O}^{\circ} \mathrm{I} \mu$, with one exception, the $T$. equinum of caderas, in which the centrosome is only $\frac{1}{4} \mu$ in diameter, and is difficult to recognise at the extremity of the flagellum. Often the centrosome is surrounded by a clear space or halo, which makes it appear the more distinct. When the centrosome is fairly large this halo is not visible; the centrosome then occupies the whole width of the parasite, and sometimes appears even to extend beyond it, doubtless owing to its refractile character in stained specimens.

The centrosome divides in a simple fashion. It elongates, becomes halter-shaped, and finally the two halves separate. Sometimes, as in $T$. lewisi, it elongates transversely to the long axis of the body; more often, as in T. brucei and others, it lengthens longitudinally, so that the two resulting centrosomes are situated the

1 Wasielewski and Senn have represented, in a case of multiple division of $T$. lewisi, nuclear division with equatorial plates. We have never seen anything like this. [We shall see later, however, that more complex forms of nuclear division have been described in the case of $T$. noctuce (Schaudinn), T. lewisi, and T. brucei (Prowazek).]

${ }^{2}$ [This little body, the significance of which has been much discussed (see Section 5), is also called the micronucleus, the blepharoplast, and the kinetonucleus by other authorities.] 
one in front of the other in the long axis of the parasite (Fig. 22, Chapter VI.).

In the species which we have placed in the genus Trypanoplasma, the two chromatic masses are about equal in size, each being as large as the nucleus of an ordinary trypanosome. They are laterally placed, often along two transverse diameters near together. They differ a little in their staining reactions. Thus, according to Miss Plehn', who has recently described in the carp a new species of this genus, the chromatic body in connection with the flagella (which we identify with the centrosome of Trypanosoma) stains deep violet, like the nuclei of the carp's erythrocytes; the other body (the true nucleus) stains lilac, like the nuclei of the leucocytes and the bloodplatelets. Amongst the numerous trypanosomes which we have examined we have never seen an arrangement intermediate between that found in Trypanoplasma and that observed in Trypanosoma.

Originating in the centrosome of the true trypanosomes is a very clear filament, which runs along the edge of the undulating membrane, and is then usually prolonged beyond the end of the body into a free part. We shall call this filament throughout its whole length the flagellum. In it three parts can be distinguished: the first part or root, which is very short, and extends from the centrosome to the undulating membrane; the second, which runs along the edge of the undulating membrane; while the third part is the free flagellum. The flagellum has the same appearance and the same chromatic reaction throughout. It stains purple, exactly like the chromatic granules of the nucleus, differing slightly from the deep violet colour of the centrosome.

The connection between the flagellum and centrosome is undoubted. In those forms in which the centrosome is surrounded by a clear space, the flagellum appears to end at the border of that clear area; but it is, nevertheless, connected with the centrosome, as the examination of other trypanosomes in stained preparations will demonstrate. The flagellum, isolated from the undulating membrane and the rest of the body of the parasite, nearly always remains attached to the centrosome.

The undulating membrane appears as a ridge or crest extending laterally from the body of the parasite along a variable part of its length. Its longitudinal extent along the body of the parasite obviously depends upon the position of the centrosome. If the latter is near the posterior extremity, as is usually the case, the undulating membrane extends along the greater part of the body of the parasite; if, on the other hand, the centrosome is near the middle of the body,

1 M. Plehn, Arch. f. Protistenkunde, v. 3, 1903, pp. 175-180. Miss Plehn has since written us (March 26, 1904 ) that she was mistaken about the chromatic body in relation with the undulating membrane. We transpose, therefore, what the author says about these two bodies. The same facts have also recently been made out by Léger (C.R. Acud. Sciences, v. 138, March 28 and April 4 , 1904), and agree with our own recent observations. 
the undulating membrane will be found only along the anterior half of the trypanosome.

The undulating membrane is always very thin, much more so than its border, which is constituted by the flagellum. We think it is always composed of protoplasm identical with that of the body itself; we have never made out any special protoplasm, periplast or otherwise. It is always homogeneous; we have never seen any reinforcing striæ as occur, for example, in the Trichomonas (Fig 2, 5). ${ }^{1}$

The variations in the membrane amongst the different species depend largely upon the number of folds which it exhibits. The largest number of folds is seen in $T$. rotatorimn (Fig. 2, 3 and $f$ ), T. avium (Fig. 6o, Chapter XIV.), and T. gramulosum of the eel. The pathogenic trypanosomes of mammals of the type brucei have membranes more folded than the non-pathogenic trypanosomes of the type lewisi. Finally, there are forms ( $T$. dimorphon, etc.) in which the flagellum is so closely applied to the body that the membrane is reduced almost to nothing. These few examples are sufficient; a study of the numerous figures in this book is of more value than any description.

The undulating membrane is often prolonged anteriorly beyond the body itself; or, in other words, the body is represented anteriorly only by the continuation of the undulating membrane, of course with the flagellum along its edge. Generally a free portion of the flagellum, of variable length, extends beyond this part (longer, for example, in $T$. lewisi than in the pathogenic trypanosomes of mammals), the dimensions of which furnish specific characters of a certain value. Sometimes the flagellum has the same thickness right up to the end; at other times it gradually tapers off. This free part of the flagellum may be absent, however, as in $T$. dimorphon (Fig. 28, Chapter VII.) and T.johnstoni (Fig. 6I, I., Chapter XIV.). It is also the case in a species which Broden has recently found in mammals (sheep and donkey) on the Congo. A similar appearance is observed in certain forms of $T$. rotatorium, in which case it seems to be due to a change of shape-into a spherical form, with retraction of the undulating membrane-in a certain number of the parasites after leaving the frog's bloodvessels.

Even amongst species of the type brucei where the majority of the parasites have a free flagellum, some occasionally have no free flagellum. This is probably connected in some way with repeated subdivision (vide infra).

Taking all the known forms into consideration, it is impossible to attach great importance (e.g., a generic importance) to the presence or absence of a free flagellum. For example, we should not on that account alone put $T$. dimorphon in a genus different from the $T$. gambiense or $T$. brucei.

1 [Since Schaudinn's original description of the ectoplasmic origin of the undulating membrane in $T$. noctua, and of the occurrence of reinforcing strix in this membrane, several observers have described a similar structure in other trypanosomes.] 
In forms without free flagellum the anterior extremity of the body tapers off just as much as in those with flagellum.

[Since the interesting and very revolutionary observations of Schaudinn upon the blood parasites of the owl (Athene noctua; see Section 4), in which he describes sexual forms as well as a highly complex structure of the parasites, Ziemann, Prowazek, and others, have described analogous sexual and 'indifferent' forms, and also great complexity of structure, in several of the mammalian trypanosomes. Prowazek's first observations were upon $T$. lewisi, and in Chapter IV., Section 3, the reader will find an account of the results obtained by Prowazek with this trypanosome as regards the sexual forms, the presence of an ectoplasm and of myonemes, and the complexity of the nucleus.]

In Trypanoplasma the undulating membrane runs along the whole length of the parasite, but it is only clearly recognizable by its border (formed by the flagellum), which follows the convex surface of the body. The membrane has few folds, is not prominent, and in certain places is very closely applied to the body. Anteriorly, after coursing along the anterior rounded end of the body (see Fig. 3, 4), it turns back again along the concave side of the body to within a short distance of the centrosome, where it appears to be inserted. Posteriorly, the thickened edge of the membrane gives rise to the free posterior flagellum. Another free flagellum is seen anteriorly, which is inserted in front of the centrosome, like the undulating membrane. Are the relations between the centrosome and the flagella as close as in the case of the true trypanosomes? We have no definite observations on this point. ${ }^{1}$

The longitudinal division of the flagellum in trypanosomes has been studied with the greatest detail. We may note in passing that it is the first definite observation of division of the flagellum amongst the flagellates.

Division always starts in the centrosome. Two modes of division are clearly recognisable: (I) That seen in $T$. lewisi, where only the root of the flagellum is duplicated. This root is seen to broaden out at first, then to divide into two. (2) That seen in the majority of the other trypanosomes, where the duplication of the flagellum and the undulating membrane proceeds gradually from the centrosome to the extremity of the undulating membrane. Sometimes the free flagellum is also duplicated.

In the first case the short flagellum thus formed grows in a centrifugal manner, beginning from its centrosome.

[From Schaudinn's observations upon $T$. noctua of the owl, it appears that the whole of the new flagellar apparatus is developed-

I. [In Trypanoplasma borreli Léger has described a centrosomic granule ('diplosome') at the base of each flagellum ; and Keysselitz suspects the presence of similar granules in Trypanophis. I'rowazek also frequently figures such a centrosomic granule, situated between the centrosome and the base of the flagellum, in both $T$. lewisi and $T$. brucei.] 
independently of the original flagellum-from the daughter centrosome. Prowazek states that this is also the case with T. lewisi and and T. brucei (see Chapters IV. and VI.). McNeal has described the same phenomenon in culture forms of $T$. brucei. There is some doubt, therefore, whether the flagellum of trypanosomes does really split, although blood-films in which fission forms occur certainly give one the impression that the flagellum is actually splitting.]

There remains little to be said about the multiplication of trypanosomes, since we have already spoken about the division of the nucleus, centrosome, and flagellum. The simplest mode of division, as seen in the pathogenic trypanosomes of mammals and the trypanosomes of fishes, is a longitudinal division into equal or subequal parts. It begins in the centrosome, ${ }^{1}$ follows in the nucleus and undulating membrane, and ends in the protoplasm (Fig. 22, Chapter VI.).

In $T$. lewisi, and also undoubtedly in certain trypanosomes of birds, the process of division is apparently more complicated. We shall be content to mention here only those points which appear to us to be the most important for the comprehension of the type, Trypanosoma, leaving the consideration of less important details to the chapter dealing specially with $T$. lewisi.

The trypanosomes first enlarge considerably, and then divide longitudinally into two unequal parts. One of the resulting trypanosomes has a nucleus and centrosome exactly like those of its congener, but has less protoplasm, and a short flagellum without undulating membrane. Those forms without undulating membrane can divide again, and give rise, by longitudinal and equal division of all their parts, to others similar to themselves-namely, small fusiform parasites, with the centrosome near, and sometimes even in front of, the nucleus, and without undulating membrane. In cultures only these forms are found. These forms in their turn can give rise to others of the adult type with undulating membrane, probably by displacement backwards of the centrosome and the elongation and lateral displacement of the flagellum, which comes to be the border of a projection from the side of the body. As we shall see later on, these rat trypanosomes thus pass through a stage which is the adult condition in other flagellates-the genus Herpetomonas.

All the flagellates parasitic in the blood known up to the present can be grouped in two genera :

I. Trypanosoma, Gruby, I843 (Laveran and Mesnil, Igor, amended): ${ }^{2}$ Flagellates with fusiform body, presenting laterally an undulating membrane, whose thickened edge ends posteriorly, in the posterior half of the body, in a centrosomic corpuscule (clearly differing from the mucleus in structure), and anteriorly is usually prolonged as a free

${ }^{1}$ [Although the centrosome is usually the first to divide, division may sometimes begin in the nucleus.]

${ }^{2}$ We reproduce here, with very slight alteration, the description we gave in Igor (C. R. Acad. Sciences, v. 133, p. I3I). 
flagellum. Binary longitudinal division into equal or unequal parts. Certain species pass through a stage without undulating membrane. Parasitic in the blood of all classes of vertebrates. ${ }^{1}$ A very large number of species known.

2. Trypanoplasma, Laveran and Mesnil, Igor (amended Igo4): Flagellates with elongated body, presenting laterally an undulating membrane, whose thickened edge is prolonged posteriorly as a flagellum, and turns back anteriorly to reach a mass (centrosome) which is as large as, and has up to a certain point the same structure as, the nucleus. An anterior free flagellum has its origin in the same mass. Multiplication is probably by binary equal longitudinal division. Parasitic in the blood of fishes. ${ }^{2}$ Several species known. ${ }^{3}$

The differences between the characters of the two genera are so clear and sharply defined ${ }^{4}$ that the necessity of dividing the flagellated hæmatozoa into these two genera is unquestionable.

Is it necessary to create a larger number of genera? The various species $^{2}$ of the genus Trypanoplasma are closely allied, therefore no difficulty arises on that point. As to the species of the genus Trypanosoma, there are (as we have already shown in previous pages, and as a study of our figures will also demonstrate) all stages of transition from one to another. A further generic subdivision, based on any single characteristic or group of characteristics, appears to us quite impossible. ${ }^{5}$

In another paragraph we shall justify the generic names we use.

[Woodcock ${ }^{6}$ places the flagellate of the owl, the life cycle of which has been so fully worked out by Schaudinn, in a new genus (Trypanomorpha).

Lühe, in his article on the blood-parasites in Mense's 'Handbuch der Tropenkrankheiten,' v. 3, Igo6, 'creates the new genus Trypanozoon (with flagellum anterior) for all the mammalian trypanosomes. On the other hand, he thinks (without giving reasons for so doing) that the

1 [We shall see later that trypanosomes have been described in tsetse-flies, though, of course, it is possible that the flies had obtained their trypanosomes by sucking the blood of some vertebrate which contained the parasites.]

2 [Léger has since described (in $C$. R. Soc. Biol., v. 58, I905, p. $5 \mathrm{II}$ ) an intestinal trypanoplasm in fishes. In the same paper he expresses the opinion that Keysselitz's new genus Trypanophis (see footnote 4) is so like Trypanoplasma that the former should be included in the latter.]

${ }^{3}$ [In the original it is stated that two species are known, but since that was written several other species have been described and named (see Chapter XVII.).]

${ }^{4}$ Poche has recently discovered (Arbeit. a. d. zool. Inst. zu Wien, v. I4, 1903, p. 307) amongst pelagic Cœlenterata of the group Siphonophora, a flagellate with undulating membrane which he has called Irypanosoma grobbeni. The subjecı has recently been further studied by Keysselitz at Rovigno. In his paper, which has recently appeared (Arch. f. Protistenkunde, v. 3, 1904, p. 367), he regards this flagellate as the type of a new genus Trypunophis, characterized by: a short anterior flagellum and a long flagellum posteriorly ruıning along the whole length of the body, accompanying an undulating membrane. The centrosome (or blepharoplast), situated quite in front, is much smaller than the nucleus. Evidently this new genus is closely allied to Trypanoplasma, according to the modified description we give above.

5 Perhaps the flagellated hematozoon seen by Dutton and Todd in the blood of mice in the Gambia Colony (sce Chapter V.) is neituer a Tryptimosoma nor a Trypanoplasmet. According to the observers, it had no undulating membrane, and reminded one of a Herpetomonus. As their observations were made only on fresh specimens, it is as well to keep an open mind upon the subject.

${ }^{6}$ [Woodcock, Quart. Journ. Micr. Sc., v. 50, part ii., 1906, pp. 283-289.] 
trypanosomes of fresh-water fishes have the flagellum posteriorly situated, and he puts them in the genus Hamatomonas, created for them by Mitrophanov. He does not commit himself upon the trypanosomes of other vertebrates, and therefore leaves us in doubt as to the morphological orientation of the type species, $T$. votatorium ' (Mesnil). $\left.{ }^{1}\right]$

\section{Section 2.--Biology of the Trypanosomes.}

The general survey we are making of the trypanosomes would be incomplete without referring briefly to their behaviour in the blood or body-fluids of infected animals immediately after leaving the body and in vitro under different conditions.

Nutrition And Movement.-Trypanosomes derive their nutriment by osmosis. No digestive vacuoles or solid bodies of any kind are ever seen in them. Trypanosomes displace the red corpuscles by their movements, but we have never seen them attack the red corpuscles, either to absorb them or to penetrate into their interior. Nevertheless, judging from McNeal and Novy's attempts to cultivate them, hæmoglobin seems to be useful, if not indispensable, to them.

[Goebel ${ }^{2}$ has studied the osmotic properties of $T$. brucei by adding a drop of blood rich in trypanosomes to I c.c. of saline solutions of various strengths. In strong solutions the trypanosomes are rendered motionless at once, and lose their virulence, but retain their shape. In isotonic solutions they remain motile, while in weak solutions they become deformed and involuted. Trypanosomes immobilized by strong solutions remain alive for some time (e.g., one hour in a normal solution of $\mathrm{NaCl}$ ), and on diluting sufficiently, regain their mobility and virulence.

The action of the saline solutions upon the trypanosomes is almost the same as on the red corpuscles; perhaps the latter are a little more sensitive than the trypanosomes.]

The movements of trypanosomes are due chiefly to the undulating membrane and the free flagellum. When moving sluggishly in loco one can study in detail the movements of the membrane, which consist of undulations, first in one direction then in another. It is possible to make out also that the free flagellum has a lashing movement, oscillating alternately to the right and left. When the trypanosomes move from place to place, with the flagellum foremost, which is their usual mode of progression, this movement of the flagellum to right and left can again be distinguished if the parasite is not moving too quickly. When it is very active, however, one can see only a darting, arrow-like movement of the whole of the parasite.

Besides these movements of the undulating membrane and flagellum, there are others due to a contraction of the whole of the protoplasmic body. This contraction is apparently dependent upon a kind of myocyte layer, such as has been observed in gregarines and other Sporozoa, [or upon the somewhat spirally-arranged myonemes described by Prowazek and others in several of the trypanosomes.] Finally, there are amoboid movements localized in

1 [Mesnil, in Bull. Inst. Past., v. 4, 1906, pp. 793-795.]

2 [Goebel, Ann. Soc. méd. de Gand, 1906, p. II ; abstract by Mesnil, in Bull. Inst. Past., v. 4, 1906, p. 256.] 
particular parts of the body especially the posterior part (see section on Morphology for variations in shape of this part of the body).

One or other movement predominates, according to the species. Amongst the mammalian trypanosomes it is the flagellar motion, but this varies in degree in the different species. For example, $T$. lewisi easily traverses the field of the microscope with an arrowlike motion; trypanosomes of the type brucei hardly move from the spot where first seen, except $T$. evansi, which is sometimes seen to travel across the field of the microscope, but always more slowly than $T$. lewisi. ${ }^{1}$

The contractile movements of the protoplasmic body are often seen in the trypanosomes of fishes - for example, the parasites of the eel or of the Selachii, which are often contorted and coiled up on themselves. The trypanoplasms also move about very actively, so as to resemble a piece of drapery or muslin thrown into a succession of folds or curves. Lastly, $T$. rotatorium of frogs exhibits marked amoboid movements, consisting mainly of contractions of the extremities of the body, especially the posterior, with constant changes of shape.

Infectivity and Virulence.-Trypanosomes develop in the body of an animal owing to their power of reproduction. By the pathogenic trypanosomes this power is retained throughout the illness, but is more or less developed according to the species of animal infected and the period of the disease. For example, in a horse with nagana the trypanosome's power of reproduction coincides with the febrile attacks. In the case of the non-pathogenic trypanosomes, the period of multiplication of the parasite is limited to a certain number of days at the beginning of the infection. Later on only adult forms of the parasite are seen in the blood and internal organs, developmental forms being absent.

Even in the case of the non-pathogenic trypanosomes the power of infection may vary within certain limits. Such is the case, for example, with $T$. lewrisi, where the variation depends upon both the origin of the trypanosome and the rats inoculated. We shall see, moreover, that this species is in certain cases really virulent, the rats dying from it not showing any more trypanosomes than other rats which, infected with another strain of parasite, are unaffected thereby. Apparently, therefore, infectivity and virulence may be independent of one another.

${ }^{1}$ [I have very often seen the human trypanosome, both in human blood and cerebro-spinal fluid and in the blond of inoculated animals, travel very rapidly, so that it was with difficul'y kept under observation by moving the slide about. The same rapidity of movement was seen in the case of several animal trypanosomes found in Uganda. Possibly the difference between my own and the authors' observations may he due to the fact that in our laboratory in Uganda the temperature was generally about $80^{\circ} \mathrm{F}$. and the air very moist, and that the cover-glasses were always ringed with a layer of vaseline at once to prevent evaporation.

Ziemann has also described. in the blood of cattle in Cameroon, a very active trypanosome, which, on account of its great activity, he calls T: viz'ax. (See Chapter VI.)] 
For a given pathogenic trypanosome the virulence varies, first, with the species of animal inoculated. Every chapter of this book furnishes so many instances of this that there is no necessity to insist upon it here. Secondly, it varies with the race of the mammal; thus it seems clear that races acclimatized to the countries where such trypanosomiases are endemic are less susceptible than corresponding races of unaffected areas-e.g., dogs imported into South Africa or India, cattle from Madagascar, etc.

Thirdly, it varies with the origin of the trypanosomes, and thus seeins to depend on conditions we are unable to determine accurately. All the evidence brought forward hitherto goes to show that all the human trypanosomes seen in Africa belong to a single speciesT. gambicnse, yet the parasite studied by Brumpt and Wurtz was clearly more virulent than that from the Gambia studied by Dutton, Todd, Annett, and ourselves. The latter was more virulent than that of Bruce, Nabarro, and Greig from Uganda, and finally Manson, with a trypanosome from another source (Congo), was unable to infect any animals.

Fourthly, virulence apparently varies, though only to a slight extent, with passage through certain definite species of animals. ${ }^{2}$ To this undoubtedly must be attributed the different results obtained by Kanthack, Durham, and Blandford in England, and by ourselves in France, with the same strain of trypanosome, and with very closely allied races of mammals. Other instances of this will be found later on in this volume, and we shall see that Koch and Schilling at one time thought that a method for vaccinating bovines against $T$. brucei might be based upon this diminution of virulence by passage.

Preservation of Trypanosomes in vitro.-Trypanosomes can be kept alive for a time in the defibrinated or citrated blood of an infected animal. The addition of physiological saline to defibrinated blood rather favours the preservation of trypanosomes, while the addition of ordinary or distilled water brings about their death more or less rapidly, according to the proportion of water added.

A large number of chemical substances are harmful to trypanosomes in vilvo. A list of them will be given in the account of our attempts at the treatment of nagana.

Trypanosomes are very sensitive to heat. Few pathogenic trypanosomes can stand for even a very short time a temperature of $40^{\circ} \mathrm{C}$. or above. Exact data will be found in the chapter on $T$. brucei. $T$. lewisi is more resistant to temperatures above $40^{\circ} \mathrm{C}$., but its sensitiveness is still very marked, and it never lives longer than twelve hours under those conditions.

Trypanosomes are better able to resist a lowering of temperature.

1 [The only exception is that of Plimmer, to which reference is made in Chapter XII., but which has not been confirmed by any other observer.]

2 [We shall see later on, however, that the 'genealogy' of a parasite-that is to say, the number of times it has been passed through animals and the different species of animal through which it has passed-may have a decided effect upon the virulence, often resulting in a virus-fixe. (See Chapter VI., part ii.) J 
This is particularly the case with $T$. lewisi, which has been kept for two months at a temperature of $5^{\circ}$ to $7^{\circ} \mathrm{C}$. This parasite can live between the extremes of temperature $5^{\circ}$ and $40^{\circ} \mathrm{C}$. On the other hand, pathogenic trypanosomes do not live for more than five or six days at $5^{\circ} \mathrm{C}$.

Trypanosomes show a certain amount of resistance to temperatures below zero, but some die very rapidly. The lower the temperature, the more rapidly does death take place. Jürgens has shown that $T$. lewisi kept for two hours at $-I 7^{\circ} \mathrm{C}$. is no longer virulent. Under very similar conditions we have found a few trypanosomes still motile and highly infectious for the rat.

After a quarter of an hour's exposure to liquid air ( $-\mathrm{Ig1}^{\circ} \mathrm{C}$.), all the trypanosomes with which we have experimented (various pathogenic trypanosomes and $T$. lewisi) were found still virulent. It is true the large majority had died, and only a very few were found motile. After an hour and a quarter's exposure (on two occasions) $T$. lewisi was still able to infect a rat. Blood containing $T$. dimorphon was no longer infectious after an hour's exposure, although motile parasites were still visible. After twenty-four hours' exposure $T$. lewisi and $T$. dimorphon were all completely destroyed or spherical in shape; neither was virulent on inoculation.

Before dying the trypanosomes undergo various changes of form, of which the following are the most important: The appearance of vacuoles in the protoplasm, especially at the centrosomic end, granular degeneration of the protoplasm, detachment of the flagellum from the undulating membrane, and conversion of the protoplasmic body into spherical forms, passing through intermediate stages, such as tadpole forms, in which a little protoplasm still accompanies the flagellum. Some of these degenerative stages are seen in vivo, as, for example, during the last hours of the life of an infected animal; also, in a marked degree, in an animal treated with human serum or with arsenic.

Another very curious phenomenon exhibited by trypanosomes is agglutination. But in this case, contrary to what has been observed in the case of bacteria, agglutination is not preceded by loss of motility. The results of agglutination are very peculiar: rosettes composed of a variable number of parasites, all joined together by their posterior extremities, and with the undulating membranes and flagella still carrying on their different movements.

These phenomena, which take some time to appear in the case of trypanosomes kept in the defibrinated blood, occur very rapidly and to a marked degree on adding to defibrinated or citrated blood serums obtained from species of animals different from that which has furnished the trypanosome, or, as in the case of $T$. lewisi, a specific serum from the same animal species.

Cultures.-Cultivations of trypanosomes are best made in the water of condensation in tubes of a solid medium, consisting of 
nutrient agar, to which is added, when at a temperature of $50^{\circ} \mathrm{C}$., as much defibrinated blood as possible (up to two or three times the volume of the agar). This method of cultivation has been particularly successful in the case of $T$. lewisi. Inoculations from one tube into another succeed, and sometimes give rise to a copious growth, in which the parasites are typical in appearance. Generally, however, the culture forms have no undulating membrane, and the flagellum is inserted in front of the nucleus. The parasites are of all sizes, from I to $2 \mu$ in length (without flagellum). Some parasites seem to be able to pass through a Berkefeld filter, for the filtrates of cultures are infectious.

Results obtained with the pathogenic trypanosomes are much less satisfactory, and the culture forms, containing as they do numerous protoplasmic granules, give the impression of being under unfavourable conditions.

Cultures do well at $25^{\circ} \mathrm{C}$., or even at the ordinary laboratory temperature. The organisms grow slowly, but remain alive and virulent for a very long time. At $34^{\circ}$ to $37^{\circ} \mathrm{C}$. growth takes place more rapidly, but the cultures die in a few dars. Temperatures above $37^{\circ} \mathrm{C}$. are quickly fatal. These facts are analogous to those which we have noted in connection with the preservation of trypanosomes in vitro.

\section{Section 3.-Historical Survey of the Genera of Trypanosomes.}

Neither Valentin, who discovered the trypanosome of the trout in I84I, nor Gluge, who discovered that of the frog in I842, thought of giving new specific or generic names to these parasites. The former author simply classes his hæmatozoon with the Proteus or Ancebe of Ehrenberg. Maver, in July, I8 43 , gave specific names to the parasite of the frog (Amceba rotatoria, Parancecium loricatum and costatum), but, nevertheless, placed it in two old genera, Amaba and Paramocium, where it evidently could not remain.

It is therefore undoubtedly Gruby ${ }^{1}$ who, in November, I $8_{+3}$, was the first to give a new generic name to the organisms we are studying, and that name, Trypanosoma, should be retained to designate the trypanosome of the frog (species, sanguinis, Grubr, antedated by rotatorium, Maver) as well as the other flagellates, which ought to be included under the same generic name as the species of MayerGruby. We have shown in a preceding paragraph that all the hæmoflagellates known up to the present should be so included, with the exception of the new species of parasite found in fishes, for which a new generic name is necessary. These points admitted, we shall inquire into the synonyms of the genus Trypanosoma.

In I87 I Ray Lankester, ${ }^{2}$ unacquainted with the work of Gruby and his predecessors, rediscovered the $T$. rotatorium of the frog and

1 Gruby, C. R. Acad. Sciences, r. I7, November, I8+3, p. I134.

2 Ray Lankester, Quart. Journ. Micr. Sc., v. I I, I871, p. 387. 
described it under the name of Undulina ranarum. Undulina is, therefore, the first synonym of Trypanosoma.

Saville Kent, in vol. i. of his 'Manual of Infusoria' (r88o-I88I), classifies the genus Trypanosoma Gruby with two species: (I) T. sanguinis of the blood of Rana esculenta and R. temporaria (with Undulina ranarum Lank. as synonym); (2) T. eberthi (n. sp.) for the parasite of the cæcum of birds described by Eberth in I86I. ${ }^{1}$

In another part of his book Kent creates the genus Herpetomonas for the form figured by Stein in his 'Infusionthiere' (part iii., 1878) under the name of Cercomonas musca-domesticae and identified with the Bodo musca-domestice of Burnett and the C. muscarum of Leidy. The parasite seen by Lewis in the blood of rats in India is included provisionally by Kent in this new genus Herpetomonas; he calls it Herpet. lewisi. This inclusion of the flagellate of rats in the genus Herpetomonas was admitted in I 884 by Bütschli (article 'Protozoa' in the work 'Tierreich'), and was retained until recent years, at least provisionally, by Wasielewski and Senn and by ourselves in our earlier publications. $^{2}$ For example, Senn in I9oo, in the article dealing with the Flagellata in the 'Pflanzenfamilien' of Engler and Prantl, gives the following differential diagnosis of the two genera Trypanosoma and Herpetomonas :

Undulating membrane thickened in the form of a flagellum along the outer border, and not reaching to the posterior extremity of the cell $\ldots$... $\quad \ldots \quad \ldots$

Undulating membrane not thickened along the edge, and running from the anterior to the posterior extremity of the cell ...

Herpetomonas

Trypanosoma

But recent researches have shown that the retention of the genus Herpetomonas to designate the flagellates of the type lewisi is impossible for two reasons: First, the Herpetomonas musca-domestica, the type-species of the genus Herpetomonas, from its morphology (whether one accepts the description of Léger or the more recent one of Prowazek) should belong to a genus different from that containing the flagellate of the blood of rats (constant absence of the undulating membrane in the case of the parasite of the fly, etc.). Secondly, the study we made in Igor ${ }^{3}$ of the type-species of the genus Trypanosoma showed that this species did not differ, in any essential character having a real generic value, from the species lewisi (it has, in fact, all the characters which Senn gives to his genus Herpetomonas), and that there was no reason to classify that species, and all the allied species, in a new genus different from Trypanosoma.

All the facts discovered since that time, establishing, as they do, the existence of a series of trypanosomes intermediate in all respects

1 Eberth, Zeitschr. f. Wiss. Zool., v. II, 186I, p. 98. It is twenty-five years since Leuckart called attention to the fact, as Stein had already done, that this supposed trypanosome is probably a Trichomoncrs. We also agree with this view.

2 Since then certain authors (IJanilewsky, Chalachnikor, Balbiani, Iaveran) have included under the same generic name the parasites of the rat and frog.

${ }^{3}$ Laveran and Mesnil, $C: R$. Soc. Biol., June 22, I90I. 
between $T$. rotatorimm and $T$. lewisi, have confirmed our view, which has, moreover, been accepted by Senn ${ }^{1}$ and all competent authorities. The genus Herpetomonas, with the modified meaning given to it by Senn, ought to disappear. It should be retained in its original sense and with its type-species.

In I882 Grassi, ${ }^{2}$ while accepting the genus Trypanosoma Gruby, created a genus Paramcecioides to designate a special parasite of the blood of Rana esculenta, with undulating membrane, but no free flagellum. We shall show, when speaking of $T$. rotatorium, in agreement with the majority of authors (Bütschli, Danilewsky, etc.), that the form for which Grassi created a new name is a special form of $T$. rotatorium. The genus Paramcecioïdes should therefore also be considered a synonym of Trypanosoma.

In I883 Mitrophanov ${ }^{3}$ introduced a new name-the genus Hamatomonas - for two species of hæmatozoa of fishes with an undulating membrane and anterior flagellum. The author's description and drawings show clearly that the organisms in question were what we understand by the name Trypanosoma (as opposed to Trypanoplasma).

[It has already been mentioned that Lühe also places the trypanosomes of fresh-water fishes in the genus Hamatomonas of Mitrophanov. He has further created the new genus Trypanozoon for all the mammalian trypanosomes.]

This name Hamatomonas was applied by Crookshank, ${ }^{4}$ in I886, to the trypanosomes of Lewis and Evans, but Crookshank only regarded it as a sub-genus of Trichomonas Donné, I837. It is only necessary to glance at Fig. 2, 5, which represents a Trichomonas, to be convinced that such a generic assimilation is untenable. No one else has adopted Crookshank's view.

Of the work of Danilewsky (I885-I 889$)^{5}$ we shall say but little here. He disregards the Linnean rules of nomenclature and never troubles about rules of priority. He designates the flagellated hæmatozoa generally under the name of Trypanosoma, but he creates names of generic value to designate certain phases in their development. Thus he calls the flagellated forms of Trypanosoma without undulating membrane Trypanomonas. We shall find this name Trypanomonas used by Labbé as a generic term to designate a parasite from the digestive canal of leeches.

Doflein, ${ }^{6}$ in his book which appeared in July, rgor, places all the hæmatozoa with undulating membrane in the single genus Trypanosoma, but he divides it up as follows:

1 Senn, Arch.f. Protistenkunde, v. I, I902, p. 344.

2 Grassi, Arch. ital. de Biol., v. 2 and 3.

3 Mitrophanov, Biol. Centralbl., v. 3, I883, p. 35.

4 Crookshank, Journ. Roy. Micros. Soc., December, 1886, p. 91 3.

5 Danilewsky, Biol. Centralbl., v. 51, 1885, p. 529, and 'La parasitologie comparée du sang,' i., Charkov ( 1888 in Russian, 1889 in French).

6 Doflein, 'Die Protozoen als Parasiten und Krankheitserreger,' Jena, Fischer, I çoI, p. 57 . 
Chief flagellum absent, or very short and thick

Chief
present $\left\{\begin{array}{c}\begin{array}{c}\text { Undulating membrane prolonged } \\ \text { as a posterior flagellum (two } \\ \text { flagella present) }\end{array} \\ \begin{array}{l}\text { No posterior flagellum. Undu- } \\ \text { lating membrane terminating } \\ \text { with the body, or even not } \\ \text { reaching the end of the body }\end{array}\end{array}\right.$

Sub-genus Trypanosoma (sensu stricto).

Sub-genus Trypanomonas

(Danilewsky-Labbé).

\section{Sub-genus Herpetosoma}

(new).

The name Herpetosoma replaces Herpetomonas, to which Doflein restores its original significance. The sub-genera Trypanosoma and Herpetosoma correspond to the two genera Trypanosoma and Herpetomonas in Senn's classification.

In the sub-genus Trypanosoma Doflein places three species: Trypanosoma sanguinis Gruby, type-species of the genus, T. eberthi Kent, and $T$. balbianii Certes, I883. We have already stated (see p. 32, footnote) that $T$. eberthi was, in all probability, a Trichomonas. The T. balbianii was discovered by Certes $^{1}$ in 1883 , and studied anew by Möbius, Certes himself, and Lustrac. It is found in the digestive tube and crystalline style of oysters and of other bivalves. These various authors have described it as a very motile organism, with undulating membrane along the whole length of the body, but no free flagellum. We have shown ${ }^{2}$ that it was not of the nature of a flagellated Protozoon, but of a Bacterium. The chromatin substance is not condensed into a definite nucleus, but scattered throughout the protoplasm of the body. Moreover, there is, strictly speaking, no undulating membrane, but rather a loose sheath, in which the body floats, joined to the body by its two extremities. There is certainly no thickened edge to the membrane as in Trypanosoma.

[This parasite, which is perhaps a true Spirochata, and ought to be called S. balbianii, has recently been closely investigated by Perrin. ${ }^{3} \mathrm{He}$ describes sexual and 'indifferent ' forms of the parasite, as well as encystment of the female and indifferent forms. Many of his figures, however, do not readily suggest the interpretation which Perrin places upon them. Vles ${ }^{4}$ describes flagella in bunches, but recognises-as, indeed, some of his figures suggest-that these 'flagella' may be due to fragmentation of the membrane.]

Finally, Doflein gives an inexact definition of the Trypanosoma sanguinis. That definition amended, and the two species cbcrthi and balbianii omitted, the sub-genus Trypanosoma of Doflein blends with his sub-genus Herpetosoma, and, as we have already had occasion to mention, the creation of these two sub-genera is no longer justified.

As for the sub-genus Trypanomonas, its definition is the same as, only shorter than, that of the genus Trypanoplasma, which we created some months later.

1 Certes, Bull. Soc. zool. France, v. 7, 1882, p. 7.

2 Laveran and Mesnil, C. R. Soc. Biol., October 19, I9or.

${ }^{3}$ [W. S. Perrin, Proc. Roy. Soc., Series B, v. 76, 1905, pp. 368-375; Arch.f. Protistenkunde, v. 7, 1906, pp. I 3I-I 56.]

+ [Vles, C. R. Soc. Biol., November 16, '1906, p. 408.] 
We come next to the creation by ourselves, in October, Igor, ${ }^{1}$ of a new genus, Trypanoplasma, with the characters we have given above. We shall now give our justification for this new generic name.

It is quite probable that organisms answering to the characters of the genus Trypanoplasma had been previously seen by others. Chalachnikov has described varieties of trypanosomes in fishes (especially of the carp) with two flagella, anterior and posterior. There was some doubt about this description of the trypanosome of the carp until M. Plehn described a trypanoplasm of the carp. There is little doubt that Plehn saw and described the parasite seen by Chalachnikov. The latter did not create a special generic name for his bi-flagellate species. Since then other bi-flagellate trypanosomes have been described, but we have such scanty details about these parasites that their existence can hardly be considered as proved. The trypanosome of the guinea-pig of Kunstler ${ }^{2}$ is figured with two flagella, but the figure is not accompanied by any description. There were possibly also two flagella - the author is not very explicit on the point-in a trypanosome found by Labbé ${ }^{3}$ in the digestive tract of leeches which had sucked mammalian blood (from the horse or ass, Labbé thinks). Labbé compares this trypanosome with the forms described by Danilewsky under the name Trypanomonas, and he calls it Trypanomonas danileweskyi. But we now know that Trypanomonas is a particular evolution form of certain species of the genus Trypanosoma which never has two Hagella. Hence, even if the trypanosome of Labbé were really bi-flagellate, it would not be correct to adopt the generic name Trypanomonas for the bi-flagellate trypanosomes, as Doflein does. The name Trypanomonas should disappear from the nomenclature, since, taken in its original sense, it designates only particular forms of Trypanosoma.

In conclusion, we may say that the existence of parasites with undulating membrane and two flagella had not been established before our discovery, in Igor, of the hæmatozoon of the red-eye or rudd, and, in any case, it was necessary to create a new genus for these organisms, since no existing name was qualified to designate them.

\section{Section 4.-Position of the Trypanosomes amongst the Flagellates.}

The position of the trypanosomes (genera Trypanosoma and Trypanoplasma) in the classification of animals is still far from being definitely established. Let us, therefore, give the views of the best authorities.

In I88o Saville Kent ${ }^{4}$ made the Trypanosomata the first order of

${ }^{1}$ Laveran and Mesnil, C. R. Acad. Sciences, v. 133, October 29, I90I.

2 Kunstler, Bull. scientif. France et Belg., v. 31, I898, p. 206.

3 A. Labbé, Bull. Soc. zool. France, v. I6, I891, p. 229.

4 Saville Kent, 'A Manual of Infusoria,' v. I, I880-I88I. 
the class Flagellata. Thus he gives them an isolated position in his classification. We may add that this order comprises the single genus Trypanosoma, and that the parasite of the rat's blood is placed in the genus Herpetomonas (vide supra), which forms part of the family Cercomonadina.

We find almost the same thing in the article 'Protozoa,' by Bütschli ${ }^{1}$ in the 'Tierreich' of Bronn. Trypanosoma and Herpetomonas (including the parasite of the rat) are placed correctly in the same sub-order, Monadina Bütschli ; but Trypanosoma there occurs as an appendix to the family Rhizomastigina of Saville Kent (flagellates which emit pseudopodia), whilst Herpetomonas forms part of an allied family, Cercomonadina (also of Kent).

Danilewsky, ${ }^{2}$ in 1888 , insists upon the affinities of the trypanosomes, especially in their simple form (without undulating membrane) Trypanomonas, with the Herpetomonas and Leptomonas of Kent.

Senn, ${ }^{3}$ in Igoo, places the two genera Herpetomonas and Trypanosoma (with the descriptions given above) side by side in the family Oicomonadacea (=Cercomonadina of Kent), of the order Protomastigince, the simplest of the Flagellata. This family, characterized by the presence of a single flagellum, and the absence of a lip-like or collar-like process of protoplasm at the anterior extremity, is made up as follows:

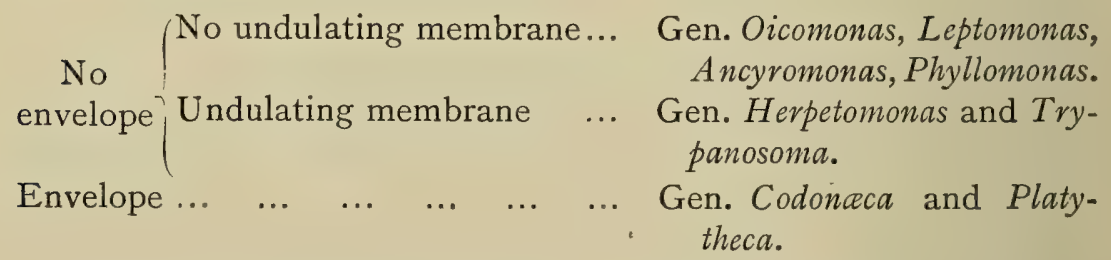

Later, in $1902,{ }^{4}$ in a review of recent researches upon the trypanosomes, he admits the identity of Herpetomonas (as he understood it in I9oo) and Trypanosoma, and he recognizes the existence of the new genus Trypanoplasma. But he thinks this genus should be placed in a family distinct from the Oicomonadacea-namely, the family Bodonace, characterized by two anterior flagella. He adds that Trypanoplasma is not exactly like any particular member of the Bodonaca; it should be placed near Bodo as a genus differentiated through having acquired a parasitic habit.

In the interval, and before the discovery of the genus Trypanoplasma, Doflein, in I9or, ${ }^{5}$ placed the trypanosomes in the order Protomonadina of Blochmann. He divides the Protomonadina into three families: (I) Trypanosomida, comprising the single genus

1 Bütschli, Bronn's 'Tierreich,' 'Protozoa,' v. I, fasc. 2, 'Mastigophora,' pp. $811-813,1884$.

${ }^{2}$ Danilewsky, 'La parasitologie comparée du sang,' i., Charkov, 1888 and I 889 .

${ }^{3}$ G. Senn, 'Die natiurlichen Pflanzenfamilien von Engler und Prantl,' Parts 202 and 203, Leipzig, 1900.

${ }^{4}$ Senn, Arch. f. Protistenkunde, v. I, 1902, p. 353.

5 Doflein, 'Die Protozoen als Parasiten, etc.' loc. cit. 
Trypanosoma; (2) Cercomonadince, with the genus Herpetomonas Kent, amended Doflein (excluding the parasite of blood of rats); and (3) Bodonida.

[Minchin ${ }^{1}$ divides the Flagellata into (a) Choanoflagellata (with one flagellum and one or two collars at its base) and (b) Lissoflagellata (flagellum single or multiple, no collar, and sometimes an undulating membrane). The Lissoflagellata comprise three orders: (I) Monadidea, (2) Euglenoidina, and (3) Phytoflagellata. All forms of parasitic Flagellata hitherto known are referred to the first of these orders, the Monadidea. Minchin gives three sub-orders of this order-namely, (I) Pantastomina, (2) Protomastigina, and (3) Polymastigina. The sub-order Protomastigina is the most important from our point of view, including as it does (I) the genus Trypanosoma and its allies (Trypanoplasma, and perhaps Trypanophis), grouped together as the family Trypanosomatida; (2) the genus Spirocheta and allied forms; and ( 3 and 4 ) the genera Herpetomonas and Crithidia.]

Léger, in his publications of 1902 and $1903,{ }^{2}$ in which he gives precise cytological details concerning Herpetomonas and creates a new genus, Crithidia (body pyriform or like a barleycorn, instead of fusiform, as in Herpetomonas), also draws attention to the close connection between these genera and the genus Trypanosoma, based no longer upon a simple external resemblance, but upon a resemblance in the cytological details. Moreover, as he has found some of these forms in the intestine of blood-sucking insects, he has suggested that they were perhaps stages in an evolutionary cycle in the life-history of the Trypanosoma.

[During the past few years flagellates of the genera Herpetomonas and Crithidia have been described by various observers in several other invertebrates.]

[In I898 Ross ${ }^{3}$ found 'amœbulæ and flagellulæ' in mosquitoes (Culex fatigans, and once in Anophelina) in India. Their habitat was chiefly the intestinal canal of the larva, pupa, and imago, and Ross states that many of them resembled trypanosomes. Since then the same or a similar organism has been seen in Anophelina by Chatterjee, Stephens and Christophers, and by Léger, who has given it the name Crithidia fasciculata.]

[The Sergents ${ }^{4}$ describe a new flagellate, which they call Herpetomonas algeriense, from the intestine of many Culex pipiens, and one Stcgomyia fasciata reared from larvæ in their laboratory. Two forms were seenelongated motile forms and spherical motionless forms-but they both differed from Crithidia fasciculata in having the centrosome posterior to the nucleus. The Sergents also found in the digestive tube of a larva of Anopheles maculipennis a flagellate closely resembling Herpetomonas jaculum Léger.]

1 [Minchin, article on the 'Parasitic Protozoa,' in Allbutt and Rolleston's 'System of Medicine,' v. 2, part 2, I907. I am indebted to Professor Minchin for allowing me to see the proof-sheets of this article before publication.]

${ }^{2}$ Leger, C. R. Acad. Sciences, March, 1902 ; C. R. Soc. Biol., April, 1902 ; Arch. f. Protistenkunde, v. 2, 1903 .

3 [R. Ross, Journ. Hyg., v. 6, 1906, pp. I0I-108.]

4 [Ed. and Et. Sergent, C. R. Soc. Biol., v. 60, I906, pp. 291-293.] 
[Similar parasites have been found in a blow-fly by Prowazek ; in the gut of a sheep-louse (Melophagus ovimus) by Pfeiffer ; ${ }^{2}$ in the body-cavity of the silkworm (Bombyx movi) by Levaditi $; 3$ in fleas which had fed on jerboas harbouring the Hamogregarina balfouri, by Balfour $; 4$ in the gut of Stomoxys, in Uganda, by Gray ${ }^{5}$ and in the intestine of Tabamus glaucopis by Léger, ${ }^{6}$ who has named this parasite Herpetomonas subulata.]

[Novy, McNeal, and Torrey ${ }^{7}$ examined over 800 mosquitoes, after allowing them to feed on rats, guinea-pigs, and pigeons, which were certainly free from hæmatozoa, and found that I 5 per cent. had an intestinal flagellate infection. In some cases masses of rosettes, with centrally disposed flagella, were met with. Different forms of flagellates were found, the most common being Crithidia fasciculata, and a species probably identical with Herpetomonas subulata. From their researches on the cultivation of the trypanosomes of birds, Novy and McNeal maintain that the flagellates ('trypanosomes') seen in the stomach and digestive tract of mosquitoes, tsetse-flies, lice, leeches, etc., are 'cultural ' forms in vivo, corresponding to those obtained in vitvo.]

[Most, if not all, of the parasites above mentioned occur in two forms: (I) a flagellated monadine form (the body being long and acicular in most of the Herpetomonas, pyriform in the Crithidia, and rarely intermediate between these two forms as in Herpetomonas (or Crithidia) minuta of Tabanus tergestinus ; (2) a gregariniform resting stage, with a rudimentary or no flagellum and two chromatic corpuscules, the parasite resembling the Leishman body of kala-azar and, to some extent, the piroplasms.]

[As has already been mentioned, rosettes of parasites occur in some cases, and, moreover, in $H$. subulata, H. bombycis, and in the parasite of the sheep-louse (Melophagus ovimus), a rudimentary undulating membrane has been described.]

[We come lastly to the interesting fact that flagellates (trypanosomes) have been found in the gut of tsetse-flies. This is important in view of the statements of Gray and Tulloch and of Koch that ingested mammalian trypanosomes (T. gambiense, $T$. brucei) undergo developmental (? sexual) changes in tsetse-flies. Novy and Minchin, Gray, and Tulloch, from an extended study of the trypanosomes of Glossina palpalis (their results are given in detail in Chapter XVIII.), have come to the conclusion that the trypanosomes found in freshlycaught tsetse-flies have nothing to do with $T$. gambiense. Novy ${ }^{8}$ thinks that they are 'cultural' forms of harmless non-parasitic flagellates, corresponding to the equally harmless Herpetomonas and Crithidia observed by him in mosquitoes.]

1 [Prowazek, Arb. a. d. kaiserl. Gesund., v. 20, part 3 ; trans. in Journ. Trop. Med., v. 8.]

${ }_{2}$ [Pfeiffer, Zeitschr.f. Hyg. und Infektionskrank., v. 50, 1905, p. 324.]

3 [Levaditi, C. R. Acad. Sciences, v. I4I, 1905, p. 63I.]

${ }^{4}$ [Balfour, "Second Report of the Wellcome Research Laboratories at Khartoum,' 1906, pp. 103-110.]

${ }_{5}^{5}$ [Gray, Sleeping Sickness Comm. of the Roy. Soc., Rep. No. 8, 1907, Art. 21, App. 3, p. I33; also Proc. Roy. Soc., Series B, v. 78, 1906, p. 254.]

${ }^{6}$ [Léger, C. R. Soc. Biol., v. 58, 1904, p. 6r3; abstract in Bull. inst. Past., v. 3, I905, p. 190.]

7 [Novy, McNeal, and Torrey, Journ. Hyg., v. 6, 1906, p. I10.]

8 [Novy, Journ. Infect. Dis., v. 3, 1906, pp. 394-4II.] 
Schaudinn ${ }^{1}$ has also suggested that Trypanosoma is closely related to both Herpetomonas and Trypanoplasma.

To sum up, all authors are agreed in recognising the connection between Trypanosoma and the genera without undulating membrane, such as Herpetomonas of Kent and Crithidia of Léger, which are nearly all parasitic in the intestine of insects.

Indeed, everything tends to show that this is the correct view. It is only necessary to compare the figures which Léger gives of Herpetomonas and Crithidia (two of them are reproduced in Fig. 4,

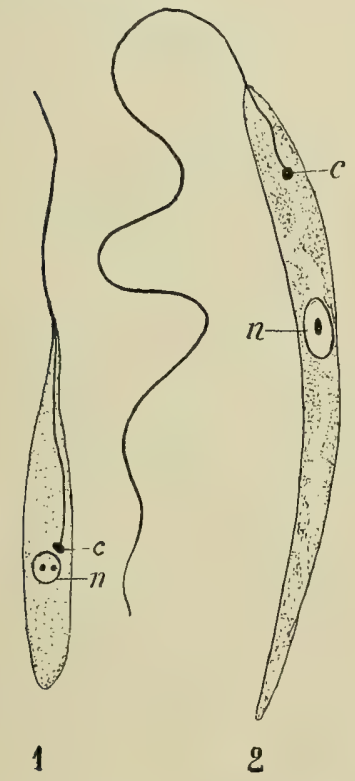

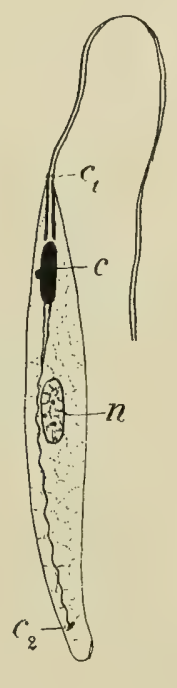

3

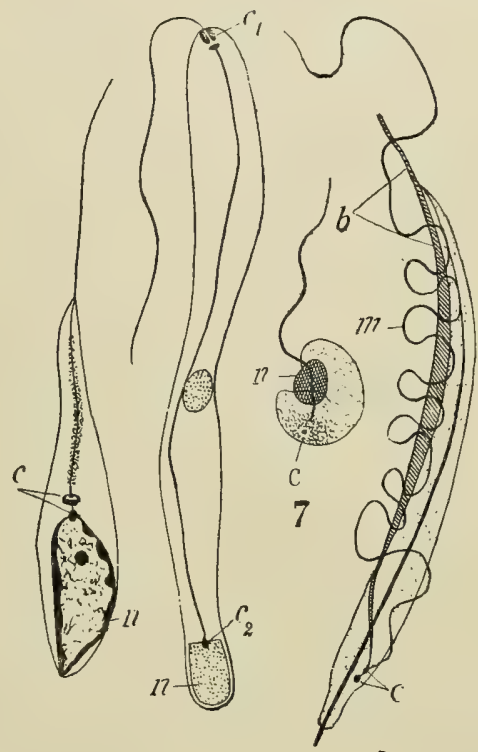

5

6

Fig. 4.-I-3, Flagellates: 4-6, Spermatozoa; 7 , Flagellated Spore of NOCTILUCA.

I. Crithidia minuta Léger, from the gut of Tabanus tevgestinus (after Léger). 2. Herpetomonas jaculum Léger, from the gut of Nepa cinevea (after Léger). 3. Herpetomonas musce-domestice Burnett (after Prowazek). 4. Immature spermatozoon of Rana fusca (after Broman). 5. Immature spermatozoon of the snail, Helix pomatia (after von Korff). 6. Adult spermatozoon of Bombinator (after Broman). 7. Flagellated spore of Noctiluca (after Ishikawa). $n$, Nucleus ; $c, c_{1}, c_{2}$, centrosomes ; $b$, supporting rod; $m$, undulating membrane.

$I$ and 2) with those of the developmental or cultural forms of $T$. lewisi, to be struck by their great similarity.

The difference in the adult forms consists only in the presence of an undulating membrane in Trypanosoma and its absence in the genera studied by Léger. ${ }^{2}$ This is to be regarded primarily as a character of adaptability, and correlated with the parasitic mode of existence of trypanosomes. It has appeared, doubtless for analogous reasons, in a group of organisms very different from the trypano-

1 Schaudinn, Arb. a. d. kaiserl. Gesund., v. 20, 1904, p. 387.

2 [We have seen, however, that a rudimentary undulating membrane has since been described in certain species of Herpetomonas. This makes the resemblance of these organisms to Trypanosoma still greater.] 
somes-that containing Trichomonas. We cannot agree with Kent, Danilewsky, and Doflein, who see in it a sufficient reason for placing Trypanosoma in a separate family, and we adopt the view of Senn, who regards Trypanosoma as a special genus of the family Oicomonadina or Cercomonadina.

Amongst all the members of that family, trypanosomes included, movement takes place with the flagellum foremost. It is, therefore, natural to suppose that in all of them, including trypanosomes, the flagellated end is morphologically the anterior end. Recently, however, it has been suggested by some that the morphologically anterior end of Trypanosoma is the non-flagellated one. ${ }^{1}$ That opinion is based on: (I) a consideration of Trichomonas, ${ }^{2}$ in which the undulating membrane appears as a flagellum directed backwards, its free end being posterior (see Fig. $2,5 f$ ); (2) the fact that in Herpetomonas, as figured by Léger, the centrosome is close to the anterior extremity, and that it should be the same in Trypanosoma. Facts do not lend themselves to such an interpretation. The development of $T$. lewisi shows us, amongst other things, forms identical with the Herpetomonas of Léger, where the centrosome, at first situated in front of the nucleus, travels gradually towards the posterior end of the body, taking along with it the flagellum, which elongates and at the same time becomes separated laterally from the body of the parasite, so that it comes at last to be joined to it by merely a thin ridge-the undulating membrane. There is thus no evidence of a turning backwards of the flagellum ; and, as it is evident that the anterior extremity of the Herpetomonas-form is likewise the anterior extremity of the adult form, it must be agreed that it is always the flagellated end.

[Léger, ${ }^{3}$ Lühe, and others regard trypanosomes as diphyletic in origin. Those with a morphologically anterior flagellum-recognisable by their becoming attached or fixed by the flagellated end-would be derived from a Herpetomonas or Crithidia, by the migration backwards of the centrosome and the gradual development of the undulating membrane; whereas those trypanosomes with a morphologically posterior flagellum - such parasites becoming fixed by their non-flagellated end-would be derived from a Trypanoplasma by the loss of its anterior flagellum. Mesnil ${ }^{4}$ does not agree with this view, but regards all trypanosomes as having the flagellum morphologically anterior, and therefore considers the genus Trypanosoma monophyletic. In support of his view, he states that no importance can be attached to the pole of fixation, since in the same species one may find rosettes of trypanosomes with the flagella directed centripetally, and others with the flagella at the periphery. (See later under $T$. lewisi.)]

It remains now to review the genus Trypanoplasma. Senn places it in Bodonace; Schaudinn, on the other hand, regards it as closely

1 Sambon, Journ. Trop. Med., v. 6, July I, 1903, p. 205, note; J. Guiart, ibid., v. 7, January $\mathrm{I}$, 1901, p. 4. [See also Lïhe, Minchin, and Woodcock, in the articles previously quoted.]

2 One can add, in the light of Léger's recent work, ' and of the Trypanoplisma.'

3 [Léger, C. K. Soc. Biol., v. 58, 1904, pp. 613-617.]

4 [Mesnil, Bull. Inst. Past., v. 3 , 1905, p. 100.] 
allied to Trypanosoma. He even regards Trypanoplasma as the most primitive form of all the hæmoflagellates. The trypanosomes are derived from it by the atrophy of one of the flagella, and by the diminution in size of one of the originally equal chromatin masses. ${ }^{1}$ The genus Trypanophis (see p. 26, footnote 3) would represent one of the stages in the evolution : one of the flagella is reduced in size, and the two chromatin masses are no longer equal.

The Trypanosoma noctuce of the owl would represent another stage: the two chromatin masses are unequal, the one being derived from the other by heteropolar mitosis; but the smaller mass has still a true nuclear structure, and is not reduced almost to a dot, as it is in Trypanosoma. In other respects $T$. noctuce is a true Trypanosoma.

As for the genus Herpetomonas, Schaudinn, basing his opinion on the recent researches of Prowazek, ${ }^{2}$ who describes two flagella very close together at the anterior extremity (Fig. 4, 3), regards it as being probably derived from the primitive form Trypanoplasma, by the approximation of the flagellar apparatus leading to the fusion of the opposite poles.

The recent researches of Léger $^{3}$ lead to the conclusion that Trypanoplasma should be regarded as a Bodo (see the figures of Bodo lacerte recently published by Prowazek ${ }^{4}$ ), of which the flagellum, directed backwards, is closely applied to the body, just as Trichomonas may be looked upon as a Trichomastix with the flagellum directed backwards and applied to the body.

As to the affinities of Trypanoplasma and Trypanosoma, they appear to us, in the present state of our knowledge, difficult to define accurately. The argument of Léger, who looks upon the trypanosomes as trypanoplasms which have lost their anterior flagellum, and conquently as organisms with the flagellum morphologically posterior, cannot, in our opinion, prevail against that based, on the one hand, upon the development of $T$. leverisi (vide supra), and, on the other hand, upon the comparative morphology of different species of the genus (e.g., transvaaliense and rotatorium), which leads to the conclusion that the flagellated end is the anterior.

It is thus possible that the relations of Trypanoplasma and Trypanosoma are not so close as Schaudinn and Léger suppose them to be, and that the points of similarity are partly of the nature of adaptability to environment.

In conclusion, the trypanosomes appear to us to be the representatives of one of the simplest and most primitive groups of the flagellates.

We cannot leave this subject without making reference to the

1 We have already made this suggestion as to the origin of the centrosome in trypanosomes. We shall refer to it again later.

2 Prowazek, Arb. a. d. kaiserl. Gesund., v. 20, 1904, p. 440.

3 Léger, C. R. Acad. Sciences, v. 138, March and April, 1904

4 Prowazek, Arb. a. d. kaiserl. Gesund., v. 2 I, 1904 (Plate II., Figs. 43-46). 
recent work of Schaudinn ${ }^{1}$ (loc.cit.), in which he regards the trypanosomes (and also the spirochætes which, according to him, have fundamentally the same structure as trypanosomes) ${ }^{2}$ as forming part of the developmental cycle of intracorpuscular hæmatozoa (Hæmocytozoa). That view is based upon observations made upon two particular species of intracorpuscular parasites of the little owl (Athene noctua), which both complete their evolutionary cycle in the body of the common mosquito, Culex pipiens.

In one species (Trypanosoma noctuce), the ookinete, or product of fertilization of a female by a male element, is transformed into a true trypanosome in the stomach of the mosquito. Fig. 5, copied from Schaudinn, shows the different stages of this transformation, which is accomplished in the following manner: The nucleus

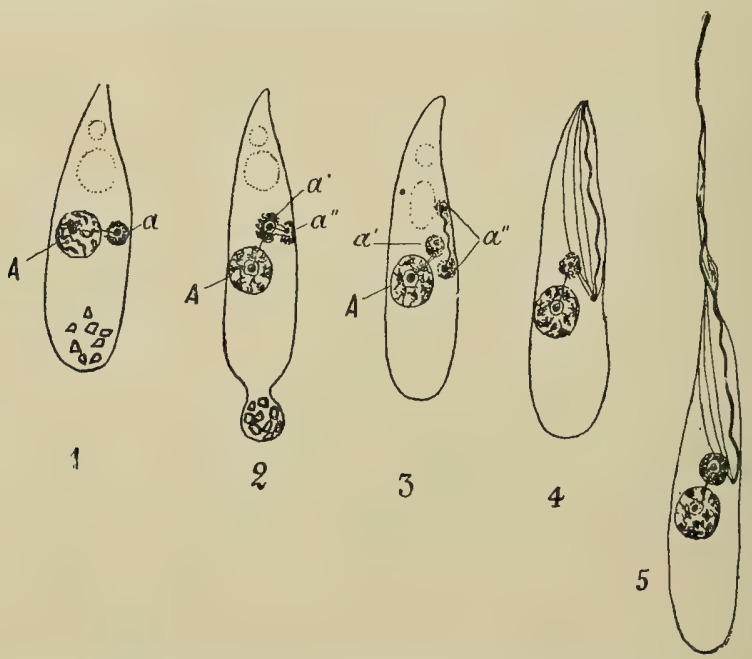

Fig. 5. - Transformation of an Ookinete of an Intracorpuscular Parasite into a Trypanosome (after Schaudinn).

divides, by heteropolar mitosis, into $A$ and $a$ (Fig. $5, I$ ) the central filament of the division spindle persisting. The part $a$ divides in its turn into $a^{\prime}$ and $a^{\prime \prime}$ (Fig. 5, 2), the new central filament again persisting. $A$ is the principal nucleus, $a^{\prime}$ the accessory nucleus or blepharoplast. Finally, $a^{\prime \prime}$ divides by mitosis, and gives rise to the whole of the flagellar apparatus (Fig. 5, 3, 4, 5). The edge of the undulating membrane and the free flagellum are the central filament of the spindle greatly elongated (which explains how it is the flagellum has a chromatin-staining reaction). The peripheral filaments of the division spindle also persist.

${ }^{1}$ [A complete translation of this paper by Schaudinn appeared in the numbers of the Journ. Trop. Med., June I to November 1, 1904. A very full analysis of it is given by Woodcock in his article in the Quart. Journ. Micr. Sc., v. 50. At the express wish of the authors, I have simply translated what they wrote in the original of their book, without making any allerations. I have, however, made some additions to the authors' original short résumé.-ED.]

2 [But Schaudinn afterwards abandoned this view that all spirochetes would be found to have fundamentally the same structure as trypanosomes.] 
In the other species ${ }^{1}$ there is formed on the surface of the ookinete a large number of small trypanosomes (like those represented in Fig. 6, $I$, very highly magnified). These trypanosomes elongate, divide longitudinally (Fig. 6, 2), often forming pairs joined by their posterior extremities (Fig. 6,3), and in the same straight line. Thus are produced, says Schaudinn, parasites indistinguishable from true spirochætes, having the power when they are in pairs of moving equally well in either direction, and capable of further subdivision (Fig. 6, 4 and 5). They are of all sizes (Fig. 6, 2, 3, and 6 to 8), and some are so small that they are recognisable under the microscope only when agglomerated into rosettes, and one can easily imagine, says Schaudinn, that they could pass through a Chamberland filter.

[In the above brief résumé of Schaudinn's paper several interesting facts have been omitted by Laveran and Mesnil. (A) Trypanosoma noctua.-

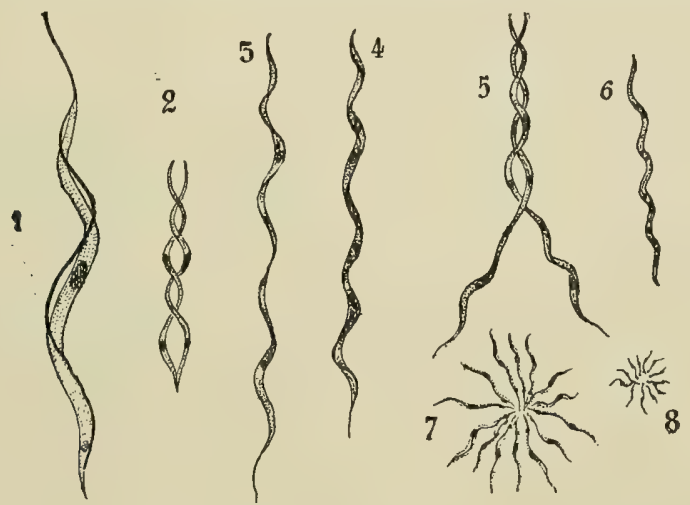

Fig. 6. - Transition of Trypanosomes into Spirochetes (after Schaudinn).

(I) When an infected mosquito feeds on an owl, it injects into the blood of the bird 'male,' young 'female,' or ' indifferent' forms of the trypanosome. The male forms rapidly die; the indifferent forms multiply; and the young females mature, the last two passing through alternate intracorpuscular and extracorpuscular phases. For six days and nights the intracorpuscular (Halteridium) stage in the owl alternates with the extracorpuscular or trypanosome stage, the former occurring in the peripheral blood by day, the latter in the internal organs by night. The 'indifferent' forms of the parasite are fully developed at the end of this six days' alternation, but the 'female' forms mature more slowly. (2) Female gametocytes may multiply by parthenogenesis, and give rise to ' indifferent' trypanosomes, or to 'male' or 'female' forms. To undergo their normal development, however, the male and female gametocytes must be swallowed by a mosquito with a meal of blood. Both forms of gametocyte contain melanin pigment, which is got rid of during the subsequent development of the zygote. (3) Eight slender microgametes (of complex structure) are formed from the microgametocyte. (4) The female gametocyte, soon after arriving in the mosquito's stomach, undergoes

${ }^{1}$ [This parasite of the white corpuscles of the owl has been called Spirochata, or Trypanosoma ziemanni (Schaudinn), Hamamoeba ziemanni (Laveran), and Leucocytozoon ziemanni (Lühe).] 
nuclear reduction, and becomes a ripe macrogamete. It remains spherical, but develops no flagellar apparatus, and is fertilized by a microgamete, with fusion of the two nuclei. When fertilization is complete, the resulting ookinete may develop in one of three ways : it may become an 'indifferent" or a 'female' trypanosome, or it may give rise to eight small 'male' forms. These male trypanosomes soon die off in the stomach of the mosquito, as they also do in the blood of the owl. The 'indifferent' and 'female' forms gradually pass along the gut of the mosquito, and eventually break through the gut wall, getting into the body-cavity. Most of the trypanosomes eventually reach the proboscis, ready to infect a bird the next time the mosquito sucks blood; but some of the parasites may be carried to the mosquito's ovaries, and so give rise to hereditary transmission of the infection.]

[(B) Spirochata ziemanni.-In addition to the spirochætiform and trypaniform stages described above, Piroplasma-like resting stages-which resemble the Leishman body of kala-azar-are figured by Schaudinn; also very large sexual forms, which after conjugation produce a zygote, and ultimately, by sporulation, an enormous number of spirochætes. These spirochætes flood the gut and Malpighian tubes of the mosquito, and multiply by fusion, as shown in Fig. 6.]

These facts are not in agreement with the ordinarily accepted views, and require confirmation.

According to Schaudinn, trypanosomes or spirochætes represent the asexual multiplication forms (produced by binary longitudinal division) of the intracorpuscular hæmatozoa in question, both in the mosquito and in the bird.

We shall confine ourselves to this résumé of Schaudinn's work, referring the reader to the original memoir for a more complete account of the facts and of the conclusions drawn from them by the author.

[Since Schaudinn published his paper giving the remarkable lifehistory of these two parasites of Athene noctua, some of his results and conclusions have received confirmation in certain quartersnotably from the work of the Sergents-but have been severely criticized by Novy and McNeal, Ross, and others. ${ }^{1}$ There are two possible sources of error in the experiments of Schaudinn : (I) Some of the mosquitoes may have been-and probably were-infected with intestinal flagellates (Herpetomonas or Crithidia), which, as we have already seen, frequently occur in species of Culex. After the ingestion of the blood of an owl these flagellates, if present in the mosquito's gut, would multiply. This might lead to the erroneous conclusion that the parasites found in the mosquito after feeding were derived from others present in the blood of the bird. (2) The observations of Novy and McNeal, Thiroux, Billet, ${ }^{2}$ and others show

${ }^{1}$ [The following are some of the papers bearing on Schaudirn's work on the 'Alternation of Generations and Change of Host': Billet, C.R. Soc. Biol., v. 57 , 1904, p. 16r; Brumpt, ibid., v. 57, p. 165, and v. 61, 1906, p. 167; Ed. and Et. Sergent, ibid..v. 57. p. 164, and v. 58, p. 57 -all abstracted by Mesnil in Biull. Inst. Past., v. 2, 1904, pp. 724, 725; Novy and McNeal, 'On the Trypanosomes of Birds,' Journ. Infect. Dis., v. 2, 1905, pp. 256-308; also 'Trypanosomes of Vlos. quitoes,' Journ. Hyg., v. 6, 1906, p. IIO; Ross, ibid., p. 96 ; 'Tniroux, Ann. Inst. Pest., 1905, pp. 65-83.]

2 [Billet, C. R. Acad. Sciences, October 10, 1904.] 
that birds, frogs, etc., frequently suffer from a double or even a multiple infection, as with one or more species of trypanosomes together with one or more species of Hæmocytozoa. The trypanosomes, moreover, may be very scanty in the blood, so that only one or two are found on examining several slides, or they may only be detected by cultivating the blood arificially in vitro, or in the gut of some blood-sucking insect (mosquito, leech). Two conclusions are possible from these observations: (I) That the flagellates are stages in the life-cycle of the intracorpuscular parasites, as believed by Schaudinn and his followers; or (2) that the flagellates and the intracorpuscular parasites are quite distinct, and that the animals harbouring them are suffering from a mixed infection, as believed by Novy and McNeal, Ross, Thiroux, and their adherents. When such blood containing both parasites is artificially cultivated or taken up by an insect and only flagellates are found subsequently, the absence of Halteridium, Drepanidium, etc., would be due to the fact that these die off, owing to unfavourable conditions.]

[Novy and McNeal conclude from their observations that Schaudinn has wrongly interpreted his facts in recognising a genetic relation between trypanosomes and Cytozoa. They interpret his results quite differently, and say that ' he has cultivated trypanosomes in vivo, and has obtained forms which agree fully with those obtained by us (Novy and McNeal) in artificial culture in vitro.']

[Mesnil ${ }^{1}$ makes the following remarks in this connection: 'In our opinion, this is going too far. It is highly probable-and this is, we know, Schaudinn's opinion - that there are avian trypanosomes which always occur in the trypanosome form; similarly, there are Cytozoa without trypanosome-like stages. But we cannot conclude from Novy and McNeal's interesting observations that Schaudinn's conclusions are incorrect, obtained as they are by the zoological method and based entirely upon observations made on mosquitoes. There was no doubt about the transformation of an ookinete into a trypanosome, the melanin pigment helping to trace the stages in the evolution; it cannot be said, therefore, that in this case it is the trypanosomes from the bird's blood which have grown.']

[The SPIRochætes.-These organisms are possibly closely related to the family of trypanosomes, and may in some cases-as appears to follow from Schaudinn's observations upon Spirochata (Trypanosoma or Hamamoba) ziemanni-even be a stage in the lifecycle of a trypanosome. At first Schaudinn suggested that on careful examination, all spirochætes would be found to be similarly allied to true trypanosomes. In a later paper ${ }^{2}$ he recognises, however, that the 'spirochætiform' stage of this parasite of the owl is far

1 [Mesnil, Bull. Inst. Past., v. 3, 1905, pp. 363-367.]

2 [Schaudinn, Deutsche med. Wochenschr, October 19, 1905, p. I665; abstract by Mesnil in Bull. Inst. Past., v. 3, p. 879.] 
removed from the true spirochætes (such as Sp. plicatilis Ehrenberg, which is the type-species), and that it has only very distant phylogenetic relations with them.]

[Numerous 'spiral' organisms are now known, but there is still much difference of opinion as to their true nature, especially in the case of the mammalian parasites. This is due to the fact that some of them closely resemble the genus Spivillum of Bacteria, and further, being very slender organisms, it is exceedingly difficult to make out the details of structure-such as the presence of flagella or of an undulating membrane--which are relied upon to distinguish between the genera Spirillum and Spirochata. Thus, to take one example, the organism of relapsing fever, for a long time known as the Spirillum or

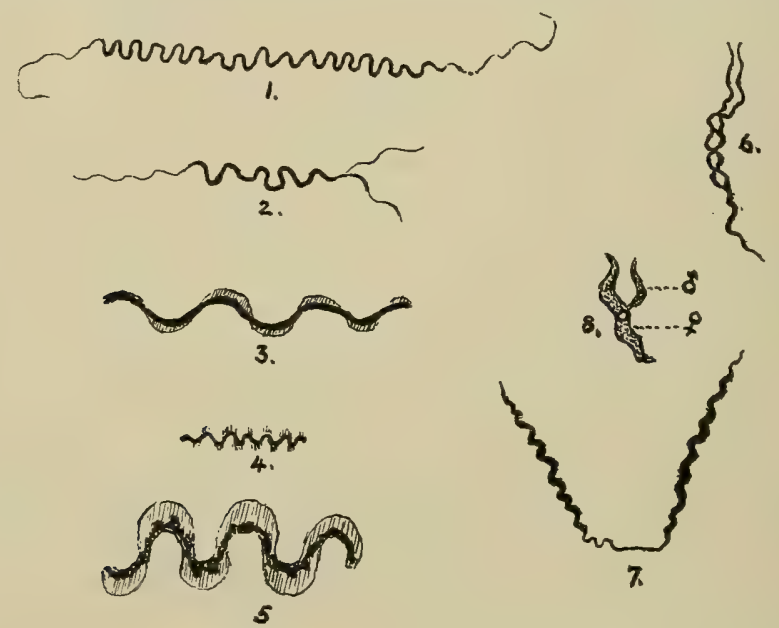

Fig. 7.-Treponema pallidum and various Spirochetes.

I. Treponema pallidum, ordinary or 'indifferent' form. 2,6, and 7 . Stages in division of the Spirocheta-form of Treponema-pallidum. 3. Spirochsta refringens. 4. Spirochata of ulcerated cancers (note the blunt ends and the undulating membrane). 5. S. plicatilis, the end of a long parasite. 8. Conjugation of the male and female elements (Trypanosoma-form). The small clear spaces in these sexual forms are regarded as representing the nucleus. (I-5, after Schaudinn ; 6-8, after Krzysztalowicz and Siedlecki).

Spirochata obermeieri, had come to be regarded as a true spirochæte ; recently, however, Borrel and Zettnow have described flagella in $S p$. obermeieri and in the Sp. gallinarum of fowls, in which case these organisms should be regarded as Bacteria and not as Protozoa. ${ }^{1}$ Novy and Knapp, ${ }^{2}$ in a recent comprehensive paper upon $S p$. obermeieri and allied forms, express this opinion and recognise as a distinct species, to which they give the name Spirillum duttoni, the parasite of African tick fever. Breinl and Kinghorn, ${ }^{3}$ while retaining the generic name Spirochata, agree with Novy and Knapp that these are distinct species, but Koch regards the two organisms as identical.]

1 [Other details of structure, such as the absence of undulating membrane and of chromatin, point to the same conclusion.]

${ }_{2}^{2}$ [Novy and Knapp, Journ. Infect. Dis., v. 3, 1906, part iii.]

3 [Breinl and Kinghorn, Liverpool Sch. Trop. Med., Memoir 21, I906, pp. I-52, with full bibliography.] 
[Fig. 7, 5 shows the end of a Spirochata plicatilis, the type-species of the genus. The characteristic features (according to Schaudinn) of a true spirochæte are exhibited by this organism-blunt ends, no flagella, presence of undulating membrane. The chromatic substance extends along the whole length of the body.]

[The most interesting and important of this group of organisms is the one first described by Schaudinn and Hoffmann ${ }^{1}$ in syphilis, and called by them Spirochata pallida. A later study of this organism led Schaudinn to conclude that S. pallida differs from all the other spirochætes studied by him, viz., S. plicatilis, S. refringens (Fig. 7, 3), which often accompanies $S$. pallida in scrapings of superficial syphilitic lesions, the spirochæte of Vincent's angina, the spirochæte of ulcerated cancers (Fig. 7, 4), etc., in that it shows no trace of an undulating membrane, its transverse section is circular, and it has a long flagellum at each end. In one case (see Fig. 7,2$)$ two flagella were seen at one end, suggesting longitudinal division.]

[All the other spirochætes (Fig. 7, 3 to 5) have a well-developed undulating membrane, the body is flattened, and flagella are entirely absent. Schaudinn therefore thinks that $S$. pallida should be removed from the genus Spirocheta, and has created the new genus Treponema, ${ }^{2}$ so that the organism is now known as the Treponema pallidum. It may be mentioned that Castellani has discovered a similar parasite in yaws, to which he has given the name Spirocheta pertenuis.]

[Treponema pallidum (Fig. 7, $, 2,6$ and 7 ) is a very delicate, actively mobile organism, almost transparent in the living condition. It has a long, slender, corkscrew-like body with pointed ends, and according to Schaudinn, there is a long slender flagellum at each end. The length of the organism varies from $4 \mu$ to $I 4 \mu$; its curves vary from ten to twenty or more in number. The curves are narrow, sharp, and corkscrew-like, and are always more numerous than in S. refringens, in which the curves are less abrupt and are broad and undulating.]

[To show the Treponema in scrapings of syphilitic lesions, the films should be fixed in absolute alcohol for ten minutes, or, better, in osmic acid vapour for a few seconds (Schaudinn), and then stained by the Giemsa or Borrel-blue method (see Chapter II.).]

[Krzysztalowicz and Siedlecki ${ }^{3}$ describe trypanosome-like forms (which they regard as sexual phases) in addition to the ordinary spirochætiform parasites, which they say are asexual. The latter divide by longitudinal fission (Fig. 7, 2 and 6), and frequently the two daughter individuals may remain attached by one end. Other appearances these authors interpret as phases of conjugation (Fig. 7,8$)$. Convinced of the trypanosomal nature of this parasite, they propose the name Trypanosoma luis for it. In

1 [Schaudinn and Hoffmann, Deutsche med. Wochenschr, May 4, I905, p. 7 II.]

2 [Spironeme was suggested by Vuillemin and adopted by Schaudinn, but was found to be preoccupied.]

${ }^{3}$ [Krzysztalowicz and Siedlecki, Bull. Acad. Sc. Cracovie, November, 1905, pp. 713-728 ; abstract by Mesnil, in Bull. Inst. Past., v. 4, 1906, p. 204.] 
the case of such a delicate organism as the Treponema pallidum, one has to be careful not to mistake adventitious objects, such as shreds of tissue or fibrin, or fragments of chromatic substance from the nuclei of leucocytes, for phases of the parasite. As Minchin remarks, these observations urgently need confirmation.]

[It is an interesting fact that dourine, a trypanosome disease of horses and mares (see Chapter X.), is also conveyed by coitus, and that the nervous symptoms seen during life, as well as the microscopic lesions found post-mortem in the nervous system, very closely resemble those seen in syphilis (Mott):]

[The Leishman-Donovan Body.-This organism, the parasite of kala-azar, tropical splenomegaly, and cachexial fever, was first discovered by Leishman in I9oo, in smears of the splenic pulp of a patient who had died of Dum-dum fever. Leishman's first account was published in $1903^{1}$ and since then the parasite has been found in similar cases in many other parts of India, ${ }^{2}$ in Arabia and Egypt, ${ }^{3}$ in China, ${ }^{4}$ and in the Sudan ${ }^{5}$ and other parts of Africa. Similar organisms have been found in the tropical skin lesion known as tropical ulcer, Delhi boil, bouton d'Alep, etc. $\left.{ }^{6}\right]$

[The parasite is usually intracellular and occurs in greatest numbers in the spleen, then in the liver, bone-marrow, and the lymphatic glands. It is much more rarely found in the other organs. Statham, ${ }^{7}$ who studied its distribution in the organs very carefully, could not find the parasite in the blood, urine, fæces, or small cutaneous ulcers during life. He says the parasite is never free or inside the red corpuscles, but other observers (Laveran and Mesnil, Donovan) describe endoglobular as well as free forms-the latter being possibly liberated by rupture or disintegration of the cells containing them. Statham found them in the endothelial cells of the capillaries, and also in the reticular cells, myelocytes, and mononuclear and polymorphonuclear leucocytes of the organs previously mentioned. In the spleen the parasites often occur in very large cells, 'macrophages' (see Fig. 8, I), which may contain as many as ${ }^{5} \mathrm{O}$ or more of these bodies.]

[The Leishman-Donovan body is rounded, oval, or pyriform, and measures $2 \mu$ to $3{ }^{\circ} 5 \mu$ in length by ${ }^{\circ} 5 \mu$ to $2 \mu$ in breadth. The cytoplasm is described as being finely granular and is sometimes vacuolated. There are two chromatic corpuscules, the larger of which is more or less spherical and stains faintly, while the smaller

1 [Leishman, Brit. Med. Journ., May 30, 1903, p. 1252.]

2 [Donovan, Lancet, September I0, I904, and January 2I, I905, giving the earlier bibliographical references on the subject; Christophers, Sc. Mem. of the Govt. of India, Nos. 8, I1, and 15, 1904, 1905; James, ibid., Nos. 13 and 19, I905; Rogers, Brit. Med. Journ., May 28, 1905, p. 1249; Discussion at the B.M.A. Meeting, Brit. Med. Journ., September 17, 1y04, pp. 642-658 and 687,688.]

${ }^{3}$ [Phillips, Journ. Trop. Med., August I, 1904, p. 236.]

4 Kerr, Journ. Trop. Med., v. 8, I905, p. 220.]

5 [Neave, Brit. Med. Journ., May 28, 1904, p. 1252.]

6 [Wright, Journ. Med. Research, Boston, v. 10, 1903, p. 472 ; Mesnil, Nicolle, and Remlinger, C. R. Soc. Biol., v. 57, 1904, p. I67.]

${ }^{7}$ [Statham, R. A.M. C. Journ., v. 5, August and September, 1905.] 
is usually rod-shaped and stains very deeply. Multiplication takes place commonly by simple binary fission, but multiple division may also occur.]

[The interesting point about this organism, and the reason for describing it in association with the trypanosomes and other flagellates, is that in cultures of the Leishman-Donovan body typical
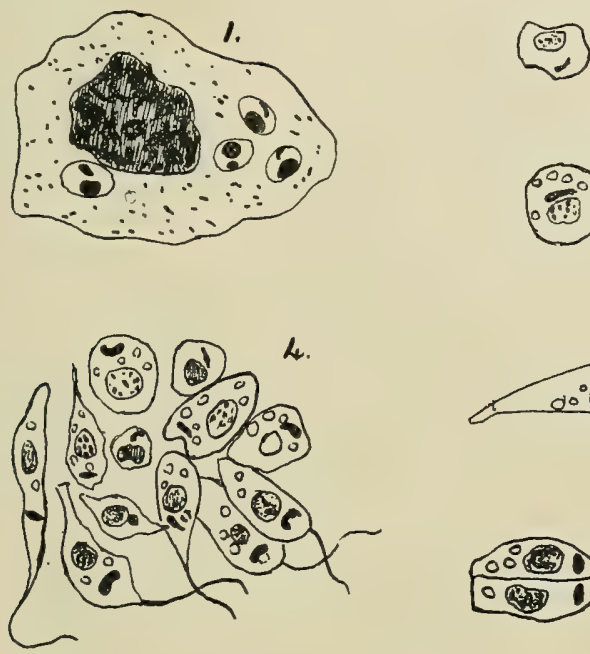

2
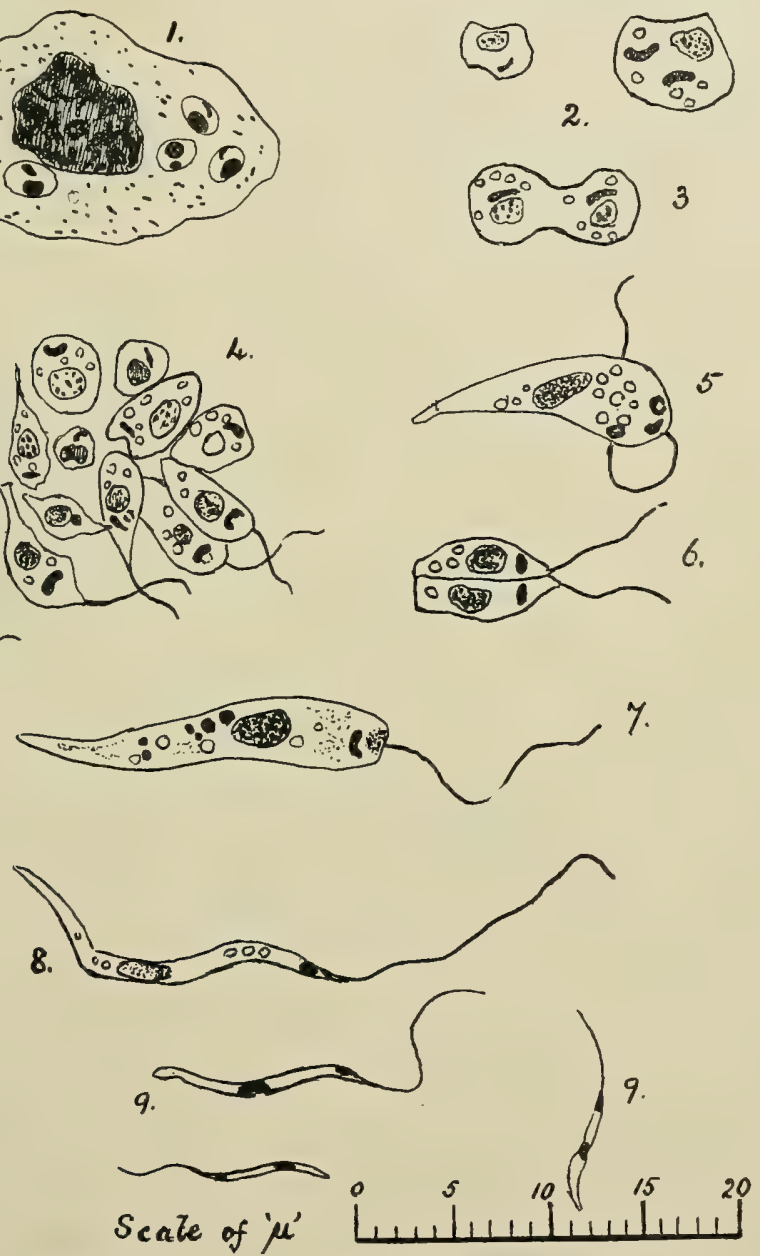

Fig. 8.-The Development of the Leishman Bodies in Cultures (After LEISHMAN AND STATHAM).

I. Large splenic cell containing parasites. 2, 3. Stages of development in cultures, 3 showing a stage of division. 4. Group of young parasites, some flagellated. 5, 8. Fully developed flagellated parasites. 6. Division forms. 7. A fully-developed parasite, showing the disposition and shape of the chromatic granules. 9. Small flagellated 'spirillar' forms.

flagellates are produced. Rogers ${ }^{1}$ first discovered this fact, and his observations have been confirmed by Chatterjee, ${ }^{2}$ Christophers, and

1 [Rogers, Quart. Journ. Micr. Sc., v. 48, I904, p. 367 ; Lancet, I904, ii., p. 215 ; ibid., June 3, I905, pp. 1484-1487: Proc. Roy. Soc., Series B, v. 77, I906, pp. $284-293$; Brit. Med. Journ., and Lancet, February 23 and March 2 and 9, I907.]

2 [Chatterjee, Lancet, December 3, I904, and January 7, I905.] 
Leishman and Statham. ${ }^{1}$ Rogers has found that the best conditions for culture are citrated human splenic blood made slightly acid with citric acid, and kept at $22^{\circ} \mathrm{C}$. According to Rogers, the fact that an acid reaction is found to favour the extracorporeal development of these organisms supports the hypothesis that the second host of the parasite is an insect, such as a mosquito or a bug, with acid stomach contents. $\left.{ }^{2}\right]$

[The stages in the development of the Herpetomonas- or Crithidialike forms from the small ovoid splenic bodies are well shown in Fig. 8, which is taken from Leishman and Statham's paper. The parasites enlarge (2), then multiply by fission (3), and soon become pyriform (4). At this stage many of the parasites become flagellated (4), and multiplication now occurs by simple longitudinal fission (6).]

[The flagellum appears to develop rapidly, but in a curious

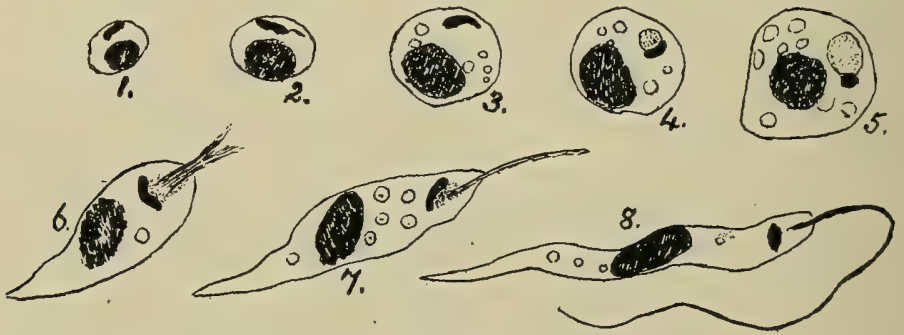

Fig. 9.-Stages in the Development of the Flagellum in Cultures of the Leishman Body (From Leishman and Statham).

I. Ordinary form of spleen parasite. 2. Slightly enlarged parasite from a young culture. 3. Further stage of orowith, vacuolation of the protoplasm, 4. Development of the "flagellar angle, Ml 130 the micronucleus. 5. Increase in size of

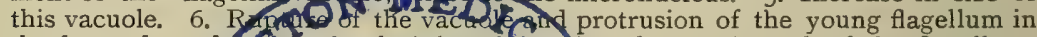
the form of a tuf gunch ofopint-atain threads. 7. Growth of the flagellum, the thickened base being inserted in the eollapsed flagellar vacuole. 8. Fullydeveloped form (69h long fre 10 givos. (Scale of $\mu$ as in Fig. 8.)

manner (see Fig. 9). A pipk-staining area, or 'flagellar vacuole,' appears at the side of the smatter ohromatic body (micronucleus). This gradually enlars 18 Bbst the size of the nucleus; then, by the rupture of the rim of the body, a small flagellum is extruded, together with a tuft of pink-staining substance from the vacuole. The flagellum is at first short and straight, but afterwards enlarges and becomes curved, and apparently is not directly continuous with the smaller nucleus. There is no trace of an undulating membrane ]

[Some of the mature flagellated forms had a curious mode of unequal longitudinal division. Leishman describes a cleavage of thin, sickle-shaped, 'spirillar' forms from the parent Herpetomonaslike individuals. Occasionally two such spirillar forms may split off simultaneously. At first these small forms have no flagellum, but

\footnotetext{
1 [Leishman and Statham, R.A.M.C. Journ., v. 4, 1905, p. 32 T.]

2 [Captain Patton, 1.M.S., has recently found that the parasites are numerous in the circulating blood in the later stages of the disease; also that the parasite is able to complete its development into the typical flagellate culture form in the stomach of bed bugs fed on kala-azar patients.]
} 
subsequently a flagellum is developed and the parasite becomes very active (see Fig. 8, 9). No conjugation was observed, and experiments on animals (by inoculation and feeding with cultures) gave negative results.]

[The systematic position of this parasite is still far from being settled. Laveran and Mesnil ${ }^{1}$ and Donovan, who describe endoglobular forms of the parasite, regard it as a Piroplasma, and Laveran has called it $P$. donovani. Rogers at first agreed with Leishman's original view that the parasite was a degenerated trypanosome, but recently he has proposed to place it in the genus Herpetomonas. ${ }^{2}$ From the resemblance of the Leishman body to the small oval forms of Crithidia fasciculata, with nucleus and centrosome, described by Léger, Birt ${ }^{3}$ suggests that possibly Leishman's body is the gregariniform stage of a Herpetomonas. Ross has suggested the name Leishmania donovani, regarding the parasite as a distinct genus. Further investigations will show which is the correct view; for the present we may adopt Ross's name Leishmania donovani for the parasite of kalaazar, and may include in the same genus the similar organism of tropical ulcer, Delhi boil, etc., under the name L. tropica (Wright).]

\section{Section 5.-Significance of the Basal Corpuscule of Trypano- somes (Posterior Chromatic Body). Its Centrosomic Nature.}

We have hitherto always called this little body the centrosome. We now give our reasons for attributing to it such a significance.

In 1899 Rabinowitsch and Kempner ${ }^{4}$ regarded it as a mucleolus, Plimmer and Bradford ${ }^{5}$ as a micronucleus. The former name is based solely upon the chromatic reactions of this little body (which are those of a karyosome rather than of a nucleolus), and as used by the authors it gave no idea of the true significance of this body. Why should we, when dealing with trypanosomes, introduce the new idea of an extranuclear nucleolus, or karyosome, or, in other words, change the ordinary denotation of those tẹrms?

The name micronucleus, hitherto reserved for the reproductive nucleus of ciliated Infusoria, can only be allowed for the small chromatic body of trypanosomes so long as it has the same physiological function. Up to the present time we are unaware that this little body plays the physiological part which is so definite in the case of a similar structure in the Infusoria. Moreover, the nucleus of trypanosomes cannot be regarded as a macronucleus.

These names were suggested at a time when the close connection between the chromatic corpuscule and the flagellum had not been

${ }^{1}$ [Laveran and Mesnil, C. R. Acad. Sciences, v. I37, 1903, p. 957 ; ibid., v. I38, I904, p. I87.]

2'Rogers, Proc. Roy. Soc., Series B, v. 77, I906, pp. 284-293.]

3 [Birt, R.A.M.C.Journ., v. 6.]

4 Rabinowitsch and Kempner, Zeitschr.f. Hyg., v. 30, I899.

5 Plimmer and Bradford, Proc. Roy. Soc., v. 65, I899, p. 274 ; Centralb. J. Bakter., I, v. 26, 1899, p. 440. 
demonstrated. Since then, however, none of the authorities who have adopted the term micronucleus has adduced any convincing argument in favour of that term.

The researches of Wasielewski and Senn (Igoo), ${ }^{1}$ confirmed and extended by our own (Igoo-IgOI), ${ }^{2}$ have established the following facts, which we have given in an earlier paragraph, and which we summarize here :

The flagellum is always associated with the chromatic corpuscule in question. In dividing forms, the longitudinal division of the old flagellum is always preceded by that of the corpuscule, and always begins in this corpuscule. In those parasites which undergo unequal binary division, such as $T$. lewisi, the newly-formed flagellum, or at least the greater part of it, clearly grows from this little chromatic body. The intimate relations of the flagellum with this chromatic body are thus evident, and they persist throughout the life of the parasite. Hence the name Geisselwurzel, or root of flagellum, given to this corpuscule by Wasielewski and Senn, which is a noncommittal name, and but the expression of observed facts. In view of the meaning attributed to the word blepharoplast by Webber, ${ }^{3}$ who introduced it - a meaning which has been unanimously adopted by writers-the Geisselwurzel is a blepharoplast.

[Woodcock (loc. cit., p. 2I5) adopts the term kinetonucleus for this corpuscule of trypanosomes, "in view of its essential nuclear character and the fact that it serves as the directive centre for the locomotor activities of the cell.']

[Schaudinn, ${ }^{4}$ from his later observations upon fertilization in Protozoa, concludes that " in all the Protozoa in which the evolutionary cycle, and especially fertilization, are accurately known, a duality of the somatic and reproductive nuclear substances, comparable with that realized in Infusoria, is recognisable at a given stage of development.']

[Mesnil ${ }^{5}$ summarizes this paper of Schaudinn in the following terms: - Schaudinn applies the conclusion mentioned above to the structure of trypanosomes, especially $T$. noctua. According to this observer, the flagellar apparatus of a trypanosome (except the micronucleus or blepharoplast at its base) is comparable physiologically with the macronucleusthat is, the vegetative nucleus-of Infusoria. The two chromatic masses (the true nucleus and the blepharoplast or centrosome) are both comparable, up to a certain point, with the micromucleus of Infusoria, for both of them are formed of reproductive substances. During its evolution the large nucleus eliminates its vegetative substances in the form of chromidia, whereas the small nucleus does so by giving rise to the locomotor apparatus, which may be regarded as a highly specialized chromidium. ${ }^{\circ}$ Both, therefore, have a part comparable with the macronucleus of Infusoria, and Schaudinn thus arrives at the idea of a double nuclear dimorphism in trypanosomes.'

'It is well known that Schaudinn differentiates the ookinetes of Halter-

1 Wasielewski and Senn, Zeitsihr. f. Hyg., v. 33, 1900.

2 Laveran and Mesnil, C.K.Soc. Biol., November 17, 1900, March 29, I901, etc.

3 Webber, Botun. Gaz, June, 1897.

* [Schaudinn, Verhandl. d. dentsch. zool. Gesellsch, 1905, pp. 16-35.]

5 [Mesnil, Bull. Inst. Past., v. 4, 1906, pp. 103, 104.]

6 [llesnil, ibid., v. 3, 1905, pp. $318,319$. 
idium noctua - which in the mosquito give rise to trypanosomes-into female, indifferent, and male. The female trypanosomes are very large, and have a large nucleus; the locomotor apparatus with its blepharoplast is feebly developed. In the males, which are small, the reverse obtains. Schaudinn thinks, therefore-and represents it by a series of ingenious diagramsthat the large nucieus contains chiefly female substances, the small nucleus mainly male substances. This sexual differentiation of the nuclear apparatus starts at the very beginning of the development of the ookinetes. In all of them there is a division of the single 'synkaryon' (fertilization nucleus) into a larger and smaller part. The former aborts in the male forms, the latter in the female forms, while both persist in the indifferent forms, which are intermediate in all respects between the male and female.']

We thus arrive at a question of gèneral cytology: Are the corpuscules which are found at the root of the cilia or flagella centrosomic in nature?

Amongst the opinions of authorities in cytology, we have elsewhere ${ }^{1}$ already alluded to that of Henneguy, that the centrosomes ought to be regarded, not only as kinetic centres for the internal movements of the cell, but also as kinetic centres for external movements $;^{2}$ and to that of Guignard, that the blepharoplasts of Webber are of the same nature as centrosomes. ${ }^{3}$ We may add, in this connection, that recent observations, such as those of Meves and von Korff, ${ }^{4}$ diminish the weight of the objections raised by Webber and Strasburger against this idea, in so far as the plant spermatozoids are concerned.

On the other hand, it should be understood that we have no right to generalize and to say that these basal corpuscules are always centrosomic in character. For example, this appears not to be the case with the corpuscules at the base of the cilia of the ciliated Infusoria, or of certain epithelial ciliated cells of Metazoa (in the latter instance a centrosome independent of the basal corpuscule has been demonstrated). However this may be, the centrosomic nature of this little body in the case of the male sexual elements is certain, and the only question that arises is to determine whether the blepharoplasts of the Flagellata are of the same nature as those of the animal spermatozoa or of the plant spermatozoids.

There are striking analogies between the centrosomes of the mesial part of spermatozoa and the small chromatic bodies of trypanosomes. We have shown that the centrosomes of the male elements of the earthworm, during the transformation of the spermatids into spermatozoa, stain a deep violet by the eosin-Borrelblue-tannin method, exactly like these corpuscules of trypanosomes.

The structure of the various flagellates which have a corpuscule at the base of the flagellum or flagella is quite comparable with that

${ }^{1}$ Laveran and Mesnil, Soc. Biol., November 17, I900; Arch. f. Protistenkunde, v. I, 1902 .

2 Henneguy, Arch. Anat. micros., v. I, r.897-1898, p. 495.

${ }^{3}$ Guignard, Ann. Soc. Nat. Botan., v. 6, p. I77.

4 Meves and von Korff, Arch. f. mikr. Anat., v. 57, I901. 
of spermatozoa, and especially of their immature forms. This is quite obvious on comparing Fig. $4, I$ to 3 , representing, as we have already seen, flagellates allied to the trypanosomes, with Fig. 4, 4 and 5 , representing two immature spermatozoa. ${ }^{1}$ One also finds spermatozoa with an undulating membrane which closely resemble trypanosomes, especially as regards the relation of the edge of the membrane to the basal corpuscule, as is the case, for example, with the spermatozoa of Bombinator (Fig. 4, 6). Contrary to what is usually seen in spermatozoa, the centrosomes are situated side by side quite close to the head of the spermatozoon, and they serve as a point of origin, the one for the edge of the undulating membrane $m$, the other for the supporting filament $b$ of that membrane (a structure which is not represented in trypanosomes, but is in Trichomonas).

Moreover, it is known that the axial filament of the tail of spermatozoa, or, in other words, their flagellum, is developed in connection with one of the centrosomes. In certain cases (cartilaginous fishes, Helix, Fig. 4, 3) the anterior centrosome gives rise to an axial median filament, the posterior to the axial filament of the tail. Lastly, in those spermatozoa which have an undulating membrane it appears, from the description and figures given by Broman of the development of the spermatozoa of Bombinator, that the edge of the undulating membrane $m$ and the supporting $\operatorname{rod} b$ both have their origin in the centrosomes $c$ (Fig. 4, 6). ${ }^{2}$

These similarities between the centrosomes of spermatozoa and the chromatic bodies of trypanosomes are alone sufficient, in our opinion, to establish a homology. ${ }^{3}$ But there is to be found among the flagellated Protozoa themselves an argument still more convincing.

The nuclear divisions preparatory to the sporing of Noctiluca are all of the mitotic type, and attraction spheres at the two poles of the division spindle have been demonstrated. When the nucleus has finished dividing, and the small spores are differentiated, Ishikawa ${ }^{4}$ has seen the flagellum of the young Noctiluca grow from the attraction sphere, and probably even at the expense of the latter. This flagellum remains in relation with the centrosomic corpuscule (see Fig. 4, 7).

The homology between this little body in Noctiluca and that of

1 The juxtaposition of the centrosome to the nucleus is not essential ; it is a secondary phenomenon. In any case, it also occurs amongst certain flagellates (Polytoma, for example) which have a corpuscule at the edge of the nucleus and another at the periphery of the protoplasm, as in the spermatozoon of Helix.

2 For a fuller account of this subject and for the bibliography see E. B. Wilson, 'The Cell in Development and Inheritance,' second edition, 1900; Korschelt and Heider, 'Lehrbuch der vergleich, Entwickelungsgeschichte der wirbellose Thiere,' general part; Prenant, P. Bouin, and Maillard, 'Traité d'Histologie,' v. $x$; and especially Fr. Meves, 'Struktur und Histogenese der Spermien,' in Engebn. $f$. Anat. u. Entwickel. Gesch., for 1901, 1902.

3 The same comparison is well brought out hy Dangeard (Le Botaniste, 7 th series, 6 th fasc., April 10, 1901). He denies a centrosomic significance to the corpuscules of Flagellatc, and therefore also to those of spermatozoa.

4 Ishikawa, Journ. Coll. Sci., Tokyo, v. 12, I899. 
trypanosomes appears evident. The part the former plays in mitotic divisions of the nucleus shows its centrosomic nature. ${ }^{1}$ It seems to us that we have no right, a priori, to conclude that the corpuscule of trypanosomes is different in nature.

The objection which Senn (loc. cit., I902) raises to our centrosomic view is based on his observation that the Geisselwurzel stains like the periplast, and is distinct from the rest of the protoplasm, which would not be the case with a centrosome. We must inquire, therefore, into this 'periplast' of Senn. According to him, ${ }^{2}$ it is a special layer of protoplasm (recognizable by its different staining reaction) which surrounds the body of trypanosomes and functions as an organ of movement. ${ }^{3}$ We have never seen such a layer, although our preparations have been as carefully stained as those of Senn. We quite agree that the undulating membrane stains a little differently, ${ }^{4}$ but the rest of the periphery of trypanosomes has shown us no further differentiation, neither in $T$. lewisi (the only species Senn studied), nor in the larger trypanosomes, such as those of Rana esculenta. In the latter we have also shown that the centrosome is sometimes situated deep down in the body in a layer which Senn could certainly not regard as periplastic. This is constantly the case in T. transvaaliense (see Fig. 45). But even in the case of $T$. lewisi, Senn himself mentions facts which are opposed to his theory. ${ }^{5}$ He has noted and drawn the migration of his Geisselwurzel into the interior of the protoplasm each time division of the body occurs. He has seen it come to lie close to the nucleus, and he even asks the question whether sometimes it does not become intranuclear. To sum up, then, we may say that the existence of the so-called periplast of Senn seems to us quite unproven, and the blepharoplast of trypanosomes can, like a centrosome, migrate into the interior of the body of the parasite. Senn's objection to our view appears, therefore, to be groundless.

On the other hand, our view has received support from other quarters. Léger, who has described blepharoplasts in the flagellated microgametes of certain gregarines, ${ }^{6}$ and later in flagellates allied to trypanosomes, but without undulating membrane, ${ }^{7}$ regards these

1 There is no doubt about this, but similar evidence is wanting in the case of the trypanosomes. In them the nuclei divide amitotically, so that we cannot actually demonstrate a centrosomic function of the chromatic corpuscule. [We have seen, however, that in $T$. noctuce. and perhaps in other trypanosomes, heteropolar division of the nucleus has been described.]

Wasielewski and Senn, Zeitschr.f. Hyg., v. 33, I900, p. 459.

3 [Prowazek and others also describe an ectoplasmic layer, in $T$. lewisi, $T$. brucei, and $T$. theileri, in which delicate myonemes or muscle-fibrille are said to occur.]

4 The colour is generally lilac, like that of the nucleus. On the other hand, in most species of Trypanosoma the colour of the Geisselwenrzel is deep violetdifferent from that of the nucleus and undulating membrane.

5 Wasielewski and Senn, loc. cit., p. $46 \mathrm{I}$.

- Léger, C. R. Acad. Sciences, v. 132, January 10, 1901, and Arch.f. Protistenkunde, v. 3 .

7 Léger, Soc. de Biol., March 22 and April 12, 1902. 
blepharoplasts as centrosomes. Schaudinn, ${ }^{1}$ in his excellent work upon the coccidia of moles, draws attention to the fact, when discussing our view, that already in 1894 he had described a special body at the base of the cilia of the gametes of Hyalopus (Gromia) dujardini, and had suggested its possible centrosomic nature. It is also admitted by R. Hertwig. ${ }^{2}$ At present, therefore, we cannot but adhere to our original conclusions.

The study of Trypanoplasma has brought out an interesting factnamely, the existence in that genus of two bodies of the same size and structure, both, apparently, of nuclear nature. One of these bodies is in relation with the flagella. In that region the undulating membrane is wanting, and the body in question is obviously deep down in the cytoplasm which forms the whole mass of the Trypanoplasma. There can be no question here, still less even than in the case of Trypanosoma, of a body essentially periplastic. It seems to us rational to regard this body as being of the same nature as its analogue in trypanosomes.

This view leads to various other interesting points. The question of the homologies of the centrosome and of its phylogenetic origin has been much discussed during the past ten years. In $18 \mathrm{~g} 6$, at the congress of the "Deutsche zoologische Gesellschaft,' ${ }^{3}$ a very interesting discussion was raised by a communication of Schaudinn's upon the central body (Centralkorn) of the Heliozoa. Schaudinn, and afterward Lauterborn, in studying the phylogeny of the centrosome, have expressed the opinion that it is the final stage of a series of similar bodies which were developed originally from a true nucleus. They find the starting-point for these progressive morphological changes $^{4}$ in the $A$ maba binucleata, which has two identical nuclei. The next stage is exemplified by the Paranceba eilhardi, where the two masses already show differentiation, the one playing the part of the nucleus, the other of the internal kinetic centre. According to Lauterborn, we come next to the centrosome of Diatomaceæ, and perhaps of Noctiluca, and finally to that of the Metazoa. ${ }^{5}$ It seems to us that Trypanoplasma also presents a stage, possibly anterior to that reached by the Paramaba eilhardi, in which the two bodies are similar morphologically, but one of which has already developed the function of a blepharoplast. Nuclear bodies during their evolution along centrosomic lines have thus been able to acquire independent

1 Schaudinn, Arb. a. d. kaiserl. Gesund., v. 18, 1902, see p. 395.

2 R. Hertwig, Arch.f. Protistenkunde, v. I, I902, see p. 23.

3 See Verhandlungen (Transactions), p. I1 3 et seq.

4 R. Sand (Bull. Soc. belge Micros., v. 24, 1899, p. $6+$ et seq.), who gives an excellent outline of all the discussions, makes the appropriate remark that it is a question of morphological, and not phylogenetic, changes.

5 According to Lauterborn, a second morphological series starts from the Amaba binucleatc, one of the nuclei giving rise to the macronucleus of the Ciliata, the other to the micronucleus. Schaudinn also regards the micomuclius of the Ciliata as being possibly in series with the centrosome. The blepharoplast of trypanosomes could therefore be both a centrosome and a micronucleus. But (vide supra) there is no reason to give it this second significance. 
functions as internal and external kinetic centres for the cell. These two functions coexist in the centrosome of Noctiluca; the centrosome of Trypanosoma is only the centre for external movements. ${ }^{1}$

In conclusion, it is seen that our view harmonizes perfectly with the most satisfactory theories which have been brought forward concerning the origin of the centrosome, and that, to a certain extent, it amplifies them.

1 These remarks, borrowed almost verbatim from our paper in the Arch.f. Protistenkunde (1902), find confirmation in the recent observations of Schaudinn on the origin and structure of the blepharoplast of $T$. noctua. It is derived from the nucleus by heteropolar mitosis; it has still all the properties of a complete nucleus, and, like the small chromatic body of other trypanosomes, gives rise to the flagellar apparatus. It is, adds Schaudinn, a proof of the soundness of our view, expressed above, as to the nuclear nature of the blepharoplast of Trypanoplasma. The blepharoplast of $T$. noctuce is thus a distinct morphological stage between that of Trypanoplasma and that of Trypanosoma (sensu stricto). 
CHAPTER IV.

\section{'TRYPANOSOMA LEWISI'-PARASITE PECULIAR TO RATS}

\section{Section 1.-Historical Review and Geographical Distribution.}

THE first mention of a trypanosome in mammals was, as we have already stated, as far back as I845, when Gros, ${ }^{1}$ in Russia, discovered in the blood of field-mice and moles numerous motile vermicules, 'so small that they were hardly visible with a magnification of 400 diameters.' Was this trypanosome of field-mice the same as T. lewisi? This seems doubtful, for we shall see later that, according to our experiments, the field-mouse (Mus sylvaticus)' is insusceptible to intraperitoneal injections of true $T$. lewisi.

It would therefore be Chaussat ${ }^{2}$ who first saw this species, in I850, at Aubusson, in the blood of Mus rattus. The parasites were not often found in young rats, but nearly always in full-grown rats. Chaussat took them to be young nematodes. Much later, in I877, Lewis ${ }^{3}$ rediscovered them in Calcutta, and afterwards at Simla, in Mus decumanus and Mus rufescens. Lewis recognized clearly that he was dealing with a flagellated Protozoon. In I88I S. Kent called it Herpetomonas lewisi. ${ }^{4}$ All authors now agree in calling it Trypanosoma lewisi (see Chapter III., section 3).

This discovery of Lewis was soon followed by that of Evans of the trypanosome of surra in the Equidæ and the Camelidæ of India.

The almost simultaneous discovery in the same country of two trypanosomes, difficult to distinguish from one another in the living condition and by the methods of staining then in vogue, was the starting-point of a series of confusions which we find first in Lewis's ${ }^{5}$ work published in I884, then in the successive papers by Lingard, ${ }^{6}$ and it is not always easy to make out in the mass of papers published by that indefatigable worker what refers to the one species and what

1 Gros, Bull. Soc. Nat., Moscow, I845, p. 424.

2 Chaussat, Thise Firc. Méd., Paris, 1850 , No. 192.

3 T. Lewis, "Fourteenth Annual Report of San. Com. with Govt. of India, 1878, Appendix; and Quart. Journ. Micr. Sci, v. 19, 1879, p. 109.

4. Sent, 'A Manual of Infusoria,' v. 1, 1880-188r.

5. Lewis, Quart. Journ. Mirr. Sci., v. 24, p. 357.

6 Lingard, 'Report on Horse Surra,' Bombay, i., I893; ii., 1899. 
to the other. The incorrectness of Lingard's view was first clearly pointed out by Koch and later by Rogers.

Crookshank ${ }^{1}$ was the first to give a description at all accurate of the trypanosome of rats. He saw clearly the undulating membrane and its relation to the flagellum. Interesting details are also to be found in the memoirs of Carter ${ }^{2}$ (I887), of Danilewsky ${ }^{3}$ (I886-I889), and of Chalachnikov ${ }^{4}$ (I888).

One may say, however, that the morphological and experimental study of this trypanosome, as well as the rtiological study of the infection which it produces in rats, dates from the year I899, when the important researches of L. Rabinowitsch and W. Kempner ${ }^{5}$ were published.

Since then numerous papers on $T$. lewisi have appeared. Amongst them are: (I) The researches of Wasielewski and Senn ${ }^{6}$ (I900), almost entirely devoted to the morphology of the parasite; (2) the morphological and experimental studies of Laveran and Mesnil $^{7}$ (I9O0-I9OI), of Jürgens ${ }^{8}$ (IgO2), and of Francis ${ }^{9}$ (I903); and (3) the comparative study of $T$. lewisi and T. brucei by Martini. ${ }^{10}$ Amongst other researches special mention should be made of the paper by McNeal and Novy upon the cultivation of the trypanosome of rats ${ }^{11}$ (June, I903), [and of that by Prowazek ${ }^{12}$ upon the structure and life-history of $T$. lewisi both in rats and in the rat-louse (Hamatopimus spimulosus), in the latter of which sexual conjugation was observed (I905).]

Wild rats, particularly sewer rats; are often infected with trypanosomes. The occurrence of the parasite has been noted in all parts of the world.

Crookshank, in London, found trypanosomes in 25 per cent. of the rats examined. [Petrie, at Elstree, found about 30 per cent. of the wild rats infected. Smedley found 5 out of 13 rats infected in a certain locality near Cambridge.] In Paris, one of us, in $1892,{ }^{13}$ showed that the infection is not infrequent among rats. According to our observations, sewer rats (Mus decumanus) were not often

1 Crookshank, Journ. Roy. Micr. Soc., November, I886, p. 9r 3.

2 Vandyke Carter, 'Scientific Memoirs by Medical Officers of the Indian Army,' I887, v. 4, p. 5 o.

3 Danilewsky, Arch. slaves de Biol., I886 I887; and 'Recherches sur la parasitologie comparée du sang,' Charkov, I888-1889.

${ }^{4}$ Chalachnikov, 'Rech. sur les parasites du sang chez les animaux à sang froid et à sang chaud,' Charkov, I 888.

5 Rabinowitsch and Kempner, Zeitsch.f. Hyg., 1899, v. 30, p. 251.

6 Wasielewski and Senn, Zeitsch. f. Hyg., 1900, v. 33, p. 444.

7 Laveran and Mesnil, C.R. Soc. Biol., October and November, Işoo; Ann. Inst. Past., v. 15, 1901, p. 673.

8 Jürgens, Arch. f. Fiyg., v. 42. 1902, p. 265.

9 Francis, Bull. No. II, Hyg. Lab. U.S. Pub. Health and Mar. Hosp. Serv, Washington, February, 1903.

${ }_{10}$ E. Martini, 'Festschrift to R. Koch,' 1903, p. 220.

11 McNeal and Novy, 'Contrib. to Med. Research,' dedicated to V. C. Vaughan, June, I903, p. 549; also Journ. Infect. Dis., v. 1, 1904, pp. I and 517.

12 Prowazek, Arb. a. d. kaiserl. Gesund., v. 22, 1905.

13 Laveran, Arch. méd. expér., March I, I892. 
infected; only 3 out of 50 examined by us (I900-1903) were found infected. Railliet, ${ }^{1}$ at Alfort, found a large proportion of black rats and of Mus decumamus infected. At Lille, Calmette found that more than 50 per cent. of sewer rats had trypanosomes in the blood. At Bordeaux, Buard ${ }^{2}$ examined I5 sewer rats; all were infected. [In the same city, Sabrazés and Muratet ${ }^{3}$ examined I4 Mus rattus, and found them all infected, while none of the $M$. decumamus (49 examined) or $M$. musculus ( 36 examined) had trypanosomes in the blood.]

At Krommenie (Holland), Schoo found 90 per cent. of the Mus decumamus infected. ${ }^{4}$ Rabinowitsch and Kempner found nearly 42 per cent. of the wild rats in Berlin infected. They noted no marked difference in the various parts of the city. In Southern Russia, Danilewsky and Chalachnikov found a considerable percentage of the Mus decumanus and rufescens infected. In St. Petersburg, Tartakovsky ${ }^{5}$ found infected rats, but only in certain parts of the city. At Rovellasca, in Italy, Grassi failed to find infected rats. [In Lisbon, Alvares ${ }^{6}$ found $4^{\circ} 7$ per cent. of the sewer rats infected.]

In India, T. R. Lewis found 29 per cent. of the Mus decumanus and Mus rufescens in Calcutta to be infected. He also found the parasite in rats in Simla. Vandyke Carter found I2 per cent. of the rats in Bombay with trypanosomes in their blood. Lingard found 35 per cent. infected in Bombay and Poona. The percentage varies according to the season; during the rainy season (June to October) it is 42 per cent., during the dry season (November to May) it falls to 28 per cent. ${ }^{7}$

In Java, Penning, during his investigations upon surra (vide infra), found rats naturally infected with $T$. lewisi. In Manila, according to Musgrave and Clegg, ${ }^{8}$ trypanosomes occur in 20 to 65 per cent. of the animals examined. The proportion varies with the season and locality.

In Japan, Kitasato found many rats infected (mentioned by Musgrave and Clegg).

In Africa, Koch ${ }^{9}$ found parasites in Io out of 24 rats caught in different houses in Dar-es-Salaam. This rat infection occurs also in the Gambia Colony (Dutton and Todd), in Cape Town (Edington),

1 Railliet, 'Traité de zoologie méd. et agricole,' v. I, I893, p. 164.

2 Buard, C. R. Soc. Biol., I902, p. 877.

3 [J. Sabrazés and L. Muratet, C. R. Soc. Biol., v. 59, 1905, p. 441.]

4 Private information.

5 Tartakovsky, Arch. sc. vétérin., I90 I (in Russian).

${ }^{6}$ [C. D. Alvares, Journ. Soc. sc. medicas de Lisboa, v. 70, I906, p. 99 ; abstract Bull. Inst. Past., v. 4, 49 r.]

${ }^{7}$ [Lingard has recently described a new trypanosome in the blood of rats (Mus niveizenter and decumanus) in India. The characteristic fenture of this trypanosome is the long and tortuous posterior extremity of the body, looking like a posterior flagellum, longer even than the true anterior flagellum. This postcentrosomic part is about $19 \mu$ long, as compared with $6 \mu$ in $T$. lertisi. In other respects-e.g., the anterior position of the nucleus-this new trypanosome, which Lingard calls $T$. longoiaudense, resembles $T$. liwisi (Lingard, Journ. Trop. Vet. Sci., v. 1, 1906, pp. 5-14).]

8 Musgrave and Clegg, Biol. Labor., Manila, 1903, No. 5, p. 170.

9 Koch, Reiseberichte, etc., Berlin, I808, p. 70. 
in Mombasa, British East Africa (Stordy), and at Harrar, in Ethiopia (Brumpt). [Bruce and Nabarro found it amongst the wild rats in Entebbe, Uganda, and Ziemann ${ }^{1}$ also in Cameroon.] It does not occur in Pretoria (Theiler), or in Constantine (Rouget).

At Tananarivo, Madagascar, 9 out of ro rats (from the houses as well as from the rice plantations) had trypanosomes in their blood (Thiroux). At St. Denis, in Réunion, a natural infection of rats is common (Vassal).

In America T. lewisi is also found. In the United States, Francis, McNeal, and Novy ${ }^{2}$ have obtained them for their investigations. [Kendall ${ }^{3}$ found the rats in Panama infected with $T$. lewisi; Thomas ${ }^{4}$ has found $T$. lewrisi in rats at Iquitos, in Peru.] At Rio Janeiro, I in 20 of the sewer rats is infected (Fajardo). At Buenos Aires the infection is rare, or, rather, is restricted to certain parts of the town. It was discovered there by Sivori and Lecler, who found that 30 per cent. of the Mus decumamus caught near the lunatic asylum were infected. ${ }^{5}$

We see, therefore, that $T$. lewisi is found in nearly all parts of the world where it has been looked for at all carefully.

[As a rule, a spontaneous infection with $T$. lereisi is found only in 'wild' rats. Laveran and Mesnil ${ }^{6}$ in Igo4 came across two tame rats spontaneously infected, and in 1905 Terry ${ }^{7}$ recorded an epidemic of trypanosomiasis amongst white rats which had been kept in contact with grey rats. This possible infection of white rats must be borne in mind in experimental work, as Laveran and Mesnil point out.]

\section{Section 2.-The Course of Experimental Infection.}

INFECTION OF RATS.-Rabinowitsch and Kempner have shown that intraperitoneal inoculation is the most reliable. By it they had only two failures out of fifty tame rats' inoculated. Out of a hundred white or speckled rats we had only three failures. Of these three rats, two were absolutely refractory, one receiving five and the other eleven inoculations without showing trypanosomes in the peripheral blood. The third rat died nine days after inoculation without having shown a blood infection. ${ }^{8}$ Finally, in a fourth case, one inoculation

1 [Ziemann, Centralb. f. Bakter., I, Orig., v. 38, 1905, p. 3II.]

2 Francis states that not $\mathrm{I}$ of 60 house rats examined in $W$ ashing ton was infected. Of 107 wild rats examined by McNeal and Novy at Ann Arbor (Michigan), 5-all taken from the same loft-were infected. The infection occurred also in Detroit, [Philadelphia, Lincoln, and San Francisco (McNeal, Journ. Infec. Dis., I904, v. I, pp. 517-543). Hultgen (Trans. Chicago Path. Soc., Ig05, v. 6, p. 369) found I $5{ }^{\circ}$ per cent. of sewer rats in Chicago infected].

3 [Kendall, Journ. Infec. Dis., v. 3, 1906, pp. 228-231.]

4 [H. W. Thomas, 'First Report of the Expedition to the Amazon,' 1905, Liverpool School of Trop. Med. ; also Lancet, September 8, I906, p. 668.]

5 Sivori and Lecler, An. d. Min. de Agricult., v. I, I902, pp. $62-66$.

${ }^{6}$ [Laveran and Mesril, C. R. Soc. Biol., v. 57, 1904, pp. 247-249.]

7 [B. T. Terry, Trans. Chic. Path. Soc., v. 6, 1905, pp. 264-267.]

8 These three rats were not pregnant, as were the refractory rats of Rabinowitsch and Kempner. Jürgens has seen the trypanosome disappear suddenly 
produced no effect, but a second gave rise to an intense and prolonged infection.

The infection of tame rats by the peritoneal method comprises three distinct stages. During the first stage, which lasts three to four days, the trypanosomes are actively multiplying in the abdominal cavity. This increase scarcely starts until twenty-four or thirty-six hours after injection; it attains its maximum during the third day, and soon ceases. The parasites then entirely disappear from the peritoneal cavity, and do not reappear subsequently during the infection. ${ }^{1}$ The importance of this first stage has been shown by Rabinowitsch and Kempner to be very considerable from the point of view of the diffusion of the parasite. At this period all the multiplication forms described in the chapter on morphology are found in the peritoneal cavity, the small rosette forms predominating.

The passage of the trypanosomes into the circulatory system is more or less rapid in different cases. Rabinowitsch and Kempner, Wasielewski and Senn observed it from the third to the seventh day as a rule, only exceptionally on the first day. In our experiments, on the other hand, as in those of Francis, blood infection usually occurred in twenty-four hours; in several cases in which the blood was examined only five or six hours after inoculation a considerable number of trypanosomes were found in it. ${ }^{2}$ But side by side with these cases of rapid generalization of the parasite, there were others in which trypanosomes were not found in the blood until the second or third day, or even later (up to the seventh day), after inoculation. This was usually the case with old rats Rapid infection of the blood occurs in about half the old rats, whereas in young rats (weighing 30 to Ioo grammes) it nearly always occurs.

Undoubtedly the different results obtained by other investigators and by ourselves are to be partly explained by the difference in

from one of his rats the day before the animal had young. This was the only one of his rats in which the infection lasted only some days. All the pregnant rats we have inoculated have been susceptible. Francis has found the same thing. Further, Lingard, in India, gives statistics of Ioo rats, showing clearly that pregnant females are found infected as olten as other rats.

1 According to Jürgens, trypanosomes are present in the peritoneal cavity during the whole of the infection. That has never been the case in any of our numerous experiments. Moreover, we have shown that infected rats, which no longer have any trypanosomes in the peritoneal cavity, do not contract a new infection as the result of a repeated intraperitoneal inoculation. The divergent results of Jürgens must depend, we think, upon the special virulence of the try. panosome he employed (vide infra).

[Byloff (Sitzungsbericht, d. kaiserl. Akad. d. Wissensihuften in Wien. Mathem.natur. Klasse, Ig04) also states that the trypanosomes disappear only slowly from the peritoneal cavity, and appear in two to four days as multiplication and young forms in the blood.]

2 According to Jïrgens, these differences clepend upon the kind of trypano some injected. When the trypanosomes are still undergoing development the incubation period is only one to two days; when the trypanosomes are all adult forms the incubation is three to four days. We doubt very much whether this is the sole explanation of the different results obtained by investigators. 
weight of the rats used in the experiments. The number of trypanosomes injected has only a slight influence upon the rapidity of the blood infection, provided that at least $\frac{1}{50}$ c.c. of blood be injected.

The earliest trypanosomes to appear in the blood are thin adult forms; these are probably parasites which were injected. Very soon, however (often after forty-eight hours), there appear swollenout forms about to divide. As a rule, it is on the fourth day that the trypanosomes are numerous in the blood, and that many reproduction forms are present (second stage). Multiplication in the blood thus succeeds intraperitoneal reproduction, but there are always relatively. fewer reproductive forms in the blood than in the peritoneal cavity, and particularly of the small rosette forms. Apparently, as Rabinowitsch and Kempner suggest, the peritoneal fluid is a better medium for the growth of the blood-parasites in question than the blood itself. This, however, does not prevent us from regarding the blood as playing an important-and perhaps even the most important-part in the multiplication of these parasites.

Reproductive forms of trypanosomes are usually seen in the blood until the end of the eighth day, sometimes a little later, especially if the parasites were late in appearing. From that time until the end of the infection only thin adult trypanosomes are found in the blood. We ourselves have never seen multiplication forms after that time, but Jürgens says they may occasionally occur. It should be remarked, however, that he was dealing with a pathogenic trypanosome (vide infra), which was never so in our own cases. This is the third stage, the duration of which is very variable.

Sewer rats, which are found to be naturally infected, are nearly always in the third stage. Of all observers, Sivori and Lecler alone have described the presence of reproductive forms in young sewer rats naturally infected. [Among the wild rats examined by Petrie at Elstree, two of the young rats naturally infected had, in their blood, numerous dividing forms, including rosettes of small parasites.]

The three stages are thus: (1) intraperitoneal multiplication; (2) multiplication in the blood; (3) the stage of the fully-developed infection.

This account of the infection in rats applies particularly to the cases, usually numerous, in which the blood contains many trypanosomes for a long period-for example, one parasite to every two or three red corpuscles, or sometimes even, though rarely, one or two trypanosomes to every red corpuscle. In such cases the infection lasts at least twenty days, generally two months, occasionally four months or more. Sometimes it ends abruptly; at other times the trypanosomes disappear gradually (up to one month). Occasionally relapses occur during the decline of an infection.

The infection may be a mild one, as is frequently the case in old rats. The trypanosomes then take a long time to appear in the blood; 
they are never very numerous, and disappear in from two to eight or ten days. These rats, nevertheless, acquire an active immunity, ${ }^{1}$ as we shall see in a subsequent section.

Subcutaneous injections, as a rule, also give positive results, provided-so say Rabinowitsch and Kempner-sufficient blood be inoculated. Failures, however, are not uncommon. The stage of intraperitoneal multiplication is absent; the infection of the blood is rapid, but less so than after intraperitoneal inoculation.

Contrary to the results of Rabinowitsch and Kempner, Francis obtained positive results: (I) by injecting infective blood into the stomach (eleven out of twelve rats so treated became infected); (2) by making rats swallow all the blood of an infected rat (five successes with seven white rats; five successes with wild rats). All precautions were taken to avoid, as far as possible, any wound of the mouth or digestive tract which might serve as a point of entry for the virus. We have repeated the second series of experiments of Francis, but without success.

The experiments which we have made with sewer rats enable us to say that the course of the infection in them is identical with that which we have just described for white rats.

Infection with Preserved Blood.-If blood kept in the refrigerator (see below) still contains a fair number of active trypanosomes, the time which elapses between its inoculation and the onset of the blood infection is not materially altered. But when the blood has been kept for some time, and on microscopical examination only a few trypanosomes (or none at all) are seen, the incubation period is longer.

Blood kept for forty-seven days on ice, as the result of which the parasites were diminished in number, gave rise to a prolonged but mild infection in one rat out of two injected. Trypanosomes did not appear in the blood until the sixth to the ninth day. The same blood, kept on ice for fifty-one days and showing no trypanosomes under the microscope, infected a rat in a similar fashion. The parasites appeared in the blood at the end of seven days.

Jürgens has obtained similar results with blood kept for thirtytwo and fifty-three days.

The trypanosomes in cultures were virulent in most of the experiments of McNeal and Novy, [of Smedley], and in our own.

Control experiments made by ourselves with traces of fresh blood proved that the increased length of the incubation period did not depend solely upon the small number of parasites injected; it depends more upon their condition.

Infections obtained by the use of preserved blood are as intense

1 The accounts given by different observers of the duration of the infection agree more or less with our own. Jiirgens found it to be one to two months, rarely more (more than seven months in two cases), and less in one single case. Francis has seen infections lasting from seven to fourteen days These authors make no mention of the weight of their animals. 
as those with fresh blood. Indeed, the longest infection we have obtained was one brought about by the injection of blood kept on ice for thirty days. The period of incubation was five days, and the duration of the infection five and a half months (diminution at the end of three months).

Lastly, we may add that trypanosomes retain their power of infection in agglutinating serums, specific or normal, as well as they do in salt solution.

Symptoms in Infected Rats. - On this point authors differ. Let us take first the case of tame (white or speckled) rats. Rabinowitsch and Kempner say there is no fever, and that the animals are only less lively during the first twenty-four hours after the injection. We have ourselves scarcely ever seen any symptoms. In a number of young rats in which the infection was unusually severe-for several days there were three trypanosomes to one red corpuscle-we noticed that the animals failed to go on gaining in weight, and they even lost weight during the first week, while other young rats, passively immunized, continued to grow. Soon, however, the other rats became quite normal again. Musgrave and Clegg are equally of opinion that $T$. lewisi is not pathogenic for the rat, judging from their observations upon thousands of animals.

On the other hand, Jürgens has observed a severe illness, attacking only young rats. The animal is quiet, and no longer gains in weight. Later on dyspnœa, œdema of the hind limbs, and subcutaneous hæmorrhages are present. Death generally supervenes in the second week after inoculation. Old rats show no symptoms, and the younger rats do not all suffer. In all, Jürgens had sixteen rats sick out of forty-seven. At the autopsy there was congestion of the lungs, with pneumonic patches varying in size from that of a lentil to that of a haricot-bean. The spleen was much enlarged, and the lymphatic glands larger than normal. The parasites were still multiplying even in a rat dying on the twenty-fifth day.

Francis lost several of his rats, presumably from the infection. $\mathrm{McNeal}$ and Novy noticed that the trypanosomes obtained from one source were not pathogenic, whilst those obtained from another source were clearly so. We must conclude, therefore, with these authors that differences observed in experiments depend on variations in virulence of the trypanosomes used.

Even rats which withstand the infection well show enlargement of the spleen. The weight is generally doubled, as Lingard's careful statistics on this point show.

[Of the white rats which were found by Terry (loc. cit.) to be spontaneously infected in the Chicago University Laboratory, 90 per cent. had gangrene of the nose or paws; but Terry was unable tc say whether the trypanosomiasis stood in any causal relation to the gangrenous condition.]

In the case of wild rats precise details are wanting. It seems 
that they are able to stand a natural infection well, [but Hultgen (loc. cit.) has recently stated that the infected rats found by him in Chicago appeared to be ill, and Ziemann noticed the same thing in naturally infected rats in Cameroon]. In all probability it is the same with an artificial infection. We should add, however, that Rabinowitsch and Kempner maintain that, contrary to what they found in the case of white rats, the infection is fairly severe in wild rats, and sometimes causes death. Details are not given.

GuINEA-PIGs - Abortive Infections.-In their memoir on nagana Kanthack, Durham, and Blandford ${ }^{1}$ state that the rat trypanosome is met with in small numbers in the blood of guinea-pigs from the fifth to the seventh day after inoculation. But in order to be able to affirm that there is a true infection, it is necessary to observe reproduction of the parasite in the body of the guinea-pig. We have seen this occur in the peritoneal cavity from the second to the fifth day after inoculation, ${ }^{2}$ but it is rarely seen in the blood, a fact which no doubt explains why the infection is never of long duration. ${ }^{3}$

The reader is referred to Section 3 for an account of the morphological changes which $T$. lewisi undergoes in the body of the guineapig. The parasites appear in the blood in the first twenty-four hours after inoculation, and remain there for five to seven days. They increase in number at first, so as to equal one-fiftieth to one-twentieth of the number of the red corpuscles, then diminish and finally disappear. The disappearance of the numerous parasites (mainly multiplication forms) from the peritoneum generally precedes by twenty-four hours the disappearance of the trypanosomes from the blood. It takes place fairly suddenly, and it appeared to us to coincide with an accession of leucocytes, the peritoneal exudate becoming more abundant. Under these conditions we have seen trypanosomes being taken up by the phagocytes. The trypanosome lies in the long axis of a depression formed by processes of the leucocyte, the part of its body still free being very actively motile.

In stained preparations we have most distinctly seen all phases of the digestion of trypanosomes by the guinea-pig's mononuclear leucocytes, the only leucocytes which seem able to take up these parasites. The trypanosome first becomes spherical, and is contained within a vacuole. Its protoplasm, nucleus, and centrosome are quite normal ; the flagellum alone stains less deeply. Later on disintegration of the protoplasm occurs, the nucleus and centrosome

1 Proc. Rey. Soc., v. 64, 1898, and Hyg. Rundschau, 1898, No. 24.

2 All our injections were made intraperitoneally, and in guinea-pigs of roo to 400 grammes in weight. We inoculated at least I C.c. of blood containing many parasites. Even under these conditions there were many failures, but more than half the guinea-pigs became infected.

${ }_{3}$ Rabinowitsch and Kempner and Francis failed entirely in their attempts to infect the guinea-pig. Per contri, Musgrave and Clegg state that they succeeded, like ourselves, in producing with $T$. lezvisi a mild infection, which was transitory and unaccompanied by any symptoms. 
still remaining quite recognisable. Next the centrosome disappears, for leucocytes containing only the nucleus of a trypanosome are often to be seen. The appearance of this nucleus, however, shows that it is undergoing digestion; some chromatic granules are alone visible, the nuclear fluid having disappeared, undoubtedly on account of the destruction of the nuclear membrane. The destruction of trypanosomes in the guinea-pig is thus brought about by a process of phagocytosis - the trypanosomes, surrounded while still living and motile, are digested by the guinea-pig's mononuclear leucocytes.

[McNeal ${ }^{1}$ does not agree with the view that phagocytosis plays the chief part in the final destruction of trypanosomes. He repeated Laveran and Mesnil's experiment of injecting trypanosomes into the peritoneal cavity of (immunized) guinea-pigs, but could find no evidence of phagocytosis. The parasites became motionless and were gradually 'dissolved,' like the process of bacteriolysis in Pfeiffer's reaction. McNeal thinks, therefore, that trypanosomes disappear as the result of the action of cytolytic ('trypanolytic') agents, and not by phagocytosis.]

Lingard says that the blood of Indian bandicoots (vide Chapter V.) containing trypanosomes is infectious for the guinea-pig, parasites appearing in the blood on the fourth, fifth, sixth, and eighth days after inoculation. On the other hand, it is not infectious for the mule, ass, or rabbit. This fact is interesting in view of the almost identical degree of susceptibility of the guinea-pig to the rat trypanosome.

Other Animal Species. -All other species of animals appear to be quite refractory to inoculations with $T$. lewisi.

It is true that Lingard states that he succeeded in infecting various species of animals with the rat trypanosome, but he confused this trypanosome with that of surra, and the horses which he claims to have infected with the rat trypanosome had simply become naturally infected with surra. In our opinion we must only take into account those of his experiments which relate to field-rats (Nesokia providens). Two of these rats showed parasites in their blood seven days after inoculation, and the trypanosomes were constantly present until the death of the animals, which occurred 102 and I68 days later. In two other Nesokia trypanosomes did not appear in the blood until after twenty-four and thirty-nine days respectively.

Koch, Rabinowitsch and Kempner, and we ourselves, have tried to inoculate this trypanosome into different animals, but without success. Mice (grey and white), field-mice (Mus sylvaticus and Arvicola arvalis), rabbits, dogs, goats, and horses were all experimented with. Even the hamster, which is often found to be infected with a trypanosome closely allied to $T$. lewisi, is refractory.

When the blood of infected rats is inoculated into any of the above animals, some trypanosomes can be found in the blood of the

1 [McNeal, Journ. Infec. Dis., v. I, 1904, pp. 526, 527.] 
inoculated animals for twenty-four or forty-eight hours, but they do not multiply and rapidly disappear.

After injecting blood rich in trypanosomes into the peritoneal cavity of white mice, we found that the parasites could be recovered from the abdominal cavity and from the blood after twenty-four hours. At the end of forty-eight hours, however, they had all disappeared. That was also found to be the case with monkeys (Macacus) inoculated in India by Vandyke Carter and Lingard. The former found some trypanosomes in the blood on the second and third days, the latter on the third day. After that trypanosomes could no longer be found.

\section{Section 3.-Study of Trypanosoma lewisi.}

Trypanosoma lewisi IN THE BODY OF THE RAT.-First we shall study the parasite as it occurs in the living body: (I) the fullydeveloped parasite; and (2) the multiplication forms.

(I) Adult Form of T. lewisi.-In fresh blood, examined either in film or hanging-drop preparations, $T$. lewisi appears as a very active worm-like body. It is the most motile of all the trypanosomes. It moves about very actively amongst the red corpuscles, to which it imparts varied movements without changing their shape in any way. It is often seen to travel, flagellum foremost, right across the field of the microscope like an arrow, which is quite unusual with trypanosomes. When it moves more slowly slight oscillations of the flagellum to the right and left alternately can be made out. In preparations which have been made some time the movements become still slower, and one can then see the wave-like undulations of the undulating membrane, which occur first in one direction, then in another.

[Some authors (Rabinowitsch and Kempner, Prowazek) state that $T$. lewisi can also move with the flagellum posterior. Prowazek, in describing the movements of $T$. leweisi, says: 'In addition to movements of the body through contractions of the myonemes, which often cause a slight spiral motion of the body, one observes also a forward movement of the parasite through the vibrations of the undulating membrane and flagellum.']

Fine granules can be seen in the protoplasm, and often, towards the posterior extremity, a very refractile granule corresponding to the centrosome. The nucleus is not visible.

$T$. lewisi measures $24 \mu$ to $25 \mu$ in length, including flagellum, by about $I^{*} 5 \mu$ in breadth.

After staining by the eosin-Borrel-blue and tannin method mentioned in Chapter II., the structure of the parasite is distinctly seen (Fig. IO, I, and Fig. I of the plate). The protoplasm, which is stained pale blue, contains the nucleus (a) stained lilac, and a much smaller chromatic body $(b)$, situated towards the posterior end, 
stained a deep violet. Along the free border of the undulating membrane $c$ is a filament stained lilac, continuous at the one end with the flagellum $d$, whilst at the other it leads to the corpuscule marked $b$ in the figure. The undulating membrane is unstained except its thickened edge, which is continuous with the flagellum.

The protoplasm often contains fine granules.

The nucleus, usually situated much nearer the anterior than the posterior extremity, is oval in form. It contains granules staining more intensely than the general chromatic body of the nucleus.

The centrosome, which is deeply stained, is usually in the middle of a clear space, at the border of which the flagellum generally ends, or appears to end. There is no doubt, however, that the flagellum and centrosome are connected. When trypanosomes break down without being entirely destroyed (in blood which has been kept for some time), stained preparations often show altered trypanosomes reduced to a flagellum and centrosome, representing, so to speak, the skeleton of the parasite (Fig. II, I6), and in such cases the continuity of the flagellum with the centrosome is quite apparent (Laveran and Mesnil, Francis).

[From a recent study of T. lewisi, Prowazek ${ }^{1}$ concludes that the structure and life-history of the parasite are much more complicated than is usually supposed. As Wasielewski and Senn (loc. cit.) had originally observed, Prowazek confirms the presence of a peripheral ectoplasmic layer, or 'periplast,' all round the parasite. He also describes a number (eight) of fine fibrils-myonemes-like those seen by Schaudinn in T. noctue, but much more delicate and difficult to see. The nucleus is very complicated, possessing a reticulum and karyosome, and with the chromatin concentrated into eight minute granules or chromosomes; there is also said to be a filament joining the nucleus and centrosome.]

[Prowazek distinguishes three forms of parasites in the blood: (I) 'indifferent' forms, with many granules, and the nucleus not sharply outlined; (2) 'male' forms, smaller than (I), often staining more deeply, with an elongated nucleus rich in chromatin; and (3) 'female' forms, with a larger and rigid-looking body, and a reticular pale-staining cytoplasm. He describes also in the blood of the rat various nuclear changes-'auto-synthesis' of the nuclear karyosome (division into four, two parts disappearing and the other two joining up again), and nuclear reduction (Mesnil, in Bull. Inst. Past.).]

(2) Multiplication Forms of T. leverisi.-Authors are not agreed upon the manner in which $T$. lewisi multiplies. ${ }^{2}$

1 [Prowazek, Arb. a. d. kaiserl. Gesund., v. 22, 1905; abstract by Mesnil in Bull. Inst. Past., v. 3, 1905 , pp. 55 I-554.]

2 As has been stated in a preceding paragraph, in order to study multiplication forms, it is necessary to examine the blood of a rat inoculated tour to eight days previously; after that time only adult trypanosomes are found in the blood. This is the condition found in most of the sewer rats infected naturally, and, as a rule, after a sufficient lapse of time multiplication forms are not seen. The 
According to Danilewsky, two forms of multiplication must be distinguished-(I) longitudinal division which occurs while the trypanosome is actively motile; and (2) multiplication by segmentation. In the latter mode of division the flagellum and undulating membrane disappear, the parasite becomes spherical, and the nucleus by repeated subdivision gives rise to a variable number of young forms.

Rabinowitsch and Kempner recognize-(I) longitudinal division, (2) transverse division, and (3) segmentation; in the last case the flagellum and undulating membrane entirely disappear.

Wasielewski and Senn hold that all the processes of division of trypanosomes are reducible to a longitudinal division, as is the case with the other flagellates, except that in dividing trypanosomes the mother cell is always recognizable by being larger than the daughter cell or cells. The parent cell and the daughter cells may remain together for some time, forming a kind of rosette. These authors are somewhat reticent upon the subject of simple multiple segmentation. They give a tigure, however, illustrating multiplication of the parasite, in which it is impossible to distinguish the parent cell, as they say can always be done.

The division forms of $T$. lewisi are very varied, and on first examining a specimen of blood in which these multiplication forms are numerous, one can hardly recognise them as such. Very large forms are seen side by side with very small ones; some are practically normal in appearance, others may assume all varieties of shape.

In fresh blood it is easy to make out, by means of these variations in shape, whether the trypanosomes are multiplying or not; but it is only in well-stained preparations that the evolution of these parasites can be satisfactorily studied. Fresh blood containing dividing forms reveals the interesting fact that the trypanosomes while dividing continue to move, only less actively than usual.

On examining a large number of dividing forms in a well-stained preparation, they are seen to be divisible into two groups-group $a$, represented by Fig. Io, 2 to 5; group $b$, by Fig. IO, 6 to $I O$.

Group a.-A parasite about to divide enlarges in all dimensionsin length often to $35 \mu$, in width to three or four times the normal (Fig. IO, 2). At the same time the nucleus and centrosome enlarge, the latter becoming elongated, and the root of the flagellum thickens. Finally, the nucleus and centrosome come to lie close together.

Later on the nucleus and centrosome divide, sometimes the one, sometimes the other dividing first. With the division of the centrosome, the thickened base of the Hagellum divides (Fig. IO, 3).

[McNeal and Prowazek maintain, however, that the original flagellum never divides, but that the entire flagellar apparatus of the daughter trypanosome is developed from the new centrosome.]

peritoneal fluid of a rat inoculated intraperitoneally one or two days previously also contains numerous multiplication forms, but this exudate does not lend itself to the cytological study of these parasites. 
The newly-formed flagellum separates from the old one before the latter has divided in its whole length, so that at this stage there is a large trypanosome with two nuclei, two centrosomes, and two flagella, of which one is much longer than the other. The new flagellum rapidly grows. The protoplasm next divides, giving rise to a young trypanosome with short flagellum, attached more or less closely to the parent trypanosome. Before the young parasite is liberated it may subdivide, and the parent trypanosome may also give rise to other parasites; in this way is brought about the rosettelike arrangement sometimes seen with one large trypanosome and several smaller ones.

Group b. - The multiplication forms in this group differ from the preceding in that the parent trypanosome can no longer be

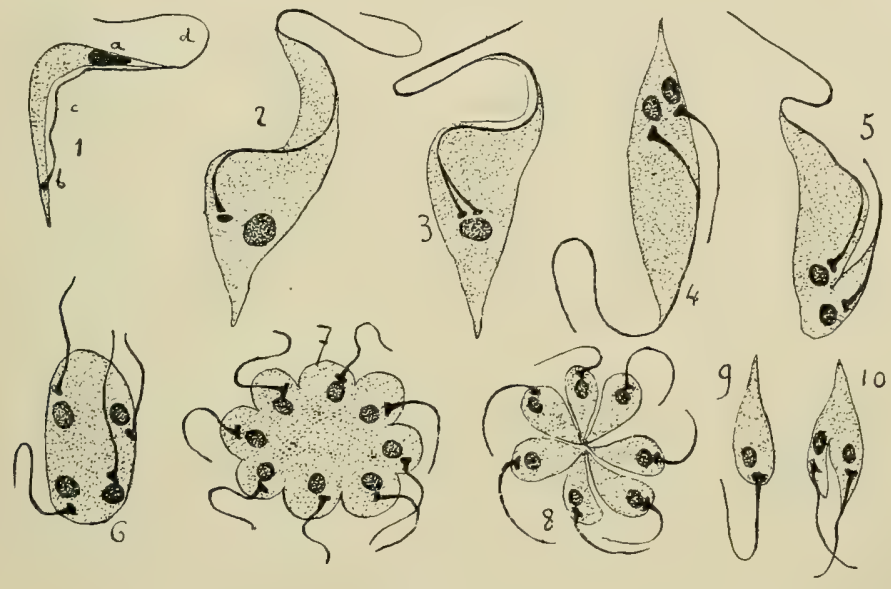

Fig. io.-Multiplication Forms of T. lewisi.

I. Adult trypanosome : $a$, nucleus; $b$, centrosome; $c$, undulating membrane; $d$, flagellum. 2-5. Trypanosomes in process of division ; 5 shows a small parasite about to separate from its parent cell. 6-8. Other kinds of multiplication forms. 9. A young free parasite. Io. Division of a young form. (Magnified about I, 700 diameters.)

distinguished. These forms were certainly seen by Rabinowitsch and Kempner, but with the flagella unstained. Wasielewski and Senn doubt the existence of them without absolutely denying it. [McNeal ${ }^{1}$ has recently stated that the characteristic feature of this group $b$ does not exist in reality, and that 'unbroken multiplication rosettes show the mother cell.' Petrie ${ }^{2}$ also thinks that multiplication takes place in every instance by unequal longitudinal division.] We were the first to describe these forms accurately. Rabinowitsch and Kempner ${ }^{3}$ have recently confirmed our observations in every detail.

The parasites vary in size and are spherical, ovoid, or irregular in shape. In the protoplasm is seen a variable number of nuclei

1 [McNeal, Journ. Infec. Dis., v. I, 1904, p. 523.]

2 [Petrie, Journ. Hyg., v. 5, 1905, p. 194.]

3 Rabinowitsch and Kempner, Centralh. f. Bakter., I, Orig., v. 34, 1903, p. 804. 
with centrosomes near them, and, arising from the centrosomes, small flagella of equal length.

The nuclei may number $2,4,8$, or 16 . The centrosomes may be twice as numerous as the nuclei, because division of the former precedes that of the latter.

At first, although the nuclei, centrosomes, and flagella are multiple, there is no trace of segmentation of the protoplasm (Fig. I0, 6). Later on the periphery of the parasite becomes indented (Fig. 10, 7, and Fig. 2 in coloured plate), and finally the protoplasm divides into as many parts as there are nuclei and centrosomes (Fig. I0, 8). The nuclei always multiply by simple division. ${ }^{1}$

In the parasites undergoing multiplication the flagella are always seen in well-stained preparations. During the earlier stages of our researches upon T. lewisi, we often obtained results like those figured by Rabinowitsch and Kempner in which the flagella had apparently disappeared. Since we have learnt to stain our preparations better we never get such forms, the flagella always being visible, and this is in agreement with what we said above about the motility of trypanosomes which are undergoing division.

The multiplication forms in group $b$ are evidently derived from those in group $a$. When the young trypanosome (Fig. Io, 5) has separated from the parent form, its nucleus, centrosome, and flagellum continuing to divide without concomitant division of the protoplasm, one can easily understand the formation of such forms as those represented in Fig. I0, 6, 7, and 8 . The small forms (Fig. Io, 9 and Fig. 3 in the plate), arising from the breaking up of the rosettes, may further subdivide into two (Fig. IO, IO), thus accounting for the existence of very small forms.

To sum up, the method of multiplication of the rat trypanosome is always the same: there is always a division of the nucleus, centrosome, and root of the flagellum, ${ }^{2}$ but the variations in appearance which result from the simple or repeated subdivision of these elements and from the early or late segmentation of the protoplasm, are numerous.

Trypanosoma lewisi IN THE GUINEA-PIG - In the guinea-pig T. lewisi shows certain differences which are probably attributable to a process of involution. The multiplication forms occurring in the peritoneal cavity after inoculation are abnormal. They are still more varied than they are in the rat, very small forms predominating. After twenty-four or forty-eight hours, the trypanosomes in the peritoneal fluid and blood show a highly refractile spot, which at first sight, on account of its constant proximity to the posterior end of the

1 [Wasielewski and Senn, however, have described a form of mitosis in a case of multiple division of $T$. lezvisi (see footnote, p. 2I); Prowazek (see p. 69) and Byloff (loc. cit.) have also described complex nuclear divisions. $]$

2 [It is possible that the flagellum does not divide (see p. 70).] 
body, might be mistaken for the centrosome. In stained preparations, however, it is easy to see that the centrosome has its usual appearance, and that by its side there is a rounded, unstained vacuole corresponding with the refractile spot seen in the living trypanosome.

Fig. II, II shows a trypanosome seen in the fresh blood of a guinea-pig: Fig. II, $I 2$ a stained parasite from the blood of the same animal.

Differential Characters of Trypanosoma lewisi.-T. lewisi differs considerably from the pathogenic trypanosomes of mammals. ${ }^{1}$ The size is practically the same, but the general appearance is different. $T$. lewisi is thinner and more pointed, and its undu-
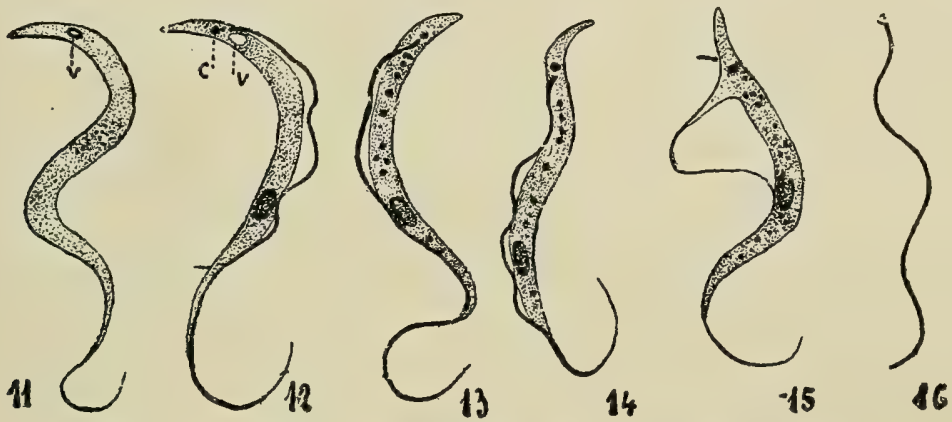

Fig. II.-T. lewisi in the GuInea-PIG. Involutron Forms.

II. Trypanosome in the fresh blood of the guinea-pig: $v$, refractile vacuole. 2. Trypanosome in a stained specimen of guinea-pig's blood: $c$, centrosome; $v$, vacuole. I3 and $\mathrm{I}_{4}$. Stained trypanosomes after being kept for twenty days in the ice-chest. I5. Deformed trypanosome after nine days in hanging-drop preparation (blood and serum of fowl). I6. Centrosome and flagellum, remains of a trypanosome in stained specimen.

lating membrane less folded. It is more active, and may often be seen to travel across the field of the microscope without difficulty; this is rarely seen in the case of the pathogenic trypanosomes, except with $T$. evansi. ${ }^{2}$

The protoplasm of $T$. lewisi stains faintly, and never contains the large and numerous granules so common in the pathogenic trypanosomes. The nucleus, except in those parasites about to

1 In his recent work Martini (loc. cit.) has drawn particular attention to these differences.

${ }^{2}$ [I have myself often seen human trypanosomes from cases of "trypanosome fever ' and of sleeping sickness, as well as animal trypanosomes from various diseased mammals in Uganda, very actively motile in fresh specimens of cerebrospinal fluid or blood. In fact, it was sometimes difficult to keep the parasites under observation, so rapidly did they travel across the field of the microscope. This difference between my own observations in Uganda and those of the authors is probably to be explained by the fact that, whereas I was able to study the parasites directly in the blood of the naturally-infected individuals, the trypanosomes studied by the authors had been many times passed through experimental animals. T. vivax (Ziemann) is also very active (see p. 200).-ED.] 
undergo multiplication, is not situated in the middle of the parasite, as it is in the case of the trypanosomes of the type brucei, but is always near the anterior end of the body. The posterior extremity of $T$. lewisi is usually very pointed, but too much importance must not be attached to this characteristic. Lastly, the multiplication forms are very different.

Rats infected with $T$. lewisi are just as susceptible to $T$. brucei or $T$. evansi as are healthy control rats, as Koch (op. cit.), Rogers, ${ }^{1}$ and we ourselves have shown. In fresh blood-films of doubly infected rats it is very difficult to distinguish the two species of parasite, but in stained specimens the diagnosis presents no difficulty.

Sivori and Lecler have repeated the experiment with $T$. equimum of mal de caderas with analogous results.

$T$. lewisi further differs from the pathogenic trypanosomes in question by the length of time it can be kept alive at low temperatures, and the relative ease with which it can be cultivated artificially (vide infra).

The morphological differentiation of $T$. lewisi from the nonpathogenic trypanosomes of small mammals (which probably constitute so many distinct species or, what amounts almost to the same thing, varieties specially adapted to their particular host) is much more difficult, and we shall see in the next chapter that the differential characteristics of $T$. lewisi and of the trypanosomes of the rabbit (Petrie), of the Indian squirrel (Donovan), [of the mouse (Thiroux), and of the bat.(Petrie, Sergent)] are not always easy to define.

Preservation of Trypanosoma lewisi.-The time that trypanosomes can be kept in hanging-drop preparations or in blood preserved in sterilized tubes is very variable.

Danilewsky observed living trypanosomes in rat's blood kept for eight or nine days in a pipette at the room temperature. Young trypanosomes, he says, can live a little longer, up to ten or twelve days.

Our own observations, confirmed by Jürgens, Francis,' Musgrave, and Clegg, have shown that the temperature has a marked influence upon the length of time $T$. lewisi can be kept alive.

During the summer trypanosomes kept in the laboratory hardly ever survived longer than four days. In winter we kept blood containing trypanosomes (mixed with serum from the rat, fowl, or pigeon) in hanging-drop at the laboratory temperature for eighteen days. At the end of that time the blood was profoundly altered, but several active trypanosomes were still visible.

Trypanosomes so kept gradually become granular and their movements become sluggish. Fig. II, 15 represents a trypanosome in a stained preparation made from blood mixed with fowl serum, and kept for nine days in hanging-drop. Most of the parasites in this 
specimen of blood were deformed. In other cases the trypanosomes assumed characteristic 'tadpole' forms.

At the temperature of the ice-chest $\left(5^{\circ}\right.$ to $7^{\circ} \mathrm{C}$. above zero), $T$. lewisi can be kept alive for a considerably longer time. ${ }^{1}$

We have found active trypanosomes in defibrinated blood mixed with sait solution and kept in the ice-chest for $30,36,44,47,49,50$, $5 \mathrm{I}$, and 52 days. Francis has kept them alive under the same conditions for $8 \mathrm{I}$ days. On coming out of the refrigerator the trypanosomes are always sluggish, but become more active as their temperature rises. The number of living trypanosomes, however, diminishes in proportion to the length of time they are kept in the cold, and those which survive are not as active as they were originally. Examined fresh they are seen to be distinctly granular. Large granules appear in trypanosomes kept in the cold for a fortnight or longer. These granules stain like the centrosome, and are often as large, but their size and distribution vary. Fig. II, I3 and If, show two trypanosomes stained after being in the refrigerator for twenty days.

Lastly, agglomeration of the parasites, in twos or more, may occur after as short a time as two or three days in the ice-chest. The parasites are always joined by their posterior extremities. In this way rosettes may be formed, and, as the trypanosomes are still active, each organism in the rosette continues to move its flagellum and undulating membrane. The number of free trypanosomes diminishes the longer they are kept in the cold, but side by side with the agglomerated parasites one nearly always finds some free ones even after a month or more. (For the details of agglomeration, see later.)

Specimens of blood containing trypanosomes kept in the ice-chest for $44,47,5 \mathrm{I}, 52$, and 53 days were still virulent, but the incubation period in rats inoculated with such blood was longer than usual (vide supra). The length of time trypanosomes can be thus preserved is much diminished if the blood is not collected aseptically and if many bacteria develop in it.

This great vitality of $T$. lewisi at low temperatures is one of the most characteristic features of this species. It enables us to distinguish it, and, if necessary, to separate it from the pathogenic species of the type brucei. The effect upon the other non-pathogenic trypanosomes should be studied. We shall see later that Petrie has shown that the trypanosome of the rabbit lives for a month in the ice-chest.

Jürgens says that these trypanosomes live only two to four days at $37^{\circ} \mathrm{C}$. We have shown that they can withstand a temperature of $4 \mathrm{I}^{\circ} \mathrm{C}$. quite well. At $50^{\circ} \mathrm{C}$. the motility rapidly diminishes, and at the end of five minutes no motile trypanosomes can be seen. Jürgens has, however, found that they are still infective after being

1 Laveran and Mesnil, C. R. Soc. Biol., October 6 and November Io, 1900. 
heated for two hours to $50^{\circ} \mathrm{C}$. and then slowly cooled down. They cannot withstand the same length of exposure to $58^{\circ} \mathrm{C}$.

In a general way, one may say that $T$. lewisi is affected by temperatures above $40^{\circ} \mathrm{C}$., but certainly less so than are the trypanosomes of the type brucei.

T. lewisi can also withstand low temperatures well. Thus Jürgens has kept it alive in slide preparations for at least seven days at a temperature of $-5^{\circ}$ to $-8^{\circ} \mathrm{C}$. When he warmed the blood for the daily examination he noticed that the trypanosomes, at first motionless, gradually regained their motility.

The same observer found that blood kept for two hours at $-\mathrm{I} 7^{\circ} \mathrm{C}$. was no longer infective. We have repeated this experiment under almost identical conditions. A tube of citrated rat's blood was placed for two hours in a mixture of ice and sea-salt, the temperature varying from $-155^{\circ}$ to $-18.5^{\circ} \mathrm{C}$. At the end of that time we found on warming the blood that the majority of the trypanosomes were motionless, but several were still quite active. Half a c.c. of this blood injected intraperitoneally into a rat produced a severe infection after an incubation period of six and a half days.

We may add, finally, that $T$. lewisi is able to withstand for a certain time the temperature of liquid air. Some citrated blood, kept for a quarter of an hour at a temperature of $-\mathrm{IgI}^{\circ} \mathrm{C}$.- (very few of the trypanosomes remained active; the others were, as a rule, well preserved, but often granular)-infected a rat on injection of $\frac{1}{2}$ c.c. intraperitoneally (incubation five days; infection severe). The same blood cooled to $-\mathrm{I}_{91}{ }^{\circ} \mathrm{C}$. for a quarter of an hour, then warmed up and again exposed to liquid air for one hour (on microscopical examination of the blood, one active trypanosome was found after a long search), infected a rat again after injection of $\frac{1}{2}$ c.c. intraperitoneally (incubation six days; infection severe). In blood exposed to liquid air for twenty-four hours the trypanosomes were all spherical and the blood was not infective.

Action of Radium.-T. lewisi in hanging-drop was submitted to the action of radium through the cover-glass and the sheet of mica which closed the tube containing the radium. Several experiments showed that it required about twelve hours to render the trypanosomes motionless. Trypanosomes are, therefore, more sensitive to the action of radium than are bacteria.

[Russ ${ }^{1}$ exposed $T$. lererisi to the action of $\mathrm{X}$ rays, but could detect no change in the parasite under the microscope.]

Cultivation.-Hitherto we have dealt only with the preseriation of $T$. lewisi. We are now going to show how this trypanosome may be artificially cultivated. At different times various observers have claimed to have obtained, either accidentally or otherwise, the growth in vitro of various trypanosomes. Thus Danilewsky and 
Chalachnikov have described in detail the changes of shape (into spherical forms with loss of flagella) and the segmentation of the trypanosomes of birds, Batrachia, and fishes, when preserved in pipettes.

In the case of $T$. lewisi, Chalachnikov has claimed to have obtained cultures by inoculating blood containing trypanosomes into dog's serum. On looking at the author's tigures of the culture forms, there is no doubt that some of them, at any rate, represent agglutination rosettes.

More recently Jürgens (loc. cit., p. 286) mentions having seen, in hanging-drop preparations of rat's blood containing ' young ' trypanosomes, and kept at $37^{\circ} \mathrm{C}$., ' division forms and developmental stages which were not seen the day before.' But even if we admit that the author was not the victim of a mistake, we cannot say that the problem of the cultivation of trypanosomes was solved.

There is now, however, no doubt that the problem is solved, especially so far as $T$. lewisi is concerned, by the researches of McNeal and Novy, which we have been able to repeat.

In the chapter on Technique we stated under what conditions trypanosomes could be cultivated; it is unnecessary, therefore, to refer again to the subject here.

$T$. lewisi was the first trypanosome to be cultivated. This is comparatively easily done in the water of condensation of the bloodagar medium of McNeal and Novy. By inoculating several tubes with blood from an infected rat, one nearly always succeeds in obtaining a culture, and subcultures are equally easy to obtain.

According to McNeal and Novy, T. lewisi grows in media containing I part of blood to 5 and even 10 of agar. But it prefers media rich in blood, the best proportion being apparently 2 of blood to I of agar. In their earliest experiments these observers generally used a medium containing I part of blood to 2 of agar.

At the laboratory temperature growth is very slow, especially if the medium is inoculated with few parasites. ${ }^{1}$ At the end of a certain time one sees, in addition to isolated trypanosomes, rosettes, which become more and more numerous and are often composed of a large number of parasites-as many as I,000. All these trypanosomes are actively motile. Later on the number of living trypanosomes diminishes. In the middle of the rosettes numerous spherical degenerated forms are seen, and finally they all die. This final stage is reached at the end of a variable time, which may be several months when the medium has been freshly prepared and inoculated with few trypanosomes. By modifying the type-medium we have described, which served for the cultivations in series of tubes, McNeal and Novy succeeded in keeping trypanosomes alive in the same tube for as long as 306 days.

The medium in this case was composed of 2 parts of agar, I part

1 [As previously stated (p. 14), Mathis has recently found that growth occurs more rapidly in media that have been heated for some time to $75^{\circ}$ to $100^{\circ} \mathrm{C}$.] 
of rat's blood, and I part of a solution containing I per cent. of glycocoll and I per cent. of sodium asparaginate. After the tube had been cooled some defibrinated rabbit's blood was added to the water of condensation. Defibrinated rabbit's blood alone seems to be an excellent medium for the growth and preservation of $T$. lewisi. Lastly, good results may be obtained by using ordinary sloped agar tubes, in which some defibrinated blood is added to the water of condensation.

At the laboratory temperature McNeal and Novy obtained between May I6, 1902, and May I9, 1903, eleven generations of cultures, using a medium containing 2 parts of agar to I of defibrinated blood. A little of the growth of the tenth generation, inoculated intraperitoneally into two rats, infected them after an incubation period of four days.

[In May, I904, Novy, McNeal, and Hare ${ }^{1}$ stated that this culture had passed through twenty-six generations in two years. After a year, says $\mathrm{McNeal}^{2} \mathrm{~T}$. lewisi has become so well adapted to its new conditions of growth that true colonies, white and glistening like the colonies of bacteria, often appear on the agar. Smedley ${ }^{3}$ has subcultivated $T$. leveisi through nine generations in nine months, and 'the last generation multiplied rapidly and was quite as infective as the preceding ones.']

At a temperature of $34^{\circ}$ to $37^{\circ} \mathrm{C}$. growth is more rapid-attaining its maximum in eight to twelve days-but is otherwise similar in all respects to that occurring at the room temperature. After fifteen to twenty days all the parasites are dead. McNeal and Novy attribute this rapid death to the transformation of the hæmoglobin into hæmatin, which occurs rapidly at the body temperature.

From December 4, I902, to May 23, I903 (I70 days), McNeal and Novy obtained a series of twenty-two subcultures at the body temperature. It should be added, however, that the seventh and ninth tubes of the series were grown at the room temperature. The sixth tube having accidentally been kept at $40^{\circ} \mathrm{C}$. for several hours, its trypanosomes rapidly degenerated, and subcultures made when this degeneration was noticed would not grow at $35^{\circ} \mathrm{C}$., but only at the room temperature. The eighth tube of the series, incubated at $37^{\circ} \mathrm{C}$., did not grow well, and the ninth tube would not grow at all at that temperature. It was, therefore, necessary to use a tube of the ninth subculture grown at the room temperature.

These details show clearly how badly cultures of trypanosomes can withstand temperatures of $37^{\circ} \mathrm{C}$. and above.

Trypanosomes from three tubes of the twentieth subculture of the series were inoculated into rats. One animal developed only a very mild infection, but the other two showed parasites in the

1 [Novy, McNeal, and Hare, Journ. Amer. Med. Assor., May 28, 1904.]

2 [McNeal, Journ. Infec. Dis., r. I, 1904, pp. 517-543.]

3 [Smedley, Journ. Hys., v. 5, 1905, p. 24.] 
blood after an incubation period of five to six days. The infection was very severe and both rats died. McNeal and Novy also found that, of two tubes of the tenth generation grown at the body temperature, one (containing an alkalized medium) did not produce an infection in a rat, while the other (containing ordinary medium, non-alkalized) gave rise to a normal infection. It is impossible to draw any definite conclusion from these facts, for the rats which resisted infection may have been naturally immune.

We have given a mild infection to a rat with a culture of the second generation grown for twenty-two days at $20^{\circ} \mathrm{C}$. Two other rats became severely infected by intraperitoneal inoculation of $\mathrm{I}$ drop of an original culture thirty-five days old, in which for about twelve days a copious growth of bacteria and various moulds had developed.

' The $T$. lewisi as found in cultures varies greatly in size. It is
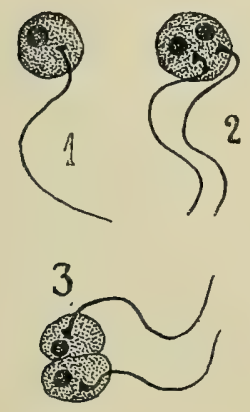
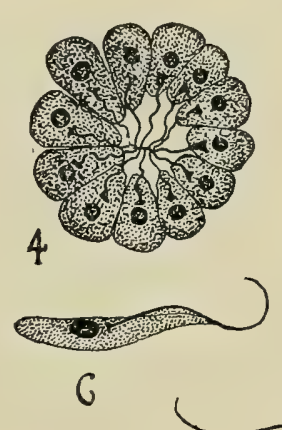

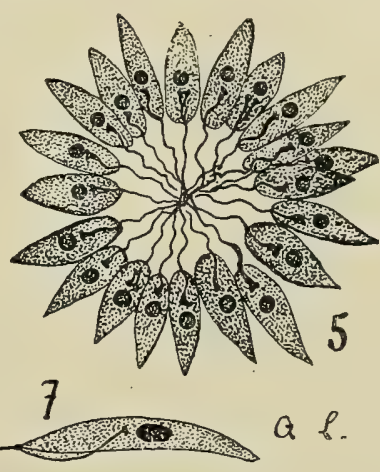

Fig. 12.-Culture Forms of T. lewisi.

not unusual to find minute forms which, not counting the whip (flagellum), are but I to $2 \mu$ in length. There are others which are typical in form, but are not much longer than the diameter of a red corpuscle. While most of the spindle-shaped cells range from ${ }_{15} \mu$ to $20 \mu$ in length, some trypanosomes can be found at times which are $50 \mu$ to $60 \mu$ long. The existence of the small form accounts for the fact that we have repeatedly been able to infect rats with Berkefeld filtrates of such cultures' (Journ. of Infect. Dis., v. I, p. 27).

By means of its long flagellum the parasite is able to travel with great rapidity and almost in a straight line.

Cultures of $T$. lewisi contain numerous colonies of rosettes, composed of hundreds of fusiform parasites, having rather a stiff or rigid appearance. In these rosettes, contrarily to what is seen in the agglutination rosettes, ${ }^{1}$ all the flagella are turned towards the centre.

${ }^{1}$ [Most authorities are agreed upon the central arrangement of the flagella in the culture rosettes. Prowazek, on the other hand, describes rosettes in culture media with the flagella peripherally situated, and suggests that the centrosomes secrete, and pass out on to the surface of the body, a viscid substance, which causes the end of the body to become sticky. McNeal (Journ. Infec. Dis., v. I, pp. 517-543) has also ascribed rosette formation and agglutination to a stickiness of the body of the trypanosomes.] 
In the culture forms the centrosome is on the same side of the nucleus as the flagellum, instead of at the other end, as in the forms found in the blood. It is, therefore, nearer the centre of the rosette than is the nucleus.

Figure I2 gives an idea of the forms met with in cultures. (I) Free forms, rounded or spindle-shaped. Some ( $I$ to 3) show undoubted division forms (equal binary division); others ( 6 and 7 ) are interesting because, although the centrosome is on the same side of the nucleus as the flagellum, the latter is joined to the body by a short undulating membrane. This is an intermediate stage between the Herpetomonas without undulating membrane and the adult form seen in the blood, and its existence proves that the undulating membrane is not formed by the attachment to the protoplasmic body of a flagellum folded backwards. There are all stages between these different types.

(2) Rosette forms - some (4) of rounded or pear-shaped parasites, with short flagella; others (5) of fusiform parasites, with welldeveloped flagella. The former appear to be an early stage of the latter. Indications of division; especially of the centrosome and flagellum, are numerous in these rosette forms.

[McNeal (loc. cit., p. 528) studied living cultures by making a Ranvier slide preparation of I loopful of an actively-growing culture with 2 loopfuls of fresh condensation fluid from a blood-agar tube. On watching small rosettes-say of four or five cells-he found that at $30^{\circ} \mathrm{C}$. complete division of a cell took about one hour, and that the cell may proceed to a second division within about four hours.]

[Smedley (loc. cit., p. 33) states that Laveran and Mesnil's eosin-Borrelblue stain gives the best results with cultures; and that 'Romanowsky's stain and similar stains deposit masses of precipitate, and fail to bring out any detail in the organisms.']

\section{Section 4.-Agglomeration of Trypanosoma lewisi. ${ }^{1}$}

T. lewisi is, of all the trypanosomes, the one which lends itself best to the study of the phenomena of agglomeration. On account of the interesting nature of these phenomena, we are devoting to them here a section, reproduced almost verbatim from our paper published in Igor.

Under certain conditions $T$. lewisi forms very characteristic and regular colonies. The conditions which bring about the formation of these colonies may be classified under two distinct headings :

I. Agglomeration is produced in defibrinated blood kept for a shorter or longer time in the refrigerator. In this case the phenomenon is always partial, and lasts until the death and degeneration of the parasites (see Chapter X.).

1 These phenomena, which we were the first to describe in $1900(C . R$. Sinc. Biol., October 6 and November 10, 1900), and in greater detail in 1901 (Ann. Inst. P(ust), have since been studied in the case of the rat trypanosome by Jiirgens and Francis. 
2. When defibrinated blood or serum ${ }^{1}$ containing trypanosomes is acted upon by the serum of certain animals, and particularly of rats which have had one or more injections of blood containing the parasite, a rapid and often complete massing together of the trypanosomes is observed. Agglomeration may occur in a few minutes. Sometimes it persists until the death of the parasites; sometimes the agglomerated masses break up again. All the phenomena in the second category have this in common-that they are brought about by substances which, by analogy with what is known of the agglutination of bacteria and red corpuscles, may be called agglutinins, from their behaviour to heat.

Formation and Morphology of the Agglutinates.-To study in detail the manner in which these masses of trypanosomes are formed, it is best to select cases in which the various phases occur very slowly and the agglutination is never very marked, as, for example, when physiological saline is added to blood containing trypanosomes.

The first fact to note, and certainly the most important as underlying all the peculiarities we shall point out, is that the agglutination of the trypanosomes is not preceded by any loss of motility. The trypanosomes which agglomerate are as active as those which remain isolated, either in the same or in control preparations.

Agglomeration always begins in the same way: two trypanosomes join by their posterior non-flagellated ends ${ }^{2}$ (Fig. I3, $r$ ). The area of contact is very small, but suffices to keep together the two parasites, which form a straight line, and show considerable activity. ${ }^{3}$

Generally the process does not end there, but other parasites come and join the first two, forming a rosette of a variable number of individuals, all arranged with the posterior ends towards the centre of the group, the free and active flagella towards the periphery (Fig. I3, 2). In this way masses of a hundred or more parasites may be formed, and they are very interesting to watch. Each trypanosome retains its movements independently, and seems to be trying to escape from its fetters, which in some cases it succeeds in doing. Sometimes, especially in the case of cooled blood, there is a leucocyte or a group of blood-platelets more or less damaged, in the centre of the agglomerated mass.

1 Francis recommends allowing trypanosome-containing blood to coagulate, when the parasites pass out into the serum, and one is then not troubled by the red corpuscles. He took a precaution which we have never found necessary. As the blood of infected rats often contained an auto-agglutinin (vide infra), Francis got rid of this by filtration through porcelain and frequent washings with distilled water. The trypanosomes collected thus on the filter were suspended in a quantity of liquid equal in volume to that of the original serum. It seems to us that these operations would greatly alter the character of the trypanosomes.

${ }^{2}$ In examining fresh specimens, there may be some doubt as to which ends are in contact; in stained specimens there is never any doubt. Figs. $13, r$ and 2 , are reproduced from stained smears.

${ }^{3}$ When we study the trypanosome of nagana we shall quote cases in which the agglutination is reduced to this pairing of the parasites. 
The agglomerated masses are not always so slowly formed. When due to true agglutinins, one sees a large number of trypanosomes joining together almost immediately to form a mass. In such cases the trypanosomes approach one another without the least regard to orientation, and often this stage is so short that there is no opportunity of watching it. Soon, however, the parasites arrange themselves in proper position; all the posterior ends come together, and produce the rosette formation we have described.

When dealing with highly agglutinating, and especially with specific, serums, the process may be more complicated. A number of rosettes become grouped together so as to form enormous secondary masses (Fig. I3, 3), which, by reason of the movements of all their component elements, scatter the red corpuscles by which they are surrounded. Such a condition is quite visible to the naked eye in hanging-drop preparations, as clear areas with a greyish centre on a red background.

Finally, when agglutination persists, it is seen that the trypanosomes in the centre of these secondary masses become motionless and degenerate, those at the periphery alone remaining motile.

The parasites in these masses are often so adherent to one another that the blood can be spread in thin films without disturbing the agglomeration. In this way very pretty stained preparations may be obtained. We have never seen any morphological changes in recently agglomerated trypanosomes.

Agglomeration of Dead or Paralyzed Trypanosomes.As we have said above, agglomerated trypanosomes usually retain their motility. What happens with trypanosomes previously rendered motionless? We have been able to answer this question in two ways: (I) by killing the parasites with chloroform or formalin; and (2) by studying the action of several specific serums which in big doses paralyze the trypanosomes.

If a thick film of blood containing trypanosomes be exposed to the vapour of chloroform, all the parasites die in from five to fifteen minutes, and become granular; their outlines become less distinct, so that the parasites are less easily seen. A trace of formalin added to the blood gives better results: the trypanosomes are well fixed, and retain their refractile appearance.

Serums which agglutinate living trypanosomes agglutinate dead ones equally well, and ilice icrsit, but the agglomerations differ from those we have described above. The individual parasites in thim are disposed aithout any attompt at resularity.

Thus, in the case of trypanosomes killed by chloroform there are masses in which the parasites form a network, with meshes more or less chesely interworen. In the case of formalin-fixed trypanosomes the masses are very compact, but the parasites show no definite arrangement in them.

Our parralyzing serums in hig doses do not render the trypano- 
somes quite motionless, but only considerably less motile. Under these conditions the masses formed are like those obtained with
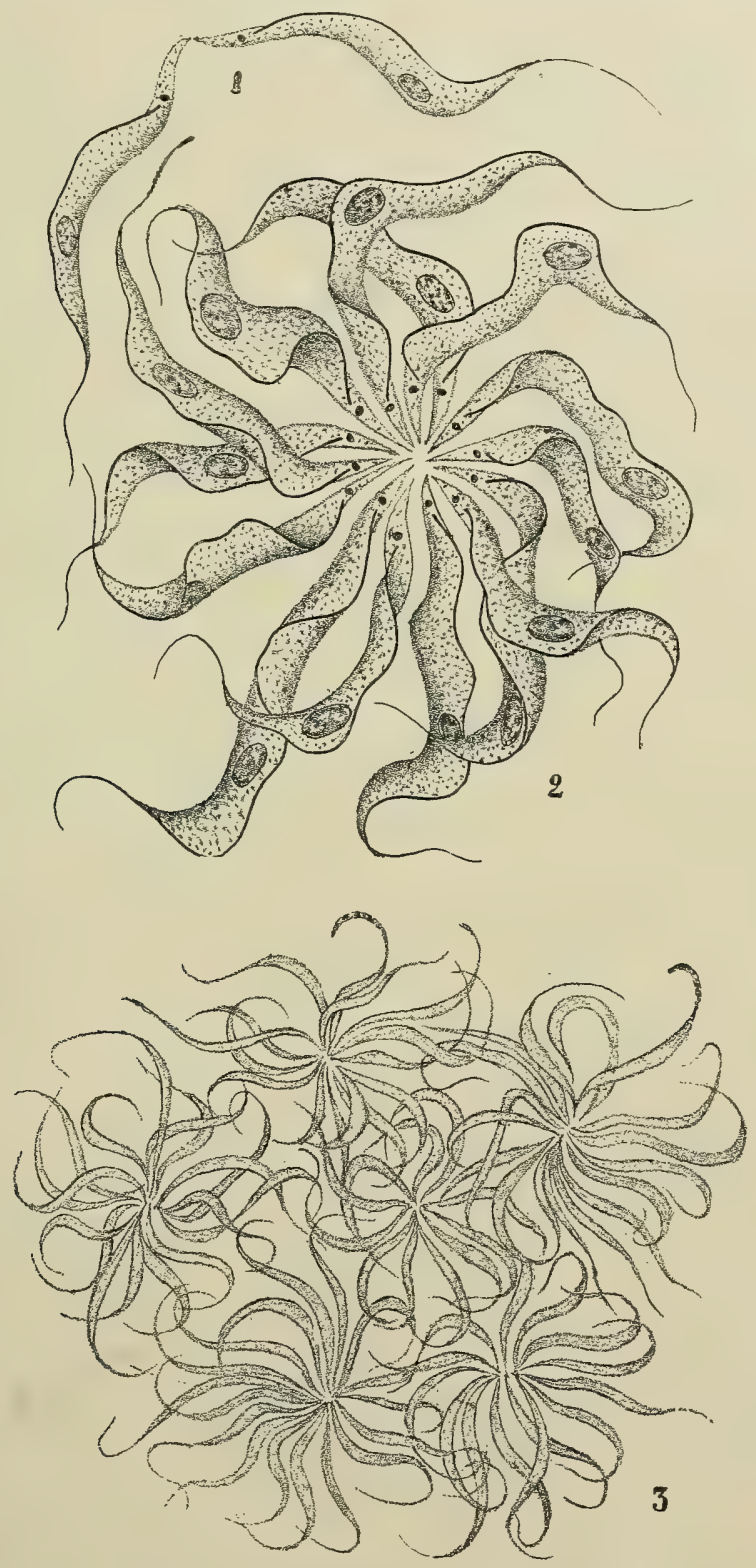

Fig. I3.-AGgLutination of $T$, lewisi.

I and 2. From stained specimens. 3. From fresh specimens. I. Two trypanosomes joined by their posterior extremities. 2. Rosette of trypanosomes (primary agglutination). 3. Colony or mass of rosettes (secondary agglutination).

trypanosomes killed with formalin, when they are often in the form of sheaves. In small doses these serums do not exert any 
paralyzing influence, but still have great agglutinating power. In such cases rosettes are formed.

We think that all these facts admit of only one interpretation. The particular form the agglomerate assumes in ordinary cases depends upon the motility of the trypanosomes. In a colony which is in process of formation each trypanosome tries to escape flagellum foremost; the condition of equilibrium which results is, therefore, represented by a rosette, in which all the parasites have their posterior extremities directed towards the centre and the flagella towards the periphery.

DISAGGLOMERATION.- The motility of trypanosomes also accounts for a curious phenomenon often seen in agglomerated masses of the parasites, but never seen in bacterial agglutinates.

Trypanosomes agglomerated almost immediately on coming in contact with a serum may subsequently become free and isolated again. The secondary masses disaggregate, the rosettes either completely breaking up or losing a large number of their component parasites. ${ }^{1}$ This fact is very confusing when these phenomena are first studied. It is not brought about by all serums, and with any particular serum it is more marked with small than with large doses of the serum. On the other hand, it does not occur unless the trypanosomes are actively motile. Those parasites which are not too tightly held succeed in freeing themselves, and one might imagine that if the motility of the trypanosomes diminished, the phenomenon of agglutination might reappear. We have, indeed, seen this happen on several occasions. Disagglomeration may be prevented by putting the preparations in the refrigerator; for, as we have shown, under these conditions the trypanosomes retain their vitality for a long time, but their activity is diminished. Looked at from another point of view, it is seen that, if our explanation of disagglomeration is correct, its intensity should be in inverse ratio to the agglutinating power of the serum used.

Vitality and Virulence of Agglomerated Trypanosomes. -We have made a large number of observations on this point, particularly with specific serums, of which we always carefully studied the agglomerating properties before using them for prophylactic purposes. For example, we used the same trypanosome-containing blood mixed either with salt solution or with the serum of a healthy rat. The mixtures were kept in hanging-drop preparations at the room temperature $\left(15^{\circ} \mathrm{C}.\right)$, and in tubes plugged with cottonwool in the refrigerator.

The agglutinated mixtures always showed living trypanosomes as long as the 'control' preparations, and, moreover, these parasites were just as infective.

There remains one observation to be made in this connection.

1 The observations were made in hanging-drop preparations at about $15^{\circ} \mathrm{C}$. 
We have already stated that, in the case of persistent secondary agglutination masses, the trypanosomes in the interior of the mass die very quickly. That, however, is not to be looked upon as an exception to the general rule. The trypanosomes die as the indirect result of the agglomeration, because in the interior of the mass they find the conditions unfavourable for their vital processes. The survival of the peripheral parasites is in favour of this interpretation.

Let us now inquire into the details of agglomeration as it occurs in different cases.

Specific Agglutinins. - The serum of normal rats-white or speckled rats, or sewer rats (Mus decumamus) - does not agglutinate. ${ }^{1}$ As a result of successive inoculations with trypanosome-containing blood, rat's serum acquires agglutinating properties, which become more marked in proportion to the immunity produced. ${ }^{2}$ Even after a single inoculation, there is distinct agglutinative power when the infection is at an end. The serum of a rat even during the progress of the infection may agglutinate slightly, sometimes agglutinating its own trypanosomes, as we shall see later on.

After five to ten intraperitoneal inoculations the rat's serum has an agglutinating value varying from 5 to 50 - that is to say, it is necessary to add to a given quantity of defibrinated blood at least one-fifth or one-fiftieth of its volume of the serum, in order to produce agglutination of its trypanosomes. Francis records a case in which the agglutinating value of the serum was 200 . When minimal doses of serum are used, agglutination masses are formed, which subsequently break up again almost completely. In larger doses and with highly-agglutinating serums, most of the masses remain agglutinated, and this is always the case when the agglutinating value of the serum is higher than Io.

With highly agglutinating serums used in quantities of at least double the minimal dose, secondary agglomeration masses are usually formed. Lastly, we have already mentioned the case of rats (which had had more than ten inoculations) whose serum, in a dilution of I in 20 (it was not tested in higher dilutions), produced persistent clumps, and in stronger doses had a distinctly paralyzing effect. One of these rats, which in seven months had been inoculated thirteen times, had a serum so paralyzing that, with a dilution of $I$ in $I 0$, rosettes of the agglutinated trypanosomes were no longer produced.

The paralyzing power of specific serums thus develops very slowly during immunization, contrary to what occurs with antibacterial serums, in which the paralyzing power is always associated with the power of agglutination.

1 We have, moreover, noted that, on adding to defibrinated trypanosomecontaining blood some rat's serum instead of salt solution, the trypanosomes did not agglutinate when placed in the ice-chest.

2 It is curious that Rabinowitsch and Kempner, who were the first to prepare a specific serum, state that their serum had no agglutinating power. 
Specific serums contain a true agglutinin. Heating to $55^{\circ}$ to $58^{\circ} \mathrm{C}$. for half or three-quarters of an hour does not diminish their agglutinating value, but the clumps produced by heated serums are, as a rule, not so marked nor so persistent as with non-heated serums. Serum heated to $63^{\circ}$ to $65^{\circ} \mathrm{C} .^{1}$ for half an hour loses its agglutinating power.

The serum of a guinea-pig which had been inoculated with trypanosomes on several occasions was slightly agglutinating; that of a normal guinea-pig has no agglutinating power whatever.

Agglutinins in Different Normal Serums.-The serum of the guinea-pig, white mouse, man, pigeon, or frog has no agglutinating properties for $T$. lewisi. That of the sheep, goat, dog, or rabbit is slightly agglutinating. In order to obtain definite results it is necessary to use equal volumes of serum and of blood containing trypanosomes. Even then agglutination is never complete, and, as a rule, the rosettes contain only few parasites. With the serum of the rabbit disagglomeration is almost complete at the end of some hours. The agglomerates are more persistent with the serum of the sheep or dog, and with the latter secondary agglomeration masses may even occur.

The serums of the fowl, horse, and, according to Francis, of the cat, are much more agglutinating than the above-mentioned, their value being from 2 to Io. One drop mixed with one or even two drops of blood containing trypanosomes brings about complete agglomeration of the parasites. The rosettes contain a very large number of component trypanosomes, and enormous secondary masses are formed, quite as large as in the case of specific serums. The only difference is that in the case of these normal serums the clumps separate again more or less completely. The agglutinin present in these serums is affected by heat in exactly the same way as that of specific serums.

There is a certain parallelism between the agglutinating power of these various serums for the rat's red corpuscles and for trypanosomes. Thus, of the mammalian serums which have been investigated, that of the horse is the most active in both cases. Fowl's serum is also very potent, while the pigeon's is inactive. The agglutinating value is always much higher for the red corpuscles than for trypanosomes; thus, the serum of a fowl had an agglutinating value of 20 for red corpuscles, whilst for trypanosomes its value was only between 4 and 5 .

In these different serums trypanosomes, whether agglutinated or not, remain alive for a long time. The serum of the fowl and pigeon appear to us to possess remarkable propertics in this respect (see p. 74).

1 Rat's scrum is coagulated at that temperature. It must, therefore, be mixed with an equal volume of salt solution. After heating, a very opalescent liquid is obtained. 
The Agglutinating Power of the Body Fluids of Infected RATS FOR THEIR OWN TRYPanosomes.-This phenomenon is particularly well seen when the peritoneal fluid of actively or passively immunized rats injected with trypanosomes is examined in hangingdrop; but in such cases only small rosettes are formed, which may be permanent or temporary.

Sometimes, when the blood of a rat is examined during the course of an infection, the trypanosomes in an ordinary slide preparation show a distinct tendency to arrange themselves into groups, ${ }^{1}$ occasionally forming definite rosettes, but we have never seen such clumping persist. ${ }^{2}$

Only a small proportion of our rats have at any time shown this phenomenon. It appears to have been present in all Francis's rats, and, moreover, the agglutinins must have been very active, for that observer was able to get stained specimens showing persistent rosettes. Francis has observed, and we can confirm his observation, that the appearance of what he calls 'auto-agglutinins' in the blood precedes by a few days the ultimate recovery of an infected rat. The rats of $\mathrm{McNeal}$ and Novy also frequently showed the presence of auto-agglutinins. [McNeal thinks that agglutination and rosette formation are due to increasing difficulty in division, and to a gradually increasing stickiness of the body of the trypanosome, and that these phenomena indicate commencing antagonistic action of the host.]

In conclusion, the fact which dominates the phenomena of the agglomeration of trypanosomes, and which gives them so special a character, is that the trypanosomes remain active. ${ }^{3}$ From the point of view of the general conception of the phenomenon of agglutination, this fact proves that in the case of actively motile organisms loss of motility does not necessarily precede agglomeration, or, in other words, that the paralyzing and agglutinating substances are different. Certain facts suggest the coexistence of these two substances in the case of motile bacteria, but in the case of trypanosomes it is distinctly proved.

During immunization the agglutinating power of the serum appears rapidly, even while the infection lasts; on the other hand, the paralyzing power is manifested only by highly immunized animals, and we shall see subsequently that the preventive power develops much later.

1 If one were not aware of this phenomenon, one might think it was the first stage of conjugation. Stassano (Soc. de Biol., January, I9OI) has indeed given it this interpretation.

${ }_{2}$ Laveran and Mesnil, Ann. Inst. Past., v. 15, p. 696.

3 Ledoux-Lebard (Ann. Inst. Past., v. I6, I902) has recorded analogous results when blood-serum (from the guinea-pig, for example) acts upon Paramacia. These Infusoria retain their motility and agglomerate into rosettes, with their posterior ends towards the centre of the rosette. The Infusoria are kept agglutinated by a viscid substance, which they eliminate. 


\section{Section 5.-Natural Modes of Infection in Rats.}

The natural infection in grey wild rats seems to be conveyed by means of fleas and lice, which, after sucking the blood of infected animals, bite healthy animals.

On crushing lice caught on infected rats, we have found, in well-stained specimens of the stomach contents, absolutely normal trypanosomes amongst the red blood-corpuscles. $\left[\mathrm{McNeal}^{1}\right.$ has also found trypanosomes in lice fed on infected animals; and in one experiment such lice infected a healthy rat. He was unable to make out any developmental stages in the lice. Prowazek's ${ }^{2}$ observations are very interesting and important, because he was able not only to distinguish trypanosomes in various stages in the body of a rat-louse (Hamatopinus spinulosus), but also to observe the formation of sexual forms, as will be described more in detail later. From these experiments of Prowazek it seems to follow that the Hcematopinus is a true alternate or definitive host, and not merely a mechanical carrier of the parasite. This is the first time that a sexual cycle of development in a mammalian trypanosome has been observed.]

Sivori and Lecler have found living $T$. lewisi in fleas. Rabinowitsch and Kempner did not succeed in finding trypanosomes in fleas caught on infected rats, but when they crushed some of these insects in salt solution, and inoculated the mixture into the peritoneal cavity of healthy rats, an infection occurred in five out of nine cases. Sivori and Lecler have successfully repeated this experiment. Rabinowitsch and Kempner have, in addition, made the following observations :

I. An infected white rat was kept together with healthy rats. At the end of eleven to fifteen days trypanosomes appeared in the blood of the healthy animals. (Sivori and Lecler and we ourselves have confirmed this observation.)

2. About twenty fleas caught on infected rats were placed upon a healthy rat. At the end of two to three weeks trypanosomes appeared in its blood.

These experiments tend to show that fleas play a part in the transmission of $T$. lewisi analogous to that of the tsetse-fly in the transmission of nagana. The transmission by fleas and lice, which are apterous insects, explains why the infection in rats in any particular locality may be very localized; for example, the loft in Ann Arbor where McNeal and Novy found all the rats infected.

Attempts to produce infection by the mouth or stomach have given negative results with all observers except Francis (iide supra). It is certain that rats may become infected by eating food mixed with blood containing trypanosomes or by devouring infected rats, but only when there is an abrasion of the muzzle or buccal mucous

1 IIcNeal, Journ. Infic. Dis., 1. I, 1904, pp. 517-543.]

2 [I'rowazek, Arb. a. d. kaiserl. Gesund., v. 22, 1905.] 
membrane. It is impossible to say whether this mode of infection plays any part under natural conditions.

[In lice which had fed on infected rats Prowazek saw trypanosomes first in the stomach or fore-gut, in which he succeeded in studying the peculiar sexual changes, and even, though rarely, saw the actual process of conjugation and subsequent development. At the second meal the trypanosomes are forced towards the middle-gut, and later into the hindgut, where they accumulate in the region of the Malpighian tubes. The parasites then get into the blood-stream, and, as in the case of the mosquito studied by Schaudinn, reach the pharynx. From there they are inoculated into the body of the host the next time the louse bites. Prowazek only once found trypanosomes in an ovum, so that hereditary transmission of the infection appears to be exceptional in the louse.]

['Various forms were observed in the louse, of which the following appear to be the most interesting: Forms with a chromatic reduction of
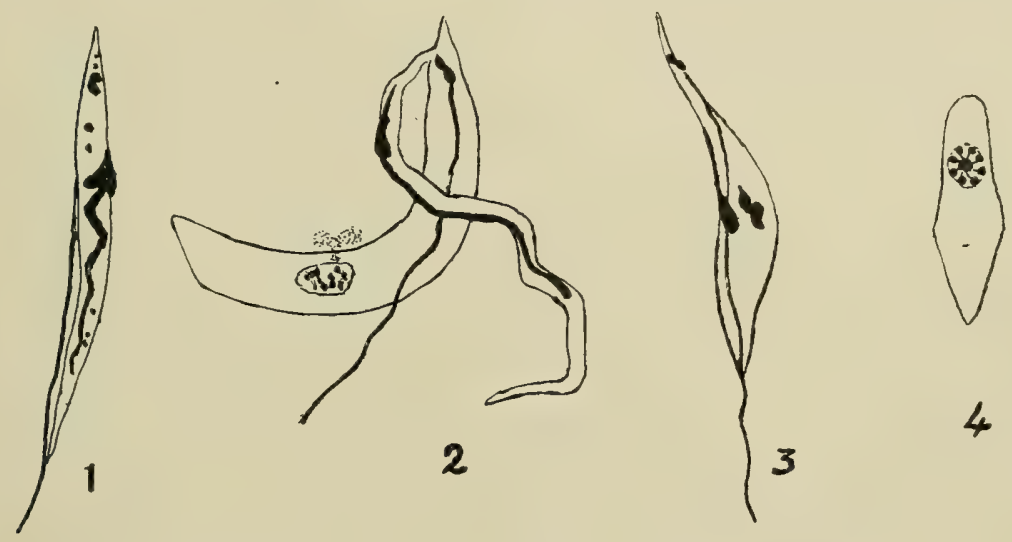

Fig. I4.-Sexual and Conjugation Forms of T. lewisi.

r. Male (sexual) form. 2 and 3 . Stages in conjugation of $T$. lewisi. 4. Ookinete (zygote). (After Prowazek.)

both nucleus and centrosome, each of these dividing twice, so that the final nucleus has only four chromosomes. Later, the male and female forms are differentiated. The male forms are smaller and the nucleus very elongated and band-like. Prowazek was able to observe, though very rarely, different stages in conjugation. Fig. 14 shows two stages in conjugation, and the final result-the ookinete with a single nucleus and no flagellar apparatus-exactly resembling those of the Plasmodium or Hamamceba. One finds all stages between these zygotes (ookinetes) and flagellated forms, in which the centrosome is in front of and near the nucleus, and which in some respects resemble culture forms, but, unlike the latter, would have a sexual origin. Prowazek maintains that, during the development of the flagellated forms from the zygotes, the centrosome is derived from the nucleus of the zygote by heteropolar mitosis, as was described by Schaudinn in T. noctua." ${ }^{.1}$ ]

The parasites do not seem able to pass through the placenta. When pregnant females are infected trypanosomes are not found in

1 [From Mesnil's abstract of Prowazek's paper, Bull. Inst. Past., v. 3, 1905 , pp. 55I-554.] 
the blood of the fotus. Chaussat had already noted this fact, which has been confirmed by Lewis, Lingard, Rabinowitsch and Kempner, and by ourselves.

\section{Section 6.-Active Immunity : its Mode of Production.}

In the short paragraph in their report setting forth the differential characteristics of $T$. lewisi (which they call $T$. sanguinis), and of the parasite of nagana, Kanthack, Durham, and Blandford state that rats which have had one infection are refractory to a second inoculation. These observers, therefore, deserve the credit of having shown the existence of an active immunity against a trypanosome. But it was Rabinowitsch and Kempner who drew particular attention to the fact and insisted upon its importance. They say that a rat which has recovered from a first infection never becomes reinfected, no matter how large a dose of infective blood be injected into the peritoneum. This was confirmed by us, then by Jürgens and Francis. There are, however, exceptions to this rule.

Thus, of thirty rats which we carefully studied from this point of view, only two became reinfected as a result of a second injection of blood containing trypanosomes. Perhaps we should mention also the case of a rat which resisted a first inoculation, but became severely infected (the infection lasting at least two and a half months) after a second inoculation. Lastly, one of our rats, which was severely infected (three and a half months) after an injection of blood mixed with $\frac{1}{2}$ c.c. of a slightly active specific serum, developed a mild infection, lasting about twenty days, after a second inoculation, and a very severe infection after a fourth inoculation. On the other hand, we have already referred to two rats which were quite refractory to the first and all subsequent inoculations (p. 6r).

Francis has also recorded two exceptions to this rule in the case of the two rats which received their first infection, the one by way of the stomach (?), the other through the mouth.

These exceptions, however, do not affect the generality of the law laid down by Rabinowitsch and Kempner. A first infection, even though it last only two days and there be very few trypanosomes in the blood, confers an active immunity upon rats.

Are the Young born of Immunized Mothers themselves IMmune? - A female rat immunized by us had two litters. The sole survivor of the first litter resisted all inoculations, but the eight young of the second litter were as susceptible as normal rats. All the young of two other females were susceptible. Finally, of two offspring of a fourth immune female, one resisted the first inoculation, but became severely infected after the second inoculation, whereas the other was susceptible to the first injection. These facts show that immunization through the placenta or by lactation is exceptional, if 
it occur at all. We also found that the agglutinating substance does not pass through the placenta.

Francis likewise found, on infecting five pregnant females, that the young ones, born without a blood infection, had no particular resistance to subsequent inoculation.

The immunity of the guinea-pig after a first infection does not appear to be so easily acquired or so complete as that of the rat. Thus, of four guinea-pigs two resisted all inoculations subsequent to the first, the trypanosomes not reappearing in the blood, while the two others became freshly infected after the third injection. These observations are, however, too few in number to enable one to draw any conclusions from them.

The Mode of Production of Active Immunity in Rats. What becomes of the trypanosomes injected into the peritoneal cavity of an immune rat? Only very rarely do they appear in the blood, and in such cases they are present in it only for a very short time and in small numbers. The trypanosomes, therefore, are destroyed in the peritoneal cavity itself.

It takes a variable time for all the parasites to disappear. In large rats, which have already received several injections, one hour, or even less, is often sufficient for the complete destruction of all the trypanosomes contained in $\frac{1}{2}$ or I c.c. of blood very rich in parasites (one trypanosome to one to three red corpuscles).

How is this destruction of the parasites effected? In order to ascertain this, a little fluid is removed from the peritoneal cavity of rats at varying intervals and examined in hanging-drop. It is seen that all the trypanosomes in the fluid remain motile, normal in appearance, and isolated until they completely disappear. It is absolutely necessary to examine the fluid directly it is withdrawn from the peritoneal cavity, in order to see that the parasites are unclumped in the fluid, for in a few minutes after removal from the body small clumps are formed. The body fluids of immunized rats are agglutinating, but in our experiments this property has always been only feebly developed (see Section 4).

The peritoneal fluid withdrawn contains many leucocytes, of which about two-thirds are polymorphonuclear and one-third mononuclear. One is struck by the fact that the trypanosomes are often adherent by one of their extremities to a leucocyte. The parasites are often seen in process of absorption by the leucocytes. The leucocyte sends out a prolongation shaped like a truncated cone, in the long axis of which is seen a part (either the anterior or posterior end) of the body of a trypanosome. The free part of the parasite remains quite motile, and, if this be the flagellated end, the flagellum moves about vigorously. The lencocytes of immunized rats, therefore (and we have made many control experiments with normal rats with negative result), engulf living trypanosomes.

These observations, made at the moment that the fluid is removed 
from the abdominal cavity, may be continued in the hanging-drop preparations. The engulfment of the trypanosomes goes on uninterruptedly if the slide is placed on a warm stage at $15^{\circ}$ to $20^{\circ} \mathrm{C}$. under the microscope, and can be seen after an interval of one to two hours. At $37^{\circ} \mathrm{C}$. the engulfment of a trypanosome is seen to take place with great rapidity. At first the parasite is simply adherent to the white corpuscle, and it often happens that the trypanosome succeeds in getting away again. Sometimes, however, the leucocyte gains the upper hand; it sends out processes all round the trypanosome, which in a few minutes is drawn in towards the centre of the leucocyte. Here it rapidly loses its characteristic shape, and becomes mixed up with the more or less granular protoplasm of the leucocyte, so that in a very short time it is indistinguishable (see
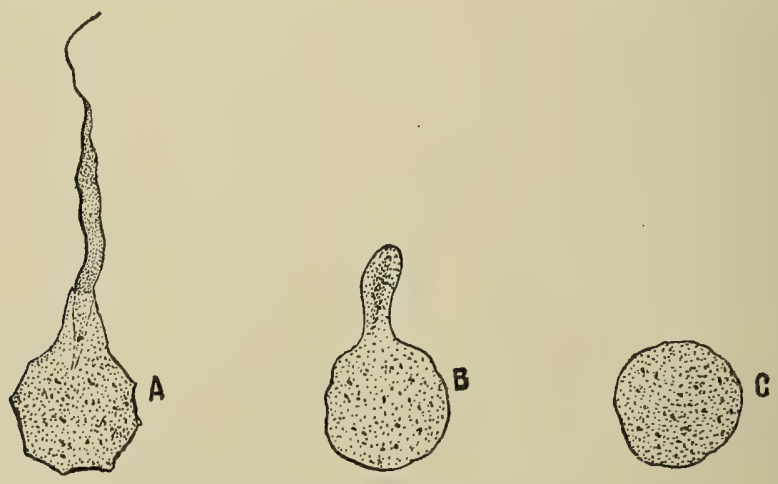

Fig. 15.-Engulfment of a Trypanosome by a Leucocyte.

The figure represents various stages in the engulfment of a trypanosome by a leucocyte in the peritoneal exudate of a rat, four hours after intraperitoneal injection of infective blood. A. The free part of the trypanosome is still distinctly motile, but sluggish; the part already engulfed is less distinct than the rest of the parasite. B. The same corpuscle at the end of five minutes: the trypanosome is seen merely as a process of the corpuscle, the nature of which could be easily mistaken if one had not followed the stages of the engulfment of the parasite. C. The same after another five minutes: the trypanosome has been completely swallowed up. and the leucocyte has resumed its normal appearance.

Fig. I5, A to C: which represents successive stages, drawn at intervals of five minutes, in the engulfment of a trypanosome by a leucocyte). At a temperature of $15^{\circ}$ to $20^{\circ} \mathrm{C}$. engulfment takes place in a similar manner, but much more slowly (the stage represented in Fig. I5, A is very often met with; the conical process is sometimes much more elongated).

To sum up, we may say that the engulfment of trypanosomes by the leucocytes is the only mode of destruction we have seen in cases of active immunity, and we have no hesitation in saying that this is, in our opinion, the only method which occurs. ${ }^{1}$

We wished to find out, by means of stained specimens, how the trypanosomes were digested by the leucocytes. In this we have not

1 [McNeal does not agree with this view. (See addition made on p. 69.)] 
been so fortunate as with the peritoneal exudate of the guinea-pig. Probably the destruction of the parasites occurs very rapidly, ${ }^{1}$ for we have already seen that observations on the absorption of trypanosomes in vitro show that the parasites rapidly become deformed. Undoubtedly the protoplasm is rapidly digested, and only the nucleus and centrosome remain. Inside the leucocytes, especially the mononuclear, chromatic bodies are often seen, frequently a large one associated with a smaller one, which we think are the remains of a trypanosome which has been swallowed up.

We have obtained the best results, showing phagocytosis in vitro, by mixing together in hanging-drop some exudate (obtained by injecting intraperitoneally some fresh broth twenty-four hours previously) from an immune rat and blood containing trypanosomes. On making smears some hours later we have thus observed several trypanosomes beginning to be engulfed.

The manner in which trypanosomes are engulfed by the leucocytes of the rat closely resembles the processes described and figured by Sawtchenko for the incorporation of the spirochætes of relapsing fever by the leucocytes of the guinea-pig. ${ }^{2}$ In that case, however, the spirochætes can be seen a long time afterwards in large vacuoles, especially on staining with methylene blue intra vitam. These peculiarities can be accounted for by the differences in the structure of trypanosomes and spirochætes.

\section{Section 7.-Passive Immunity. Protective Value of the Seruin of Immunized Rats. Attempts at Treatment. The Mode of Production of Passive Immunity. The Results of Passive Immunization.}

It is to Rabinowitsch and Kempner that we owe the important discovery that the serum of rats which have received several injections of infective blood is protective. In their experiments I c.c. of serum injected into rats, either at the same time as the infective blood or twenty-four hours before or after, completely protects the animals from infection, whereas control rats contract a short infection (four to seven days). The serum of a normal rat, of a rat passively immunized, of a hamster infected with trypanosomes (of the hamster), and of a dog, did not show any protective power. The same is true of emulsions of brain, spleen, liver, and bone-marrow of rats whose serum was protective. This last observation is interesting, inasmuch as it creates a strong presumption in favour of the leucocytic origin of the protective substance. We have already seen that the trypanosomes are destroyed and digested by the leucocytes.

1 On adding methylene blue, or, better still, neutral red, to a hanging-drop, one is struck by the large number of leucocytic inclusions which are stained; but only on two or three occasions have we seen trypanosomes within the leucocytes, still recognizable by their shape, stain with neutral red.

2 Sawtchenko, 'Archives russes de pathologie et de bactériologie' (Podwyssotzky), I900. 
As to the mode of production of this passive immunity, Rabinowitsch and Kempner make the very unlikely suggestion of an antitoxic action. T. lewisi, which is so feebly pathogenic, is certainly not a parasite one would associate with any toxic action.

We have repeated the experiments of Rabinowitsch and Kempner, and have confirmed their observation that the serum of rats immunized against $T$. lewisi has protective properties. ${ }^{1}$ All our experiments were made with young rats of 30 to 125 grammes. With these rats the infection is always long and severe, and there are three well-marked stages in the evolution of the parasite. In such cases one can easily see the effect of the inoculation of a serum upon this evolution of the parasite, even when it does not entirely stop the infection.

In our control experiments we used the serum of various animals : normal rats, sheep, rabbit, horse, and fowl. In most of the experiments the different serums were mixed in the syringe itself with blood containing trypanosomes, and the mixture was at once injected into the peritoneal cavity of rats. In doses of $0^{\circ} 5$ to $x^{*} 3$ c.c. the various normal serums were unable to prevent an infection from following the inoculation.

As to the specific serums, their action varied with the rat furnishing the serum, and particularly with the number of injections the animal had received. Usually the serum of rats, which had been inoculated at least five times with infective blood, was active in doses of 0.5 c.c. when it was injected intraperitoneally mixed with trypanosomes. Under those conditions the parasites disappeared from the abdominal cavity in from a few to forty-eight hours without undergoing any development, and did not appear in the peripheral blood at all. Our most potent serums were furnished by rats which had received thirteen and ten injections respectively.

The serum of the former of these rats was active, when mixed with the virus, in doses of O.I c.c.; but this was evidently the minimal dose, for the trypanosomes injected took twenty-four hours to disappear from the peritoneum, and the rat had a very mild blood infection lasting four days. In doses of $\frac{1}{4}$ c.c. the blood infection was prevented, and the trypanosomes took only four hours to disappear from the peritoneum.

The Action of Heat upon the Protective Properties of THE SERUM.-Heated for half or three-quarters of an hour to $58^{\circ} \mathrm{C}$., and even to $64^{\circ} \mathrm{C}$., the serum still retains its protective power; but in these cases larger doses must be given, and the destructive action on the parasites is slower. At temperatures of $58^{\circ}$ to $64^{\circ} \mathrm{C}$., therefore, the serum loses part of its power. When the serum is heated to $55^{\circ}$ to $58^{\circ} \mathrm{C}$., its value is reduced to about one half the original, and on heating to $64^{\circ} \mathrm{C}$. it is reduced still more. ${ }^{2}$

${ }^{1}$ Francis states that he has also confirmed the discovery of Rabinowitsch and Kempner.

2 As we have said previously, the serum, before being heated to $64^{\circ} \mathrm{C}$, must be diluted with an equal volume of salt solution. 
Action of the Serum Unmixed with the Virus.-The serum also acts when it is injected subcutancously at the same time that the trypanosomes are injected intraperitoneally; but in that case, even though a much larger dose is given, the action is slow, and a mild blood infection follows. Injected twenty-four hours after the trypanosomes, the serum can stop an infection already started, provided it be given in big doses (I c.c.), but the trypanosomes do not disappear at once. Our experiments have also shown that the serum injected twenty-four hours before the trypanosomes prevents an infection, but for this purpose it is necessary to use slightly larger doses than are used in mixtures of serum and infective blood.

We have also injected the serum intraperitoneally instead of subcutaneously in doses of I c.c., twenty-four and forty-eight hours after the commencement of an infection. In all cases the blood infection was cut short, but sometimes there was a subsequent very short relapse. These cases should be looked upon as instances of cure rather than of prevention, because the trypanosomes were already numerous in the blood, and showed abundant signs of multiplication in the peritoneum, particularly in the forty-eight-hour experiments.

Attempts at Treatment.-We have been less successful in our attempts to stop an infection in the declared stage. Rabinowitsch and Kempner have similarly failed, judging by the following sentence (op. cit., p. 282): 'In the case of rats whose blood contained many parasites, serum injected intraperitoneally for a week showed no antiparasitic properties.'

We have tried the effect of serum by injecting it into rats on the eighth day after infection (the beginning of the third stage), on the thirteenth, thirty-fourth, and fifty-first days. On each occasion there were many trypanosomes in the blood, and in none of the rats did the infection show any signs of diminution.

Some rats received, in several injections, as much as 4 c.c. of serum from immunized rats, control rats receiving similar doses of serum from normal rats. The results were by no means constant. In some of the animals the number of trypanosomes diminished in the blood immediately after the injection, and the parasites disappeared in a few days. In others a transitory diminution in the number of trypanosomes occurred, and in fresh specimens the movements were seen to be sluggish, with an occasional tendency to agglutination. Lastly, in half of the rats treated we could not detect any antiparasitic action even on injecting 4 c.c. of serum from immunized rats in some cases. The serum of normal rats never had any effect.

The serum, therefore, has a slight action in some cases, but this beneficial effect is not by any means constant, nor is it rapidly produced.

We have tried, but unsuccessfully, to treat our rats with sodium arsenite, trypanred, and human serum, which all have a decided effect upon the animal trypanosomiases. 
The Mode of Production of Passive Immunity.-We have seen in Section 4 that in vitro a specific serum is always agglutinating, rarely paralyzing, and never microbicidal. As a rule, the agglutinating and preventive properties of a serum develop pari passu. Our paralyzing serums had the greatest protective powers.

Do these agglutinating and protective properties play any part in passive immunity? This is, a priori, hardly likely when the following facts are borne in mind: (I) Heat affects these properties differently; the agglutinating power is not appreciably altered at temperatures below $58^{\circ} \mathrm{C}$., but has completely disappeared at $64^{\circ} \mathrm{C}$., whereas the protective power, though reduced one half at $58^{\circ} \mathrm{C}$., is not entirely lost at $64^{\circ} \mathrm{C}$. (2). Highly agglutinating serums of normal animals (fowl, horse) have no protective power, whereas a specific serum, heated to $64^{\circ} \mathrm{C}$., whereby it is deprived of all its agglutinating properties, is still protective.

A close study of what happens in the peritoneal cavity proves that passive immunity is not humoral in origin, but cellular. On removing a little of the peritoneal fluid at varying intervals, from immediately after the injection until the complete disappearance of the parasites, and examining it in hanging-drop and stained preparations, the following changes are seen :

The trypanosomes in the peritoneal cavity always remain very active; they are never motionless, and do not appear altered at all. The only thing noticed in hanging-drop preparations is slight agglutination of the parasites, which is not surprising seeing that the serum injected is highly agglutinating. This agglutination which is seen to occur under the microscope is not to be compared with that described in Section 4, and, moreover, it is always incomplete. Lastly, it occurs quite as markedly in the exudate of rats injected with certain normal serums which have no protective power, such as the serum of the sheep. Agglutination can, therefore, play only an unimportant part in the protection of the body from trypanosomes.

It is not uncommon to find, on first examining a hanging-drop preparation, that the majority of the trypanosomes, though very active, are closely associated with the leucocytes, and that some are in process of being engulfed by them. On examining the preparation for some time, all the stages of the engulfment of a very active trypanosome by a phagocyte may be observed. The details of the process are identical with those we have already described in connection with active immunity.

Stained specimens are less instructive. As in the case of active immunity, the trypanosomes are destroyed very rapidly, and only the chromatic remnants-very numerous, it is true-are seen in the mononuclear and, less frequently, in the polymorphonuclear leucocytes in the exudate. ${ }^{1}$ Passive immunity is, therefore, phagocytic

1 This contains two-thirds polymorphonuclears and one-third mononuclears. 
in origin. In active immunity, as in passive, there appears to be a stimulation of the leucocytes.

Results of Passive Immunization.-Are rats which resist inoculation as a result of the action of a prophylactic serum really immune? We have inoculated all our passively immunized rats during the second week after 'immunization.' About one half of them were refractory; the others became infected, but the infection was always mild and of short duration. Moreover, we have noted in the majority of these rats that, in hanging-drop or ordinary fresh preparations of the blood, the trypanosomes show a distinct tendency to agglutination, sometimes forming small rosettes. We have already referred to this. It follows, therefore, that the agglutinating substance inoculated persists longer in the body-fluids of the rat than does the preventive substance. This phenomenon is not absolutely distinctive of rats passively immunized; we have seen it, though very rarely, in rats which have never received any serum, but Francis states that he has seen it constantly in such cases.

We may add that the rats which were refractory to a second inoculation had nearly always contracted a mild infection after the first inoculation with infective blood, plus prophylactic serum. 


\section{CHAPTER V}

\section{DIFFERENT TRYPANOSOMES OF SMALL MAMMALS}

THERE are a certain number of trypanosomes parasitic in the blood of various mammals, and apparently not pathogenic. The best known of them resemble T. lewisi-(I) in structure; (2) in that, as with $T$. lewisi in rats naturally infected, one does not as a rule find multiplication forms in the blood; and (3) in that these trypanosomes can develop only in a single species or in a small number of allied species of animals.

Such trypanosomes have been met with in the following species of mammals :

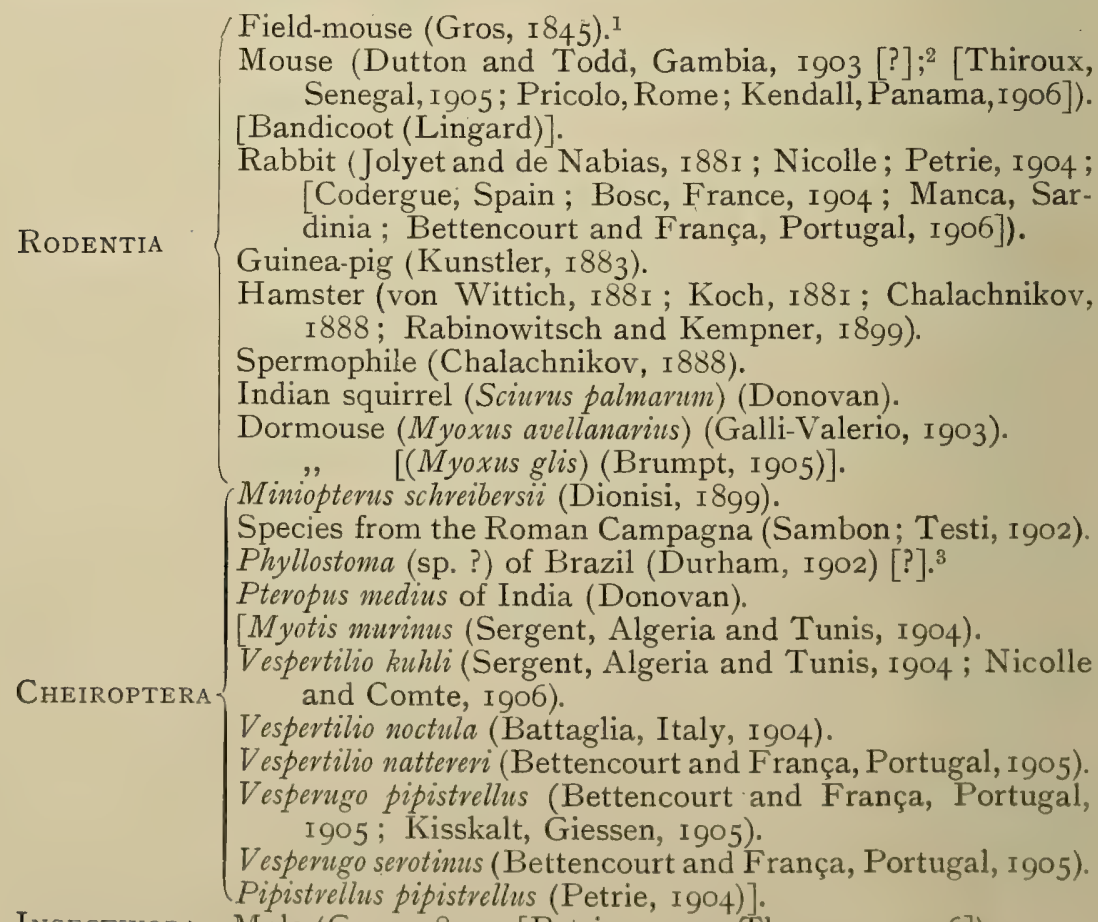

Insectivora-Mole (Gros, I 845; [Petrie, I904; Thomson, I906]).

[Carnivora - Badger (Bettencourt and França, Portugal, rgo5). ${ }^{4}$ ]

1 Gros, Bull. Soc. Natur. Moscow, First Series, v. 18, 1845, p. 423. Gros writes as follows: "The blood of a field-mouse showed worm-like bodies which were so numerous that the red corpuscles appeared endowed with movement. The parasites were so small as to be hardly recognizable when magnified 400 diameters. The blood of moles often presents the same appearance.'

These parasites have not been seen since.

${ }^{2}$ [ It is not quite certain whether the flagellate seen by. I)utton and Todd was a trypanosome or not.]

${ }^{3}$ [There is some doubt about this observation. See later.]

+ Finally, Zicmann (Arih. f. Silhifts u. Tropinhy's., v. G, No. Io, October, 


\section{Section 1.--Trypanosome of the Mouse.}

\section{[T. duttoni, Thiroux.]}

Whereas rats in different parts of the world are so frequently infected with trypanosomes, mice appeared until recently to be quite free from these hæmatozoa. The discovery recently made by Dutton and Todd of a flagellate in the blood of mice (species?) found in houses on MacCarthy Island, in the River Gambia, ${ }^{1}$ is all the more interesting on this account.

This flagellate, however, cannot be a trypanosome, or, to be more exact, a species of the genus Trypanosoma.

Unfortunately, it has hitherto only been studied in the living condition. It has the form of a long spindle with rounded ends, the thinner end having attached to it a flagellum a little longer than the body. Its discoverers could not see any trace of an undulating membrane. The flagellum is the only organ of locomotion the animal possesses, as one can easily verify by watching the movements of these parasites in a blood preparation. This flagellum acts chiefly as a propelling organ, but at the same time, by its whip-like movements, it pushes aside the red corpuscles or other solid bodies. which might get in the way of the body of the parasite, which it drags after it.

The body is $20.8 \mu$ long by $3.2 \mu$ in greatest width. At about $5 \mu$ from the anterior extremity there is a very refractile spot from which the flagellum starts. Other refractile granules exist in the anterior half of the body. The body is flexible, and often takes the form of the letter S.

Of fourteen mice examined, three showed the parasite in their blood but always in very small numbers, and the animals were apparently not ill. Field-mice in the same locality were free from infection (twenty examined).

A rat inoculated with infective blood remained free from infection.

'This parasite,' Dutton and Todd conclude, 'closely resembles Hevpetomonas (Leptomonas) biitschlii (Kent). The published figures of this organism very closely resemble our flagellats, except that in the latter the non-flagellate end is not so conical in shape.' Herpetomonas bitschlii inhabits the gut of a nematode (Trilobus gracilis).

[Thiroux ${ }^{2}$ was the first to describe a true trypanosome in the blood of the domestic mouse (Mus musculus), and the followirg account is taken from Thiroux's paper. This trypanosome was found in one out of thirty-three domestic mice ( $M$. musculus) caught in St. Louis (Senegal). Morphologically, it closely resembles the trypanosomes of other small mammals-rat, rabbit, and squirrel.]

1902) has seen trypanosomes (in very small numbers) in the blood of a chimpanzee in the French Congo, resembling in appearance the 'stumpy' form (preparatory to division) of $T$. lewisi, with this difference, that the free flagellum was a quarter as long again. The undulating membrane was very easily seen. The after-history of the chimpanzee was not followed. Perhaps this was the human trypanosome of Dutton.

1 Dutton and Todd, 'Trypanosomiasis Expedition to Senegambia,' Johnston and Thompson Yates Labor. Reports, v. 5, 1903, pp. 56, 57.

2 [Thiroux, Ann. Inst. Past., v. I9, 1905, pp. 564-572.] 
[Morphology.-Examined in the living condition, T. duttoni is seen to be very actively motile, always moving flagellum foremost, and often getting out of the field of the microscope. When its movements become more sluggish an undulating membrane is visible. When stained, its total length is $25 \mu$ to $30 \mu$, width $2.5 \mu$. The centrosome is fairly large, stains a deep violet by the Borrel-blue method, and is $5 \mu$ from the posterior end. The nucleus is $3.3 \mu$ long by ${ }^{\circ} 6 \mu$ to $2 \mu$ broad, stains well, and is $6 \mu$ to $8 \mu$ from the centrosome. There are sometimes fine chromatic granules in the protoplasm in the posterior half of the body. Anteriorly the body appears to end abruptly about $3^{*} 3 \mu$ from the nucleus, but on careful inspection the protoplasm is seen to be prolonged along the flagellum for a variable distance, which may be as much as $5^{\circ} 6 \mu$. The free flagellum measures $6.6 \mu$ to Io $\mu$, and there is a thin undulating membrane starting from the centrosome.]

[MULTIPLICATION.-Multiplication forms are seen only in animals that are severely infected. Parasites showing binary longitudinal fission are then not uncommon, and all stages of division may be seen. Rarely multiple division forms with several nuclei and flagella may be met with. The division forms of $T$. duttoni seen in the blood of mice closely resemble those seen in rats infected with T. lewisi.]

[CULTUREs. - In a special medium (a slight modification of that of Novy and McNeal), containing I part of bouillon-peptone agar to 2 parts defibrinated rabbit's blood, ${ }^{1}$ Thiroux succeeded in growing $T$. duttoni artificially at $25^{\circ}$ to $26^{\circ} \mathrm{C}$. Growth was slow in the first three generations, taking ten to fifteen days, but the fourth generation showed definite signs of growth on the fourth day. The parasite evidently takes some time to become accustomed to the altered conditions, as Thiroux had previously found to be the case with cultures of $T$. padda.]

[The trypanosomes develop in zooglœal masses (of rosettes or colonies), which usually float on the surface of the water of condensation. Growth is scanty in the earlier cultures, but in the later ones these masses are much more numerous, and colonies of the parasite, resembling those of $B$. coli, may even develop on the agar itself. The parasites seen in cultures are slightly smaller than in the blood. They vary much in shape-round, oval or pyriform, and fusiform. Thiroux does not agree with Novy and $\mathrm{McNeal}$ in thinking that these different forms are sexually different, for all intermediate stages are seen. On staining, it is seen that the centrosome is anterior to or at the side of the nucleus, and that there is no undulating membrane. $T$. duttoni thus resembles $T$. lewisi in giving rise to Herpetomonas-forms in culture. Reproduction takes place by binary fission or by division into three, but the commonest is multiple division, which gives rise to pretty rosette forms. As in cultures of $T$. lewisi, the flagella are always directed towards the centre of the rosette.]

[INocelation Experiments. $-T$. duttoni is inoculable into domestic mice (M. musculus), white mice, and harvest mice 1 [For cletails of the preparation of this medium, see Thiroux's paper, loc cit., pp. $566,567$. 
(M. minutus). The incubation period is four to nine days, and the infection lasts two to four weeks. The infection may be slight or severe, but the trypanosome is not pathogenic, and the presence of large numbers in the blood does not inconvenience their host. Intraperitoneal injections of cultures of the third and fourth generation, fifteen and twenty-five days old, were found to be as efficacious as injections of infective blood.]

[White rats (two), ordinary grey rats ( $M$. rattus) (six), guinea-pigs (four), and an owl (species ?), were found to be insusceptible.]

[The blood of cured animals does not agglutinate $T$. duttoni in the blood of mice. The spontaneous formation of rosettes in cultures prevents one seeing whether the blood of cured animals causes agglutination. Such blood does not agglutinate $T$. lewisi in rat's blood.]

[It is impossible to say with certainty whether T. duttoni is the same as the parasite seen by Dutton and Todd, as these investigators studied their flagellate only in the living state, and consequently gave incomplete details of its structure. T. duttoni is peculiar to mice, but appears to be inoculable into all species of mice. In spite of its morphological and cultural resemblances, it cannot be confounded with $T$. lewisi, because neither trypanosome is inoculable into the other's host.]

[Kendall ${ }^{1}$ has described, under the name T. musculi, a trypanosome which he found in about 8 per cent. of mice (M. musculus) in Panama. He says that morphologically, in its great motility, and in its mode of division it resembled T. lewisi. It was Io $\mu$ to $16 \mu \mathrm{long}$ by $3 \mu$ wide; the posterior end was pointed, and the nucleus, which was round in young forms and more elongated later, was situated in the centre of the body. This trypanosome was not pathogenic for the mouse, and was not inoculable into rats.]

[T. musculi is much smaller than $T$. duttoni, which is $25 \mu$ to $30 \mu$ long, so possibly it may be a distinct species.]

[Pricolo ${ }^{2}$ found trypanosomes in at least forty out of a hundred grey mice ( $M$. musculus) caught in the garden of a laboratory in Rome. In its general morphology (total length about $3.5 \mu$, of which free flagellum is about I2 $\mu$ ), its great motility, and its inoculability into white mice, this trypanosome resembles $T$. duttoni. The number of parasites in the blood was found to vary from time to time. A mild infection did not seem to incommode the mice, but Pricolo found that mice harbouring many trypanosomes in the blood when caught, usually died in twenty-four or forty-eight hours. Postmortem, there was anæmia of all the organs and serous effusion into the pericardium.]

[Trypanosomes were found in fleas caught on the infected mice, but no developmental stages could be made out in them.]

1 [A. I. Kendall, Journ. Infec. Dis., v. 3, 1906, pp. 228-231.]

2 [A. Pricolo, Centralb. f. Bakter., I, Orig., v. 42, Part 3, I906, pp. 23 I-235.1 
[Pricolo describes some interesting observations in connection with this trypanosome. When fresh blood swarming with trypanosomes was allowed to stand for ten hours, nearly all the parasites had disappeared, and were replaced by rounded or oval bodies, free or intracorpuscular. These bodies were also seen in stained specimens; they were mostly endoglobular, and all stages in the formation of these bodies were seen. Pricolo regards them as resistant forms of the trypanosome, but it is more likely that they. were involution or degenerative forms than resistant forms.

[Pricolo found that this trypanosome was able to pass through the placenta and to multiply in the foetus, the latter showing a number of different forms, which he considers as part of the cycle of development of the trypanosome. Among the various multiplication forms were some undergoing binary longitudinal fission; others in the form of rosettes, with the centrosomes towards the centre and the flagella peripheral; others, again, which seemed to divide transversely; and, finally, many forms of multiple division. In some the protoplasm had nearly or entirely disappeared, leaving only a rod-like centrosome and a nucleus, which gave rise to forms closely resembling the Leishman body. Pricolo looks upon these as phases in the progressive development of the bodies seen by him in the blood. In additicn, he describes bodies of the nature of oöcysts, in the interior of which young parasites, with nucleus and centrosome, are developed. Holmes ${ }^{1}$ had previously drawn attention to similar forms seen by him in the spleen and other organs in cases of surra. Moore, ${ }^{2}$ in a cattle trypanosomiasis observed in Nigeria, also describes coccus-like bodies occurring free in the plasma. After pairing, they are said to invade the red corpuscles, in which they become snail-shaped. These are again extruded into the plasma, and this is followed by the appearance of many trypanosomes in the blood, and by the extrusion of the 'tail-spot' of the parasites-apparently the same as the free coccus-like bodies.]

\section{Section 2.-Trypanosome of the Bandicoot.}

\section{[T. bandicotti, Lingard. $\left.{ }^{3}\right]$}

According to Lingard, young and half-grown bandicoots [Mus (Nesokia) giganteus] in Bombay and the Dekkan are infected with trypanosomes. The adult animals are not infected. The proportion of infected animals is 25 per cent. Lingard has not shown whether this trypanosome of the bandicoot is the same species as that of $M$. rattus and $M$. decumamis. It is, however, very likely that they are the same, for Lingard infected another species of Ncsokia (N.providens) with $T$. leacisi. Musk rats (Sorex carulcus) and mice (M. spicatus) in the same districts are never infected. ${ }^{4}$

[We have seen (p. 69) that Lingard succeeded in infecting guinea-pigs with the bandicoot trypanosome, but that the mule, ass, and rabbit proved refractory. The fact that guinea-pigs died of the infection tells against the identity of $T$. bandicotti and $T$. lowisi.]

\footnotetext{
${ }^{1}$ [Holmes, 'Evolution of T. idensi,' Journ. Comp. Peth, and Thirip., v. 17, INO

2 [Moore, Lancet, 1904, v. 2, p. 950.]

[Lingard, Indian Med. Gazetle, December, 1904, p. 445.]

- The above paragraph appeared as a footnote on p. 56 of the original.]
} 


\section{Section 3.-Trypanosome of the Rabbit.}

\section{[T. cuniculi, Blanchard.]}

This trypanosome was discovered by Jolyet and de Nabias in I89r. ${ }^{1}$ It was not rare in Bordeaux, at least at that time, for these observers found it in four out of ten rabbits examined.

Jolyet and de Nabias give a good description of the movements of the parasite, both of translation-flagellum end foremost-and in loco.

They also describe agglomeration of two or three parasites joined by their posterior ends, but still showing considerable motility. In January, at a temperature approaching zero, the parasites remained alive at least five days in an ordinary slide preparation, the edges of the cover-glass having been ringed with vaseline. They appeared to live very well in rabbit's blood kept at $4 \mathrm{I}^{\circ}$ and $42^{\circ} \mathrm{C}$.

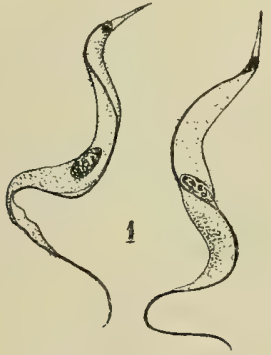

FIG. I6.-TRYPanosomes
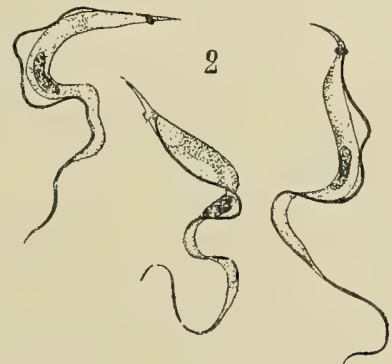

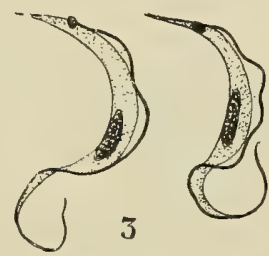

3

$$
\begin{aligned}
& \text { OF } M . \text { Rattus, OF THE RABBIT } \\
& \text { INDIAN SQUIRREL (DONOVAN). }
\end{aligned}
$$

I. Two $T$. lewisi of $M$. vattus. 2. Three trypanosomes of the rabbit (from a preparation of G. F. Petrie). 3. Two trypanosomes of Sciuvus palmarum (from a preparation of C. Donovan). All these trypanosomes were drawn with the cameva lucida under the same magnification, I,400 diameters.

Blood fixed with osmic acid, then dried and stained with concentrated alcoholic solution of dahlia-violet or fuchsine, enabled Jolyet and de Nabias to study the structure of the parasite, which is $30 \mu$ to $3^{6} \mu$ long (free flagellum I5 $\mu$ ) by $2 \mu$ to $3 \mu$ wide. These observers saw the undulating membrane, the nucleus, and probably also the centrosome, but they did not recognize the intimate relation of the latter body with the undulating membrane.

'The health of the animals does not seem to be affected by the presence of this animalcule, even though it be present in the blood in very large numbers, at least hundreds of thousands, each drop of blood containing more than fifty. It should be added, however, that the parasite is to be met with more often in rabbits which are wasted and ill, or in those which have suffered from diarrhœa, than in healthy rabbits.'

1 F. Jolyet and B. de Nabias, Soc. d'Anat. et Physiol. de Bordeaux, February 16, I891 ; Journ. de Méd. de Bordeaux, March I8, I901; and Travaux du labor. de M. Jolyet, v. I, I89I, pp. 39-42. 
Dr. M. Nicolle some years ago, in Constantinople, described a trypanosome in the blood of cachectic rabbits. This trypanosome was kept alive for a considerable time in the laboratory by passage through rabbits. The inoculated animals showed the parasite in their blood, became cachectic, and died. The trypanosomes were never abundant in the blood, and only appeared in it at intervals. The trypanosome of Nicolle was therefore pathogenic, but, unfortunately, it has been lost. (Unpublished communication of M. Nicolle.)

In I904 Petrie, ${ }^{1}$ at Elstree, Hertfordshire, found three perfectly healthy tame rabbits spontaneously infected with a trypanosome (Fig. I6, 2) which closely resembled $T$. lewisi of the rat--(I) in its morphology (it is, however, a little smaller, and its post-centrosomic end is very short and slender); (2) by its agglutination with the serum of the horse, I in $\mathrm{ro}$; and (3) by remaining alive for at least a month in the refrigerator.

The parasite was abundant in the blood of Petrie's rabbits, one of which had the trypanosome in its blood for six months.

The animals (ten rabbits, three guinea-pigs, one rat) inoculated intravenously, intraperitoneally, and subcutaneously with this trypanosome, did not become infected; but, nevertheless, this trypanosome-containing blood appears to be toxic, for four out of the ten rabbits died, without any sign of bacterial infection.

[Although it was in tame rabbits that Petrie ${ }^{2}$ first found the trypanosome, further observations have shown that the infection is rare in tame rabbits (230 examined, not a single one infected), but commoner in wild rabbits (four infected out of forty examined). Petrie was unable to transmit the infection to tame rabbits or to white rats. He succeeded in cultivating the wild-rabbit trypanosome on rabbit-blood agar, forms similar to those of $T$. lewisi being obtained.]

$\left[\mathrm{Bosc}^{3}\right.$ found a rabbit spontaneously infected at Montpellier. The trypanosome resembled that described by Petrie, but was slightly larger. Bosc thinks that the centrosome and chromatic protoplasmic granules are nuclear in origin. He also figures early stages of division and conjugation forms, but many of his figures are unconvincing (Mesnil). $\left.{ }^{4}\right]$

[The infection could not be transmitted to guinea-pigs, rats, or to three rabbits out of four. The fourth rabbit had a very mild infection of short duration.]

[Bettencourt and França ${ }^{5}$ found rabbits spontaneously infected in Portugal, and Manca"i a young wild rabbit (Lepus cuniculus) in Sardinia. The animals did not appear ill. Bettencourt and França succeeded in infecting two rabbits out of five inoculated intraperitoneally, the infection lasting twenty days. Mice, rats, and guinea-pigs were refractory, which proves the individuality of $T$. cuniculi.]

1 G. F. Petrie, Centralb. f. Bakter, I, Orig., v. 35, January 16, 1904, pp. $484-486$.

2 [Petrie, Journ. Hyg., v. 5, 1905, p. 193.]

3 [Bosc, Arch. f. Protistenk., v. 5, 1904, pp. 40-77.]

4 [Mesnil, Bull. Inst. Past., v. 3, I905, p. 144.]

5 [Bettencourt and França, Arch. Inst. roy. de Bucter. Camarn Pestana, v. I, I906, p. 167.]

${ }_{6}^{6}$ [Manca, C. R. Soc. Biol., v. 60, I906, p. 494.] 


\section{Section 4.--Trypanosome of the Guinea-pig.}

Kunstler recorded the existence of this trypanosome in $1883,{ }^{1}$ without giving a description of it. In 1898 he gave a figure of it, ${ }^{2}$ but without any description or context, nor was the magnification mentioned. The figure represents a very long organism with a much-folded undulating membrane extending from one end of the body to the other. Posteriorly (?) it terminates in a flagellum which is about one-third as long as the body. Anteriorly there is also a flagellum quite as long as the first, but which does not appear to be continuous with the undulating membrane. In the neighbourhood of the anterior extremity there is a small oval granule, which is the only detail of the internal structure of the parasite that Kunstler figures. According to this figure the parasite is rather a Trypanoplasma than a trypanosome properly so called.

Kunstler writes to us: "It is so rare that I have only found it in a few animals out of, perhaps, several hundreds which I have had the opportunity of examining.'

We may just recall the fact that we obtained an abortive infection with $T$. lewrisi in the guinea-pig.

\section{Section 5.--Trypanosome of the Hamster.}

\section{[T. rabinowitschi, Brumpt; $;^{3}$ Trypanozoon criceti, Lühe. $\left.{ }^{4}\right]$}

This trypanosome, discovered by Koch $^{5}$ and von Wittich ${ }^{6}$ in I $88 \mathrm{I}$ in the blood of the hamster (Cricetus frumentarius), has since been further investigated by Chalachnikov ${ }^{7}$ in I 888, and by Rabinowitsch and Kempner ${ }^{8}$ in 1899 .

The latter authors state, without giving any details, that the trypanosome of the hamster is morphologically hardly distinguishable from that of the rat, but they find that the trypanosome of the hamster cannot multiply in the body of the rat and vice versâ. They conclude that the trypanosomes of the hamster and rat constitute at least two varieties of the same species, if not two distinct species. They found that several rats which had been inoculated intraperitoneally with the trypanosome of the hamster, but unsuccessfully, were able to resist a subsequent inoculation with $T$. lewisi. Other rats, on the other hand, did not acquire any resistance to the

1 Kunstler, C. R. Acad. Sciences, v. 97, I883, p, 755.

2 Kunstler, Bull. Scientif. France et Belgique, v. 31, p. 206.

3 [Brumpt, C. R. Soc. Biol., v. 60, I906, p. I62.]

4 [Lühe, in the article on Blood Parasites in Mense's 'Handbuch der Tropenkrankheiten,' 1906, v. 3, pp. 69-193.]

5 Koch. Mittheil. a. d. kaiserl. Gesund., v. I, I88I, p. 8.

'Von Wittich, Centralb. f. mediz. Wissensch., 1881, No. 4. These two references are taken from Rabinowitsch and Kempner's paper.

7 Chalachnikov, 'Researches upon the Parasites of the Blood of Cold-and Warm-blooded Animals,' Charkov, I 888 (in Russian).

8 Rabinowitsch and Kempner, loc. cit., pp. 275 and 277. 
$T$. lewisi as a result of a previous inoculation with the trypanosome of the hamster.

Most of the inoculation experiments with this trypanosome have been done on rats. Von Wittich inoculated several guinea-pigs unsuccessfully, but as the inoculations were made subcutaneously and not intraperitoneally, no definite conclusions can be drawn from these experiments.

\section{Section 6.-Trypanosome of the Spermophile.}

In the same work in which he speaks of the trypanosome of the hamster, Chalachnikov mentions (p. 88) having found in the blood of Spermophilus musions and guttatus, of the district of Cherson (South Russia), a trypanosome which he regards as being morphologically identical with the trypanosomes of the rat and hamster. It measures ahout I $\mu$ wide by $30 \mu$ to $40 \mu$ or more long. It lives in broth and 0.6 per cent. salt solution for six days. Twelve per cent. of the spermophiles were infected.

\section{Section 7.-Trypanosome of the Indian Squirrel. (Sciurus palmarum.)}

[T. indicum, Lühe, Igo6.]

We have been able to make a careful study of the adult form of this trypanosome, thanks to the preparations which Dr. Donovan, of Madras, has been good enough to send us.

Of eleven squirrels examined in August, I903, two only were infected; in one the parasites were fairly numerous, in the other they were scanty. The trypanosome of the squirrel is closely allied to $T$. lewisi. In the preparations which were sent us only those forms, which in the case of $T$. lewisi we call 'thin adult forms,' were present; we did not see a single parasite undergoing division.

The general shape of the body (Fig. 16,3 ) is the same as that of $T$. lewisi. The posterior end is a little shorter, but the centrosome is equally large and striking. The edge of the undulating membrane is almost straight, and as in T. lcwisi, and contrary to what is found in the group brucei, it is only slightly folded. The nucleus is situated nearer the middle of the body than in the lowisi, in which it is anteriorly placed. The total length, including Hagellum, is I $8 \mu$ to $20 \mu$, which is a little less than that of lewisi. Fig. I6, 3 gives a sufficiently clear idea of the trypanosome of the Indian squirrel.

To sum up, a differential diagnosis between this trypanosome and $T$. lcreisi is possible, although the differences are slight. We cannot be sure that we are dealing with two morphologically distinct species, for these differences are hardly greater than those which may occur in the same species in different mammals (for example, $T$. brucci in the horse and mouse).

But it is very probable that the two species are at least phy'siolusicully' distinct, like the trypanosomes of the rat and hamster. 


\title{
Section 8.-Trypanosome of the Common Dormouse. (.Myoxus "vellanavius.)
}

\author{
[T. myoxi, Blanchard; T. blanchardi, Brumpt.]
}

Galli-Valerio $^{1}$ has recently seen in the unstained blood of Myoxus avellanarius, probably of Swiss origin, two specimens of a flagellate. He writes thus upon the subject: "They present an elongated body with one of the ends rounded, the other end prolonged into a flagellum joined to the body by a thin membrane slightly folded and motile by reason of its undulatory movements. The spherical nucleus is situated towards the rounded end of the body. Sometimes these two parasites became shrunken and assumed a spherical form, but one could distinguish at the periphery a portion of the membrane and of the flagellum. They were about $22 \mu$ long.' Galli-Valerio concludes that the characters presented by these flagellates show that they are closely allied to the trypanosomes.

[Brumpt ${ }^{2}$ has found trypanosomes in the common dormouse, Myoxus glis. Young animals were most frequently infected; animals a year old rarely harboured the parasite and appeared to be actively immunized. Morphologically this trypanosome, to which Brumpt has given the name $T$. blanchardi, closely resembles $T$. lewisi, and, like it, has the nucleus nearer the anterior end.]

[After intraperitoneal injection, the trypanosomes multiply rapidly, and from the third to the eighth day multiplication forms, similar to those of $T$. lewisi, are seen in the blood. After the eighth day, all the parasites are adult forms, no longer able to divide.]

[Infected animals do not appear ill. T. blanchardi is not inoculable into rats, nor $T$. lewisi into the dormouse.]

\section{Section 9.--Trypanosomes of Bats.}

\section{[T. vespertilionis, Battaglia.]}

Dionisi, ${ }^{3}$ in 1899 , recorded the occurrence of trypanosomes in an Italian bat, Miniopterus schveihersii. He found them frequently in bats infected with intracorpuscular hæmatozoa, as well as in healthy bats. F. Testi ${ }^{4}$ has also recorded them in bats in the Agro Grossetano. Sambon has recently told us that he found them in two bats in the Roman Campagna.

Trypanosomes undoubtedly exist also in the small bats of the genus Phyllostoma, occurring in Brazil (Para). Durham ${ }^{5}$ having placed a Stegomyia fasciata with one of these Cheiroptera, found the bat dead on the following day and the mosquito gorged with blood.

1 Galli-Valerio, Centralb. f. Bakter., I, Orig., v. 35, p. 85.

2 [Brumpt, Revue Scientif., I905, second semestre, pp. 32 I-332.]

3 Dionisi, Atti d. Soc. p.g. Studi. di Malaria, v. I, I 899, p. 145.

4 F. Testi, Boll. Soc. Zool. Ital., rgoz, quoted from the Centralbl. f. Bakter., Referate, v. 34, p. 66.

5 Durham, 'Report of the Yellow Fever Expedition to Para,' I900, p. 79. 
In this blood were numerous trypanosomes, which, according to Durham, were different from those of the rat and of nagana. ${ }^{1}$

Dr. Donovan informs us that he has observed trypanosomes in the blood of a large frugivorous bat, Pteropus medius, from the neighbourhood of Madras. The infected animals were not very common, and hitherto Donovan has not been able to find them again or to send us preparations of their blood.

[Since then trypanosomes have been found in various other species of bats in different parts of the world. In Vespertilio kuhli (Io out of 26 infected) and in Myotis murimus ( 7 out of 35) by Ed. and Et. Sergent ${ }^{2}$ in North Africa; in Vespertilio noctula ( 2 out of 6 ) by Battaglia ${ }^{3}$ in Italy; in Pipistrellus pipistrellus (3 out of 8) by Petrie ${ }^{4}$ at Elstree; in Vesperugo pipistrellus ( 5 out of 40 ) by Kisskalt ${ }^{5}$ at Giessen in Germany, and in 2 out of 9 by Bettencourt and França ${ }^{6}$ in Portugal; in Vesperngo serotimus ( $I$ out of 4 ) and Vespertilio nattereri (3 out of 14), also by Bettencourt and França. Nicolle and Comte $^{7}$ found nearly a quarter of the adult Vespertilio $k u h l i$ in Tunis infected; the young bats were never found infected.]

[The Sergents describe two forms of trypanosomes in bats. A smaller one, rather like $T$. lewisi, with very pointed posterior end. Total length $20 \mu$ to $24 \mu$, width $\mathrm{I}^{\circ} 5 \mu$, free flagellum $4 \mu$ to $5 \mu$. Like $T$. lewisi, it is very motile, the nucleus is rather nearer the anterior end, and the centrosome is some distance from the posterior extremity. They call this trypanosome $T$. nicolleorum, but it may be the same as $T$. vespertilionis (Battaglia, I904), in spite of certain differences in the description given. In two of the bats $(V$. kuhli) examined, much larger trypanosomes $(25 \mu$ to $30 \mu$ long by $6 \mu$ wide) were seen, in fresh films only. The name $T$. vespertilionis was given to these large forms, but as this had been previously used by Battaglia for the small trypanosome described by him, a new name will have to be found, if it be proved eventually that these large forms are a different species. The Sergents themselves recognised the possibility of these being individuals about to divide, though they say no intermediate forms were seen. Woodcock ${ }^{8}$ suggests that they may be sexual (female) individuals.]

[The trypanosome seen by Battaglia, which he calls $T$. vespertilionis (I904), was I $2 \mu$ to I $5 \mu$ long by $2 \mu$ to $3 \mu$ wide; the undulating membrane feebly developed, no free flagellum, and a large centrosome quite at the posterior end of the body." He thinks that $T$. vespertilionis, as well as $T$. lewisi, multiplies by means of 'spores' which originate from the nucleus by division.]

1 [In the light of recent observations (see p. 37), it is quite possible that the flagellates seen by Durham were parasites peculiar to the mosquito, especially as the described trypanosomes of bats are so like T. lewisi, and Durham states that those seen by him were different from those of the rat. See note by Mesnil, Bull. Inst. Past., v. 4, 1906, p. 606.]

2 [Sergent, C. R. Soc. Biol., v. 58, 1905, pp. 53-55.]

3 [Battaglia, Ann. di med. navale, v. 2, 1904, fasc. 5.]

+ [Petrie, Journ. Hyg., v. 5, 1905, pp. 191-200.]

"[Kisskalt, Centrulb.f. Bakter., 1, Orig., v. 40, 1905, pp. 213-217.]

" [Bettencourt and França, C. R. Soc. Biol., v. 59, 1905, pp. 305.307 ; Arch.

Inst. roy. de Bacter. Camara Pestana, v. I, 1906, pp. 187-194.]

7 [Nicolle and Comte, C. R. Soc. Biol., v. 6o, 1906, pp. 736.738.]

8 Woodcock, Quart. Journ. Micr. Sci., v. 50, 1906, p. 292.]

9 [Mesnil, Bull. Inst. Past., v. 3, 1905, p. 286.] 
[The trypanosome seen by Petrie resembles $T$. nicolleonum (Sergent). In the fresh condition it is very active and shows more contortions than $T$. lewisi. In stained films it often assumes a characteristic circular or oval form, and hence it is difficult to measure accurately. One was I $6 \mu$ long, including free flagellum $8 \mu$ (see Fig. 17,3 to 6 ). On rabbit-blood agar at room temperature there were signs of commencing multiplication in eight days, but the tube then became contaminated.]

[Kisskalt says that the trypanosome seen by him was probably the same as Petrie's. It was smaller and more slender than T. lewisi, and in fresh films some of the parasites looked almost like spirochætes. When stained, the centrosome was found quite at the posterior end, and seemed half out of the body. One of the bats harboured, in addition to the trypanosomes, small ring-shaped endoglobular parasites containing one or more chromatin dots. This parasite was found in eighteen other bats. Kisskalt was unable to trace any connection between this parasite and the trypanosome.]

[Bettencourt and França originally thought that their trypanosome was
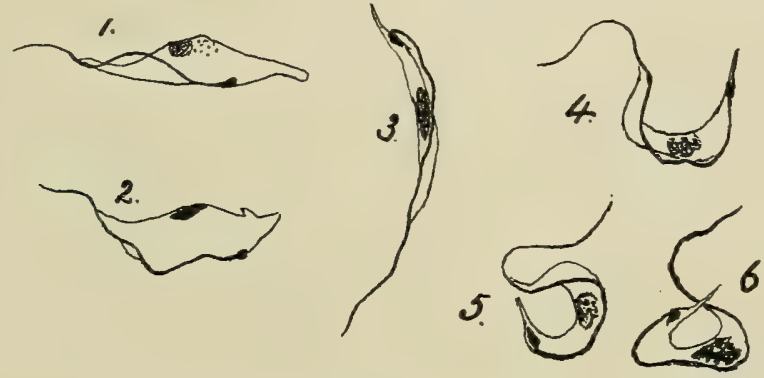

Fig. i7.-Trypanosomes of the Mole ( $T$. talpa) and Bat ( $T$. vespertilionis). (After Thomson and Petrie.)

I and 2. Trypanosomes of the mole, with nucleus and centrosome compact and sharply defined; in 2 the posterior end is partially retracted (Thomson). 3.6. Trypanosomes of the bat, showing frequent contortions of the body (Petrie).

a new species, and called it $T$. dionisii, but in their later paper (loc. cit.) they come to the conclusion, with which Mesnil agrees, that all the described species of bat trypanosomes should be included in a single species, T. vespertilionis, Battaglia, I904.]

[Nicolle and Comte in Tunis studied, in stained films, the two forms of trypanosomes described by the Sergents. The larger form they found to be $24 \mu$ by $4 \mu$; free flagellum very short, $3 \mu$ at the most. On account of the association or the alternation of the two forms in the same bat, these observers think that they belong to the same species.]

[These trypanosomes appear to be non-pathogenic for bats, and are not inoculable into rats, mice, guinea-pigs, or rabbits. Nicolle and Comte succeeded in only one out of twenty experimental inoculations into healthy bats.]

[The carrier of the parasite is unknown; Kisskalt examined two fleas and many mosquitoes with negative results.]

[We may mention here that Laveran ${ }^{1}$ inoculated a bat, Pteropus medius, subcutaneously with the Mauritian surra trypanosome. The bat became severely infected after five days' incubation, and died nine days after inoculation-that is, sooner than the average rats and mice inoculated with the same virus.]

1 [Laveran, C. R. Soc. Biol., v. 58, I905, p. 8.] 
Nicolle and Comte ${ }^{1}$ injected two bats (Vespertilio kuhli) intraperitoneally with the trypanosome of dromedaries in Algeria (see Chapter VI.). The bats became infected after an incubation of two days, the trypanosomes were most numerous at the end of four days, and disappeared after six days. The bats died in eighteen and twenty-two days (undoubtedly through captivity) without again showing trypanosomes in their blood. A control rat, inoculated at the same time as the bats, died of trypanosomiasis in thirteen days.]

\section{Section 10.-Trypanosome of the Mole. (Talpr europea.)}

[Trypanosoma talpa, new species.]

[Petrie, ${ }^{2}$ at Elstree, found six out of twenty moles infected with trypanosomes. They were always scanty in the blood, and in size and form resembled $T$. lewisi. Satisfactory stained preparations were not obtained. A white rat was inoculated intraperitoneally with infective blood, but was refractory.]

[Thomson, ${ }^{3}$ in May and June, Igo6, found trypanosomes in three out of fourteen moles examined at Elstree. All three moles harboured in addition intracorpuscular parasites, but this may have been merely a coincidence. Thomson was more successful than Petrie in obtaining stained specimens of the parasite. Only adult forms were seen, and they were fairly uniform in size and shape. The most striking feature of this trypanosome is the long, pointed, proboscis-like posterior end, looking sometimes almost like a posterior flagellum. The nucleus is relatively small $(2 \mu$ long) and lies close to the ventral border. There appears to be a narrow, slightlyfolded undulating membrane (see Fig. I7, I and 2). The average dimensions are as follows: Total length $27^{\circ} 6 \mu$, free flagellum $5^{\cdot 2} \mu$, post-centrosomic end $9{ }^{\circ} 3 \mu$, greatest width about $3{ }^{\circ} 5 \mu$. Intraperitoneal inoculation into a white rat and attempts at cultivation proved unsuccessful.]

[This parasite of the mole appears to be a new species, for which I propose the name Trypanosoma talpa.]

\section{[Section 11.-Trypanosome of the Badger. (Meles tuxus.)]}

\section{[T. pestanai, Bettencourt and França, Ig05.]}

[This trypanosome was found by Bettencourt and França ${ }^{4}$ in two badgers (Mcles taxus) out of four examined from the neighbourhood of Cintra, Portugal. It is $30 \mu$ to $32 \mu$ long by $5 \mu$ to $6.5 \mu$ wide, and is therefore relatively stumpy. The posterior extremity is long and thin, the centrosome being a considerable distance (Io $\mu$ ) from the tip. There is a well-developed and actively motile undulating membrane. The infected animals seemed quite well.]

1 [Nicolle and Comte, C. R. Soc. Biol., v. 58, 1905, pp. 245, 246.]!

2 [Petrie, loc. cit., p. 194.]

3 [J. D. Thomson, Journ. Hyg., v. 6, 1906, pp. 574-579.]

1 [lettencourt and Franca, Arih. Inst. Mry. Birt. Cimll. Pist., r. I, Igor, pp. 73-75; also C. R. Soc. Biol., v. 59, 1905, pp. 305, 306.] 


\section{CHAPTER VI}

\section{NAGANA AND ALLIED AFRICAN DISEASES}

Historical Review and Geographical Distribution of Nagana, AND OF THE Allied African TRYpanosomiases

Historical.- 'Nagana, or the fly disease,' says Bruce, ${ }^{1}$ who discovered its parasite, 'is a specific disease which occurs in the horse, mule, donkey, ox, dog, cat, and many other animals, and varies in duration from a few days or weeks to many months. It is invariably fatal in the horse, donkey, and dog, but a small percentage of cattle recover. It is characterized by fever, infiltration of coagulable lymph into the subcutaneous tissue of the neck, abdomen, or extremities, giving rise to swelling in these regions, by.a more or less rapid destruction of the red blood-corpuscles, extreme emaciation, often blindness, and the constant occurrence in the blood of an infusorial parasite' - a trypanosome.

The excellent ætiological and experimental study made by Bruce in Zululand will remain the fundamental work upon the subject of nagana.

An infected dog was sent by Bruce to England in November, I896. This was the starting-point of the researches of Kanthack, Durham, and Blandford, ${ }^{2}$ which were carried on first in London, then in Cambridge, from November, I896, to August, I898. A résumé of this joint work on the tsetse-fly disease was published at the end of I898. It contains numerous interesting experimental facts. It is to be regretted that the complete work has not appeared.

Plimmer and Bradford ${ }^{3}$ continued these researches in London, and occupied themselves mainly with the morphology of the

1 David Bruce, 'Preliminary Report on the Tsetse-Fly Disease, or Nagana, in Zululand,' Ubombo, Zululand, December, I895. ' Further Report,' etc., Ubombo, May 29, I896; London, I897. Nagana is a Zulu word which, according to Bruce, alludes to the state of depression and weakness of the sick animals.

2 Kanthack, Durham, and Blandford, Proc. Roy. Soc., v. 64, I898, p. Ioo.

3 Plimmer and Bradford, Proc. l'oy. Soc, v. 65, I899, p. 274 ; Centralb. $f$. Bakter., I, v. 26, 1899, p. 440 ; and Quart. Journ. Micr. Sci., v. 45, February, 1902. 
hæmatozoon, which they called Trypanosoma brucei ${ }^{1}$ (1899), and with its distribution in the bodies of infected animals.

It was also the $Z$ ululand parasite, for which we were indebted to Miss Florence Durham and to Dr. W. Mitchell, which we used in the series of investigations which we have carried out upon Trypanosoma brucei. ${ }^{2}$

Nocard used the same parasite for the experimental demonstration of the non-identity of nagana and dourine, ${ }^{3}$ and for his inoculation experiments with cattle and sheep, and it was this parasite again which Novy and McNeal used for their cultivation experiments. ${ }^{4}$

At the present time the study of this trypanosome has advanced more than that of any other pathogenic trypanosome, and it may therefore serve as a type.

The nagana of $Z$ ululand, or an allied disease, occurs in many other parts of Africa. Its existence, as we shall see later on, is associated with that of the tsetse-fly (genus Glossina), or, more accurately, with that of one or a few species of that genus. It is mainly, therefore, in the terrible accounts which explorers in South and Central Africa have given us of the ravages caused by this fly that it has been necessary until quite recently to study the distribution of the group of diseases with which we are dealing. All references to the tsetse-flies in these accounts have been analyzed and collated in the excellent monograph by Austen called 'Monograph of the Tsetse-Flies,' from which we shall have occasion to quote frequently.

Since the discovery by Bruce of a trypanosome as the causal agent of nagana, the microscope has helped materially in the elucidation of these 'fly diseases,' and at the present time papers upon the microscopic examination of the blood of sick animals and experiments upon laboratory mammals are becoming quite numerous.

We may mention the works of $\mathrm{Koch}^{5}$ upon the trypanosome of German East Africa; of Theiler ${ }^{6}$ upon that of the Northern Transvaal; of Brumpt ${ }^{7}$ upon that of Ogaden; of Schilling, ${ }^{8}$ Ziemann, ${ }^{3}$

1 [Plinmer and Bradford spelt it brucii, but Laveran and Mesnil state that, according to the rules of nomenclature, the spelling should be brucei, which is the form used by most authors.]

2 Laveran and Mesnil, C. R. Soc. Biol., March 23, 1901 ; Ann. Inst. Past., 1902, v. 16, pp. I-55 and 785-817; and Bull. Acad. Méd, June 3, 1902, p. 646.

3 Nocard, C.R. Soc. Biol., May 4, 1901.

4 Novy and McNeal, Journ. Amer. Med. Assoc., November 21, 1903; and Journ. Infec. Dis., v. I, I904, p. I.

5 Koch, Reiseberichte, etc., Berlin, 1898, pp. 65-72, 87, 88; and Deutsch. Kolonialblatt, No. 24, rgor.

${ }^{6}$ Theiler, Schweizer Archiv f. Thierheilkunde, v. 43, I901, p 97.

7 Brumpt, in Blanchard, Bull. Acad. Mit., Third Series, v. 46, October 29, 1901 ; and C. R. Soc. Biol., April 23, r9o4, p. 673.

8 Schilling, Centralb. f. Bakter., I, Orig., v. 30, October 30, I901, p. 545 : v. 32, April 16, 1902, p. 452; v. 33. January, 1903, p. 184 ; and [Arb.a. d. kaise $\%$. Gesund., v. 21, 1904].

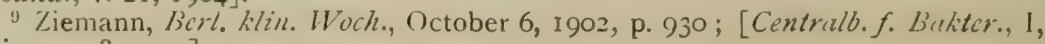
Orig., v. 38,1905$]$. 
and Martini ${ }^{1}$ upon that of Togo ; of Dutton and Todd ${ }^{2}$ upon that of horses in the Gambia; and of Broden ${ }^{3}$ upon that of the Congo. ${ }^{4}$

But a new question has arisen: Do all these 'fly diseases' of different parts of Africa constitute a single morbid entity, or is there, rather, a group of diseases closely allied, but each one possessing its own individuality? This question is far from being answered, but one may say that a priori a large number of facts point to a plurality of fly diseases. There are, on the one hand, the variations in virulence of the trypanosomes of different countries, and, on the other hand, the experimental proof which we furnished in June, I903, that surra and nagana, which were very generally considered to be identical, are really two distinct diseases.

Indeed, we must already consider the trypanosomiasis of horses in the Gambia, discovered by Dutton and Todd, as a separate morbid entity: its trypanosome has special morphological characters, and experiments to which we shall allude later on prove that animals immunized against the nagana of Zululand are susceptible to this trypanosome. A special chapter will be devoted to this trypanosomiasis.

As to the other trypanosomiases, we shall for the present include in one chapter all the African epizootics of Equidæ and of ruminants due to trypanosomes, of which the occurrence in a district appears to be associated with the presence of tsetse-flies, or possibly of some other biting insect, and of which the individuality is not yet established. But in the experimental, morphological, and biological study which we shall make of the trypanosomes of these different epizootics, we shall consider them separately according to their origin.

Geographical Distribution. ${ }^{5}$ - Let us examine in order the river basins of Eașt and West Africa. Nagana, properly so called, exists in Zululand, particularly in the low and humid regions, where malaria also occurs. As wè have already said, it was there that Bruce studied the disease. Cape Colony, Natal, Transvaal, and Orange River Colony appear free from disease, except Northern Transvaal, where Theiler studied the disease. All the countries around the Transvaal to the north, east, and west are infected (Bechuanaland, Matabeleland, Mashonaland, Mozambique). $\quad\left[\right.$ Theiler $^{6}$ writes (I905): 'In South Africa nagana has become a negligible quantity. It exists only in certain districts, not numerous, in Zululand and Rhodesia. The Transvaal and the coast of Delagoa Bay have become free, which is principally due to the ravages of cattle plague for several years ; as a result nearly all the big game is destroyed.']

1 Martini, Zeitschr.f. Hyg., v. 42, 1903, pp. 341-350; Deutsche mediz. Wochenschr., August 6, 1903, pp. 573-575 ; [Zeitschr.f. Hyg., v. 50, 1905].

2 Dutton and Todd, 'Trypanosomiasis Expedition to Senegambia'; Johnston and Thompson Yates Labor. Reports, v. 5, 1903.

3 Broden, Bull. Soc. études colon., February, 1903, and February, 1904.

4 [The more recent investigations upon the trypanosomiases of various parts of Africa are considered in detail in the second part of this chapter.]

6 See Africa on map, Fig. I, p. 4.

6 ['Theiler, Bull. Inst. Past., v. 3, I905, p. 663.] 
Foà ${ }^{1}$ has given a good account of the distribution of nagana on: the road from Pretoria to Lake Nyassa. Already before him the English travellers Sir W. C. Harris (I839), Gordon Cumming (I850), and James Chapman (I869), ${ }^{2}$ had given excellent accounts of the presence of the disease in the basin of the I.impopo, and of its. course in cattle and horses.

Further north, in the basin of the Zambesi, the disease has been known for a long time, and it was there that it was observed by. Livingstone, ${ }^{3}$ who described it with a precision truly scientific and remarkable considering the time he wrote. Judging from the enormous area occupied by the tsetse-fly, the disease must be very widely spread in that region, as it extends along the western shore: of Lake Nyassa. The flies are also very abundant all around Lake Tanganyika, and in the valley of the Luapula, which flows towards. the north and empties itself in Lake Moero. This region will be referred to again when speaking of the Belgian Congo.

In German East Africa the nagana of cattle and its geographical. distribution (valley of the Ruaha, etc.). have been closely studied in recent years, continuing Koch's. researches of 1897 , by Schmidt, Stuhlmann, Sander, Kummer, etc. (see the second part of this: chapter). [Ochmann ${ }^{4}$ has recently described a spontaneous trypanosomiasis of pigs in German East Africa. This is the first time that a natural infection in pigs has been observed. It is as yet uncertain whether the disease is nagana or not.]

In British East Africa, according to Stordy, between Mombasa and the Victoria Nyanza, the 'fly belt' commences about one-third. of the way from the coast. The same observer has recorded the existence of nagana on the island of Mombasa. ${ }^{5}$

Uganda is relatively free. ${ }^{6}$ However, Bruce, Nabarro, and Greig. have recently discovered in Entebbe, on the shore of the Victoria Nyanza, a disease in cattle due to a trypanosome. Unlike the trypanosome of nagana, it was not pathogenic for the dog. The trypanosome of this epizootic is likewise different from that of galziekte, for Bruce, who knew this trypanosome well, saw no. resemblance between them.

1 Foà, 'Du Cap au Lac Nyassa,' Paris, i 897.

2 J. Chapman, 'Travels in the Interior of South Africa,' London, I 868 (quoted from Austen).

3 Livingstone, 'Missionary Travels and Researches in South Africa,' first edition, 1857 ; 'The Last Journals,' London, 1874.

${ }^{4}$ [Ochmann, Berl. Tierärztl. Wochenschr., 1905, No. 19, pp. 337, 338. The animals were brought down from the cool uplands of Usambara, (ierman East Africa, to the hot coast districts, when several became ill and died. Five of the pigs looked very ill and were unable to stand; the respiration was hurried and there was high fever (IO5.8 F.). In three of the five animals many trypanosomes were present, and in one the spleen was much enlarged the day betore death. The trypanosome was very short and broad, and had a very short flagellum. If this should prove to be a new species, Ochmann suggests the name $T$. suis.]

"Stordy; The Veterincricn, v. 72, January, 1899, pp. II-20, and June, I899, pp. $385-388$.

"[The later observations of Nabarro and Greig in Uganda show that animal trypanosomiases are more prevalent there than was at first supposed (see Section 8 ; second part of this chapter.) $]$ 
In the country of the Somalis and the Gallas fly diseases have been recorded at different times (Donaldson Smith in I894, Prince Nicholas D. Ghika in I898), especially along the banks of the River Webi-Shrebeli. In the same country, in the district of Ogaden, Brumpt, naturalist of the mission of the Bourg of Bozas, observed an epizootic due to a trypanosome amongst-the camels of the mission. This trypanosome is said to be carried by the Glossina longipennis. The Somalis call this camel disease, as well as the fly which conveys it, Aino. [Theiler ${ }^{1}$ has also recorded the presence of this epizootic in thirty-four out of thirty-six camels that were brought from Somaliland to Pretoria.]

Our knowledge of these diseases further to the north is much less exact, but is being gradually extended.

Agatarchides and James Bruce have recorded the presence of flies, probably tsetse-flies, in Abyssinia. During the English expedition in Abyssinia (I867) a large number of the horses died. The veterinary surgeon, Hallen, who afterwards went to India, was struck by the likeness of this Abyssinian epizootic to surra. ${ }^{2}$

According to Savouré, in a recent paper in the Journal d'A griculture tropicale, surra occurs in cattle in Abyssinia. The disease appears to have been imported from Bombay by the Italians. It is therefore possible that in Abyssinia and Ogaden the trypanosomiasis is surra, and not nagana. That is not the opinion of Brumpt, who thinks that this disease is the same as that occurring in the Juba Valley and in East Africa to the south of Juba River. He bases his opinion upon the commercial relations of Ogaden with these regions.

[Memmo, Martoglio, and Adani ${ }^{3}$ have described a trypanosome epizootic, particularly virulent for cattle, sheep, and goats, in Erythrea, the Italian colony along the south-west shore of the Red Sea. Guinea-pigs, rabbits, dogs, and monkeys appear to be refractory to this trypanosomiasis, which in this and other respects. resembles the disease described by Cazalbou in the French Sudan (Haut-Niger, see later) under the name Souma.]

$\left[\right.$ Balfour ${ }^{4}$ and $\mathrm{Head}^{5}$ have described trypanosomiases amongst donkeys, mules, and cattle in the Anglo-Egyptian Sudan. These are possibly the same diseases as those recorded by Nabarro and Greig in the animals in Uganda.]

Finally, we may quote the opinion of Westwood, that the tsetse, extending beyond its ordinary limits, gave rise to the fourth plague of the Egyptians. 'A host of very dangerous flies came into the houses of Pharaoh, of his servants, and all over Egypt' (Exod.

1 [Theiler, Revue gén. méd. vétér., v. 7, 1906, p. 298.]

2 Brumpt thinks it was horse plague, and not a trypanosomiasis (Soc. Biol., April 23, 1904, p. 675.).

3 [Memmo, Martoglio, and Adani, Ann. d' Igiene Sper., I905, pp. I-46.]

4 [Balfour, Brit. Med. Journ., 1904, No. II, pp. 1455, 1456 ; Edin. Med. Journ., N.S., v. I 8, 1905, p. 202 ; Journ. Path. and Bact., v. II, 1906, p. 209.]

5 [Head, Journ. Comp. Path. and Therap., v. I7, I904, p. 206.] 
viii. 24). The fifth plague, the murrain of animals, would thus have been the result of the fourth. ${ }^{1}$

We shall now examine the distribution of the fly diseases in the river basins of West Africa.

Exact accounts of the existence of nagana in the Congo Free State are still scanty. Bruce refers to Schloss on this point. Broden (loc. cit.) has published observations upon trypanosome epizootics occurring amongst cattle, sheep, and donkeys on farms in the Free State around Leopoldville. ${ }^{2}$

At the south-eastern extremity of the Free State, in the district of Katanga - that is to say, quite in the interior of Africa-the presence of the tsetse-fly has been recorded by different explorers. ${ }^{3}$ These flies were said to be quite harmless to cattle, donkeys, etc.; but a little further north, in the neighbourhood of Lake Moero, Drs. Ascenzo and Derclaye have recorded a great mortality amongst cattle and donkeys. All these observations, however, lack scientific precision.

In Cameroon, ${ }^{4} Z$ iemann has noted the existence of trypanosomiases along the whole shore of the German colony. Cattle, sheep, goats, donkeys, horses, mules, and dogs are attacked. According to this author, the trypanosomes differ more or less from those which he observed in Togo in previous years, and the trypanosomes in the blood of a sheep were specifically distinct from the trypanosomes of Togo.

[After a more extended study of the trypanosomiases in Cameroon, $Z$ iemann ${ }^{5}$ has come to the conclusion that, in addition to nagana,

1 Westwood, quoted by Laboulbène, art. 'Tsetse' in the 'Diction. encyclop. des Sciences médic.'

2 Broden saw seven head of cattle of the herd succumb to nagana (a trypanosome morphologically almost identical with that of Bruce). He used the blood of a cow to infect a goat, which still lives; the trypanosomes are numerous in its blood, and the temperature is raised frequently to $41^{\circ} 5^{\circ} \mathrm{C}$. (I06 ${ }^{\circ} \mathrm{F}$.). On the same farm Broden saw two sheep die with trypanosomes in their blood. These trypanosomes were only $10^{\circ} 5 \mu$ to $15^{\circ} 5 \mu$ long by ${ }^{\circ} 7 \mu$ to $2{ }^{\circ} 5 \mu$ wide ; the flagellum had no free portion. Broden suggests the name $T$. congolense for this trypanosome, which he thinks is a new species. These trypanosomes killed a macaque monkey in twenty-five days and a guinea-pig in twenty-six days. In the monkey the trypanosomes presented a pleomorphism with forms identical with those of the sheep and others, $20^{\circ} 5 \mu$ long, with free flagellum. The trypanosome in the guineapig appeared to differ hardly at all from $T$. brucei; the validity of the species congolense is, therefore, somewhat questionable. On the same farm a young donkey contracted a trypanosome infection, the parasites resembling those found in the sheep. [In a later paper (Bull. Acad. roy. Belgique, 4th sér., v. 20, I906, pp. 387-416), Broden retains the name $T$. congolense provisionally. Since writing his earlier paper, he has found trypanosomes in Bovidae in Stanleypool and the region of the equator, and in dromedaries in Leopoldville. All the trypanosomes were similar to those previously found in the sheep and donkey. Mesnil (Bull. Inst. Past., v. 4, p. 1026) thinks that the trypanosome is $T$. dimorphon.]

${ }^{3}$ Captain Lemaire, Bull. Soc. belge Góogr. Commerc, 1900. Letter from the Secretary-General of the Department of Finances of the Congo Free State, June 20, 1903, and Letter from the President of the Special Committee, Katanga, December I9, 1902 (communicated to one of us). We have examined the flies. They are Glossina morsitans and Gl. longipalpis.

4 Ziemann, Deutsche mediz. Wochenschr., 1903, April 9, p. 268 ; April 16, p. 289.

${ }^{5}$ [Ziemann, Centralb. f. Bakter., I, Orig., v. 38, 1905, pp. 307 and 429.7 
which is mainly confined to the hinterland, there is an infection very prevalent along the coast and in the river valleys, due to a trypanosome, which he calls $T$. vivax, on account of its extraordinary motility. This trypanosome is morphologically much like $T$. evansi, the parasite of surra, and $Z$ iemann agrees with Laveran and Mesnil in thinking that possibly $T$. vivax may be the same as $T$. evansi, but is a species distinct from $T$. brucei.]

[The fly diseases in Cameroon have also been studied by Diesing, ${ }^{1}$ who attempted to immunize cattle against nagana, and was to some extent successful.]

[In I903 Ziemann (loc. cit., p. 3I 4 ) found a trypanosomiasis prevalent in Lagos amongst the domestic animals, the horses often dying of the disease.]

In I899 Christy recorded a trypanosome epizootic amongst horses in Upper Nigeria. ${ }^{2}$,

Likewise Hewby has noted the existence of a disease which appears to be nagana along the north bank of the river Benue, in Northern Nigeria. ${ }^{3}$ It was caused by the Gl. palpalis, and attacked horses (which died in from three to ten weeks) and cattle. The disease is caught solely during the rainy season.

[W. F. Gowers ${ }^{4}$ caught many specimens of tsetse-flies ( $G l$. morsitans) near Yola, on the bank of the River Loko, a small tributary of the Benue. The local natives all agree that cattle, horses, and donkeys are killed by the bite of the fly, while it is harmless to sheep and dogs. Gowers adds: 'I fancy that the tsetse-fly will be found to be pretty well distributed throughout the southern portion of Northern Nigeria.' The same observer found Gl. tachinoides along the Benue River, between Lau and Lokoja, in Northern Nigeria, and states that, with the exception of one or two small spots, no horses or cattle can be kept in this area. Above Lau, however, the river banks swarm with cattle, especially in the dry season (October to April), when the fly is scarce.]

[In Southern Nigeria, Moore ${ }^{5}$ has observed a fly disease in cattle which presents certain peculiar symptoms not usually noted in nagana.]

1 [Diesing, Arch.f. Schiffs u. Trop. Hyg., v. 9, 1905, pp. 427-431.]

2 Appendix H in Austen's monograph, p. 3 Io.

3 In Dutton and Todd, 'Trypanosomiasis Expedition to Senegambia,' p. 44.

${ }^{4}$ [See in Austen, 'Supplementary Notes on the Tsetse-Flies,' Brit. Med. Journ., September 17, 1904.]

5 [Moore, Lancet, 1904, v. 2, p. 15. The following symptoms were noted: Diminution of milk, unwillingness to eat (due probably to a loosening of the teeth), running from the eyes, and puffy swelling of the legs. The animals had difficulty in passing urine, 'hunching' themselves, and straining very much during the act. The flow of urine was intermittent, suggesting loss of contractility of the bladder. They wasted rapidly; the lymphatic glands rapidly enlarged; there was moderate intermittent fever, and during the early stage the respiration was increased and there was a dry, hacking cough.]

[After a month drowsiness supervened, deepening into profound slumber, which lasted thirty-six hours, and ended in death. Most animals, however, died before the sleepy stage from obstinate constipation and retention of urine (due probably to paralysis of intestine and bladder). Trypanosomes were found in the blood by Chichester, and one animal showed numerous and active spirochætes. (For the appearances described by Moore in the blood, see p. Io2). Fowler's solution by the mouth did no good, but hypodermic injections of (alkaline) sodium arseniate gave good results.] 
In Togo the existence of epizootics due to trypanosomes was first established by Koch $^{1}$ in 1895 by the examination of blood-films which had been sent to him. This epizootic was studied with the greatest care by Schilling (loc. cit.), who has written about it in three papers, to which we shall have occasion to refer later. There are in Togo three species of tsetse-fly, of which Gl. morsitans is one, but neither the flies nor the disease exist at the coast, both being confined to the hinterland. The epizootic of Togo has also been studied by $Z$ iemann (loc. cit.) [and by Martini, ${ }^{2}$ and Laveran and Mesnil ${ }^{3}$.

[Ziemann states, in his paper already referred to, that 'Schilling has in Togo ... in addition to the ordinary $T$. brucei, rediscovered the parasite $T$. vivax,' which Ziemann had found in the Cameroons in I903, and had already differentiated from T. brucei. Schilling thinks that $T$. vivax is not distinct from $T$. brucei, and that all the trypanosomiases in Togo are nagana. Laveran and Mesnil have recently concluded from their experiments on serum diagnosis that Schilling's Togo virus is probablv not nagana, and that Martini's Togo virus is probably neither nagana nor surra.]

The French veterinary surgeon Cazalbou has recently noted that the region of the Bani (a big tributary of the right bank of the Niger) is badly infected. According to native reports, it is impossible to keep alive any domestic animal along the banks of this river. Four horses taken along the banks of the Bani to 45 kilometres to the south-east of Ségou and kept there-two for one day and two for four days-all became infected with trypanosomes. The insects, which were caught in large numbers upon the horses, included ordinary flies, tsetse, and horse-flies. Cazalbou thinks that the trypanosomiases which developed in these horses were not of the same species (different diseases from both the clinical and experimental points of view); he admits that one of these species is very probably nagana.

[Laveran ${ }^{4}$ has examined a number of animals (sheep and dogs) which were inoculated at Ségou by Cazalbou with the blood of equines or bovines suffering from trypanosomiases, and recognises the existence of three distinct infections. A dog inoculated from a dromedary showed a trypanosome identical with that of mbori and of surra, $T$. evansi; a sheep showed a trypanosome closely resembling $T$. dimorphon; another sheep showed a trypanosome which appeared to be that of souma. Laveran thinks this is a new species, which he calls $T$. cazalbour. The Massina appears to be the principal focus of souma, but this disease has also been observed at Bamako and at Kati. According to Cazalbou and Pécaud, the disease is propagated by horse-flies (Tabinidæ). The trypanosome which resembles $T$. dimorphon also appears to be a new species, for which Laveran has recently proposed the name $T$. pecaudi $\left.{ }^{5}\right]$

[In I90, Major F. Smith ${ }^{6}$ discovered a small trypanosome (about

1 Koch, Reiseberichte, etc., Berlin, I898, p. 66.

2 [Martini, Zcitschr.f. Hyg. u. Infektionskrank, v. 50, 1905, pp. I-96.]

3 Laveran and Mesnil, C. R. Acad. Sciences, v. I42, I go6, see pp. 1486, I487.]

4 [Laveran, C. R. Acad. Sciences, v. 143, 1906, pp. 94-98.]

6 Ibid., v. 144, 1907, pp. 243-247.]

${ }^{6}$ [See R.A.M.C. Journ., v. 3, 1904, p. 330.] 
I3 $\mu$ long) in a blood-film taken from an ox post-mortem in Sierra Leone. Possibly this may have been the small form of $T$. dimorphon. Smith states that Gl. palpalis is found all over the colony.]

In I904 one of us ${ }^{1}$ observed trypanosomes which have the greatest resemblance to the trypanosome of nagana (general appearance, structure, size) in blood-films taken by Dr. Tautain, SecretaryGeneral of French Guinea, at the autopsy of a horse which died at Konakry. This horse, which Tautain only saw the day before death, staggered and was almost completely blind.

[Martin, ${ }^{2}$ in 1905 , found numerous trypanosomiases existing in Lower Guinea, the mountainous districts of Fouta Djalon and of Labé, and along the banks of the Niger. These diseases were acute or chronic, and Martin found spontaneous infections in the horse, ass, mule, ox, sheep, goat, dog, and pig. Nearly all the trypanosomes had common characters, and appeared to belong to the type dimorphon. The trypanosome of souma is also prevalent in various parts of French Guinea.]

Information about the trypanosome epizootics in Gambia is furnished by Dutton and Todd (loc. cit.), who collected it at the same time that they were making their extended inquiries into the distribution of human trypanosomiases, about which we shall have more to say in another chapter. The disease amongst horses exists at the coast, at Cape St. Marie, and along the whole extent of the Gambia, It is very common, and is largely responsible for the great mortality amongst horses in the colony. This trypanosome of the horse is different from that of nagana, and will form the subject of a special chapter (Chapter VII.). At Maka, in French Senegal, quite close to the frontier of Gambia, I50 miles inland, Dutton and Todd also observed two horses infected with trypanosomes, which must have been caught in English territory. From the similarity of the symptoms they thought that the so-called ' malaria' of horses which is seen at St. Louis, in Senegal, was the same trypanosome epizootic. The existence of a similar epizootic, endemic in the region of Upper Senegal, between Kayes and the Niger, had already been rendered probable by the works of the French veterinary surgeons Dupuy, ${ }^{3}$ Lascaux, Richard, and Pierre. ${ }^{4}$ Dutton and Todd also saw cases of this disease, but were unable to discover a trypanosome in association with it.

- A. Laveran, C. R. Soc. Biol., February 27, I904, pp. 326, 327.

2. [G. Martin, Ann. Hyg. et Méd. Colon., v. 9, 1906, pp. 304-314; C. R. Soc. Biol.; v. 6I, 1906, pp. 107-109.]

3 Dupuy, Rec. Méd. Vétér., 1888 , pp. 535 and 594 ; 1889, p. 253.

4 Pierre, see report, Cadiot, Bull. Soc. cent. méd. vétér., March 3o, I896. p. I48. Concerning the parasite Pierre writes thus: "Almost constantly we have met with in our preparations a crescentic element, thickened and pigmented at the summit of the arc; a transparent zone clearly marked off from the rest of the little body sometimes joins the extremities of the crescent. The disappearance of the parasites amongst the red corpuscles is sometimes very rapid, and if one does not take care to fix the blood previously upon the slide they disappear before there is time to make out their characters' (pp. 154, I55). Pierre thought the disease identical with human malaria and Indian surra. 
Observations in Cameroon and in Gambia show that these trypanosome epizootics may exist close to the coast. We find them also occurring in the heart of the African continent. Thus Dr. Morel, medical officer of the colonial forces, has furnished accurate information upon the distribution of trypanosome diseases in the region of Lake Chad. The tsetse-flies (probably Gl. morsitans from the specimens which we have been able to examine) and nagana only exist along, and close to, the banks of the River Shari. The disease occurred during the rainy reason, and attacked horses, donkeys, and cattle. A favourite habitat of the fly is a large sensitive plant, Mimosa polyacantha. Along the shores of Lake Chad there is no mimosa, no fly, and no nagana. ${ }^{2}$

In blood-films taken by Morel from sick animals we found a trypanosome closely resembling $T$. brucei in shape and size.

More recently another colonial surgeon, Decorse, has observed, also in the Shari Valley (at Kousri and Fort Archambault), epizootics amongst horses and cattle. In blood-films which he has given us we have seen a trypanosome differing markedly in its dimensions from $T$. brucei. Its average length is $5 \mu$ (instead of $25 \mu$ to $30 \mu$ ), and its width is in proportion. There seems to be no free flagellum, but we cannot be quite certain about this. It resembles $T$. dimorphon of Gambia (see Chapter VII.). In this region Decorse caught many specimens of $G l$. tachinoides $^{3}$ and also some of Gl. morsitans. $\mathrm{He}$ noted that the tsetse-fly exists there independently of the mimosa.

The trypanosomiases of camels and cattle in the regions of Timbuctoo and of Massina have formed the subject of careful investigations by Cazalbou, who has written several papers on them in the Bull. Acad. Méd. ${ }^{4}$ These trypanosomiases will be considered in a special paragraph later (see also p. II 8).

Do trypanosome epizootics, allied to nagana, occur in Algeria? It appears to us that this is no longer a debatable point, since the discoveries by Szewczyk ${ }^{5}$ and Rennes ${ }^{6}$ of a trypanosome epizootic amongst the horses of the Spahees in the extreme south-west of Algeria (valley of the Zusfana), and by the brothers Sergent ${ }^{7}$ of a

1 Morel, Ann. Hyg. and Méd. colon, v. 5, 1902, p. 305, and v. 6, I903, p. 264.

2 [Brumpt (C. R. Soc. Biol., April I6, 1904, p. 629), however, makes the following statement: "Gl. decorsei (i.e., Gl. tachinoides) has been found only in the Shari basin and along the shores of Lake Chad' (italics not in the original), 'where it seems to exist exclusively at the water's edge.']

${ }^{3}$ [In the original of Laveran and Mesnil's book this is called $\mathrm{Gl}$. palpalis, var. tachinoides (Austen), but from the study of further material Austen has come to the conclusion that Gl. tachinoides (Westwood) is in reality a perfectly distinct species. See Austen, 'Supplementary Notes on the Tsetse-Flies,' Brit. Med. Journ., September 17, 1904.] It is undoubtedly the same species which Brumpt (Soc. Biol., April It, 1904, p. 628) has recently described under the name Gl. decorsei, n. sp.

"See the reports of Laveran, Bull. Acad. Míd., June 30, 1903, p. 307; April 26, 1904, p. 348 .

i Szewczyk, Bull. Soc. centr. méd. vétér., April 30, 1903, p. 218.

${ }^{6}$ Rennes, ibid., September 30, 1903, p. 424, and April 30, 1904, p. 248.

7 Ed. and Et. Sergent, C. R. Soc. Biol., January 23, 1904, p. 120. 
disease amongst dromedaries in the district of Constantine. In discussing later on the characters of these epizootics and their relation to nagana, we shall show that it is difficult to identify them with dourine, the only trypanosome epizootic known in the North of Africa down to 1903. The case of the horse seen by Chauvrat in I892 must also, in all probability, be classed with these diseases allied to nagana. ${ }^{1}$

In the following pages we shall be concerned solely with the nagana of $Z$ ululand and its trypanosome discovered by Bruce. We shall then consider the trypanosomes of the African diseases allied to nagana, about which we have more or less accurate information, drawing particular attention to the points in which they resemble, and differ from, nagana.

\section{NAGANA.}

Pathogenic Agent : Trypanosoma brucei, Plimmer and Bradford, I899.

Section 1.-Animals susceptible to Nagana. Refractory Animals. Man. Symptoms and Course of the Disease amongst the Different Species of Animals.

Whereas the majority of protozoal diseases (malaria, piroplasmosis, etc.) are confined to a particular animal species or to a small number of allied species, nagana, like almost all the trypanosome diseases, can occur in a large number of species of mammals.

The list of species susceptible to nagana, whether naturally or experimentally, is already a long one, and even now is far from complete. One may say that, with very few exceptions, all mammals are susceptible to nagana. A certain number of species, especially ruminants living in the wild state, appear to have great tolerance, for they can harbour the T. brucei in their blood without being inconvenienced thereby; but they are not really refractory. We shall have occasion to refer to these cases again later, especially in the Section dealing with the spread of nagana.

Fortunately man is refractory to this terrible disease. Livingstone, Foà, and all the explorers who have travelled in countries where nagana is prevalent, relate that they have been bitten thousands of times by tsetse-flies without experiencing anything more than slight irritation analogous to that produced by mosquitoes. ${ }^{2}$

Other than mammals, we do not know of any animal susceptible to nagana. Birds are particularly refractory, the destruction of the trypanosomes inoculated apparently depending upon the high tempera-

1 Chauvrat, Rec. méd. vétér., I 896, p. 344 .

2 The recent discovery of a human trypanosomiasis does not invalidate this assertion, since the trypanosome is different from $T$. brucei. 
türe of birds (vide infra). [Schilling ${ }^{1}$ has, however, succeeded in infecting geese experimentally with his Togo virus, which he calls nagana, but which Laveran and Mesnil think is probably not nagana, but an allied disease. Mesnil and Martin, ${ }^{2}$ who repeated Schilling's experiments, found a goose susceptible to the original Zululand T. brucei. Goebel, ${ }^{3}$ using Laveran and Mesnil's virus, recently succeeded in infecting fowls.]

Nagana can be easily inoculated, and inoculations always succeed provided they are made beneath the epidermal or epithelial layers. When blood containing trypanosomes is injected into a vein or into the peritoneal cavity, infection takes place more quickly than when the inoculation is made in the subcutaneous tissue, but by the latter method infection occurs just as certainly as by the former.

The duration of the incubation period-that is, the time which elapses between the inoculation and the appearance of the parasites in the blood-varies with the species of animal, with the number of trypanosomes injected, and with the stage in which the parasites are on injection.

[Several observers have also found that the virulence of a particular trypanosome for the same species of animals may become increased by a series of passages, until finally a stage of vinus-fixe is reached. This increase in virulence is evidenced by a shortening of the incubation period, but more especially by a diminution in the duration of the disease. Thus, working with the same strain of parasite (the Zululand T. brucei), Laveran and Mesnil found the virulence for guinea-pigs greater than Kanthack, Durham, and Blandford had done, as regards both the incubation period and the total duration of the infection. Jakimoff, again, found the same parasite more virulent than Laveran and Mesnil had done. Nabarro and Stevenson, with the same strain of $T$. brucei which they obtained from the Lister Institute, London, in a rat, found that at first guinea-pigs lived for eight to eighteen weeks after inoculation, but that after a considerable number of passages through guinea-pigs, a stage of virus-fixe was reached, which killed the animals in fourteen or fifteen days. Martini found, in his experiments with the Togo virus, that a low virulence was often associated with a short form of trypanosome' without free flagellum. After several passages through animals, trypanosomes with free flagellum appeared, and this was accompanied by an increase of virulence. This relation of virulence to length of flagellum was by no means constant, however.]

When the fluid injected contains a very small number of parasites, the incubation period is longer than when blood rich in trypanosomes is injected. The incubation period is also prolonged when the trypanosomes have been kept for one or two days at the room temperature, or exposed for one or two hours to a temperature of $40^{\circ}$ or $4 \mathrm{I}^{\circ} \mathrm{C}$. This will be referred to again later. The diminution in virulence is manifested only by this prolongation

\footnotetext{
1 [Schilling, Arb. a. d. kaiserl. Gesund., v. 2 I, 1904, p. 476.]

2 [Mesnil and Martin, C.R. Soc. Biol., v. 6o, 1906, p. 739.]

3 [O. Goebel, ibid., v. 61, 1906, p. 321.]
} 
of the incubation period, the infection itself being just as severe. ${ }^{1}$ In the experiments to be described, infection was always produced, except when otherwise stated, by the injection of numerous trypanosomes in a normal condition.

In certain animals, especially rats and mice, $T$. brucei multiplies rapidly and regularly, so that the microscopical examination of the blood is sufficient to study the progress of the infection. In other animals the trypanosomes are very scanty, at least at certain periods of the disease, so that the diagnosis cannot be made by a simple blood examination. It is then necessary to inoculate $I$ to 3 or more c.c. of blood from the suspected animal into an animal in which the evolution of nagana is rapid and progressive. Throughout this volume we shall come across numerous instances showing that it is often indispensable to have recourse to inoculation experiments in order to be certain whether an animal is infected or not. (See especially the observations upon the pig, sheep, goat, and ox.)

The duration of the disease produced by $T$. brucei varies greatly with the animal species; from this point of view animals may be divided into three groups :

(I) Animals in which nagana runs an acute course : mouse, rat, field-mouse (Arvicola), marmot, hedgehog, dog, monkey, [cat, fox, squirrel];

(2) Animals in which nagana runs a subacute course: rabbit, guinea-pig, field-mouse (Mus sylvaticus), garden dormouse (Eliomys quercinus), equines, pig ;

(3) Animals in which nagana runs a chronic course: cattle, goat, sheep, [goose, fowl].

The three forms of nagana may be studied in the above order. ${ }^{2}$ [The table ${ }^{3}$ on p. I24 summarizes the results cbtained by different investigators in various animals infected with nagana.]

1 The following table shows clearly the influence of the number of trypanosomes injected, and of the mode of entry, upon the length of the incubat on period.

\begin{tabular}{|c|c|c|}
\hline \multirow{2}{*}{ Fluid inoculated. } & \multicolumn{2}{|c|}{$\begin{array}{l}\text { Incubation Period in the Mouse inoculated with } \\
\qquad \frac{\frac{1}{20} \text { c.c. Fluid. }}{}\end{array}$} \\
\hline & Intraperitoneally. & Subcutaneously. \\
\hline $\begin{array}{l}\text { Blood rich in trypanosomes, } \\
\text { diluted } \mathbf{I} \text { in } 5 \\
\text { The same, diluted } \mathrm{I} \text { in } 500 \ldots \\
\text { The same, diluted } \mathrm{I} \text { in } 50,000\end{array}$ & $\begin{array}{l}\text { I day (died in } 3 \text { days). } \\
2 \text { days (died in } 4 \text { days). } \\
4 \text { days (died in } 6 \text { days). }\end{array}$ & $\begin{array}{l}2 \text { days (died in } 5 \text { days). } \\
4 \text { days (died in } 6 \frac{1}{4} \text { days). } \\
5 \text { days (died in } 7 \text { days). }\end{array}$ \\
\hline
\end{tabular}

2 For a number of details see Laveran and Mesnil, Bull. Acad. Méd., June 3, Iyo2, p. 646.

${ }^{3}$ [This table is not in the original French edition.] 


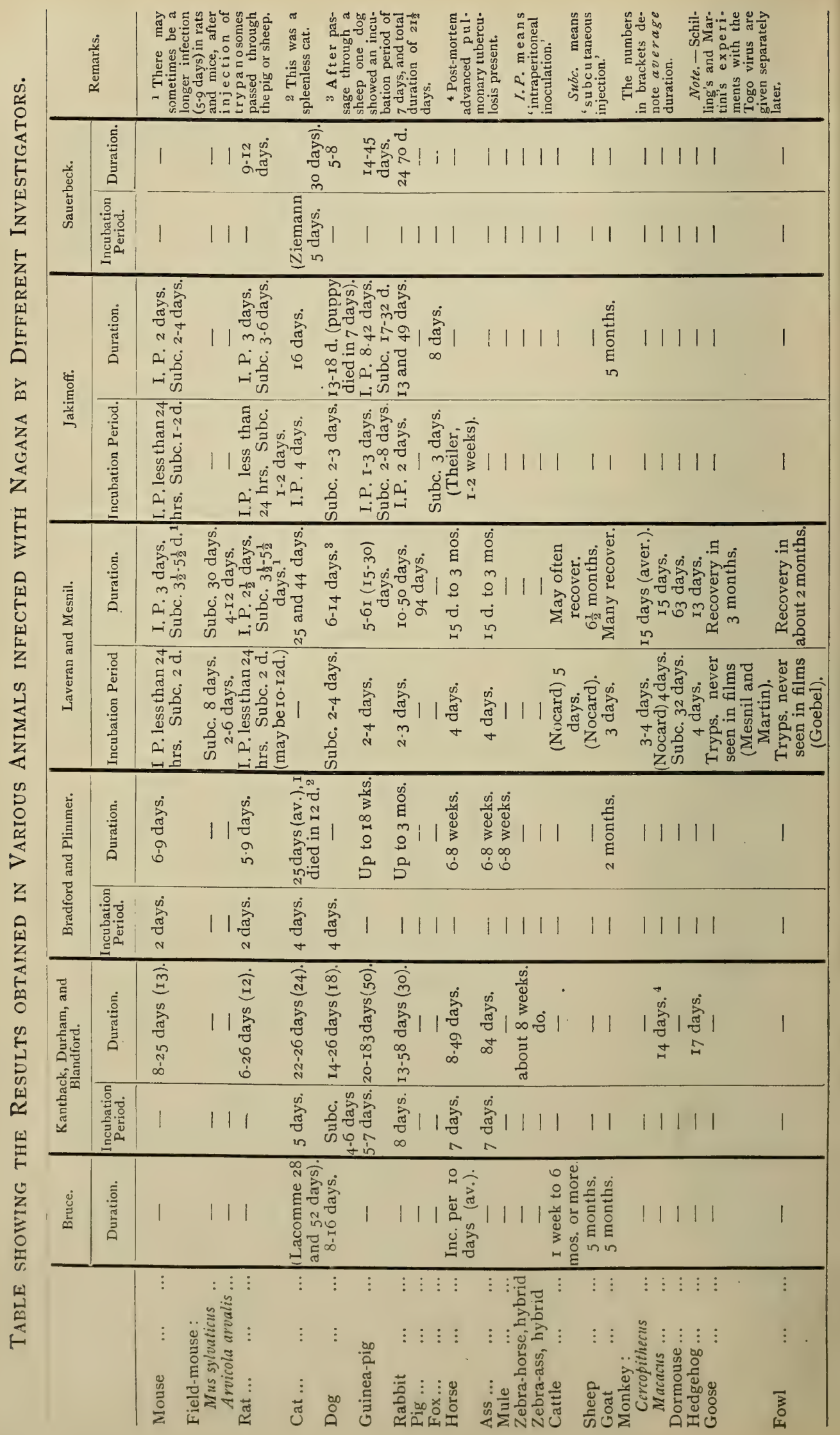




\section{(1) Animals in which Nagana runs an Acute Course.}

RATS And MicE.-The course of the disease is the same in tame rats (white and speckled), in sewer rats (Mus decumanus), and in tame or wild mice.

A rat from which the spleen had been removed eight days previously behaved exactly like a control rat, as did also rats highly immunized against $T$. lewisi.

When an animal receives subcutaneously a moderately large dose of trypanosomes-for example, $\frac{1}{20}$ c.c. of blood containing many parasites-the incubation period is about two days; with a smaller dose the incubation period is longer, and may even reach ten to twelve days. The duration of the incubation period gives important information about the number of trypanosomes contained in a specimen of blood when the microscopic examination is negative.

After intraperitoneal inoculation of a fairly large dose of the parasites, the incubation period is less than twenty-four hours, and, together with a multiplication within the blood-stream, there occurs an intraperitoneal reproduction of the parasite.

Upon this question of incubation period we are quite in accord with English observers. We do not agree with them, however, concerning the total duration of the infection: Kanthack, Durham, and Blandford give, for rats, from six to twenty-six days (average twelve) ; for mice, from eight to twenty-five days (average thirteen); Bradford and Plimmer give from six to nine days for rats and mice. ${ }^{1}$ Starting, as these observers also did, with an incubation period of two days, we found the total duration of infection, incubation included, to be only three and a half to five and a half days. After intraperitoneal inoculation, there is an incubation period of some hours, and the animal dies in two and a half days or in three days (rat and mouse respectively).

From the time that the trypanosomes appear in the blood, their numbers increase constantly and regularly until death, when they are as numerous as, if not more so than, the red blood-corpuscles. If the incubation period has been prolonged, the animal usually succumbs, as in the previous cases, two or three days after the first appearance of the parasites in the blood.

There are some exceptions to this rule. Animals inoculated with the blood of a pig (in the third month of the disease) or of a sheep (in the fourth and sixth months of the disease) sometimes suffer from a long infection (five to nine days instead of two or three). The trypanosemes, after having appeared in the blood, diminish in number for a time and then increase again. The trypanosomes of these animals suffering from a mild infection, inoculated into other rats and mice, are of ordinary virulence. We had a similar result on

${ }^{1}$ [One of Bradford and Plimmer's rats died in five days; loc. cit., p. 463.] 
inoculating into a rat trypanosomes which had been kept in contact with toluidin blue until they were completely motionless. ${ }^{1}$

The temperature in the case of two of our rats showed no definite rise. During life there is no lesion or morbid symptom ${ }^{2}$ noticeable, but the animals appear in good health until the approach of death. The majority of mice and a certain proportion of rats appear drowsy in the last hour of their life and die without convulsions. Some mice and the majority of rats, on the other hand, while apparently perfectly healthy, become very excited; this is followed by convulsions immediately preceding death. ${ }^{3}$

[Jakimoff ${ }^{4}$ found that rats and mice inoculated by him behaved similarly. For details of the incubation period and duration of the infection, see Table, p. I24].

FIELD-MICE (Avvicola arvalis).-We have experimented upon six field-mice, two inoculated intraperitoneally and four subcutaneously. Although it is difficult to keep these animals alive long when in captivity, fairly definite results were obtained with them. Two of them behaved exactly like rats or mice which served as controls: the trypanosomes appeared rapidly in the blood and quickly increased in numbers, the animals dying in four days, with very many trypanosomes in the blood. The spleen was three times the normal size. The other field-mice contracted a less acute infection; the trypanosomes appeared in the blood quickly or only after four to six days, but in all cases they were scanty in it. The animal then died in about a week, but death was undoubtedly hastened by captivity. The spleen was enlarged.

MARMOTS.-According to Blanchard, ${ }^{5}$ who used the same virus as we did, the marmot, when awake and also when very lethargic, suffers from an acute infection with many trypanosomes in the blood, death occurring in nine to fourteen days. On the other hand, when sound asleep the marmot is either less susceptible or entirely refractory. Unfortunately, the experiment quoted by Blanchard does not lead to any definite conclusion.

Hedgenogs.-Kanthack, Durham, and Blandford inoculated a hedgehog. Infection occurred rapidly, and the animal died seventeen days after inoculation, having lost a quarter of its original weight.

We have also experimented upon a hedgehog weighing

1 One of our rats survived after having shown trypanosomes in its blood. This rat was inoculated intraperitoneally on April 26, I90I, with a mixture of equal volumes of toluidin blue and of trypanosome-containing blood injected after being mixed for fifteen minutes, when the trypanosomes were quite motionless. On May I trypanosomes were present in the blood; on May 3 they were very scanty, after which the examinations were always negative; tested later this rat was not immunized.

2 Kanthack, Durham, and Blandford note lesions of the eyeball in their rats.

3 Our researches include observations upon more than 500 rats and mice.

4 [W. L. Jakimoff, Centralb. f. Bakter, I, Orig., v. 37, p. 668.]

${ }^{6}$. R. Blanchard, C. R. Soc. Biol., July 25, 1903, p. I122. 
420 grammes, which was inoculated subcutaneously on January 7 , I903.

Trypanosomes appeared in its blood after four days. They were scanty for several days, then very numerous from January i 6 to I 8 , and more so on the Igth and 2oth, when the animal died. The disease lasted thirteen days. At the time of death the animal weighed only 340 grammes. Spleen weighed ro grammes, undoubtedly much enlarged.

Dogs.-The dog is very susceptible to nagana. The incubation period, after subcutaneous injection, was from four to six days in the experiments of Kanthack, Durham, and Blandford, from two to four days in our own. Fever is often slightly marked; in the majority of cases the temperature is raised from the third to the fifth day, but

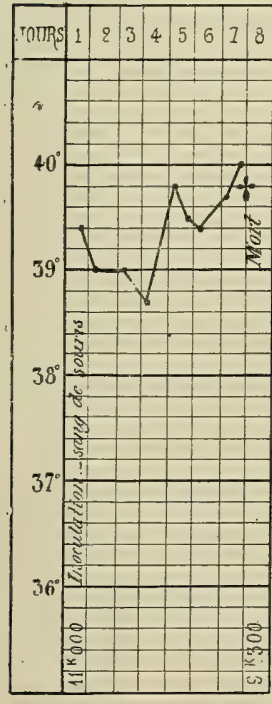

A

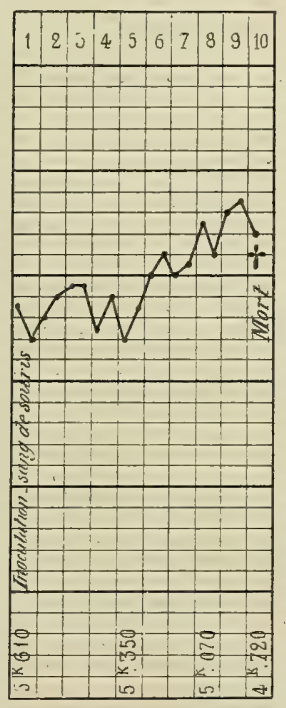

B

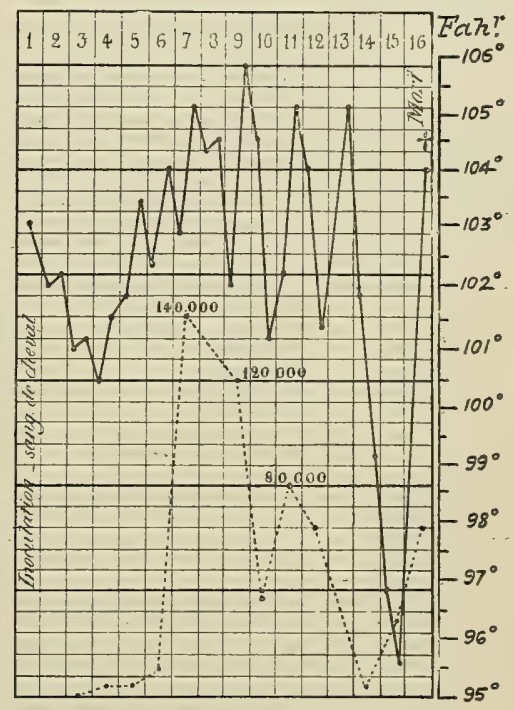

C

Fig. I8. - Temperature Charts of Three Dogs with Nagana.

$A$ and B. Our own observations. C. After Bruce. In the case of $\operatorname{dogs} A$ and $B$ the weight is noted. In the case of $\operatorname{dog} \mathrm{C}$ the dotted lines represent the variations in the number of trypanosomes present, the figures indicating the number of parasites per cubic millimetre of blood.

never goes beyond $40^{\circ} \mathrm{C}$. [10 $\left.4^{\circ} \mathrm{F}.\right]$. Bruce noted continued fever with exacerbations to $40^{\circ}$ and $4 \mathrm{I}^{\circ} \mathrm{C}$., and we have had similar cases (see charts, Fig. I8).

The total duration of the infection was from eight to sixteen days in Bruce's experiments; from fourteen to twenty-six (average eighteen) in those of Kanthack, Durham, and Blandford; from six to fourteen in the experiments of Nocard and in our own. In one instance one of our dogs died twenty-one-and a half days after inoculation, the incubation period lasting seven days. It had been inoculated with blood containing few trypanosomes, probably of modified virulence by passage through a sheep.

During the last hours of its life the dog appears very weak, and it 
dies without apparently suffering. Edema of the genital region (sheath, testicles), with considerable hypertrophy and congestion of the inguinal glands, is frequently, but not constantly, present. Less frequently, transient œdema of the face, particularly of the eyelids, and opacity of the cornea are observed. We have never seen, as have Bruce, Kanthack, etc., blindness following these corneal troubles; nor have we ever seen the pustular eruption described by Bruce. The animal rapidly wastes and often presents transient paresis.

When the course of the disease is rapid (six to nine days), the trypanosomes constantly increase in number from the time that they appear in the blood, or, rather, their number remains stationary for several days and increases later. When the disease lasts twelve days there is generally a diminution in the number of parasites, followed by an increase, which lasts until death. Finally, in our dog which lived for twenty-one and a half days there were several such periods of diminution, followed by an increase.

Speaking generally, one may say that, after the rat and mouse, the dog shows most trypanosomes in its blood during the course of the disease.

CATs.-The cat appears less susceptible than the dog to nagana, and perhaps, when nagana in the cat has been more studied, it will be necessary to classify this animal with those in which the disease runs a subacute course.

Kanthack, Durham, and Blandford give five days as the incubation period. Death occurs in twenty-two to twenty-six days. The animal has fever; trypanosomes are present in the blood, but their number shows marked daily fluctuations. As with the dog, an affection of the aqueous humour, with fibrinous deposits in the anterior chamber, and opacity of the cornea, are observed.

Plimmer and Bradford state that trypanosomes are less numerous in the cat than in the dog. A cat from which the spleen had been removed died twelve days after inoculation with nagana.

We have never experimented on cats, and can only quote the following observation, for which we are indebted to Dr. Chantemesse.

On October 20, I903, a cat ate an infected mouse which had just died. A month later the cat was losing weight, its head was swollen, and its eyes affected. These troubles became more marked, and the animal died on December 3, forty-four days after this accidental inoculation. The blood swarmed with trypanosomes, the spleen was considerably enlarged, (35 grammes for an animal of 2,040 grammes); the liver was also enlarged. The animal was completely blind owing to opacity of the cornea and lens. All round the eyes the hair had fallen away and scabs . had formed.1

1 Since then we have observed another cat, which died twenty-five days after having eaten an infected guinea-pig, with practically the same symptoms as the preceding; during the last days of its life trypanosomes were numerous in the blood. 
[Ziemann ${ }^{1}$ inoculated a cat with $T$. brucei from a chronic case in a horse; the incubation period was five days, and the disease lasted thirty days. Lacomme ${ }^{2}$ has recorded two cases of nagana in cats which had eaten rats just dead of the disease; the cats died in twenty-eight and fifty-two days. For other experiments on cats see table, p. I24.]

Monkeys.-Monkeys, at least the genus Cercopithecus, are easily infected, trypanosomes rapidly multiplying in the blood, where they are met with in large numbers. The disease had an average duration of fifteen days, and terminated fatally in each of three cases. A baboon inoculated by us proved refractory.

The chief symptoms are: fever, anæmia, œdema (very slight in the case observed by us), and general weakness, which becomes very marked in the last stages of the disease. About the fourth day after inoculation the temperature rises from the normal $\left(37^{\circ} 5^{\circ} \mathrm{C}\right.$. in the monkey ${ }^{3}$ ) to about $40^{\circ} \mathrm{C}$., and after remaining up for several days, falls again to normal. The monkey, of which a detailed account is given below, died with a remarkably low temperature; the day before death the rectal temperature was only $28.5^{\circ} \mathrm{C}$. $\left[83^{\circ} 4^{\circ} \mathrm{F}.\right]$.

Kanthack, Durham, and Blandford experimented upon a Macacus rhesus; it survived the inoculation a fortnight, and died in 'an advanced condition of pulmonary tuberculosis . . . ; very many hæmatozoa were present in the blood until death occurred.'

Nocard experimented upon 'an old Macacus, active and very spiteful, which received under the skin of the tail several drops of the blood of a mouse. Four days later the monkey was quiet, refused its food, and allowed itself to be handled with impunity. Its temperature rose to $4 \mathrm{I}^{\circ} \mathrm{C}$. [IO6 ${ }^{\circ} \mathrm{F}$.] , and the blood contained an enormous number of trypanosomes.' This monkey-so Nocard kindly informed us-died fifteen days after inoculation; it had fever, œdema of the eyelids and pouches, and very many parasites in the blood from the fifth day onwards. At the time of death the parasites were more numerous than the red corpuscles.

A Cercopithecus we inoculated with nagana died in thirteen days. As it showed certain symptoms resembling those produced by the inoculation of $T$. gambiense, we reproduce the detailed record of the monkey as we presented it to the Académie de Médecine in Igoz.

A monkey, Cercocebus fuliginosus (Cercopithecus), was inoculated on March 23, I9O2, under the skin of the abdomen with the blood of a mouse which had just died of nagana. At the time of injection the trypanosomes were very motile.

Rectal temperature of the monkey before injection, $37^{\circ} 6^{\circ} \mathrm{C}$. $\left[99^{\circ} 6^{\circ} \mathrm{F}\right.$.]. Weight, 2,040 grammes.

1 [Ziemann, Centralbl. f. Bakter., I, Orig., v. 38, p. 314.]

2 [L. Lacomme, Journ. de Phys. et Path. gén., v. 8, I906, pp. II5-I I7.]

3 [In Uganda we found the average normal temperature of monkeys (Macacus and Cercopithecus $)$ to be $102^{\circ} 2^{\circ} \mathrm{F} .\left(39^{\circ} \mathrm{C}\right.$.).] 
March 26: Few trypanosomes in the blood.

March 27: Trypanosomes very numerous. In the evening the temperature rose to $40^{\circ} \mathrm{C}$. [104 ${ }^{\circ} \mathrm{F}$.] ; the monkey does not appear ill, and has a good appetite.

March 28: Trypanosomes numerous; E. $40^{\circ} \mathrm{I}^{\circ} \mathrm{C}$. $\left[\mathrm{IO}_{4} \cdot 2^{\circ} \mathrm{F}.\right]$.

March 29: Trypanosomes numerous; E. $39^{\circ} 9^{\circ} \mathrm{C}$. [103.8 $8^{\circ} \mathrm{F}$.].

March 30: Trypanosomes have appreciably diminished; M. $36.7^{\circ} \mathrm{C}$. $\left[98^{\circ} \mathrm{F}.\right]$. The monkey is less lively than usual, but eats well.

March 31 : Trypanosomes very scanty; M. $38^{\circ} \mathrm{I}^{\circ} \mathrm{C}$. [100 $\left.6^{\circ} \mathrm{F}.\right]$.

April I : Trypanosomes scanty; M. $374^{\circ}$ C. $\left[99^{\circ} 4^{\circ}\right.$ F. $]$; E. $39^{\circ} 8^{\circ} \mathrm{C}$ [103. $\left.6^{\circ} \mathrm{F}.\right]$.

April 2: Trypanosomes scanty; M. $364^{\circ} \mathrm{C}$. $\left[97^{\circ} 6^{\circ} \mathrm{F}.\right]$; E. $36.3^{\circ} \mathrm{C}$ $\left[974^{\circ} \mathrm{F}.\right]$. The monkey is getting weaker; remains quiet almost continually, with his head between his legs. A little œedema of the right eyelids present ; appetite diminished.

April 3: Trypanosomes scanty; weakness increasing, subnormal temperature very marked; M. $34^{\circ} 4^{\circ} \mathrm{C}$. $\left[94^{\circ} \mathrm{F}.\right]$; E. $33^{\circ} 4^{\circ} \mathrm{C}$. $\left[92^{\circ} 2^{\circ} \mathrm{F}\right.$.]

April 4: The monkey is so weak that it can hardly maintain the sitting posture; it constantly reels and falls on its side. The temperature continues to fall, reaching only $28.5^{\circ} \mathrm{C}$. $\left[834^{\circ} \mathrm{F}\right.$. $]$ in the rectum. Trypanosomes very scanty. The blood is very fluid, and flows freely from a small cut in the ear, which was made to obtain blood for examination. The blood fixes badly, and the red corpuscles are greatly diminished in number.

The monkey was found dead on the morning of April 5. It weighed I,920 grammes; the spleen, I9.5 grammes. The tissues were anæmic; the blood watery, as in the severe anæmias; no œedema or infiltration of the serous membranes; urine normal.

[Fox.-Jakimoff injected a fox subcutaneously. The incubation period was short-three days-and the trypanosomes were very numerous, coming even to exceed the red corpuscles in number. The disease ran a very acute course, death resulting on the eighth day].

[SoUIRREL.-Mathis ${ }^{1}$ has shown that the squirrel is susceptible to experimental inoculation. A common French squirrel (Sciurus vulgaris) became infected after an incubation period of seven days, and died thirty-eight days after injection. In an Annamese squirrel (Sc. griseimanus) the incubation period was four days, and the total duration of the infection nineteen days. Trypanosomes were very numerous in the blood, and the animals did not appear ill until a few days before death. In one case there was opacity of the corneæ and marked wasting. Both animals were drowsy shortly before death. Post-mortem the spleen was much enlarged in one of the animals.].

\section{(2) Animals in which Nagana runs a Subacute Course.}

Rabirts.-The duration of the incubation period noted by Kanthack, Durham, and Blandford is about eight days. In our experiments we frequently observed, with intravenous or subcutaneous inoculation, that it was only two or three days.

The temperature runs a very variable course. Sometimes in ${ }^{1}$ [C. Mathis, C. R. Soc. Biol., October 13, 1906, v. 6r, p. 273.] 
cachectic rabbits, which succumb quickly, the temperature rises when the parasites appear in the blood. In more resistant rabbits during the eight or ten days which follow the inoculation the temperature remains below $40^{\circ} \mathrm{C} .\left[\mathrm{IO}^{\circ} \mathrm{F}.\right]$; then there is intermittent fever, with irregular rises to $40^{\circ}$ and $4 \mathrm{I}^{\circ} \mathrm{C}$. [IO $4^{\circ}$ to IO $5^{\circ} 8^{\circ} \mathrm{F}$.], rarely above $4 \mathrm{I}^{\circ} \mathrm{C}$. These exacerbations of temperature do not appear necessarily to coincide with an increase in the number of the parasites. We have sometimes seen the first rise of temperature coincide with the appearance of œdema.

The total duration of the infection varies, according to Kanthack, Durham, and Blandford, from thirteen to fifty-eight days (average thirty days). It may be as long as three months (Bradford and Plimmer). According to our observations, it varies from ten to fifty days, not taking into account those rabbits which were wasted at the time of inoculation.

In rabbits which survive more than twenty days certain local symptoms occur. At first there is a little blepharo-conjunctivitis with coryza; very soon follow œdemas, localized especially to the head (the root of the ears more particularly), to the anal mucous membrane, and to the external genital organs. Congestion of the testicles or true orchitis may be present. The hair falls out around the eyes and nose and at the root of the ears, sometimes also in different parts of the abdomen and back. If the animal survive a long time, these bald patches may ulcerate and exude a purulent fluid. In these cases, too, the blepharo-conjunctivitis increases and becomes purulent, the eyelids are stuck together, pus accumulates beneath them, and the cornea is rapidly affected. It becomes opaque, and blindness may ensue. [Two rabbits inoculated by Jakimoff died in thirteen and forty-nine days respectively; both of them had purulent eye lesions-conjunctivitis, keratitis, and iritis.]

In animals which succumb rapidly trypanosomes are frequently seen on microscopical examination, but they do not become very numerous until two or three days before death. In those cases in which the disease runs a relatively slow course, microscopical examination may be constantly negative, but inoculation into a mouse proves the presence of trypanosomes in the blood.

Guinea-Pigs.-Kanthack, Durham, and Blandford give the incubation period as five to seven days. In our experiments it was from two to four days.

Throughout the disease the guinea-pigs have continued fever with occasional intermissions, the temperature being as a rule above $40^{\circ} \mathrm{C}$. Kanthack, Durham, and Blandford's guinea-pigs died in from 20 to 183 days (average fifty days). The disease may last eighteen weeks (Bradford and Plimmer); the majority of our guineapigs died in from fifteen to thirty days after subcutaneous or intraperitoneal inoculation; some died five or six days after, while two survived forty-six and sixty-one days. When the disease lasts 
more than twenty days, the animal generally shows lesions of the eyes, such as loss of hair around the eyes, and a little purulent conjunctivitis, œdema of the vulva or sheath, and of the anus.

Except in those cases in which death occurs rapidly, the number of parasites in the blood does not increase with any regularity. On examining the blood, parasites may be found for several days, then they may disappear, and after some days reappear again. This may be repeated several times. As a general rule, the parasites are more numerous than in the blood of the rabbit, and sometimes they are very numerous, or may even swarm for several days together. In the two guinea-pigs which survived forty-six and sixty-one days the parasites were seen under the microscope from the fifth to the eleventh day after inoculation, then they were not seen again until the last days of the disease.

Field-Mouse.-We have experimented upon only one fieldmouse (Mus sylvaticus), weighing I5 grammes; it proved very resistant.

It was inoculated subcutaneously on December 2I, I902. Trypanosomes did not appear in the blood until December 29, and were scanty until January Io. They were very numerous from the IIth to the I3th (on the I 3 th there was marked tendency to agglutination); they were scanty from January I3 until death (January 2I). No external symptoms. Spleen was enlarged, and weighed $0^{\circ}$ I 7 gramme; the duration of the disease was thivty days.

One may note the great difference in susceptibility between the field-mouse and the mouse, although so closely related.

Dormouse.-We have experimented upon a dormouse (Eliomys quercinus), which throughout the experiment was kept in the incubator at $22^{\circ} \mathrm{C}$.

From November I8, I902-when inoculated subcutaneously-to December 20, the daily examination of the blood was negative.

On November 25 two drops of blood did not infect a mouse; later (November 30, December 6 and 19) blood inoculated in doses of 2 to 4 drops infected mice with an incubation period of four to six days. From December 20 to January Io trypanosomes were seen in the blood on three occasions. The animal was very lively, and ate well; its weight increased from 43 to 48 grammes. From January Io to 20 the animal lost weight. Trypanosomes were found almost every day (always in small numbers) on microscopic examination. There was marked weakness, especially of the right side of the body. The animal was found dead on January 2I. It weighed only 30 grammes; spleen was not enlarged, weighing $0^{\circ} 08$ gramme. The disease lasted sixty-three days.

EQUIDE.-We have numerous accounts of the course of the disease in equines, for it is these animals which are specially liable to be attacked by the natural disease in South Africa, and, moreover, Bruce experimented upon a certain number of them. The horse, ass, mule, and, according to the experiments of Kanthack, Durham, and Blandford, zebra-horse hybrids (male zebra and mare, stallion and female zebra) and zebra-donkey hybrids (male donkey and female zebra) are susceptible. The zebra is refractory according to 
the accounts given by explorers, but we know that animals have often been considered refractory when really the disease runs a very chronic course in them. ${ }^{1}$ Recent experiments of Grothusen and of

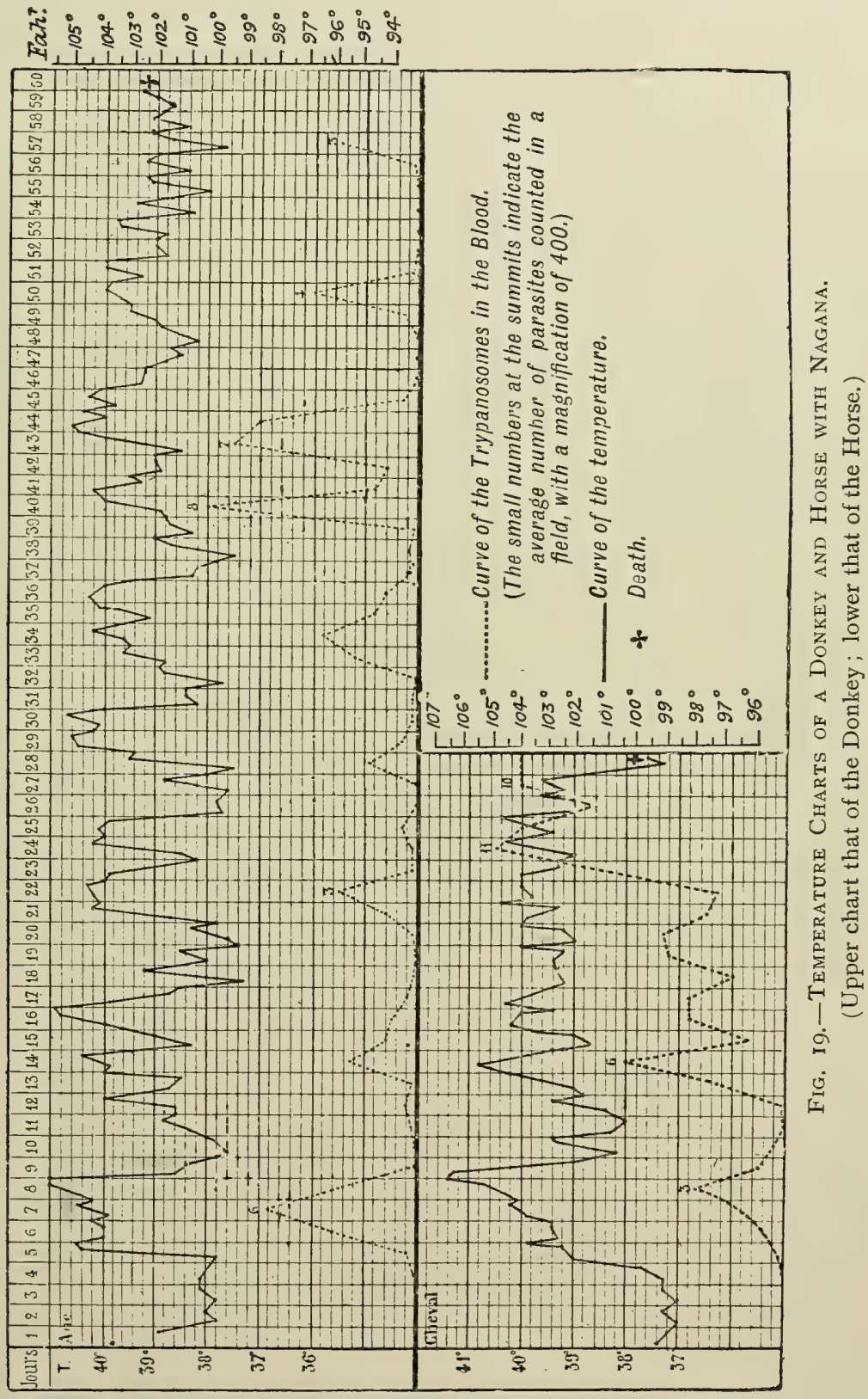

Martini with the German East African and Togo parasites, about which we shall speak later on, have proved the susceptibility of the

${ }^{1}$ [When at Naivasha, British East Africa, I had the opportunity of examining the blood of several zebras, which had been tamed by Mr. R. J. Stordy, P.V.O., but no trypanosomes were ever found.-ED.] 
zebra to these parasites. The same thing is probably true for the original Zululand trypanosome of nagana.

When the disease in equines is contracted as a result of the bite of tsetse-flies, the incubation period is on an average ten days. Kanthack, Durham, and Blandford record an incubation period of seven days, while in the horse and donkey with which we experimented the incubation period was four days. In these experimental cases the amount of the virus injected was considerable compared with that which even a large number of tsetse-flies can convey. [Theiler gives three to twelve days as the incubation period.]

Fever occurs simultaneously with the appearance of trypanosomes in the blood. It is always remittent or continued, and lasts

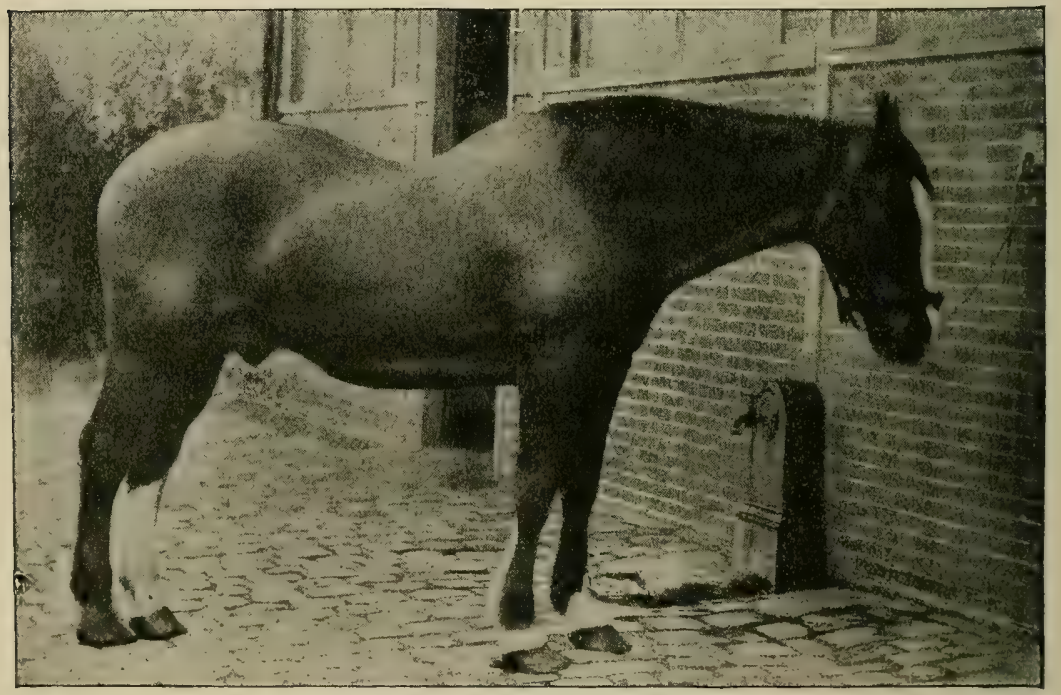

Fig, 20.-A Horse sUfFERING From NAGANA.

until death. In some cases there may be hyperpyrexia just before death.

The first rise of temperature generally exceeds $4 \mathrm{I}^{\circ} \mathrm{C}$. $\left[\operatorname{Io6}^{\circ} \mathrm{F}\right.$. $]$, and may reach $42^{\circ} \mathrm{C}$. $\left[107^{\circ} 6^{\circ} \mathrm{F}\right.$. , but later rises rarely exceed $4 \mathrm{r}^{\circ} \mathrm{C}$. We shall see later on that various ruminants show this great initial rise of temperature. The accompanying charts (Fig. I9) from our experiments with a donkey and a horse show the two types of fever, remittent and continued.

Death occurs in from fifteen days to two or three months, and depends in great measure upon the resisting power of the animal. Our horse, which was an old one, lived twenty-seven days, while the donkey, which was young and active, lived fifty-nine days. The zebra-horse and zebra-donkey hybrids succumbed in eight weeks. [Theiler, in the Transvaal, found the disease run a very acute course in equines-one to two weeks.] 
Equines suffering from nagana may show, during the course of the disease, a number of characteristic symptoms as recorded by Bruce :

'The first appearance of a horse being affected by nagana is that his coat stares and there is a watery discharge from the eyes and nose. Shortly afterwards a slight swelling under the belly or a puffiness of the sheath may be noticed, and the animal falls off in condition.

'The hind extremities also tend to become swollen; and these various swellings fluctuate, one day being marked, another day being less marked or having disappeared. During this time the animal is becoming more and more emaciated; he looks dull and hangs his head; his coat still stares, becoming harsh and thin in places; the mucous membranes of the eyes and gums are pale, and probably a slight milkiness of the cornea of the eyes is observable. In severe cases and in the last stages a horse presents a miserable appearance. $\mathrm{He}$ is a mere scarecrow covered with rough harsh hair, which has fallen off in places. His hind extremities and sheath may be more or less swollen, sometimes to a great extent, and he may have become quite blind. At last he falls down unable to rise, his breathing becomes shallower and shallower, and he dies of exhaustion. During his illness he has shown no symptoms of pain, and up to the last day has had a fairly good appetite.'

Our two animals did not show all these symptoms. The horse had great œdema of the whole of the ventral region, as shown in Fig. 20. The ass had no odema, but during the last three weeks of its life it was profoundly lethargic, which was commented upon by all who saw the animal.

We have mentioned the coincidence of the appearance of trypanosomes in the blood and the first febrile paroxysm. Our charts show better than any description the striking parallel between the curve of the temperature and that of the parasites in the blood. ${ }^{1}$ Trypanosomes could be found almost every day on microscopic examination, but they were never very numerous. The red corpuscles gradually diminished, and at the time of death were reduced to one half their original number or even less.

PIG.-We showed, at the same time as Plimmer and Bradford, the great susceptibility of the pig to nagana. ${ }^{2}$ We shall here merely

1 This parallelism is particularly well seen in the case of the ass. One notes the great regularity with which the maximum number of trypanosomes occurs at intervals of about seven days; also the striking parallel (much more marked than in the case of the horse) between the temperature curve and that of the parasites. It should be noted that the maximum number of parasites generally preceded the maximum temperatures by twenty-four hours. The disappearance of the parasites during the last four days of the illness is probably due to two injections of sodium arsenite.

2 [Bradford and Plimmer state (loc. cit., p. 462): 'The pig shows the organism in the blood very rarely and in very small numbers, and dies with pulmonary symptoms.' Nocht and Mayer, in Kolle and Wassermann's 'Handbuch der pathogenen Mikro-organismen,' state that the pig is fairly resistant. Schilling and Martini also found that the pig is not very susceptible to the Togo virus.] 
refer to the case of the young pig which we inoculated, and which died ninety-four days later (see detailed account in Bull. Acad. Méd., January, I902). Its temperature fluctuated between $39^{\circ} 5^{\circ}$ and $40^{\circ} 5^{\circ} \mathrm{C}$. [103 ${ }^{\circ}$ and $105^{\circ} \mathrm{F}$.] (which is a little higher than the normal temperature of a young pig), except during the last few days of life.

During the first forty days the animal showed no symptoms, but gained steadily in weight. Trypanosomes were never found in the blood on microscopic examination, but a few drops of the blood inoculated intraperitoneally into a mouse always infected it. About the fortieth day there was marked weakness of the limbs. This weakness rapidly increased, and soon the animal was no longer able to stand. Its anterior extremities, which were constantly bent, became œdematous and ulcerated at the knees. There was marked arching of the back at the level of the lower dorsal vertebræ.

During the last month the animal lay either on its belly or on its side, and it was necessary to support it in order that it might eat. The left cornea became opaque. During the last three days the temperature fell, and was markedly subnormal when the animal died. Two days before death the microscopic examination of the blood was positive for the first time. On the day of death trypanosomes were numerous.

Lignières, ${ }^{1}$ at Buenos Ayres, infected two pigs, one of which was immunized against caderas. At the end of fifteen days and of one month the blood of the two pigs was virulent; after two months the blood of the pig immunized against caderas was no longer virulent; after three months the blood of the other pig was also non-virulent. These two animals, therefore, made a rapid recovery.

\section{(3) Animals in which Nagana runs a Chronic Course.}

Bovid死. - 'There is,' says Bruce, 'a great difference in the duration of the disease in cattle: a few will die within a week of taking the disease, many die within a month, and others linger on for six months or longer. The general opinion among the traders and natives in $Z$ ululand is that only a very small percentage recover.

- The general symptoms in cattle are less marked than in horses and dogs. They gradually waste away; the hair, at first harsh and staring, tends to fall off; there is the same trickling of a watery fluid from the eyes and nose, and a tendency to diarrhœa, which, however, I have never found marked. In many cases the dewlap becomes swollen and baggy, but I have not found the same tendency to the swelling of the under surface of the belly or the extremities as in the other animals, nor have I ever seen blindness occur in cattle. The hrematozoa are also much less numerous in the blood of cattle than in that of horses and dogs, and often require to be looked for on several days in succession before they can be demonstrated.'

${ }^{1} \mathrm{~J}$. Lignières, Bol. de Agrric. y Gunad., third year, No. 50, February I, 1903. 
The fever, which is continued, is less marked than in horses, bearing in mind the higher normal temperature of cattle (about $384^{\circ} \mathrm{C}$. or $\operatorname{IOI}^{\circ} \mathrm{F}$.), but the temperature sometimes rises above $4 \mathrm{I}^{\circ} \mathrm{C}$. $\left[\mathrm{IO}_{5} \cdot 8^{\circ} \mathrm{F}\right.$. $]$.

The accompanying chart, taken from Bruce's paper, gives an idea of the course of the temperature and of the number of trypanosomes. In this case the number of red corpuscles diminished from $5,260,000$ to $\mathrm{I}, 800,000$ per cubic millimetre. When death occurs more rapidly the diminution is less marked.

At Alfort, Nocard inoculated several Breton cows subcutaneously. They all survived, and exhibited only the slightest symptoms of ill-health. There was a rise of temperature above $40^{\circ} \mathrm{C}$. five days

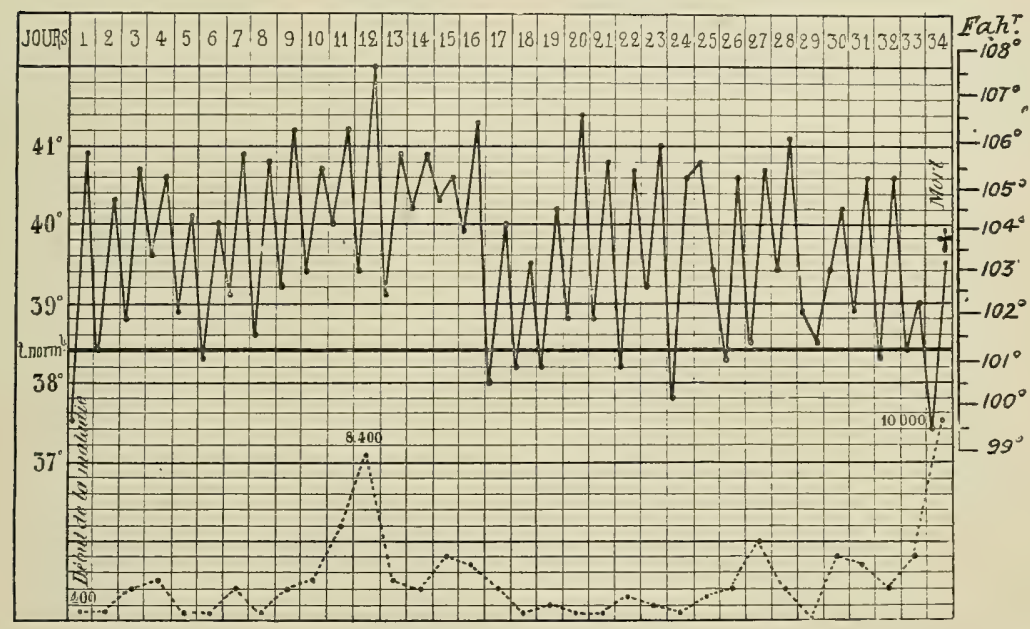

Fig. 2i.-Chart showing the Temperature and Number of Parasites in a Cow With Nagana (AFter Bruce).

As it was a case of spontaneous infection, the curves start from the day on which the disease was recognised. The normal temperature of the cow is about $1014^{\circ} \mathrm{F}$.

after inoculation, and on that day a few trypanosomes were visible in the blood. Two days later the temperature was normal, and remained so until the end of the infection. The general condition of the animals was good. Microscopical examination of the blood was always negative, but the blood remained infective for mice for five months. At the end of that time the blood was no longer infective, and the cow was immunized against nagana.

Is this marked resistance of Breton cows to trypanosomes a question of race, or is it due to the fact that the virus injected had, through long disuse with cattle, lost its virulence for them? Further experiments are necessary in order to decide this point. We may note that Argentine cattle are also only slightly susceptible to our virus (Lignières).

Nocard introduced infective blood ( 2 c.c. of rat's blood rich in trypanosomes) into the teat of a cow which had recently calved. 
This did not infect the animal, nor was there any fever, mastitis, or alteration of the milk.

SheEP AND GOATs.-According to Bruce, the native goats and sheep of South Africa are less susceptible than other mammals, the disease as a rule running a chronic course and lasting five months. In Bradford and Plimmer's experiments a goat died in two months. Nocard has given us a complete account of the disease in a sheep which died in six and a half months (I97 days). The animal had on the sixth day after inoculation a rise of temperature to $4 \mathrm{I}^{\circ} \mathrm{C}$., after which it fell to between $39^{\circ}$ and $40^{\circ} \mathrm{C}$. On the twenty-fourth day it rose again to $4 \mathrm{I}^{\circ} 5^{\circ} \mathrm{C}$. [ Io6. $8^{\circ} \mathrm{F}$.] , and remained for a considerable time at about $4 \mathrm{I}^{\circ} \mathrm{C}$. There was œdema of the face and eyes, then of the testicles. It was only during this period that trypanosomes could be found on microscopical examination, and for about a week several could be found in each field of the microscope. The swellings increased, and extended as far as the hind quarters and shoulder by the end of the third month, after which they rapidly disappeared. During the fourth, fifth, and first half of the sixth months, the animal had apparently recovered; its temperature was about $39^{\circ}$ or $40^{\circ} \mathrm{C}$., but its blood was still infective. During the last month it wasted rapidly, and after death there were found signs of profound cachexia, and gelatinous exudation about the throat, pericardium, and pleuræ.

Some of our sheep died after infection with nagana without showing any local symptoms, but as they were all somewhat cachectic, it is possible that nagana was not the sole cause of death. On the other hand, two of our sheep recovered. One of them was carefully watched from day to day; its history was as follows :

The infection lasted six to seven months. The disease started two days after inoculation with a rise of temperature, which on the third day reached $4 \mathrm{I}^{\circ} 5^{\circ} \mathrm{C}$. $\left[106.8^{\circ} \mathrm{F} .\right]^{1}$; on the same day trypanosomes were seen on microscopic examination, but this was the only day on which the microscopical examination was positive.

For the next four months the temperature remained high, with several rises to $4 \mathrm{I}^{\circ} \mathrm{C}$., and the animal wasted considerably. At first the blood was almost always virulent for the mouse in doses of a few drops; the incubation period for the mouse was four to eight days, so that the trypanosomes inoculated must have been very few. During the fourth month the blood was no longer infective in doses of I c.c., but it became infective again later. The fifth month, although trypanosomes were still present in the blood, and the temperature was considerably raised (about $40^{\circ} \mathrm{C}$.), the sheep gained in weight. At the end of the sixth month its condition improved, the temperature coming down to $39^{\circ} \mathrm{C}$., and the blood became gradually less infective, so that sometimes there was not a single trypanosome in I c.c. At the end of the seventh month 5 c.c. of blood failed to infect two rats, so that the animal may be considered cured. It was immunized. During the six and a half months that the disease lasted the animal did not show the least sign of cedema.

1 This great rise during the first week occurred with all our goats and sheep. 
Lignières, working with our virus, found that Argentine sheep become infected, but recovered in less than three months.

Bradford and Plimmer's goat which died in two months had œdema of the genital organs and opacity of the eyes.

We have inoculated a nanny goat and a billy goat. The billy goat succumbed very rapidly after a severe infection, but we cannot say with certainty whether death resulted from the infection or was due to some other unknown cause.

The history of the nanny goat is particularly interesting, as it furnishes a proof of the non-identity of nagana, caderas, surra, and the Gambian horse disease. The goat ended finally by succumbing to the last-named infection. We append a short account of the nagana infection in this goat.

Nanny goat, weighing $24 \frac{1}{2}$ kilogrammes, inoculated October 25 , Igor, with the blood of a mouse with nagana. A very mild infection followed, the symptoms being fever (rises to $4 \mathrm{I}^{\circ} \mathrm{C}$.), wasting (the weight fell to 20 kilogrammes), and the presence of trypanosomes in the blood. The parasites were always very scanty; they could be seen on examining the blood only on October 28 and 29 , and then only in very small numbers. Afterwards, in order to demonstrate their presence, it was necessary to inoculate the blood into a rat or a mouse. In November or December two or three drops of blood were sufficient. On January $3 \mathrm{I}$ and February 6 , I902, I c.c. of blood was no longer infective. Animals inoculated on February 24 and March Io with $\frac{3}{4}$ and 2 c.c. of blood respectively contracted the infection.

From April I injections of the goat's blood failed to produce an infection, in spite of large doses (up to 3 c.c.), and of the fact that the goat was frequently reinjected with blood containing many trypanosomes. Its cure and its immunity from nagana were established.

On April 23 the goat was inoculated subcutaneously with I c.c. of diluted blood from a mouse. From May 8 to October 4 it received in all fifteen injections of blood from infected dogs. On one occasion 6o c.c. of blood was injected; frequently the dose was 50 c.c., and altogether the goat received 500 c.c. of infective blood. During July and August inoculations were few and far between, the reason being that the goat had a very large abscess, which took a long time to heal.

Later on we shall come across this goat again in connection with other trypanosomiases. (Goat I.). Here we may just mention that the animal was still immune against nagana in May, I903, after having recovered from an infection of caderas, and in December, I903, after having recovered from an infection of surra.

Many other ruminants are susceptible to nagana. Thus Plimmer and Bradford have seen a springbok (Antidorcas euchore) succumb at the end of four weeks, after having shown parasites in its blood and having exhibited nervous symptoms. The duration of the disease was remarkably rapid if it were due entirely to the virus inoculated.

The wild ruminants of $Z$ ululand are susceptible to nagana, as shown by Bruce's experiments, about which we shall speak in the paragraph on propagation. Bruce infected dogs with nagana by 
inoculating them with blood from the African buffalo and various Zululand antelopes: Tragelaphus scriptus sylvaticus (bushbuck), Catoblepas gru (wildebeest), Strepsiceros capensis (koodoo). These animals had been hunted and shot, and trypanosomes were never visible in their blood on microscopic examination. It is, therefore, probable that, although they have $T$. brucei in their blood, the disease in them is extremely mild. ${ }^{1}$

There is a belief, very prevalent amongst explorers and sportsmen, that animals born in the 'fly country' are much less susceptible than animals of the same species which are imported, and this is said to apply not only to ruminants, but also to horses and dogs.

[GEESE.-Until Schilling, ${ }^{2}$ in 1904 , succeeded in infecting geese with the Togo virus, all authors, with the exception of Voges, were agreed that birds were refractory to the pathogenic mammalian trypanosomes. Schilling's positive results were obtained with the Togo virus, and will be considered more fully in the section dealing with the Togo trypanosomiases. Mesnil and Martin ${ }^{3}$ repeated these observations on geese and fowls, using the following trypanosomes: (I) T. brucei (Zululand strain); (2) T. evansi (Indian); and (3) T. equimum (caderas). One goose inoculated with $T$. brucei became mildly infected-the infection lasting about three months, and resulting in the immunity of the bird-but all the other experiments yielded negative results. In the only positive experiment, the trypanosomes were never found on microscopical examination of the blood, but the blood was infective for guinea-pigs.]

[Goebel ${ }^{4}$ has recently succeeded in infecting fowls with the Zululand $T$. brucei by injecting 2 c.c. doses of virulent guinea-pig blood. The fowls never showed trypanosomes in blood-films, but the blood was virulent for guinea-pigs as long as fifty-five days after the birds were inoculated. The fowls became immunized, and when their blood was no longer infective, reinjection failed to reinfect.]

\section{Section 2.-Pathological Anatomy.}

Nagana is one of those diseases in which very few lesions are found post-mortem. Most authors mention hypertrophy of the spleen as the only constant lesion. It is always present in rats, mice, ${ }^{5}$ and dogs, ${ }^{6}$ but is rather rare in guinea-pigs and rabbits. According to Bruce, it is equally present in the larger domestic animals. As a

1 Compare the susceptibility of the buffalo to the trypanosome of Togo noted by Martini (second part of this chapter).

2 [Schilling, Arb. a. d. kaiserl. Gesund., v. 21, pp. 476-536.]

3 [Mesnil and Martin, C. R. Soc. Biol., v. 6o, 1906, p. 739.]

4 [Goebel, C. R. Sioc. Biol., v. 6r, 1906, p. 321.]

5 The spleen of a normal mouse is about 100 of its weight ( 7 centigrammes in a mouse weighing 20 grammes); that of a mouse which has died of nagana is 100 of its weight. It is, therefore, increased threefold. The spleen of a rat with nagana is very variable in weight: sometimes its weight is trebled, at other times it is increased tenfold.

6 The normal spleen of a dog is about $\frac{\pi}{500}$ of its weight. In a dog with nagana it is sometimes doubled, in others quadrupled in weight. 
general rule, we may say that enlargement of the spleen is most marked in those animals which have shown a very large number of trypanosomes in their blood during life. [Occasionally in guineapigs the enlarged spleen may become so soft that it ruptures, either spontaneously or as the result of the animal being handled. $]^{1}$ In the rat hypertrophy is due to congestion of the organ, no appreciable histological changes being visible on microscopical examination.

In smears of the spleen and liver, the numerous parasites present are often deformed, if the autopsy has not been made immediately after death. The parasites are often arranged in groups suggesting reproduction forms.

Kanthack, Durham, and Blandford, and Plimmer and Bradford, have drawn particular attention to the enlargement of the lymphatic glands, especially of those nearest to the site of inoculation.

This hypertrophy is indeed marked, but, contrary to the opinion of the two last-named authors, it does not appear to us to be associated with a considerable multiplication of the parasites in situ: as a matter of fact, they are rather scanty in the glands.

In a spleenless rat which died of nagana the lymphatic glands on the side inoculated were considerably enlarged, but not more so than those of a control rat with nagana. It is said that animals which become infected by eating virulent material always show enlargement of the glands in the region of the head or neck, which proves-so say these authors-that the infection first enters the system through an abrasion of the mouth or nostrils. At the autopsy on horses which have died of nagana hypertrophy of the spleen and liver, yellowish gelatinous infiltrations under the skin and mucous membranes and between the muscles, pleural and pericardial exudations, and subpericardial ecchymoses are found.

At the autopsy on our horse, made eleven hours after death, the lesions were insignificant. The spleen did not appear enlarged (weight $=3$, I50 grammes), but its surface was uneven and covered with dark brown mottling; 200 c.c. of pale red fluid was present in the pleural cavities, and I5o c.c. in the pericardium, both containing a few trypanosomes. There were some ecchymoses under the pericardium and endocardium; the myocardium and all the other organs were normal.

[Martini made the interesting observation that the cerebro-spinal fluid may be increased in equines dying of tsetse-fly disease, and that many trypanosomes may be found in the cerebro-spinal fluid immediately after death. In this respect, as well as in the typical bent attitude, the tsetse-fly disease of the horse and donkey resembles human trypanosomiasis (sleeping sickness). Martini also found trypanosomes in the fluid in the anterior chamber of the eye in animals with corneal opacity (dog, cat, rabbit). Jakimoff found

${ }^{1}$ [Laveran and Mesnil, Markl, Nabarro and Stevenson, and Sauerbeck have seen this happen.] 
several of the body fluids of experimental animals infective on inoculation - the cerebro-spinal fluid, pleural, pericardial, and peritoneal exudations, bile, and subcutaneous œdema fluid. The urine was not infective.]

At the post-mortem on the young pig which died of nagana after showing very marked paralytic symptoms we found the following condition present. The bones of the spinal column were softened so that the vertebræ could easily be cut with a knife. This softening of the skeleton, a true osteomalacia, explains how, under the influence of the paralysis and of the faulty position of the hind limbs which followed it, a very marked arching of the back in the dorsal region was produced. The lower part of the spinal cord was surrounded by a gelatinous exudate, a partially coagulated serous effusion analogous to that found in the pericardium and subcutaneous tissue, but there was no spinal meningitis. The spinal cord removed in its entirety and carefully examined did not show any macroscopic changes - there were no patches of softening. The medulla oblongata, cerebrum, and cerebellum appeared normal. The spleen was not enlarged. There were 20 c.c. of straw-coloured fluid present in the pericardium, and a little in the pleural and peritoneal cavities.

Finally, we may note that from the time an infected animal dies, and sometimes even during the agony, the trypanosomes which it contains diminish in vitality. Twenty-four hours after death, especially in small animals, there are no longer any active parasites in the blood or internal organs. They may, however, be present in very small numbers, for the blood is sometimes still virulent.

[Since I904, numerous investigations have been carried out on the changes produced in the blood, and on the microscopical appearances seen in the various organs of animals infected with nagana. Amongst those dealing with the blood changes may be mentioned the researches of Goebel and Demoor, ${ }^{1}$ Markl, ${ }^{2}$ Massaglia, ${ }^{3}$ Mayer, ${ }^{4}$ Nissle, ${ }^{5}$ Sauerbeck, ${ }^{6}$ and Jakimoff. ${ }^{7}$ The papers dealing with the pathological histology of, and the distribution of trypanosomes in, the internal organs are those of Baldwin, ${ }^{8}$ van Durme, ${ }^{9}$ Halberstaedter, ${ }^{10}$

${ }^{1}$ [O. Goebel and A. Demoor, Ann. Soc. de Mád. de Gand, I906, pp. I37-I48. See also Mesnil, abstract in Bull. Inst. Past., I906, v. 4, p. 667.]

2 [Markl, Centralb. f. Bakter., I, Orig., v. 37, p. 530.]

3 [A. Massaglia, Boll. R. Accad. med. di Genova, 2 rst year, No. I., I904; see abstract by Mesnil, Bull. Inst. Past., v. 4, 1906, pp. 667, 668.]

${ }^{4}$ [Mayer, Zeitschr.f. Path. u. Ther., v. I, 1905.]

5 [Nissle, Arch.f. Hyg., v. 53, I505, pp. I8I-204; and v. 54, I 905, pp. 343-353.]

6 [Sauerbeck, Zeitschr. f. Hyg. u. Infektions., V. 52, 1905, pp. 31-86; see also abstract by Mesnil, Bull. Inst. Past., v. 4, I906, pp. 317, 318 . In the Ergebn. d. allgem. Path. $u$. pathol. Anat. des Menschen. u. der Tiere, v. Io, I906, pp. $305-$ 379 , Sauerbeck gives a long résumé of our knowledgre of the trypanosomes, as well as an extensive bibliography.]

7 [W. L. Jakimoff, Centralb. f. Bakter., I, Ref., v. 38, I906, pp. 13-16.]

8 [Baldwin, Journ. Infec. Dis., v. I, 1904. pp. 544-550.]

9 [Van Durme, Ann. Soc. de Míd. de Gand, 1905, p. 23I ; Arch. de Parasit., v. 10, 1906, p. 160.]

10 [Halberstaedter, Centralb. f. Bakter., I, Orig., v. 38, 1905, pp. 525-532.] 
Massaglia, ${ }^{1}$ Neporojny and Jakimoff, ${ }^{2}$ Prowazek, ${ }^{3}$ Rodet and Vallet, ${ }^{4}$ and Sauerbeck ${ }^{1}$.

[BLood-Changes.-Coincident with the first appearance of the trypanosomes in the blood and with their multiplication in it, there is a diminution in the number of the red blood-corpuscles, which in extreme cases may fall to one-third of the original number. As a rule, the corpuscles themselves look normal, but Nissle has described various pathological changes in them (alterations in shape, poly. chromatophilia), and also very varied endocorpuscular forms of the parasite. These, he says, are to be seen in the blood when the trypanosomes are undergoing destruction either spontaneously or as the result of injections (B. prodigiosus, trypanred, etc.)]

[The leucocytes are always increased, but Goebel and Demoor state that at the outset there is a marked leucopenia. At first the polymorphonuclears are increased and the mononuclears relatively diminished, but soon the polymorphonuclears are diminished (from 78.5 to 38 per cent. in a dog, Jakimoff), and the mononuclears are increased ( $5_{5}$ to $4^{8}$ per cent. in the same dog, Jakimoff). There is no eosinophilia.]

[Jakimoff found that the alkalinity of the blood is diminished in dogs infected with nagana, caderas, and dourine. Martini found that the blood of nagana animals is diminished in coagulability. The blood-proteids undergo the same changes as in bacterial infections, the ratio of albumin to total globulins falling from $I^{*} 5$, or 2 to I (Mayer). At death there may be lipæmia (Massaglia), as Mayer has also shown in the cases of dogs with caderas.]

[The Distribution of the Trypanosomes in the Body.-As we have already seen, the trypanosomes, once they appear in the blood, may steadily and progressively increase in number until death -as in rats and mice; or they may be present in large and small numbers alternately, being sometimes very numerous and at other times very scanty, or even absent in blood-films-as in guinea-pigs ; or the parasites may be very scanty in the blood throughout the infection, except, perhaps, just before death-as in rabbits. During the remission stage (in the guinea-pig) Goebel and Demoor found the trypanosomes fairly numerous in the lymphatic glands, and very abundant in the testicles, but none could be found in the other organs.]

[Van Durme, who examined the blood and freshly teased-up organs of rabbits killed at varying intervals after the onset of the infection, found that the parasite multiplies first at the site of inoculation. Thus, after intraperitoneal inoculation, trypanosomes are present for ten days in the peritoneum. Soon after there is an increase in the blood, and it is at this stage of the onset that the

1 [See references on p. 142.]

2 [Neporojny and Jakimoff, Centralb.f. Bakter., I, Ref., v. 35, 1904, pp. 457, 458. ]

3 [Prowazek, Arb. a. d. kaiserl. Gesund., v. 22, 190;; see abstract by Mesnil,

Bull. Inst. Past., v. 3, 1905, pp. 553, 554.]

${ }^{4}$ [Rodet and Vallet, Arch. méd. expér., v. I8, July, I905, p. 450-494.] 
parasites are most numerous in the blood. Shortly after this they are found in the testicles, which explains the orchitis and other lesions of the genitalia so commonly seen in rabbits. The glands are next infected, then the conjunctiva, the skin in the region of the œdematous swellings, and the nasal mucous membrane. In all these organs and tissues the number of trypanosomes increases progressively and then diminishes, pari passu with the well-known clinical affections shown by them. The order given by van Durme corresponds almost exactly with the order of the clinical symptoms. The secretions (semen, conjunctival pus) are free. The spleen, salivary glands, liver, kidneys, suprarenal capsules, lungs, brain and spinal cord, lachrymal and thyroid glands, thymus, bone-marrow, and ovary were always negative (except once in the case of the first two). In the rabbit, therefore, $T$. brucei may be more abundant in certain organs than in the blood, and these organs are precisely those which present functional derangements and macroscopic lesions. $\left.{ }^{1]}\right]$

[Halberstaedter obtained somewhat similar results in his rabbits, except that he never found trypanosomes in the testicle. In the œedematous swellings about the eyes and nose, trypanosomes were found in the altered skin cells and between the cells under the epithelium, as well as down in the subcutaneous tissues, but never in the bloodvessels. In mice, on the other hand, he found trypanosomes in all the bloodvessels in the internal organs, especially the liver, and in the skin.]

[In other animals Prowazek and Neporojny and Jakimoff found masses of parasites, causing capillary embolism in the brain, lungs, and liver.]

[Nabarro and Greig, in Uganda, nearly always found subserous petechial hæmorrhages (pleural, pericardial, and endocardial) in their experimental animals (monkeys, dogs, guinea-pigs) dying of various animal trypanosomiases. Smears of these petechiæ usually contained masses of trypanosomes (many more than were present in the blood of the animal), which led these observers to conclude that the petechiæ were really embolic areas, the emboli being the masses of the parasites. $\left.{ }^{2}\right]$

[The Place and the Mode of Destruction of Trypanosomes.Most authorities are agreed that trypanosomes are destroyed by the phagocytic action of various cells.]

[Prowazek found all stages of destruction of the parasites in the polymorphonuclears (and exceptionally in the eosinophiles), as well as in the mononuclears.]

[Sauerbeck, working with rats, guinea-pigs, rabbits, and dogs, could not find any degenerating forms in the blood, but saw them easily in the internal organs, especially in the spleen, lymphatic glands, bone-marrow, liver, and, to a less extent, in the lungs. These involution forms, 3 SauerI906.]

1 [The above account is taken from Mesnil's abstract in Bull. Inst. Porst., v. 4,

2 [They made a somewhat analogous observation in the case of dogs dying of piroplasmosis. These animals also showed petechial hremorrhages, especially under the pleura, and smears of these areas showed many more infected red corpuscles and more parasites in the corpuscles ( 8 to Io were sometimes seen), than did the peripheral blood-films.]

[Saucrbeck draws attention to the similarity of the commonest involution forms to the Lcishman body, but this morphological resemblance is not sufficient to warrant our putting the trypanosoniases and tropical splenomegaly in the same categrory, as Satuerbeck, Marchand, and others would do. See Mesnil's abstract in Iiull. Inst. P'ist., v. 4, 19o6, pp. 317, 318.] 
beck thinks, explain the so-called amœboid and plasmodial forms of Bradford and Plimmer. The involution forms of trypanosomes in the organs are, as a rule, intracellular. The hypertrophy and the histological changes in the organs are due to a phagocytic proliferation. In the lymphatic glands it is the cells of the lymphoid tissue which destroy the trypanosomes; in the spleen, the cells of the pulp and, to a less degree, those of the follicles; in the bone-marrow, the meduilary cells; in the liver-unlike the foregoing organs-it is the endothelial cells of the capillavies; and, lastly, in the lungs, the alveolar epithelium. The cells concerned become very large and amoboid. In them one or more degenerating trypanosomes, easily recognisable in the form of Leishman's bodies, may be found; but often there are only remnants, or even only empty vacuoles, doubtless marking the place where a parasite has been digested. The cellular hypertrophy is followed by hyperæmia of the phagocytic organ, and the two together give rise to enlargement of the organ. Secondarily there are thromboses and catarrhal desquamation of the lungs. ${ }^{1}$ ]

[Rodet and Vallet, ${ }^{2}$ on the other hand, ascribe the extensive destruction of trypanosomes in the spleen to an extracellular trypanolysis. The method they employed-that of making smears-is not a good one (Mesnil), as many intracellular parasites may become liberated thereby.]

[It has been found that injection of a culture of B. prodigiosus (Nissle) or an intercurrent microbic infection, as with streptococci (Massaglia), causes a rapid disappearance of the trypanosomes from the blood. It is possible that the scarcity of trypanosomes in the blood of patients in the later stages of human trypanosomiasis (sleeping sickness) is also due to the concomitant bacterial infection, which is frequently present as a terminal phenomenon.]

[Changes in the Organs.-Microscopic changes have been described in several of the tissues and organs.]

[Baldwin found the spleen most affected. Microscopically there were congestion accompanied by hyperplasia of the reticulum of the pulp, and a variable degree of hyperplasia of the cells resembling the myelocytes seen in the blood in certain diseases. There were also present some red blood-corpuscles and giant cells like those of the bone-marrow. The myelocyte-like cells and the giant cells could be seen developing from endothelial cells of the pulp. There was also an abundant deposit of the iron-containing pigment, hæmosiderin, which Baldwin suggests is formed by a hæmolytic action of a soluble toxin of the trypanosomes. The other hæmatopoietic organs, the glands, and the bone-marrow also showed hyperplasia. The lungs were engorged, and the alveoli often contained desquamated epithelial cells and granular exudate.]

[Neporojny and Jakimoff found the spleen enlarged in all their animals. Microscopically some Malpighian bodies were enlarged, and others were undergoing degenerative changes. The lymphatic glands were enlarged in chronic cases, and in such cases the bone-marrow was red. The lungs showed considerable microscopic changes, the capillaries being filled with trypanosomes, and sometimes even the larger vessels were thrombosed from the same cause. The kidneys and the liver showed the most marked changes. In the former there was congestion of the cortex, often with

1 [This catarrhal desquamation of the lungs may explain why catarrhal pneumonia is so frequently a terminal phenomenon in human and several animal trypanosomiases.]

2 [A. Rodet and G. Vallet, C. R. Acad. Sciences, v. I42, I906, pp. I229-123I ; also Arch. méd. expér., July, Igo6.] 
petechial hæmorrhages, which microscopically were found to have their seat in the glomeruli. The authors think that this lesion of the kidneys explains the fact that Jakimoff constantly found albumin in the urine of infected animals, and they make the suggestion that possibly the frequent œdemas, fits, and drowsiness seen clinically are uræmic in origin.]

[The liver is usually enlarged and finely granular on the surface. The changes in it seem to occur as follows: Owing to masses of trypanosomes in the capillaries, the blood-flow is sluggish, and in places completely stopped. As a result, degenerative changes take place, with atrophy, fatty degeneration, necrosis, and karyolysis of the liver cells and of the capillary endothelium. Around these degenerating areas are zones of inflammatory reaction with karyokinetic figures in the liver cells and in the endothelial cells. The latter are definitely phagocytic, engulfing and digesting trypanosomes and leucocytes.]

[Halberstaedter, on examining the characteristic testicular swellings in rabbits, found a marked round-cell infiltration between the tubules; the epithelium of the latter was degenerating, but no trypanosomes were seen.]

[Massaglia always found the kidneys most affected, and in a condition of subacute hæmorrhagic parenchymatous nephritis, with marked degeneration of the epithelium of the tubules. The liver showed subacute hepatitis with fatty degeneration ; the spleen, lymphatic glands, and bone-marrow showed lesions similar to those already described.]

\section{Section 3.-Résumé of Symptoms and Pathological Anatomy.}

The variations in the symptoms and course of nagana in different species of animals will be clearly seen from the following accounts.

If we were not acquainted with the pathogenic agent it would be difficult to believe that the very varied conditions produced by $T$. bruce $i$ in the rat and mouse, in the horse, pig, ox, and goat, were due to the same disease. We have seen that nagana may sometimes occur as an acute or subacute disease which is always fatal, sometimes as a chronic disease which may end in recovery.

Amongst the most constant symptoms should be mentioned fever, anæmia, and œdema, but here again there are great variations. in the different species of animals.

In rats there is no fever or œdema, even when, by means of appropriate treatment (vide infra), the life of these animals is prolonged for two months or more. In the rabbit the febrile paroxysms are very irregular. In the guinea-pig and dog the fever is often of a continued type, with more or less marked morning remissions. The horses and cattle of Zululand suffer from remittent fever during the whole course of the disease. In European cattle, after an initial rise, the temperature falls to normal and remains so. In the $\mathrm{dog}$ the temperature is usually raised until death occurs. Other animals, such as the pig and monkey, die with a subnormal temperature. In the monkey, the account of which we have given above, the temperature fell below normal several days before death, and on the day the animal died the rectal temperature had fallen to $28.5^{\circ} \mathrm{C} \cdot\left[83^{\circ} 4^{\circ} \mathrm{F}.\right]$.

The oedematous swellings, which are usually very marked in the 
rabbit and horse, are less marked or entirely absent in the guineapig, dog, sheep, and goat. Blepharo-conjunctivitis, coryza, ulcerations of the skin, and partial loss of hair, are common symptoms in the rabbit, but rare in other animals. Opacity of the cornea is seen in certain species of animals and not in others. In the horse and donkey, and sometimes in dogs, there is paresis of the posterior extremities towards the end of the disease. The pig with which we experimented had almost complete paralysis of all four limbs and osteomalacia. Rats often die suddenly with convulsions; this mode of death is much rarer in the mouse, and has not been observed in any other species of animal.

The way in which the trypanosomes multiply in the blood also varies considerably.

In the mouse and rat they increase rapidly and regularly, so that at the time of death the number of parasites may be at least equal to that of the red corpuscles. In the dog and monkey the trypanosomes multiply fairly rapidly, but less regularly than in rodents, and rarely to so great an extent. In the rabbit trypanosomes are almost always scanty, even at the time of death. In the guinea-pig there is no regular increase in the number of the trypanosomes. In equines the curve of the trypanosomes shows great oscillations, almost parallel with those of the temperature. In the pig trypanosomes are so scanty that they are not found on microscopical examination, and it is only during the last days of the disease, when the temperature is subnormal, that the parasites multiply in the blood. In the cow, sheep, and goat, especially in our experiments, the trypanosomes are nearly always so scanty that it is necessary to inoculate susceptible animals to show that the blood is infective.

The pathological anatomy of the disease also differs in different animals; thus splenic hypertrophy, which is constant and well marked in rats and mice, is very generally absent in rabbits.

Why does nagana present such variable symptoms in different animal species?

There are evidently many factors which may increase or diminish the virulence of $T$. brucei. The facts we have adduced above suggest that the virulence of $T$. brucei for rodents has increased as the result of numerous passages through these animals which, in preference to others, are used in laboratories for the preservation of the parasite, and that at the same time the virulence has diminished for other species-Bovidæ, for example.

The virulence of trypanosomes may be somewhat attenuated by passage through different species. We have quoted instances of this-e.g., nagana in mice, rats, and dogs, produced by a trypanosome which had been for a long time in the pig or the sheep; but $T$. brucei readily adapts itself to its new conditions in passing from one species to another, and in all cases it rapidly regains its natural 
virulence. We shall return to this subject in the paragraph upon immunization.

The trypanosome of nagana evidently finds the blood of certain animal species a more favourable medium for its multiplication than the blood of other species; but this explanation merely alters the form of the question. Why is the blood of certain mammals a better medium for $T$. brucei than that of other mammals?

The temperature of the animal seems to play a part. The trypanosome of nagana cannot withstand long exposures to a temperature of $40^{\circ}$ to $4 \mathrm{I}^{\circ} \mathrm{C}$. in vitro, and no doubt the same thing is true in vivo.

When the temperature of an infected animal is raised $\left(40^{\circ}\right.$ to $4 \mathrm{I}^{\circ} \mathrm{C}$.), the trypanosomes are seen to diminish in number, and when the temperature falls, the parasites begin to multiply again in the blood. This explains the almost parallel oscillations; in the Equidæ, of the number of trypanosomes and of the temperature; after the febrile paroxysms the curve of the trypanosomes falls.

In the young pig of which an account has already been given the effect of temperature is evident. The pig had a normal rectal temperature of $40^{\circ} \mathrm{C}$., and during the whole of the disease the trypanosomes were so scanty that they could not be seen on microscopic examination, but some days before death the temperature fell, and at the same time the trypanosomes multiplied in the blood.

The high temperature of birds appears to play an important part in rendering them refractory to nagana; [but, as we have seen, Schilling, Mesnil and Martin, and Goebel have succeeded in infecting geese and fowls, so we can no longer consider birds absolutely refractory to mammalian trypanosomes].

The effect of temperature upon $T$. brucei only partially explains the facts; it is certain that other factors help to modify the course of nagana.

Human serum injected into infected animals causes the trypanosomes to disappear, at least for a time, and this property of human serum no doubt depends upon the natural immunity of man against nagana. One may ask whether the leucocytes of certain animals do not contain a substance analogous to that which gives to human serum its remarkable properties, but in too small a quantity to give the serum of these animals an action upon $T$. brucci comparable with that of human serum.

How does the T. brucei act? An infection so intense as that found at the moment of death in rats, mice, and certain other animals, leads to the natural supposition that such an enormous number of parasites must produce serious and even fatal effects upon the general nutrition. But we must not forget that rats infected with $T$. leroisi may also have a very large number of parasites in their blood without apparently suffering any inconvenience. And, moreover, in the experiments on treatment with arsenic, of which we 
shall speak later, rats have lived for more than a fortnight with almost as many trypanosomes as red blood-corpuscles in their blood.

In animals which survive for a considerable time there is marked anæmia, but it is never sufficiently pronounced to be the sole cause of death. [Jakimoff also thinks that the blood-changes found by him are not sufficient to account for the fatal issue. He says death may be due to a number of causes: (I) Well-marked histological changes in all the parenchymatous organs and plugging of the capillaries with trypanosomes; (2) poisoning by the toxins of the trypanosomes; (3) oligocythæmia; and (4) uræmia, for during the disease he always found albumin in the urine.]

The trypanosomes, when they occur in very large numbers in the blood (as in the last stage of the disease in rats and mice), may act mechanically by plugging the small vessels of the brain or bulb. In this way is probably to be explained the rapid death, accompanied by convulsive movements, which is common in rats. But this is not always the case, ${ }^{1}$ and moreover, in many animals which die of nagana trypanosomes may be very scanty during the whole course of the disease, as, for instance, in the rabbit. Consequently one is almost forced to admit that the trypanosomes produce a toxin which gives rise to the febrile paroxysms, the pareses, the disorders of nutrition, the apathetic condition, and finally death. It is possible that the nervous system of certain animal species is more susceptible than that of others to this toxin, and this would explain the predominance of a particular symptom in a particular species of animal.

Kanthack, Durham, and Blandford, and later we ourselves, have tried to prove the existence of such a toxin.

(I) The fresh serum of infected animals filtered through a Berkefeld filter; (2) blood or serum kept for several days in order that all the trypanosomes should be dead; (3) blood in which the parasites had been killed by heating to $50^{\circ} \mathrm{C}$.; (4) extracts of the organs of animals which had died of nagana; and (5) extracts of trypanosomes from the blood of the dog or rat separated, as much as possible, from the red corpuscles by centrifuging, then exposed to a temperature of $42^{\circ} \mathrm{C}$. for sixteen hours or dried over sulphuric acid, did not, even in enormous doses, produce any toxic symptoms in animals when inoculated, either under the skin, or, as we have done, into the brain. Blood very rich in trypanosomes, enclosed in a collodion sac, and inserted into the peritoneal cavity of a guineapig and of a cat, produced no effect. The whole of the blood of a very sick rabbit was inoculated into a healthy rabbit without producing any immediate symptoms of acute intoxication.

$\left[\mathrm{McNeal}^{2}\right.$ has obtained some evidence of a toxin formation in cultures of $T$. brucei. He found that the first two or three subcutaneous injections

${ }^{1}$ [The trypanosomes might possibly be present in the small vessels in some other form-e.g., like the Leishman bodies- which is not very easily recognisable in sections.]

${ }_{2}^{2}$ [McNeal, Journ. Infec. Dis., v. 1, 1904, p. 538.] 
of an attenuated culture into guinea-pigs produced no local effect, but only a slight general effect. Later injections, however, produced both local effects (ulceration) and general effects (rapid rise of temperature).]

[Mayer ${ }^{\mathrm{I}}$ was unable to prove the presence of a soluble toxin, but found that precipitins occur in the blood of nagana dogs. T. brucei treated with trypsin for three days at $37^{\circ} \mathrm{C}$. furnishes on filtration a liquid which, when mixed with the serum of a nagana dog ( $0^{\circ}$ I to 0.5 extract with $0^{\circ} 5$ serum), gives a turbidity in less than an hour, and later a precipitate. Trypanosomes not treated with trypsin do not furnish this 'precipitinogen.' The same 'precipitinogen' liquid remains clear when mixed with the serum of a caderas dog, thus showing the specificity of the reaction. Mayer thinks this reaction may be of use in comparing allied trypanosomiases.] ${ }^{2}$

Amongst the factors which may influence the course of the disease and its severity must be mentioned the race of the animals, and, probably, heredity in countries in which nagana is prevalent. The wild animals of Central Africa-buffaloes, antelopes, etc.-although, susceptible to nagana, can multiply in those districts in which the tsetse-fly abounds.

Finally, it may be mentioned that animals which are old, badly nourished, or in a feeble condition, live a shorter time. Horses and cattle suffering from nagana live for a considerable time if not allowed to work.

It would be a mistake to attribute the survival of animals, such as cattle and goats, to the effects of treatment or to the use of an attenuated virus, without bearing in mind the variable susceptibility of animals for $T$. brucei, and the fact that cattle and goats may recover spontaneously.

Animals which recover are immune. This fact alone is sufficient to show the necessity for studying the evolution of nagana in different species of animals. That study should serve as a basis for researches having as their aim the treatment or the prevention of the disease.

\section{Section 4.-Morphology of Trypanosoma brucei. Effect of External Agents (Heat, Cold). Cultures. Agglomeration of the Trypanosomes. Involution Forms.}

$T$. brucei in THE BLOOD OF INFECTED ANimALs. - In fresh blood $T$. brucei appears as a small, very motile worm-like body, with an undulating membrane and flagellum. When its movements become sluggish, as happens quickly in ordinary preparations, the wave-like undulations of the undulating membrane are well seen. The parasite usually moves with the flagellar end foremost, so that this must be regarded as the anterior extremity. The posterior extremity varies in shape: sometimes it is drawn out, sometimes rounded off or truncated. The movements which are produced by the undulating membrane and the flagellum are not very extensive. The

1 [Mayer, Zeitschr.f.exp. Ther. u. Pathol., v. I, I905.]

2 [Quoted from Mesnil's abstract of Mayer's paper, Bull. Inst. Past., v. 3, 1905, p. 681.] 
parasite does not move about much in the field of the microscope; neither does it show the darting, arrow-like movement of $T$. lewisi.

In the fresh condition neither the nucleus nor the granules, which are clearly seen after staining, can be distinguished. Nevertheless, neutral red, toluidin blue, and methylene blue stain the granules in the interior of living trypanosomes; but when the action of these substances is continued for a certain time, the parasites are killed, and the staining becomes general.

All the trypanosomes are approximately of the same length in the blood of any particular animal. Very small forms are not seen side by side with the large ones, as in the case of $T$. lewisi during the stage of multiplication. The only variation noticeable is that certain trypanosomes are broader than others, and that they have two undulating membranes; these are forms in process of division, with which we are not concerned at the present moment.

According to Bruce, the trypanosome of nagana assumes different forms in the blood of different animal species. In the dog the parasite is relatively short and stumpy, with a blunt posterior extremity; in the horse it is almost twice as big, and the posterior extremity drawn out and pointed.

According to Plimmer and Bradford, the dimensions of the parasite vary with the period of the disease and the species of animal: the largest forms are seen in the rat at the moment of death, the smallest in the rabbit during the early days of the disease. We have studied $T$. brucei in different animal species-rat, mouse, guinea-pig, rabbit, dog, horse, donkey, sheep, and goat-and we have not seen such marked differences as are recorded by those observers.

When the parasites are examined only in fresh blood, it is easy to fall into error with regard to their size on account of the variation in size in the different animal species of the red corpuscles, which one uses instinctively for the purpose of comparison. Thus, in the goat the diameter of the red corpuscles is only $4 \mu$ to $4.5 \mu$, in the mouse $5.5 \mu$ to $6 \mu$, and in the rabbit $6 \mu$ to $7 \mu$; consequently, if one examine trypanosomes in the blood of these three animals, the tendency will be to regard the parasites as larger in the goat and mouse than in the rabbit. In order to obtain an accurate estimate of the size of trypanosomes, it is necessary to measure them in blood preparations well fixed and stained. In that way we have shown that $T$. brucei has approximately the same size in the rat, mouse, guinea-pig, rabbit, and dog-namely, $26 \mu$ to $27 \mu$ long (flagellum included), by $I^{\circ} 5 \mu$ to $2 \cdot 5 \mu$ wide. In the horse and donkey the parasite is longer, varying from $28 \mu$ to $33 \mu$, its width being about the same. ${ }^{1}$

1 This passage is quoted from our paper in the Ann. Inst. Past., January, I902. We have never altered our opinion on this point, as might be supposed from a sentence in the recent paper of Rabinowitsch and Kempner. In our note of November 17,1900 , we gave the length as $30 \mu$ to $34 \mu$, as at that time we had seen the trypanosome only in the blood of the horse. In our note of March 23, I90I, we gave $25 \mu$ to $30 \mu$ as the length of the trypanosomes seen in the blood of the rat, mouse, dog, and rabbit. The figures we have given since are simply more exact. 
When the blood is centrifuged, the trypanosomes gather together at the upper part of the layer formed by the red corpuscles. Kanthack, Durham, and Blandford recommend this procedure when looking for the parasites in a blood in which they are very scanty. If the blood contains very many trypanosomes, the parasites form an obvious whitish layer. In this way the parasites can be obtained almost pure.

Trypanosoma brucei in Stained Preparations (Fig. 4 in coloured plate).-After staining by the method given in Chapter II., the structure of $T$. brucei can be well made out.

The protoplasm stains a deep blue, and, as a rule, very large deeply-staining. granules are seen in it, especially in the anterior half of the body (Fig. 22, I). The posterior extremity of the parasite often has the appearance of a truncated cone. The nucleus, situated about the middle of the body, is elongated; in its interior are numerous granules staining more deeply than the chief chromatic mass (Fig. 22, a) ${ }^{1} \quad$ Near the posterior extremity is the centrosome $(b)$, staining more intensely than the nucleus; it is often surrounded by a small clear space.

The flagellum, free anteriorly $(d),{ }^{2}$ is continued posteriorly along the whole length of the undulating membrane $(c)$, of which it shows up the folds, and extends back to join the centrosome. Although the flagellum appears to be separated from the centrosome by a small clear area, there is no doubt that it is continuous with it. In studying the involution forms later on, we shall see that isolated flagella, still attached to the centrosome, are frequently met with when the protoplasm and the nucleus have disappeared.

[The trypanosomes seen in stained films are not always of the same type. Prowazek ${ }^{3}$ differentiates three kinds of parasites in the blood, which are very similar in appearance to those described in the case of $T$. levoisi, and are regarded by him as indifferent, male, and female

1 [Quite recently Ronald Ross and J. E. S. Moore have stated (Brit. Med. Journ., January 19, 1907, p. 138), that on staining liquid blood containing T. brucei and $T$. equiperdum with nuclear stains, such as basic fuchsin and thionin, the whole of the nucleus does not become coloured, as it does by the various modifications of the Romanowsky method. A much smaller central sphere stains by basic fuchsin, etc., and it is suggested that this is the true chromatin portion of the nucleus of the trypanosome. The peripheral part of the nucleus is regarded as a vesicle bounded by the nuclear membrane, and usually filled up by the red colouring matter of the Romanowsky reagents. Ross and Moore think that their observation tends to reopen the cytology of trypanosomes and allied organisms.]

2 One sees a certain proportion of trypanosomes in which the protoplasm extends along the flagellum right to the end; in such cases one cannot, strictly speaking, talk of a free part of the flagellum. Those forms are undoubtedly associated with repeated subdivision. Moreover, after the division of a trypano. some with free flagellum, as a rule one of the two individuals retains the free part of the original flagellum; the other has, therefore, at first a flagellum without a free portion. The occurrence of such forms is not the rule with $T$. brucci, although is, as we shall see later on, with $T$. dimorphon (Chapter VII.).

${ }_{3}$ [Prowazek, Arb. a. d. kuiserl. Gesund., v. 22, I905.] 
forms. Prowazek also describes the same complexity of structure in T. brucei as in T. lewisi (see p. 69).]

Multiplication Forms.-Bruce states that multiplication takes place by longitudinal division, but he dismisses the subject in a few words.

Kanthack, Durham, and Blandford did not see any multiplication forms of the parasite. They record the occurrence in the lymphatic glands, in the bone-marrow, and in the spleen, of small forms I $\mu$ to $2 \mu$ in diameter, oval in shape, and with or without a short flagellum, which they look upon as young forms of the parasite.

According to Plimmer and Bradford, there are two modes of reproduction for the trypanosome of nagana: (I) direct division (longitudinal or transverse); and (2) reproduction preceded by conjugation,
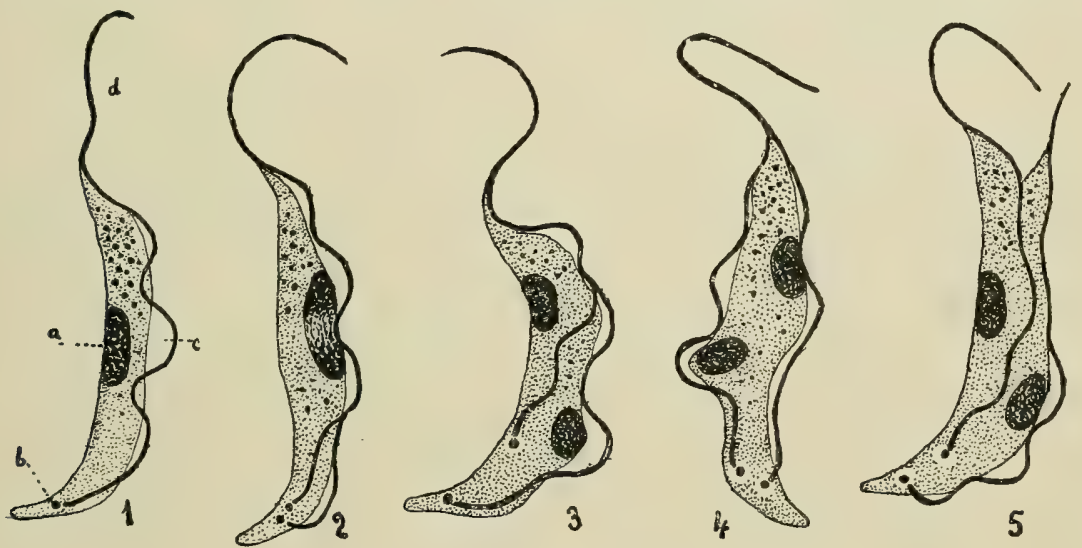

Fig. 22.-Multiplication of $T$. brucei.

I. Trypanosome of nagana $(a$, nucleus; $b$, centrosome; $c$, undulating membrane; $d$, flagellum). 2. The same trypanosome at the commencement of division (there are two centrosomes, and the flagellum and nucleus are undergoing division). 3. 4, 5. More advanced stages of division. (Magnified about 2,000 diameters.)

resulting in the formation of amœboid and plasmodial masses which are found in the spleen. These latter forms are said to give rise to flagellated parasites by a process of segmentation. The second mode of multiplication is stated to be commoner than the former.

We have seen above that, on examining fresh blood, large forms are seen which have two undulating membranes, and sometimes two flagella. The simple observation of fresh blood shows, therefore, that multiplication by longitudinal division takes place. To study the different phases of division it is necessary to examine stained preparations. In animals infected with nagana dividing forms are always seen in the blood. The study of those forms is, therefore, easier than in the case of $T$. lewisi, which has a very limited period of multiplication, after which only adult parasites are seen in the blood. 
Fig. $22(2,3,4$, and 5$)$ represents different stages in the division of $T$. brucei. ${ }^{1}$ A trypanosome about to divide increases in size and enlarges in all dimensions. The centrosome, flagellum, nucleus, and protoplasm successively divide, the centrosome always dividing first.

I. Division of the Centrosome and Flagellum. ${ }^{2}$-The centrosome becomes elongated, then divides into two small rounded bodies, placed, as a rule, one above the other (Fig. 22, 2); at the same time the adjacent part of the flagellum thickens and divides. Fig. 22 $(2,3$, and 4$)$ shows different stages in the duplication of the flagellum. In Fig. 22, 4, the new flagella are separate up to the point where the flagellum becomes free, but sometimes the flagellum divides throughout its whole length.

2. Division of the Nucleus.-The nucleus increases in size, and becomes elongated; the chromatin collects at the extremities (Fig. 22, 2), and finally the two daughter nuclei separate (direct division). At first close together, the nuclei soon separate from one another (Fig. 22, 3 and 4). They are usually oval in shape.

3. Division of the Protoplasm. - The protoplasm divides into two almost equal parts around the nuclei. In well-stained preparations this division is very obvious; a clear space separates the two masses of protoplasm, which are stained blue.

The two parasites remain close together for some time, and this explains the large forms with two undulating membranes seen in fresh blood.

The parasite remains active throughout the whole period of division, motility being only slightly diminished.

The division of the cytoplasm may begin at the anterior part of the body, as in Fig. 22, 5, or at the posterior. It sometimes happens that the two parasites resulting from the division of a trypanosome themselves subdivide before the division of the cytoplasm is complete, but such cases are very rare.

We have carefully examined the peritoneal fuid, the lymphatic glands, and the spleen of animals inoculated intraperitoneally, but we have never seen either multiplication forms or the small forms which Kanthack, Durham, and Blandford have described as young T. brucei. Moreover, we have not met with the amœboid or plasmodial forms of Plimmer and Bradford. When studying the agglutination of the trypanosome of nagana and its involution forms later on, we shall have occasion to return to this question, and shall show how Plimmer and Bradford were led to believe in the existence of conjugation and plasmodial forms.

The method of multiplication of $T$. brucei is in reality very simple; it always occurs by longitudinal division, and after the two

${ }^{1}$ Laveran and Mesnil, 'On the Mode of Reproduction of the Trypanosome of Nagana,' Soc. de Biol., March 23, I90I.

2 [For recent accounts of the details of division of 7 : brucei see later.] 
resulting trypanosomes separate, they are almost of the same size. The multiplication forms in fresh blood differ from the ordinary forms only by their greater width, which explains how observers, who had not at their disposal a method of staining by which they could study the different stages of fission, came to err regarding the mode of multiplication of these parasites. One is always inclined to look for small forms as multiplication forms of a parasite, but in this case, as a result of the particular mode of subdivision, the young forms are almost as large as the adult.

Schilling and Martini, ${ }^{1}$ who have studied the Togoland virus, have, like ourselves, observed only one mode of division, namely, longitudinal fission.

[According to Prowazek's recent researches (loc. cit.), the details of the division of $T$. brucei, as of $T$. lewisi, are very complicated. The

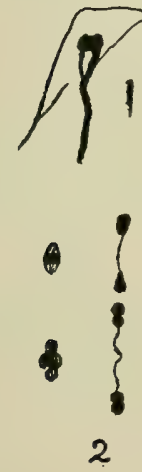

2

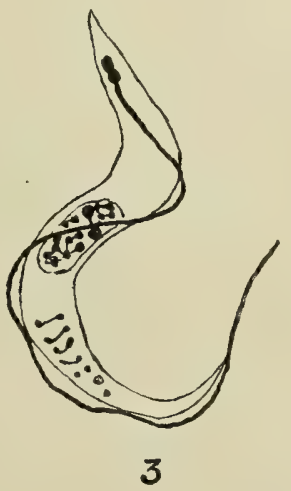

3

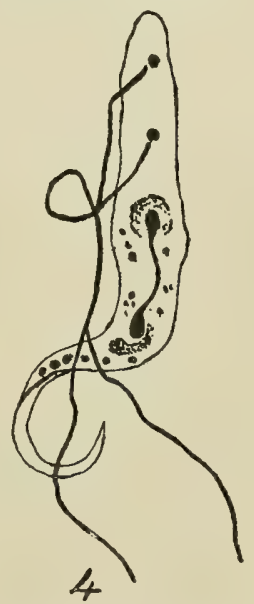

Fig. 23.-Details in the Division of Nucleds and Centrosome of $T$. brucei.

I, 2. Division of the centrosome. 3, 4. Division of the nucleus. (After Prowazek.)

centrosome becomes thickened and elongated, and subsequently dumb-bell shaped. The two thicker terminal portions then separate, but remain connected by a slender thread (see Fig. 23, $I, 2$ ). The nucleus also enlarges; its chromatin becomes grouped into eight rod-shaped chromosomes, which divide in a similar fashion to the centrosome. The nuclear karyosome (karyocentrosome) has usually divided at this stage. Fig. 23, 4, shows the karyocentrosome drawn out with the chromatin grouped around its two ends. The chromatic granules in the protoplasm may also divide, as in Fig. 23, 3.]

[As in the case of $T$. leweisi, Prowazek maintains that the flagellum does not divide, ${ }^{2}$ but that the whole of the flagellar apparatus is derived from the new centrosome. $\mathrm{He}$ found that the nucleus,

${ }_{1}$ Martini, Zeitschr. f. Hyg., v. 42, 1903, pp. 341-350, and Festschrift zum 60 Geburtstage von R. Koch, Jéna, 1903, p. 219.

2 [McNeal says the same thing of $T$. brucei in cultures.] 
centrosome, flagellum, periplast or ectoplasm, and the special 'myoneme,' or fibrillary layer surrounding the body, withstand the digestion of phagocytosis the longest. From this observation he concludes that all these structures are of nuclear nature and origin.]

[Although the sexual phases of this trypanosome would normally only occur in the appropriate tsetse-fly, Prowazek occasionally, though rarely, observed the maturation process of female parasites in the blood. This, he states, consists of two divisions of both nucleus and centrosome, only one of the four elements of each kind persisting. In one case, in the blood of a guinea-pig just dead, Prowazek saw the process of conjugation completed on the slide, and he found several stages in a stained specimen of the blood. $\left.{ }^{1}\right]$

[The Morphology of the Trypanosomes in the Tsetse-Fly. - Koch ${ }^{2}$ has described two well-marked forms of the parasite in the digestive-tube of infected tsetse-flies (Glossina morsitans and Gl. fusca). (I) Large forms, with abundant cytoplasm staining dark blue, and with a large, round, reticulated nucleus; and (2) thin, elongated forms, with feebly-staining protoplasm, and a long rod-shaped nucleus composed of a homogeneous mass of chromatin. These Koch regards as female and male forms respectively. Koch states, moreover, that the sexual forms of $T$. brucei in Gl. morsitans and Gl. fusca are distinct from those of T. gambiense in Gl.palpalis, and that these differences in the evolution forms will serve to differentiate the pathogenic mammalian trypanosomes.]

$\left[\right.$ Novy, ${ }^{3}$ from his observations on the flagellates of mosquitoes and of tsetse-flies, thinks that the forms described by Koch as resulting from the development of $T$. brucei and $T$. gambiense in Glossina, have really nothing to do with these flagellates. As Minchin had previously shown, they are probably 'wild' or native trypanosomes of the tsetse-flies.]

Differential Diagnosis of T. brucei from Allied Trypanosomes.In Chapter IV. we drew attention to the differences between $T$. brucei and $T$. lewisi. The reader is referred to subsequent chapters for the differences between $T$. brucei and the other pathogenic mammalian trypanosomes.

The Preservation of $T$. brucei in the Blood in vitro.According to Bruce, the blood of animals infected with nagana is still infective four days after it has been collected in vitro, provided it is not allowed to dry. Dried blood was sometimes still infective at the end of twenty-four hours, but that was exceptional.

Kanthack, Durham, and Blandford found that $T$. brucci may remain alive in vitro from one to three days, exceptionally from four to six days.

In preparations of biood ringed with vaseline Plimmer and

1 [From Mesnil's abstract in Rull. Inst. Past., v. 3, 1905, pp. 553, 554.]

2 [Koch, Sitzungsber. d. K. pr. Akad. d. Wiss., v. 46, 1905, p. 958.]

3 [Novy, Journ. Infec. Dis., v. 3, 1906, pp. 394-411.] 
Bradford sometimes found living trypanosomes at the end of five to six days. Blood collected aseptically and preserved in contact with oxygen remains virulent for at least three days, according to the same observers.

Blood containing T. brucei collected aseptically, mixed with citrated salt solution, and kept at the temperature of the laboratory, may be still virulent at the end of three days. Such a result is not constant, however, and blood kept for only forty-eight hours may sometimes be found to have lost its virulence.

We have already seen that in animals inoculated with trypanosomes preserved in vitro the appearance of the parasites in the blood is considerably delayed. It is very important to remember this fact in these experiments, otherwise one may record as negative inoculations the results of which are really only delayed.

Trypanosomes keep well and remain active for a longer time in blood mixed with serum than in pure blood. We have seen trypanosomes still active at the end of three days in defibrinated rat's blood mixed with an equal quantity of horse serum, whereas in pure blood, even at the end of twenty-four hours, no living trypanosomes could be seen. Human serum and that of animals resistant to nagana (birds) are just as useful for the preservation of trypanosomes as the serums of the most susceptible animals (see later 'Cultures').

[Jakimoff ${ }^{1}$ has recently made some similar observations. Defibrinated trypanosome-containing blood after being kept for two days at $0^{\circ}$ to $5^{\circ} \mathrm{C}$., or for six days at $20^{\circ} \mathrm{C}$., was still infective for mice. The addition of horse serum to the blood had no effect, the mixture being infective for mice after keeping for six days at $20^{\circ} \mathrm{C}$. The addition of 6 per cent. $\mathrm{NaCl}$, on the other hand, was distinctly harmful. He found that blood diluted even 50,000 times can still infect; the incubation period is prolonged to fifteen days, and death occurs three days later. Different germicides killed the trypanosomes very rapidly: potassium permanganate, I per cent., in one minute; $\mathrm{HgCl}_{2}$, O.I per cent., in two to three minutes; carbolic acid, 5 per cent., in four to five minutes.]

The Action of Cold.-We have already drawn attention to the long time that $T$. lewisi can be kept alive in the ice-chest. T. brucei does not possess the same property, for it does not keep better in the ice-chest $\left(5^{\circ}\right.$ to $7^{\circ} \mathrm{C}$. above zero) than at the temperature of the laboratory.

Blood kept for three to five days in the refrigerator has on several occasions given us negative results on inoculation. Such negative results may even be obtained when on microscopical examination some slightly active trypanosomes are still found.

In blood which is kept in the ice-chest the trypanosomes rapidly alter their shape. Later on we shall describe these changes of form, which are produced in other ways, as well as by prolonged exposure to cold (see 'The Involution Forms'). Cold diminishes the activity

1 [Jakimoff, Centralb. f. Bakter, I, Ref., v. 38, I906, pp. I3-16.] 
of the trypanosomes, but on taking them out of the ice-chest their activity becomes more marked.

Although the trypanosomes of nagana cannot resist the prolonged action of moderate cold, they show great resistance to sudden falls of temperature to $-50^{\circ} \mathrm{C}$., $-55^{\circ} \mathrm{C}$., and even $-\mathrm{Ig} \mathrm{I}^{\circ} \mathrm{C}$., as the following experiments will show.

In all these experiments we used rats' blood containing many $T$. brucei, diluted with citrated salt solution.

Experiment I.-Blood exposed for half an hour to a temperature of $-\mathrm{I} 5^{\circ}$ to $-18^{\circ} \mathrm{C}$.

Experiment 2.-Blood exposed for twenty minutes to $-15^{\circ}$, and afterwards for eight minutes to $-25^{\circ}$ to $-30^{\circ} \mathrm{C}$.

Experiment 3.-Blood exposed for half an hour to $-15^{\circ}$, and afterwards for five minutes to $-50^{\circ}$ to $-55^{\circ} \mathrm{C}$.

Experiment 4.-Same as preceding, except that the blood was warmed up quickly, and not slowly, as in Experiment 3.

In all these cases at the end of two hours many normal active trypanosomes were found in the blood, when thawed and warmed up again to the temperature of the laboratory.

Mice inoculated subcutaneously with these trypanosomes (two mice for each experiment) died of nagana as quickly as control mice, except in Experiment 4, in which the control mouse died first.

Experiment 5.-Rat's blood containing many T. brucei, diluted with citrated salt solution, was exposed to the temperature of liquid air. At the end of five minutes the tube was withdrawn and allowed to warm up again. One-quarter c.c. was used to inoculate mouse I; the remainder was again exposed for ten minutes to $-19 \mathrm{I}^{\circ} \mathrm{C}$.; after being warmed up again it was used to inoculate mice 2 and 3. Finally the blood kept for twentyfive minutes at $-\mathrm{I} g \mathrm{I}^{\circ} \mathrm{C}$. was used to inoculate mouse 4 . On cursory examination, the trypanosomes in these experiments appeared motionless. They were, however, still virulent.

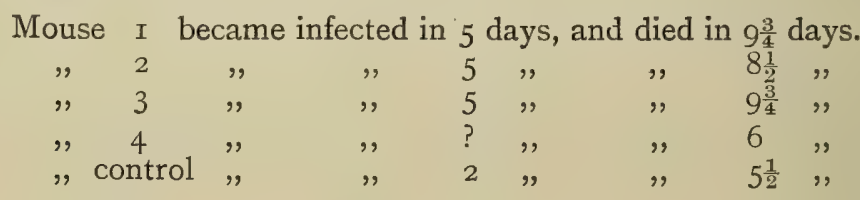

Action of Heat.-Two factors must here be considered: (I) the temperature, and (2) the time during which the blood is exposed to the raised temperature.

Specimens of blood heated for three hours to $40^{\circ} \mathrm{C}$, and for one hour and twenty minutes to $42^{\circ} \mathrm{C}$., were still virulent; other specimens heated for forty minutes to between $4 \mathrm{I}^{\circ}$ and $44^{\circ} \mathrm{C}$., and for twenty minutes to $44.5^{\circ} \mathrm{C}$, did not produce an infection in animals on inoculation. Heating to $44^{\circ}$ to $45^{\circ} \mathrm{C}$., therefore, very rapidly kills the trypanosomes, whilst with temperatures of $40^{\circ}$ to $43^{\circ} \mathrm{C}$. prolonged exposure is necessary. When one examines the blood which has been exposed for an hour to a temperature of $4 \mathrm{I}^{\circ} \mathrm{C}$., at first sight it appears as though all the trypanosomes were destroyed; the parasites are motionless, deformed, and almost unrecognisable by anyone who has not often seen them in this condition. The majority are spherical and appear dead, but blood inoculated into a rat or a mouse still produces an infection, only after a slightly longer incubation period. 
In animals inoculated with blood heated to $40^{\circ} \mathrm{C}$. from one hour and fifty minutes to three hours we have seen trypanosomes appear in the blood on the fifth or sixth day.

We have already seen that the number of trypanosomes in the blood of different species of animals infected with nagana varies considerably. The temperature of the blood secms to play an important part in this connection. When the temperature goes up to $40^{\circ}$ or $4 \mathrm{I}^{\circ} \mathrm{C}$., the trypanosomes diminish gradually in number in the general circulation. In the case of the pig recorded previously the rectal temperature was, as a rule, about $40^{\circ} \mathrm{C}$., and under these conditions the parasites were never seen in the blood.

We have not only studied the action of heat and cold upon the trypanosomes of nagana, but we have submitted these parasites, in vitro, to the action of a large number of chemical substances, in order to find out, from the point of view of treatment, the toxic effect, if any, of these substances upon the T. brucei (see Section 6).

[Action of X Rays and other Agents.-Exposure of liquid preparations of blood containing active trypanosomes to the action of Roentgen rays, Finsen rays, and radium emanations for half to one hour was found by Ross ${ }^{1}$ to have no harmful effect upon the parasites. De Nobele and Goebel had previously shown that X-rays have no action in vitro upon trypanosomes, and more recently they have found that radio-therapy has no effect upon the course of experimental nagana in animals (see Chapter XIII. on 'Treatment').]

[Goebel ${ }^{2}$ has studied the action of cobra venom on T. brucei. $\mathrm{He}$ finds that at $37^{\circ} \mathrm{C}$., I C.C. of a I per cent. solution of venom in physiological saline mixed with O'I c.c. suspension of trypanosomes from guineapigs' blood, causes hæmolysis and trypanolysis in fifteen minutes. At $19^{\circ} \mathrm{C}$. it takes two hours, and at $0^{\circ} \mathrm{C}$. there is no action. The trypanosomes are rapidly destroyed, and no intermediate involution forms are seen.]

Cultures.-The brilliant results obtained by Novy and McNeal in the cultivation of $T$. lewrisi led them to attempt to cultivate pathogenic trypanosomes. ${ }^{3}$ They succeeded with $T$. brucei. The methods used being the same as for $T$. lewisi, the reader is referred to the account given in Chapter II. The authors lay stress upon the fact that $T$. brucei grows only exceptionally in the condensation water of an agar medium containing only a half, or less than half, its volume of blood. The best results appear to be obtained with mixtures containing 2 to 3 parts of blood to I of agar. ${ }^{4}$ Even on these media only one or two out of a large number of tubes show any sign of growth; and, moreover, nearly all the trypanosomes inoculated succumb, and the cultivation always starts, at the end of about twenty days, from a very small number of parasites which have survived-

1 [Ross, Brit. Med. Journ., I906, v. I, p. 798.]

2 [O. Goebel, Ann. Soc. Méd. Gand, 1905, 3rd fasc.; abstract by Mesnil in Bull. Inst. Past., v. 3, ig05, p. 7 14.]

${ }^{3}$ Novy and McNeal, Journ. Amer. Med. Assoc., November 2I, I903; Journ. Infect. Dis., v. I, January 2, I904, pp. I-3o.

${ }^{4}$ We have seen that $T$. lezvisi grows in media containing I part of blood to 2 , 5, or even Io, of agar, but it prefers media containing 2 parts of blood to I of agar. 
'survival of the fittest.' In order to give an idea of the difficulty, it is sufficient to state that only four out of fifty attempts to cultivate trypanosomes from the blood of animals suffering from nagana (obtained from Zululand, Bruce) were successful. But once a culture begins to grow the difficulty ceases, for subcultivations are easily made.

[In their first paper (loc. cit.) Novy and McNeal state that one of their cultures had grown through eight generations of subcultures in Ioo days. In a later paper ${ }^{1}$ they stated that one of the cultures, started on August 27, I903, was then in its twenty-seventh generation, but no mention is made of its virulence].

Cultures grow at the room temperature, at $25^{\circ} \mathrm{C}$. and at $34^{\circ} \mathrm{C}$., but the higher the temperature the sooner does the culture die. At the room temperature cultures have been found still living after forty-five days.

Trypanosomes which have been kept at $25^{\circ} \mathrm{C}$., and have really multiplied, are rarely as virulent as parasites occurring in the blood. As an exception it may be mentioned that trypanosomes of the third generation, which had been outside a host for sixty-two days, killed mice in three to five days, like trypanosomes passed through animals. As a rule, trypanosomes in cultures kill rats and mice in seven to ten days, instead of three to five days. Cultures twenty-two days old may still be virulent.

If cultures growen at $25^{\circ} \mathrm{C}$. are heated to $34^{\circ} \mathrm{C}$., they generally lose their virulence in less than forty-eight hours, although the trypanosomes may remain alive for several days.

In culture tubes, even without multiplication taking place, the parasites inoculated sometimes remain alive for ten and even eighteen days. These inoculated trypanosomes, before losing their motility, lose their virulence, generally after five days, but exceptionally only after ten days. Before losing their virulence they become somewhat attenuated, as is shown by the prolongation of the incubation period. These are analogous to the results obtained with preserved trypanosomes, but the disease, once declared, is always fatal. An inoculation not followed by infection does not confer any immunity.

Attempts at immunization by non-virulent cultures did not give any appreciable result (merely a delay of one or two days on subsequent inoculation of the virus), probably because 'the material injected in the first inoculation was too feeble' (Novy and McNeal).

When grown at $25^{\circ} \mathrm{C}$., T. brucei shows two large, highly refractile granules in the anterior half of the body. At $34^{\circ} \mathrm{C}$. these granules increase in number and size, and may attain a diameter of I $\mu$. They probably stand in some relation to an alteration in vitality of the parasites, for they are not found in the blood of animals inoculated with these cultures. This fact, together with all we have said about the difficulty in obtaining cultures, of the loss of virulence in cultures,

${ }^{1}$ [Novy and McNeal, Journ. Amer. Med. Assoc., May 28, 1904.] 
especially when heated to $34^{\circ} \mathrm{C}$., proves, as Novy and McNeal state, that the ideal medium for the cultivation of $T$. brucei has yet to be discovered.

In cultures of $T$. brucci the parasites are either in pairs, joined by their posterior extremities, or in colonies of Io to 20 long, thin parasites showing wriggling movements, with the flagella apparently arranged around the periphery. The parasites vary a little in size, measuring from I $5 \mu$ to $I_{7} \mu$ in length (flagellum, undoubtedly, not included). $T$. brucei is longer and proportionately narrower than $T$. lewisi; its flagellum is very short.

'The motion of $T$. brucei is slow and wriggling, and only exceptionally is a slowly progressive form observed. The wave motion slowly passes along the thick undulating membrane, and gives the appearance of a spiral rotation to the entire cell ' (Novy and McNeal).

$\left[\mathrm{McNeal}^{1}\right.$ and $\mathrm{Smedley}^{2}$ have recently made further observations upon the cultivation of T. brucei. McNeal recommends a modification ${ }^{3}$ of the original blood-agar medium for isolating the trypanosome from an infected animal. For subcultures he used a blood-agar containing four times as much meat extractives. Transplantations from the first culture were made as early as the fourteenth day and as late as the fifty-second day. He found $25^{\circ} \mathrm{C}$. to be the best temperature, though the room temperature is fairly good.]

[By a special method of treatment, ${ }^{4} \mathrm{McNeal}$ was able to show that the new flagellum was not produced by a splitting of the original flagellum, but that it developed from the new centrosome by the side of the old flagellum.]

[Smedley $^{2}$ succeeded in cultivating $T$. brucei on rabbit-blood agar three times out of ten. In young cultures he found the trypanosomes to possess very active movements, and to move across the field fairly quickly. The cultural forms were somewhat smaller than the forms seen in the blood, the average dimensions in stained films being $18 \mu$ to $23 \mu$ long (including free flagellum, which measured $3 \mu$ to $5 \mu$ ) by $2.5 \mu$ to $3.5 \mu$ wide. The undulating membrane was well developed, and the centrosome was nearly always posterior to the nucleus (unlike $T$. lewisi in cultures). The colonies of $T$. brucei were unlike those of $T$. lewisi, being much smaller and much less numerous; the flagella are directed peripherally in them, and secondary massing together of the colonies does not occur to any extent. The cultural characteristics, therefore, are sufficient by themselves to distinguish $T$. brucei from $T$. lewisi.]

AgGLOMERATION.-Under certain conditions the trypanosomes of nagana become arranged in a regular fashion, like those of the

1 [W. J. McNeal, Journ. Infec. Dis., v. I, I 904, pp. 517-543.]

2 [R. D. Smedley, Journ. Hyg., v. 5, I 905, pp. 24-43.]

3 [Add 2 volumes sterile defibrinated rabbit's blood to I volume of the following medium, previously sterilized in tubes or flasks and cooled to $60^{\circ} \mathrm{C}$.: The extractives of 125 grammes of beef in I,O00 c.c. distilled water; agar, 20 grammes; peptone, 20 grammes; common salt, 5 grammes; normal solution of sodium carbonate, 10 c.c. $]$

4 [Add a drop of defibrinated rat's blood, rich in trypanosomes, to a mixture of $\frac{3}{4}$ c.c. defibrinated rabbit's blood and $\frac{1}{4}$ c.c. of a molecular solution of normal sodium phosphate. Let it stand for half an hour, make films, and stain. 'I he alkaline phosphate dissolves away the undulating membrane which sheaths the flagellum, and this is then set free.] 
rat. They often join together in pairs. ${ }^{1}$ Sometimes they form primary agglomeration rosettes, but the large secondary agglomerations, which are common in blood containing $T$. lewisi, are rarely seen with this trypanosome. The trypanosomes are always joined by their posterior ends, as can be readily made out in stained specimens. Fig. 24, I2, represents trypanosomes agglomerating in rosette form, as seen in fresh blood; Fig. 24, 13, shows two trypanosomes in a stained specimen joined by their flattened posterior extremities, so that the line of separation between the parasites is hardly visible.

These trypanosomes united in pairs suggest conjugation, but such an interpretation is inadmissible. Agglomeration is not seen in pure, fresh blood, but is produced only under abnormal conditions, and, moreover, the number of parasites which agglomerate varies very much.

With $T$. brucei, as with $T$. lewisi, one may observe the rosettes break up after a variable interval.

We have seen agglomerations of trypanosomes form in pure blood a half to one hour after removal from the heart; in peritoneal exudates after intraperitoneal injection into rats or mice of blood containing many parasites; and in blood mixed with salt solution kept for twenty-four hours on ice, or heated for half an hour to $4 \mathrm{I}^{\circ} \mathrm{C}$.

On mixing equal parts of horse serum and defibrinated blood from a rat or mouse rich in trypancsomes, we have obtained very beautiful persistent agglomeration masses. At the end of some hours, however, the trypanosomes lose their characteristic shape. On mixing I part of horse serum with Io of blood, agglomeration masses are not produced. Pig serum also agglomerates well. Sheep serum mixed with an equal volume of the blood of a rat or mouse rich in $T$. brucei gave in one case well-marked agglomeration masses, but in another case the agglomeration was less marked and not persistent. The serum of a goat gave, with an equal volume of blood, small and non-persistent rosettes. Human serum was neither agglutinating nor microbicidal.

The following serums, mixed with an equal volume of the blood of a rat or mouse containing many $T$. brucei, did not exhibit any agglutinating properties : the serum of a rat, either normal or immunized against $T$. lewisi, and agglutinating those trypanosomes; the serum of a normal fowl, and that of a fowl inoculated on several occasions with $T$. brucei; the serum of a normal goose, and that of a goose inoculated on several occasions with blood rich in T. brucei.

On adding a drop of water slightly acidulated with acetic acid to

1 These combinations of two parasites are particularly abundant in the peritoneal fluid of rats or mice inoculated intraperitoneally.

[McNeal (loc. cit.) also saw numerous pairs of trypanosomes, joined by a considerable portion of their posterior ends, in the blood of mice shortly before death. The contact was so intimate that there was no line of demarcation and the centrosomes showed such various forms (fusion, dumb-bell shapes, and tetrads) that it would seem to indicate an actual conjugation, as described by Bradford and Plimmer.] 
several drops of blood containing many T. brucei, the trypanosomes agglomerate and soon change their shape. On the other hand, a drop of water made faintly alkaline with soda does not produce agglomeration.

Dead trypanosomes also tend to agglomerate, but in such cases agglomeration is very irregular.

Involution Forms.--When the trypanosomes of nagana are under unfavourable conditions, which, however, are not sufficient to bring about rapid death of the parasites, they show involution forms, which it is important to recognise. We have seen these forms produced under various conditions : rat's blood rich in trypanosomes mixed with the serum of other animals, and kept for several hours in hanging-drop preparation or in ordinary fresh films; blood heated

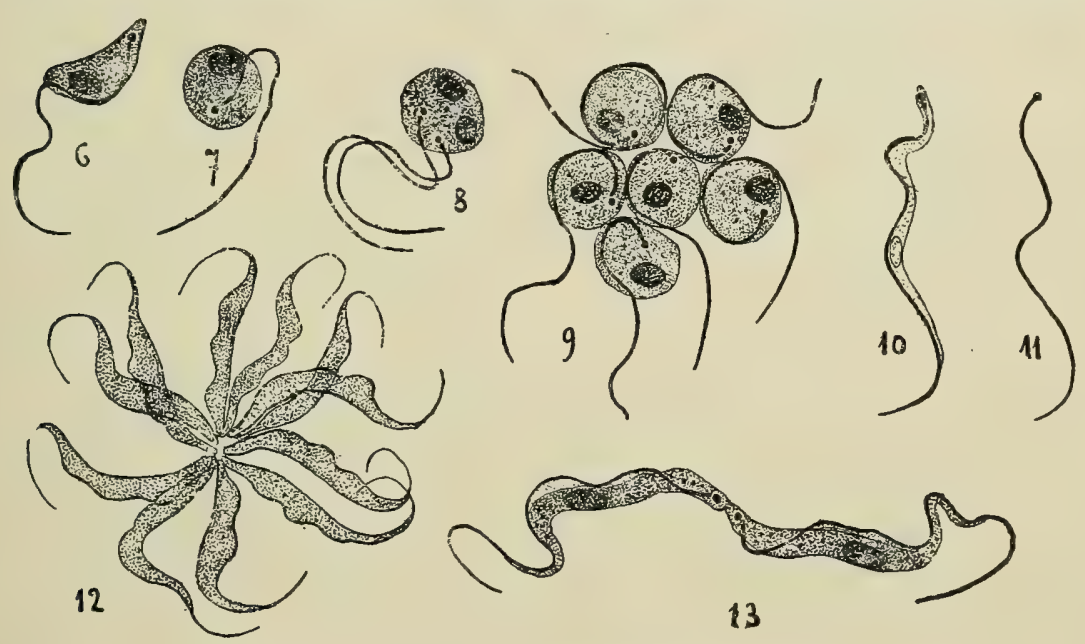

Fig. 24.-Involution and Agglomeration Forms of T. brucei.

$6,7,8$. Spherical forms of trypanosomes in blood mixed with horse serum; 8 shows a trypanosome which was undergoing division when it became spherical. 9. Agglomeration of spherical trypanosomes. Io. Trypanosome undergoing disintegration ; the trypanosome has almost entirely disappeared, and the nucleus is very pale. II. Free flagellum with centrosome. 6-II were drawn under a magnification of 1,400 diameters. I2. Trypanosomes, agglomerated in the form of a rosette, seen in fresh blood ( $\mathrm{I}, 000$ diameters). I3. Two trypanosomes, joined by their posterior ends, seen in a specimen of stained blood ( 1,600 diameters).

to $4 \mathrm{I}^{\circ}$ or $42^{\circ} \mathrm{C}$. for an hour or more; blood injected into the peritoneal cavity or into the connective tissue of birds, and examined at the end of one to three hours; blood kept in the ice-chest or frozen; blood of rats treated with arsenic, etc. Fig. 24, 6 to $I I$, shows different involution forms of $T$. brucei.

The trypanosome becomes short and stumpy (Fig. 24, 6), then spherical (Fig. 24, 7). On staining, spherical forms of the parasite are seen, containing a nucleus, centrosome, and flagellum. Fig. 24, 8, represents a trypanosome which was undergoing division when it became spherical; two nuclei, two centrosomes, and two flagella can be seen in it. 
The spherical forms may produce small agglomeration masses (Fig. 24, 9). It is very probable that it was these masses which Plimmer and Bradford saw and described as plasmodia giving rise to flagellated parasites.

[Rodet and Vallet ${ }^{1}$ regard these abnormal parasites not as true involution forms, which arise gradually as the result of unfavourable conditions, but as parasites which have undergone a sudden and active change in shape in response to an abnormal stimulation. By pricking an infected animal through a drop of I per cent. osmic acid, they found that the trypanosomes so obtained were all of this 'amœeboid' type. Rodet and Vallet suggest that possibly the chemical agents used to fix tissues may account for the amœboid forms seen in sections of organs.]

Trypanosomes which are spherical and no longer show any signs of movement are not always dead, however, for on injecting blood containing this form of the parasite into a rat or mouse, the trypanosomes sometimes appear in the blood after a lengthened incubation period, varying in length with the degree of alteration in the parasites injected.

If the trypanosomes remain exposed to harmful conditions, they die and undergo profound alterations, which are seen most clearly in stained preparations.

The protoplasm disappears first, and can no longer be stained; the outline of the parasite becomes considerably narrowed (Fig. 24, IO), and the nucleus stains badly. Later on the protoplasm and nucleus have disappeared altogether, and the flagellum is seen either alone or with the centrosome forming a small swelling at one end (Fig. 24, $I I$ ).

\section{Section 5.-—Etiology of Nagana. The Rôle of the Tsetse- Fly and of the Big Game.}

Bruce's experiments have placed beyond all doubt the rôle of the tsetse-fly in the spread of nagana. ${ }^{2}$ It is true the part played by this fly had long been suspected, and Livingstone in particular had clearly recognised and well described the effects of the bite of the tsetse upon domestic animals; but for a long time we were mistaken in our idea of the exact manner in which the bite of this fly produced its harmful effects. It was thought that the fly itself was poisonous, and several observers looked, but in vain, for poison glands in the insect. It was Bruce who showed that the tsetse-fly is not poisonous, and that if its bite is, as a rule, so dangerous, it is because the fly sucks alternately the blood of animals suffering from nagana and of healthy animals, and that it inoculates into the latter the pathogenic trypanosomes.

It is, nevertheless, right to state that other observers had suspected the truth. 'The poison germ,' wrote Livingstone in $1857, \ldots \ldots$ 'seems capable, although very minute in quantity, of reproducing

1 [Rodet and Vallet, Arch. méd. expér., v. 18, July, 1906.]

- For the biology, morphology, and classification of the tsetse-flies, see Chapter XVIII. 
itself ... In I875 Mégnin stated that the tsetse-fly carries a virus, and does not inoculate a poison of its own. In 1879 , the day after the discovery of the rôle of the mosquito in filariasis, J. J. Drysdale suggested that the fly ' might be an intermediate host of some ... blood parasite, or the means of conveying some infectious poison.' In 1883 Schoch expressed himself almost in the same terms. In I88 + Railliet and Nocard, who also suspected this, found that inoculations with the proboscis of the tsetse were harmless.

We come next to Bruce's experiments, which prove conclusively that a tsetse-fly which has bitten an infected animal, and afterwards bites a healthy animal, can convey the disease to the latter. Bruce used dogs in his experiments. First he showed that flies fed on infected animals, then kept in captivity for several days, and afterwards placed on two dogs, did not infect. The tsetse, therefore, cannot give rise per se to a local or general disease in susceptible animals. Secondly, flies fẹd on a sick dog and immediately afterwards on a healthy dog conveyed the disease to the latter. The flies were infective for twelve, twenty-four, and even forty-eight hours after having fed on an infected animal; but under these conditions infection was not easily produced, many and repeated bites being necessary.

That point being proved, Bruce tried to find out whether the fly acted as a simple carrier or as a true intermediate host. He found that the blood of an infected animal after drying on threads was still infective, though rarely, after twenty-four hours-never after fortyeight hours. On the other hand, blood kept moist in an aseptic condition was still infective at the end of four days, but not after seven days. As the proboscis of the fly to a certain extent prevents the blood from drying up, it follows, if the fly acts as a simple carrier, that the figures found by Bruce for the time that the virus remains active in the fly should be intermediate between those obtained with dried and with liquid blood.

It is, therefore, by means of the fly that animals living in infected areas contract nagana. Horses were taken for several hours during the day into such districts; they were not allowed to eat or drink during that time; they were bitten by the tsetse-flies and contracted the disease. Drinking-water and food, which had often been suspected, could not have played any part in the causation of the disease in this experiment, so that it must have been the flies which communicated the disease.

A final experiment proves that this is the case. Flies were collected every day in a district where nagana was prevalent, and were placed upon a healthy animal living in a district where the tsetse was not found. Care was taken to collect these flies from healthy animals, yet they gave the disease to animals (a horse and a dog) upon which they were allowed to feed. 
We may, therefore, conclude, with Bruce, 'that it is proved that the tsetse-fly does commonly, in a state of nature, convey the disease from animal to animal, and that, on the other hand, there is no proof that the drinking-water or the eating of soiled herbage plays any rôle in the process.'

Can all the species of the genus Glossina play such a part? Bruce speaks only of $G l$. morsitans, but Austen states that he has confused two species, the true Gl. morsitans and a closely allied species, Gl. pallidipes (the photographs in Plate II. in Bruce's paper represent the latter species).

The question is far from being settled. By analogy with what is found in malaria and Texas fever, certain authors, such as Stuhlmann and Schmidt, think that Gl. morsitans is the only species which is able to convey nagana. One might immediately raise the objection that the trypanosomiasis of camels in Somaliland is most probably spread by Gl. longipennis; but as the identity of this disease with nagana is doubtful, the objection has no weight in the present state of our knowledge.

There is, however, in this connection another point of considerable importance. It is hardly questionable that the species of Glossina have a special action upon the African trypanosomes which other flies do not possess. We cannot do better than reproduce the following passage from Bruce's paper:

'That all blood-sucking flies are not capable of transferring the fly disease from affected to healthy animals is, I think, shown by the fact that up here at Ubombo, where we have several species of these pests, no single instance of the disease arising spontaneously has occurred, although healthy horses, cattle, and dogs, have been constantly and closely associated with those suffering from the disease. Why this should be so is at present a mystery, and it is to be hoped that some point may be discovered which will throw light on the subject. There may be some anatomical peculiarity in the tsetse which enables it to act as carrier, or there may be some undiscovered fact in the life-history of the parasite associating it with this particular species of fly.'

These questions remain unanswered at present. Considerations based upon the comparative geographical distribution of the tsetsefly and of the trypanosomiases of the type of nagana do not allow any definite conclusion to be drawn. However, if we note, on the one hand, the very close agreement existing between the distribution of nagana and of Gl. morsitans (we have already disposed of the objection in the case of the trypanosomiasis of dromedaries in Somaliland), ${ }^{1}$ and, on the other hand, the fact that nagana does not

1 'At the present moment.' says Austen, 'there is no definite information of the presence of nagana in a locality where Gl. morsituns does not occur. The unexpected discovery of Gl. morsituns in Togo, where the existence of a trypanosomiasis resembling nagana has been recognised, is an important fact in this 
appear to occur in regions infested by other tsetses, we have evidence, not absolutely amounting to proof, but very strongly in favour of the hypothesis that Gl. morsitans (and undoubtedly also Gl. pallidipcs, which appears to have the same geographical distribution) is the carrier of nagana, to the exclusion of the other species of Glossina.

The study of the other trypanosomiases has lately received such an impetus that facts in connection with them accumulate rapidly, and no doubt very soon all these questions will be answered.

[From the evolution which the malarial parasite is known to undergo in the body of the mosquito, it seemed probable that trypanosomes would be found to undergo an analogous development in the body of some bloodsucking insect or insects. Schaudinn, Prowazek, Koch, and others would seem to have shown that this is the case with certain trypanosomes. Schaudinn has described the sexual development of $T$. nocture of the owl in the body of mosquitoes; Prowazek that of T. lewisi in the rat-louse; and Koch that of T. brucei in the tsetse-fly. We have seen, however, that Novy, Ross, Minchin, and others, have found flagellates in mosquitoes and tsetse-flies which had not been fed experimentally on infected animals, so that the conclusions drawn by Schaudinn and Koch as to the evolution of $T$. nocture and $T$. brucei in Culex and Glossina respectively may prove to be incorrect.]

[Koch ${ }^{1}$ found that the trypanosome of tsetse disease in German East Africa is carried by Gl. morsitans, Gl.pallidipes, and especially Gl. fusca. The other species of Glossina-Gl. tachinoides, Gl. palpalis, and probably also Gl. longipennis-found there are not concerned in the transmission of the disease. By pressing on the bulb at the root of the proboscis, a small drop of liquid can be expressed, and in this trypanosomes are found (fiftyeight times in fusca, once each in morsitans and pallidipes), evidently ready to be inoculated. These trypanosomes are in various stages of development, and no red blood-corpuscles are mixed with them. Koch concludes from this that there is an undoubted development in the fly. In the intestines the trypanosomes are rare or absent, nor are they found in other organs. In the stomach of the fly the trypanosomes multiply and increase very much in size. There is, then, a well-marked difference between the large and the thin, elongated forms (described on p. I56), which Koch regards as female and male respectively. In the posterior part of the stomach very large forms are seen, with only one centrosome and one flagellar apparatus, but usually four-sometimes two or eight-nuclei. These large forms may possibly be fertilized females. Koch did not - follow out the subdivision of these forms, but he supposes that the elements with nucleus and without centrosome are derived from them. Eventually Herpetomonas-forms, with the centrosome in front of the nucleus, arise.]

[In the liquid expressed from the proboscis all stages in the development of the trypanosomes are seen, and some are like the ordinary forms found in the blood. It is these which determine the infection of a fresh mammal, and Koch was unable to infect rats with the trypanosomes from the flies' stomachs. Flies fed on recently infected cattle did not become infected, but they did when fed on oxen and mules with a chronic infection,

connection. Several localities are known in which the Gl. morsitans occurs without nagana being present. This is quite comparable with the fact that Anopheles may occur in districts where malaria is absent.'

1 [Koch, Deutsche mediz. Wochenschr., November 23, I905, pp. I865-I869; abstracted in Brit. Med. Journ., January 6, I906, pp. 31, 32, and in Bull. Inst. Past., v. 4, I906, p. 34, 35, by Mesnil.] 
and with only a few trypanosomes in their blood. Koch suggests that probably the blood trypanosomes must be in a certain phase of development, which is found in slightly susceptible animals, such as the buffalo and antelope, in order to be carried over. (Novy's criticism of Koch's conclusions will be considered in the section dealing with the trypanosomes of tsetse-flies, Chapter XVIII.)]

The proof that the trypanosome of nagana is conveyed by the tsetse-fly is not sufficient by itself to solve the problem of the rtiology of the disease. For example, the disease may be contracted in a district where not a single sick domestic animal exists. Whence does the fly obtain the virus which it inoculates? Bruce again has answered this question for us.

When he arrived in Zululand the Europeans were of opinion that it was the fly which caused the disease, and we have seen that they were not wrong. The natives, on the other hand, thought that the disease was caused by the presence of big game, the wild animals contaminating the herbage or water by their saliva or their excretions. $A$ priori, the two hypotheses are not mutually exclusive, and the fact that the association of the tsetse and wild game is confirmed by all explorers tends to reconcile them.

Bruce was successful in combining the two hypotheses by proving that the blood of the wild animals in Zululand may contain the trypanosome of nagana. It is true the parasite is always very scanty, for Bruce says in his report that he never saw it on microscopical examination, ${ }^{1}$ but this blood in doses of 5 to Io c.c. was infective for dogs. Thus, the blood of one out of eight buffaloes, of three out of thirteen wildebeest (Catoblepas gmu), and of three out of four koodoo (Strepsiceros capensis) examined, was found infective on injecting into dogs. Finally, the blood of a bush-buck (Tragelaphus scriptus sylvaticus) and of a hyena was found similarly infective.

These animals, therefore, constitute a reservoir of the virus from which the fly may obtain its supply. As we have already stated in a previous paragraph, the infection appears to be chronic in these animals and hardly to affect their health. Nevertheless, it makes them a source of great danger to these susceptible domestic animals which are introduced into their neighbourhood.

Is the spread of nagana by means of the fly the only way in which this disease may be propagated ?" That appears to be certainly the case with herbivorous animals. Carnivorous animals, such as the dog, may often contract the disease through eating animals which have recently died of nagana, and whose blood

i Later, however, he found them in the blood, according to a verbal communication made to Theiler, and published by the latter author.

2 [Markl (Cintralb. f. Bukter., I, Orig., v. 37, p. 530) states that in guinea-pigs the trypanosomes do not seem able to pass through the placenta. This is in agrecment with the observations of Chaussat, Laveran and Mesnil, and others, on the rat trypanosome, but we have seen that Pricolo observed the passage of trypanosomes ( $T$. duttoni?) through the placenta of infected mice and their development in the foetus (see p. 102) ] 
swarms with virulent trypanosomes. Bruce quotes instances of dogs having contracted the disease in this way. It is well known that in infected districts the disease in dogs especially affects the sporting dogs which are introduced into those parts. We have quoted the case of two cats which became similarly infected; [also the two cases in cats recorded by Lacomme. In Uganda, Nabarro and Greig had a jackal which developed a fatal infection after devouring a monkey with many trypanosomes in its blood. Lignières ${ }^{1}$ has recorded instances in which the infection has been conveyed by the bite of infected animals (capybara) to dogs. $\mathrm{He}$ concludes from this that the saliva itself may contain trypanosomes and be infective, but it is more likely that the trypanosomes present were due to an admixture of blood from the gums. $\left.{ }^{2}\right]$

It is quite easy to understand that this method of propagation is much less important than the first method. In a country which is immune it is impossible for the disease to become endemic or even epidemic in this way. From the point of view of prophylaxis, we need concern ourselves only with the former mode of propagation.

\section{Section 6.-Treatment.}

\section{Treatment by Means of Chemical Substances.}

Sodium Arsenite.-Stimulated by Lingard's experiments upon the arsenical treatment of surra, Bruce in Zululand treated several horses and donkeys by adding to their food each day a dose varying from 6 to I 2 grains $\left(0^{*} 384\right.$ to $0^{\circ} 768$ gramme) of arsenic in the form of sodium arsenite. ${ }^{3}$ In the most favourable cases the parasites disappear from the blood (on microscopical examination) a few days after the commencement of the treatment, ${ }^{4}$ and rarely reappear. There is no anæmia; the animals often remain strong and healthy, and can do work, which is quite impossible in the case of infected horses which are untreated. Treated animals survive an appreciably longer time than control animals, but the treatment has always to be discontinued, as the animals can no longer tolerate it, and the trypano-

1 [Lignières, Bull. et Mém. Soc. centr. vétér., v. 83, June 3o, 1906, pp. 363366 ; also Report presented to Section of Pathology, Eighth International Congress of Veterinary Medicine, Budapest, September, 1905. Abstracts by Mesnil in Bull. Inst. Past., vols. 3 and 4,1905 and I906.]

2 [Lignières also records the case of a rabbit, vaccinated against dourine, which became infected with nagana by licking the sore places and eating the crusts of a rabbit with nagana kept in the same cage. He thinks this seems to prove that T. brucei can pass through intact mucous membranes, but Mesnil does not agree with him.]

3 This arsenical treatment of nagana was in vogue long before we were acquainted with the cause of the disease. See, in this connection, James Braid, G. W. Balfour, Livingstone (Brit. Med. Journ., 1858, pp. I35, 214, 360).

4 Bruce quotes an experiment in which 5 C.c. of the blood of a horse under treatment did not infect a dog. 
somes then reappear in the blood. Only one donkey appears to have been cured, and he did not acquire immunity. ${ }^{1}$

Horses and a donkey which were saturated with arsenic rapidly contracted nagana when taken into a fly area. In a dog prophylactic treatment with arsenic was equally ineffectual. Bruce concludes from his investigations that arsenic is quite useless in the prevention of nagana, but that it is of use in prolonging the life of animals after the disease has once started.

The indication was obviously to study, in laboratory animals, the effect of arsenic compounds which had given, in the hands of Lingard and Bruce, more or less favourable results in the treatment of surra or nagana. We have studied the arsenic treatment, particularly in the rat, mouse, and dog. For the treatment of rats we use the following solution:

$\begin{array}{lllll}\text { Arsenious acid } & \ldots & \ldots & \ldots & \text { I gramme } \\ \text { Sodium carbonate } & \ldots & \ldots & \ldots & \text { I gramme } \\ \text { Distilled water } & \ldots & \ldots & \ldots & 500 \text { c.c. }\end{array}$

Boil.

Syringes holding I c.c. are graduated as a rule in twenty divisions, which gives $0^{\circ} I$ milligramme of arsenious acid for each division of the syringe.

For the treatment of mice it is advisable to dilute this solution to half the strength; on the other hand, in the treatment of dogs a stronger solution may be used.

At first in the dog we used intravenous injections of the arsenic solution, but we found that subcutaneous injections gave quite as good results as intravenous, and since that time we have used exclusively the hypodermic method, which is not only more convenient, but is the only one applicable to very small animals.

The subcutaneous injections of arsenic in the abdominal region of rats and mice often give rise to abscesses. This may be avoided, however, by injecting the fluid into the muscles of the shoulder. The hair is cut and the skin washed with perchloride of mercury. The injection is a little painful, and produces in rats and mice an obvious malaise, which sometimes lasts several hours.

The action of arsenious acid upon the trypanosomes of nagana is very remarkable, if the drug is used in sufficient doses. In the rat and mouse we have found that it is necessary to use $0^{\circ} \mathrm{I}$ milligramme of arsenious acid per 20 grammes of animal.

Should a rat of Ioo grammes be infected with nagana and have numerous trypanosomes in its blood, 5 milligrammes of arsenious acid are injected into the muscles of the shoulder. One to two hours

' [Moore (Lanict, I904, part 2, p. I5) states that in the treatment of tsetse-fly disease in cattle (? nagana) in Nigeria, sodium arseniate ( 1 ounce of a 1 per cent. solution, made alkaline with sodium carbonate) given hypodermically once a week had very good and lasting effects, even in apparently hopeless cases. He does not state, however, whether the animals were permanently cured.] 
after the injection the blood examination shows no change, but at the end of five to six hours there is an obvious diminution in the number of parasites, and at the end of twenty-four hours they can no longer be found in the blood, or, if present, they are extremely scanty.

When trypanosomes are swarming in the blood at the time the treatment is commenced their disappearance is less rapid. It may not take place in less than thirty-six or forty-eight hours, and sometimes even a second injection is necessary. Unfortunately, this disappearance of the parasites from the blood is only temporary, and at the end of forty-eight hours, or at the most of three to four days, the parasites reappear, at first in small numbers, and rapidly multiply if a second injection is not given. By repeating the injections of arsenic at intervals of two to three days, the life of the animal may be considerably prolonged, but a permanent cure never results. There comes a time when the arsenical treatment can no longer be tolerated: diarrhœa, wasting, neuritis, and œedema at the seat of inoculation are produced. The animal dies of nagana if the treatment is stopped, or of arsenical poisoning if the treatment is persisted in.

Whereas rats infected with nagana and untreated die on an average in five days, those treated with arsenic may live for two or two and a half months. The longest time that we have kept them alive has been seventy-eight and seventy-nine days. ${ }^{1}$

In mice arsenic produces the same results as in rats, only it is more difficult to use in their case, because the drug has to be pushed more in mice than in rats before improvement occurs. In mice weighing I4 to 20 grammes $O^{\prime} I$ milligramme of arsenious acid may be injected.

Arsenic has given us less satisfactory results with dogs than with rats. The treatment obviously prolongs the life of the animal, but much less so than with rats. We have injected into dogs from 2.5 to 3.5 milligrammes of arsenious acid per kilogramme of animal.

The earliest injections of arsenic cause the trypanosomes to disappear more or less completely from the blood, but they soon reappear, and the animals die in spite of repeated injections. It seems that arsenious acid loses its effect more rapidly in the dog than in the rat. In nine dogs infected with nagana ${ }^{2}$ and treated with arsenious acid, the average duration of the disease was twentyfive and a half days, the maximum being forty-six days. In dogs which were untreated the duration of the disease was from six to sixteen days (average ten and a half).

The Mode of Action of Arsenious Acid upon the Trypanosomes of Nagana.-When blood rich in trypanosomes is diluted with solution of

1 For the details of these experiments; see Ann. Inst. Past., v. 16, p. 792.

2 By a virus which was not obtained from a ruminant. 
citrate and mixed in vitro with the arsenical solution, the trypanosomes are rapidly killed. If, for example, to I $_{5}$ drops of blood one adds I drop of a 2 per I,Ooo solution of arsenic, the trypanosomes are seen to become motionless at the end of fifteen to twenty minutes, sometimes even more rapidly, whilst in control preparations (blood without the addition of arsenical solution) mobility persists.

Arsenious acid is therefore toxic for the trypanosome of nagana in vitro, but from that we cannot conclude that it has the same action in vivo. Some substances which are highly toxic for the trypanosome in vitro-formalin, for example-have no effect upon it in vivo.

In order to study the action of arsenious acid upon the trypanosome of nagana in vivo, we have examined the blood of infected rats at varying intervals after the injection of solutions of arsenic. The blood was examined fresh and after staining in the ordinary way. As a rule, involution forms are found among the trypanosomes two hours after the injection; at the end of four to five hours an obvious diminution in the number of parasites has occurred, and at the end of twenty-four hours they have entirely disappeared.

The involution forms in rats treated with arsenic are the same as those which are seen when blood containing trypanosomes is exposed to a temperature of $4 \mathrm{I}^{\circ}$ to $42^{\circ} \mathrm{C}$. for some time. The movements of the trypanosomes become less marked, the parasites are deformed and granular, and finally assume the characteristic spherical form. Later on the protoplasm and nucleus disappear, so that in stained preparations isolated flagella, sometimes with an adherent centrosome, are seen.

In certain cases there is slight leucocytosis. We have looked in vain for signs of phagocytosis of living trypanosomes, but have often seen leucocytes which had engulfed the remains of trypanosomes, sometimes the nucleus and centrosome being quite distinguishable.

We think it may be concluded that arsenious acid is microbicidal for the trypanosome of nagana, and that the leucocytes take up only dead trypanosomes, or those in an advanced stage of involution.

We have endeavoured to ascertain whether the susceptibility of trypanosomes to arsenic in vitro is diminished in rats previously treated with this drug for some time. On mixing the arsenical solution in the same doses (I) with the blood of an infected rat which had been treated for a long time with arsenic, and (2) with the blood of an infected rat which was untreated, no appreciable difference could be observed; the trypanosomes were destroyed very rapidly in both cases.

How is it that arsenious acid, although it has an obvious microbicidal effect upon the trypanosomes of nagana, never cures infected animals? The trypanosomes which are present in the general 
circulation are destroyed, but those in certain out-of-the-way parts of the body ${ }^{1}$ evidently escape the action of the drug. When the bulk of the arsenious acid has been eliminated or fixed by the tissues, these trypanosomes come out again into the blood, and there multiply rapidly. One might suppose that the spleen was a place of refuge for the trypanosomes of nagana, as it is for the malarial parasite. Experiments have shown, however, that arsenical treatment gives no better results in splenectomized animals than in normal animals.

TRYPANRED (TRYPAnRoT). ${ }^{2}$ - We shall see (Chapter IX.) that Ehrlich and Shiga have introduced a red dye of the benzo-purpurin series, which they call 'trypanrot,' for the treatment of mice infected with caderas. According to these authors, this substance is of less value in the case of mice infected with nagana. The disappearance of the trypanosomes is never permanent, but a relapse always occurs.

We have been able to verify this action of trypanred, used in doses recommended by Ehrlich and Shiga, upon mice and a rat having numerous $T$. brucei in their blood. If treatment be not too long delayed, the trypanosomes disappear from the blood in twentyfour to forty-eight hours; the diminution in number and the appearance of involution forms beginning to take place at the end of twenty-four hours. The action of this substance is, therefore, slower than that of arsenic or of human serum (vide infra).

In vitro trypanred is only feebly microbicidal. At the end of three hours' exposure to trypanred (I part of a I per cent. solution mixed with an equal volume of citrated blood), the trypanosomes are exactly the same as in a control preparation. One can therefore understand why it is that involution forms appear very slowly in the blood of animals treated with this substance. In the leucocytes the chromatic remains of the parasites acted upon by the trypanred are seen. ${ }^{3}$

We have obtained unsatisfactory results in attempts to treat rats, mice, or dogs, by subcutaneous injections of the following substances: cacodylate of sodium, arrhenal (dimethylarseniate of sodium), liquor iodi, iodide of potassium, salts of quinine, toluidin blue, and methylene blue, all of which have a slight action in vitro upon trypanosomes; and of perchloride of mercury, the double iodide of mercury and arsenic, salts of silver (lactate, fluoride or

1 [Possibly the trypanosomes take refuge in the bone-marrow, or they may assume a more resistant form, which is different from the typical form, and not easily recognisable.]

2 [For Mesnil and Nicolle's experiments with other dyes, and for the recent attempts at treatment, see special article on 'Treatment,' Chapter XIII.]

3 A mouse weighing I9 grammes had, on April 7, 1904, numerous trypanosomes in its blood, and received $\frac{1}{2}$ c.c. of a I per cent. solution of trypanred. On the 9th the trypanosomes had disappeared, but had become very numerous again on the I 5th. A fresh injection of $\frac{1}{2}$ c.c. was given, and the parasites again disappeared. On April 24 trypanosomes were again numerous, and $\frac{1}{2}$ c.c. fluid was injected. The next morning the mouse died, with very few parasites in its blood. 
tachiol, and caseinate or argonine), and formalin, all of which have a considerable effect upon trypanosomes in vitro.

Amongst the drugs which we have tried without success in nagana may be added : carbolic acid, salicylic acid, chinosol, essence of garlic, solution of calcium chloride, chloral, and, more recently, colloidal silver (collargol). ${ }^{1}$ The glycerinated bile of dogs which have died of nagana is also useless for treatment.

\section{Attempts at Serum-therapy.}

Our researches in this direction include the action of normal serums and of the serum of animals immunized against nagana. Amongst the normal serums, human serum is the only one which has any microbicidal effect upon $T$. brucei. ${ }^{2}$

Human Serum.-Human serum, injected in sufficient doses into infected animals, has a marked effect upon the course of the disease. ${ }^{3}$ The trypanosomes disappear from the blood, at least for a time, the progress of the disease is delayed, and sometimes even complete cure may result.

The serum of adults is much more active than that of the newly-born. ${ }^{4}$

Human serum retains its power for some time when it has been collected aseptically, but serum in which moulds or bacteria have developed loses its activity rapidly.

Dried human serum retains the properties of the liquid serum for at least six months; O.I gramme of dried serum corresponds to about I c.c. of liquid serum, and when used should be dissolved in I c.c. of distilled water.

Pleural fluid is less active than blood-serum, and ascitic fluid is still less active than pleural fluid.

Our experiments upon the treatment of nagana with human serum have been carried out almost exclusively upon rats and mice. In these days, when bleeding is rarely resorted to, it is difficult to obtain human serum, and we have had to make the most of the small quartities of serum we have succeeded in obtaining. We have made, however, certain experiments upon dogs.

In mice of Io to 20 grammes the dose of human serum used was, as a rule, from $\frac{1}{2}$ to I c.c. ; in rats of 50 to Ioo grammes it was I to 2 c.c. If into a mouse of 20 grammes with $T$. brucci in its blood $\frac{1}{2}$ to I c.c. of human serum is injected, one finds, at the end of twenty-four to thirty-six hours, that the trypanosomes have dis-

1 Blanchard has also tried without success the effect of this drug upon the trypanosome of nagana in the marmot.

2 [Laveran has since shown (C. R. Acad. Sciences, v. 139, 1904, pp. 177-179) that the serum of the baboon (Cynocephalus) has a similar efiect upon $T$. brucei (as well as on $T$. evornsi, $T$. cquinum, and $T$. gambicnsc), but that it is distinctly less potent than human serum.]

:Laveran, Acud. des Sciences, April I, 1902, and July 6, 1903 ; Laveran and Mesnil, Ann. Inst. Past., November 25, 1902.

$\$$ Compare this fact with others recorded by Halban and Landsteiner (Münch. mediz. Wochenschr., 1902, No. 12), who have shown that the maternal serum is more hremolytic, more bactericidal, etc., than the foetal. 
appeared from the blood. The same result is obtained in a rat of Ioo grammes, on injecting I to 2 c.c. of human serum.

The disappearance of the parasites is less rapid when they are very numerous at the time of injection. Account must also be taken of the difference in activity of serums. We have had serums which, in doses of $\frac{1}{4}$ and even $\frac{1}{10}$ c.c., have caused the disappearance of the trypanosomes from the blood of a mouse weighing I5 grammes.

It sometimes happens that the trypanosomes do not reappear, but this is exceptional. Although we have treated a large number of infected rats and mice with human serum, we have had only four cases of cure in mice after one or two injections. Two of these mice were reinoculated twenty-five and thirty days after they had recovered, and contracted an ordinary trypanosome infection.

As a general rule, human serum causes only a temporary disappearance of the trypanosomes from the blood, and after a variable time the parasites reappear, and require a fresh injection of serum to make them disappear again. In rats and mice with nagana the trypanosomes often disappear for four to eight days, after one injection of human serum; and in some of our mice treated with human serum the parasites disappeared from the blood for twelve and even eighteen and nineteen days. When the trypanosomes have reappeared in the blood of an animal undergoing treatment, they rapidly multiply and swarm as in untreated animals, and death soon follows if the serum is not injected again.

In this way frequent injections of human serum will considerably prolong the life of an animal suffering from nagana. In those cases in which recovery has taken place it has always been as the result of one or two injections, and it is noteworthy that animals which had to be treated for a long time did not recover.

One might think that human serum frequently injected into an infected animal would lose its effect upon the trypanosomes. That is indeed so, but the diminution of activity occurs only very slowly, so that an animal may be successfully treated in this way for two or three months.

Usually in animals treated with human serum we have waited for the reappearance of the parasites in the blood before giving a fresh injection, but sometimes we have made the injections every two or three days without waiting for the parasites to reappear. We have not obtained better ultimate results by so doing.

Animals tolerate these injections of human serum quite well; thus we have injected, without any ill effects, as much as 2 c.c. into a mouse weighing 15 grammes. In rats and mice the injections are made under the skin or into the muscles of the shoulder, with the usual antiseptic precautions. With a human serum of good quality it is quite easy to prolong the life of rats and mice for two months, control animals untreated dying of the disease in four to five days. In some cases animals have survived for three months when treated.

By injecting arsenious acid and human serum alternately we 
have obtained still more favourable results. A rat lived 127 days and a mouse Io3 days; but we have obtained no cures by using these two substances, either alternately or simultaneously.

Mode of Action of Human Serum.-It is difficult to study in vitro the action of human serum upon the trypanosome of nagana, because the movements of this trypanosome very rapidly become sluggish. On mixing equal parts of trypanosome-containing blood and human serum the trypanosomes become less active at the end of half to one hour, and as a rule they are motionless at the end of two to three hours; but practically the same thing happens in blood which has not been mixed with human serum. The action of human serum upon these trypanosomes is not sufficiently rapid to be studied in vitro, as is that of arsenious acid.

Human serum does not agglutinate $T$. brucei, whereas the serums of the guinea-pig, goat, sheep, and pig, which do not possess any curative property, give rise to well-marked agglutination rosettes. We see once again, therefore, that the agglutinating power may exist without any microbicidal power.

On carefully examining the blood of animals treated with human serum, the mode of action of the serum can be studied in detail. The examination should be made at short intervals during the twenty-four hours immediately following the injection of the serum into a severely infected animal.

During the first few hours after the injection the trypanosomes remain normal, but at the end of four to five hours it is seen that in fresh blood, and, better still, in stained preparations, many of the trypanosomes are changed in shape, and have assumed a tadpole or spherical form. Later on the protoplasm and the nucleus disappear in those parasites undergoing involution. These changes which the trypanosomes undergo are, therefore, similar to those seen in animals treated with arsenious acid.

There is no marked leucocytosis. Leucocytes containing recognisable remnants of trypanosomes (such as nucleus and centrosome) are seen, but phagocytosis appears only to occur with trypanosomes which are dead or markedly involuted. We see, therefore, that human serum has a microbicidal effect upon the trypanosome of nagana analogous to that of arsenious acid.

Human serum heated to $56^{\circ} \mathrm{C}$. for one hour loses about one half its power, but good results may, nevertheless, be obtained with it by increasing the dose a little. A mouse treated only with serum which had been heated to $56^{\circ} \mathrm{C}$. lived for forty-three days. On heating to $62^{\circ} \mathrm{C}$., human serum becomes almost entirely inactive.

No doubt there is a close relation between man's immunity from nagana and the microbicidal action of human serum upon T. brucci. Probably the active principle of the serum is produced by the leucocytes, which would account for the fact that the serum is more potent than pleural fluid, and that ascitic fluid, which is very poor in leucocytes, is almost inactive. In one experiment citrated human 
plasma was found only slightly active compared with the serum; but it is conceivable that human plasma may contain sufficient of the active principle to protect the body against the invasion of the trypanosomes without being sufficiently potent to have a curative effect when it is injected into animals, and is thus diluted with a relatively large volume of inactive plasma.

Perhaps, also, the microbicidal substance of human serum is, in the circulating blood, contained solely in the leucocytes, thus rendering them able to destroy the trypanosomes of nagana. It was of interest to ascertain whether repeated injections of human serum into an infected animal might not give rise to the formation of an antibody which would ultimately neutralize the active principle of the serum. Experiments which have been made do not confirm this hypothesis.

Normal Serum of Animal Origin.-The serum of none of the lower animals has an effect upon the trypanosome of nagana at all comparable to that of the serum of man. ${ }^{1}$ The following serums injected into rats or mice with nagana gave us negative results: fowl, goose, horse, sheep, goat, and pig.

It is interesting to compare the action of monkey's serum upon T. brucei with that of human serum. The serum of a Cercopithecus was found to be inactive, which is in agreement with the marked susceptibility of these monkeys to nagana. The serum of a chimpanzee, even in doses of I c.c., had no effect upon the course of the infection in a mouse.

The Serum of Animals Refractory to Nagana, after Repeated Injections of Blood containing $T$. brucei.-We have made repeated injections of trypanosomes into birds (goose, fowl), but the curative power of the serum of birds thus treated has always been nil. [Mesnil and Martin were able to confirm this observation in the case of the geese which they found refractory to nagana, surra, and caderas. The serum of a goose which contracted a mild infection with nagana, from which it recovered, was found equally inactive, although the goose had become immunized.]

The Serum of Animals which have acouired Immunity AGAinst NAGANA.- Nagana, which always runs a fatal course in a large number of mammals, is less serious in certain species. Goats, sheep, and cattle show a fairly large percentage of cures, and animals so cured are immunized against nagana.

In this way we have cured (vide supra) a goat and two sheep. After ascertaining that these animals had quite recovered and were immunized against nagana, we made experiments to see whether their serum possessed any curative properties. These serums were only found active when mixed with blood containing $T$. brucei, ${ }^{2}$ but

1 [But see footnote, p. I74, re the action of the serum of Cynocephalus.]

2 We may note once more that this slight activity as a rule disappears at the end of a few days in serums kept in the ice-chest, contrary to what occurs with human serum. 
on injection into infected animals they never arrested the march of the infection. Similarly, they produced no effect whatever when injected simultaneously with the infective blood, but into some other part of the body.

We endeavoured to increase the immunity of the goat and sheep by injecting very large doses of infective blood into them; thus, the goat received sixteen injections of dog's blood containing trypanosomes (vide supra). Its serum, tested for curative power upon mice, in doses of $I$ and even 2 c.c., always gave a negative result.

[Martini, Schilling, and Kleine and Möllers succeeded in immunizing animals against the Togo virus, and Diesing immunized asses against the Cameroon virus. The serums of these immunized animals had preventive and agglutinating properties, but were rarely, if ever, curative. As the identity of these diseases with the nagana of $Z$ ululand is questionable, the details of these observations will be considered later (see second part of this chapter)].

In conclusion, we can say that arsenious acid, trypanred, and human serum, are the only therapeutic agents which have any appreciable effect in the treatment of nagana. ${ }^{1}$

Arsenious acid may be of use in prolonging the life of draught animals, but, except in these special cases, its use cannot be recommended. Animals thus treated are hardly ever cured, and, moreover, may spread the infection amongst healthy animals, and finally, it would be dangerous to give the large doses of arsenious acid which are necessary to combat nagana to animals destined for the food of man.

Human serum, which in our hands has quite cured several mice, in most cases cannot do more than prolong the animal's life for a time. Moreover, the treatment of large animals with human serum is impracticable on account of the enormous doses of serum it would be necessary to use.

\section{Section 7.-Prophylaxis.}

In order to protect animals against a microbic disease, immunizing serums may be used, or the animals may be inoculated with microbes of diminished virulence-that is, an attenuated virusso as to produce mild attacks of the disease, which lead to immunity. In these two ways we have tried to immunize animals against nagana.

I. ATtempts to PREPARE AN IMMUNIZING SERUM.--Human serum has slight preventive power against nagana. If one inoculates into a mouse $\frac{1}{10}$ c.c. of infective blood mixed with to c.c. or more of human serum, the animal does not become infected. Sometimes infection is prevented when the virus and the serum are injected simultaneously in different parts of the body, but in that case the

${ }^{1}$ [This statement requires modification, for certain dyes, notably o-dichlorobenzidine + acid $H$, as well as one or two arsenic derivatives, notably atoxyl, have given better results as regards prevention and cure than trypanred and arsenious acid respectively (see Chapter XIII.).] 
result is not constant; infection may occur after a slightly longer incubation period.

If a rat be inoculated with I c.c. of human serum, and twenty-four hours later with infective blood, the animal contracts the disease, but the incubation period is prolonged, the trypanosomes appearing in the blood at the end of five to nine days. On injecting $T$. brucei, together with blood from an animal which has been recently treated with human serum, one finds in the same way that the incubation period is prolonged, but once the trypanosomes appear in the blood the disease runs its ordinary course.

Nagana has developed in mice inoculated with trypanosomes which had been mixed with human serum and then washed; there was merely a delay in the appearance of the trypanosomes in the blood. Mice inoculated with a mixture of infective blood and human serum, in which an infection does not follow, do not acquire any immunity against nagana.

The serum of the dog, sheep, goat, horse, fowl, or goose, mixed with trypanosomes, does not prevent an infection occurring in animals injected with the mixture, and, moreover, the incubation period is not prolonged. This property of human serum seems, therefore, to be peculiar to it. ${ }^{1}$

One might have hoped that the serum of animals which had recovered from nagana, and were thus immunized against the disease, would have possessed immunizing properties superior to those of human serum. Here again our expectations have not been realized, as the following observations show : ${ }^{2}$

The serum of a billy goat infected with nagana forty-seven days previously, and still suffering from the disease, showed slight preventive power. Two mice inoculated with a mixture of $\frac{1}{1} \frac{1}{0}$ c.c. of infective blood and I or 2 c.c. of serum survived. The serum of this goat was active, however, only when mixed with the infective blood injected.

The serum of a nanny goat which had recovered from nagana, and so acquired immunity against the disease, and which was frequently injected with blood rich in trypanosomes (see p. I39), was tested four to eight days after each inoculation. It wa's found to possess only a very slight preventive power, comparable with that which it rapidly acquires during the course of an infection. This power did not increase, however, although the goat was reinoculated every week with 30 to 40 c.c. of dog's blood. The serum of this immunized goat, mixed in vitro with trypanosome-containing blood, had no definite microbicidal action. On examining the mixture kept for one to two hours at the room temperature, and then placed

1 [As has already been stated, Laveran has found that the serum of the baboon has an action similar to that of human serum, but that it is distinctly less potent.]

2 [Martini, Kleine and Möllers, and Diesing have shown that the serums of calves and asses immunized against the diseases of Togo and Cameroon, closely allied to nagana, have preventive properties (see second part of this chapter).] 
in the ice-chest for twenty-four hours, we found that the majority of the trypanosomes were still living, but less motile. This serum, therefore, behaved like a normal serum, and was no more agglutinating than the serum of a normal goat.

This serum was only found active when mixed with the virus, and even then only in the big dose of I c.c. for a mouse. In smaller doses it merely prolonged the incubation period one or two days. Serum injected twenty-four hours before the virus, or even at the same time, but in some other part of the body, had no effect or merely prolonged the incubation period one or two days.

We obtained similar results with the serum of the two sheep which had recovered from nagana, and which had received, the one six and the other three, inoculations of nagana blood. The serum of the latter sheep protected a mouse in doses of $\frac{1}{2}$ c.c. mixed with the virus, and in doses of 2 c.c. inoculated in some other part of the body.

According to Schilling, ${ }^{1}$ cattle which have acquired immunity as a result of repeated injections of infective blood furnish a serum possessing microbicidal properties for $T$. brucei. He quotes the following experiment: A bull which had apparently recovered from an attack of nagana was injected subcutaneously with I9 c.c. of defibrinated horse blood containing numerous trypanosomes. On the ninth day after injection a few trypanosomes were found in the blood of the bull, and they had disappeared by the twelfth day. A fresh injection of trypanosomes was given intraperitoneally, and it was then found that the serum of the bull had a specific action in vitro upon the trypanosomes of nagana, which became motionless thirty and even twenty-one minutes after being mixed with this serum. ${ }^{2}$

Nocard was not able to confirm this observation. He inoculated into a cow in one week 852 c.c. of blood from a cat or dog very rich in trypanosomes. The serum was found to have a marked agglutinating effect upon the trypanosomes, but was not microbicidal. In vivo this serum, mixed with trypanosome-containing blood, very appreciably delayed the appearance of the parasites in inoculated mice, but unless used in mixture it had no effect.

The serum of this cow ${ }^{3}$ actively immunized against nagana was therefore only slightly prophylactic, although attempts were made to increase the immunity by means of intraperitoneal injections of blood rich in trypanosomes. None of the mice used in this series of experiments had acquired any resistance whatever to the virus.

The serum of a fowl and goose which had been injected with

1 Schilling, Centralb. f. Bakter., I, v. 3I, I902, p. 452.

2 [We shall see later that Martini, Kleine and Möllers, and Diesing have succeeded in obtaining similar results.]

3 This cow, like the one mentioned on $\mathrm{p}$. 137, showed a rise of temperature to $40.8^{\circ} \mathrm{C}$. $\left[104^{\circ} \mathrm{F}.\right]$ three days after inoculation, and several trypanosomes were seen on microscopical examination. The next day the temperature was normal again. From that day the cow received enormous doses of virulent blood. It remained, however, in excellent condition, and its blood was not virulent for more than two months. 
blood rich in trypanosomes showed very slight, if any, preventive power, as the following experiment shows: A mouse inoculated with $\frac{1}{2}$ c.c. of serum of an inoculated fowl mixed with infective blood lived for fifteen days, whereas a control mouse died in a week. The trypanosomes inoculated in this experiment, however, were only slightly motile at the time of injection.

[Even the goose which Mesnil and Martin ${ }^{1}$ succeeded in infecting with nagana did not, after its recovery from the infection, furnish a prophylactic serum. Mice inoculated subcutaneously with $\frac{3}{4}$ c.c. of goose serum mixed with trypanosomes did not live longer than control mice.]

The serum of rats with nagana which had received from three to seven injections of arsenic showed only slight preventive properties. We have observed, as Bruce had done, that arsenic has no preventive action whatever upon nagana. Animals treated with arsenic as a prophylactic become infected quite as easily and as quickly as control animals, and the course of the disease is not altered in any way.

2. Attempts to produce Attenuation of the Virus.-We have tried in several ways to attenuate the virulence of $T$. brucei.

The blood of animals suffering from nagana very rapidly loses its virulence when kept on ice or at the room temperature. At the end of several hours the trypanosomes become much less active, while at the end of twenty-four hours most, and sometimes all, of them are motionless.

When such blood is inoculated the incubation period is considerably longer than when fresh blood containing very active parasites is injected; but the alteration in the trypanosomes has no other effect upon the course of the disease. From the time that the parasites appear in the blood of inoculated animals they develop with the usual rapidity, and the disease loses none of its severity.

We have experimented with blood heated to different temperatures and for variable lengths of time. On examining blood rich in trypanosomes, which has been heated for an hour to $4 \mathrm{I}^{\circ} \mathrm{C}$., it is seen that the parasites are motionless, deformed, and spherical. Blood thus heated gives rise to an infection after a markedly increased incubation period, but the disease is just as severe as usual. A more prolonged exposure to $4 \mathrm{I}^{\circ} \mathrm{C}$. or a shorter exposure to a temperature of $44^{\circ} \mathrm{C}$. kills the parasites. We see, therefore, that by heat it is not possible to attenuate the virulence of nagana, or, rather, that attenuation of the virus results only in a prolongation of the incubation period.

We have seen (p. I6o) that the trypanosomes in Novy and McNeal's cultures ceased to be virulent some days before they died. This loss of virulence was produced in forty-eight hours on heating to $34^{\circ} \mathrm{C}$. cultures grown at $25^{\circ} \mathrm{C}$. Mice inoculated with these non-

${ }^{1}$ [Mesnil and Martin, C. R. Soc. Biol., v. 60, 1906, p. 739.] 
virulent trypanosomes were almost as susceptible to virulent parasites (even although slightly attenuated by culture) as control mice.

The virulence of $T$. brucei may be diminished by mixing a I per cent. solution of toluidin blue with the blood of an infected animal. Animals inoculated with this mixture show a longer incubation period than animals inoculated with the blood alone.

In two rats inoculated with a mixture of 8 parts of trypanosomecontaining blood and I part of toluidin blue, which was allowed to stand twenty minutes after mixing, trypanosomes appeared in the blood only on the seventh or eighth day. In a guinea-pig inoculated with a mixture of infective blood and I per cent. toluidin blue (injected two minutes after mixing) the parasites did not appear in the blood until the twelfth day.

As a general rule, the diminished virulence of the trypanosomes is shown only by this increase in the incubation period, for the disease, once it has declared itself, runs a normal course. There are, however, exceptions.

In a mouse inoculated with a mixture of trypanosomes and toluidin blue the parasites appeared only on the eighth day after inoculation, and the disease ran quite an unusual course. In a rat similarly inoculated trypanosomes appeared in the blood on the fifth day after inoculation, and permanently disappeared at the end of three to four days. This experiment is quite unique for nagana in rats. The rat which thus suffered from a mild form of the disease was not immunized by it.

This last experiment resembles those cases of reinfection in mice cured of nagana by means of human serum (p. I75). It seems proved that an attack of nagana induced by an attenuated virus or modified by treatment is not sufficient to confer immunity. We have observed immunity against nagana only in those animals (goat, sheep, cow) which, after a prolonged infection, have been cured spontaneously. From this it may be concluded that it is difficult to immunize animals against nagana by injecting into them trypanosomes of diminished virulence, and so producing in them a mild attack of the disease. ${ }^{1}$

Preliminary inoculations with infective blood kept for several days on ice or for several hours above $40^{\circ} \mathrm{C}$., or kept for a time in contact with a dye by which the virulence is destroyed, do not prevent an infection resulting from the injection of a fresh or of an attenuated virus under the conditions we have just mentioned, and do not in any way modify the course of the disease.

1 A female rat, inoculated September 23, I902, and treated alternately with human serum and arsenic starting from September 25 , gave birth on October i4 to seven well-cleveloped young ones, which she reared. On November 10 the young rats were able to eat. Two of them were inoculated subcutaneously with infective blood at the same time as two young ones of about the saine weight born of a healthy rat. The four young rats showed the same susceptibility to nagana, which was a little greater than that of adult rats. 
We shall see (pp. IgI and I95) that Koch and Schilling have tried to attenuate for cattle the virulence of trypanosomes allied to that of nagana, by passing these parasites through different species of animals, and that they claim to have obtained certain favourable results in this way. ${ }^{1}$ The facts quoted by these authors do not appear to us to be conclusive. Koch is said to have obtained his 'vaccine' by two passages only - the first through the rat, the second through the dog.

We cannot form any definite conclusion from the cure of Koch's two oxen, for it is by no means rare for cattle to recover spontaneously from nagana and surra, and animals which so recover are immune, as were Koch's cattle.

In his first paper Schilling stated that he had vaccinated two calves by inoculating them with a virus of the third passage through dogs. But he very quickly recognised that two or even three passages through the dog or rat were not sufficient to attenuate the virus for cattle; and in his paper, published in January, I903, he speaks of a virus which had been passed seven times alternately through the dog and rat, then from eighteen to twenty-one times through dogs alone. That is a very different thing from the two passages of Koch. Thirty-six oxen were inoculated with this virus, each one on two or three occasions, with the result that they contracted a mild and temporary ${ }^{2}$ infection, and were then taken into a fly area. What happened to these cattle we shall see in the second part of this chapter.

This process, as Schilling himself acknowledges, is useless for the vaccination of horses.

According to our own observations the virulence of $T$. brucei may be slightly attenuated by passage through different species of animals, but the trypanosome loses little of its virulence in passing from one animal species to another, and in all cases rapidly regains its virulence, as the following experiment shows:

On April 24, I902, a dog was inoculated with the blood of a sheep infected with nagana for six months. On May I trypanosomes had appeared in the dog's blood. On May 2 one rat and two mice were inoculated subcutaneously with several drops of the dog's blood considerably diluted with citrated salt solution. On May 4 parasites appeared in the blood of the rat and mice; on the 6th they were very numerous, and all the animals were found dead on the morning of the 7 th.

1 [Martini's experiments on the same lines seem to have been to some extent successful. Diesing has obtained from immunized asses a serum which is able to produce a temporary passive immunity in animals, sufficient to enable them to pass through a fly belt.]

2 About half these cattle showed parasites in the blood ten days after inoculation. At the end of the first month microbicidal properties were found to be present in the serum of five out of eight cattle examined from this point of view. The trypanosomes in the peritoneal exudate of an infected dog, mixed with an equal volume of ox serum, were killed by the serum in twenty minutes. We have already quoted a similar result, p. I8o. 
Trypanosomes which had been passed through the sheep and then through the dog thus gave rise in a rat and in mice to infections just as acute as those produced by parasites inoculated direct from rat to rat or from mouse to mouse.

It is possible, however, that attenuation of the virus for a particular species of animal may be produced as a result of numerous passages through another species. That is, perhaps, the case with the virus we have used in our experiments. Although cattle suffer severely from nagana in Africa, our virus, inoculated by Nocard into three cows, produced in them only a mild infection. It may be due to the difference in race of the cattle, but it may also be due to attenuation, resulting from the innumerable passages through different mammals other than ruminants, which the virus has undergone since 1896 , when it was imported from Zululand into Europe. It would be very important to test the virulence of these trypanosomes, which we have at the present moment under observation, upon South African cattle. It is just possible, if our second hypothesis is correct, that this virus has become a vaccine which might be used in Africa to protect cattle against the natural infection.

In conclusion, we must admit that hitherto we have succeeded no better in preventing nagana than we have in our attempts to cure it, but we must not regard the conclusions at which we have arrived as final. We hope to continue our investigations, and no doubt other observers will do the same. Perhaps experiments made with other animal species than those which we have had at our disposal might yield better results. It would be interesting, for example, to experiment upon African antelopes or buffaloes, which, although often infected with nagana, are very resistant to the disease. Such experiments could only be carried out in Africa.

In the actual state of our knowledge we may say that we are not acquainted with a certain and practical remedy for nagana, nor with any reliable process of immunizing animals against the disease.

[The experiments of Thomas with atoxyl, and those of Mesnil and Nicolle with atoxyl and various benzidine derivatives, are a step in the right direction. Mesnil and Nicolle were able to prevent, as well as cure, an infection of nagana in mice with atoxyl and a particular derivative of benzidine. Possibly these drugs will be found useful in the prevention and treatment of nagana in cattle and horses. Full reference to these experiments is given in Chapter XIII., on Treatment.]

Prophylactic measures intended to limit the areas in which nagana is endemic, and to prevent its introduction into countries still immune, are therefore of considerable importance. The dangerous areas should be mapped out accurately. This is relatively easy, because we know that it is only certain species of tsetse-fly which 
propagate the disease; but it would be as well to find out exactly which species of tsetse convey the parasite, by fresh experiments on the lines of Bruce's.

The invariable result of the civilization of a country is to destroy or to drive away the big game. In this way, then, we may hope that the areas of nagana will become narrowed in proportion to the advance of Europeans into the African continent, of which until recently only the coast regions were known, but which is already being intersected at various points by railways into the interior. In fact, Foà and Theiler have already observed that the destruction of the big game has rendered the fly areas more healthy. At the present moment, at any rate, there is no question of attempting to exterminate the fly.

When the fly and nagana areas are well known, it is often possible to take effective precautions, if it is merely a question of passing through those areas. Perhaps the best precaution is to travel only at night, as the tsetse-fly does not, as a rule, bite at night. [Diesing recommends injecting the serum of highlyimmunized animals, by which a temporary passive immunity is produced, sufficient to allow the susceptible animals to pass through the fly belt. He obtained fairly good results with cattle in this way.] Then, it has been recommended to rub different substances, particularly creolin, over the animals one wishes to protect. In the hinterland of Togo the natives rub the juice of a plant, Amomum melegueta, over the animals, in order to protect them against the bite of the tsetse. Smoke drives away the flies, and may be used, for example, in encampments. Good results have been obtained by placing horses in boxes in which the apertures are closed with gauze, or in stables in which horse manure is constantly burnt. ${ }^{1}$

Precautionary measures are, or should be, taken by the sanitary authorities to prevent the importation of nagana, particularly in fly districts which are free from the disease. (See Prophylaxis of Surra, p. 284.)

\section{AFRICAN DISEASES ALLIED TO THE NAGANA OF ZULULAND.}

[Section 1.-General Considerations. Classification of the Trypanosomiases. Methods of Differentiating and Identifying the Pathogenic Trypanosomes. $\left.{ }^{2}\right]$

[The African trypanosomiases, which resemble the nagana of Zululand more or less closely, may be divided into two groups-those occurring in districts where tsetse-flies are found, and which are probably spread by means of these flies, and those occurring where the tsetse-fly is absent and which are probably spread by other biting flies, such as Tabanidæ or

1 A Report by Pitchford, of Natal, analyzed in Bull. Soc. d'éludes colon., June, 1903.

2 [The whole of this section, down to p. 190, has been added.-ED.] 
Stomoxys. In the first group would come the trypanosomiases of cattle and pigs in German East Africa (described by Koch, Ochmann, and others); of dromedaries in Ogaden, Somaliland (Brumpt, Theiler); of donkeys, mules, and cattle in the Anglo-Egyptian Sudan (Balfour, Head); of cattle, mules, and dogs in British East Africa, Uganda, and adjacent territory (Stordy, Bruce, Nabarro, Greig, and Gray); of cattle in the Congo Free State (Broden); of Equidæ and cattle in Cameroon (Ziemann, Diesing); of cattle and horses in Nigeria (Christy, Hewby, Moore); of cattle and horses in Togo (Koch, Schilling, Ziemann, Martini); of oxen in Sierra Leone (Smith); and perhaps a trypanosomiasis of horses in French Sudan (Cazalbou). The second group would include the Zusfana disease of horses in Algeria (Szewzyck, Rennes, Roger and Greffuhle); Debab, a disease of dromedaries in Algeria (Sergent); Mbovi, a disease of dromedaries in French Sudan and Timbuctoo (Cazalbou); Souma, of cattle and horses in French Sudan (Cazalbou) and in Guinea (Martin); the trypanosomiases of cattle and Equidæ in Erythrea (Memmo, Martoglio, and Adani); of cattle and horses in Abyssinia (Savouré and others); of cattle, sheep, and goats in Cameroon (Ziemann); and of oxen and Equidæ in Haut and Moyen-Niger (Pécaud).]

[In the present state of our knowledge it is impossible to speak with certainty about the nature of many of these diseases. Some of them have been called nagana, others surra, and others again, such as Souma, are thought to be distinct diseases. The fact that certain of these diseases occur in tsetse-infested districts, while others are found in the absence of the tsetse, suggests the classification adopted above into the 'tsetse group' of trypanosomiases and those not dependent on the tsetse for their transmission. Many of the latter may eventually prove to be varieties of surra. This has been shown by Laveran to be the case with mbori. It is extremely probable that further investigation may show that nagana and surra are not two individual diseases, but rather two distinct groups of diseases, the one group (nagana) being dependent on the tsetse-fly for its transmission, the other (surra) on Tabanidæ, Stomoxys, or other biting flies.]

[The subject is rendered all the more difficult by the fact that the trypanosomes of these various diseases present only very slight morphological differences. These differences between the various trypanosomes are not greater than those seen in the same trypanosome in the blood of different animal species. Sometimes, indeed, one finds greater morphological differences in the same trypanosome in the various species of animals than are found between the trypanosomes of nagana and of surra. This has led Koch and many other German authorities to regard nagana and surra as identical. This view of Koch, Musgrave, and others, is almost certainly erroneous, as various recent methods of differentiating trypanosomes prove ('cross-immunization' experiments, cultivation of the respective trypanosomes, etc., see later). Moreover, as a result of his recent researches in German East Africa on the development of trypanosomes in Glossina-results which can, however, be interpreted otherwise than Koch has done-Koch appears to be modifying his original 
views, and to regard at least $T$. brucei and T.gambiense as distinct species.]

[Koch's Classification of Trypanosome Diseases. - Koch ${ }^{1}$ divides the trypanosome diseases into two groups :

Group I., including the trypanosomiases of rats and of cattle (Theiler). In these the trypanosomes are constant in their more important characters, and are sharply separated from the other mammalian trypanosomes. The most important properties, according to Koch, are (I) the morphological characteristics, (2) the virulence, and (3) their relations to the domestic animals. The trypanosomes in this group are said to be constant in all these characters, and to show no variation. This is not absolutely true, however, for several observers have shown that the virulence of $T$. lewisi for rats may vary.

Group II. includes human trypanosomiasis, nagana, surra, and mal de caderas, but no mention is made by Koch of dourine or of the Gambian horse disease. These trypanosomiases are said to be in a stage of mutability. In the three important properties mentioned above the trypanosomes are fluctuating and variable; thus they are not sharply differentiated morphologically from each other, their virulence fluctuates over a wide range, and they are not found exclusively in one host. From this Koch argues that they are not yet developed into distinct species.]

[Methods of Differentiating and Identifying the Pathogenic Trypanosomes.-Attempts have been made by different observers to differentiate these trypanosomes by their morphology-e.g., by the presence or absence of vacuoles or of chromatic granules in the protoplasm, the length of the body and of the free flagellum, the shape of the posterior end, and so on; but it has been pointed out that such variations may occur in the same trypanosome, consequently they are valueless in the determination of species.]

[I. Animal Inoculations. - The morphological characters failing to differentiate these trypanosomes, the next step was to inject the parasites into various species of animals. In certain closely allied diseases, such as nagana, surra, and several other less definite African trypanosomiases, the effects produced in animals are not sufficiently characteristic to enable us to state, from animal inoculations alone, that these are distinct morbid entities. But some results of animal experiments are fairly constant-e.g., nagana in rats and mice is usually very rapidly fatal, and at death the blood generally swarms with trypanosomes, while $T$. gambiense sometimes fails to infect these animals altogether, and the trypanosomes are never so numerous as in nagana; dourine produces characteristic cutaneous 'plaques,' which are rarely seen in the other trypanosomiases.]

[Animal inoculations are made subcutaneously, intraperitoneally, or intravenously, an infection usually following any of these procedures. Dourine is to be distinguished from the other trypanosomiases by the fact that infection may occur-both naturally and experimentally - through intact mucous membranes.]

[2. 'Cross-Immunization' Experiments (Laveran and Mesnil).This method has been used with success by Laveran and Mesnil for

${ }^{1}$ [R. Koch, Deutsche med. Wochenschr., November I7, I904, pp. I705-I7II ; translation in Brit. Med. Journ., November 26, 1904, pp. I445-1449.] 
differentiating several of these trypanosomiases. In this way they showed the individuality of surra, of nagana, of caderas, and of Gambian horse disease; also the identity of Indian and Mauritius surra and mbori. Nocard and Lignières found that dogs immunized against dourine were as susceptible to nagana and caderas as normal animals.]

[This method is rather long and tedious. Resistant animals, such as sheep, goats, and calves, must be used as a rule, and it takes a long time for these animals to recover from their chronic infection, and to become immunized. When their blood, on inoculation into small animals, is found to be no longer infective, the original trypanosome, on reinoculation, does not give rise to a fresh infection (or sometimes there may be a slight transient blood infection), whereas a different species of trypanosome produces the same effect as it does in a normal animal.]

[Koch (loc. cit.) has criticised this method of differentiating trypanosomes, and has stated that the experiments upon which Laveran and Mesnil base their opinions are inconclusive, for the following reasons: (I) The experiments were made upon goats, which are only slightly susceptible to surra, and therefore ill adapted to the study of the disease. (2) Animals which appear to have acquired immunity against a trypanosomiasis retain the parasites for long periods in their blood, and if the blood of a goat immunized against nagana, and subsequently inoculated with surra, becomes again virulent, it is perhaps because the animal is still infected with nagana. (3) The fact that animals immunized against one trypanosome are susceptible to a trypanosome from another source does not show conclusively that these parasites are different species, animals which resist a variety of trypanosomes of feeble virulence being capable of infection by a more virulent variety of the same trypanosome.]

[Laveran and Mesnil ${ }^{1}$ have answered these criticisms of Koch as follows: (I) In order to obtain animals immunized against surra or nagana it is, of course, impossible to employ species in which these infections are always fatal. One is, therefore, obliged to use animals, such as goats, in which these trypanosomiases may end spontaneously in recovery. Moreover, surra and nagana do not produce in goats such mild infections as Koch seems to think, and several goats died during the course of the experiments. Further, these goat experiments have been confirmed by others with bovines. Vallée and Carré ${ }^{2}$ have shown that a cow immunized against nagana was susceptible to surra. Vallée and Panisset ${ }^{3}$ immunized bovines against surra, and found that they were then not susceptible to mbori.]

[The same method has given good resuits with other trypanosomiases, and goats were not the only animals used. A goat and sheep immunized against nagana were as susceptible to caderas as normal animals. ${ }^{4}$ An ox, sheep, and pig immunized against caderas were normally susceptible to nagana (Lignières). ${ }^{5}$ Lastly, dogs immunized against dourine were as susceptible to nagana and caderas as normal dogs (Nocard and Lignières) $\left.{ }^{6}\right\rceil$

1 [Laveran and Mesnil, C. R. Acad. Sciences, v. 140, March 27, 1905, p. 831.]

[Vallée and Carré, Ibid., October I9, 1903.]

[Vallée and Panisset, 1bit., November 21, 1904.]

[Laveran and Mesnil, 1bid., November 17, 1902.]

[Lignières, Bol. Agricolt. y Ganad., Buenos Aires, February I, 1903.]

- [ Nocard, Soc. Biol., May 4, Igor ; Lignières, Riv. Soc. med.'Argent., v. 1o, 1902, Pp. I12-II4] ] 
[(2) Koch's statement that animals apparently cured of a trypanosome infection, and which have thereby acquired an apparent immunity, may nevertheless retain trypanosomes in their blood for a considerable time is quite true, but it is the exception, not the rule. Thus as much as $50 \mathrm{c}$. c. of blood from such an animal has been injected into dogs without producing an infection, which proves that the blood cannot have contained any trypanosomes. Laveran and Mesnil showed also that the blood of rats immunized against $T$. lewisi, as the result of an attack of this trypanosomiasis, was no longer infective.

[(3) The statement that an animal which has resisted a feeble virus sometimes becomes infected when inoculated with a stronger virus of the same species is also true, but this again appears to be the exception rather than the rule. Laveran and Mesnil forestalled this objection by inoculating the 'weaker' virus into animals immunized against the 'stronger' virus. Thus their goat immunized against nagana became infected with surra; so also did Vallée and Carré's bovine, although the Zululand nagana experimented with is undoubtedly more virulent than Mauritian surra.

[3. Serum-Diagnosis. - The 'cross-immunization' method of diagnosis being a long and tedious one, and the practical difficulty of always having immunized animals available being great, Laveran and Mesnil $^{1}$ have attempted to elaborate a method of serumdiagnosis. They had found previously ${ }^{2}$ that the serum of sheep cured of nagana had an action upon $T$. brucei, but not upon T. equinum. Similarly, Kleine and Moellers ${ }^{3}$ have shown recently that the serum of a donkey, which had a considerable action upon Martini's Togo virus, is without action upon T. gambiense.]

[Laveran and Mesnil used the serums of three cured goats : one (N) cured of nagana (Zululand), another (NS) of nagana and of Indian surra, and the third (S) of Mauritian surra, then of the Annamese horse trypanosomiasis (see Chapter VIII.). The usual method was to mix the serum with the virus, in vitro, one or two minutes before injecting subcutaneously into mice. The serum of goat $\mathrm{N}$, in doses of $\mathrm{O}^{\circ} \mathrm{I}$ to $\mathrm{O}^{\circ} 5$ c.c., completely protected the mice from nagana; even dried serum kept for two months was found to be protective. When injected apart from the virus, either twentyfour hours before or at the same time, this serum was much less active. The serum had little or no action upon the virus of caderas, of Indian surra, of Annam, and of Togo. We may add that goat $\mathrm{N}$ was inoculated with Schilling's Togo virus, to which it succumbed in a month.]

[Similar results were obtained with the serums of the other two goats. Laveran and Mesnil conclude that such serums acquire specific properties, which can be used as a means of identifying trypanosomes. There are, however, certain fallacies ${ }^{4}$ which considerably diminish its value as a means of diagnosis. Bearing in

1 [Laveran and Mesnil, C. R. Acad. Sciences, v. I42, 1906, p. 1482.]

2 [Laveran and Mesnil, 1bid., v. I 35, I902, p. 838.]

3 [Kleine and Moellers, Zeitschr. f. Hyg., v. 52, I905, p. 229.]

4 [The serum of goat $\mathrm{N}$ in one experiment had an action upon the surra trypanosome. The serum of goat $S$ immunized against Mauritian and Annamese surra had very little action upon the trypanosome of the latter.] 
mind these fallacies, Laveran and Mesnil think it follows from their experiments that (I) the Annamese trypanosomiasis is different from the Indian; (2) Schilling's Togo virus is not nagana, but a species of trypanosome very virulent for goats, killing them in one to two months; and (3) Martini's virus is neither nagana nor surra.]

[4. Cultural Characteristics.-We have seen that T. lewisi and $T$. brucei, when grown artificially on Novy and McNeal's medium, show considerable differences ( $T$. lewisi is much more motile, forms large colonies of rosettes, flagella are directed towards the centre, etc.; $T$. brucei is less active, has an undulating membrane, colonies are small, et.c.), by which these two trypanosomes can be easily distinguished (see pp. 79, I6I). Novy, McNeal, and Hare have cultivated the Philippine surra trypanosome, and find that the cultures differ from those of $T$. brucei in several important respects.]

[5. The 'Precipitin' Reaction.-Mayer has found (see p. I50) that a specific precipitin is present in the serum of nagana animals, and he thinks that this reaction may be a means of diagnosing allied trypanosomes.]

[6. Fly Experiments.-Koch suggests that the different evolution forms of trypanosomes seen in the appropriate species of Glossina may be a help in diagnosis. It must be remembered, however, that a certain percentage of 'fresh' flies may harbour 'wild' trypanosomes, and Novy and Minchin are of opinion that some of Koch's 'evolution' forms of T. brucei and T. gambiense have no relation to these parasites, but are trypanosomes peculiar to the tsetse.]

[Nabarro and Greig, by dissecting flies-Glossina and Stomoxysat varying intervals after feeding on infected animals, found that trypanosomes from various sources (human trypanosomiasis, Uganda cattle disease, Abyssinian dog disease, and Uganda mule disease) remain actively motile in the stomach-contents of flies for very different lengths of time (see Section 8). This may prove to be a means of differentiating species of trypanosomes, where live tsetse-flies are to be obtained.]

[7. 'Chromo-Therapentic' Reactions.-Mesnil and Nicolle, Wenyon, and Aubert have made numerous observations upon the action of various dyes in animals inoculated with different pathogenic trypanosomes (see Chapter XIII.). From the results obtained it seems probable that the 'chromo-therapeutic' reaction will be a means of differentiating the pathogenic trypanosomes.]

\section{Section 2.-Trypanosomiasis of German East Africa.}

It is to Koch ${ }^{1}$ that we are indebted for the earliest accounts of the trypanosomiasis of German East Africa. He observed it there in 1897 , and found that it affected chiefly cattle. The incubation

1 Koch, 'Reiseberichte,' etc., Berlin, I898, pp. 65-72, 87, 88, and Deutsches Kolonialblitt, No. 24, 1901. Stuhlmann has published a map giving (I) the exact areas in which the disease has been found; (2) the routes along which herds of cattle have become infected. The disease exists in the region of the heights of 
period is from nine to twelve days. At the outset the disease is manifested by a rise of temperature and the appearance of the hæmatozoa in the blood; then the animal becomes weak, anæmic, and thin, and death occurs more or less rapidly. Nearly all the cattle die.

According to Sander, ${ }^{1}$ this trypanosomiasis of cattle may present itself in an acute or chronic form.

Veterinary Surgeon Schmidt ${ }^{2}$ states that the incubation period is from five to six weeks. The presence of the parasite in the blood coincides with the rises of temperature. The number of parasites, the condition of the animal, and the amount of work it is made to do, all help to affect the course of the disease. Cattle which are allowed to be idle may live for years, whilst those which work suffer from a very acute form of the disease. Cattle are more susceptible than mules.

Koch made a certain number of experiments during his short stay in the German colony. We reproduce here those of his experiments which he quotes in support of his method of vaccination, already mentioned (p. I83).

Blood containing many trypanosomes from a spontaneously infected ox was inoculated into the following animals:

\begin{tabular}{|c|c|c|c|}
\hline Animal. & Dose. & & Result. \\
\hline I Masai donkey .. & 5 c.c. & $\ldots$ & Remained healthy. \\
\hline I cow & 5, & .. & Died in 39 days. \\
\hline calves & 5, & & ,, . 4I and 49 days. \\
\hline monkeys (sp. ?) & 4, & $\cdots$ & Remained healthy. \\
\hline guinea-pigs & 2, & & \\
\hline rats & 2, & $\ldots$ & and 52 day \\
\hline $\operatorname{dog}$ & 5, & ... & " I9 days. \\
\hline
\end{tabular}

The blood of one of the two rats in the previous experiment was inoculated into :

One rat, which died in 68 days (trypanosomes in its blood I3 days after inoculation), and one dog, which died in 42 days.

The blood of the dog was inoculated into:

Two dogs, which died in 19 and 26 days.

Three rats, which died in 67,73 , and 80 days.

Four Masai donkeys, which remained well.

Two oxen, in which the parasites appeared from the roth to the I3th day, and remained present for 3 to 4 weeks; after that they were no longer seen. The oxen were cured and were immune. They had shown no sign of illness at any time.

U'sambara (northern boundary of the colony near the sea) ; in the region of Dar-esSalaam and the island of Mafia; from the sea (the disease, perhaps, exists on the island of Mafia itself, according to Panse, Zeitschr. f. Hyg., v. 46, 1904, p. 376) to Usagara and along the whole valley of the Ruaha; opposite Kiloa; in the valley of the Rovuma, which serves as the southern boundary of the colony, from the sea right up to Lake Nyassa ; and finally, quite in the interior along Lake Tanganyika, in latitude about $5^{\circ} \mathrm{S}$.

1 L. Sander, Deutscher kolon. Kongress, 1902.

2 Schmidt, in Stuhlmann, 'Ber. üb. Land. u. Forstwirtschaft in DeutschOstafrika,' v. I, June, 1902, p. 137. 
We have seen that Koch regarded this experiment with cattle as the basis of his method of vaccination against nagana.

These statements of Koch relative to the immunity of Masai donkeys (or Masai and Muscat hybrids) have been received with a certain amount of scepticism, although Koch has founded his statements upon current opinion as well as upon his experiments.

The objection has been raised that he simply placed blood containing trypanosomes in contact with an abrasion on the ears of the donkeys, a method of inoculation which is not infallible.

Since then Koch's statements have been disproved by means of more exact experiments. Stuhlmann ${ }^{1}$ has seen trypanosomes in the blood of sick Masai donkeys at Mombo, at the foot of the heights of Usambara. Kummer ${ }^{2}$ has observed a heavy mortality amongst the donkeys of German East Africa whenever they have been taken into fly areas, and he has found trypanosomes in the blood of the sick animals. He adds that the differences in race of the donkeys used in this district are not recognisable by a European. Grothusen ${ }^{3}$ also states that Masai donkeys are susceptible to nagana. He rubbed some blood from one of these donkeys into a wound on the ear of a zebra. The zebra died seventeen days later, after showing symptoms of nagana, and with many trypanosomes in its blood. This result, confirmed by that of Martini, obtained with a virus coming from Togo, is important, for it proves that the zebra, which has been regarded as refractory to the African trypanosomiases, is susceptible to these diseases.

The various experimental data given by Koch show a considerable difference in the virulence of the German East African and Zululand parasites. For example, we see that monkeys and guineapigs are refractory, or only very slightly susceptible; that in rats the disease is very chronic, instead of fatal in a few days; and that the disease in dogs is also more protracted than is the nagana of $Z$ ululand.

As to the morphology of the trypanosome, we possess only a very diagrammatic drawing which Koch has published in his ' Reiseberichte' sufficient to differentiate it from T. lewisi, which Koch had in his mind at the time, but not for a critical comparison with $T$. brucei of $Z$ ululand.

Further observations are obviously necessary before we can come to a definite conclusion as to the relation between the nagana of Zululand and this disease of German East Africa.

It would appear that Glossina morsitans carries the infection.

[Koch has since made a further study of this East African disease, and considers its trypanosome to be $T$. brucei. It was with this trypanosome that he made the observations on the evolution of the parasite in Glossina, mentioned on p. ${ }_{5} 6$. He states that in German East Africa this trypanosome is carried by Gl. mersitans, Gl. pallidipes, and especially by Gl. fusca].

\footnotetext{
1 Stuhlmann, loc. cit.

2 Kummer, Tropenpflanzer, 1902, No. 10, pp. 525, 526.

3 Grothusen, Archiv f. Schiffs u. Tropenhys., v. 7, August, 1903, p. 387.
} 
[A natural infection of pigs has been observed by Ochmann in German East Africa, but whether it is true nagana or an allied disease is uncertain (see p. II4).]

\section{Section 3.-Trypanosomiasis of Togoland.}

The existence of a trypanosomiasis in Togoland was recognised by Koch, who in 1895 examined in Berlin two specimens of the blood of a horse coming from this German colony on the Gulf of Guinea.

Since then some important papers upon the nagana of Togoland have been published by Schilling and Ziemann, who carried on their investigations in the colony itself, and by Martini, whose researches originated in the discovery of a trypanosome pathogenic for mammals in the blood of a pony imported from Togo into the Zoological Gardens in Berlin.

According to Schilling ${ }^{1}$ the disease affects horses, donkeys, and cattle throughout the whole hinterland of Togo. Fever is intermittent, and there is no definite relation between the temperature and the number of parasites in the blood. The symptoms are not constant, and the parasite is often absent on microscopical examination.

In a horse which he had under observation during the last ten days of its life Schilling noted wasting, swelling of the testicles, penis, joints, and belly, and a purulent discharge from the eyes and nose; its appetite remained good until the end. Seven days before death its hæmoglobin was only 25 to 30 per cent. No morbid conditions were found post-mortem. Neither in this nor in any of the other horses did Schilling find enlargement of the spleen.

Trypanosomes may be absent from the blood and the peritoneal cavity at the time of death, but, according to Schilling, they are always present in the marrow of the bones. Anæmia would appear to be due, not to a destruction of the red corpuscles in the peripheral circulation, but to diminished production in the bone-marrow.

Schilling inoculated various animals: horses, donkeys, oxen, goats, pigs, and dogs. They were all susceptible except the pig. The course of the experimental disease in the horse differs from the natural infection in that the symptoms are less marked, the swellings being almost entirely absent.

The disease in the horse may be acute or chronic, death occurring after an interval of from forty-three days to more than eight months. Sudan donkeys appear more susceptible than the horse, for two of them died in eleven and eighteen days respectively after inoculation.

In his first paper Schilling describes the evolution of the parasite in several dogs inoculated subcutaneously or intraperitoneally. In dogs inoculated intraperitoneally, and also in a dog in which an abrasion on the ear was smeared with blood containing trypanosomes,

1 Schilling, Centralb. f. Bakter., I, Orig., v. 30, October 30, I901, p. 545 ; v. 32, April I6, I902, p. 452, and v. 33, January, I903, p. I84. 
there occurred intraperitoneal as well as intravascular multiplication of the parasite. A dog inoculated in the peritoneum with I c.c. of horse blood containing few trypanosomes died in twentyeight days.

Schilling also infected a dog by allowing it to be bitten on several occasions by a tsetse-fly which had previously fed upon a dog with numerous trypanosomes in its blood.

Two dogs inoculated in Berlin with Schilling's trypanosome by Stähelin, ${ }^{1}$ with the object of studying the metabolism of animals with nagana, died in twenty-four and twenty-eight days respectively. During the course of the disease they suffered from remittent fever, chemosis, corneal trouble, hypopyon, and wasting, and during the last two days of life they had a subnormal temperature.

[Schilling's ${ }^{2}$ later observations on the trypanosomiasis of Togo are summarized by Mesni1 ${ }^{3}$ as follows: The trypanosome appears not to differ from the type brucei, but we have seen that Laveran and Mesnil, from their experiments on 'cross-inoculation' and serum-diagnosis, conclude that this Togo disease is probably not true nagana. Schilling describes a form of the parasite-possibly a sexual form-which is fairly wide and shorter than the ordinary form, especially as regards the flagellum. The cytoplasm is pale-like the undulating membrane-and is poor in granules.]

[In the horse this disease is less acute in Togo than in South Africa. The incubation period is 6 to 12 days; the average duration, 50 days. The hæmoglobin may fall to 25 per cent. of the normal.]

[In the donkey the incubation period is 4 to 5 days, and the average duration Io to 18 days. In bovines the incubation period is 4 to 9 days, and the symptoms are slight, the disease usually ending in recovery.]

[Berlin pigs are susceptible, but the parasite is only visible at the beginning of the infection, and the animals recover from the infection. Schilling had previously found that the Togo pig was refractory.]

[Most of the experiments on rodents and birds were done with a virus passed twenty-five times through the dog. White rats died in about 28 days, but they lived considerably longer with a virus passed through the pig (54 days) or goose ( 80 days). White mice died in about 37 days, a grey mouse (M. musculus) died in Ioo days, and a $M$. agravius in I22 days. Schilling thinks these long survivals are due to the passage of the virus through dogs, for with a divect virus he killed rats in 4 to Io days in Togo. The 'pedigree' of the parasite would therefore appear to be of considerable importance; and the virulence of trypanosomes for the same species of animals may vary enormously, according to the 'genealogy' of the parasites. This may possibly account for the fact that Schilling was able to infect a goose, which no previous investigator had succeeded in doing. $\left.{ }^{4}\right]$

[Four animals were inoculated in Berlin and developed a mild infection, with only febrile symptoms at the outset. These included two cones inoculated (the first on three occasions) with a virus of the twenty-sixth to thirtyfirst passage through dogs; a young bull, inoculated with the blood of the second cow; and a calf of the first cow (born during the infection, but free from it), inoculated when forty-five days old with the bull's blood. The serum of these four animals did not show the bactericidal properties noted

1 Stähelin, Arch. f. Hyg., v. 50, 1904, p. 77.

2 [Schilling, Arb. a. d. kaiserl. Gesund., v. 21, 1904.]

3 [Mesnil, Bull. Inst. Past.. I905.]

4 [These experiments, together with those on other animals, are summarized in the table on p. I97.] 
in the Togo animals similarly treated. These microbicidal serums had no action in vivo.]

[Passage through resistant animals did not attenuate the virus for the dog; but a virus of the third passage through the goose was much attenuated for the donkey.]

Schilling has paid particular attention to the vaccination of cattle. An ox inoculated with the virus after five passages through cattle died in forty-one days. On the other hand, oxen or calves inoculated with a virus which had been passed a certain number of times through the dog or rat (three times from dog to dog in the first experiment; seven times alternately through dog and rat, then exclusively through the dog up to the eighteenth and twenty-first passage, in a second experiment) contracted a mild and temporary infection, which rendered them immune. We have already discussed (p. I83) the significance of these facts.

[In recent papers Schilling ${ }^{1}$ gives the after-history of the 36 cattle vaccinated by him at Sokode in 1902 (see p. I83). Seventeen were sent into fly areas, and of these I 3 died, probably of nagana, doubtless, thinks Schilling, because they were exposed to the fly too soon. Of the 4 survivors, I died of nagana after a fresh journey, and 3 were in perfect health 3 years after their vaccination.]

[Of the I9 cattle kept at Sokode, 2 died fairly quickly-perhaps from the vaccination; 4 out of 8 tested were still infected at the end of I I months (Io c.c. blood injected into dogs).]

[At the end of 14 months I 8 vaccinated cattle were taken to the coast: 3 died ( 2 of nagana) and 2 others became infected, but recovered. Three of these cattle were inoculated 2 years after their vaccination with fairly virulent trypanosomes. A very mild infection followed, and at the end of 3 months the blood was no longer infective in doses of 5 to Io c.c., while at the end of Io months only I was infective (40 c.c. blood injected into a dog). Schilling insists on the importance of this observation, which seems to prove the reality of cures-contested by Koch-and which in any case shows that 'cured' animals are not a great source of danger as reservoirs of infection. Schilling thinks it is advisable to vaccinate animals when very young.]

[A final observation of Schilling's shows how carefully one must interpret the results of this method of vaccination, and that it is not of much value practically. Six cattle (3 vaccinated, 3 unvaccinated) were taken through a very bad fly belt : 5 became infected, and of these 2 died ( $\mathrm{I}$ vaccinated) $; 2$ were still infected 9 months later ( $\mathrm{I}$ vaccinated), and I recovered quickly (unvaccinated). Of the 3 vaccinated animals, therefore, I died, I was still infected after 9 months, and the third did not become infected at all.]

In rgoo Ziemann ${ }^{2}$ had under observation two horses and one dog $q$ which were spontaneously infected. The blood of the dog was used to infect a goat which became ill. This goat showed numerous trypanosomes on the eighth day, few on the eleventh, many on the twelfth and thirteenth, few on the fourteenth and sixteenth, and very few on the seventeenth days, after which it was rare to find even one under the microscope, and the animal was cured.

1 [Schilling, Deutsch. Kolonialbl., No. I, I904; Zeitschr.f. Hyg., v. 52, 1905, pp. I49-160; abstract by Mesnil in Bull. Inst. Past., v. 4, I906, pp. I85, I86, from which the above account is taken.]

2 Ziemann, Berlin klin. Wochenschr., October 6, I902, p. 930. 
The other animals-a duck, a fowl, a pigeon, and a sucking-piginoculated by Ziemann remained healthy. This last observation confirms that of Schilling, ${ }^{1}$ but differs from the results of experiments obtained with the $Z$ ululand virus (see p. I36). We may add that Martini found the pig susceptible to the Togo virus. ${ }^{2}$

Martini has also inoculated his virus into a female Egyptian buffalo and a zebra from Kilimanjaro. The buffalo died in six weeks of typical trypanosomiasis (severe anæmia, etc.), with an enormous number of parasites in its blood. The zebra lived for a longer time, and, although trypanosomes were present in the blood from the fourteenth day after inoculation, the animal remained in good health for three and a half months. It died, however, a fortnight later, after having shown the symptoms characteristic of nagana in the Equidæ. ${ }^{3}$

Martinit has given a very detailed account of the morphology of his Togoland trypanosome, which appears not to differ in any essential point from the typical $T$. brucei.

[Martini ${ }^{5}$ has made an exhaustive study of the trypanosomes of two Togo ponies which were brought to Berlin. ${ }^{6}$ The trypanosome of the stallion was very virulent and deadly, while that of the mare was originally only slightly virulent. Martini thought at first that the more virulent strain was morphologically different from the less virulent one, the former having a long flagellum, the latter a short flagellum. Later he found, however, that the more virulent strain could have a short flagellum and still retain its virulence. The less virulent parasite, after passage through the horse and dog, became more virulent for a number of animals, and at the same time the flagellum increased in length. As a rule, the shortflagellated forms of both strains appeared more frequently in those animals which were less susceptible to the fly disease, and which presumably had protective bodies in their serum. This relation of length of flagellum to virulence was, therefore, by no means constant.]

[The Trypanosome of the of Pony.-This trypanosome killed the original stallion in 100 to 120 days. The blood of this animal was injected into a number of animals, with the results shown in the table on p. I97. Martini tried especially to create a series of particular strains by repeated passages through the same animal species. Passage through Equidæ (horse and ass) and cats did not affect the virulence much, but passage through rats and mice resulted in the formation of a vinus-fixe, which killed

1 [We have seen, however, that Schilling has since found Berlin pigs susceptible to his Togo virus.]

${ }_{2}$ Martini, Arch. f. Schiffs u. Tropenhyg., v. 7, November, 1903, p. 499.

3 Martini, Diutsche med. Wochenschr, August 6, 1903, pp. 573-575. It should be noted that this susceptibility of the zebra (see p. 192) to experimental inoculation contrasts with the fact, which appears well established, that the zebra may pass with impunity through fly areas where nagana occurs, and that its blood never contains trypanosomes and is not infective for susceptible animals. Martini recognises that further investigations are necessary upon this point-for example, upon animals living for a long time in fly areas and made to do work.

* Martini, Zeitschr. f. Hyg., v. 42, I903, p. 341, and Koch's 'Festschrift,' Jena, I903, p. 219.

[Martini, Zeitschr.f. Hyg.u. Infec., v. 50, 1905, pp. 1-96; abstract by Mesnil, Bull. Inst. Past., v. 3, 1905, pp. 463-466.]

${ }^{6}$ [Practically the whole of the following 14 pages, down to the end of Section 8, has been added.-ED.] 


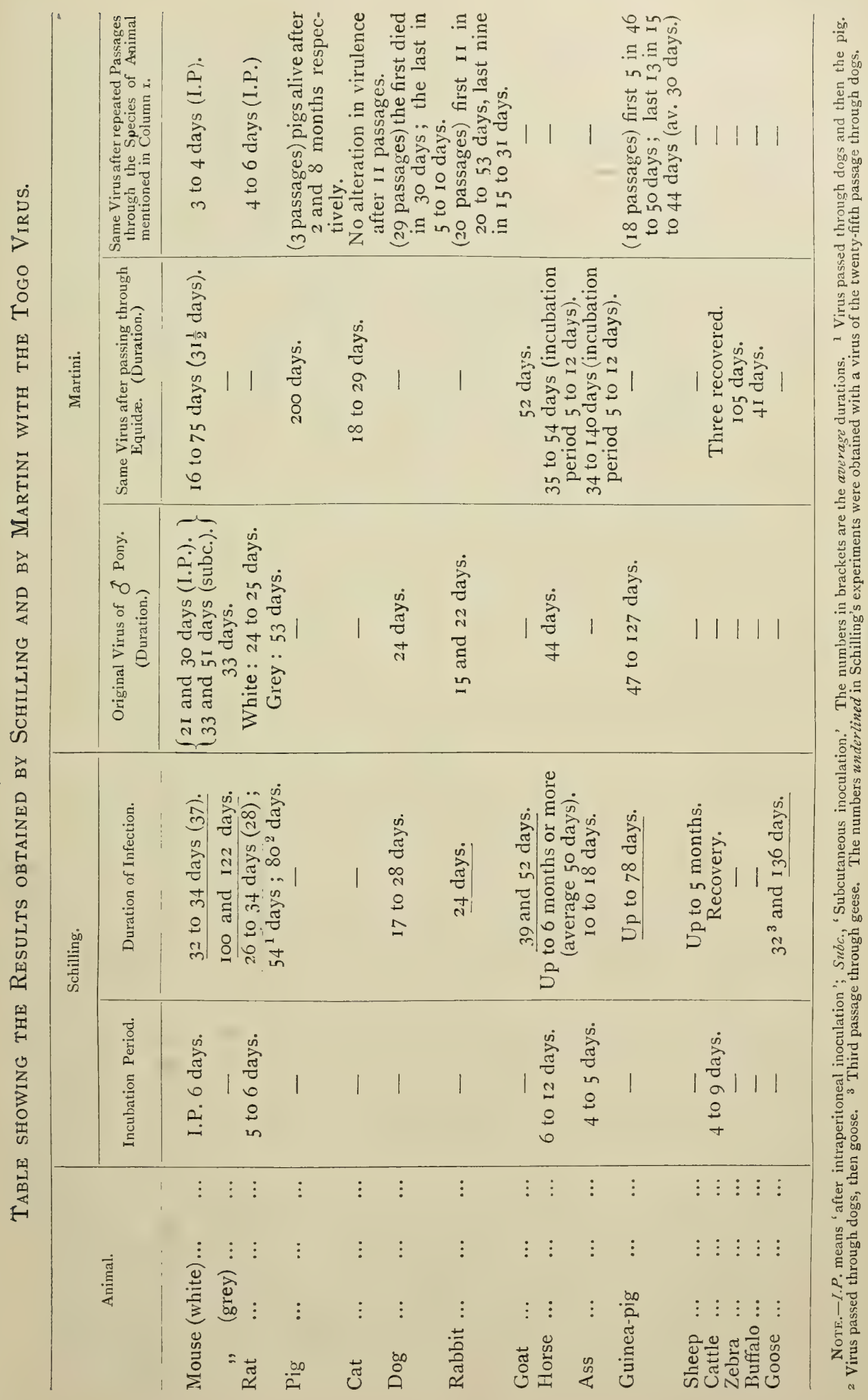


very rapidly, not only animals of the same species as that which had furnished the virus, but even those of other species. The vinus-fixe of the rat and mouse was very virulent for the dog, and that of the dog was also as virulent for rats and mice as their own virus-fixe. On the other hand, the viruses of passage through Equidæ, rabbit, and pig were very slightly virulent for rats, mice, and dogs (see Table, p. I97).]

[Martini's aim in creating these various strains of the parasite was to perfect a method of immunizing cattle against the fly disease in the manner outlined by Koch and Schilling. The results were variable. The viruses of passage through the dog, cat, pig, and rat were as virulent for the ass as the original virus, or the virus of passage through Equidæ. The viruses of passage through the rabbit and guinea-pig were even more virulent. On the other hand, the virus of passage through grey mice took 275 days to kill a donkey, while two donkeys inoculated with a virus of passage through white mice were alive and well I 3 and I 6 months after inoculation. They had a definite but slight trypanosome infection, from which they recovered, apparently immunized, as we shall see later.]

[The Trypanosome of THE o Pony.-This animal was scarcely ill at all. There were occasional rises of temperature to $38.5^{\circ}$ or $39^{\circ} \mathrm{C}$. (Ior ${ }^{\circ}$ to $I O 2^{\circ}$ F.). Some of the dogs injected with 2 to IO c.c. of its blood contracted a mild infection; only one dog died (in IO2 days). A donkey, pony, and gelding contracted moderately severe infections, from which they recovered.]

[Passages through dogs, starting direct from the Togo mare, gave very discordant results - some dogs died in I 5 to 20 days, others later, and some had only a slight infection. On the other hand, a well-marked series was obtained starting from the gelding, which was infected from the mare direct. The earliest dogs of this series died in 13 to 28 days, the later ones in 3 to 12 days. This virus of passage through dogs was also raised in virulence for rats and mice, for the initial virus did not kill these animals. It also killed a donkey "in 85 days. A virus was thus obtained identical with the original virus of the $\delta$ pony.]

[MoRPhology OF THE TRYPANosomes.-The strain obtained from the $\sigma$ pony was like the typical $T$. brucei, but we have already seen that Laveran and Mesnil think that this virus of Martini was neither true nagana nor surra. Mention has also been made of the changes in length produced by passage through different animals, and of the accompanying alterations in virulence noted in certain cases.]

[Attempts at Vaccination.-Equidæ and dogs cured of a mild infection, caused by the strain from the $q$ pony, did not acquire any immunity against the $\delta$ pony strain. These animals, including the original Togo $q$ pony, succumbed to the second infection.]

[On the other hand, asses which had contracted a mild infection after inoculation with the $\delta$ strain passed through mice (see above) survived a virulent inoculation, which killed controls in 96 and 54 days. This is an interesting observation; and it remains to be seen whether animals thus vaccinated will prove equally resistant to infection by the bites of tsetseflies. Martini thinks, with Koch, that this method of vaccination has a grave drawback, the animals thus immunized retaining the trypanosomes indefinitely in their systems. It could, therefore, only be used under certain definite conditions.]

[The serums of vaccinated calves and asses had preventive and agglutinating properties. Mixed with the virus in doses of 0.5 c.c. for the mouse, it prevented an infection. Similar results were obtained with dogs. These results are analogous to those previously obtained by Laveran and Mesnil, and Martini was equally unable to 
exalt the potency of his serum. Kleine and Möllers ${ }^{1}$ succeeded in increasing the potency of the serum of these asses by giving them intravenous injections of trypanosomes (the first injection was with guinea-pig's blood, later ones with rat's blood, at intervals of a fortnight.) The maximum potency was reached after the second injection with these rat trypanosomes. The serum then protected mice in doses of $\frac{1}{2}$ c.c., injected either twenty-four hours before the virus or at the same time as the virus, the serum being injected under the skin, the virus in the peritoneum. Injected twenty-four hours after the virus, the serum still protected, provided the injections of $\frac{1}{2}$ c.c. were repeated several times. In certain cases (early use of the serum in mice infected with a virus from the guinea-pig) the serum was able to cure mice, but in other cases it only prolonged life up to twenty-five to forty days. A dog treated with doses of 20 c.c. ass's serum from the fifth day after infection had its life prolonged by about ten days.]

[This serum was found to have no action upon mice infected with the human trypanosome, so that the action is specific, as Laveran and Mesnil have also found in many of their experiments.]

[It is an interesting fact that the donkeys, which throughout the experiment were in a cachectic condition, eventually died, and that their blood was infectious for the dog in 20 c.c. doses.]

\section{[Section 4.-Trypanosomiases of Cameroon.]}

[These infections have been studied by $Z$ iemann ${ }^{2}$ and by Diesing. ${ }^{3}$ A short account of Ziemann's earlier observations is given on p. II6. In his later publication Ziemann describes in detail a trypanosome infection due to a parasite which he thinks may be a new specieshe calls it $T$. vivax, on account of its unusual motility-but which, he says, is certainly distinct from T.brucei. Schilling, however, who saw the same trypanosome in Togo, maintains that it is T. brucei, showing an unusual motility in sheep and calves, but not in the rat.]

[In addition to $T$. vivax infection in Cameroon, Ziemann met with(I) true nagana; (2) T. lewisi in rats, some of the infected rats appearing ill ; (3) sporadic cases of human trypanosomiasis; and (4) a trypanosome in birds (kingfisher).]

[True nagana was found with certainty by Ziemann in two horses, one mule, two humped cattle (zebus), and one donkey. A1! these animals except the last had come from the interior, but Ziemann does not deny that nagana may occur in the coast regions. Gl. morsitans and longipalpis are not met with in the coast regions, so that the carrier of $T$. brucei is wanting. This explains why horses and mules do well in these parts. In the hinterland nagana appears to be propagated by $G l$. tachinoides.]

1 [Kleine and Möllers, Zeitschr.f. Hyg., v. 52, I905, pp. 229-237.]

2 [Ziemann, Deutsche med. Wochenschr., April 9 and I6, I903; Centralb. $f$. Bakter., I, Orig., v. 38, I905, pp. 307, 429.]

3 [Diesing; Arch.f. Schiffs u. Tropenhyg., v, 9, I905, p. 427.] 
[Infection with Trypanosoma vivax.-According to Ziemann, this infection is much commoner in Cameroon than nagana. It is very prevalent among cattle, sheep, and goats along the whole forest region of the coast and in the river valleys. Dogs, cats, and pigs were found not to suffer from the natural infection. Animals brought from immune districts into infected areas died in large numbers of this trypanosomiasis. The infection seems to vary with the seasons, epidemics occurring at the height and towards the end of the rainy season.]

[MoRphology of THE Trypanosome.-In the living state $T$. vivax is distinguished from other pathogenic trypanosomes by its extraordinary activity, which is as great as that of $T$. lewisi. Stained specimens resemble $T$. evansi (of surra) so closely that Laveran could see no difference between them. Ziemann gives several characteristics by which $T$. vivax may be distinguished from $T$. brucei, one being the great difficulty in differentiating the sexual forms of $T$. vivax.]

[The Natural and Experimental Infection in Animals.-The natural disease in cattle, sheep, and goats may be very acute, killing in a few days; or it may be very chronic, lasting a year or more. Such chronic cases in sheep and goats may end in recovery, but the animals are not immune, for relapses may occur, which are rapidly fatal, or may again end in recovery. Cattle, apparently, never recover from the disease. There is irregular fever, loss of appetite, and in very acute cases in sheep and goats there may be tonic and clonic spasms. In chronic cases the appetite may remain good ; in cattle and artificially infected asses the coat was harsh and staring. In acute cases the cattle, apparently well the day before, fell down obviously very ill, the eyes dull and glassy, and mucus often dribbling from the mouth. The pulse and respiration are increased; before death the temperature is often subnormal. Unlike I:c. " ndemas and petechial hæmorrhages are absent, even in chronic ce. Anrmia was marked in all cases, and in the acute cases was pr. (rod corpuscles, 2, I30,000; hæmoglobin, 22 per cent.).]

L... isease did not appear to be inherited, nor were the offspring of infected mothers immune against the natural or experimental infection.]

[The incubation period is five to eight days in the natural or experimental infection.]

[Post-mortem there are signs of anæmia, yellowish gelatinous masses on the pericardium and kidneys, and some fluid in the serous cavities. The liver and spleen are not enlarged in acute cases. Inoculated grey rats died in 8 to II days; donkeys died in 52 to 290 days, the disease being much more chronic in them than true fly disease. A dog and a native sucking-pig developed a slight temporary infection. White rats, geese, ducks, native hens, young turkeys, a native cat, and an old pig, were all refractory.]

[Mode of Transmission.-Tsetse-flies are absent from the districts where this disease is most prevalent, so that $T$. vivax cannot depend on Glossina for its transmission. Ziemann incriminates Tabanidæ or Stomoxys, but gives no conclusive evidence in support of this. From certain facts observed, he thinks the carrying insect, whatever it may be, acts as a definitive host for the trypanosome, which it allows to multiply in its body.]

[Prophylaxis is difficult, if not impossible, owing to the character of the country and the ignorance and distrust of the natives. General hygienic measures should be adopted, and attempts made to exterminate biting flies and other insects. Ziemann also suggests inoculating very young animals, so as to give them a mild infection, which in some cases he found conferred immunity.]

[This trypanosomiasis may belong to the group of nagana-like diseases not dependent on the tsetse for their transmission, or it may be a variety of surra.] 
[Diesing (loc. cit.) has given a general account of the fly disease in Cameroon. In the coast regions the disease may remain latent for a time. It affects especially the overworked and badly-fed horses and the stallions during the pairing season; it is rapidly fatal. The Adamaoua asses, on the other hand, recover from the infection. The serum of these cured and immunized asses, injected into nagana cattle and horses, produces an improvement in their condition, which lasts about a month. Injections of the serum ( 40 to 50 c.c.) into healthy cattle give rise to a temporary passive immunity lasting about a fortnight, sufficient to enable the animals to pass through a fly belt.]

\section{[Section 5.-Trypanosomiasis of Somaliland.]}

[Brumpt ${ }^{1}$ has recorded a trypanosome epizootic of dromedaries in Ogaden (see p. II5), known locally as Aino. It would appear to be propagated by $G l$. longipennis.]

Camels (dromedaries) are very susceptible to the natural disease; nevertheless they can live for a very long time if they do not work. As a local symptom there is little else than odema of the supra-orbital fossa. The animal dies with a subnormal temperature. At the autopsy a large quantity of peritoneal fluid and a little pericardial fluid are present; but no hypertrophy of the spleen. A mule died of the same disease, also with a subnormal temperature, three weeks after its arrival in the infected area. Inoculations were made into a donkey and a young camel. At the end of three days parasites appeared in the blood. The donkey died in less than thirteen days; the camel was still alive at the end of that time. The Cercopithecus sabaus is susceptible; a baboon (Theropithecus gelada) ' inoculated subcutaneously proved to be refractory.' A bitch two months old inoculated intravenously showed the parasites very quickly; she died in three and a half months, after having exhibited a sero-purulent bullous eruption on the abdomen (at the end of a fortnight), anæmia, wasting, an ulcer of the left eye, and finally, two days before death, paralysis of the posterior extremities. The trypanosomes 'closely resemble those of nagana.' ${ }^{2}$

[Theiler ${ }^{3}$ found the same disease in thirty-four out of thirty-six camels that were brought from Somaliland to Pretoria.]

[One camel died before arrival, and six soon after arrival, in Pretoria. The wasting, anæmia, and absence of parasites from the blood on microscopical examination suggested a trypanosomiasis, which was confirmed in all the cases but two by injecting some blood into dogs. In six of the cases the dogs did not become infected until after a second injection (20 c.c. of blood used). This shows how difficult it is sometimes to make a diagnosis; moreover, in some cases the dogs did not become infected until two and a half months after inoculation. The virus, after passage through the dog, was not very virulent, killing a horse in 66 days, a rabbit in I08 days, a rat in 30 days, and dogs in 12 to 90 days.

I [Brumpt, C. R. Soc. Biol., v. 56, I904, pp. 673-675.]

2 [In the original this paragraph was a footnote to p. I IO.]

3 [Theiler, Rev. gén. méd. vétér., v. 7, 1906, pp. 298-303; abstract by Mesnil, Bull. Inst. Past., v. 4, I906, p. 453. Also Theiler, in Transvaal Agric. Journ., Ig06, of which an abstract is given in Vet. Journ., v. 13, I906, pp. 214-218.] 
An inoculated mule, ass, ox, sheep, and goat were still alive Ioo days later.]

[On account of its feeble virulence, Theiler concludes that the disease is mbori (a variety of surra), and not nagana. The disease may be true nagana, the feeble virulence being due to the 'genealogy' of the trypanosome (Mesnil), or it may belong to the tsetse group of nagana-like trypanosomiases. In all probability it is not surra, because in Somaliland it appears to be conveyed by a tsetse-fly. Attempts to transmit the disease from Equidæ to Equidæ by Hippobosca and Stomoxys failed.]

\section{[Section 6.-Trypanosomiases of Abyssinia and Erythrea.]}

[As stated on p. Ir5, Hallen and Savouré have observed a trypanosomiasis in Abyssinia-the former amongst horses, the latter amongst cattle. Both these observers regarded this trypanosomiasis as surra, but Brumpt thinks it the same as that found in the Juba Valley, and in British and German East Africa-a true tsetse disease.]

[A dog which accompanied the Abyssinian Boundary Commission (I903) developed a trypanosomiasis, which was studied by Nabarro, Greig, and Gray (see Section 8).]

[In Erythrea there is a bovine infection, called giaan by the natives, which has been investigated by Memmo, Martoglio, and Adani. $\left.{ }^{1}\right]$

[The disease in cattle resembles nagana or surra, but œdemas and skin lesions are absent. Towards the end of the disease nervous symptoms are very marked, and the temperature may be subnormal. Abyssinian sheep and goats are very susceptible to this trypanosome infection, dying in twelve days. An inoculated horse and ass became infected and died in a year and five months respectively after inoculation. The mouse, rat, guinea-pig, rabbit, dog, fowl, and monkey appear to be insusceptible to this infection. They all failed to show trypanosomes on microscopical examination, except the rat during the first few days.]

[One animal, which during the last week of its illness showed a typical clinical picture of 'sleeping sickness,' had areas of cerebral softening containing numerous trypanosomes, but no trypanosomes were found elsewhere in the body.]

[The trypanosome is morphologically like T. brucei or T. evansi, but is not more than $24 \mu$ long, free flagellum, which is fairly long, included. It is extremely motile, like $T$. vivax of Ziemann. This trypanosomiasis appears to be very virulent for ruminants, and thus differs from typical surra. In many respects it resembles the disease described by Cazalbou in French Sudan under the name Souma. There is no tsetse in the infected area, and the suspected fly is a Tabanus or a Hippobosca.]

${ }^{1}$ [Memmo, Martoglio, and Adani, Annali d' Igiene sperim., I905, pp. I-46.] 


\section{[Section 7.-Trypanosomiases of the Anglo-Egyptian Sudan.]}

[Balfour ${ }^{1}$ and Head ${ }^{2}$ have made a study of these diseases, which they found in cattle at Kodok (formerly Fashoda), and in Equidx from the Bahr-el-Ghazal province.]

[Six mules were taken from Khartoum to the Bahr-el-Ghazal; they remained there for five months, and were then taken back to Khartoum. From thirty to fifty days after their return four of the mules began to show signs of a trypanosome infection (progressive anæmia and fever), and they all died in fourteen days after the onset of the symptoms (Head).]

[Balfour found trypanosomes in a donkey and in mules also from the Bahr-el-Ghazal province. Tsetse-flies were first recorded there by the Pethericks in 1869, and in 1903 Colonel Griffiths found Gl.morsitans on the Pongo River, at Runbek, which is the most northerly point of occurrence yet recorded for the Sudan.]

[Trypanosomes were also found in cattle brought from the Shilluk country. The cattle appeared ill, and on examination trypanosomes were found in the blood. The disease is particularly prevalent in the valley of the Sobat and of its tributary, the Baro, especially at Itang, in Abyssinian territory; three mules coming from Itang were also infected. An infected ox was discovered fifty miles to the north of Kodok (Fashoda).]

[Laveran ${ }^{3}$ has described the morphology of these trypanosomes. (I) The trypanosomes of cattle are all small-Io $\mu$ to I $4 \mu$ long by $x^{\prime} 5 \mu$ to $2 \mu$ broad, with very narrow undulating membrane and no free flagellum. These characters, together with the non-pathogenicity for dogs, rabbits, and monkeys, point to the probability of this being a new species of trypanosome, which Laveran calls $T$. namum. It is possible that some of the Uganda cattle were infected with the same trypanosome.]

[(2) The trypanosomes found in the mule's blood are of two distinct types: (a) Small forms, I $2 \mu$ to I $4 \mu$ long, without free flagellum, but with a well-developed undulating membrane, and resembling the small forms of T. dimorphon; (b) large forms, 2I $\mu$ to $30 \mu$ long by $2 \mu$ wide, with free flagellum, and resembling $T$. evansi. Laveran thinks it is either a case of mixed infection or infection with $T$. dimorphon. Balfour inclines to the latter view, but notes that his inoculated dogs developed eye troubles, whereas Dutton and Todd never observed this.]

[The affected cattle usually die profoundly anæmic and cachectic, but sometimes the trypanosomes disappear from the blood, the animals appear well nourished, and possibly, if well looked after, they may recover spontaneously. In fatal cases Balfour often found a congested or ulcerated condition of the stomach, spirilla being found in the lesions present. ${ }^{4}$ Animals - rabbit, dog, and monkey (Cercopithecus sabaus)inoculated with this trypanosome, $T$. namm, were found to be refractory.]

[The mule trypanosome-probably $T$. dimorphon - was pathogenic for various animals. Dogs showed the usual symptoms, and always developed corneal opacities. Two dogs which were untreated died in twenty-three and twenty-five days. Some dogs had gastric ulceration with spirilla in the blood-clots. A Cercopithecus sabaus died in fourteen days;

1 [A. Balfour, Brit. Med. Journ., 1904, No. 2, pp. 1455, I456; Edin. Med. Journ., N.S., v. I8, I905, pp. 202-212; Journ. Path. and Bact., v. I I, March, I906 pp. 209-236.]

2 [A. S. Head, Journ. Comp. Path. and Therap., v. I7, I904, pp. 206-208.]

3 [Laveran, C.R. Soc. Biol., v. 58, February 18, 1905, pp. 292-294.]

4 [Balfour suggests that the spirilla in the gastric lesions may indicate the way the trypanosomes leave the body-per anum; but there is no proof that the stools can convey the infection.] 
jerboa (Jaculus govdoni) in six days; gerbils (Gevbillus pygargus, the common desert mouse) in fourteen days. A rabbit and a goat also became infected.]

[Chrysoidin and the serum of a 'water-buck' were valueless in the treatment of this trypanosomiasis.]

\section{[Section 8.-Trypanosomiases of Uganda and Adjacent Parts of East Africa.]}

[During the course of their investigations on sleeping sickness in Uganda, Bruce, Nabarro, and Greig discovered several other trypanosomiases in cattle, a mule, and a dog. The trypanosomes present in these diseases and their effects upon experimental animals were fully worked out by Nabarro, Greig, and Gray. ${ }^{1}$ These trypanosomiases included-(I) a disease among transport oxen in Entebbe, Uganda; (2) an epizootic affecting a herd of cattle brought from the Bukedi country (Wamia's District, south-west of Mount Elgon) to Jinja, Busoga; (3) an infection in a dog which had accompanied the Abyssinian Boundary Commission; and (4) an infection in a mule in Entebbe.]

(i) Trypanosomiasis of Oxen in Entebie.-Of three sick oxen, markedly emaciated and with coat staring, two showed trypanosomes in the blood. Thinking this might be the parasite of nagana, Bruce, Nabarro, and Greig injected 2 c.c. of blood containing many trypanosomes into a dog. The dog did not become infected. A further injection of 5 c.c. infective blood from the same ox was made two and a half months after the first injection, and with the same result. It is therefore very unlikely that this was $T$. brucei. ${ }^{2}$

[These oxen came from East Africa about three years before they became obviously ill. They remained quite well in Entebbe, until they were placed near the forest to graze. In this forest, which is near the shore of the Victoria Nyanza, many tsetse-flies (Gl.palpalis) are found.]

[This disease of cattle appears to run a chronic course. Progressive wasting is the most obvious sign, and in the later stages the lymphatic glands are greatly enlarged, and there are daily rises of temperature to $104^{\circ}$ or $105^{\circ} \mathrm{F}$. $\left(40^{\circ}\right.$ to $41^{\circ} \mathrm{C}$.). The parasites, which are numerous in the blood at first, may disappear for several weeks before death. In one case they could not be found in the blood during the last six weeks of life, even on centrifuging ro c.c. A dog and a monkey injected with this blood, taken immediately after death, failed to become infected.]

[Post-mortem the superficial lymphatic glands are enlarged and congested, and all the organs are somewhat anæmic. The spleen is only slightly enlarged.]

[This disease is known locally as Mukebi. It is almost certainly not nagana, as its trypanosome is not pathogenic for the dog. Its exact nature is uncertain, and unfortunately the trypanosome

1 [Bruce, Nabarro, Greig, and Gray, 'Reports of the Slceping Sickness Commission of the Royal Society, London,' Nos. I., IV., V., and VI., 1903-I905; abstracts of V. and VI. in Bull. Inst. Past., v. 3, 1905, pp. $771-777$.

" [This paragraph is in the original of Laveran and Mesnil's book as a footnote to p. 110.$]$ 
was lost, owing to its non-pathogenicity for experimental animals. Several other oxen in Entebbe were subsequently examined, but this trypanosome was not seen again. The trypanosome resembled T. gambiense in appearance, so this disease is probably not the same as that seen by Balfour in cattle in the Anglo-Egyptian Sudan, due to $T$. nanum. It may, however, be the same as that described by Memmo in Erythrea, by G. Martin in French Guinea, and by Cazalbou in French Sudan, under the name Souma.]

[(2) Trypanosomiasis of Cattle brought from Bukedi to Jinja, BUSUGA. - On their way from Bukedi to Jinja these animals halted at several places, in all of which a tsetse-Hly (Gl. pallidipes) is found. When first brought into Jinja they were apparently healthy, but later on died at the rate of five or six a day. Over 60 per cent. of the cattle showed trypanosomes in their blood when first examined, and within a month 50 out of 2 10 (24 per cent.) had died. The duration of the disease, as gathered from local information, is possibly two to three months; a bullock injected with the virus, which had been passed through a monkey and then a dog, died two and a half months after inoculation. A year after the discovery of this disease the cattle were again examined, and 50 per cent. had trypanosomes in the blood, yet the animals appeared well and were not dying of the infection. The virulence of the epizootic had become greatly modified, either through attenuation of the parasite or, as is more likely, through the animals having become immunized.]

[The native name of the disease is Sutoko, and it has been regarded as an internal form of $M u k e b i$. When only the external glands are enlarged many cases are said to recover.]

[The symptoms observed in naturally infected cattle are often not very marked. There is some wasting and enlargement of the lymphatic glands; usually there is pronounced anæmia. The animals often die quite suddenly and in fairly good condition. In more chronic cases they waste considerably, and are moribund for several days.]

[Post-mortem the supraclavicular and cervical glands are enlarged and congested, sometimes hæmorrhagic. In some cases there is excess of fluid in the serous cavities. At the base of the heart there is yellowish gelatinous material, which is also found at times in the subcutaneous tissue and at the root of the lungs. In most cases petechiæ are seen under the serous membranes, especially the pericardium and, less frequently, the endocardium. The blood and organs are pale, but otherwise normal; the spleen is somewhat enlarged and congested.]

Morphology of the Trypanosome.-The trypanosomes seen in the blood of the naturally-infected cattle varied considerably in size and shape: (I) Short, stumpy forms, average length about I $2 \mu$ to I $3 \mu$, including free flagellum, $2 \mu$ to $4 \mu$. Occasionally very short parasites, only $7 \mu$ long, were seen. (2) Longer, more slender forms, $20 \mu$ to $25 \mu$ long, including free flagellum, $8 \mu$ to Io $\mu$. In one animal a very long parasite was seen-total length, $44 \mu$; free flagellum, I $8 \mu$-about to undergo division (the centrosome had just divided). ${ }^{1}$ In the short forms the posterior end was usually very blunt, while in the longer forms it was more drawn out. Similar variations in size and shape were seen in inoculated animals. In an ox,

1 [This was probably T. theileri; see Chapter XI. This trypanosome is figured in the Roy. Soc. Sleeping Sickness Rep., No. V., I905, Plate I.] 
sheep, goat, and dog the trypanosomes were usually short and stumpy, with a very short flagellum. In monkeys and a guinea-pig they were long and thin, but sometimes the one form and sometimes the other form predominated in the blood of the same animal.

[Experimental Inoculation into Animals.-This trypanosome was found to be pathogenic for the following animals: monkey ${ }^{1}$ (Cercopithecus sp.), dog, rat, rabbit, guinea-pig, sheep, goat, ox, and Masai donkey; the dog-faced baboon was refractory. In white rats the incubation period was 4 to 8 days, average 5 days; the total duration of the disease Io to 29 days, average 20 days. In guinea-pigs the incubation period was 6 to I9 days, average 9 days; and the total duration 9 to 93 days, average 35 days. In both these species of animals death often occurred very suddenly. In monkeys (Cevcopithecus) the average incubation period was 6 days (limits, 4 to Io days), and the disease lasted 56 days (limits, 28 to 86 days). (Gray and Tulloch.) $\left.{ }^{2}\right]$

[A bullock injected with trypanosomes (after passage through a monkey and then a dog) became infected after an incubation period of twelve days. During the course of the disease there was well-marked intermittent fever, the temperature rising to $105^{\circ}$ or $106^{\circ} \mathrm{F} .\left(4 \mathrm{I}^{\circ} \mathrm{C}\right.$.) every day, and sometimes even exceeding $107^{\circ} \mathrm{F}$. $\left(4 \mathrm{I} \cdot 6^{\circ} \mathrm{C}\right.$.). The symptoms were slight: some weakness and wasting, also slight opacity of both corneæ. Trypanosomes were constantly present, and two days before death the animal appeared obviously ill, lying down and unable to rise. It died two and a half months after inoculation, the evolution of the disease, as well as the post-mortem appearances, closely resembling those observed in the natural infection. The brain was not unlike that seen in sleeping sickness cases, but careful study of it by Mott ${ }^{3}$ failed to reveal any meningo-encephalitis or bacterial infection. The ganglion cells, however, showed chromolytic changes, and there were many minute capillary hæmorrhages. The vessels were full of bodies resembling Leishman's bodies.]

[The disease appears to be propagated by tsetse-flies, $G l$. pallidipes and $G l$. palpalis occurring where the disease is endemic. Gl.palpalis fed on an infected monkey, and six and twenty-four hours later on healthy monkeys, were able to convey the disease to the latter. In the stomach contents of $\mathrm{Gl}$. palpalis actively motile trypanosomes were found Ioo hours after a meal of infective blood. In one of the flies dissected seventy-one hours after a feed the trypanosomes were very numerous and very actively motile. When stained they appeared quite different from the cattle trypanosomes, and were probably 'wild' tsetse-fly trypanosomes (see Chapter XVIII., "The Trypanosomes of the TsetseFly'). No developmental or sexual stages could be made out in the stomach contents of the flies; the changes in the trypanosomes all appeared to be degenerative. In the stomach contents of Stomoxys this variety of trypanosome was found active only twenty-four hours after a feed.]

[Greig and Gray think that this trypanosome 'most closely approaches the classical African type (nagana), and is probably identical with it.'

1 [One of the inoculated monkeys (Cercopithecus) which showed the trypanosome in its blood eight days after inoculation harboured filaria embryos which appear to be a new species. They were about $200 \mu \operatorname{long}(189 \mu$ to $218 \mu$ ), had a pointed tail and no sheath. There was a clear space at the head, and the break in the continuity of the protoplasm was about $46 \mu$ from the head end. A Stomoxys fed on this monkey had actively motile filaria, as well as trypanosomes, in its stomach twenty-four hours after feeding. No trace of the parent worm could be found post-mortem.]

2 [Gray and Tulloch, S. S. Comm. Rep., No. 8, 1907, pp. 7 1.80.]

3 [Mott, Proc. Roy. Soc., Ser. B, v. 76, I905, pp. 239-241.]

4 [Greig and Gray, loc. cit., p. 194.] 
From the morphological appearances seen in the naturally infected cattle and in some of the inoculated animals, it rather appears to resemble T. dimorphon.]

[(3) Trypanosomiasis of the Abyssinian Frontier.-This trypanosome was obtained from an English dog whicl had accompanied the Abyssinian Boundary Commission. Other animals had succumbed to the same disease-namely, eleven Boran and Abyssinian ponies, several camels, and five dogs. None of the Abyssinian donkeys or mules were affected, and an Abyssinian dog which had accompanied the English dog throughout the expedition remained quite healthy. ${ }^{1}$ From the boundary the route taken was via Lake Rudolph and Baringo to Nakuru. Two ponies died of the disease at Nakuru, and as the journey from Baringo to Nakuru occupied only four days, the infection probably occurred at or near the boundary, and certainly some distance north of Baringo.]

[The affected $\operatorname{dog}^{2}$ was half Airedale and half bull terrier. It had previously been famed for its pluck, but afterwards became very nervous. The dog developed opacity of both corneæ, and became emaciated. Towards the end there was irregular fever, and the number of trypanosomes in the blood varied considerably from day to day. The total duration of the disease in this dog could not be ascertained exactly, but it was probably two and a half to three months.]

[Post-mortem the only noteworthy lesions were considerable enlargement of the spleen, slight enlargement of the abdominal lymphatic glands, and small embolic areas under the pleuræ. All the other organs and cavities were normal.]

[Morphology of the Trypanosome.-In the naturally infected dog and in injected animals the trypanosomes were long and slender; total length, $20 \mu$ to $30 \mu$; free flagellum, $6 \mu$ to $8 \mu$. The posterior end was, as a rule, sharply pointed. On one occasion, when the parasites were very numerous in the dog's blood, many 'paired' forms were seen, in which the posterior ends overlapped considerably, and appeared to be fused.]

[Animal Inoculations.-Dogs, monkeys, a guinea-pig, rats, a sheep, goat, ox, Masai donkey, and dog-faced baboon were inoculated with this trypanosome. The dogs, monkeys, guinea-pig, rats, and donkey showed trypanosomes in their blood, and all succumbed to the infection. The disease was more chronic than that produced by the 'Jinja-cattle' trypanosome-the infection in the guinea-pig lasting for nine months, for example. The ox, sheep, goat, and baboon never showed trypanosomes in their blood, but a dog injected with the ox blood became infected, showing that the ox was not immune against this variety of trypanosome. Without having tested by inoculation into dogs whether the blood of the sheep or goat was infective or not, Greig and Gray inoculated these two animals with the Jinja trypanosome, with fatal results. They conclude from this experiment that the Jinja and Abyssinian trypanosomes are

${ }^{1}$ [This dog was susceptible, however, as it became infected after experimental inoculation with the trypanosome.]

${ }^{2}$ [The disease in this dog was diagnosed by Mr. R. J. Stordy, Chief Veterinary Officer of the Uganda and East Africa Protectorates. We take this opportunity of thanking Mr. Stordy for sending the dog to us, and so enabling us to study this trypanosomiasis.] 
different. As Mesnili points out, this conclusion is not justified, because (I) it had not been proved by injection into dogs that the animals were not infected with the first trypanosome, and the second trypanosome may have been a more virulent strain of the same species; and (2) it had not been proved that the sheep and goat were cured and immunized against the first trypanosome when the second was injected. Even if they were cured and immunized against the Abyssinian virus, the objection might still be raised that this vaccine was too feeble to immunize against the more virulent strain (the Jinja variety).]

[The chief symptoms in the susceptible animals were irregular fever, wasting, and, in some cases, rapid anæmia. Edema, glandular enlargements, and opacity of the cornea were absent.]

[Post-mortem the spleen was somewhat enlarged, and small hæmorrhages were present under the pericardium and pleuræ. There were no other lesions.]

[The disease may possibly be carried by tsetse-flies. Austen states that Gl. fusca has been found on the north-eastern shores of Lake Rudolph, but we have no information as to the presence of Glossina at or near the Abyssinian boundary, where the disease was probably contracted by the Commission's animals. Gl. palpalis fed on an infected monkey, and six and twenty-four hours later on healthy monkeys, conveyed the disease to the latter. In the stomach contents of Gl.palpalis sluggishly-motile trypanosomes were found only five and a half hours after feeding on an infected animal ( $c f$. the Jinja trypanosome - Ioo hours). In Stomoxys active trypanosomes were found as long as twelve hours after a meal.]

[(4) Trypanosomiasis of a Mule in Entebbe.-The mule in which the disease was discovered had been in Africa about five years. At first it was in the 'fly belt' in the East Africa Protectorate. At the time it came under observation it had been in Uganda about eighteen months, and remained well until it had fever and swelling of the glands in the groin.]

[It was first noticed to be sick on July 3, I903, but was not brought up for examination until September 9. It then had fever $\left[105^{\circ}\right.$ to $107^{\circ} \mathrm{F}$. $\left(40^{\circ} 5^{\circ}\right.$ to $\left.\left.4 \mathrm{I}^{\circ} 6^{\circ} \mathrm{C}.\right)\right]$, was rather wasted, and the glands in the groin were slightly enlarged. There was no œedema or swelling of the sheath. Its appetite was good. A few very vacuolated trypanosomes were found in blood-films. Three days later the blood was very pale and watery, and no trypanosomes could be found in films. The next day the animal fell down and was unable to rise again; its breathing was very hurried, and it died shortly after. During the last four days of life the temperature was raised ( $105^{\circ}$ to $107^{\circ} \mathrm{F}$.).]

[Post-mortem nothing characteristic was found; the organs were pale and the spleen was enlarged. No trypanosomes could be found on centrifuging Io c.c. blood taken half an hour after death, but this blood was found to be infective on injection into monkeys.]

[Morphology of the Trypanosome.-The parasites were very scanty in the blood of the mule four days before death. Those seen in blood-films were rather short and very vacuolated. On injecting the blood into a dog, however, the parasites which appeared in the latter were long and thin. In another dog injected with the blood of an infected monkey, which at times had very broad, stumpy trypanosomes in its blood, the trypanosomes were long and thin-average total length $27 \mu$, free flagellum $7 \mu$ to Io $\mu$.

1 [Mesnil, Bull. Inst. Past., v. 3, 1905, p. 776.] 
The undulating membrane and flagellum were well stained, and the posterior extremity was truncated. A very broad form (? sexual) was seen-total length, I7 $\mu$; free flagellum, $6 \mu$; greatest width, nearly $5 \mu$. Similar variations were seen in other animals, the short, stumpy forms, with vacuolated protoplasm, being common in monkeys and a guineapig.]

[Animal Inoculations.-Monkeys, dogs, rats, a rabbit, jackal, baboon, guinea-pig, Masai donkey, ox, sheep, and goat were used. The monkeys, dogs, rats, jackal, guinea-pig, and donkey became infected and died. The rabbit, after an incubation period of fifty-seven days, showed trypanosomes in its blood on two occasions, but was still alive a month later (at the time the report was written). The ox, sheep, goat, and baboon never showed trypanosomes in blood-films, but the blood of the ox was found infective for a dog on injection. The other animals were not tested in this way. The sheep lived nearly nine months, but trypanosomes were never found in its blood. There was no fever, and the animal died in good condition. There was no œdema or opacity of the cornea. Postmortem there was some effusion into the pericardium, and much jelly-like material around the base of the heart. The spleen was not enlarged. From the course of the disease, the post-mortem appearances, and the omission to test the infectivity of the blood for dogs, it is by no means certain that this sheep died of a trypanosome infection, as Greig and Gray think was the case.]

[The ox and goat were subsequently injected with the Jinja variety of trypanosome, which produced a fatal infection in both cases. Greig and Gray conclude from these experiments that the Jinja and mule trypanosomes are different, but the same objections may be raised as in the case of similar experiments with the Abyssinian trypanosomes (see p. 208).]

[The jackal experiment was interesting. The animal devoured an infected monkey, and thirty-six hours later had very many trypanosomes in its blood. The parasites continued present throughout the disease, and the jackal died fifteen days after eating the monkey.]

[Most of the injected animals had irregular fever, and varying degrees of anæmia and wasting. Edema, glandular enlargement, and corneal opacity were absent. Post-mortem the only constant lesion was splenic enlargement. In the brain of the ox which was injected with the mule trypanosome, and later with the Jinja variety, Mott ${ }^{1}$ found a few chromatin bodies, resembling Leishman's bodies, in the small vessels, as well as trypanosomes and forms like the amœboid forms of Bradford and Plimmer.]

[The disease may possibly be conveyed by tsetse-flies. The infected mule had been in the 'fly belt' of East Africa, and we know that Gl. palpalis is present in Entebbe. Gl. palpalis fed on an infected monkey, and twenty-four hours later on a healthy monkey, conveyed the disease to the latter. In the stomach contents of these flies sluggishly motile trypanosomes were found twenty hours, and in the stomach of Stomoxys as long as thirty hours, after a meal of infective blood.]

[This mule trypanosomiasis resembles the Abyssinian Boundary disease in being less virulent for experimental animals than the Jinja cattle disease. This was most marked in the case of ruminants, which never showed the first two trypanosomes in blood-films, yet on subsequent inoculation with the Jinja trypanosome succumbed to the infection. As has already been stated, this

1 [Mott, Proc. Roy. Soc., Ser. B, v. 76, I905, p. 240.] 
does not prove conclusively that the trypanosomes are specifically distinct; it may be that the Jinja strain is merely more virulent than the other two strains.]

[The behaviour of these trypanosomes in the stomach contents of biting flies (Glossina and Stomoxys) suggests that they may be at least two distinct species. It was found, moreover, that the Abyssinian and Entebbe mule trypanosomes remained active for a longer time after a meal in the stomach of Stomoxys than of Gl.palpalis. Perhaps these two diseases are more allied to surra than to true nagana, although Nabarro, Grieg, and Gray found that Gl.palpalis was able to convey all three trypanosomes from infected to healthy monkeys. Similar feeding experiments-with intervals of eight and twenty-four hours between the successive feeds upon the infected and the healthy animals-were all negative with Stomoxys. Nevertheless, Stomoxys conveyed the disease directly to several of our experimental dogs, the flies biting the sore and bleeding places on the dogs' ears, and then immediately alighting on similar sore places on the other dogs.]

[Finally, it is possible that the Abyssinian and the Entebbe mule trypanosomiases may be one and the same disease, and identical with the mule disease described by Balfour in the AngloEgyptian Sudan (see Section 7).]

\section{Section 9.-Trypanosomiases other than Dourine, found in Algeria.}

The first authentic record of a trypanosome in Algeria is that of Chauvrat, made in $1892 .{ }^{1}$ This veterinary surgeon discovered it in the blood of a horse suffering from profound anæmia, the horse dying some days later. Chauvrat regarded it as a case of surra; but it has since been looked upon as one of dourine, as a result of the discoveries of Rouget and of Schneider and Buffard of a trypanosome in horses suffering from that disease. It appears, now that the existence of Algerian trypanosomiases other than dourine has been demonstrated, that we must give up this retrospective diagnosis of dourine $;^{2}$ but a certain doubt will always exist, as Chauvrat made no experiments with the parasite.

We shall reserve for the chapter on dourine the discussion of

1 Chauvrat, Rec. méd. vétér., 8th series, v. 3, No. I 1, June I5, I8g6, p. 344. Chauvrat published this note immediately after the announcement by Legrain of Bougie of the discovery of a trypanosome "in a kind of varicose swellings on the outer surface of the pericardium of a cow killed in the slaughterhouse' (Rec. méd. vếér., April I 5, I896. p. 266).

2 The horse, which came from Barika, was brought to Batna, where it died six days later. It was a hide-bound skeleton, suffering from extreme anxmia, and tottering upon its stiff and swollen legs; the abdominal region was also swollen. The day before death its temperature was $39^{\circ} 2^{\circ} \mathrm{C}$. $\left[102^{\circ} 6^{\circ} \mathrm{F}\right.$. . At the autopsy made immediately after death Chauvat found in the heart blood a large number of very active trypanosomes about $60 \mu$ long (?), often joined by their posterior ends. Chauvrat recorcled it as a sporadic case of surra or nagana. The discovery of many trypanosomes in the blood is rather against its being dourine. 
the question whether the trypanosome found by Rouget in I89t, and described by him in 1896 , is or is not the parasite of dourine.

There can be, however, little doubt about the trypanosomiasis of horses in the extreme south of Algeria, in the Oran district, recorded in 1903 by the military veterinary officers Szewzyck ${ }^{1}$ and Rennes, ${ }^{2}$ or about the trypanosomiasis of dromedaries in the department of Constantine, recently described by the brothers Sergent. ${ }^{3}$ Both these trypanosomiases appear to be distinct from dourine.

Trypanosomiasis of Horses.-The disease of horses described by Szewzyck and Rennes was observed amongst the horses of the Spahees of Sidi-Medjaheb and of Beni-Ounif (valley of the Zusfana). Rennes suggests the provisional name mal de la Zusfana for this disease.

The microscopical diagnosis was made by Schneider from bloodfilms which were sent to him by Szewzyck. In these films he found very many trypanosomes, differing (?) morphologically from the trypanosome of dourine. The disease, says Rennes, resembles the chronic forms of trypanosomiasis, but a special character is given to it by certain nervous symptoms, and the almost complete absence of the odemas which are so common in similar affections.

The disease comes on slowly and insidiously. At the outset anæmia is shown only by pallor of the mucous surfaces, loss of vigour when at work, and rapid fatigue. The temperature is a little raised-morning, $38^{\circ} \mathrm{C}$. [100 $4^{\circ} \mathrm{F}$.] ; evening, $385^{\circ}$ to $39^{\circ} \mathrm{C}$. [IOI $4^{\circ}$ to $102^{\circ} 2^{\circ} \mathrm{F}$.] There is progressive wasting, accompanied by general weakness. From time to time there are extreme but very evanescent rises of temperature-in a few hours the temperature may rise from $38^{\circ}$ to $42^{\circ} \mathrm{C}$., or even $43^{\circ} \mathrm{C}$. [Iog. $4^{\circ} \mathrm{F}$.]. The appetite is poor. Sometimes hæmaturia is present as a passing symptom, lasting one or two days and reappearing at varying intervals.

Although weak and thin, the animal may live for months. Weakness of the hind limbs, with pronounced knuckling-over at the fetlock, leads eventually to paraplegia. Finally the animal falls down, the death agony lasting from three to five days, with paralysis of the rectum and bladder. The total duration of the disease is from four to six months.

But in certain cases there are marked nervous symptoms. Rennes has seen a case of true ataxy and one of temporary paraplegia followed by inco-ordination, and later by an attack of vertigo, which carried off the animal.

Rennes has succeeded in inoculating the grey mouse, the jerboa or jumping mouse, and the dog. ${ }^{4}$ The grey mouse dies two or three

1 Szewzyck, Bull. Soc. centr. méd. vétér., 8th series, v. 10, April 30, 1903, p. 220.

2 Rennes, Ibid., September 30, I903, p. 424; April 30, I904, p. 248.

3 Ed. and Et. Sergent, C. R. Soc. Biol., v. 56, January 23, I904, p. I20.

4 [Rennes subsequently infected several other animal species: see later.] 
days after intraperitoneal inoculation, fifteen to twenty days after subcutaneous inoculation. In the latter case the actual duration of the disease (complete paralysis of the posterior part of the body, generalized contractures, and intense dyspnoea) is very short, but the incubation period is prolonged. The parasites swarm in the blood when the earliest symptoms appear. At the autopsy there is considerable enlargement of the spleen.

In the jerboa the disease takes the same course as in the mouse.

The $d o g$ is more resistant; for example, a dog inoculated on April I2, I903, was still alive on May I6. During that time it showed first some congestion at the point of inoculation, and later wasting, pallor of the mucous membranes, and eye lesions (conjunctivitis and milkiness of the cornea, and later on turbidity of the aqueous humour). Trypanosomes were seen in the blood from the sixth day after inoculation.

In other dogs, whose after-history was followed for a longer time, Rennes noted, apart from œdema at the site of inoculation, febrile paroxysms, anæmia, and wasting (which are constant symptoms of the trypanosomiases), general hyperæsthesia, particularly in the lumbar region, and, above all, very marked signs of drowsiness and hebetude. Rennes looks upon these latter symptoms as the most characteristic in the course of the disease. At the end of three months the animals were still alive. Rennes believes that some of these dogs are becoming cured. He draws particular attention to the absence of the characteristic lesions of dourine, and to the analogy between the symptoms of this disease in the dog and sleeping sickness in man.

[The further observations of Rennes ${ }^{1}$ upon the pathogenicity of the Zusfana trypanosome for various animals are summarized by Mesnil ${ }^{2}$ as follows: The experimental disease in $d_{0}$ gs lasts from forty days to more than seven months. When the duration is very prolonged, the animals show the ordinary symptoms of chronic trypanosomiasis-numerous and transitory œdemas, ocular lesions, loss of hair, and extreme cachexia. The infection runs a very similar course in the jackal. Cats die in fifteen to sixty days with the same signs and symptoms as the dog; there is hypothermic lethargy before death.]

[Ruminants are also susceptible. A bull died in fifteen days after intravenous injection. There was an initial rise of temperature, and the blood was infective on inoculation, but no trypanosomes were seen in films. A goat died in six months. At the outset there was fever, and later wasting and slight corneal opacity. Trypanosomes were seen in the blood only once-on the fifth day. A sheep had cedema of the throat and became wasted, but subsequently 100.]

${ }^{1}$ [Rennes, Bull. et Mím. Soc. contr. méd. vítír., February, I \$̧05, pp. 95[Mesnil, in Bull. Hist. Past., v. 3, 1905, pp. 340, 34I.] 
recovered; its blood was infective, although no trypanosomes were seen.]

[A young pig showed no symptoms for five months, but its blood was still infective at the end of that time.]

[A donkey ( $q$ ) became infected after inoculation, but trypanosomes were seen in the blood on only three occasions. At first weakness and wasting were marked, but seven months later the animal appeared well, though its blood was still infective.]

[Rabbits died in thirty to thirty-five days with characteristic symptoms of trypanosomiasis. Trypanosomes were always scanty in the blood. Guinea-pigs died in thirty to forty days. Parasites were more numerous in them than in rabbits. One guinea-pig proved refractory. Rats and mice died in eight to thirty-five days after subcutaneous inoculation.]

' The almost complete absence of œdemas, the complete absence of cutaneous plaques, and of the loss of hair, the special nervous symptoms, and lastly the spread of the disease apart from coitus, clearly differentiate,' says Rennes, 'mal de la Zusfana from dourine.' The last diagnostic feature seems to us to be important, as well as the observation of Schneider that the trypanosomes were very numerous in the blood of naturally infected horses, which is not the case in dourine.

Rennes draws attention to the resemblance of mal de la Zusfana to mal de caderas. The diseases undoubtedly resemble one another, but we cannot say at present that they are identical. It is to be regretted that no mention is made of the size and appearance of the centrosome in the trypanosome of the Zusfana disease, for the mere mention of these characteristics would have been sufficient to answer the question.

It is not very likely that the trypanosome of Szewzyck and Rennes has the characters of the parasite of caderas. Schneider, who has studied it in stained preparations, and who records the differences between it and the trypanosome of dourine-differences rather difficult to appreciate, such as its larger dimensions and the larger number of protoplasmic granules-would assuredly not have omitted to draw attention to so marked a difference in the centrosomes if such had been present.

[Rennes, in his later publication quoted above, says that the trypanosome cannot be distinguished from that of surra or nagana. $\mathrm{He}$ adds that the susceptibility of ruminants to this trypanosome distinguishes it from that of dourine, but Mesnil and Rouget ${ }^{1}$ have recently shown that ruminants are susceptible to the virus of dourine, so this diagnostic point between dourine and the other Algerian trypanosomiases can no longer be relied upon.]

[Roger and Greffulhe ${ }^{2}$ have described, under the name North African surva, a similar, if not identical, disease in horses at Mécheria (Oran).

1 [Mesnil and Rouget, Ann. Inst. Past., v. 20, September, I906, pp. 689 697.]

2 [Roger and Greffulhe, C. R. Soc. Biol., v. 58, March 4, 1905, p. 396 ; May 20, I 905, pp. $826,827$. 
Probably mal de la Zusfana is prevalent in many other parts of Algeria, but its distribution, the mode of transmission, and the animal constituting the reservoir of the virus, all require further investigation. Tsetse-flies do not occur in Algeria, so that this is not true nagana or one of the nagana-like diseases propagated by Glossina; but until more is known of the disease and its trypanosome, it is better not to use the name North African surra.]

Trypanosomiasis of Dromedaries or EL DEBab. ${ }^{1}$ - In October, Igo3, the Sergents detected the presence of trypanosomes in the blood of several dromedaries at Oued-Athménia, department of Constantine. In a herd of twenty animals three were found infected, of which two were thought to be ill by the camel-drivers. One of them, an old female, had aborted each time she had become pregnant. She had been ill for two years, but, except for marked wasting, there was no obvious sign of disease-no ulceration of the vulva or anus, nothing in the belly, eyes, or lips. The mouth temperature was $38.9^{\circ} \mathrm{C}$. [ $\left[\mathrm{IO}^{\circ} \mathrm{F}.\right]$. As many as three parasites were seen in a field with the immersion lens. The third animal was a camel six months old, which showed no sign of disease. There were two or three parasites in each preparation of its blood. It seems, then, that the only symptom is progressive wasting, which ends in death.

The morphology of this trypanosome resembles that of the parasites of nagana and surra, and, moreover, presents no characteristic differences. [The Sergents state, however, in a later paper ${ }^{2}$ that the average size of their trypanosome is I9 $\mu$ long by I' $5 \mu$ broad, the average length of the nagana and surra parasites being $25 \mu$.] The centrosome stains well, and division forms are seen in the peripheral blood. The Sergents inoculated the trypanosome from the blood of the old camel into different laboratory animals. They also made series of passages through rats and mice, by which the virus became altered in virulence.

From the outset white rats were consistently susceptible to the trypanosome. The disease lasted on an average sixteen days after subcutaneous inoculation, and nine and a half days after intraperitoneal inoculation. The incubation period was respectively three days and one day. Three or four days after their appearance in the blood of the rats the parasites diminished in number or disappeared completely for some days, then reappeared and were constantly present. When death occurred, the trypanosomes had either become very numerous or they diminished in number during the last few days of the animal's life.

1 That is the name which the Sergents suggest. In publications of the period I $850-1860$, particularly in Vallon's paper upon the dromedary, this disease of dromedaries, which at that time formed the subject of several works, was thus designated. El debab means the fly, or horse-fy. According to information obtained from the Arabs by the Sergents, the disease is contracted in the Tell, where the horse-flies occur, during the short period of summer (forty days only). See in this connection Railliet, Zool. médic. et agricole, p. 793.

${ }^{2}$ [Ed. and Et. Sergent, Ann. Inst. Past., v. 19, 1905, pp. 17-48]. 
At the autopsy the only lesion noted was an enormous hypertrophy of the spleen, which in some cases weighed ten times as much as the normal.

Sewer rats reacted as a rule in the same way as white rats, but some of them lived for a longer time. Thus, the Sergents had a sewer rat which lived for five and a half months after inoculation. It showed trypanosomes in its blood at first nearly every day, then at fairly long intervals, and continued to do so until it died.

White mice seem to be a little less susceptible than white rats. Some of them died in twelve days with the parasites swarming in the blood, but in others the disease was more prolonged, and on some days the parasites were absent from the peripheral blood. The average duration of the incubation period was four days after subcutaneous inoculation, and two days after intraperitoneal inoculation. At the autopsy there was very considerable enlargement of the spleen.

Grey mice reacted very irregularly, some of them not becoming infected after inoculation with virulent blood. Others developed a mild infection, which was only temporary, and these animals appear to have recovered.

By repeated passages through rats and mice, the virulence, which at first varied somewhat for different animals of the same species (particularly mice), became more constant and at the same time more exalted. Inoculations with such an exalted virus produce a form of the disease which progresses, with constant increase in the number of parasites in the blood.

[In later experiments it was found that this debab virus, which at first killed mice in about twelve days, became less virulent for mice after being passed through rats for two years. Thus, five white mice, after injection with rat's blood, developed only a slight infection, from which they recovered cured, but not permanently immunized. That they were cured is shown by the fact that the blood and extracts of the organs of two of them-killed one year after inoculation-did not infect rats. Two others of these cured mice were not immune, but were much more resistant than control mice. The latter died in seven to eighteen days, the two former in fifty-four and sixty-one days.]

Rabbits reacted to inoculation with this trypanosome, as they do with the other pathogenic mammalian trypanosomes. The disease followed an irregular course; the trypanosomes increased from time to time in the blood, the increase coinciding sometimes, but not always, with a rise of temperature. There were also œdema of the genital organs and anus, loss of hair about the tail and the base of the ears, and purulent conjunctivitis. In three rabbits the average duration of the disease was nineteen days, but other rabbits survived fifty days. ${ }^{1}$ After subcutaneous, intraperitoneal, and intra-

${ }^{1}$ [One hundred and fifty days is the maximum given in a later publication of the Sergents.] 
venous inoculation, the incubation period was eight and a half days, six days, and two days respectively.

In guinea-pigs the shortest duration of the disease was twelve days, but some lived for more than four months, two guinea-pigs, still alive, having been infected for four and a half and seven and a half months. [One guinea-pig ( $q$ ) lived eleven months after inoculation. ${ }^{1}$ She gave birth to two uninfected young ones, which were found to be normally susceptible to the virus.] The incubation period was, on several occasions, only three days after subcutaneous inoculation, but was sometimes much longer. After intraperitoneal inoculation it was about four and a half days. The parasites were, as a rule, numerous in the blood of the guinea-pig, and the animal sometimes lived in that condition for several weeks. The external lesions so characteristic in the rabbit were not seen in the guinea-pig.

In the $d o g$ the course of the disease was rather irregular, the appearance of the parasites in the peripheral circulation coinciding with the rises in temperature. In a dog which died thirty days after inoculation the parasites swarmed in the blood towards the end of the animal's life, and this was accompanied by a marked fall of temperature. The other dogs, which died in thirty-five and thirty-seven days, did not have a subnormal temperature.

A Macacus died two months and eight days after subcutaneous inoculation. The parasites were fairly numerous from the fourth to the seventh day, when the monkey had a subnormal temperature. The trypanosomes then disappeared from the blood, and became fairly numerous again from the fifteenth to the seventeenth day; but from that time they were very scanty or even absent on microscopical examination, particularly during the last month. As a rule, the monkey's temperature was subnormal, and the presence of trypanosomes definitely concided with the rises of temperature above $37^{\circ} \mathrm{C}$. $\left[98^{\circ} 6^{\circ} \mathrm{F}.\right]$. The monkey died with a subnormal temperature, after having slept for two or three days. ${ }^{2}$ On the last day the temperature was below $25^{\circ} \mathrm{C}$. $\left[77^{\circ} \mathrm{F}\right.$. , yet trypanosomes were present.

A goat weighing 34 kilogrammes became infected after subcutaneous inoculation with the blood of a rat. At the end of five day's trypanosomes were seen on microscopical examination, and subsequently they were only seen again, and then only in very small numbers, from the tenth to the fourteenth day. After that time microscopical examination was always negative; but rats inoculated with 5 c.c. of the goat's blood one month and two months after the inoculation of the goat very rapidly became infected. The goat, which appeared on the road to recovery, died very suddenly three months after inoculation. During the progress of the disease its weight had fallen to 2 I kilogrammes, but at death it had risen again to $27 \frac{1}{2}$ kilogrammes.

1 [This is the maximum duration given in a later publication of the Sergents.]

${ }^{2}$ [In the Sergents' paper, Ann. Inst. Past., v. 19, Ig05, it is stated that the monkey slept for a fortnight before death.] 
Lastly, the Sergents inoculated a horse, which died in three and a half months. It had intermittent fever, with rises of temperature to about $40^{\circ} \mathrm{C}$. from the eighth to the eleventh day and from the sixteenth to the eighteenth day, and to between $40^{\circ}$ and $4 \mathrm{I}^{\circ} \mathrm{C}$. $\left[\mathrm{IO}_{4}{ }^{\circ}\right.$ to $\mathrm{IO} 5^{\circ} 8^{\circ} \mathrm{F}$.] from the twenty-third to the twenty-sixth day, from the thirty-fourth to the thirty-eighth, forty-fifth to forty-eighth day, and on the sixty-sixth, seventy-seventh, and ninety-fifth days. In the intervals the temperature was generally normal. These rises of temperature coincided with increases in the number of parasites in the blood, which were, as a rule, only visible in films during these febrile attacks. During several of the paroxysms they were numerous in the blood; they were scanty or absent during the few days before death. The course of the disease closely resembled that of surra.

At the end of a week the horse had a patch of œdema the size of a hand in the abdominal wall, and also œedema of the sheath. This odema spread in the form of a longitudinal band, occupying the whole ventral region. During the course of the second month the horse passed dark brown or reddish urine, but neither red corpuscles nor hæmoglobin could be detected in it. The horse died in a wasted condition.

[Several bats (Myotis murinus) were inoculated by the Sergents intraperitoneally, but they died in six days without becoming infected. Nicolle succeeded, however, in infecting bats (Vespertilio tuhli) with this trypanosome. The infection was a mild one, and the bats recovered from it (see p. IIO).]

This trypanosomiasis of dromedaries does not appear to be dourine. The pathogenicity of its trypanosome for the goat and monkey, ${ }^{1}$ and the fact that the young camel which had never had connection became infected, make this diagnosis improbable. Moreover, the characteristics of the disease experimentally produced in the horse differ markedly from the normal course of dourine. In the former disease (I) the febrile paroxysms are not restricted to the onset of the disease; and (2) trypanosomes are often present, and even numerous, on microscopical examination, which is never the case in dourine.

The trypanosome of the Sergents is evidently different from that of caderas, since its centrosome is easily visible.

What is the relation of this trypanosomiasis of dromedaries to nagana and surra? This is a question requiring further study for its elucidation. It is particularly important to compare this disease with the trypanosomiasis of dromedaries in Timbuctoo. The disease attacks the same animals, and the natives call it by the same Arabic name $(e l d e b a b)$ in the two districts. The disease is spread most

1 [This argument no longer holds good, since Mesnil and Rouget have shown that monkeys and goats are susceptible to the virus of dourine; nevertheless, the observations of the Sergents recorded later prove almost conclusively that debab and dourine are different diseases.] 
probably by means of these debab, which are similar, if not identical, flies (horse-flies).

These are the only two African diseases in which insects other than the tsetse are incriminated. ${ }^{1}$ Finally, if intercourse by means of caravans between Timbuctoo and Twat is not frequent, it none the less exists, and it is quite likely that the Algerian and Sudanese diseases in question may have a common origin.

[The recent observations of the Sergents ${ }^{2}$ have materially increased our knowledge of these Algerian trypanosomiases. In addition to dourine, there are two diseases-(I) the well-defined disease of dromedaries, called debab; and (2) the isolated cases or limited epizootics amongst horses called by Rennes mal de la Zusfana. The natives in various parts of Algeria have long recognised these diseases of camels and horses, which they say are occasioned by the bites of horse-flies. The horses in Constantine are said to become infected in the spring-when these flies are very prevalent-and never to survive the succeeding winter. The symptoms usually appear with the cold season. There is marked dragging of the legs, but wasting and loss of appetite and strength are absent. In the Oran animals, which become infected in the Tell district (see footnote, p. 214), the symptoms are more marked. They include loss of appetite, fatigue, wasting, hanging of the head, staring of the coat with partial loss of hair, and œdemas; there is no marked weakness of the hindquarters or limbs. The disease is always fatal in horses, and lasts a shorter time than debab does in dromedaries.]

[The examination of the blood of animals from various parts of Algeria shows that the disease in dromedaries is very widely distributed. Of $35^{2}$ dromedaries examined during I 904 and I905, 33 (9*4 per cent.) were found to be infected, whereas of 594 Equidx examined during the same period only I ( $0^{\circ} I 7$ per cent.) was found infected.]

[Big game cannot be the reservoir of the virus in Algeria; therefore it is probably the dromedaries which serve as the source of infection for camels and horses. The occurrence of these small epizootics among the horses of the Spahees is explained by the occupation of oases by cavalry, and by the habits of the Tabanidæ, which prick several horses - sufficiently to inoculate them with the virus-before finally settling on one to feed.]

[The Sergents think that the two diseases, as observed in dromedaries and in horses, are probably identical. Taher and tmerdjin are two local names for the horse disease, but these should be merged into debab if certain cross-inoculation experiments still in progress show that the diseases are identical.]

[The Experimental Study of the Viruses.-The taher virus killed white vats in six to forty days, and a vabbit in five months. Guinea-pigs were easily infected, but showed no lesions. In a sheep no trypanosomes were found, but the blood was still infectious for rats on the thirty-third day.]

[Oran debab killed white rats in sixteen to seventy-five days, and white mice in seven to thirty days. Guinea-pigs and sheep reacted as with the taher virus.]

[Constantine $d e b a b$, which was the virus used by the Sergents in the experiments already recorded (see pp. 2I4-2I 7 ), was passed through rats for a year, then inoculated into a goat, in which it was found ten months later, and afterwards again passed through rats. Two sheep were still infected a year after inoculation, and two goats ten months after inoculation.]

1 [We have seen, however, in various parts of this chapter, that several other trypanosomiases, resembling nagana, have been described in districts where the tsetse-fly has not been found.]

${ }^{2}$ [Ed. and Et. Sergent, Ann. Inst. Past., v. 20, August, 1906.] 
[INFECTION EXPERIMENTS.-Tabanidæ seem to be the carriers of the infection. Two species-Atylotus (Tabanus) nemoralis (Meigen) and At. (Tab.) tomentosus (Macquart) - are specially incriminated. The Sergents were able to convey the disease to healthy animals by a single prick, without suction of blood, of a single fly. These positive results were obtained when there was no interval between the pricking of the diseased animal and that of the healthy animal, and in one case after an interval of twentytwo hours. Motile trypanosomes were never found in the stomach of Tabanidæ one hour after a feed. Stomoxys and Hamatobia are never found on camels, and are consequently of no importance in the propagation of the disease.]

[Similar experiments with the same species of Tabanidæ gave positive results with the trypanosomes of nagana (five out of nine positive), mal de la Zusfana (one out of six), and dourine (two out of two). These positive results were all obtained when the pricking of the infected and of the healthy animal took place without an interval. All the other experiments were negative.]

[Experiments with young ticks, hatched from eggs laid by a mother living on heavily infected animals, were negative. Inoculation experiments by way of intact mucous membranes (conjunctival and genitalmale and female) gave positive results with dourine in four cases out of eight. Similar experiments with debab (six in number) were all negative.]

\section{Section 10.-Trypanosomiases of the French Sudan. ${ }^{1}$}

Trypanosomiasis of Dromedaries or Mbori.-Early in the year I903 the military veterinary officer Cazalbou was commissioned to investigate the disease of the dromedary known as mbori, or the fly disease, which causes the death of most of the camels coming from the Sahara to the Sudan.

Cazalbou, at Timbuctoo, examined seventeen dromedaries which were said to be infected. The animals were wasted, and sixteen of them showed trypanosomes in the blood.

The sick animals have rises of temperature to about $40^{\circ} 5^{\circ} \mathrm{C}$. $\left[105^{\circ} \mathrm{F}.\right]$, and it is when the temperature is high that the trypanosomes are most numerous in the blood. Fever and wasting are the only constant symptoms of the disease. There is no œdema, swelling of the limbs, or paralysis. Frequently there is a copious watery discharge from the eyes, and sometimes diarrhoa is present.

The disease usually ends fatally after an average duration of five to six months. Animals which recover are immune.

Cazalbou took to Ségou two dogs and two sheep which had been inoculated at Timbuctoo with the blood of camels suffering from mbori. He was thus able to study the course of the disease in different species of animals. A dog which was brought to Paris enabled one of us to corroborate and to complete Cazalbou's experiments.

1 [A. Laveran, 'Reports to the Académie de Médecine,' June 30, I903, and April 26, 1904. [L. Cazalbou, Rec. de méd. vétér., v. 81, October I 5, 1904, p. 61 5 ; C. R. Soc. Biol., v. 58, April 1, 1905, pp. 564, 565; Revue gén. méd. viét., v. 8, I906, pp. 240-248. Pécaud, C. R. Soc. Biol., v. 60, I906, pp. 58, 59.] 
The results of the researches upon this trypanosome may be summarized as follows:

Rats and Mice.-In the grey rats of the Sudan which were inoculated by Cazalbou, subcutaneously or intraperitoneally, with blood rich in trypanosomes the parasites appeared in the blood on the second or third day, and gradually increased in number until death, which occurred on the eighth or ninth day. In white rats in Paris the parasites appeared in the blood on the third or fourth day, and became increasingly numerous until death, which occurred from the thirteenth to the sixteenth day. The spleen was greatly enlarged, weighing $2 \frac{1}{2}$ to 3 grammes in rats of 165 to Igo grammes.

In the grey mice (? species) of the Sudan the trypanosomes appeared from the third to the sixth day after inoculation. At first they were numerous in the blood on several occasions, but later on they were present only in small numbers and at longer or shorter intervals. Of four mice inoculated at Ségou, two died II 5 and I4I days after inoculation; the other two were still alive 138 and 148 days after inoculation.

Two wild grey mice in Paris died in eight and nine days. Three white mice (Paris) died in seven, fourteen, and twenty-four days. Trypanosomes appeared in the blood two to three days after inoculation, and steadily increased in number until death occurred.

In the 'giant rat' of the Sudan the parasites appeared on the seventh day, and increased in number until the eleventh to the thirteenth day, when death occurred. Adult rats were more resistant than young rats.

Guinea-pigs and Rabbits.-Of four gninea-pigs inoculated in Paris only one showed many parasites in its blood; in the other three the parasites were always scanty. Three of these animals died in twenty-seven, twenty-eight, and forty days; the fourth was still alive two months after inoculation, [but died on the r26th day with numerous trypanosomes in its blood]. ${ }^{1}$ Post-mortem the spleen was only slightly, if at all, enlarged; [but in the animal which survived 126 days the spleen weighed 3 grammes, the total body-weight being 500 grammes]. ${ }^{1}$ Two rabbits inoculated-the one intraperitoneally, the other subcutaneously-became infected. One month later the trypanosomes were very scanty in the blood, and one of the rabbits was very thin and was suffering from double blepharo-conjunctivitis. Of these two rabbits, one died on the fifty-first day of the disease; the other was still alive two months after inoculation, but the trypanosomes were extremely scanty in its blood. [This rabbit died ninety-six days after inoculation; its weight was 1,360 grammes, that of the spleen being I gramme]. ${ }^{1}$

Dogs.-Four Sudanese dogs died thirty, forty-eight, fifty-six, and seventy-five days after inoculation. The dog in which the virus was brought from the Sudan to France died in sixty-five days. Of two

${ }^{1}$ [Information kindly furnished by the authors.] 
dogs inoculated in Paris, one died in twelve and the other in seventeen days after inoculation, with many parasites in their blood. The chief symptoms of the disease in the Sudanese dogs were febrile paroxysms, coinciding with increases in the number of the parasites, wasting, and swelling of the intermaxillary space and throat (in three out of four cases). Postmortem there was serous infiltration of the connective tissue of the intermaxillary space and throat with enlargement of the cervical lymphatic glands. The spleen was much enlarged in two out of four cases. In the Sudanese dog which was brought to France the spleen weighed I6o grammes (weight of $\operatorname{dog} 8,300$ grammes); in the dog weighing $9 \frac{1}{2}$ kilogrammes, which died in twelve days, the spleen weighed 60 grammes.

Cats.-A kitten four months old inoculated by Cazalbou on July 24, I903, showed the trypanosome in its blood on the seventh day and afterwards only at intervals. On January I, I904, I6I days after inoculation, the cat was still alive.

[Panisset, ${ }^{1}$ at Alfort, inoculated a cat with the trypanosome of mbori; the cat died in four months. Mbori took considerably longer to kill cats than did the Mauritian surra].

Sheep and Goats. - Two rams inoculated at Timbuctoo with the blood of a dromedary died in twenty and twenty-one days, but it is not certain that they succumbed to the trypanosomiasis. A ram inoculated on May 20, I903, had a series of febrile paroxysms, the temperature going up to $40^{\circ} 3^{\circ}$ to $40^{\circ} 9^{\circ} \mathrm{C}$. [ $\mathrm{IO}_{4} 6^{\circ}$ to $\mathrm{IO} 5^{\circ} 6^{\circ} \mathrm{F}$.] Seventy days after inoculation this animal's blood was still infectious for rats, but Ioo days after it was no longer so, and the ram must be regarded as having recovered from the disease.

A nanny goat injected on August 6, I903, was still infected on November Io (ninety-seventh day). The chief symptoms were rises of temperature and enlargement of the pharyngeal lymphatic glands. Trypanosomes were never found on microscopical examination of the blood, and it was always necessary to inject rats in order to show the presence of the infection. The after-history of the disease in this goat is unknown.

A sheep and a billy goat inoculated in Paris became infected. The trypanosomes were very scanty in the blood of these animals, which never had any rise of temperature.

[The sheep died six and a half months after inoculation. The goat was cured eleven months after inoculation, and had then acquired immunity against mbori, for on reinoculation with the virus of mbori the animal did not become reinfected. ${ }^{2}$ Two months after being unsuccessfully reinoculated with mbori, the goat was subcutaneously inoculated by Laveran with the Mauritian surra trypanosome (see Chapter VIII.). There was a marked febrile reaction-for a fortnight the temperature was about $40^{\circ} \mathrm{C}$. [10 $4^{\circ} \mathrm{F}$.],

1 [Panisset, C. R. Soc. Biol., v. 58, 1905, p. I6.]

2 [Laveran, C. R. Acad. Sciences, v. I4I, December 26, I905, p. I 204.] 
and on three occasions reached $40^{\circ} 8^{\circ} \mathrm{C}$. [105.5 $\mathrm{F}$.]. The blood, however, never showed trypanosomes, nor did any of the test animals inoculated ever become infected. Thus, the goat which had acquired immunity against mbori was also immune against Mauritian surra. This result confirms those of Vallée and Panisset, ${ }^{1}$ who had previously shown that bovines which were immunized against surra were likewise immune against mbori.]

Roebucks (Sudanese).-An inoculated roebuck showed very many trypanosomes in its blood on several occasions. On the I83rd day after inoculation it was still in excellent condition. Another roebuck inoculated simultaneously with the preceding died rapidly with nervous symptoms-inco-ordination of movements and convulsions. Trypanosomes were numerous in its blood.

Horses.-Three horses were inoculated by Cazalbou-two in the jugular vein and the third subcutaneously. All three became infected, trypanosomes appearing in the blood about the fifth day. They multiplied in the blood irregularly, the exacerbations usually coinciding with the febrile paroxysms.

Apart from fever, the chief symptoms observed were wasting, œdema of the scrotum and sheath, swelling of the fetlocks, profuse sweating, epiphora, and conjunctival hæmorrhages. At a later stage of the illness there was an abundant papular eruption, especially on the head, neck and shoulders, back, and hind-quarters. The lesions were covered with scabs, which, on falling off, left small round, superficial sores. One of these horses died 136 days after inoculation; the other two were lost sight of on the I44th and I84th days of the disease.

[A horse inoculated with mbori at Alfort was alive six months later, and its blood was still infectious for test animals. The average duration of surra in the horse is one to two months, so that the trypanosome of mbori is distinctly less virulent than that of Indian and Mauritian surra.]

Pathogenic Agent.-The trypanosome of mbori measures $20 \mu$ to $25 \mu$ long, by $I^{\circ} 5 \mu$ to $2 \mu$ wide at its middle. Usually it is slender, and resembles $T$. evansi more than $T$. brucei. The centrosome is about as large as in these two trypanosomes, and there is no characteristic feature by which this trypanosome can be differentiated from them.

Human serum and trypanred have a marked effect upon the trypanosome of mbori, causing it to disappear, either temporarily or even permanently, from the blood of inoculated mice and rats.

[Laveran has found that mice infected with mbori are rapidly cured by one injection of trypanred. Rats were also sometimes cured by one or two injections of this dye, and in all cases combined treatment with trypanred and arsenious acid gave excellent results in these animals. Mice and rats infected with surra were

${ }^{1}$ [Vallée and Panisset, C. R. Acud. Science, v. I39, November 2I, 1904, p. 901 .] 
more difficult to cure in this way. In mice the injections of trypanred had to be repeated or the combined treatment used; with rats the latter method had always to be employed, or there were many failures.]

Mode of Propagation.-There seems to be no tsetse in the neighbourhood of Timbuctoo, but the natives agree in saying that mbori is propagated by means of a fly called debab, which is a Tabanus.

To sum up, mbori is not sharply differentiated from nagana (in Paris dogs died in twelve days), surra, or the trypanosomiasis of dromedaries in Algeria.

Many of the symptoms of mbori occur also in nagana or in surra: irregular rises of temperature, œdema of the scrotum and sheath in horses, ecchymoses in the mucous membranes, especially the conjunctiva (which is frequently seen in surra, as in mbori), and hypertrophy of the spleen.

Other symptoms seem special to mbori: swelling of the intermaxillary space and throat in dogs, enlarged cervical glands in several species of animals, and in the horse a papular eruption similar to that seen in caderas.

[Cazalbou's original belief that mbori is a distinct morbid entity has been disproved by the experiments of Laveran and of Vallée and Panisset. From these experiments it may be concluded that the trypanosomes of surra and mbori are the same species. The trypanosome of mbori is merely a variety of $T$. evansi, a little less virulent than the trypanosome which produced the serious epizootic in Mauritius. Laveran remarks: "This is the first time that Indian surra has been recognised on the continent of Africa, a grave epizootic having previously been observed (I9O2-I9O4) in the island of Mauritius. It is possible that other African epizootics recently described as due to trypanosomes other than $T$. brucei may likewise be varieties of surra.' $]^{1}$

Trypanosomiasis of Cattle or Soumaya.-Cazalbou describes under the name soumaya or souma, borrowed from the dialect of the Bambaras, a trypanosomiasis which periodically attacks cattle in the French Sudan and kills a large number of them. In Igo3 this epizootic appears to have started in the humped cattle (zebus) of Macina, which arrived at Ségou suffering from the disease. The Macina district, which is a cattle-rearing centre situated higher up the river and to the south-west of Timbuctoo, is periodically inundated by the Niger. It is in November, when the herds of cattle are brought into the areas which are becoming dry, that the epizootic usually breaks out. From this centre it spreads to the neighbouring districts, to Guinea, and as far as the Ivory Coast and

1 [See Laveran's remarks upon Vallée and Panisset's communication in C. R. Acad. Sciences, v. I39, p. 90I.] 
Dahomey, the spread being favoured by the considerable export of cattle from Macina which takes place.

From January to July, I903, of 4,694 head of cattle taken to Ségou 676 died, the mortality being at its maximum in June (I55 per $I, 000)$. If, as is probable, infection occurred in November, the average duration of the disease in the animals which succumbed was seven to eight months.

The two races of cattle in the Sudan-those with humps (zebus), and those without humps-as well as their cross-breeds, are attacked by soumaya.

[Pécaud ${ }^{1}$ has also observed this epizootic in the neighbourhood of Bamako, the new capital of Haut-Senegal and Niger, and of Kati. It affects oxen, Algerian mules, native horses, and asses. The mortality amongst oxen is about 20 per cent. In horses there is fever and œdema of the legs, rarely œdema of the abdominal walls. Death occurs in about fifty days in horses, but in the mule the disease is more chronic, and recovery is more frequent than in the horse.]

[The disease begins in July - the middle of the rainy season-and lasts until January. Pécaud remarks that this seems to be a special trypanosomiasis propagated by the oxen of Macina.]

$\left[\mathrm{Cazalbou}^{1}\right.$ saw the disease in horses in the region of the River Bani. These horses developed the trypanosomiasis after being taken to the river banks, where they were bitten by tsetse and many Tabanidæ.]

In cattle the onset of the disease is insidious, but about the third month there is a certain degree of wasting, which gradually becomes more pronounced. The skin becomes harsh and the coat stares; there is a watery discharge from the eyes, and occasionally slight diarrhœa is present. In the zebu, which always has a well-developed dewlap, there is often odema in that region, sometimes extending to the lower part of the thorax. In the cattle without humps œedema is rare. In the later stages of the disease emaciation is extreme, and at the same time the gait becomes slow and heavy; the hair is thin, and is easily pulled out; there is marked anæmia, digestion is impaired, and diarrhœe is often present.

During the course of the disease there are irregular rises of temperature, the fluctuations being often very considerable [from $96^{\circ}$ to $106.8^{\circ} \mathrm{F}$.] ; death occurs with hyperpyrexia.

The duration of the disease is from four to twelve months, the average being seven to eight months.

Besides the emaciation, there is no constant lesion found postmortem, the spleen being very rarely enlarged.

The trypanosomes are always scanty in the blood; they very closely resemble the parasite of mbori. ${ }^{3}$

1 [Pécaud, C. R. Soc. Biol., v. 60, I906, pp. 58, 59.]

2 [Cazalbou, Rec. de méd. vétér., v. 81, October I5, 1904.]

3 [Details of morphology are given later.] 
Cazalbou thinks that soumaya is propagated by a large Tabanus, which is very common in French Sudan in wet districts, along the borders of ponds and the banks of the rivers. [Pécaud is also of opinion that the disease is propagated by horse-flies (Tabanus), and not by tsetses. Bouffard ${ }^{1}$ has recently succeeded in transmitting the disease experimentally from an infected to a healthy calf by the bites of Stomoxys. He states that large numbers of flies were caught around the different stations in this district, where the mortality amongst horses and oxen was very great. These flies were mainly Stomoxys and Hippobosca; not a single Tabanus or tsetse-fly was among them.]

Cazalbou made experiments on different animals in order to study the pathogenic effects of the trypanosome of soumaya. Three grey rats died in 27, 30, and 58 days. Mice died in 4 to 7 months after inoculation. The cat and the Sudanese $\log ^{2}$ appear to be refractory.

[Laveran, ${ }^{3}$ who has studied this disease and its pathogenic trypanosome in a sheep inoculated by Cazalbou at Ségou with the blood of a naturally infected horse, and taken to Paris in April, Igo6, has come to the conclusion that the trypanosome of souma (or soumaya) is a new species, which he calls $T$. cazalboui.]

[Morphology.-The length of this trypanosome, including free flagellum, is about $2 \mathrm{I} \mu$; its width $I^{\circ} 5 \mu$. The nucleus is oval, and is situated about the middle of the body. The centrosome is distinct, round in shape, and close to the posterior end of the body, which is rounded off. There are fine chromatic granules in the cytoplasm. The undulating membrane is poorly developed, and shows few folds, like $T$. lewisi. It is bordered by the flagellum, which becomes free at the anterior end. Division of the trypanosome is by binary fission, and usually starts in the centrosome.]

[In its pathogenic effects upon different species of animals, T. cazalboui differs markedly from allied species of trypanosomes. The natural infection occurs in Equidæ and Bovidæ. Small ruminants (sheep, goats, antelopes) are very susceptible, but rodents. (rats, mice, guinea-pigs), monkeys, and dogs are, as a rule, refractory. Pécaud also found the dog refractory.]

[In the goat and sheep the incubation period is about ten days. The principal symptoms are fever (the temperature rose to $4 \mathrm{I}^{\circ} 3^{\circ} \mathrm{C}$. $\left[\right.$ Io6 $^{\circ} 4^{\circ} \mathrm{F}$.] in one case), wasting, and general weakness. In the two goats inoculated by Laveran kerato-conjunctivitis occurred at an early stage of the infection. Trypanosomes were often found in blood-films, which is not the case in other trypanosome infections in these animals.]

1 [G. Bouffard, C. R. Soc. Biol., v. 62, 1907, p. 71.]

2 [Cazalbou originally said that dogs were susceptible and died in three to five months (see Laveran and Mesnil, Orig., p. I98), but in a letter to Laveran, dated January 5, I906, Cazalbou states that dogs inoculated with souma did not become infected. In' his earlier experiments he was evidently dealing with another trypanosome.]

${ }^{3}$ [Laveran, C. R. Acad. Sciences, v. I43, July, I906, p. 94.] 
[Cross-inoculation experiments show that souma is distinct from surra and mbori. $]$

[The Macina appears to be the principal focus of souma in the French Sudan, but Pécaud has also observed it at Bamako and Kati, in the district of Moyen-Niger. The same disease has been described by G. Martin in French Guinea (see next section), and possibly the trypanosomiasis of cattle in Erythrea (Memmo) and that of cattle in Uganda (see p. 205), are due to this trypanosome.]

[In addition to mbori and souma, there appears to be a third trypanosomiasis in the Haut-Niger district (French Sudan), for in one of the inoculated sheep brought to Paris by Cazalbou, Laveran found a trypanosome closely resembling that of the Gambian horse disease, $T$. dimorphon. $\left.{ }^{1}\right]$

[In a recent paper ${ }^{2}$ Laveran states that it is probably a new species of trypanosome, for which he proposes the name $T$.pecaudi. A sheep which recovered from the infection and proved refractory on reinoculation subsequently developed a typical infection with $T$. dimorphon. From this Laveran concludes that $T$. pecaudi and $T$. dimorphon are distinct species.]

[As in the case of $T$. dimorphon, two forms of the parasite occur in the blood of infected animals : (I) Long, slender parasites, $25 \mu$ to $35 \mu$ long, by about $I^{\circ} 5 \mu$ wide. Posterior end more or less pointed; undulating membrane very narrow; free flagellum fairly long; nucleus elongated. (2) Short, broad forms, I4 $\mu$ to $20 \mu$ long, by $3 \mu$ or even $4 \mu$ wide. Posterior end very blunt ; undulating membrane very wide and with few folds; no free flagellum; nucleus rounded. All the inoculated animals showed both forms, and intermediate stages were rarely seen.]

[Human trypanosomiasis is rare in the Haut-Niger district, but is endemic in several other parts of the French Sudan.]

\section{Section 11.-The Trypanosomiases of French Guinea.}

[In I904 Laveran ${ }^{3}$ found trypanosomes in blood-films taken from two sick horses at Conakry, in French Guinea, by Dr. Tautain. One horse belonged to the station of Télémélé, in Fouta-Djalon, the other to that of Toumanéa, on the boundary between Fouta-Djalon and the Sudan. The latter horse was taken from Toumanéa to Sanguiana (in Balaya), where it remained a month, and where it was bitten by flies called sigui tégué. On its return to Toumanéa it began to waste. The wasting increased; there was extensive œedema of the abdominal walls, and later of the hind-limbs; fever was almost continuous at this time, but for a fortnight before death, which occurred about three months after the horse was brought back to

\footnotetext{
${ }^{1}$ [In his article in the Rec. de méd. vétir. Cazalbou states that true nagana occurs along the banks of the River Bani, and another trypanosomiasis-baleri-in the Haute-Volta district.]

2 [Laveran, C. R. Acad. Sciences, v. 144, 1907, p. 243.]

3 [Laveran, C. R. Soc. Biol., February 27, 1904; C. R. Acad. Sciences, v. I39, 1904 , p. 658 , and v. 140,1905, p. 75.$]$
} 
Toumanéa, the temperature was generally subnormal. Towards the end of the illness weakness and wasting were extreme; there were no eye lesions.]

[Post-mortem the spleen and liver were enlarged and friable; small ecchymoses were present under the serous membranes, and there was gelatinous substance about the heart.]

[The trypanosomes found in the blood of these two horses were short (about I4 $\mu$ in length), and morphologically resembled T. dimorphon.]

[This observation of Laveran proves that in French Guinea an equine trypanosomiasis exists, as well as the human disease which is endemic in the greater part of Upper Guinea. But it is to the very thorough investigations of G. Martin ${ }^{1}$ that we are indebted for our present knowledge of the various trypanosomiases of French Guinea. Martin found numerous trypanosomiases existing in Lower Guinea, in the mountainous districts of Fouta-Djalon and of Labé, and along the banks of the Niger and of the Rio-Nunez. [These diseases are present throughout Upper and Lower Guinea in endemic form, and epidemics frequently arise in which the mortality is from 30 to 40 per cent.]

[These diseases were acute or chronic, and natural infections were met with in the horse, ass, mule, ox, sheep, goat, dog, and pig. There appear to be at least two animal trypanosomiases in French Guinea: 'the one, the more important, caused by $T$. dimorphon, which was found, not only in the blood of Equidæ and of Bovidæ, but very probably also in that of sheep, dogs, and pigs; the other particularly prevalent in the region of the Niger, and affecting Equidæ and Bovidæ imported from Belédougou and Macina, which is due to T. cazalboui' (Martin). The trypanosome of the goat disease also resembled that of souma ( $T$. cazalboui).]

[The more important of these trypanosomes, the $T$. dimorphon, examined fresh, showed parasites of various sizes, some very active, but the majority only slightly motile, and not travelling out of the field of the microscope. The undulating membrane is feebly developed. The trypanosomes, when numerous in the blood of a rat or mouse, show a marked tendency to agglutinate as soon as the film is made. In stained specimens, too, many paired forms are visible, the joined ends overlapping to some extent, so that the centrosomes come to lie side by side. The trypanosomes measure I3 $\mu$ to I5 $\mu, 20 \mu$ to $23 \mu$, and some even $27 \mu$ to $28 \mu$.]

[In stained films of the blood of animals inoculated with trypanosomes from different sources, Martin was unable to see, as were Laveran and Mesnil, and Thomas and Breinl (see next chapter), the long free flagellum described by Dutton and Todd. Even in the long forms of the parasite, the free flagellum is rudimentary or absent. Nevertheless, in the blood of naturally infected animals flagellated forms were sometimes seen, and these may even persist for a time in the inoculated animals. These observations confirm those of Dutton and Todd, and show that the forms

1 [G. Martin, 'Les Trypanosomiases de la Guinée Française,' Paris, I9o6, pp. I-I20, with several figures and maps, A. Maloine; also C. R. Soc. Biol., v. 6I, I906, pp. I07-109. The account given of these diseases is taken almost verbatim from Martin's summary (Chap. I.) in his monograph.] 
with free flagellum may exist for some time and then disappear. It will be remembered that Martini observed similar morphological changes in his two Togo trypanosomes.]

[Animals infected spontaneously are wasted and walk slowly and with difficulty, owing to great weakness of the posterior extremities; the hair falls off, digestion is impaired, and blindness is not infrequently observed.]

[Rats, mice, and guinea-pigs were susceptible to all the strains of $T$. dimorphon met with. Rats died in I4 to 30 days; mice died either fairly quickly ( 5 to I I days) or slowly (40 to 48 days); guinea-pigs died in I2, 39, and 57 days; a vabbit died in 23 days. Monkeys (Cercopithecus callitrichus) died in 43 days, 3 months, and 5 months. A heifer lived 54 days; cats, I9, 20, and I5I days; dogs, II, I6, 22, 30, and 97 days; a sheep 3 months and I 3 days. In all the animals the spleen was much enlarged. To put it briefly, after a fairly long incubation period, these different strains of trypanosomes produced in all the experimental animals subacute or chronic infections, resembling one another and also those observed with $T$. dimorphon.]

[Differentiation by Laveran and Mesnil's method with cured animals was not possible, for Martin's animals did not acquire immunity. A billy goat and a sheep, cured of the natural disease (blood not infective in doses of 20 c.c. and I5 c.c. for dogs), were re-inoculated with a trypanosome from a guinea-pig and a laboratory strain of $T$. dimorphon respectively. Ten days later the blood of both animals was infective for mice, and the goat and sheep themselves showed parasites in their blood in 24 and 16 days respectively after injection. No difference could be made out between the two parasites. We shall see (Chapter VII.) that Thomas and Breinl had similar cases - a sheep apparently cured of $T$. dimorphon developed a fresh infection and died. The natives in Guinea often stated that sick animals could recover, then have a recurrence the following year and die.]

[The other trypanosome found in horses, cattle, and goats closely resembles $T$. cazalboni. In fresh blood it is very motile. In the horse the total length is $26 \mu$ to $28 \mu$, free flagellum $6 \mu$ to $7{ }^{\circ} 5 \mu$; in cattle the total length is $22 \mu$ to $28 \mu$, free flagellum $6 \mu$ to $8 \mu$. This trypanosome is not pathogenic for guinea-pigs, rats, or dogs.]

[On one occasion, in a Kankaya cow, Martin found a giant trypanosome, very long and with a very active undulating membrane, but which, nevertheless, travelled very slowly, gliding between the red corpuscles without displacing them, after the manner of a spirillum. The flagellum was well developed. Only one such trypanosome was seen in several films examined. The blood of the cow was injected into a rabbit, guinea-pig, and rat without result. Centrifuging the cerebro-spinal fluid also failed to discover any more parasites. The animal was a hide-bound skeleton, and had lost nearly all its hair. Two months before it was seen it had given birth to a still-born calf.]

\section{[Human trypanosomiasis is also very prevalent throughout Guinea.]}

[Many species of Glossina have been found in this vast area. Gl. tachinoides, Gl. longipalpis, and Gl. fusca occur at Boké and elsewhere along the banks of the Rio-Nunez, but it is Gl.palpalis and Gl.morsitans that are the most widely distributed and practically universal.]

[Other biting flies found here include several species of Tabanidæ, and one or more species of Hippobosca.] 


\section{CHAPTER VII}

\section{TRYPANOSOMIASIS OF HORSES IN GAMBIA}

Pathogenic Agent: Trypanosoma dimorphon, Dutton and Todd, I904.

\section{[Section 1.-The Geographical Distribution of the Disease.]}

THIs trypanosomiasis was discovered by Dutton and Todd ${ }^{1}$ (Igo2), during their investigations upon human trypanosomiasis, which was itself discovered the year before by Dutton. These two investigators explored the Gambia from September 2, I902, to May 7, I903, and were able to examine thirty-six horses in all. Horses are very scarce in Gambia; Dutton and Todd estimate that there are not more than a hundred throughout the colony, and the majority are of Senegalese origin.

Of these thirty-six horses, ten had trypanosomes in the blood. Eight of them were in the maritime districts (five at Cape St. Mary, and three at Bathurst), whilst two were discovered a long way from the sea, at Maka, a French station a little to the north of the River Gambia. It is therefore probable that the disease occurs throughout the British colony on the Gambia, and even extends to the neighbouring regions in the basin of the Senegal.

During their stay at St. Louis and Dakar, on the Senegal, Dutton and Todd examined, but with negative results, a number of sick animals (horses, mules, cattle, and camels) coming from a region of the Sudan where there is a disease with symptoms closely resembling those of the trypanosomiasis of horses in Gambia.

Only one out of ten horses showing trypanosomes was really ill at the time of the blood examination, and it died six days later. The disease is so insidious that it escapes the otherwise acute observation of the natives, who state that the animals die of simple inanition.

[The earlier statement of the authors that 'the horse appears to be the only animal which suffers from the disease naturally, and that cattle and other domestic animals do not become spontaneously infected, although they are susceptible to experimental inoculation,'2 needs modification in the light of recent researches. We have 'already, seen that Laveran, Cazalbou, and Pécaud, in studying the

1 Dutton and Todd, First Report of the Trypanosomiasis Expedition to Senegambia (I902). Thompson Yates and Johnston Lab. Reports, v. 5, 1903.

${ }^{2}$ [Laveran and Mesnil, in the original, p. 200.] 
trypanosomiases of Haut-Niger, found a trypanosome closely resembling, if not identical with, $T$. dimorphon. ${ }^{1}$ Also that Laveran and Martin described the same trypanosome in horses in French Guinea, and that Martin found the same infection in Bovidæ, and very probably in sheep, dogs, and pigs, throughout Guinea.]

[In addition to the Gambia colony, Haut-Niger, and French Guinea, this trypanosome appears to occur in the Shari basin (Decorse). The trypanosomiasis in an ox in Sierra Leone, discovered by Smith, was probably a dimorphon infection (see p. IIg.]

[The trypanosomiasis of mules in the Bahr-el-Ghazal province, discovered by Balfour, and that of cattle in Uganda (Jinja), discovered by Nabarro and Greig, were in all probability also due to T. dimorphon.]

[Dutton and Todd found a similar trypanosome in antelopes and cattle all along the Congo from Leopoldville to Kasongo. Both long and stumpy forms of $T$. dimorphon were seen in these animals.]

[It is therefore permissible to suppose, says Martin, that a wide belt of the African continent, parallel with the Equator, and extending from Gambia and Guinea on the west to the Anglo-Egyptian Sudan and Uganda on the east, is infected with $T$. dimorphon.]

\section{Section 2.-The Course of the Disease in Horses.}

Spontaneous Infection in the Horse.-According to Dutton and Todd, the following is a résumé of the symptoms of the natural disease in horses: The first symptom is loss of strength. The temperature seldom rises above $39^{\circ} \mathrm{C}$. [ $\mathrm{IO}^{\circ} 2^{\circ} \mathrm{F}$. $]$. The parasites are scanty in the blood, sometimes ten being seen in a slide, but often they are fewer, and they may even be absent for long periods.

Two or three weeks later the animal is really ill; it wastes, hangs its head, the eyes become dim, and weakness is very apparent. At this time there are periodical rises of temperature, generally associated with the presence of parasites in the blood.

The next month wasting becomes more marked, so that the ribs are visible. It seems as though the flesh passes from the chest to the abdomen, but, in spite of this enlargement of the abdomen, there is no odema. The scrotum is pendulous, and the testicles hang so low that at first sight it seems as though they were odematous. Sometimes there is a slight watery discharge from the eyes. In none of the sick horses did Dutton and Todd find definite œdema of the abdomen, scrotum, or limbs, or staring of the coat, which are usually so pronounced in horses suffering from nagana.

The stage of the disease just described lasted ten months in one horse. During that time trypanosomes were seen in the blood only on four occasions, each time in association with a slight rise of temperature to about $39^{\circ} 5^{\circ} \mathrm{C}$. [103.2 ${ }^{\circ} \mathrm{F}$. $]$.

1 [Laveran has recently come to the conclusion, however, that this trypanosome is distinct from $T$. dimorphon (see p. 226).] 
With the progress of the disease emaciation becomes gradually more marked, so that the ribs and pelvis are very prominent. The animal is now characteristically apathetic. Edema is still absent; there is often a slight whitish discharge from the eyes, and sores appear in the lumbar region where the bones project. There are never any hæmorrhages, either from the mucous membranes or from the kidneys. During this stage trypanosomes are almost constantly present in the blood, often in large numbers. The temperature is nearly always high (up to $40^{\circ} 5^{\circ} \mathrm{C}$. $\left[105^{\circ} \mathrm{F}.\right]$ ) and fluctuating (see Fig. 25, A).

Dutton and Todd saw two horses die of this disease. One of them lingered for three days, scarcely able to raise itself owing to extreme weakness; the breathing was laboured; there was almost constant sweating, and just before death a slight convulsion. The other horse, whose temperature chart is given in Fig. 25, A, died almost suddenly. Shortly before death, but not earlier in the disease, the animals showed a diminution of the red corpuscles and of hæmoglobin.

Post-mortem there was a yellowish, gelatinous cedema of the sheath, and in the first horse also of the abdominal walls. Ambercoloured fluid was present in the peritoneal, pleural, and pericardial cavities; there was general enlargement of the lymphatic glands, the spleen was not enlarged, the liver was fatty, the lungs congested.

It is impossible to give precisely the duration of the disease. One horse was infected for more than a year. Of two colts a year old, one was alive six months after the disease was recognised in it, while the other died two months after. Possibly some horses recover from the disease. [A naturally infected horse, which was brought from the Gambia to England, was still alive and in excellent condition two years and five months after it first came under observation; but its blood was still infective, though only in larger doses than formerlyI to $1 \frac{1}{2}$ c.c. for rats, $3{ }^{*} 5$ c.c. for rabbits (Thomas and Breinl).]

The disease can only be diagnosed with certainty by finding the trypanosome in the blood. It may, however, be suspected in animals which look dejected, and have a temperature above $38.3^{\circ} \mathrm{C}$. [ IOI ${ }^{\circ} \mathrm{F}$.].

The natural disease in horses to some extent resembles nagana in its clinical history, but the symptoms are less definite, and the disease runs a much more chronic course.

By means of the virus, for which we are indebted to Dutton, Todd, and Annett, we have succeeded in infecting a certain number of animals, including a horse. ${ }^{1}$ The very complete account which we are able to give of this horse confirms, and in certain respects completes, the description of Dutton and Todd.

EXPERIMENTAL Infection of THE HoRse. - The horse we inoculated was strong and had particularly thick-set limbs. It weighed 575 kilogrammes [about 1,265 pounds]. It was inoculated on

1 Laveran and Mesnil, C. R. Acad. Sciences, v. 138, March 21, 1904, p. 732. 
November I3, Igo3, under the skin of the neck with $\frac{1}{2}$ c.c. of diluted blood of a rat containing many trypanosomes. Fig. 25, B, shows

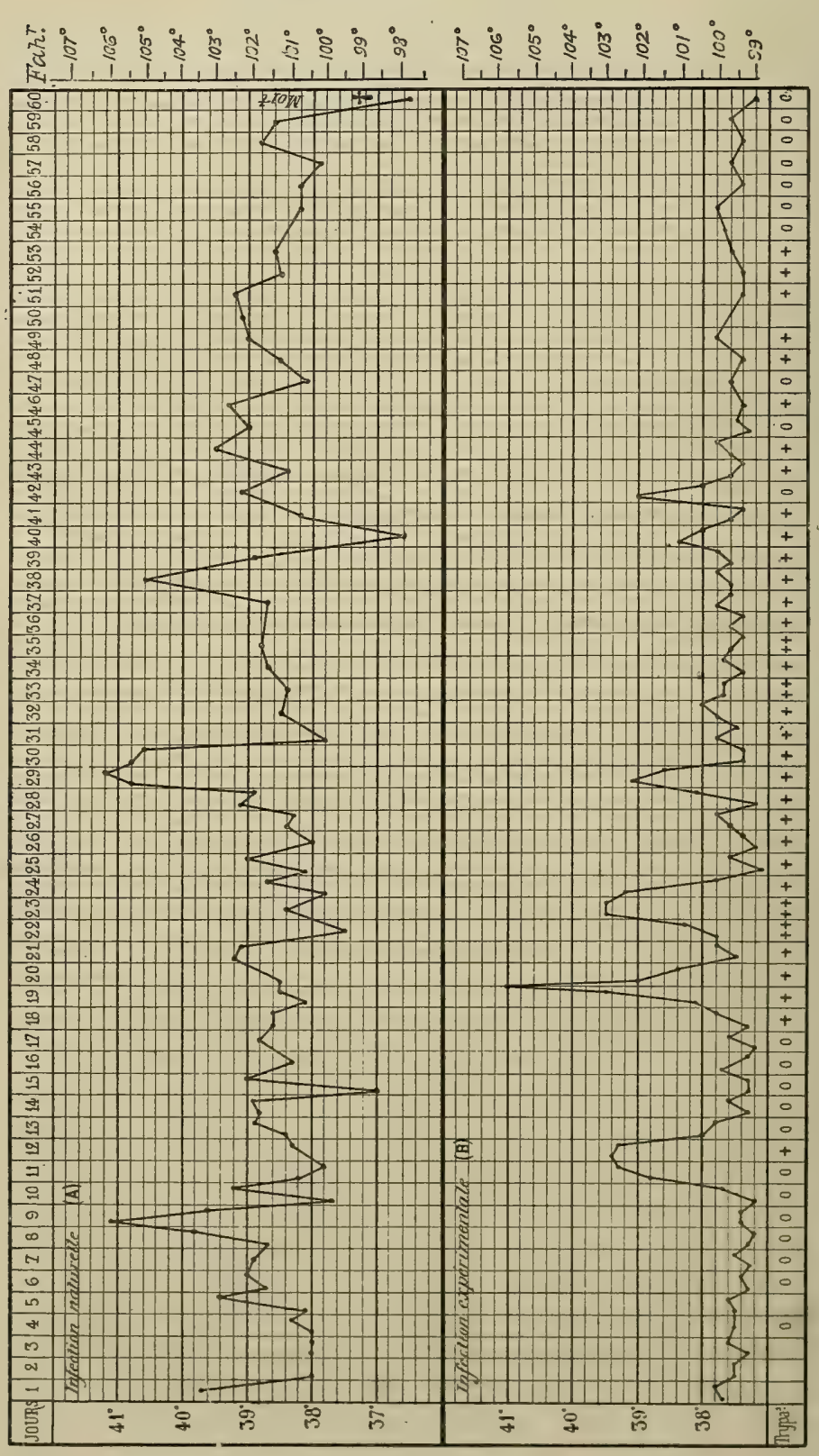

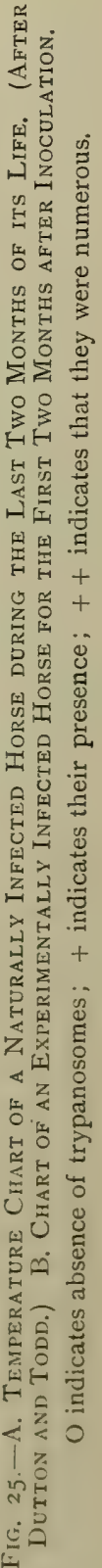

the temperature curve for two months from the day of inoculation, and also the relative number of trypanosomes in the blood.

The horse never suffered from continued fever. There was a rise of temperature to $39^{\circ} 4^{\circ} \mathrm{C}$. $\left[\mathrm{IO}^{\circ} \mathrm{F}\right.$. $]$ on the eleventh and twelfth days coincident with the appearance of trypanosomes in the blood. 
After that there was an apyretic period of seven days, during which the parasites could not be found on microscopical examination. On the Igth and 20 th days the temperature rose to $4 \mathrm{I}^{\circ} \mathrm{C}$. [ $\mathrm{IO} 5^{\circ} 8^{\circ} \mathrm{F}$.], on the $23 \mathrm{rd}$ and 24 th days to $39^{\circ} 5^{\circ} \mathrm{C}$. [ $\mathrm{IO}^{\circ} 2^{\circ} \mathrm{F}$.] , and on the 29 th day to $39^{\circ} \mathrm{I}^{\circ} \mathrm{C}$. $\left[102^{\circ} 4^{\circ} \mathrm{F}\right.$. $]$. During this period, from the $\mathrm{I} 8$ th to the 3 oth day, a few trypanosomes were always found in the blood on microscopical examination, and they continued to be present until the 53rd day, although the temperature had become normal, except for a small rise to $39^{\circ} \mathrm{C}$. $\left[\mathrm{IO}_{2} \cdot 2^{\circ} \mathrm{F}\right.$. $]$ on the $42 \mathrm{nd}$ day. From that time the temperature was practically normal, and trypanosomes were found only once, on the 8Ist day. That they were still present, however, is shown by the fact that $\frac{1}{2}$ c.c. blood, taken on the ro5th day, infected a mouse, and blood taken on the I2rst, 157 th, and 183 rd days produced, in doses of 2.5 c.c., an infection in rats after a prolonged incubation period.

About the 5oth day the scrotum was swollen, and two days later there was a very characteristic large œdematous swelling near the middle of the abdomen. This persisted for about one and a half months and then entirely disappeared. No other external sign of disease was seen, and the horse never seemed ill. [Seven months after inoculation this horse had recovered, and I5 c.c. of its blood was not infective on injection into a susceptible animal. It subsequently became infected with mbori, to which it succumbed. $\left.{ }^{1}\right]$

We would draw attention to the odema present in our horse, which is like that seen in nagana, but which, according to Dutton and Todd, does not occur in the sick horses in Gambia.

\section{Section 3.--Course of the Disease in Mammals other than the Horse.}

[Dutton and Todd, Laveran and Mesnil, Thomas and Breinl, ${ }^{2}$ and $G$. Martin have investigated the effects of this trypanosome upon various species of animals, and have found that most mammals are more or less susceptible to T. dimorphon. Martin's results with the Guinea trypanosome, as well as those of Balfour and of Nabarro, Greig, and Gray with those of the Sudan and Uganda, which were probably also $T$. dimorphon, have already been given in different Sections of Chapter VI.]

RodENTS.-Rats, mice, guinea-pigs, and rabbits are susceptible.

In the rat, according to Dutton and Todd, the incubation period varies from three to twelve days. Death occurred, with one exception, in twenty to seventy days after inoculation. Trypanosomes are nearly always present in the blood. Sometimes, when the disease runs its course in twenty to twenty-five days, the trypanosomes gradually increase in number until the end. In rats which are

1 [Information kindly furnished by the authors.]

2 [Thomas and Breinl, Thompson Yates and Johnston Lab. Reports, v. 6, part ii., I905, pp. 25-3[.] 
rather more resistant, the parasites diminish in number at times, and they may even be absent on microscopical examination. In some of these rats Dutton and Todd noticed œedema of the tunica vaginalis and of the abdominal parietes, and occasionally a slight diminution in the number of red corpuscles.

Our own observations agree with those of Dutton and Todd. We find the average duration of the disease in ordinary white or speckled rats to be twenty-three days (minimum ten, maximum fortytwo). As a rule trypanosomes are numerous in the blood. Postmortem the spleen is considerably enlarged, particularly in rats which showed a certain resistance to the disease-for example, in a rat weighing I25 grammes, which lived forty-two days, the spleen weighed 5 grammes.

[Thomas and Breinl ${ }^{1}$ obtained very similar results with their rats. One rat showed a marked degree of resistance to the parasite. It developed a mild infection after the second intraperitoneal inoculation, but it very soon recovered, and was then immune against large doses of the virus.]

[Post-mortem the spleen was considerably enlarged in chronic cases; the lymphatic glands were also enlarged and often hæmorrhagic. Hæmorrhagic nephritis was noted in two chronic cases; the urine contained a few trypanosomes, and numerous small hæmorrhages were found under the capsule and in the substance of the kidneys.]

Mice, according to Dutton and Todd, are a little more susceptible than rats; incubation two to seven days, death in sixteen days to one month. In our experiments white mice were about as resistant as rats, some, however, living for months with the disease. One animal will suffer from a fairly acute infection lasting at least a week, while another may have a chronic infection lasting sometimes more than five months, but always terminating fatally. In the one case, as in the other, trypanosomes are generally numerous in the blood during the course of the disease. In very chronic cases the enlargement of the spleen, which may be enormous, is quite characteristic. The mouse becomes deformed, and on palpation the hypertrophied spleen can easily be felt as an abdominal tumour. In two mice, weighing 24 to 25 grammes, in which the disease lasted 92 and III days, the spleens weighed 2.62 and 2.55 grammes respectively. Even when the disease runs its course in from one to two weeks, the spleen of a mouse of 20 grammes never weighs less than 04 gramme; that is to say, six times the normal weight.

One of our mice deserves special notice, as it died five and a half months after infection.

Inoculated November I2, Ig03; trypanosomes present November I 8 ; died April 24, I904. During November and December it had three

1 [Thomas and Breinl, Thompson I'ates and Johnston Lab. Reports, v. 6, part ii., 1905, pp. 25, 26.] 
injections of human serum, which caused the trypanosomes to disappear for a very short time. During January parasites were always found on examining the blood, sometimes in large, sometimes in small numbers. From the midale of February trypanosomes were always numerous or very numerous in the blood. From January I 5 the spleen was enormous; at the autopsy it weighed 3 grammes, the mouse weighing only 27 grammes.

The trypanosomes in this mouse were attenuated in virulence. Thus, a rat inoculated subcutaneously on March 2 did not show trypanosomes in its blood until April 2. Two mice were inoculated on March I8. One, inoculated intraperitoneally, showed parasites on the 3oth and died April 4, while the other, inoculated subcutaneously, did not show any trypanosomes in its blood until April 8. On different occasions we found, on microscopical examinations of stained specimens, numerous intraleucocytic trypanosomes, always spherical in form, but recognisable by their nucleus and centrosome. We never succeeded in observing the engulfment of normally shaped trypanosomes. During the last few days of life the mouse lost much of its hair.

Two guinea-pigs inoculated by Dutton and Todd died in 29 and 3 I days; incubation period 8 and 4 days respectively. The temperature was between $39^{\circ} 5^{\circ}$ and $40^{\circ} \mathrm{C}$. [103.2 $2^{\circ}$ to $104^{\circ} \mathrm{F}$. $]$. Trypanosomes were nearly always present on microscopical examination, and were numerous at the time of death. We likewise inoculated two guinea-pigs, which died in 24 and 30 days. During the last few days of life trypanosomes were fairly numerous. The spleen was found slightly enlarged.

[Thomas and Breinl (op.cit., p. 26) found the incubation period to vary from 4 to 15 days, average 4 to 6 days; the disease lasted from 9 to 60 days. The temperature was usually slightly raised when the parasites first appeared, but after that it varied little from the normal. There was moderate anæmia and loss of weight. Trypanosomes gradually increased in number, until there were as many as forty to sixty to a field. These high numbers continued for ten days before death, or there was a diminution coincident with a leucocytosis. In the more chronic cases there was marked periodicity in the number of the parasites.]

[Rupture of the spleen occurred in seven cases, five of which were of the very acute type. Enlargement of the glands was not marked in any of the guinea-pigs.]

A rabbit inoculated by Dutton and Todd had an incubation period of 13 days, and died 53 days after inoculation. Its temperature was constantly raised and even reached $42^{\circ} \mathrm{C}$. [107.6 $\mathrm{F}$.].

Two rabbits we inoculated subcutaneously died in 76 and I I 5 days, after an incubation period of about I2 days. Parasites were always scanty on microscopical examination. The first rabbit died in an anæmic and cachectic condition, while the second appeared paralyzed the day before death, but had not lost weight. The spleen weighed 20 grammes, the weight of the rabbits being about 2 kilogrammes.

[Thomas and Breinl found the incubation period in rabbits to vary from 4 to $I_{5}$ days. It was shortest after intravenous inoculation, 
longer after intraperitoneal, and longest after subcutaneous injection. The disease ran an acute course in some animals, death occurring in 26 to 35 days after intravenous inoculation. In others the disease was more chronic, and lasted 78 to 157 days. Trypanosomes were sometimes present in large numbers in acute cases, but not nearly so frequently as in the guinea-pig. In the more chronic cases there was marked periodicity in the number of parasites seen in the blood. Profound anæmia, loss of weight, staring of the coat, and slight œdema of the hind-limbs and base of the ears, were the chief symptoms noted. Discharges from the eyes, nose, and genitals were rarely seen. The temperature was raised with the first appearance of the parasites, then became irregular, often showing considerable elevations, and towards the end became subnormal. The spleen and lymphatic glands were found enlarged.]

Monkeys.-A mangrove monkey (Cercopithecus?) inoculated by Dutton and Todd showed parasites in its blood four days after subcutaneous injection of blood rich in trypanosomes. Unfortunately, it escaped the following night.

Three baboons (Cynocephalus sphinx) inoculated by Dutton and Todd were absolutely refractory, for their blood in doses of as much as 3 or $3 \frac{1}{2}$ c.c. was not infective for rats. Thomas and Linton, continuing these researches at Liverpool, succeeded in infecting a baboon.

[The temperature was irregular, and loss of weight was marked, especially when the parasites were first seen. The baboon died six and a half months (204 days) after inoculation. Its spleen and lymphatic glands were slightly enlarged. Thomas and Breinl also successfully inoculated a Cercopithecus callitrichus, a Macacus rhesus, and a Jew monkey with this trypanosome. The first died in I6o days, and the last in 75 days after inoculation. The Macacus became infected on the seventh day, but after this parasites were hardly even seen, and the monkey died finally from dysentery.]

We have injected the $T$. dimorphon into a baboon, but, like Dutton and Todd, with a negative result.

Dogs.-Interesting results have been obtained with dogs. Three young dogs inoculated by Dutton and Todd died in 19, 32, and 36 days (after an incubation period of less than 8 days, II days, and 3 days respectively). The animals had remittent fever, and trypanosomes were constantly present in the blood.

An adult dog inoculated by us behaved similarly: Incubation period Io days; death in 25 days; fever continued (see Fig. 26); trypanosomes almost constantly present on microscopical examination, and fairly numerous a few day's before death. Throughout the disease there were no obvious physical signs externally, and post-mortem the only lesion found was enlargement of the spleen, which was about six times the normal size (weight, 97 grammes in a dog of $7 \frac{1}{2}$ kilogrammes). 
Dutton and Todd inoculated a $\operatorname{dog}(q)$ which was alive ten and a half months later. At first the disease resembled that seen in the other dogs: Incubation period nine days; trypanosomes constantly present in the blood in small numbers for four months; and rises of temperature to $40^{\circ} 2^{\circ} \mathrm{C}$. [ $104^{\circ} 4^{\circ} \mathrm{F}$.] at the first apnearance of trypanosomes in the blood, and to $39^{\circ}$ or $40^{\circ} \mathrm{C}$. [102. $2^{\circ}$ or $104^{\circ} \mathrm{F}$.] with each recrudescence of the parasites. Since that time the dog has always been in good health, and has undoubtedly recovered, for in the tenth month $2 \frac{1}{2}$ c.c. of its blood were no longer infective.

[This bitch was brought to England, and was stili alive three years after inoculation. While in England it had pups, which were found

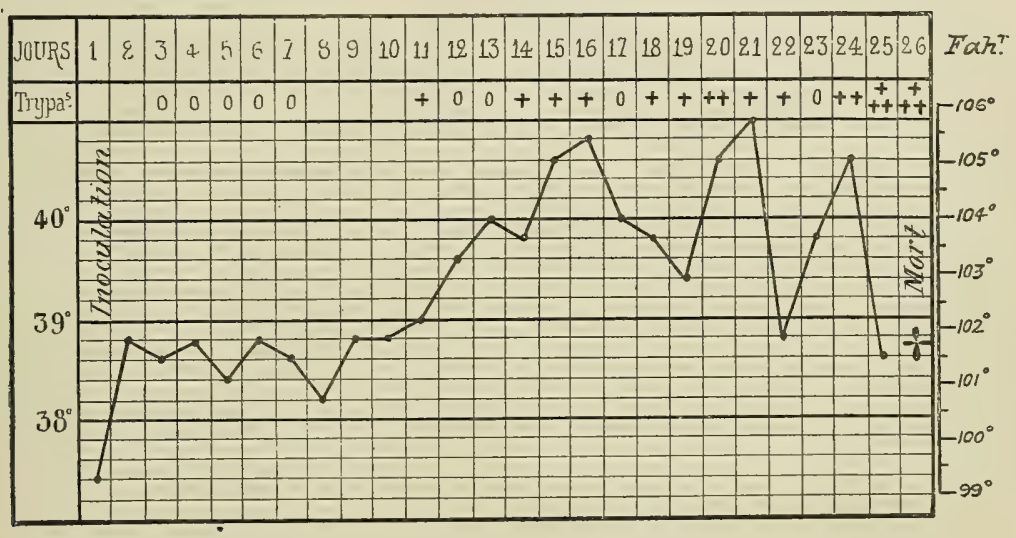

Fig. 26. - Temperature Chart of a Dog infected with $T$. dimorphon.

$\mathrm{O}$ indicates absence of trypanosomes; + , present but scanty $;++$, fairly numerous; $+{ }_{+}^{+}$, numerous.

to be quite as susceptible as normal pups, and to succumb in the usual time. The serum of the bitch was not protective.]

[Thomas and Breinl found the incubation period to be 4 to 8 days, and the total duration of the disease Io to I9 days in adult dogs, 9 to 26 days in puppies. Trypanosomes were almost constantly present; fever was irregular or continuous, the temperature being usually subnormal before death; loss of weight and anæmia were prominent features. Post-mortem the spleen was often considerably enlarged (two or three times the normal size). The lymphatic glands were enlarged and sometimes hæmorrhagic.]

[CATs.-Four cats and two kittens were inoculated by Thomas and Breinl. The incubation period was I 2 to I4 days in adult cats, and $7 \frac{1}{2}$ days in a kitten. In the former the disease was chronic ( 9 to Io months), but a kitten died in 23 days. In the full-grown animals the parasites were scanty, and appeared in the blood at irregular intervals; in the kitten they were constantly present and in greater numbers. The temperature was raised, but irregular, in all the animals, being higher in the acute disease (kitten). In the chronic form (adults) the anæmia and loss of weight were not so 
pronounced as in the acute form in the kitten. In both forms there was at times a discharge from the eyes or nose.]

[Two cats aborted during the infection, but the blood of the fœetuses was not infective on injection.]

[Post-mortem the spleen was enlarged in all cases, but more in the acute than in the chronic cases. The lymphatic glands were not much enlarged.]

CATtLE.-Dutton and Todd inoculated a calf $\left(\delta^{\top}\right)$ six months old and an ox. Nine days later in the former and twelve days later in the latter animal some trypanosomes were found on centrifuging the blood. Trypanosomes were always scanty in the blood, that of the ox, for example, showing usually only three parasites in $\frac{1}{2}$ c.c.

In the calf the temperature rose to $406^{\circ} \mathrm{C}$. [105 ${ }^{\circ} \mathrm{F}$. $]$ two days after inoculation, but fell again the next day; it was raised again on the seventh to the ninth days, and also on the eleventh day; on the eighteenth day there was another small rise. The animal died twenty days after inoculation. In the ox the temperature was below $39^{\circ} \mathrm{C}$. $\left[\mathrm{IO} 2 \cdot 2^{\circ} \mathrm{F}\right.$. $]$ for the first twenty days, but towards the end of the first month it rose to $40^{\circ} 5^{\circ} \mathrm{C}$. [ $\left[\mathrm{IO}^{\circ} \mathrm{F}\right.$.] for two days. The animal died forty days after inoculation. Cattle, therefore, appear to be very susceptible to the Gambian trypanosomiasis, yet Dutton and Todd never met with a case of natural infection in these animals.

Post-mortem there was nothing characteristic except, perhaps, enlargement of the lymphatic glands, which were congested or œdematous.

Goats.- Two goats were inoculated by Dutton and Todd, who found the incubation period to be three or four days. During the following two or three weeks a few parasites were occasionally seen in blood-films. At the first appearance of the trypanosomes the temperature rose to $407^{\circ} \mathrm{C}$. [105.2 $2^{\circ} \mathrm{F}$.] and this was followed by irregular fever. The two animals were still alive nine months and ten and a half months after being inoculated, and the blood of one of them was found to be still infective five months after inoculation.

[These two goats were brought to England in I903, and subsequently appeared to have recovered. The blood of goat I was not infective for animals in doses of 2 to 3 c.c. nine months after inoculation, but this goat had not become immunized, for it was successfully re-inoculated by Thomas and Breinl, and succumbed to the infection. Goat 2 had apparently recovered a year after inoculation, but five months later, when accidentally killed, Ioo c.c. of heart blood infected a guinea-pig after a prolonged incubation period. The serum of these goats did not cause any permanent aggrlutination.]

We have also infected two goats by injecting under the skin of the ear blood of a rat containing $T$. dimorphon. Goat I died in twelve and a half days with an acute infection. Six days after inoculation the temperature rose to $39^{\circ} 5^{\circ} \mathrm{C}$. $\left[\mathrm{IO}_{3} 2^{\circ} \mathrm{F}\right.$. ; ; on the next 
two days it was $40^{\circ} 6 \mathrm{C}$. [105 ${ }^{\circ} \mathrm{F}$.] , and on the succeeding days $41^{\circ}, 40^{\circ} 5^{\circ}$, $40^{\circ} 5^{\circ}$, and $39^{\circ} 5^{\circ} \mathrm{C}$. $\left[105.8^{\circ}, 105^{\circ}, 105^{\circ}\right.$, and $103^{\circ} 2^{\circ} \mathrm{F}$. $]$. Trypanosomes, scanty seven days after inoculation, were numerous from the eighth day until death. Goat 2 was inoculated on December 28, I903. The disease began, as in goat I, with high fever and many parasites in the blood; but later the temperature, after a series of oscillations, gradually returned to normal (see Fig. 27), and the trypanosomes became scanty in the blood. For the first month they were almost constantly present at the daily blood examination, but after that time they diminished very much in number, so that on several occasions they could not be found. [The goat lived for twenty-one and a half months after inoculation, and at death $T$. dimorphon was still present in considerable numbers.] ${ }^{1}$ The course of the disease

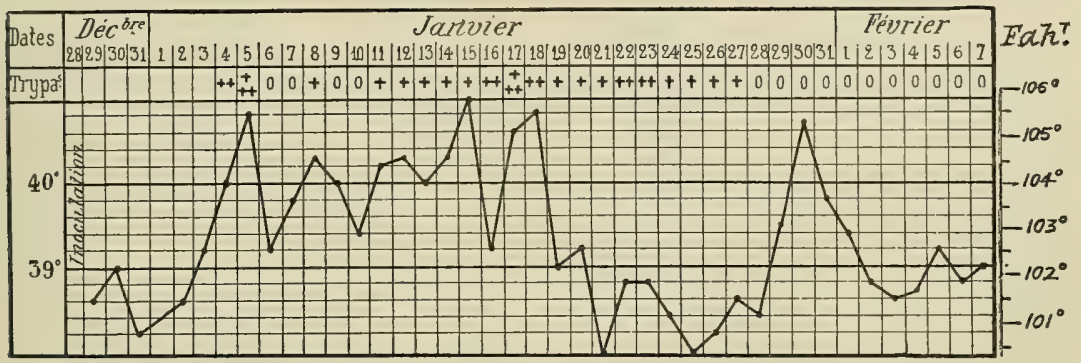

Fig. 27.-Temperature Chart of a Goat infected with $T$. dimorphon.

The signs $\mathrm{O},+$, etc., have the same significance as in Fig. 26.

in this goat closely resembled that in the two goats inoculated by Dutton and Todd.

SheEP.-Dutton and Todd infected a native sheep six months old. Trypanosomes appeared in the blood in eight days, and were numerous during the following week. Later they were still present but scanty, and finally they disappeared. With the first appearance of the parasites the temperature rose to $4 \mathrm{I}^{\circ} 9^{\circ} \mathrm{C}$. [ $\left[\mathrm{IO} 7^{\circ} 4^{\circ} \mathrm{F}\right.$.], which was followed by irregular fever, with rises to $40^{\circ} 5^{\circ}$ or $4 \mathrm{I}^{\circ} \mathrm{C}$. [ $\left[\mathrm{IO} 5^{\circ}\right.$ to I05. $8^{\circ} \mathrm{F}$.] The animal died, without showing any symptoms, I 82 days after inoculation, on its way to England.

[Thomas and Breinl inoculated a ram intraperitoneally. On the sixteenth day there was a slight rise of temperature, and trypanosomes were seen in the blood. Ten days later they were numerous (ten to forty in a field), and continued so for a week. After this they diminished, and remained scanty until death, which occurred eighty-four days after inoculation. Anæmia was constant, and the animal wasted rapidly. Post-mortem there was no enlargement of the spleen or lymphatic glands.]

BIRDs.- Two fowls were inoculated several times, but unsuccessfully, by Dutton and Todd.

1 [Information furnished by the authors.] 
In conclusion, $T$. dimorphon gives rise in those mammals which have hitherto been experimented upon either to an acute or subacute disease (as in rodents, dogs, and cattle), or to a chronic infection (as in goats, sheep, [monkeys, and cats]); but the disease is never so acute as that caused by $T$. brucei. Baboons are, as a rule, refractory.

[Changes in the Organs INDUCED By $T$. dimorphon.-These have been studied by Breinl ${ }^{1}$ in artificially infected monkeys, dogs, rabbits, guinea-pigs, and rats.]

[The leptomeninges of the brain and spinal cord were very congested in all the chronic cases. On section the grey matter was also congested, and showed many small hæmorrhages. The liver and spleen were much congested, the latter often showing hæmorrhages in its substance.]

[Microscopically, the perivascular spaces in the grey matter of the nervous system were often filled with red blood-corpuscles, pigment granules, and a few leucocytes. The spleen showed extreme congestion, especially near the periphery where frequently only red blood-corpuscles were to be seen. Many hyaline cells were present containing red bloodcorpuscles and much blood-pigment. There was great proliferation of the endothelium of the vessels, so that in some places these appeared to be obstructed.]

[The lymphatic glands presented marked changes. Very often the lymphoid tissue was much reduced; the spaces between the bands of lymphoid tissue network contained a few lymphocytes, hyaline cells with two or more nuclei, cells with a large quantity of included pigment giving the iron reaction, and very many large free pigment granules. In some cases, in which the spleen contained only traces of pigment, the glands were practically filled with it (Breinl). The degree of pigmentation increases with the duration of the infection.]

[The cells of the liver and kidneys were often degenerated, being fatty and atrophied. Blood-pigment was often found in the liver.]

\section{Section 4.--The Trypanosoma dimorphon.}

In a fresh blood-film this trypanosome can be easily identified, on account of the great difference in size of the various forms. ${ }^{2}$ There are parasites $20 \mu$ to $25 \mu$ long, which in their general appearance closely resemble $T$. brucei, the only difference being that the anterior part is less slender or drawn out, the reason of which we shall see presently. Other parasites are not more than I2 $\mu$ long; the posterior end is quite rounded off, and the body becomes gradually thinner right up to the anterior end. These forms have a very characteristic movement. They move from place to place by twisting on themselves like a tadpole, then suddenly they stop and start again in a similar fashion, the undulating membrane being only slightly visible. Between these two extreme types there are all kinds of transitional forms.

In stained specimens these various forms are also seen, but as our own observations are not entirely in agreement with those of

1 [Breinl, op. cit., pp. 87-89.]

2 [We have secn, however (p. 226), that a trypanosome, morphologically resembling $T$. dimorphom, occurs in the French Sudan, and that Laveran has concluded that these two trypanosomes are distinct species.] 
Dutton and Todd, we shall give first a résumé of their observations and then our own.

Dutton and Todd distinguish three forms of the parasite:

(I) 'Tadpole forms.' These are I I $\mu$ to I $3 \mu$ long, by $0.8 \mu$ to I $\mu$ wide. The free flagellum is very short, the centrosome almost at the end of the body, and just in front of it there is a clear space. A few chromatic granules are present. The body increases in size before dividing by fission. This form of the parasite is seen in the horse, rat, and mouse at the beginning of an infection, but tends to disappear later.

(2) 'Long forms.' These are $26 \mu$ to $30 \mu$ long, by $I^{\cdot} 6 \mu$ to $2 \mu$ wide. The body is long and slender, and there is a long free flagellum. The posterior extremity (post-centrosomic part) measures from $I^{*} 6 \mu$ to $3^{* 2} \mu$, and resembles the head of a pike. There are no bluestaining protoplasmic granules. This form predominates in the blood during the days which precede the death of the animal.

(3) 'Stumpy forms.' The body is short (I6 $\mu$ to I9 $\mu$ ) and squat $\left(3{ }^{\circ} 5 \mu\right.$ wide) ; the free flagellum is very short, and no division forms are seen. The centrosome is quite close to the rounded posterior extremity, and in front of it a clear space can usually be made out. The protoplasm contains several chromatic granules. This form is very common in the blood when the disease is not yet far advanced. It really does not differ from the so-called 'tadpole form,' and the three forms may, therefore, be reduced to two. This is the origin of the name dimorphon suggested by the investigators of the Liverpool School of Tropical Medicine to designate the trypanosome of horses in Gambia. (Letter from Dr. Annett, December I8, I903).

We ourselves have not been able to see any difference between the 'tadpole' and 'stumpy' forms. We have seen short parasites, Io $\mu$ to I5 $\mu$ long, by $0.7 \mu$ to $I^{\circ} 5 \mu$ wide, but have never seen forms $3.5 \mu$ wide in good blood-films, and the same remark applies to the spherical forms which Dutton and Todd say are derived from the 'stumpy' forms. We recognise the existence of a short form and a long form (average length $22 \mu$ ), the width of the former being about $I \mu$, and of the latter about $I^{\cdot} 5 \mu$. The short form cannot be looked upon as a young individual of the large form, because each form multiplies by longitudinal fission.

It is particularly with regard to the long form that we differ from the English investigators, who describe and figure a long free flagellum. According to our own observations, the protoplasm of the body is continued along the flagellum almost or quite to the end. As a. result, the really free part of the flagellum is very short, or even absent, in the long form as well as in the short. We have already pointed out a similar appearance in T. brucei just after division, but in the case of $T$. dimorphon all the parasites occurring in the blood show it.

[Thomas and Breinl and G. Martin agree with Laveran and Mesnil as to the absence of Dutton and Todd's long form, with 
long free flagellum, from the blood of subinoculated animals. But we have already seen (Chapter VI., Section II) that in the blood of naturally infected sheep in Guinea, Martin found trypanosomes with a long free flagellum, and that these flagellated forms even persisted for a time in the blood of experimental animals.]

As already stated, transitional forms exist between the short and long varieties (see Fig. 28, $I$ to 4 ). Some long forms have a pointed post-centrosomic end (Fig. 28, $I$ and 5); others (Fig. 28, 2) have a rounded end, like all the short forms; while Fig. 28, 6 , shows an intermediate condition. The undulating membrane is never well developed; in the small forms it is closely applied to the body proper.

Fig. 28,5 to 7 , represents the stages of division in a long form (Fig. 28, 5 and 6), and in a form transitional (Fig. 28, 7) between

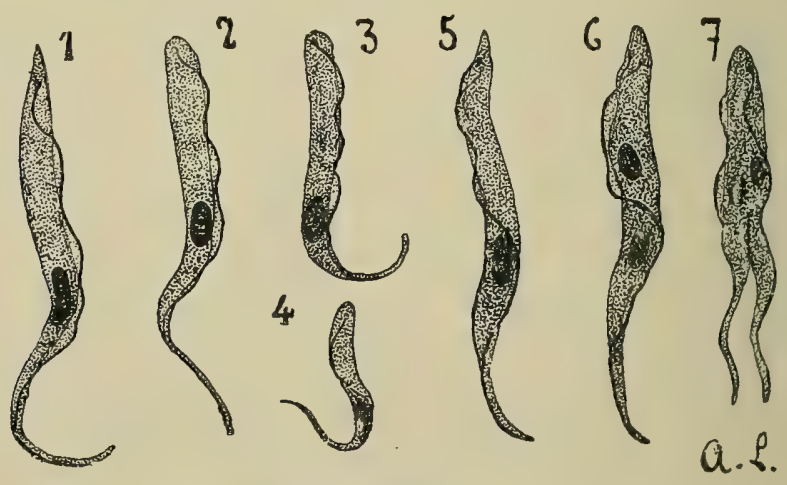

Fig. 28.-TRYPANOSOMA DIMORPHON.

I and 2. Long forms. 3. Intermediate form. 4. Short form. 5, 6, and 7. Dividing forms. (Magnified about 2,00o diameters.)

the short and long forms. The mode of division is the ordinary longitudinal fission into equal or subequal parts.

There is one other point to be mentioned, namely, that the protoplasm of all the forms of $T$. dimorphon stains a very deep blue, and only very exceptionally are protoplasmic granules visible. ${ }^{1}$

We have seen parasites with pale-staining protoplasm, as mentioned by Dutton and Todd, and compared by them with the hyaline forms of $T$. brucei of Plimmer and Bradford. We think that, in this case as in the other, they are involution forms.

The morphological differences between $T$. dimorphon and the trypanosomes of the type brucei are so obvious that it is unnecessary to dwell upon them.

An interesting feature of $T$. dimorphon is its great tendency to agglutinate as soon as the blood of a rat or mouse containing many parasites is placed on a slide and covered with a cover-glass.

1 [Thiroux and Teppaz state that they often found protoplasmic granules in this trypanosome, as Dutton and Todd had done. They also describe endocorpuscular forms of the parasite.] 
[Thomas and Breinl and Martin have also noticed this phenomenon. The first two observers saw it with the blood of rabbits and guineapigs, as well as with that of rats and mice.]

In stained specimens many trypanosomes are seen in pairs, but instead of the two posterior ends facing one anther, as is the case with $T$. brucei and $T$. leweisi, the two parasites are in contact laterally for a portion of their length, so that frequently the centrosomes are on the same level.

We have exposed to liquid air citrated and diluted rat's blood containing many trypanosomes. This blood, before the action of liquid air, injected in doses of two drops, killed mice in thirteen and fifteen days. After fifteen minutes' exposure to liquid air, most of the parasites were killed, but some survived, for the blood, in doses of $\frac{1}{2}$ c.c., killed mice in twenty-seven and thirty-two days. After one hour's exposure to $-\mathrm{I} 9 \mathrm{I}^{\circ} \mathrm{C}$. a few active trypanosomes were present, yet the blood failed to infect two mice in doses of I c.c. Blood, kept for twenty-four hours at $-\mathrm{rgI}^{\circ} \mathrm{C}$., which on microscopical examination showed only some small spherical bodies, did not infect on injection. The mice inoculated with the blood cooled for one hour and for twenty-four hours did not acquire any immunity.

We made only one attempt to cultivate $T$. dimorphon in Novy and McNeal's medium. Under those conditions we kept the trypanosomes alive for more than a month at $25^{\circ} \mathrm{C}$., but from the fourteenth day onward they constantly diminished in number. During the second fortnight we saw forms with three, and even four, flagella, which appeared to us to be true multiplication forms; but development stopped there, and subcultures were unsuccessful.

[Using a slight modification of Novy and McNeal's medium (the same as they used for T. gambiense; see Chapter XII.), Thomas and Breinl made a number of culture experiments with $T$. dimorphon. Cultures made at $22^{\circ} \mathrm{C}$. were infective for animals up to the twenty-third day; when injected in large amounts. Subinoculated, but non-virulent, cultures remained alive as long as the fifty-sixth day. In one case two feebly motile trypanosomes were found, in a second generation tube, on the seventy-sixth day.]

\section{Section 5.-Individuality of Trypanosoma dimorphon.}

The trypanosomiasis of horses in Gambia existing side by side with human trypanosomiasis, it was necessary to ascertain whether the two causal agents of these diseases, $T$. dimorphon and $T$. gambiense, were really distinct species. We agree with Dutton, Todd, and Annett in answering this question in the affirmative, for the following reasons : (r) Morphologically, $T$. dimorphon differs from $T$. gambiense as well as from the other well-defined trypanosomes. (2) Animals which have acquired immunity against $T$. gambiense are susceptible to $T$. dimorphon, as we have shown to be the case with mice. We 
may add that in all these mice (six in number) the dimorphon infection was always acute. (3) Human serum, which has no action upon $T$. gambiense (see later), has a marked effect upon $T$. dimorphon, though less than it has upon the parasites of nagana, surra, and caderas.

It was equally advisable to compare $T$. dimorphon with the parasites of the other animal trypanosomiases. The morphological differences between $T$. dimorphon and the other trypanosomes, and the details of its effect upon various susceptible mammals, already left little doubt about its individuality. After our two experiments on the goats quoted above, there can remain no doubt at all about it.

Goat I, which died so rapidly, had, as a matter of fact, become immunized against the nagana of Zululand, against caderas, and against the surra of Mauritius (see its history in Chapters VI., VIII., and IX.). It was infected with nagana from October 25, I90I, till March, I902; with caderas from November 8, I902, till April, I903; and with surra from June 5, 1903, till the end of October, 1903. After recovery from each infection it was tested with the virus from which it had just recovered. In addition, it was tested with nagana on May 20, Igo3, and December I5, I 903 , but did not become infected (on the latter occasion, however, the T. brucei remained in the blood at least a week). On December 28, I903, it was inoculated with $T$. dimorphon.

Goat 2 was immunized against caderas and surra. We have already seen that these two goats were very susceptible to inoculation with T. dimorphon, especially Goat $\mathrm{I}$, which succumbed in twelve and a half days.

\section{Section 6.-Mode of Propagation.}

Glossina palpalis ${ }^{1}$ is prevalent in Gambia along the banks of the rivers and in the brushwood as much as two miles from any water. This fly is particularly abundant in the mangrove swamps. On the other hand, under similar conditions it would appear to be absent from the Senegal.

Dutton and Todd made a certain number of experiments to show the possible part played by this fly in the transmission of the Gambian trypanosomiases. Flies caught in a locality where five horses out of six suffered from trypanosomiasis did not infect three white rats $(44,69$, and 62 flies respectively were allowed to bite these rats). Other flies were placed upon a rat after having previously fed on an infected horse and rat. All these experiments gave negative results.

Dutton and Todd had similar failures with the two species of Stomoxys, which are very plentiful on the Upper Gambia. They think that possibly their failures were due to the fact that the

1 [Mr. Austen informs me that the Gambia tsetse-fly is a local race of $\mathrm{Gl}$. palpalis, with femora and abdominal markings pale. He thought formerly that this fly was the Gl.papalis, var. tachinoides, but since examining a long series of specimens from Northern Nigeria he has come to the conclusion that $G l$. tachinoides, Westwood, is a species perfectly distinct from Gl.palpalis.-ED.] 
experiments were made during the dry season. Possibly they were also due to the animal chosen for experiment-namely, the rat.

[In French Guinea and other parts of Africa, in which the dimorphon ${ }^{1}$ infection exists, several other species of Glossina, notably Gl. morsitans, as well as other biting flies (Tabanidæ, Hippobosca), have been shown to occur. It will be remembered, too, that Nabarro and Greig, in Uganda, were able to convey the Jinja virus (probably $T$. dimorphon) from infected to healthy monkeys by the bites of Gl. palpalis.]

\section{Section 7.--Treatment.}

One of us $^{2}$ has made some experiments with arsenic, in the form of sodium arsenite, and with human serum. Both have some effect.

Human serum injected in sufficient doses into mice and rats with many $T$. dimorphon in their blood usually causes the parasites to disappear in thirty-six or forty-eight hours, but they soon reappear. In cases in which the trypanosomes are numerous, the only effect of the injection of human serum may be to diminish the number of parasites. The action of human serum is therefore quite definite, but less than in nagana, surra, and caderas.

Arsenious acid also has an effect upon $T$. dimorphon, but apparently less than it has in the case of other trypanosomiases.

We have also studied the action of trypanred upon $T$. dimorphon in mice; it causes a temporary disappearance of the parasites from the general circulation. ${ }^{3}$

1 [Thiroux and Teppaz (Ann. Inst. Past., v. 21, 1907, pp. 2ri-223) state that this trypanosomiasis is present in the region of Nianing and in other parts of French Sénégal. Mlany biting flies occur there, including Gl. palpalis and Gl. longipennis.]

2 [Laveran, C. R. Acad. Sciences, v. I 38, Feb. 22, I904, p. 450.

3 [See also Chapter XIII., on Treatment.] 


\section{CHAPTER VIII}

\section{SURRA}

Pathogenic Agent: Trypanosoma evansi, Steel, I885.

THIs name has been used from time immemorial by the natives of certain parts of India for a disease of horses characterized by profound cachexia, without any lesion post-mortem to account for this cachexia. As a result of the researches of the last twenty-five years, we now know that surra occurs naturally, not only in horses, but also in other Equidæ, in camels, and in cattle. Epizootics have also been recorded among sporting dogs in certain parts of India. According to G. H. Evans (quoted by Lingard), elephants in Burmah are also affected by the disease.

The disease closely resembles nagana, in being a kind of pernicious anæmia, with remittent or intermittent fever, wasting (in spite of a healthy appetite), œedema of the limbs and belly, frequent lesions of the eyes and eyelids, great muscular weakness, and terminal paresis. In horses the disease lasts from one to two months, sometimes less; in the camel it may run a similarly rapid course, but usually it lasts three years, whence the name Tibarsa, ${ }^{1}$ which means three years, given to the disease in camels in some parts of the Punjab.

Cattle in India are resistant as a rule, and for a long time this was looked upon as the most important difference between surra and nagana. In Mauritius, where the disease has recently been introduced from India, the mortality amongst cattle has been considerable.

\section{Section 1.-Historical. Geographical Distribution of the Disease.}

In I 88o Griffith Evans, ${ }^{2}$ working in Dera Ismail Khan (Punjab), near the Indus, discovered in the blood of horses, mules, and camels affected with surra a filiform, very motile organism, which at first he took to be a spirillum. He soon recognised, however, as did Lewis, who had just discovered the flagellate of the blood of rats, the animal nature of the parasite. He concluded that the characteristic

1 [See Pease ' 'Tibarsa Surra,' in Journ. Trop. Vet. Sc., v. 1, 1906.]

2 G. Evans, 'Report on Surra,' published by the Punjab Government, Military Department, December 3, I880. 
parasite found by him in the blood was the immediate cause of surra. By means of subcutaneous inoculation of blood he succeeded in giving the disease to the dog and horse.

J. H. Steel, ${ }^{1}$ in 1885 , found the same organism in the blood of transport mules in British Burmah. He regarded it as allied to the parasite of relapsing fever, and called it Spirocheta evansi. He transmitted the disease to the monkey (Macacus) and dog.

Crookshank, ${ }^{2}$ in London, examined films of camel's blood sent to him by Evans, and described the chief characteristics of the parasite -e.g., the undulating membrane, its relation to the flagellum, etc.

This hæmatozoon is now universally known as the Trypanosoma evansi (Steel).

The discovery of Evans and Steel was soon confirmed by a number of medical and veterinary practitioners in India-Vandyke Carter, Gunn, C. E. Nuttall, etc., and especially Lingard. ${ }^{3}$

Since then the name surra could be used in a fully specific sense, and at the same time it was necessary to widen its meaning so as to include all the pathological conditions produced by the hæmatozoon of Evans in different species of animals.

From investigations made during the past twenty years in India and Burmah, and which are summarized in Lingard's various Reports published from I893 to I899, it appears that under the heading surra must be included a large number of diseases bearing different names, according to the district in which they occur, the particular species of animal affected, and the most prominent symptom. ${ }^{4}$

[Until it was demonstrated by Vallée, Panisset, and Laveran that mbori, the trypanosomiasis of dromedaries in the Sudan, first described by Cazalbou, is merely a variety of surra, this disease was not known to occur on the continent of Africa. Further investigations may show that many of the other trypanosome epizootics described in various parts of Africa (see Chapter VI., Part II.) are true surra, or members of the 'surra' group of diseases. Novy, McNeal, and Hare, ${ }^{5}$ in their paper on 'The Cultivation of the Surra

$1 \mathrm{~J}$. H. Steel, Report on his investigation into an obscure and fatal disease among transport mules in British Burmah, 1885.

2 Crookshank, "Flagellated Protozea in the Blood of Diseased and Apparently Healthy Animals,' Journ. of the Roy. Micros. Soc., December, 1886, plate 17. Crookshank classed the parasite of surra with the Hamatomonas (flagellates in the blood of fishes) of Mitrophanov; the only mistake he made was to place the genus Hamatomonas in another genus of Flagellata, Trichomonas. Osler (Brit. Med. Journ., March 12, 1887) reintroduced the genus Hamatomonas; lastly, Balbiani (Journ. de Micrographie, 1888, p. 399) adopted the old generic name of Gruby, Trypanosoma.

3 H. Vandyke Carter, 'Scientific Memoirs by Medical Officers of the Army of India, I 887,' Calcutta, I 888. Alfred Lingard, 'Report on Horse Surra,' v. I, Bombay, 1893. Summary of 'Further Report on Surra,' Bombay, 1894.' Ibid., 1895. Annual Report of the Imperial Bacteriologist for the official year 1895-1896. Report on 'Surra in Equines, Buffaloes, and Canines, etc.,' v. 2, Bombay, 1899.

4 Consult p. I of Lingard's last Report in this connection.

5 [Novy, McNeal, and Hare, Journ. of the Amer. Med. Assoc., May 28, 1904.] 
Trypanosome of the Philippines,' even go so far as to suggest, on morphological grounds alone, that the Mauritian and Philippine trypanosomes are probably distinct species.]

In I886 Blanchard, ${ }^{1}$ in Tonkin, recorded an epizootic among mules, due, in all probability, to $T$. evansi.

In recent years surra has been observed in nearly all parts of French Indo-China (IgoI-IgO3), in the Dutch East Indies (since I899), in the Philippines (I9OI), in Mauritius (I902), [in Hong Kong ${ }^{2}$ (IgO5), and in Perak, ${ }^{3}$ Federated Malay States (I905)]. The investigations and experiments made in these different countries have materially increased our exact knowledge of this deadly epizootic.

As regards India and the countries bordering upon it, Lingard's last Report (I899) contains detailed information concerning the geographical distribution of surra, which we shall summarize briefly. The disease occurs in twenty-two out of thirty-one districts of the Punjab, in the North-West Provinces, in Kumaon (in the Himalayas), in the north-east part of the district of Jalpaiguri (Bengal), in Rajputana, and in the Bombay Presidency. In other words, nearly the whole of Northern India is affected, and through Rajputana the disease reaches as far west as Bombay. The Dekkan is almost immune. Lingard records an outbreak in 1893 at Secunderabad (Hyderabad). According to information supplied to us by Drs. Donovan and Gouzien, the disease is unknown in the neighbourhood of Madras and of the French possessions in the south-east of India ${ }^{4}$ (Pondicherry).

Outside India the disease is found in Persia, according to Haig (quoted by Lingard). It certainly occurs along the shores of the Persian Gulf, because trypanosomes have been found in horses imported into Bombay from those parts.

Nearly the whole of British Burmah, Manipur, and Assam are infected, Steel's original observations having been made at Rangoon and Taunghu. The same is true of the countries bordering on China-the Shan Provinces (Lingard) and Yunnan (Blin). We have no idea of the extent of this epizootic in China, but there are reasons for thinking that the disease of ponies and cattle recorded by W. G. Campbell in Korea (quoted by Lingard) is really surra, although microscopical confirmation is still lacking. [Gibson has recorded a case of unsuspected trypanosomiasis in a Chinese buffalo, which was brought to the abattoir in Hong Kong from the neighbourhood of Kowloon (see p. 258).]

In French Indo-China surra is not unknown. Blanchard observed it amongst the mules imported into Tonkin, and now the disease is endemic in that region.

1 See Mollereau, Bull. Soc. centrale mid. vittér., December 30, 1 S8S, p. 694.

2 [Gibson, Journ. Comp. Path. and Therap., v. 18, 1905, pp. 79, 80.1

" [Leicester, in Report of British Resident-General for the Federated Malay States, 1905.]

${ }^{+}$Donovan has, however, seen trypanosomes resembling $T$. criansi in a calf from the neighbourhood of Madras (private communication). 
From information obtained by Blin and Carougeau, veterinary surgeons at the Pasteur Institute in Nha-Trang (Annam), the regions most affected are Laos, Upper Tonkin, and Annam (especially in the neighbourhood of Nha-Trang). Horses and dogs - particularly European dogs - are affected, and in certain districts this epizootic is so widespread that horse-rearing is impossible. It is said to be propagated by horse-flies(?). The experimental investigations made by Carougeau at Nha-trang, ${ }^{1}$ and the examination of specimens of the blood of infected animals (horses and dogs) which he sent us on two occasions, clearly demonstrate the nature of this epizootic of Indo-China.

[Recent investigations have shown that trypanosomiasis is present in practically all provinces of French Indo-China in endemic form, and that from time to time it becomes epidemic. Vassal ${ }^{2}$ has made an extended study of the trypanosomiasis of horses in Annam, and has met with epizootics at Nha-trang, Khan-hoa, Vinh, and other places in Annam. Vassal states that every year there is a great mortality amongst the horses in the Laos district. Recently the districts of Vientiane, Muong - Sieng, Luang - Prabang, and Muong-Sau have been visited by the epizootic, which has spread to the adjacent Siamese provinces. The natives have for a long time noticed that the horse-flies are always very abundant at the height of the epizootic. Yersin ${ }^{3}$ thinks that Laos is an important endemic focus of the disease. At Yen-Lay, in the province of Ninh-Binh, Bodin ${ }^{4}$ came across several cases of the disease. Several foci of this equine epizootic have been met with in Tonkin by Lepinte and by Séguin (at Hanoi, Viétri, and Bac-Kan).]

Further south the disease also occurs, for recently a trypanosome epizootic in horses has been observed at Hatien, a seaport on the borders of Cochin-China and Cambodia. ${ }^{5}$ [In I905 Brau, SaintSernin, and Mutin-Boudet ${ }^{6}$ proved microscopirally the presence of surra in horses in Saigon, which had already been suspected by the veterinary officers $\mathrm{Blin}^{7}$ and Chaptal. ${ }^{8}$ It appears that the cases were always sporadic, coming either from Annam or from a focus at Cape St. Jacques and environs (Cochin-China).]

The accompanying map (Fig. 29), showing the distribution of

1 Carougeau, Bull. Soc. centrale méd. vétér., June 30, I901, p. 295.

2 [J. J. Vassal, Ann. Inst. Past., v. 20, 1906, pp. 256-295.] 1904.

3 [Yersin, Bull. économ. de l'Indo-Chine, No. 27, 1904, and Ann. Inst. Past.,

${ }_{4}^{4}$ [Bodin, Bulletin économique, No. 46, October, 1905.]

5 Kermorgant, Bull. Acad. Médecine, v. 50, November 3, I903, p. 262. Montel, Ann. dHyg. et de Méd. colon., I904, v. 7, p. 219. The presence of the trypanosome of surra in the blood of animals suffering from this disease was established by one of us.

${ }_{6}^{6}$ [Brau, Saint-Sernin, and Mutin-Boudet, Bull. Chambre d'Agriculture de Cochinchine, ninth year, February I0, I 906 , pp. 39-50. Abstract by Mesnil in Bull. Inst. Past., v. 4, I906, p. 674.]

7 [Blin, Rev.gen. méd. vét., I903, I., p. 213.]

8 [Chaptol, Rapport au Lieut.-Gouverneur de la Cochinchine, August 8, i 9.05 , Bull. Chambre d'A griculture, No. 8, August, I905.] 
surra in Asia, is borrowed, so far as India is concerned, from the R.A.M.C. Journal for January, Ig04.

In the Dutch East Indies surra was first observed among the Equidæ and buffaloes (var. Karbouw ${ }^{1}$ ) of the districts Samarang and Rembang, in Java. It appears to be spreading, for Schat ${ }^{2}$ records it in the interior of Java (in Kediri and Soerabaya), where in Igor an epizootic attacked cattle and buffaloes. Owing to the energetic measures taken - the slaughter or isolation of infected animals and protection against the bites of flies-the outbreak has been checked,

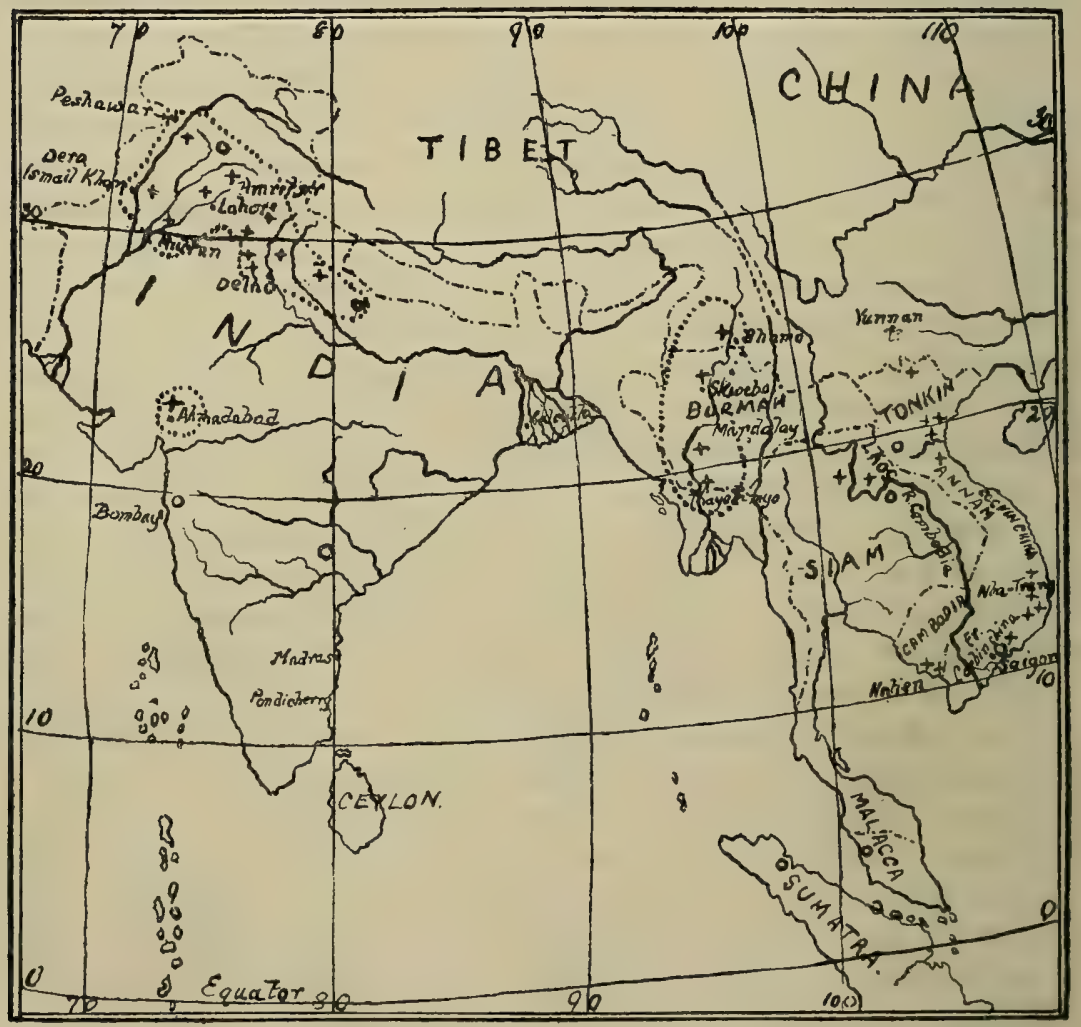

Fig. 29.-Map showing the Distribution of Surra in India and Indo-China.

The areas in which the disease is endemic are marked with a + ; those in which it has been observed only sporadically or as a temporary epizootic are marked with an $O$.

and it will doubtless be possible to prevent it from establishing itself in that region. In Sumatra a spontaneous infection of horses appears to have been seen by Vrijburg, veterinary officer at Deli. ${ }^{3}$

[Probably the epizootic of surra in Perak, investigated by Leicester in I905, was imported from Sumatra.]

1 Penning, Veeartsenijk. Bladen v. Ned. Indiè, v. 12 and 13,1899 and 1900.

2 Schat, paper published in 1902 in the Arclives de I"Industrie sucriire d Java. We are indebted to M. Van Reuth and Dr. L. Vincent for the translation of this paper, which is in Dutch.

3 Vrijburg, Veeartsenijk. Bladen v. Ned. Indii,, v. ${ }^{1} 3$, quoted by de Does, Geneesk. Tijdschr. $v$. Ned. Indië, v. 41, 1901. 
Since September, Igor, surra has been known in the Philippines, and its presence there induced the Bureau of Animal Industry at Washington to publish in its Bulletin, No 42 (IgO2), an 'Emergency Report on Surra,' by Salmon and Stiles, containing a summary of the publications on trypanosomes in general, and on surra in particular. In their work Salmon and Stiles reproduce the reports of the veterinary officer Slee, and of Drs. Allen Smith and Kinyoun. Discovered first among the horses in Manila, it was soon recognised that the epizootic occurred throughout the island of Luzon. According to Curry, it also attacked buffaloes (var. Kerabau). Musgrave and IVilliamson have given a detailed account of the disease in horses. ${ }^{1}$ They consider that the instances of infection seen by Curry in buffaloes were exceptional, and that no importance need be attached to them.

It is by no means certain how the disease originated. Salmon and Stiles incline to a recent importation of the disease. They think that Anglo-Indian troops carried it from India to China, and the Americans from China to Luzon. On the other hand, the medical and veterinary officers who have studied the disease on the spot are of opinion that the disease existed in the Philippines before the American occupation, and that it was known there among horses under the name of Calentura. The report by Maus, ${ }^{2}$ dated September, I9OI, is almost conclusive on this point. He describes what is evidently the trypanosome of the blood of horses suffering from calentura under the name of Spirillum, without suspecting that he had before him a flagellated Protozoon, which was the parasite of a well-known group of diseases.

In their recent work Musgrave and Williamson state that surra could not have existed in the Philippines before May, I9oI. Their inquiries lead them to suspect that Australian horses imported the disease. We may remark here, however, that the presence of surra in Australia has not yet been proved. Musgrave and Clegg have published an important work upon the epizootic of surra in the Philippines.

In Mauritius a trypanosome epizootic amongst horses and cattle was recorded during I9O2, at the beginning of the sugar-growing season. Already, in June, I902, according to the veterinary officer Deixonne, the mortality was appalling, the majority of the draught animals having succumbed. ${ }^{3}$

1 Musgrave and Williamson, Biolog. Laboratory, 1903, No. 3. Musgrave and Clegg, "Trypanosoma and Trypanosomiasis, with Special Reference to Surra in the Philippine Islands.' Department of the Interior, Biolog. Laboratory, No. 5 , Manila, 1903.

2 Summarized in a leading article in New York Med. Journ., February 8, 1902.

3 On the subject of the epizootic in Mauritius consult : Laveran, Acad. de Méd., October 28, 1902; Edington's Reports of August 8, 14, and 18, 1902 ; Report of the Committee of Inquiry to Study the Steps to be taken to stop the Progress of the Epizootic in Mauritius, I903; Vassal, 'Sur le Surra de Maurice,' Journ. off. de Madagascar, June 27,1903 . We have also utilized the information contained in letters sent to one of us by Dr. A. Lesur and by M. Deixonne, veterinary officer in Mauritius. 
Dr. A. Lesur wrote to one of us on June 27 , I902, as follows : - Surra has been prevalent in Mauritius since the end of last year. The markets of Madagascar, which have always furnished the colony with its oxen for transport and for food, having been practically closed to us on account of the competition of the English military authorities during the Transvaal War, our traders thought it would pay them better to import cattle from India. A cargo of them arrived in September, Igor, but a number of the animals having died during the voyage, the vessel was put in quarantine. The mortality continuing, the sanitary authority ordered post-mortems, which, however, did not reveal the cause of death. Vague and general explanations were deemed sufficient, and the animals were allowed to land. Nevertheless, the animals went on dying in the stalls where they were kept under observation, and the cause of death remained as obscure as ever. Finally, the animals which survived were sent to their consignees, who placed them in a district in the north of the island, where they became a centre of infection, which gradually spread. At the present time practically the whole of the island is infected.

' The epizootic at first appeared to be almost exclusively confined to oxen; then it attacked mules, donkeys, and horses, without, however, in any way diminishing its ravages upon cattle. The destruction of draught animals has gone on to such an extent that farmers are anxiously asking themselves whether they will be able to gather in the harvest. It is true we have already begun to import fresh animals-healthy ones this time-but as there is no law compelling the owners of sick animals to slaughter them, we are providing fresh material which will serve to perpetuate the epizootic.

'The nature of the disease was quite misunderstood at the outset, which is due to the fact that the veterinary officers in Mauritius had never seen cases of surra. The diagnosis usually made was gastro-enteritis, due to bad feeding.

'Last March my brother, Dr. Aimé Lesur, at the request of a friend whose stable was ravaged by the disease, examined the blood of sick mules microscopically, and found numerous trypanosomes present. Afterwards he also found them in the blood of cattle. Some weeks later, when the disease reached the district in which I live, I had the opportunity of making similar observations. . . .'

Since then the ruin has become complete. On January 29, I903, M. Deixonne wrote to us that the horses and mules had practically all disappeared. At Port Louis it had become necessary to hand over the work of scavenging to prisoners, who, under the supervision of policemen, were used to pull the carts.

From July to October, 1902, the death of 1,882 solipedes and I,68I cattle was recorded in Mauritius (Vassal).

The introduction of the epizootic into Mauritius by cattle from India has been questioned by Edington, but it is proved that sick 
animals coming from India were imported into Mauritius towards the end of Igor, and the arguments which have been adduced to show that surra was endemic in Mauritius before that time are quite inconclusive. The exceptional severity of the disease in the island is distinctly in favour of the idea of importation, for epidemic or epizootic diseases usually spread more rapidly, and are more severe in districts previously immune than they are in their endemic foci.

In Igor Vassal observed an isolated case of surra in Réunion, in a cow which was vaccinated against cattle plague by Turner and Kolle's method in August, IgoI, and which died on September 20, 'with very many trypanosomes in its blood, spleen, and kidneys.' Although from that time Vassal was always on the look-out for surra, he never came across another case. The origin of this isolated case remains a mystery, because the cow, Dr. Vassal told us, was born at St. Denis, in Réunion.

M. Deixonne was good enough to send us, through Dr. Vassal, living trypanosomes from animals with the Mauritian epizootic. IVe have consequently been able to study the disease in other animals.

The epizootic in Mauritius, after a period of quiescence during the last six months of 1903 , showed a marked renewal of activity in February, I904. ${ }^{2}$

[Further information concerning the Mauritian epizootic is given by Edington and Coutts ${ }^{3}$ and by Manders. ${ }^{4}$ Edington states in his report (p. 59) that during the year 1903 information had been receired from Mauritius to the effect that some of the diseased animals had completely recovered, and were doing good work; also that two oxen inoculated by him shortly after his return from Mauritius had completely recovered from the disease. Their blood was no longer infectire, and the oxen were completely' immunne, 'as ewen after a large inoculation into the rein the blood is not found to be infective when tested by inoculation into susceptible animals.']

[Manders states that the greatest mortality occurs during the months when Stomoxys geniculatus, De Bogot-the almost certain carrier of the disease in Mauritius - is most abundant. The local authorities now order the killing of any horse, ass, or mule certified by a veterinary surgeon to have surra, but cattle as a rule are isolated. In I904, 283 solipedes and $2+$ bovines were slaughtered by order of the Medical and Health Department. Between January and April, I905, 228 solipedes were slaughtered, but no bovines.]

[The latest accounts 5 of the Mauritian epizootic show that cattle are quite as susceptible as horses and mules. In r903, 2,25I cattle and 965 solipedes died of the disease; in Igot, 260 cattle and 823 solipedes.]

1 Vassal, Revue agricole de la Réunion, December, I9or.

2 Letter from M. Deixonne, dated Mauritius, February I I, I904.

3 [A. Edington and J. M. Coutts, Report of the Director of the Government Bacteriological Institute, Grahamstown, for the year I903, pp. 58-6I. Cape Town, I904.]

[N. Manders, R.A.M.C. Journ., v. 5, I905, pp. 623-626].

5 [Annual Report of the Medical and Health Department for I907, Port Louis, June 29, 1905 , quoted from Vassal.] 


\section{Section 2.-Animals Susceptible to Surra. Symptoms and Course of the Disease in Horses, Cattle, Dogs, etc.}

Like nagana, surra is inoculable into most mammals, ${ }^{1}$ but the disease occurs naturally only in the Equidæ, Bovidæ, Camelidæ, and, more rarely, in dogs. We shall first study the symptoms and course of the disease in these animals, and afterwards consider the animal species in which surra is known only as an experimental disease.

EOUID E.-The horse, mule, and donkey are very susceptible to surra. In certain parts of India surra frequently gives rise to serious epizootics amongst horses. In I8go forty ponies died of the disease at Katgodam, while fifty more died from 189 I to 1893 . In 1893

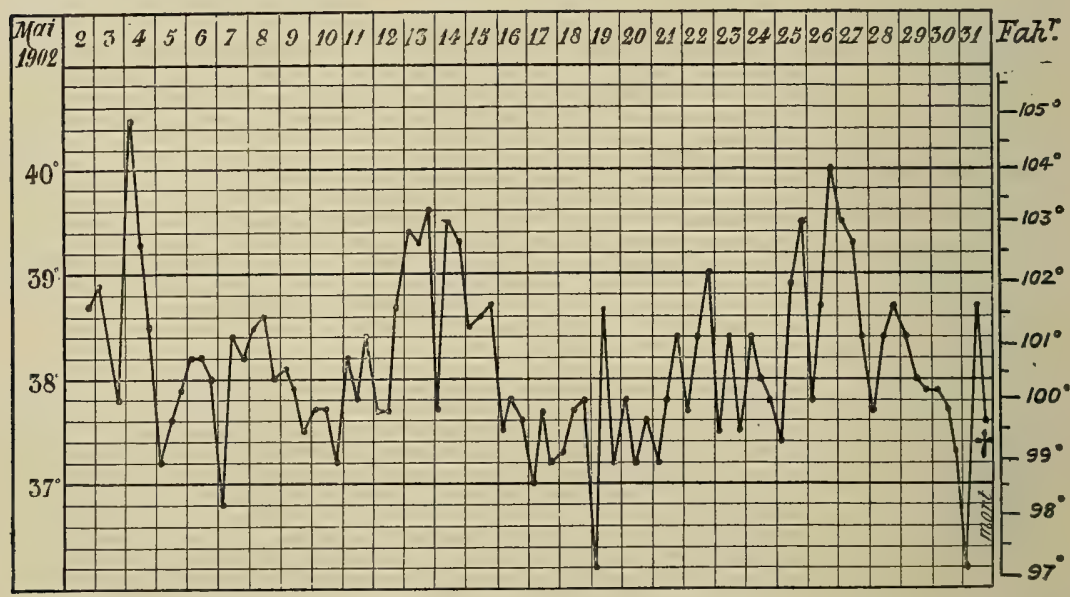

Fig, 30.-Temperature Chart of a Horse which died of Surra during the EPIZOOTIC IN Mauritius.

[The normal temperature of the horse varies from $98^{\circ} 4^{\circ}$ to $102^{\circ} \mathrm{F}\left(37^{\circ}\right.$ to $39^{\circ} \mathrm{C}$.).]

there was an outbreak in the stables between Saharanpore and Mussoorie. There have been several outbreaks of surra among the horses in Sind.

The first symptom of the natural disease is a rise of temperature. After experimental inoculation, either subcutaneous or intravenous, Lingard found that the incubation period varies from four to thirteen days. In a certain number of cases the rise of temperature is soon followed by an urticarial eruption. Petechial hæmorrhages on the mucous surfaces-particularly of the nictitating membranerunning from the eyes, and œedema also occur. The animal is dejected and shows marked loss of strength, but, as a rule, the appetite is good. The mucous membranes become very pale, and later on yellowish in colour. Anæmia is constant and progressive; there is an increase in the number of the leucocytes except of the

1 The disease does not appear to be transmissible to man. Schat pricked himself several times, with impunity, with needles which had been used to draw blood from animals infected with surra. Birds are refractory (Lingard, Penning). 
eosinophiles, which are diminished in number. The red corpuscles no longer run in rouleaux, and are deficient in hæmoglobin. Fever is remittent, or may be more or less regularly intermittent, the periods of intermission lasting from one to six days.

Contrary to what is usually the case in nagana, trypanosomes are not constantly present on microscopical examination of the blood, as Lingard has frequently observed. At first very scanty in the blood, they increase in number more or less rapidly until they reach a maximum, rarely exceeding 400 per cubic millimetre, but they may be as numerous as 350,000 per cubic millimetre. Then they diminish in number, increase again, and so on alternately. They may be absent from the blood for one to six consecutive days, and these periods coincide exactly with the apyretic intervals.

Death invariably occurs, but at a varying time after the onset of the disease. Steel gives fifty-two days for the duration of the disease in the horse, thirty in the pony. [But Pease (loc. cit.), experimenting with the camel trypanosome in India, found that ponies lived three to four months after inoculation, and that some native ponies even recovered from the infection.]

Of 35 horses experimentally infected by Lingard, 9 died in less than 6 days, 25 in from 6 to 45 days, and I in 56 days, after the first appearance of trypanosomes in the blood. Of 76 horses which contracted the disease spontaneously, 4 died in less than 6 days, 63 in from 6 to 55 days, and 9 in from 55 to IIo days, after the first appearance of trypanosomes in the blood.

[An Arab stallion, which had been in hospital for three months with a spontaneous infection of dourine, was inoculated by Pease ${ }^{1}$ with the virus of surra. This virus had been passed for some time through rabbits and dogs, and was rather attenuated. The horse was inoculated by scratching the nose and rubbing in some infective blood. The diseases, dourine and surra, ran their courses concurrently; the characteristic plaques of the former disease appeared from time to time, and the surra trypanosomes were present in the blood, with intermissions of two or three to five or six days. The horse became very weak, and was killed three months after the inoculation with surra.]

[Thomas and Breinl, ${ }^{2}$ experimenting with the Mauritian strain of surra which they obtained from Laveran, injected a horse subcutaneously with the blood of an infected rat. The incubation period was four days. The temperature rose on the fifth day, and the trypanosomes rapidly increased in numbers. The horse ate little and stood with its head hanging down. The parasites increased to 40,00o per cubic millimetre. There was rapid anæmia, the hæmoglobin falling from 95 to 63 per cent., but no great change was

1 [Pease, Vet. Journ., v. 9, 1904, p. 187.]

2 [Thomas and Breinl, Thompson Yates and Johnston Lab. Reports, v. 6, part 2, 1905, pp. 56,65. The duration of the disease is not stated.] 
observed in the numbers of the red and white corpuscles. A temporary improvement followed on injections of atoxyl, the parasites diminishing in number, the hæmoglobin increasing, and the animal gaining in weight for a time. The horse died in spite of treatment, and post-mortem all the organs were anæmic, but no other characteristic lesions were found.]

The average duration of the disease in the mule appears to be only nineteen days (Steel).

Lingard infected a donkey by subcutaneous inoculation. The incubation period was three and a half days, and the disease lasted nine days.

During the Mauritius outbreak the mortality from surra amongst the horses and mules was Ioo per cent. In some of the agricultural districts all the horses, mules, and donkeys were carried off by the disease.

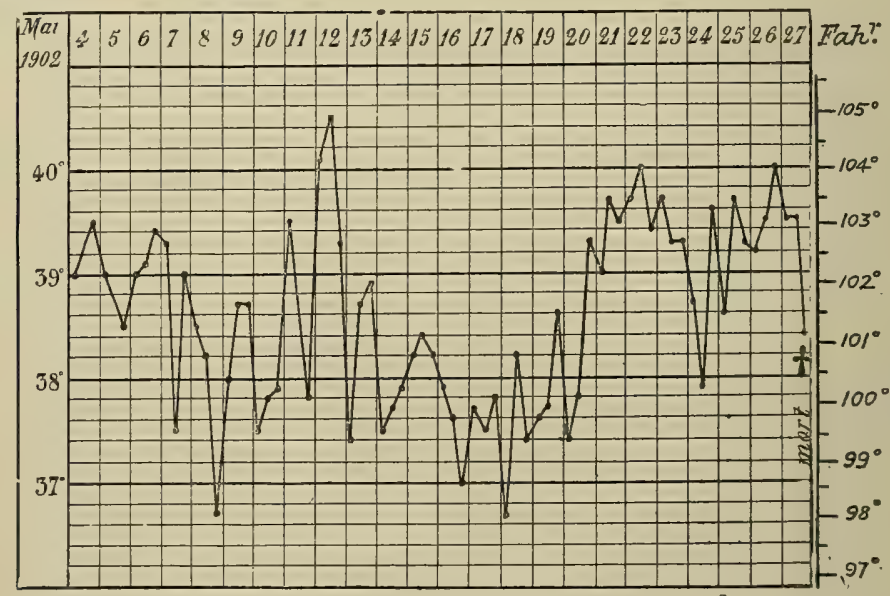

Fig. 3i. - Temperature Chart of a Horse which died of Surra during the MaURITIAN EPIZOOTIC.

In a letter dated June 27 , I902, Dr. Alfred Lesur describes the symptoms observed in Equidæ during the epizootic in Mauritius in the following words :

'The onset of the disease is insidious. The first noticeable symptom is a change in the gait. The animal no longer exhibits its customary vigour, but is slow at its work and appears to be lazy. This is often accompanied by a loss of appetite, but this is not a constant symptom.

' Fever soon makes its appearance, the temperature often rising to $4 \mathrm{I}^{\circ} \mathrm{C}$. $\left[\mathrm{Io6}^{\circ} \mathrm{F}.\right]$, or even higher. A certain number of animals die at this stage, but they are the minority. In the others the temperature, after oscillating at this high level for two or three days, falls spontaneously, or as the result of treatment. Fresh rises of temperature occur at intervals of four, five, nine, or ten days.

'Shortly after the onset of fever large odematous swellings 
appear on the chest, hypogastrium, and in the male very often in the sheath. On incising these swellings a small quantity of fluid escapes, in which many trypanosomes are found. These swellings may disappear and reappear again during the course of the disease.

' Anæmia rapidly supervenes and becomes very profound. The conjunctivæ are exsanguine, and the buccal mucous membrane has an ivory-white colour.

'At this stage of the disease the animal has lost its appetite, is very feeble, and easily falls in its stable, often never to rise again.

'Death may occur very suddenly in the first stage of the disease ; and, indeed, many animals which were not apparently ill, and which were still working, died in this way.

'Trypanosomes in appreciable numbers are present only for a short time and intermittently in the peripheral blood. For example, an animal which showed many parasites in its blood on a Tuesday did not show a single one on the following Saturday. This disappearance of the parasites, which may be only apparent, does not coincide with any improvement in the animal's condition.' ${ }^{1}$

Dr. Lesur kindly sent us temperature charts (Figs. 30 and $3 \mathrm{I}$ are reproductions of two of them) which show the characteristic rises of temperature occurring in surra.

Bovide, Buffaloes. - Bovines are much more resistant than equines. Lingard infected oxen by inoculating them with blood, but they all survived and had very few symptoms. There is swelling at the site of inoculation. As a rule, the temperature rises at the onset of the infection, and from time to time other rises may occur. The parasites are visible in the blood only from the fourth to the tenth day after inoculation, rarely later. But the blood remains infective for the guinea-pig for a very long time. In one case it was infective $I 63$ days after inoculation, but was no longer so on the 234th day. The animal recovers after having wasted very considerably. These observations of Lingard have been confirmed by Rogers. A second inoculation is not followed by any symptoms; in one case a single trypanosome (giant form) was seen under the microscope six days later. Subsequent inoculations into guinea-pigs were negative; the animal, therefore, had become immunized by recovering from the first attack of the disease.

Vrijburg, in Sumatra, has confirmed this observation in the case of the zebu.

Steel regards the ox as refractory simply from the microscopical examination of the blood; nevertheless, the ox inoculated by him showed rises of temperature, the first of which was particularly marked a few days after inoculation.

Lingard has studied the disease as it occurs naturally among oxen in India. Recovery is the rule, but the animals pass through a stage

1 Private communication from Dr. A. Lesur; see Laveran, Acad. de Méd., October.28, 1902 . 
of extreme emaciation. The blood is rich in trypanosomes during the febrile paroxysms.

Lingard has demonstrated the relatively great susceptibility of the buffalo. In two buffaloes the incubation period was five days. One animal died in 125 days, after twelve febrile paroxysms, with marked emaciation, in spite of a voracious appetite right to the end ; the second buffalo died in 5 I days.

[Two buffaloes inoculated by Pease with the camel trypanosome died, very wasted, in I20 and 46 days; a third was alive more than two years after infection, but its blood was still infective on inoculation into dogs. Pease found that the camel trypanosome, which at first killed dogs in from 63 to 288 days, became more virulent for dogs (killing them in 52 to 99 days) after passage through the buffalo. He is of opinion that in India the buffalo plays an important part in the transmission of surra.]

Penning (loc. cit., second paper) has described in detail the outbreaks of surra among the buffaloes at Samarang and Rembang (Java). The disease usually runs a chronic course, but in some cases death occurs suddenly. The usual symptoms are gradual wasting, small oscillations of temperature, and muco-purulent inflammation of the cornea, eyelids, and nose. Some animals also have œdematous swellings, especially of the abdomen.

[In Hong Kong, Gibson ${ }^{1}$ found numerous trypanosomes in a blood-film of a Chinese buffalo, which died suddenly in the abattoir into which it had been brought the previous day apparently in good health. These small buffaloes, which are slaughtered for food, are brought to Hong Kong from the mainland of China, twenty or thirty miles from Kowloon. $\left.{ }^{2}\right]$

[Shortly afterwards trypanosomes were found in a sick dog belonging to the inspector of the abattoir. These trypanosomes were pathogenic for dogs and guinea-pigs, but none of the latter died. According to Gibson, the trypanosome is morphologically indistinguishable from $T$. evansi.]

During the epizootic in Mauritius the mortality amongst cattle was rarely more than 25 to 30 per cent., whilst in equines it was Ioo per cent. (Deixonne). Animals which are well nourished and which are not made to do hard work live much longer than those which are badly nourished and overworked. In Mauritius the animals kept solely for breeding purposes enjoyed almost complete immunity from the disease, whereas in certain districts the mortality amongst cattle made to do hard work was from 75 to 8 o per cent. Overworked oxen when they become infected die of anæmia rather than of surra, for the injection of their blood in the last stage of the disease into susceptible animals often gives negative results (Deixonne).

\footnotetext{
1 [Gibson, Journ. Comp. Path. and Therap., v. 18, 1905, p. 79.]

2 [Personal communication from Dr. Bell, of the Government Civil Hospital, Hong Kong.]
} 
In Mauritius the symptoms of surra in cattle were as a rule much less constant and less marked than in equines. Frequently the only sign of the disease was the periodical febrile paroxysm, which passed unnoticed if the animals' temperatures were not taken.

Trypanosomes are scanty in the blood, and as a rule they are not seen on microscopical examination, so that it is necessary to inoculate susceptible animals in order to make certain of the diagnosis.

Sometimes, however, the disease in cattle may be very grave and run a very rapid course. During the epizootic in Java these grave forms, accompanied by complications, were particularly frequent. The following summary is from Dr. Schat's account of the epizootic: The respiration and pulse rates are greatly accelerated, the temperature rises to $40^{\circ}$ or $40^{\circ} 5^{\circ} \mathrm{C}$. [ $104^{\circ}$ to $105^{\circ} \mathrm{F}$.], the nostrils are dry, and the eyes watery, with intense redness of the conjunctivæ. There is a pustular eruption, with the formation of scabs and small superficial abscesses in different parts of the body, particularly on the neck, belly, and hind-legs. The buccal mucous membrane is covered with red patches, and the animal loses its appetite. There is almost constant diarrhoa, the dejecta consisting of reddish matter mixed with undigested food. In less severe cases the excreta are greenishyellow in colour, without any trace of blood.

In one ox there was actual sweating of blood: minute droplets of blood were seen rising up on the surface of the skin, without any traces of pricking or stinging by insects. In other cattle there were hæmorrhages from the nostrils and ears, or an abundant discharge of greenish fluid from the nose.

In the severe infections the animals died in twenty-four hours after the first appearance of symptoms ${ }^{1}$; in other cases the disease was prolonged for three or four weeks, and it might then end in recovery.

The oxen known as Madoereesche were less often attacked than the pure-bred Javanese oxen, and when they were attacked by the disease they were more resistant than the latter.

Trypanosomes have been found in apparently healthy animals, so that the disease may exist in a latent form. Schat sometimes found numerous trypanosomes in the blood of cattle. In the paper by Musgrave and Clegg previously mentioned there are interesting details of the course of surra in cattle during the outbreak in the Philippines. One ox died in twenty-four days.

Several bovines were inoculated with surra by Nocard and Vallée at Alfort, one of the animals dying of the disease. Fig. 32 gives the course of the temperature in a calf which was inoculated with surra on July 4, I903, by Vallée. The animal never showed any symptoms, but in February, I904, its blood was still virulent, and the calf was still alive in May, I904.

1 This does not mean that the trypanosome of surra can kill cattle in twentyfour hours, or even in several days, but that the disease which has been latent may suddenly manifest itself by rapidly fatal symptoms. 


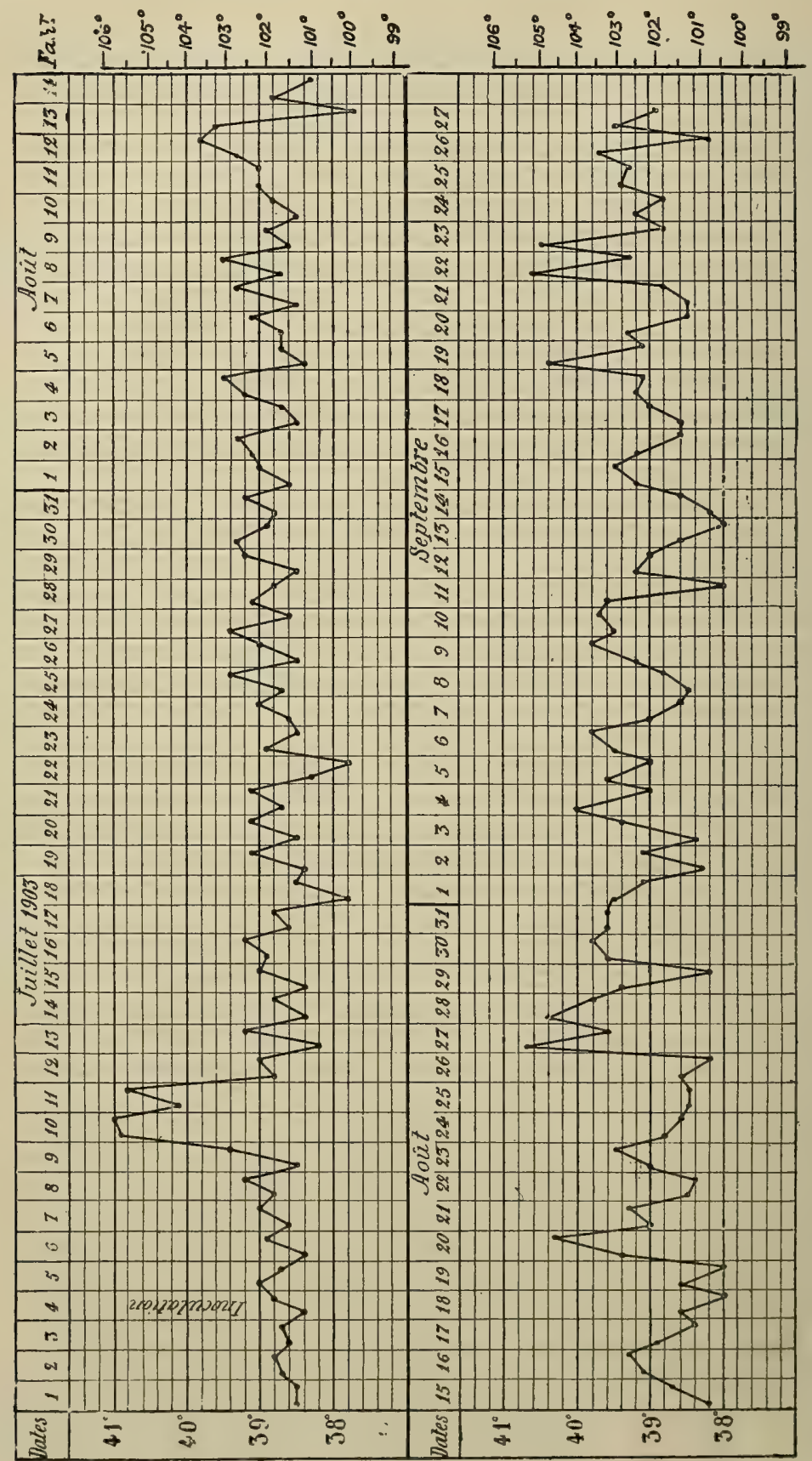

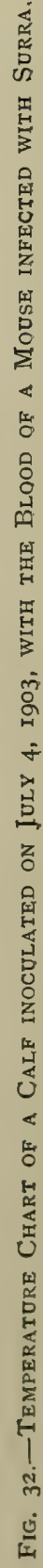

[Vallée and Panisset, ${ }^{1}$ who continued the observations upon these animals at Alfort, state that at the end of a year trypanosomes could no longer be found in the blood; and the two animals (Breton calves) were

1 [Laveran and Mesnil, C. R. Acad. Sciences, v. 140, 1905, p. 831 ; see note by Vallée and Panisset on p. 833.] 
reinoculated with the same virus on July I9, Ig04. No infection followed, thus showing that the calves had acquired immunity against Mauritian surra. Two inoculations with the virus of mbori, made on August 8 and September I9, I904, were equally unsuccessful. $]^{1}$

[On December I5, 1904, the two calves were inoculated with the Indian strain of surra, and at the same time a normal Breton cow was inoculated as a control. Blood taken on December 23 and 30 from the control cow was infective for mice and guinea-pigs in very small doses, whereas that of the two calves was not infective, even in large doses. On January I8, I905, I00 c.c. of blood were taken from each calf, and 50 c.c. injected at once intraperitoneally into dogs. All four animals so injected were still free from trypanosomes two months later. The control cow had a severe infection, to which she nearly succumbed, and her blood was still infective in March, 1905.]

['This experiment establishes indisputably the identity of Indian and Mauritian surra' (Vallée and Panisset).]

[An ox was inoculated by Edington and Coutts with the Mauritian virus on September 4, 1902. Between that date and November 2, 1903, it received seven other injections of virulent blood. The ox had become immunized. Rats inoculated with blood of this ox on November 3 and 5, I903, did not become infected, whereas some months previously the blood of the ox produced a fatal infection in dogs.]

Camels.-According to Lingard, the symptoms of the spontaneous disease in camels are: fever, swellings-of the chest, of the sheath, and scrotum in males, and of the breasts in females-which may suppurate and contain much pus, and progressive anæmia and wasting, in spite of a good appetite. The trypanosome is found in the blood only during the febrile paroxysms, when the temperature may be as high as $4 \mathrm{I}^{\circ} \mathrm{C}$. [105.8 $8^{\circ} \mathrm{F}$. . There would appear to be a small percentage of recoveries when the disease has lasted more than three years.

Elephants. - The occurrence of surra in elephants in India and Burmah is practically proved. In this connection we have only the statement of G. H. Evans, reproduced by Lingard (Summary of Further Report, I894), that in 1893 fourteen out of thirty-two elephants died of the disease in Burmah. In his Annual Report for I895-I896 Lingard incidentally mentions the trypanosomes of the elephant, as if he had seen them. It is, however, doubtful whether the elephant is to be included in the category of animals in which surra runs an invariably fatal course.

Dogs.-Surra has often been seen as a natural disease in the $d o g$. Lingard records, in his various reports, the occurrence of epizootics of surra among sporting dogs introduced from England. $\mathrm{He}$ observed outbreaks in the island of Bombay, and in other parts of the Bombay Presidency. He quotes the opinion of Evans that surra occurs among the dogs at Mandalay, and that of $\mathrm{H}$. T. Pease, that it occurs among the dogs in the district of Karnal, in the Punjab.

In I89I a disease which appears to have been allied to surra was

1 [Vallée and Panisset, C. R. Acad. Sciences, v. I39, 1904, p. 90I.] 
prevalent among several packs of hounds in Bombay. In 1893 Lingard ${ }^{1}$ saw an outbreak of surra among English foxhounds.

The following are the principal symptoms noted: Elevations of temperature, anorexia, œdema of the head and throat, injection of the conjunctivæ, and, in some cases, effusion into the joints, and corneal opacity leading to partial or total blindness. During the febrile paroxysms trypanosomes are found in the blood on microscopical examination.

We have already mentioned that spontaneous cases of surra in dogs have been reported in Indo-China [and Hong Kong ${ }^{2}$. During the epidemic in Mauritius a certain number of dogs died of surra. We shall refer to this again later. It seems that the mode of infection in

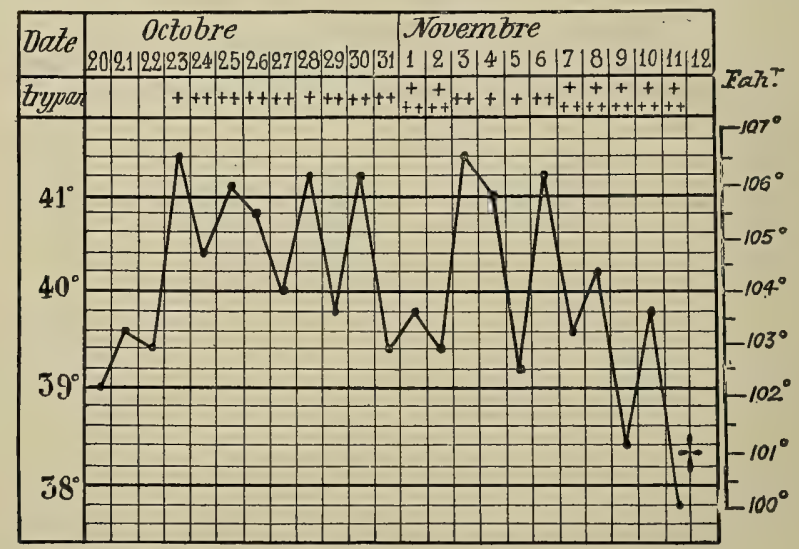

Fig. 33. -Temperature Chart of Dog No. 4, inoculated Intravenously with SURRA.

O means absence of trypanosomes on microscopical examination + , trypanosomes present, but scanty; ++ , fairly numerous ; ++ , numerous or very numerous; \&, means death.

dogs is not the same as in horses and cattle, which would account for the relative rarity of the disease in dogs.

Dogs are easily infected with surra by subcutaneous inoculation. Lingard experimentally infected eight dogs, which died in $\mathrm{I} 4 \frac{1}{2}, 2 \mathrm{I}$, $27 \frac{1}{2}, 29,34,36,47$, and 97 days after inoculation with blood obtained from different animals. The dog which lived 97 days - the incubation period lasting only 5 days - had been inoculated with the blood of a bovine suffering from the natural disease. In all cases the experimentally produced disease showed the same symptoms as the natural disease.

1 Lingard, Report of 1894 . According to Lingard, surra had been prevalent in dogs ever since 1869 in the kennels at Ootacamund, and in 1884 fourteen couples of dogs died in the packs in Madras of the same disease, the true nature of which was misunderstood until $\mathrm{r} \delta 93$.

${ }_{2}$ [Dr. Bell, of the Government Civil Hospital in Hong Kong, has sent home blood-films from a guinea-pig inoculated with the blood of a sick dog, in which I have found many trypanosomes closely resembling the parasite of surra. Professor Laveran, to whom I sent one of the slides, writes that he is also of opinion that 'these trypanosomes are very much like those of surra.'-ED.] 
In dogs subcutaneously inoculated trypanosomes appear in the blood from five to seven days after inoculation. There is a rise of temperature at the same time, and these febrile attacks are repeated from time to time during the progress of the disease (see charts, Figs. 33 and 34). The number of trypanosomes in the blood also varies. At times they are scanty, but these periods of arrest or of diminution in the multiplication of the parasites are short. During the last days of the disease trypanosomes are very numerous in the blood, but their diminished activity points to the approach of death. Surra is always fatal in dogs, the average duration of the disease being, in our experiments, twenty-eight days. Below we give the details of the observations on two dogs inoculated with surra-the first intravenously, the second subcutaneously.

Dog No. 4.-Weight, I I kilogrammes. On October 20, I903, I c.c. of diluted blood of an infected mouse was injected subcutaneously. Tempera-

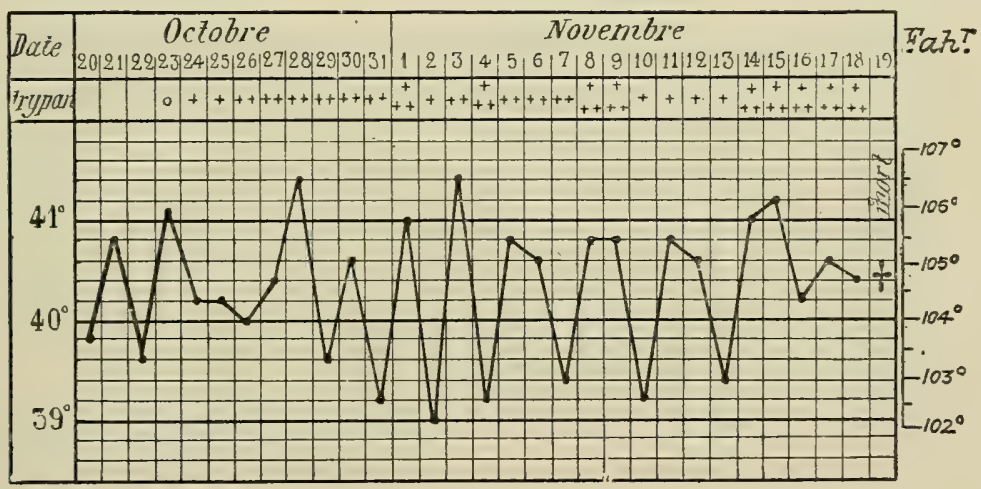

Fig. 34.-Temperature Chart of Dog No. 5, inoculated Subcutaneously with SURRA ON OCTOBER 20, I903; DIED ON NOVEMBER 19, 1903.

The symbols $\mathrm{O},+$, etc., have the same significance as in Fig. 33.

ture before inoculation was $39^{\circ} \mathrm{C}$. $\left[\mathrm{IO}^{\circ} 2^{\circ} \mathrm{F}\right.$.]. On October 23 a few parasites were seen in the blood, and at the same time there was a considerable rise of temperature-to $4 \mathrm{I}^{\circ} 4^{\circ} \mathrm{C}$. [106.6 $6^{\circ} \mathrm{F}$.]. From October 24 to $30 \mathrm{fever}$ continued with slight remissions. Trypanosomes rapidly increased in number from the 23 rd to $25^{\text {th }}$, and then remained almost stationary until the 3Ist. On October $3 \mathrm{I}$ and November I and 2 the temperature fell to between $39^{\circ} 8^{\circ}$ and $39^{\circ} 4^{\circ} \mathrm{C}$. [103. $6^{\circ}$ and $103^{\circ} \mathrm{F}$.] , but the trypanosomes became very numerous. On November 3 and 4 the temperature rose again to $4 \mathrm{I}^{\circ} 4^{\circ} \mathrm{C}$. [106. $6^{\circ} \mathrm{F}$.], while the trypanosomes became less numerous, and even scanty on November 4 . On the 5 th there was a considerable fall of temperature, but a fresh rise occurred on the 6th. After this the temperature gradually fell, so that on November I I it was only $37^{\circ} 8^{\circ} \mathrm{C}$. $\left[100^{\circ} \mathrm{F}\right.$.]. From November 7 till the night of the I Ith and I2th, when the dog died, the parasites were very numerous in the blood. There was marked wasting, and at death the animal weighed only $9^{\circ} 8$ kilogrammes. The spleen was much enlarged, its weight being 2 ro grammes-about ten times the normal weight. There were never any eye symptoms or œedematous swellings. 
Dog No. 5.-Weight, I $2 \frac{1}{2}$ kilogrammes. This dog was also injected on October 20, I903, with I c.c. of diluted blood from a mouse, but subcutaneously. On that day the temperature was $39^{\circ} 8^{\circ} \mathrm{C}$. [103.6. $6^{\circ} \mathrm{F}$.] and although it rose to $40^{\circ} \mathrm{I}^{\circ} \mathrm{C}$. $\left[\mathrm{IO}^{\circ} 2^{\circ} \mathrm{F}\right.$.] on the $23 \mathrm{rd}$, the blood examination was negative. Trypanosomes were very scanty on the 24 th, and gradually increased in number. From the 28 th onwards there was intermittent or remittent fever. On November I 8 , the day preceding death, the temperature was $40^{\circ} 3^{\circ} \mathrm{C}$. [10 $4^{\circ} 6^{\circ} \mathrm{F}$.]. Trypanosomes were present in the blood every day, their relative abundance being indicated on the chart (Fig. 34). The animal showed no eye symptoms or swellings in any part of the body. It died on November I9, the weight being $12^{\circ} 2$ kilogrammes. The spleen weighed I 70 grammes-about five times the normal weight. There was nothing else abnormal at the post-mortem examination.

[Eight dogs were inoculated by Edington and Coutts ${ }^{1}$ with the Mauritian trypanosome. The disease lasted on an average 23.6 days (minimum I3 days, maximum 39 days)].

[Thomas and Breinl, also with the Mauritian trypanosome, found the incubation period, after subcutaneous inoculation, to be seven to nine days, and the total duration of the disease sixteen to thirty days. At first the trypanosomes were scanty in the blood; they remained scanty till four or six days before death, or there were marked exacerbations persisting for a few days. In the early stages the animals presented very few symptoms, but with the rapid multiplication of the parasite decided anæmia and emaciation occurred. Death was usually preceded by a subnormal temperature. Postmortem the usual lesions met with in dogs dying of acute trypanosomiasis were found.]

[Laveran and Mesnil, ${ }^{2}$ during a series of experiments carried out with the Indian surra trypanosome ${ }^{3}$ in order to compare its effects upon animals with those of the Mauritian trypanosome, inoculated two dogs. They died in twelve and thirteen days, the incubation period lasting three days. There was continued fever, the temperature remaining at $39^{\circ}$ or $40^{\circ} \mathrm{C}$. [ $\left[\mathrm{IO} 2^{\circ} 2^{\circ}\right.$ to $\mathrm{IO} 4^{\circ} \mathrm{F}$.] and even exceeding $40^{\circ} \mathrm{C}$., until death. Trypanosomes were abundant in the blood, with a period of diminution about the middle of the disease. There were no external lesions, but wasting was extreme, the weight of the second dog falling from 7 to $4^{\cdot 8}$ kilogrammes. The spleen was enlarged, weighing 60 grammes in a dog of 7 kilogrammes.]

[Pease ${ }^{4}$ inoculated three pariah dogs with Indian surra-an equine virus passed through a rabbit-after having unsuccessfully inoculated them with dourine. The dogs died in 35,22 , and 20 days respectively, after inoculation with the $T$. evansi.]

Surra not only occurs naturally in several animal species, but its

1 [Edington and Coutts, op. cit., p. 59.]

2 [Laveran and Mesnil, C. R. Acad. Sciences, v. 140, 1905, p. 831.]

3 This strain of trypanosome was obtained from a camel by Lingard, who gave it to Dr. C. J. Martin, Director of the Lister Institute. The authors express their indebtedness to Dr. Martin for sending them an infected mouse.]

${ }^{4}$ [Pease, Veterinary Journal, v. 9, 1904, p. 187.] 
trypanosome is pathogenic for a number of other mammals. We shall consider these animals in the order of their susceptibility, beginning with the most susceptible.

MICE.-The parasites appear in the blood on an average on the fourth day after intraperitoneal, and on the fifth day after subcutaneous, inoculation. From the time that they occur in the blood they gradually increase in number, so that during the last few days of the disease they may be as numerous as the red corpuscles. Their activity becomes much diminished during the few hours immediately preceding the death of the infected mice.

The disease, if untreated, always ends fatally. During the last few days the animal loses its usual liveliness and remains very quiet and huddled up. Its coat usually becomes rough and bristling. The average duration of the disease after subcutaneous inoculation is eleven and a half days.

In several mice inoculated with the blood of a goat or dog with surra the disease ran an abnormal course. The parasites, after being present in the blood, diminished in number, or even disappeared on microscopical examination. They soon reappeared, however, and multiplied until the time of death. The disease lasted from fourteen to sixteen days, and in one case twenty-four days. It is rather curious that when three mice are inoculated under apparently identical conditions, surra may run an abnormal course in one and a normal course in the other two. The blood of a goat or sheep infected with surra usually contains very few trypanosomes, which partly accounts for the abnormal course of the disease in mice inoculated with blood from that source.

[Thomas and Breinl, using white and grey mice, found the incubation period to be three to four days, and the total duration nine to twelve days. Mice and rats inoculated with attenuated trypanosomes, such as are present in the blood of animals undergoing treatment, may only show a few parasites in their blood. Such rats and mice often acquire a chronic form of the disease, from which they frequently recover, but they are not immunized.]

[Experimenting with the Indian virus, Laveran and Mesnil found that at first the incubation period was 9 days, and the total duration $18 \frac{1}{2}$ days. After passage through a rat or mouse, the virus was exalted for the mouse, which, inoculated subcutaneously, died in $3 \frac{1}{2}$ to $8 \frac{1}{2}$ days (average 6 days), with incubation period of 2 to 4 days, and inoculated intraperitoneally died in $2 \frac{1}{2}$ to $3 \frac{1}{2}$ days, with incubation period less than I day. A grey wild mouse, inoculated subcutaneously, died in $7 \frac{1}{2}$ days; incubation period 4 days. Except in the case of a few mice which were inoculated with guinea-pig's blood, the trypanosomes never diminished in number, and at death were at least as numerous as the red corpuscles. The spleen was enlarged, and generally weighed I gramme in a mouse of 20 grammes.] 
[A jerboa (Jaculus orientalis) inoculated by Laveran ${ }^{1}$ with the Mauritian virus died in five days with an acute infection. It was, therefore, more susceptible than the rat to this virus.]

[BAT.-It has already been mentioned (p. Iog) that Laveran inoculated a bat with the Mauritian trypanosome, and that the bat died, severely infected, nine days after inoculation.]

RATs. - The trypanosomes of surra appear in the blood of white rats on the fifth or sixth day after subcutaneous inoculation. They rapidly increase in number, and are always very numerous at the time of death. The disease is invariably fatal, the average duration being eleven days. In the last stages the animals are obviously ill; they lie huddled up, and the coat is rough. We have never seen rats die suddenly with convulsive seizures, as with nagana.

[Edington and Coutts inoculated, with the Mauritian trypanosome, three white rats, which died in 4,9 , and 15 days. Thomas and Breinl found the incubation period to be 3 to $4 \frac{1}{2}$ days after subcutaneous, $2 \frac{3}{4}$ to $3 \frac{1}{2}$ days after intraperitoneal injection. The average duration of the disease was 5 to 7 days after the appearance of the trypanosomes in the peripheral blood. The course of the disease in their rats (white, black, and grey) was similar to that seen in mice.]

[Laveran and Mesnil inoculated four rats with the Indian trypanosome, two subcutaneously and two intraperitoneally. In the former the incubation period was 3 and $3 \frac{1}{2}$ days, and death occurred in $5 \frac{1}{2}$ and $6 \frac{1}{2}$ days; in the latter the incubation period was less than 24 hours, and death occurred in $3 \frac{1}{2}$ and 4 days.]

MONKEYs.-Steel showed that the monkey is susceptible to surra. He inoculated a monkey of the 'ordinary Burmese variety,' probably a Macacus, subcutaneously with a syringeful of blood. The monkey showed trypanosomes in its blood on the third day after inoculation-not on the fifth to the ninth day, but after that the parasites were again present. On the evening of the second day there was a rise of temperature, and later there were many such febrile attacks. The temperature chart given by Steel is very interesting, but refers only to the first month. One of the earliest symptoms was a reddish discharge from the vulva. The monkey became very weak and looked dejected. After two months there was great weakness of the limbs, also swelling of the feet going on to ulceration, which extended down to the bones. Finally the upper eyelids became odematous, and the animal died after some hours of coma and slight delirium.

Carougeau, Musgrave, Williamson and Clegg (loc. cit.) have also shown the susceptibility of the monkey to surra.

RABBITS.-The susceptibility of the rabbit to surra has been shown by Lingard, Carougeau, and Penning, the last-named observer ' [Laveran, C. R. Soc. Biol., v. 59, 1905, p. 250.] 
noting that rabbits with surra often show the same symptoms as rabbits with dourine.

Unlike mice and rats, rabbits never show many trypanosomes in their blood. At the beginning of the disease the animals eat well and put on flesh; towards the end, however, they rapidly lose weight. For example, a rabbit weighing 2,270 grammes was inoculated by us with surra on May 22, I903; on June I4 it did not appear ill, and its weight was 2,420 grammes, and trypanosomes were very scanty in its blood. It died on June 25 , its weight being I,695 grammes. Throughout the disease the parasites were very scanty in the blood.

At the onset of the infection, when trypanosomes first appear in the blood, there is often a slight rise of temperature. The disease lasts on an average one month, and always ends fatally.

[Edington and Coutts inoculated eight rabbits with the Mauritian virus. The average duration of the disease was thirty-five days (minimum two days (?), maximum fifty-nine days).]

[Thomas and Breinl found the incubation period to be three and a half to five and a half days after intravenous inoculation. The appearance of the parasites in the peripheral blood was usually accompanied by a slight rise of temperature, but with a severe infection the initial temperature sometimes rose to $106^{\circ}$ or $107^{\circ} \mathrm{F}$. $\left(4^{\circ}\right.$ to $4 I^{\circ} 6^{\circ} \mathrm{C}$.). During the course of the disease the fever was sometimes marked and of an irregular type; in other cases there was hardly any rise of temperature throughout. Trypanosomes were usually scanty in the blood, but in a few cases they were present in large numbers-ten to forty in a field. The temperature usually became subnormal before death; the trypanosomes were then almost absent, but in some cases were largely increased in number.]

[The symptoms were similar to those seen in rabbits with nagana and caderas. Edema of the ears and perineum, swelling of the testicles or vulva, and discharges from the penis, eyes, and nose, may all be more or less marked. Anæmia may be present, and loss of weight is constant. Young rabbits were found easier to infect than adults.]

[Two rabbits inoculated-the one subcutaneously, the other intraperitoneally - with the Indian trypanosome by Laveran and Mesnil died in twenty-seven to twenty-eight days, very wasted, but with no external lesion. Trypanosomes were very rarely seen in the blood, except during the last four or five days of the infection; but the blood was, nevertheless, infective for mice in very small doses.]

GUINEA-PIGs. - In guinea-pigs inoculated subcutaneously trypanosomes appear in the blood on the sixth or seventh day after inoculation, sometimes a little later. At the same time there is a slight rise of temperature. Thus it rose from $39^{\circ} \mathrm{C}$. $\left[\mathrm{IO}^{\circ} 2^{\circ} \mathrm{F}\right.$. $]$-the normal temperature of the guinea-pig-to $40^{\circ} 3^{\circ} \mathrm{C}$. [104. $6^{\circ} \mathrm{F}$.] in one case, and to $40^{\circ} 2^{\circ} \mathrm{C}$. $\left[104^{\circ} 4^{\circ} \mathrm{F}\right.$.] in another, the rise of temperature in each case being only of short duration and not recurring. The animals eat well and continue to gain weight-at least, during the 
early part of the disease, but in the later stages they waste. For example, a guinea-pig weighing 700 grammes was inoculated with surra on May 22, I903. On June I4 its weight was 830 grammes, and the animal appeared well, although trypanosomes were fairly numerous in its blood. The animal died on June 30 , its weight being only 670 grammes.

The increase in the number of trypanosomes in the blood is not steadily progressive, as in the case of mice and rats, but takes place irregularly. During the exacerbations the trypanosomes are numerous, whereas in the intervals between them the parasites are scanty, and may even be absent on microscopical examination. At death they are often scanty in the blood.

The disease is always fatal, and in our experiments lasted on an average 80 days (maximum I04, minimum 39).

As guinea-pigs are easily obtainable, and live a long time with the disease, they are used to keep this trypanosome alive in laboratories. By inoculating a guinea-pig every month, the virus can be kept going indefinitely.

[Edington and Coutts inoculated seven guinea-pigs with the Mauritian trypanosome. The first four died in 66, 49, 59, and $5 \mathrm{I}$ days; the last three died in Io, 30, and Io days; the average duration was 39 days.]

[Thomas and Breinl found the incubation period to be six to eight days, and the total duration forty days to four months. Trypanosomes were scanty in the blood at first, but increased later. At times they almost disappeared, then became more numerous again. There was usually a rise of temperature when the trypanosomes first appeared in the blood, but subsequently the temperature was never very high. Anæmia was not marked, but there was loss of weight, especially when many trypanosomes were present for some time. EEdema was observed in a few cases. No animal recovered without treatment.]

[Laveran and Mesnil's first four guinea-pigs, inoculated with the Indian virus, died in 76 to 88 days (average 80 days). The later ones, after passage of the virus through guinea-pigs, died soonerin 20 to 77 days. The average duration in the case of all thirteen guinea-pigs was 57 days. After an incubation period of several days, trypanosomes appeared in the blood, and remained present in variable, but often considerable, numbers until death. There were no external lesions.]

[CATs.-Thomas and Breinl infected cats with the Mauritian virus. After intraperitoneal injection the incubation period was eight to twelve days. The infection was usually chronic, lasting for six and a half to eight months, but in kittens the disease was more acute, often lasting only four to six weeks. In adult cats the symptoms were not very pronounced: slight anæmia and loss of weight, which increased towards the end of life; a discharge from 
the eyes and nose, and œdema of the perineum were occasionally observed. Trypanosomes were usually scanty in the blood of adult cats, but were numerous in the case of kittens. The chronic cases showed no characteristic lesions post-mortem, but in kittens the spleen and lymphatic glands were enlarged.]

[Panisset, ${ }^{1}$ at Alfort, inoculated the same trypanosome into cats. $\mathrm{He}$ found the incubation period to last, on an average, four days, and the total duration twenty-one days (maximum fifty-one days, minimum nine days). Wasting was usually marked, and, in the subacute cases œdemas, paresis, and ocular troubles were observed. As a rule the parasites were very numerous in the blood. Passages through cats did not produce any alteration in the virulence of this trypanosome.]

GoATs.-Steel looks upon the goat as refractory; nevertheless, the goat he inoculated had a rise of temperature to $4 \mathrm{I}^{\circ} 5^{\circ} \mathrm{C}$. $\left[\mathrm{I0} 68^{\circ} \mathrm{F}\right.$. on the ninth day after inoculation, and was markedly emaciated at the end of a month. Penning also considers the goat refractory.

Two goats inoculated by Lingard died of surra. In both cases the incubation period was six days, then the temperature went upin one goat to $42^{\circ} 2^{\circ} \mathrm{C}$. [ $108^{\circ} \mathrm{F}$. $]$-and trypanosomes appeared in the blood. The first goat had remittent fever, and died on the fiftythird day of the disease. The second goat also had remittent fever, and showed trypanosomes in its blood for a fortnight, after which they disappeared. The animal died at the end of four and a half months.

We have performed several experiments upon goats. Five or six days after inoculation with surra, the temperature goes up to $40^{\circ} \mathrm{C}$. $\left[\mathrm{IO}_{4}^{\circ} \mathrm{F}.\right]$, and then comes down again to normal. Sometimes there is a second rise a few days later. Except for these febrile attacks, which would pass unnoticed if the animal's temperature were not taken regularly, surra produces no symptoms in goats; the animals do not waste, and there are no odematous swellings. The trypanosomes are always so scanty in the blood that they are not seen on microscopical examination, but inoculation into rats or mice is necessary to ascertain whether the animals are infected or not.

After lasting about five months the disease usually ends in recovery. Goats which have recovered are immunized against surra.

[Edington and Coutts inoculated five goats with the Mauritian trypanosome. These animals lived $96,55,43,33$, and 52 days, the average being 56 days.]

[An old goat was inoculated by Pease with the trypanosome of Tibarsa surra (camel); it became infected and died in 209 days.]

[A goat ( $q$ ), weighing 3 I kilogrammes, was inoculated subcutaneously by Laveran and Mesnil with I c.c. of guinea-pig's blood containing the

${ }^{1}$ [Panisset, C. R. Soc. Biol., v. 58, 1905, p. I5 ; abstract by Mesnil, Bull. Inst ${ }^{*}$ Past., v. 3, 1905, p. 260.] 
Indian virus. Trypanosomes were found in the blood of the goat on the fourth, fifth, sixth, and ninth days after injection, but they were not seen afterwards, although the blood was examined daily for nearly a month. The blood was, nevertheless, highly infective on inoculation into mice. There was a marked febrile reaction from the fifth day onwards, and for a month the temperature rarely fell below $40^{\circ} \mathrm{C}$. [104 ${ }^{\circ} \mathrm{F}$.] ; on several occasions it rose to $4 \mathrm{I}^{\circ} \mathrm{C}$. [IO5.8 $8^{\circ} \mathrm{F}$.], and once even to $4 \mathrm{I}^{\circ} 5^{\circ} \mathrm{C}$. [IO6.8 $8^{\circ} \mathrm{F}$.] Five weeks after injection the temperature fell to $39^{\circ} \mathrm{C}$. $\left[\mathrm{IO} 2 \cdot 2^{\circ} \mathrm{F}\right.$. $]$, and the animal was in good condition.]

SHEEP.-A ram was inoculated by Lingard on two occasions, with an interval of fourteen days between them. On the sixth day after the second inoculation the temperature rose to $4 \mathrm{I}^{\circ} 9^{\circ} \mathrm{C}$. $\left[107^{\circ} 4^{\circ} \mathrm{F}\right.$. $]$ Trypanosomes were never found in the blood on microscopical examination, but eighteen days after the second inoculation the

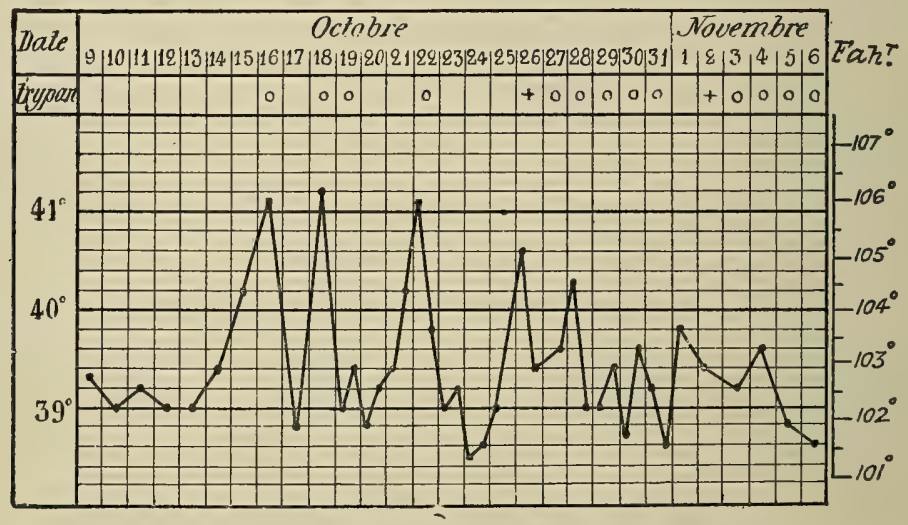

Fig. 35.-First Part of the Temperature Chart of a Sheep inoculated with SURRA ON OCTOBER I3, IgO3.

Trypanosomes were present in the blood on October 26 and November 2, I903. At the end of three months the sheep's blood was still infective.

blood was infective for a guinea-pig. On the twenty-eighth day after inoculation there was fluid in the tunica vaginalis testis, and on the forty-fifth day trypanosomes were found in this fluid. On the Io4th day the animal had paresis of the left side of the body, and it died on the I27th day.

In European sheep surra runs practically the same course as it does in goats. The only symptom is a rise of temperature to $40^{\circ}$ or $4 \mathrm{I}^{\circ} \mathrm{C}$. $\left[\mathrm{IO}^{\circ}\right.$ or $105^{\circ} 8^{\circ} \mathrm{F}$.] which may occur on several occasions. (See chart, Fig. 35). The animals take their food well, and often put on weight during the course of the disease. As a rule trypanosomes are very scanty in the blood, but they are occasionally seen on microscopical examination.

As with goats, the disease may end in recovery after a duration of five to six months, and animals which have recovered are immune. 
[BIRDS.-Edington and Coutts injected a pigeon, but unsuccessfully, with the Mauritian virus. Two geese injected by Mesnil and Martin with the Indian virus also failed to become infected. The birds remained in good health ; their blood never showed trypanosomes in films, nor was it infective, in doses of 8 c.c., on intraperitoneal inoculation into guinea-pigs.]

\section{Section 3.-Pathological Anatomy.}

In surra, as in nagana, enlargement of the spleen is always found in mice, rats, and dogs, sometimes in guinea-pigs, and only rarely in rabbits. The splenic hypertrophy seems to bear some relation to the large number of trypanosomes present in the blood at the time of death.

In mice weighing 20 grammes the average weight of the spleen was 0.8 gramme; in rats of 170 grammes the spleen weighed 3.4 grammes; in dogs weighing 20 kilogrammes the average weight of the spleen was I 80 grammes. We have already mentioned the case of the dog of 9 kilogrammes in which the spleen weighed 210 grammes-about ten times the normal weight. In guinea-pigs weighing about 465 grammes the average weight of the spleen was $2^{\prime} 23$ grammes, but the variations were very great-for example, it weighed $0 \cdot 75$ gramme and $\mathrm{r} \cdot 670$ grammes in two guinea-pigs of about the same weight ( 370 grammes). In rabbits weighing about $\mathrm{I}, 380$ grammes the average weight of the spleen was 3.5 grammes.

Apart from the splenic enlargement, surra does not as a rule produce any macroscopic lesions, but in some autopsies on rats and dogs we have seen pulmonary congestion and small subpleural ecchymoses.

In buffaloes which had died of surra Penning found enlargement of the lymphatic glands and liver, pericardial and submucous intestinal hæmorrhages, and, rarely, slight enlargement of the spleen.

[Massaglia ${ }^{3}$ has studied the changes in the organs of mice, rats, and guinea-pigs experimentally infected with $T$. evansi. The organs affected were the kidneys, liver, spleen, and heart, and, as in the case of animals infected with T. brucei, the kidneys showed the most marked changes. These organs showed many hæmorrhages, infiltration with small round cells, and degenerative changes in the cells of the tubules, such as vacuolation of the protoplasm, chromatolysis, and disintegration of the nucleus. Massaglia remarks that these pathological changes, which closely resemble those found in toxic conditions, appear to favour the idea that the trypanosome elaborates a particular toxin, which has a special effect upon the above-mentioned organs. If this be really the case, the action of this toxin must be different from that of intracorpuscular parasites. The latter affect and destroy the red corpuscles, which carry nutriment to all the tissues; the trypanosomes affect primarily the excretory organs - the kidneys and liver-and secondarily the red bloodcorpuscles. Massaglia is of opinion that his observations are opposed to Schaudinn's view that trypanosomes are a phase in the life-history of intracorpuscular parasites.]

${ }^{1}$ [A. Massaglia, Giorn. d. R. Acad. di Med. di Torino, v. I I, June 23, I905; abstract in Centralb. f. Bakter., I, Ref., v. 38 ; and in Bull. Inst. Past., v. 3, 1905.] 
[Thomas and Breinl found organic lesions similar to those seen by them in animals infected with nagana, but the amount of pigment found in the spleen was always very small.]

[In the brain of a rabbit which died three months after inoculation with surra, Mott ${ }^{1}$ found masses of trypanosomes in nearly all the vessels. 'Single trypanosomes could be seen in the capillaries; in the larger vessels solitary trypanosomes and whorls of trypanosomes and plasmodial masses, which are either degenerated trypanosomes, consisting of a zooglœal mass, in which many deeply-stained nuclei and centrosomes can be seen, or of amœboid forms, described by Bradford and Plimmer.' In spite of this severe blood infection, the vessels showed little or no inflammatory reaction. There was no perivascular cell infiltration, nor did the nervous system show any lesions beyond chromolytic changes in the ganglion cells.]

\section{Section 4.-Pathogenic Agent (Trypanosoma evansi).}

The trypanosome of surra closely resembles that of nagana in its morphology, as well as in the symptoms to which it gives rise. Most of our experiments were made with trypanosomes obtained from Mauritius; but we were able to compare these parasites with others of Asiatic origin, which were sent to us by Carougeau, veterinary officer in the Pasteur Institute at Nha-trang, Annam. The parasites coming from these two sources were found to be identical. $^{2}$

Trypanosoma evansi measures, on an average, $25 \mu$ in total length, by $I^{\cdot} 5 \mu$ in width, the free flagellum measuring about $6 \mu$. Larger forms ( $30 \mu$ long, by 2 to $2^{\circ} 5 \mu$ wide) are not uncommon, but these are always multiplication forms. Smaller forms, measuring less than $22 \mu$ in length, are not found. The largest parasites (as much as $35 \mu$ long) have been found in the blood of equines.

[Laveran and Mesnil, ${ }^{3}$ from their comparative study of the trypanosomes of Indian and Mauritian surra, have come to the conclusion that these parasites are practically identical in the blood of infected dogs and mice. They are of the same size, and the chromatic granules, when present, are neither very numerous nor very large, and are situated in the anterior half of the body. The posterior extremity varies in shape, but is usually pointed, the centrosome being generally very near the end of the body. The undulating membrane has well-marked folds, varying from three to five or even six in number.]

$T$. evansi is so much like $T$. bruce $i$ that it is unnecessary to give a detailed description of it. We shall merely indicate the various

1 [Mott, 'Histological Observations on Sleeping Sickness and other Trypanosome Infections,' Sleeping Sickness Commission's Report, No. vii., Igo6 (see p. 22). I am greatly indebted to Dr. Mott for allowing me to see a proof of his paper before publication; also for his permission to reproduce some of the plates illustrating his paper.]

2 [For the recent observations of Laveran and Vassal on the trypanosomiasis of Annam, see Appendix at the end of this chapter.]

${ }_{3}$ [Laveran and Mesnil, C. R. Acad. Sciences, v. 140, 1905, p. 831.] 
minor differences that exist between these two species of trypanosome. $T$. evansi is usually more pointed than $T$. brucei. The posterior end varies in shape, as it does in all trypanosomes, sometimes assuming the form of an elongated cone, at other times that of a truncated cone. The nucleus and centrosome are the same in the two species; the free flagellum is longer in $T$. evansi than in $T$. brucei, and the protoplasm usually contains fewer chromatic granules in the former than in the latter species. Fig. 36 shows, side by side, the trypanosomes of surra ( $I$ and 2 ), of nagana ( 3 and 4 ), and of caderas $(5$ and 6 ), the last being characterized by the small size of its centrosome.

Examined fresh in an ordinary slide preparation, $T$. evansi is more motile than $T$. brucei, and a parasite is often seen to travel right out of the field of the microscope, which is very rarely observed with $T$. brucei.

Unfortunately, none of these characteristics-which are, more-
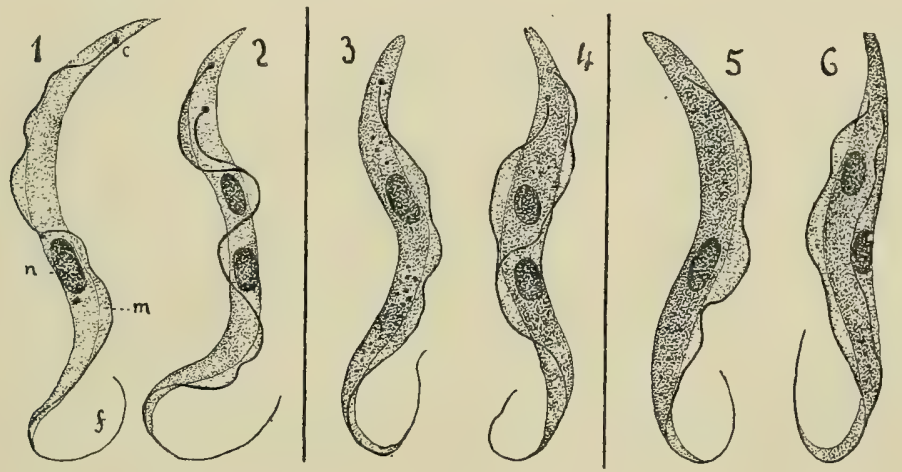

Fig. 36.-Trypanosomes of Surra, of Nagana, and of Caderas.

I and 2. Trypanosomes in the blood of a mule, Mauritian epizootic: $n$, nucleus ; $c$, centrosome; $m$, undulating membrane; $f$, flagellum. The trypanosome in Fig. 2 is undergoing division; it has two nuclei, two centrosomes, and the part of the flagellum attached to the centrosome is undergoing division. 3 and 4 . Trypanosomes of nagana, one undergoing division. 5 and 6 . Trypanosomes of mal de caderas, one in process of division. Magnified about 2,000 diameters.

over, only of minor importance-is constant, and even with the same strain of trypanosome the morphology varies slightly with the species of animal in whose blood it is examined, and also with the stage of the infection. It follows, therefore, that a differential diagnosis between $T$. evansi and $T$. bruce $i$ is impossible if based only upon the examination of a very small number of parasites. Nevertheless, with a little practice one can learn to distinguish between these two trypanosomes in well-stained preparations of blood containing many parasites.

[Novy, McNeal, and Hare, ${ }^{1}$ from a study of stained preparations of the Mauritian and Philippine trypanosomes, conclude "that the two organisms are quite unlike.' In the Mauritian trypanosome the total

${ }^{1}$ [Novy, McNeal, and Hare, Journ. of the Amer. Med. Assoc., May 28, 1904.] 
length was $33 \mu$, free flagellum I3 $\mu$, and the width (not including the undulating membrane) $I^{\cdot} 5 \mu$ to $I^{\cdot} 7 \mu_{0}$. In the Philippine trypanosome the dimensions were $22 \mu$ to $25 \mu, 8 \mu$ to Io $\mu$, and I'3 3 to I.7 $\mu$ respectively. These observers describe an enlargement at the free end of the flagellum in the Mauritian parasite, but not in the Philippine. In the former parasite the centrosome is $3 \cdot 3 \mu$ to $4 \mu$ from the posterior end, whereas in the latter it is only I $\mu$ to I.6 $\mu$. The Mauritian trypanosome has a welldeveloped undulating membrane with four distinct folds; in the Philippine trypanosome there are two or three less marked folds. Lastly, the Mauritian trypanosome has a number of deeply-stained granules in the anterior half of the body, the posterior half being quite free; the Philippine trypanosome, on the other hand, stains deeply in the posterior two-thirds.]

[As has been stated above, it is not possible to differentiate closely allied trypanosomes by relying upon morphological differences, unless a large number of individuals be examined. Although it is possible that the Philippine and Mauritian trypanosomes are distinct organisms, further experimental evidence is required before a definite conclusion can be arrived at. This evidence may be obtained by means of cultivations of the respective trypanosomes, and by immunization and cross-inoculation experiments, such as Laveran and Mesnil have used successfully in the case of nagana, surra, caderas, etc.]

Reproduction occurs by simple division, as in the case of $T$. bruce $i$; the centrosome, nucleus, and flagellum divide into two parts (Fig. 36, 2), and finally the protoplasm also divides. Sometimes further division takes place before the original protoplasm divides, and in such cases, which are rare exceptions to the rule of equal binary fission, one may find a large trypanosome containing four nuclei and four centrosomes.

[Holmes ${ }^{2}$ has studied the evolution of $T$. evansi, and distinguishes three varieties of the parasite which are recognisable especially by their posterior ends. Thus the end may be (I) very elongated-probably males; or (2) short and truncated-females; or (3) oval, like a snake's head-young females. He describes paired forms joined by their posterior ends; these he regards as conjugation forms. Of these paired forms, one is always a male parasite, with a very elongated end; the other is always a female parasite ( 2 or 3 , above). It is only the adult female (form 2) which is seen to undergo division. Division is by ordinary binary longitudinal fission or by transverse segmentation. In this way four amœboid forms may be produced, in which a flagellum develops, or which divide into smaller forms. Holmes agrees with Bradford and Plimmer that after conjugation the female form divides. He states that in the blood the amceboid bodies are few at any time, but that they are numerous in the spleen, liver, and bone-marrow post-mortem. Holmes thinks they result from the transverse division, and that they are carried to the liver, spleen, and bone-marrow, where they develop into adult forms. He thinks also that free nuclei of trypanosomes may give rise to new parasites. (Compare Pricolo's observations on the mouse trypanosome, p. I02.]

The trypanosomes of surra agglutinate under almost the same conditions as do those of nagana. IVe have obtained very good results

I [It will be remembered, howerer, that Prowazek has recently described the division of $T$. brucei as being very complicated.]

${ }_{2}$ [Holmes, Journ. Comp. Path. and Therap., v. 17, 1904, p. 210 ; abstract by Mesnil in Bull. Inst. Past., v. 2; 1904, p. 954:] 
(primary, secondary, and tertiary agglutinations) by mixing a drop of blood containing many trypanosomes with a drop of goat's serum. With the serum of the horse we obtained only loose, primary rosettes. The agglutinated parasites rapidly change, particularly in the centre of secondary or tertiary colonies of rosettes, which rapidly become granular. The parasites are agglutinated by their posterior extremities, as in the case of $T$. brucei.

When blood containing T. evansi is defibrinated or mixed with citrated salt solution, and then kept at the room temperature or in the ice-chest, the trypanosomes do not live very long. After three days in the ice-chest some normally shaped parasites are seen, but

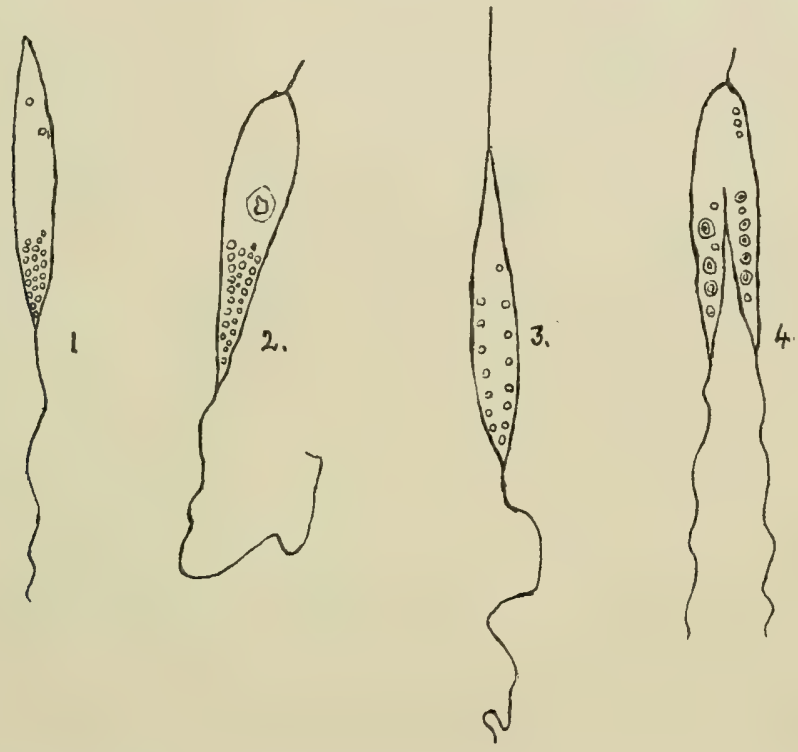

Fig. 37.-Philippine Surra Trypanosomes, from a Thirty-Eight-Day Culture, Drawn from a Living Preparation.

I, 2. Simple forms. 3, 4. Dividing forms from the same preparation. Magnified about 2,00o diameters, (After Novy, McNeal, and Hare.)

they are motionless or only feebly motile, and are no longer virulent. The blood of a guinea-pig containing $T$. evansi, exposed for a quarter of an hour to the temperature of liquid air $\left(-\mathrm{I}_{9} \mathrm{I}^{\circ} \mathrm{C}\right.$.) was still virulent, for two mice inoculated with this blood became infected in six and eight days, and died in fifteen and eighteen days respectively.

We have made attempts to cultivate $T$. evansi on blood-agar by Novy's method. We succeeded only once in six experiments, and the growth could be subcultured only once. The trypanosomes in these cultures appeared in the form of small isolated motile parasites, or of rosettes like those seen in cultures of $T$. lewisi, but much smaller. All the parasites in the rosettes had the flagella directed towards the centre of the rosettes. At the end of three months motile parasites and rosettes were still seen in the cultures. The 
culture injected into mice on several occasions gave negative results; it appears, therefore, that the trypanosome, on becoming acclimatized to artificial cultivation in vitro, had lost its virulence. The mice which were unsuccessfully inoculated with the culture products were not immunized thereby, for on subsequent inoculation with fresh virulent blood they died as quickly as control mice.

[Novy, McNeal, and Hare ${ }^{1}$ have succeeded in cultivating the Philippine trypanosomes. Tubes of blood-agar were inoculated on January I, IyO4, with the blood of an American cow suffering from Philippine surra. They were then sent from the Philippines to America, where they arrived thirty-eight days later. All three inoculated tubes contained motile and developing trypanosomes when examined in America. The cultures were quite different from those of $T$. leweisi and $T$. brucei, inasmuch as there was an entire absence of groups or rosettes. The trypanosomes were all single and actively motile, and appeared to travel equally well with the flagellum posterior or anterior. Another striking feature was the size of the parasite and the length of the flagellum. An even more marked peculiarity was the presence and arrangement of granules within the parasites, and the distinct yellowish or greenish colour of the granules and of the contents. These granules were present in large numbers, and were $0^{\circ} 3 \mu$ to $0^{\circ} 5 \mu$ in diameter. They were usually massed at the base of the flagellum, and only a few occurred in the remainder of the body (Fig. $37, I, 2)$. In the dividing forms the granules were arranged in two parallel rows (Fig. 37, 3, 4).]

[The flagellum was usually as long as, and often even longer than, the cell itself. The non-flagellated end, especially when blunt, showed a rod-like tip or stylet, $2 \mu$ to $4 \mu$, or even $6 \mu$ in length (see Fig. 37). There was no undulating membrane. The trypanosomes in culture varied considerably in length. The majority were $25 \mu$ to $35 \mu$ long, but some were $40 \mu$ or $50 \mu$ in length. The width varied from $\mathrm{I}^{\circ} 5 \mu$ to ${ }^{\circ} 5 \mu$.]

[As the cultures aged, pear-shaped or spherical highly granular involution forms appeared, and, as in the case of $T$. lewisi and $T$. brucei, these involution forms eventually gathered into large groups or masses. Living trypanosomes were found in one culture tube as late as the sixty-fifth day.]

[All attempts to inoculate animals (white mice, white rats, and guinea - pig), and to obtain subcultures on media containing varying proportions of blood and agar, proved fruitless. These failures were doubtless due to the age of the cultures (thirty eight days) when they reached America. Similar results had previously been obtained with T. bnucei; original cultures twenty-nine and thirty-eight days old failed to infect mice.]

Thomas and Brein ${ }^{2}$ also obtained several cultures of $T$. evansi at $22^{\circ} \mathrm{C}$., and at the room temperature. The organisms seen in a thirty. three day old culture resembled those described above, and were nonvirulent for animals; nor could subcultures be obtained from them. As in the case of Novy's cultures, all the trypanosomes were single, and actively motile.]

1 [Novy, McNeal, and Hare, Journ. Amer. Med. Assoc., May 28, I904.]

2 [Thomas and Breinl, op. cit., p. 45.] 


\section{Section 5.-Surra is a Distinct and Definite Disease.}

$T$. evansi and $T$. brucei resemble one another so closely, both morphologically and in their effects upon animals, that the question naturally arises whether they are not one and the same trypanosome, or at least two varieties of the same species. The general opinion was that surra and nagana were two names for the same disease. ${ }^{1}$

An experiment was necessary to decide this question-namely, to inject surra into an animal immunized against nagana, and to ascertain whether it became infected or not. It sometimes happens that a mild form of a disease does not protect against a severe form; but that objection is invalid in this particular instance, because the virulence of nagana is equal to, if not greater than, that of surra.

We have performed this experiment, and have shown that animals immunized against nagana are susceptible to surra. It also follows from our experiments that surra and caderas are two distinct diseases. $^{2}$

The animals we used to establish these facts were two nanny goats (I. and II.) and a billy goat. All three had been infected, in October or November, IgO2, with the blood of an animal suffering from caderas. They never showed any symptoms, and steadily gained in weight.

The infection with caderas lasted about five months.

Goat $I$. ( $q$ ) was highly immunized against nagana before being inoculated with caderas. It was one of the two animals we used to demonstrate that nagana and caderas are distinct diseases. The immunity of this goat against nagana was maintained, for a subcutaneous injection of infective blood made on May 20, I903, did not give rise to a fresh infection. On June 5, I903, each goat received a subcutaneous injection of $\frac{1}{2}$ c.c. diluted blood of a mouse containing many surra trypanosomes. At the same time the billy goat (intermediate in weight between the two nanny goats) was inoculated with $\frac{1}{2}$ c.c. diluted blood of a mouse containing caderas trypanosomes.

The two nanny goats reacted in the same way to the injection of surra; there was a rise of temperature to $40.5^{\circ} \mathrm{C}$. $\left[105^{\circ} \mathrm{F}\right.$.] and $40^{\circ} \mathrm{C}$. [104 ${ }^{\circ} \mathrm{F}$. $]$ on the fifth and sixth days respectively after inoculation. The next day the temperature fell to normal, and remained normal.

Trypanosomes were never found in the blood of these goats on micro. scopical examination, but intraperitoneal injection of their blood in doses of $\frac{1}{2}$ c.c. into mice gave positive results, as the following experiments show :

I. Blood obtained Five Days after Inoculation of the Goats. - The two mice injected with the blood of Goat I. showed trypanosomes in their blood five and six days respectively after inoculation. Those which were injected with the blood of Goat II. showed parasites in their blood five and seven and a half days respectively after inoculation.

2. Blood obtained Ten Days after Inoculation of the Goats. - The two mice injected with the blood of Goat I. showed trypanosomes in their blood four

1 [Even as late as 1903 Musgrave and Clegg (in Bulletin No. 5, Bureau of Government Laboratories, Manila, I903, p. I87) held that surra, nagana, mal de caderas, and probably dourine, were the same disease, and that all were caused by T. evansi (quoted from Novy, McNeal, and Hare's paper).]

2 Laveran and Mesnil, Acad. des Sc., June 22, I903. 
and five days after inoculation, while those injected from Goat II. showed them three days after inoculation.

The disease, therefore, ran a practically identical course in the two goats, so that the immunity of Goat $I$. against nagana had in no way modified its susceptibility for surra.

The billy goat, reinoculated with blood of caderas, did not contract a fresh infection, and this result confirms the fact already established that animals which have recovered from caderas are immunized against the disease. On the other hand, the experiment with the two nanny goats shows that they were both susceptible to surra, though one had previously acquired immunity against nagana.

Nocard, Vallée, and Carré showed that a cow immunized against nagana was nevertheless susceptible to surra, ${ }^{1}$ and this fact confirms the results we obtained with goats.

[As a result of their own inquiry into the relative virulence of the parasites of Indian and Mauritian surra and of mbori, and of Vallée and Panisset's immunization and cross-inoculation experiments already quoted (see p. 26I), Laveran and Mesnil conclude (I) that Mauritian surra is the same species as Indian surra; and (2) that, for the present, we may recognise three varieties of surra, which are in decreasing order of virulence-Indian surra, Mauritian surra, and mbori.]

[It is possible that further investigations may show that several other varieties of surra exist in Asia and Africa, or even that several distinct, but closely allied, diseases are included in the surra group. It has already been stated that Novy, McNeal, and Hare think the Philippine trypanosome is a distinct parasite. The trypanosomiasis of horses in Annam (see Appendix B to this chapter) is regarded by Laveran and Mesnil as a variety of surra, probably distinct from the Indian disease.]

\section{Section 6.-Ætiology. Mode of Infection.}

The ætiology of surra is still far from being elucidated. For many years the natives in various parts of India have regarded the flies they call 'burra dhang' (Tabanus tropicus and T. lineola) as the propagators of the disease. Evans, after he discovered the parasite, also thought it possible that the disease could be conveyed to a healthy animal by these horse-flies immediately after they had bitten a diseased animal. In I888 Kay Lees (quoted by Lingard), studying the disease in the Naga Hills, to the north-east of Assam, suspected the tsetse- $\mathrm{fly}^{2}$ In his report for I894, Lingard gives the following as the causes of surra in India: (I) Drinking water which is very polluted at the end of the dry season; (2) polluted food (grass, etc.), coming from flooded localities; (3) the ingestion of grain contaminated with the excretions of rats and bandicoots; and (4) in

1 Vallée and Carré, Acad. des Sc., October 19, 1903.

2 It is very unlikely that the tsetse-fly occurs in India. 
sporting dogs, the ingestion of the flesh of animals suffering from surra, and in terriers, the destruction of infected rats.

In his last report, published after Bruce's discovery of the rôle of the tsetse-fly in the transmission of nagana, Lingard mentions biting flies as an additional means of the propagation of surra, without, however, modifying his previous ideas on the subject.

In an important paper published in IgOI $^{1}$ Leonard Rogers shows that the horse-fies in India, when they have recently (less than twenty-four hours previously) bitten an infected animal, can convey surra to the dog and rabbit. He points out that the latent cases of surra in cattle may in this way often act as sources of infection. By means of convincing arguments, Rogers disproves Lingard's hypotheses as to the transmission of surra by infected water or food, particularly infection by the' excretions of rats, which, even if they contain trypanosomes, are harmless, inasmuch as the ordinary trypanosome of the rat, $T$. lewisi, has no connection with surra.

The value of Lingard's experiments is considerably diminished by the facts that they were carried out in a locality where surra is endemic, and that no precautions were taken to protect the experimental animals from biting flies.

During the Java epidemic of Igor large numbers of flies were noticed in the stables and around the sick animals, and trypanosomes were demonstrated in the flies caught upon these animals. Several drops of liquid taken from these flies and inoculated into a rabbit gave rise to a characteristic infection, the rabbit dying of surra in four weeks. The experiment repeated with flies caught on sick horses gave similar results.

A large horse-fly-probably a Tabanus-red or reddish in colour, which is very common in Java, perhaps plays a part in the spread of surra amongst horses; but this fly does not get inside the stables. According to Schat, it is the Stomoxys calcitrans which is chiefly concerned in the propagation of surra in Java, this fly attacking cattle as well as horses.

During the Mauritian epizootic flies almost indubitably propagated the disease. The tsetse-fly is unknown in the island, but a large number of other biting flies often torment the animals when working. Daruty, of Grandpré, says that in Mauritius a Stomoxys, the $S$. nigra, plays the same part in the spread of surra as the tsetse does in Africa in the spread of nagana. In certain districts where biting flies are not found the epizootic did not spread-in the Albion Dock, for example. The cows belonging to the Indians, boxed up in small, dark huts into which the flies do not penetrate, were immune.

In a letter dated November I2, I903, M. Deixonne informed us that surra had not reappeared among the horses, mules, or donkeys,

1 L. Rogers, Proc. Roy.Soc., May 4, I901 ; [also Brit. Med. Journ., I904, v. 2, p. I454.] 
and he added: 'I attribute this fact to the effect of the cold upon the hatching-out of the Stomoxys. Our winter has been very long, and the flies are still very scarce.' In another letter, dated February II, I904, ${ }^{1}$ the same observer stated that the epizootic had broken out again in Mauritius, simultaneously with the reappearance of the Stomoxys, and that the animals recently purchased were dying in great numbers.

[Manders ${ }^{2}$ states that in Mauritius the Stomoxys geniculatus, de Bogot, is almost certainly the carrier of the disease, and that the mortality amongst animals is greatest during the months when this fly is most prevalent. The fly is known locally as the 'cane-fly,' being very abundant in sugarcane fields. Manders states that de Charmoy, curator of the museum at Port Louis, had found trypanosomes in the stomach contents of flies some time after feeding. He also found that infected rabbits, placed in the same hutch as other rabbits, did not give the disease to the latter until the hutch was exposed to the access of Stomoxys.]

Musgrave and Clegg state that they succeeded in transmitting surra to monkeys, horses, dogs, rats, and guinea-pigs by the bites of flies previously fed on infected animals. They appear also to have transmitted the disease from dog to dog, from rat to rat, and from rat to dog by means of fleas. ${ }^{3}$

Carnivorous animals may contract surra by devouring the flesh of infected animals, but infection can only occur in this way if there be any wound or simple abrasion on the skin, or more especially on the buccal mucous membrane, which becomes smeared with blood. Surra is not infrequently spread amongst dogs in this way.

Steel fed a young dog on the flesh of a mule which had died of surra, and thirteen days later trypanosomes were present in the blood. The dog had only slight fever, and during the course of the disease, which lasted fifty-one days, it had enlargement of the left inguinal glands, œdema of the perineum, and swelling of the head.

Penning records the case of a dog which became infected after eating the liver and spleen of a rabbit which had died of surra.

Lingard records the following facts: On January 8, I893, four sporting dogs and a terrier were bitten by a hyena during a hunt. All five dogs became blind and died between March I2 and April I6. The animals hunted were the fox and jackal in addition to the hyena. A dog which was not present at the quarry remained quite healthy. It is probable, says Lingard, that jackals and foxes are often infected with surra. In I893, during a hunt, some of these animals appeared ill. They were unable to run away, and were devoured by the dogs, several of which contracted the disease. ${ }^{*}$

In Mauritius dogs became infected by drinking the blood of oxen

1 This was the last letter I received from M. Deixonne before his untimely death (A. Laveran).

${ }^{2}$ [Manders, Journ. R.A.M.C., v. 5, 1905, p. 623.]

3 Musgrave and Clegg, op. cit., pp. 86, 87 .

4 Lingard, Report for 1894 . 
which were bled. Deixonne, who records this fact, adds that dogs often have wounds of the buccal mucous membrane. ${ }^{1}$

If animals susceptible to surra are allowed to eat the entrails of infected animals, it is found that the disease is rarely produced, unless one previously wounds the buccal mucous membrane. The following experiment is interesting in this connection :

On October I8, I903, we fed five young rats (average weight, 65 grammes) on the viscera of a mouse containing the trypanosome of surra in large numbers. On October ig the same rats were fed on the viscera of a rat which had just died of surra, the trypanosomes being very numerous and quite active at the time of the autopsy. It is certain that each rat devoured some of the infective material.

On October 27 the blood of one of the rats contained numerous trypanosomes, and the rat died the next day. The other four rats remained free from infection.

On November I4 two of them were inoculated subcutaneously with surra, and became infected like ordinary rats.

On November I9 a slight cut was made in the upper lip of the two rats which were still healthy, and they were then given the entrails of an infected rat to eat. On November 25 many trypanosomes were found in the blood of these two rats.

It is very probable that the rat which became infected in the early part of the experiment, and died on October 28, had some abrasion of the skin or buccal mucous membrane by which the trypanosomes entered the system, and that it is incorrect to incriminate the ingestion of the trypanosomes, seeing that this produced no effect whatever in the other four rats.

In conclusion, surra is, like nagana, mainly propagated by biting flies. It is not contracted by the ingestion of fouled water or forage, but rodents and carnivores may become infected by eating infected flesh, if they have wounds or abrasions on the skin or buccal mucous membrane, such as might be produced by the devouring of bones, etc.

[Lingard ${ }^{2}$ has inquired into the manner in which $T$. evansi is carried over from one surra season to another. The infection is mildest, and sometimes ends in recovery, in Bovidæ and in camels. As has already been stated, surra may last three years in the case of camels. During the first year the blood gives rise to a fatal infection in horses, but blood taken from camels during the other two years produces a milder infection, from which the horse recovers. Nevertheless, this passage of the trypanosome through the horse increases the virulence of the virus, which is then fatal for horses. Similarly, the camel trypanosome is only feebly pathogenic for Bovidæ (ox, buffalo), and these animals show scarcely any signs of the infection. The trypanosome becomes more virulent, however, by being passed through the ox or buffalo, and it then produces a fatal infection in horses.]

1 'Report of the Commission upon the Epizootic in Mauritius,' p. I8.

2 [Lingard, Journ. Trop. Vet. Science, v. I, 1906, pp. 92-I I 2 ; Pease, ibid., pp. 7091, I27-137; abstract by Mesnil in Bull. Inst. Past., v. 4, 1906, pp. 431, 898.] 
[Pease ${ }^{1}$ is also of opinion that in India the buffalo is an important agent in the transmission of surra.]

[Cazalbou ${ }^{2}$ thinks that the distribution of surra in Africa is closely connected with the migrations of camels in their search for salt.]

\section{Section 7.-Treatment. Prophylaxis.}

Lingard, in India, tried a large number of chemical substances for the treatment of surra in horses-perchloride of mercury, iodine and potassium iodide, potassium bichromate, iodoform, turpentine, carbolic acid, the alkaloids of cinchona, the double iodide of mercury and potassium, and liquor potassæ-but without success.

Arsenic alone gave results at all satisfactory. Of twenty-one horses treated with it one was cured. In this horse the treatment was begun on the twenty-first day of the disease. 455 grains (29 grammes) of arsenious acid were given in sixty-three days, and sodium iodide in doses of 2 drachms ( 7.8 grammes) twice a day, for sixty-two days. The animal was able to resume its work in the mounted police at the end of Ioo days, and it was in good health three years and two months later.

Lingard recommends, for horses affected with surra, the administration of arsenious acid for two months in doses of I2 grains a day, followed by the double iodide of arsenic and mercury for another six months.

Trypanred, in doses of 3 to 4 milligrammes for mice and 2 centigrammes for rats, causes a temporary disappearance of the trypanosomes from the peripheral circulation, lasting from three to twenty days. We have obtained good results in rats by the combined administration of arsenious acid and trypanred. ${ }^{3}$

The double iodide of arsenic and mercury, used as a prophylactic, has not given good results.

Lingard tried in the treatment of surra, but unsuccessfully: (I) Injections of serum obtained from a blood very rich in trypanosomes by filtration through porcelain; and (2) injections of the serum of a bovine which had recovered from surra and so become immunized against the disease. ${ }^{*}$

During the Mauritian epizootic many drugs were tried, but without success : hypodermic and intravenous injections of quinine, arsenious acid and Fowler's solution, cacodylate of sodium, arrhénal, and intravenous injections of perchloride of mercury in the doses recommended by Bacelli for the treatment of aphthous fever.

1 [See footnote 2 on p. 28r.]

2 [Cazalbou, Revue gén. méd. vét., v. 8, 1906, pp. 40I-407.]

3 [See Chapter XIII. on Treatment. Mesnil and Martin obtained even better results with one of their benzidine dyes, in the case of mice infected with surra. This dye was nearly always successful, both in the prevention and cure of the infection.]

${ }^{4}$ Lingard, 'Report on Surra,' v. 2, part i., Bombay, 1899, p. 61. 
Edington had recommended, both as a prophylactic and curative agent, the bile of animals which had died of surra, diluted with a third of its weight of glycerine. The glycerinated bile was injected intravenously in doses of 20 c.c. for mules and Io c.c. for dogs. The results of this treatment were absolutely negative.

E. R. Rost speaks highly of injections of goat serum in the treatment of surra. ${ }^{1}$ According to this observer, the trypanosomes of surra die in from a half to two and a half minutes in blood to which I per cent. of goat serum has been added. This observation has not been confirmed in the case of surra, and in the case of nagana, we have shown that the serum of the goat has no special action upon the trypanosomes.

Human serum acts upon the trypanosomes of surra in the same way as it does upon those of nagana and caderas. If one inject I c.c. of serum or $0^{\circ} I$ gramme of powdered dried serum into a mouse weighing $I_{5}$ or 20 grammes, the trypanosomes are seen to disappear in twenty-four to thirty-six hours. As a rule, the parasites reappear in eight to ten days. ${ }^{2}$ A mouse cured of surra by a single injection of human serum (Io centigrammes of powdered dried serum) was not rendered immune, for a reinoculation with the virus produced a fatal infection, which ran a slightly longer course than usual. It is impracticable to use human serum for the treatment of large animals, on account of the huge doses required.

Deixonne, ${ }^{3}$ struck by the fact that the spleen is so greatly enlarged in surra, splenectomized dogs, and after they had recovered from that operation, inoculated them with surra. The result was that the spleenless dogs died even sooner than control dogs. We had obtained equally unsatisfactory results in the case of nagana (p. I25).

Therapeutic measures, therefore, are of no avail, and all our efforts should consequently be directed towards preventing the introduction of surra into clean, uninfected countries, and towards stopping its spread in those districts where it is endemic, or into which it has been introduced. The history of the epizootics of surra in Java and Mauritius shows the important effect of prophylactic measures upon the course and severity of the disease.

In Mauritius the nature of the epizootic was not recognised for several months, so that no precautionary measures were taken at the outset, which is just the time that such measures are most efficacious. Diseased animals were allowed to circulate freely, were sold at a low price, and scattered throughout the island. Animals in the last stage of the disease were left to die by the roadside. When the diagnosis of surra was eventually made, the epizootic had reached such proportions that the slaughter of all the sick animals,

1 E. R. Rost, 'Report upon the Possibility of treating Surra by Injections of an Antiparasitic Serum'; Journ. Path. and Bacter., v. 7, p. 285, June, I901.

${ }^{2}$ Laveran, Acad. des $S c$., July 6, I903.

3 Deixonne, Letter of January 29, 1903. 
recommended by one of us, presented very great difficulties. These difficulties were at first considered insurmountable, and more valuable time, during which energetic prophylactic measures might have been adopted, was lost in trying to find out methods of treatment. The Commission appointed to inquire into the methods to be taken to put a stop to the disease very wisely came to the conclusion that all diseased animals should be killed, that suspected animals should be isolated, and that for three months the importation of fresh animals into the island should be prohibited. But the report of this Commission is dated February 16, I903, and the epizootic dated from the end of Igor.

Prophylactic measures, although belated, have yielded appreciable results in Mauritius. In a letter dated April Io, I903, M. Deixonne writes: 'The disease is always most prevalent amongst the cattle of those owners who neglect the most elementary precautions. Where the systematic killing of animals and other precautionary measures are adopted, the epizootic subsides.'

In a previous letter (January 29, I903) the same observer wrote: ' I advise for horses a gauze net which protects them efficiently from flies, and for the stables double doors fitted with wire gauze. The best method would undoubtedly be the compulsory notification of the disease, and slaughter, without indemnity, in the case of horses, but with an indemnity in the case of cattle, which we know can often recover.' It is reasonable to refuse an indemnity in the case of equines, in which surra is always fatal, and to give one in the case of cattle, which might recover, but whose slaughter is for the general good, seeing that the sick animals are for several months a source of infection to healthy animals.

The flesh of diseased cattle may, without danger, be used for the food of man, when the general condition of the animals is otherwise satisfactory. In animals which have died of the disease the trypanosomes die in a period varying with the atmospheric conditions, but which probably never exceeds twenty-four hours. It is particularly during the few hours immediately after death that the bodies of animals which have died of the surra are dangerous from the point of view of the transmission of the disease. If the bodies cannot be buried at once, precautions should be taken that flies cannot suck the blood from them, and that dogs, cats, and rodents do not come and feed on the bodies of animals that have died naturally or that have been slaughtered.

During the epizootic of surra in Java in Igoo and Igor the excellent prophylactic measures which were taken succeeded in preventing the spread of the disease, and in averting such a disaster as occurred in Mauritius. The nature of the epizootic was speedily recognised, and this enabled the authorities to adopt efficient precautions in good time. Districts from which cases of surra were notified were declared infected. All the stables were inspected, the sick 
animals were slaughtered or isolated from healthy animals, and large fires were burnt in the grazing-grounds to drive away the flies. For the isolation of suspected animals localities were chosen where biting flies were unknown or very scarce, and which were not near any large farms.

In 1902 we drew the attention of the public authorities to the dangers with which the trypanosome diseases threaten our colonies, and especially Madagascar and Réunion, and we pointed out the measures it was expedient to adopt.1

We recommended that the importation of animals from districts infected with trypanosome epizootics should be prohibited or rigorously supervised. Live animals imported from suspected areas should be carefully examined by veterinary officers on their arrival at the ports, and should be killed if the existence of a trypanosome disease in them were proved.

Even when animals suffering from surra or nagana have been introduced into an uninfected district, it is still possible to adopt efficient measures to prevent the spread of the disease, provided the diagnosis be made early. The infected animals should be slaughtered as soon as the disease is recognised, and the suspected animals should be isolated.

According to Musgrave and Clegg, a certain number of sewer rats were infected with surra at Manila, and in order to prevent the spread of epizootics of surra, the destruction of rats should be recommended.

We see, therefore, that for prophylactic measures to be of value it is essential that the disease be promptly recognised, so that it is of the utmost importance to draw the attention of veterinary surgeons to the trypanosomiases, of which the prevalence in various parts of the world and the gravity are no longer open to question.

At our instigation, the Académie de Médecine proposed on July I, I902, "that the importation into France or the French colonies of animals coming from localities in which surra, nagana, or other trypanosome diseases exist, be prohibited or rigorously supervised.'

\section{Appendix A.}

Lingard ${ }^{2}$ has described and figured a very large trypanosome in the blood of cattle in India, which he used for his experiments on surra: He found it only in two animals, once in a bull, five days after a second inoculation with surra, and on four occasions in a cow, also twice inoculated with surra.

In his 'Report on Surra,' v. 2, part I, p. 83, Lingard speaks also of 'a long and active trypanosome,' which he found only once in the blood of a bull (No. 4). He makes no allusion to this observation in his paper on the giant trypanosome.

1 Laveran and Nocard, Acad. de Méd., July I, I902.

2 Centralbl. f. Bakter., I, Orig., v. 35, p. 234. 
The length of the giant trypanosome is from fourteen to twentythree times, and the width from two and a half to three and a half times, the diameter of the red blood-corpuscles of cattle (see Fig. 38,3 , which shows a red corpuscle under the same magnification as the two trypanosomes).

The body of the trypanosome (Fig. $38, I, 2)$ usually has a swelling near the posterior end, giving rise to a tadpole or spindle-shaped parasite. There is a well-developed undulating membrane. Lingard represents a nucleus about the middle of the swollen portion. If watched for a long time in a fresh preparation, the trypanosome becomes cigar-shaped and then spherical. It was not seen in stained films.

In all probability this parasite has no connection with the $T$. evansi of surra, but its presence in these two cases was a coincidence (see also Chapter XI., Section I). It appears not to be infective

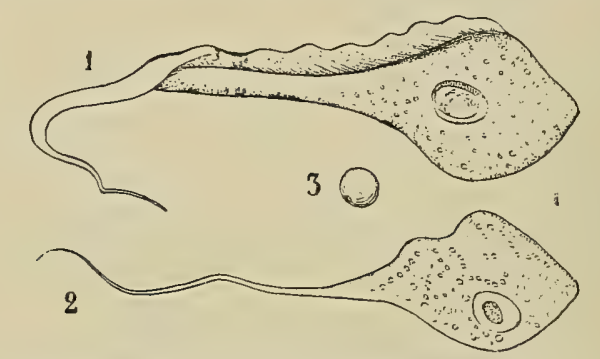

Fig. 38. - The Giant Trypanosome of Lingard.

for animals other than bovines. The blood of the cow, at the time of the second appearance of the giant trypanosome, injected into two guinea-pigs ( $\frac{1}{2}$ c.c. into each), gave rise to surra in both the guinea-pigs inoculated.

\section{APPENDIX B. ${ }^{1}$}

\section{The Trypanosomiasis of Horses in Annam.}

[This disease has been investigated locally by Vassal, ${ }^{2}$ but several other observers had previously recorded cases of trypanosomiasis in various parts of French Indo-China (see p. 248). Laveran and Mesnil, ${ }^{3}$ in Paris, have recently carried out a series of experimental investigations upon this trypanosomiasis, especially with reference to its relation to surra.]

[It has already been mentioned ( $\mathrm{p}$. 249) that the disease is endemic throughout French Indo-China, and that it becomes epidemic from time to time. It affects horses almost exclusively;

1 [The whole o1 this Appendix has been added.-ED.]

2 [J. J. Vassal, Ann. Inst. Past., v. 20, 1906, pp. 256-295.]

${ }^{3}$ [Laveran and Mesnil, ibid., v. 20, Ig06, pp. 296-303.] 
but Vassal met with one case in a cow, and Brau records its occur rence in mules.]

[Syмpтомs.- The incubation period in the case of the natural infection is unknown. In the earliest stage of the disease-the 'latent period'the animal appears perfectly well and strong, and its appetite is good; nevertheless, it has fever, and trypanosomes are present in the blood. After about eight or ten days the disease declares itself; the symptoms are remittent fever, progressive weakness and anæmia, œedema of the limbs and abdominal walls, running from the eyes and staring of the coat, wasting, and sometimes paresis of the hind limbs. A diagnostic symptom of the third or final stage of the disease is a more or less intense dyspnoea, due to hydro-pericardium. The temperature is nearly always subnormal before death. Vassal never observed diarrhœea, ophthalmia, or cutaneous lesions in this disease.]

[The curve of the trypanosomes in the blood is almost parallel with that of the temperature. In some cases the parasites disappear temporarily from the blood; in other cases they are present throughout the disease. The trypanosomes often disappear altogether three or four days before death, and post-mortem the blood may be non-infective on injection. The disease usually lasts thirty to forty days, and is always fatal.]

[Brau, Saint-Sernin, and Mutin-Boudet ${ }^{1}$ describe two forms of the disease in Cochin-China: (I) The $d v y$ form, with progressive wasting, conjunctivitis, blindness, and death in two to two and a half months; and (2) the cedematous form, with œdema of the limbs, abdomen, and genital organs, digestive disturbances-but no ocular lesions-and death in twenty days.]

[The Pathogenic Agent is a trypanosome very closely resembling that of surra. Its average length is $28 \mu$ to $30 \mu$, but in the horse, ox, and guineapig longer forms $(30 \mu$ to $35 \mu)$ are often seen. Laveran and Mesnil give its dimensions, in the blood of the rat and mouse, as $26 \mu$ long, by ${ }^{\circ} 5 \mu$ to $2 \mu$ wide; these dimensions are hardly greater than those found by them for T.evansi. The posterior end is pointed; motility is less than that of $T$. lewisi. Cultures on horse-blood agar were unsuccessful.]

[Animal Experiments.-Vassal found the trypanosome pathogenic for the rat, guinea-pig, rabbit, monkey, cat, stag, dog, civet-cat, badger, cattle, and buffalo. In rats the incubation period was two days, and death occurred in six to twelve days; trypanosomes were very numerous in the blood.]

[One guinea-pig died in eighteen days after intraperitoneal injection of horse blood, but in other cases, and after the injection of the blood of other animal species, the disease lasted sixty and even seventy days. The virulence of the trypanosome for the guinea-pig was not modified by passage through this animal.]

[Rabbits were very susceptible; the incubation period varied from four to eight days. A rabbit inoculated with the cerebro-spinal fluid of a horse became infected after nine days, and died twelve days later. The temperature and trypanosome curves were irregular; the animals lost much weight, and had œedema, especially of the genital organs.]

[Three monkeys (Macacus) became infected, and died in thirty-eight, fourteen, and thirty-four days respectively. The chief symptoms were intermittent fever, profound anæmia and emaciation, diarrhoea, and œdemas.]

[A native cat became infected in less than five days, and died in twentyfour days. Trypanosomes were very numerous in the blood, and the animal became much emaciated.]

1 [Brau, etc., Bull. Chambre d'Agric. de Cochinchine, February ı, I906, p. 39.] 
[A stag-young male $A$ xis of eight months-was very susceptible. It had remittent fever (the temperature often rising to $40^{\circ} \mathrm{C}$. [ $\mathrm{IO}_{4}^{\circ} \mathrm{F}$.]), but trypanosomes were not very numerous until the last week of the infection. The animal died twenty-three days after inoculation. Post-mortem the body was much wasted; the pericardium was full of fluid; there were many petechial hæmorrhages on the surface of the heart, kidneys, and urinary bladder. The liver and spleen were enlarged. The bladder was distended with about 2 litres of albuminous urine.]

[Vassal states that other stags are found in Annam, but he was unable to test the effect of this trypanosome upon them. If, however, it be found subsequently that they all react in the same way as the species of $A x$ is, then it will follow that the stag in Indo-China cannot play the part which Bruce has attributed to it in the spread of nagana in Africa.]

[Dogs did not appear to contract the disease naturally, even when they frequented infected stables and ate the flesh of infected horses. Blin at Tonkin, however, came across cases of spontaneous infection in dogs, especially in sporting dogs imported from France.]

[Dogs experimentally inoculated became infected in four or five days, and died in seventeen to twenty-four days later. In one case, in which the infective blood was kept in a sealed tube for twenty-four hours before injection, the incubation period was twenty days, but the subsequent course of the disease was normal, the dog dying twenty-four days after the first appearance of trypanosomes in the blood. The symptoms noted were similar to those seen in the other acute trypanosomiases in dogsirregular fever, weakness, anæmia, and emaciation. The trypanosomes were present with exacerbations, and at times swarmed in the blood; during the last few days they were very numerous. Post-mortem, the spleen was always much enlarged.]

[Three civet-cats (Pavadoxurus) died in six and a half, nineteen, and nineteen days respectively after inoculation. Many trypanosomes were constantly present in the blood, and post-mortem the spleen was enlarged, and there was much exudation in the peritoneal and pericardial cavities.]

[Three badgers (Helictis pierri and $H$. personatus) showed a marked susceptibility to the infection. The incubation period was two to three days, and death occurred in six to eight days. At death the trypanosomes swarmed in the blood of all three animals; post-mortem the spleen was enlarged in only one case.]

[Bovida.-Six bovines were successfully inoculated by Vassal. Five of the animals-a calf, two heifers, an old ox, and an old cow-died of the infection in from thirty-four days to seven months. The sixth animal, a vigorous young calf, became infected in five days, but it recovered from the disease, and ten months later its blood was no longer infective. In the other animals the incubation period was six to eight days, and the trypanosomes were visible in blood-films on the succeeding three or four days. After this they permanently disappeared from the blood, except in the case of the old cow, which was in a miserably cachectic state when injected, and in which the trypanosomes reappeared on the sixteenth, seventeenth, and eighteenth days, and swarmed in the blood on the thirtythird and thirty-fourth days, just before death.]

[The chief symptoms noted in these animals were febrile attacks, anæmia, great loss of weight -as much as 38 per cent. in thirty-nine days in the case of the old cow-and in one case cedema and coma shortly before death. Post-mortem, the usual signs of trypanosomiasis-serous effusions, subserous hæmorrhages, and gelatinous œdema-were present, but the spleen was enlarged in only one case.]

[Vassal met with one case of spontaneous infection in a young calf which did not appear ill. Trypanosomes, morphologically identical with those of the horse, were seen in the blood on two successive days, but not 
afterwards; and when the animal died, two months later, its blood was not infective for a guinea-pig or a rabbit. Such sporadic cases of bovine trypanosomiasis are very difficult to recognise, because the trypanosomes may disappear from the blood, and the latter may even fail to infect experimental animals. In Mauritius the mortality amongst cattle was very great. On the other hand, in India, as in Annam, surra is a very mild disease in cattle. Vassal concludes from this that in Annam and in India the trypanosomiasis is of ancient origin, and that the cattle in these countries have become immune.]

[A young buffalo showed trypanosomes in its blood on the sixteenth day after inoculation, and, with long intervals of absence, during the succeeding three and a half months. The buffalo gained in weight, and was cured in five and a half months; its blood was then non-virulent for rats and a guinea-pig. In India, Java, and the Philippines, surra is pathogenic for the buffalo, and the disease often terminates fatally.]

[Vassal thinks that these mild cases of the disease in cattle and buffaloes may be an important reservoir of the virus. The symptoms of the trypanosomiasis in Bovidæ are extremely slight, so that the existence of the disease is not suspected.]

[Horses. - Two horses were experimentally infected by subcutaneous inoculation. Trypanosomes appeared in the blood in seven days. In the case of an old native horse, which died in seventeen days, the parasites were almost constantly present, and gradually increased in number; the other animal, a young colt, which died in forty-five days, showed few parasites in its blood except on three or four occasions when there was high fever $\left(4 \mathrm{I}^{\circ} \mathrm{C}\right.$., or $106^{\circ} 8^{\circ} \mathrm{F}$.). The symptoms of the experimental disease in the horse are similar to those noted in spontaneous infections (see p. 287). Post-mortem the spleen was enlarged and softened; myocarditis, pericarditis, and effusions into the serous cavities were present, the blood was thin and watery, and the bone-marrow was embryonic in character.]

[Vassal found the following animals refractory: a tortoise (Testudo elongata), a native peacock, two turtle-doves (Tuvtur humilis), two pigeons, and two chickens.]

[Laveran and Mesnil found that this trypanosome killed mice in $3 \frac{1}{2}$ to I4 days, rats in 8 to I I days, guinea-pigs in 21 to 97 days, dogs in 16 to 50 days, and a rabbit in 23 days. In addition, they experimented upon three goats, two of which were immunized against Mauritian surra. Goat I., immunized against mbori and Mauritian surra, ${ }^{1}$ was inoculated with the Annam virus, and died two and a half months later, when its blood was still infective, but only slightly so. Goat II. was twice inoculated with the Mauritian surra, and subsequently twice with the Annam virus. The first inoculation with Mauritian surra produced a mild infection from which the animal recovered immunized, so that the second inoculation with the same virus failed to infect. Two months later the goat received an injection of the Annam virus, which gave rise to a very mild infection, lasting about a month. A second injection with the same virus failed to reinfect the goat, which steadily gained in weight, and looked perfectly healthy. Goat III.-a control experiment-died in about three months after subcutaneous injection of the Annam virus. Trypanosomes were never seen in the blood, but this was, nevertheless, infective, for many subinoculated animals developed a fatal infection.]

[MODE OF TRANSMISSION.-Vassal made experiments with various insects, but in every case with negative results. He used leeches, fleas, lice, ticks, and several species of biting flies.]

1 [This is the billy goat mentioned on p. $22 \mathrm{I}$, which was used to demonstrate the identity" of surra and mbori.] 
[The blood of leeches which had fed on infected animals was infective, on injection into rats, immediately after the meal of blood, but not four hours later. The trypanosomes are killed off very rapidly in the stomach of the leech.]

[Biting flies are very rare at Nha-trang and in its immediate neighbourhood. Vassal experimented with species of various genera - Hippobosca, Hamatopota, and Chrysops-but none of these flies conveyed the infection from diseased to healthy animals by biting the latter at different times (immediately, some hours, one, two, or three days) after feeding on infected animals. Vassal suggests that the rôle of Stomoxys, and also of other horse-flies in Indo-China, should be studied.]

[Whatever the carrier of the infection may prove to be, Vassal thinks it is not very abundant, although fairly widely distributed throughout Indo-China. Its existence is ephemeral, and at Nhatrang coincides with the early part of the rainy season.]

[Diagnosis and Prevention.-The recognition of the disease is often very difficult because the trypanosomes may disappear from the blood for some days before death, and the inoculation of susceptible animals may even fail to infect. This is particularly the case with cattle and buffaloes, which, as we have seen, may be only very slightly affected, and show trypanosomes in the blood on one or two occasions. It is probable, says Vassal, that the blood of oxen or of buffaloes is the reservoir of the virus, and that the epizootic spreads to the more susceptible horse, when the virus is taken in by the appropriate fly. As a precautionary measure he suggests that cattle should not be penned up near stables in which horses are kept, and that as soon as a horse is known to be infected it should be killed.]

[COMparison With SuRra. - Laveran and Mesnil $^{1}$ conclude, from the morphology of the trypanosome, as well as from the evolution of the natural disease in Equidæ and of the experimental disease in various mammals, that the trypanosomiasis of Nha-trang does not differ from surra in any essential characteristic. Like surra, it is probably conveyed by Tabanidæ. Further, the Annam epizootic is geographically connected with the Indian, by means of the endemic areas of Laos, Tonkin, Yunnan, and Burmah.]

The immunization and cross-inoculation experiments with goats, already mentioned, gave somewhat discordant results. Goat I., immunized against surra, succumbed to the Annam virus, whereas goat II., alse immunized against surra, contracted only a mild infection. The normal (control) goat died of the infection in three months, so that this virus is very pathogenic for goats, which may explain the fact that goat I. succumbed to the infection, although immunized against surra.]

[Laveran and Mesnil are of opinion that this trypanosome is allied to that of surra-either a variety or a special race-and that this trypanosomiasis is not a distinct disease, like nagana, for example. They adduce further evidence in favour of this view. The serum of goat II. (immunized

${ }^{1}$ [Laveran and Mesnil, Ann. Inst. Past., v. 20, April, 1906, p. 302.] 
against Mauritian surra and the Annam trypanosomiasis) protected mice, ${ }^{1}$ either completely or partially, against the trypanosomes of surra and of Nha-trang, but not at all against that of nagana. Conversely, the serum of a goat immunized against nagana protected mice against $T$. brucei, but not against the Nha-trang virus.]

In a later paper ${ }^{2}$ Laveran and Mesnil state, however, that the trypanosome of Nha-trang is different from that of Indian surra. This opinion is based on the fact that the serum of a goat immunized against nagana and Indian surra failed to protect mice, even partially, against the Nha-trang virus.]

${ }^{1}$ [In these experiments the serum was mixed in a tube with the trypanosomecontaining blood, and after one or two minutes the mixture was injected subcutaneously into a mouse. 'Complete protection' means that no infection followed; 'partial protection' means that the incubation period and the total duration of the disease were longer than in control mice.]

${ }_{2}^{2}$ [Laveran and Mesnil, C. R. Acad. Sc., v. I42, June 25, 1906, p. I482.] 


\section{CHAPTER IX}

\section{CADERAS}

Pathogenic Agent: Trypanosoma equimum, Voges, Igor.

Synonyms: Peste de cadeiras (Brazil); Mal de caderas, Tumby-baba or Tumby-a (Paraguay, Argentine); Flagellosis of Equidæ; Trypanosomosis of Equidæ, etc.

\section{Section 1.-Historical. Geographical Distribution.}

MAL DE CADERAS, or, briefly, Caderas, is an epizootic of equines occurring in South America. The trypanosome which is the causal agent of the disease was discovered by Dr. Elmassian, Director of the Bacteriological Institute in Asuncion, the capital of Paraguay. ${ }^{1}$ This discovery was speedily confirmed in Voges' laboratory in Buenos Aires, where the disease had been studied for some time. ${ }^{2}$ Important papers upon this subject have been published by Voges, ${ }^{3}$ Lignières, ${ }^{4}$ Sivori and Lecler, ${ }^{5}$ and Elmassian and Migone. ${ }^{6}$ Owing to the kindness of Drs. Elmassian and Lignières, who sent us infected animals, we have been able to study in Paris the trypanosome of caderas and its pathogenic action upon various animals.

According to Lacerda, caderas was imported into the island of Marajo, close to the mouth of the Amazon, and from there it spread as far as the State of Matto Grosso. This much is certain, that since 1860 caderas has caused such great ravages in this particular State that all the horses have disappeared, and the natives have been obliged to use cattle as draught animals, and even for riding, young bulls being trained for this purpose. At the present time the disease has greatly extended; it occurs in parts of Brazil and Bolivia, throughout Paraguay, in the Argentine territories of the Chaco,

1 Elmassian, Lecture given at the National Hygienic Council, May I9, I90I. Asuncion, 1901. Revista de la Sociedad med. argent., 1902, v. IO.

${ }^{2}$ Voges, Berl. tierürztl. Woch., October 3, I $90 \mathrm{I}$. Zabala (with Malbran and Voges), Anales d. Departem. nac. de Higiene, Buenos Aires, IX, November, I 901 .

${ }_{3}$ Voges, Zeitschr. f. Hyg., 1902, v. 39, fasc. 3.

4 Lignières, Revista de la Sociedad med. argent., I902, v. 10, p. $48 \mathrm{t}$.

5 Sivori and Lecler, 'Le Surra americain, or Mal de Caderas,' Buenos Aires, October, 1902 .

${ }^{6}$ Elmassian and Migone, Ann. Inst. Past., I903, p. 241. 
Formosa, and Misiones, and in the Argentine provinces of Corrientes, Santiago del Estero, and Catamarca.

The epizootic is most prevalent in the marshy districts and during the months that it rains least (April to September).

\section{Section 2.-Animals susceptible to Caderas. Symptoms and Course of the Disease in the Equidæ and other Mammals.}

Caderas occurs naturally almost exclusively amongst horses, ${ }^{1}$ but is inoculable into a large number of other mammals. The disease may run an acute or subacute course, leading to a fatal termination, or it may be chronic and end in recovery. The following mammals have been successfully inoculated with caderas, those mentioned first in the list suffering from the most acute and severe forms of the disease: mouse, rat, otter, hedgehog, dog, cat, capybara, coati, monkey, horse, mule, donkey, rabbit, guinea-pig, sheep, goat, pig, and ox. According to Voges, the disease is inoculable into fowls, ducks, and turkeys, but no other observer has succeeded in infecting birds with the trypanosome of caderas, and we ourselves always found that inoculations into fowls gave negative results.

We shall first study caderas in the horse, since it is essentially a disease of the Equidæ, and in them may give rise to very serious epidemics.

Eouide.-We have already seen that in certain parts of South America it is so difficult to keep horses alive that cattle have to be used for riding purposes. The cavalry regiments sent into the Chaco against the Indians lost a large number of horses and mules from this disease. Voges mentions the case of a regiment which in June received 600 horses, of which only roo were alive in the following November.

The mule and donkey, especially the latter, are more resistant than the horse.

The first sign of the disease in horses is wasting, which rapidly progresses in spite of a good appetite. The temperature is often raised to $40^{\circ}$ or $4 \mathrm{I}^{\circ} \mathrm{C}$. $\left[104^{\circ}\right.$ to $105^{\circ} 8^{\circ} \mathrm{F}$.]. After a variable time it is noticed that the hind-quarters are weak, and that the animal drags its legs, the hoofs grazing the ground. These symptoms increase and become characteristic, so that when the animal is made to walk it staggers along, the hind-quarters swaying from side to side. On account of this symptom the name mal de caderas, or disease of the hind-quarters, has been given to the disease. There comes a time when the animal is unable to stand : if in a stable, it leans up against a wall or seeks other support; if in the open, it staggers and falls. After thus falling to the ground an animal may still live for several days if it be fed; otherwise the inevitably fatal end is hastened by inanition.

i Elmassian, during a recent severe epizootic of caderas, saw several cases of spontaneous infection in dogs (letter of February 28, I904). 
Fever is irregular (see Fig. 39), with elevations-which may reach $4 \mathrm{I}^{\circ}$ or $4 \mathrm{I}^{\circ} 8^{\circ} \mathrm{C}$. [106 $6^{\circ}$ or $107^{\circ} \mathrm{F}$.] -and remissions, during which the temperature may fall as low as $35^{\circ}$ or $36^{\circ} \mathrm{C}$. [ $\left[95^{\circ}\right.$ to $97^{\circ} \mathrm{F}$.]

According to Elmassian, the urine is always affected, and albuminuria and hæmaturia are frequently observed. The latter, which is usually very slight and discernible only with the microscope, is sometimes severe. Sivori and Lecler never met with hæmaturia, although they studied more than thirty cases of the natural infection. Authors agree in stating that trypanosomes are not found in the urine even when there is hæmaturia. Lignières never found living trypanosomes in the urine, the parasites undoubtedly dying very quickly in this medium.

The skin, particularly of the neck, shoulders, and hind-quarters,

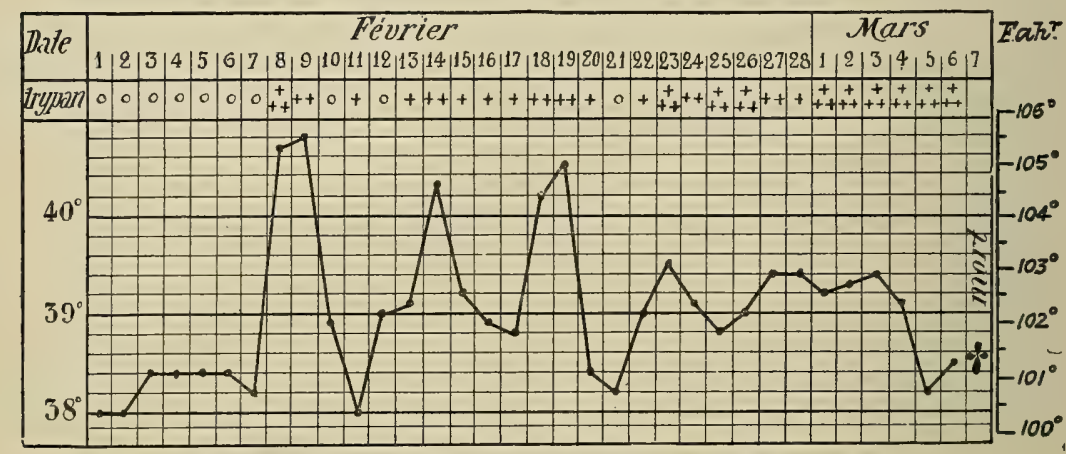

Fig. 39.-Caderas in a Horse-Acute Form.

On January 3I, I9O2, 20 c.c. horse blood, which did not show any parasites on microscopical examination, were injected intravenously, and the animal died in thirtyfour days. (Figure borrowed from Lignières.)

is often the seat of a slight weeping eruption; the patches, which are 3 to 4 centimetres [ $I$ to $I \frac{1}{2}$ inches] in diameter, are covered with small scabs, and the hair falls out in those places.

There are fugitive swellings, chiefly over the joints, but never the well-marked and persistent œdematous swellings as in nagana. There are no lesions of the genital organs even when horses are inoculated upon excoriations on the penis (Elmassian).

There is slight oedema of the eyelids, accompanied by conjunctivitis and chemosis. Interstitial keratitis, hypopyon, and plastic iritis are fairly frequent complications. Horses in which the symptoms of paresis appear usually die in one or two months.

Caderas sometimes runs a chronic course in the horse. According to Elmassian, this is the form of the disease which occurred in epizootics in Paraguay, and was known by the name of Baacy-poy. The disease is insidious, and for several months may show itself only by the wasting of the animals affected. Paresis, although occurring very gradually, is nevertheless characteristic. The microscopical examination of the blood does not show the presence of 
trypanosomes, but on injecting the blood into susceptible animals, a typical infection results. ${ }^{1}$

The disease is always fatal in the Equidæ, whether its course be acute or chronic. In horses injected subcutaneously or intravenously with virulent blood, the duration of caderas varies from one to four months. From Lignières' experiments it would appear that the shortest duration of the experimental disease in the horse is thirty-four days, the longest I34 days.

Trypanosomes appear in the blood from the fifth to the eighth day after inoculation, and this is accompanied by a considerable rise of temperature. During the course of the disease these febrile attacks may recur repeatedly, and the multiplication of the parasites occurs similarly, with exacerbations, in the intervals between which the blood examination is often negative. Elmassian and Voges have shown that the number of parasites becomes greatly diminished when the horse's temperature reaches $4 \mathrm{I}^{\circ} \mathrm{C}$. $\left[105^{\circ} 8^{\circ} \mathrm{F}\right.$.]. Anæmia is common, but is not so marked as in other hæmatozoal diseases, notably the piroplasmoses.

The severity and course of the disease depend a good deal upon the age and breed of the animal; for example, young horses, those which are suffering from other ailments, and well-bred animals are less resistant to caderas than adult healthy horses of common breeds (Lignières).

In describing the experimental infection in animals other than Equidæ, we shall follow the order mentioned above - namely, that of diminishing susceptibility to the virus.

Caderas is easily inoculated into animals, and in the susceptible species infection always follows an injection. These we have usually given subcutaneously, but intravenous and intraperitoneal injections shorten the incubation period. Only a small quantity of the virus need be inoculated, but the number of trypanosomes injected has a distinct influence upon the course of the infection. Thus if the blood of a mouse containing very few trypanosomes be used for injection, the incubation period will be a long one, and the total duration of the disease will be ten days or more. If, on the other hand, a large dose of blood containing many parasites be injected, the incubation period will be very short, and death may often occur on the fifth day after inoculation.

Mice.-After the subcutaneous injection of virulent blood into white mice, trypanosomes appear in the blood on an average at the end of two and a half days, and the duration of the disease from the time of inoculation is six days (maximum eight and a half days, minimum three and a half days). After intraperitoneal injection, parasites appear in the blood in thirty or forty hours, and the disease lasts five days. Grey mice are a little more resistant than white. When blood containing very few trypanosomes (as from a goat or

1 Elmassian and Migone, Ann. Inst. Past., April 25, 1903, p. 254. 
sheep suffering from caderas) is injected, the incubation period is prolonged to six days, and the disease lasts ten days (maximum nineteen days, minimum six and a half days).

In mice infected with caderas the trypanosomes, as a rule, increase regularly up to the time of death, when they are very numerous in the blood. We have, however, seen several exceptions in the case of mice inoculated with the blood of ruminants. The trypanosomes, fairly numerous on the seventh day after inoculation, for example, may be scanty or possibly absent on the eighth or ninth day, but soon afterwards they multiply in the usual progressive manner until death. Towards the end of the disease the mouse sits huddled up with its hair bristling, and paralytic symptoms occur shortly before the invariably fatal termination.

[Jakimoff, ${ }^{1}$ Halberstaedter, ${ }^{2}$ and Thomas and Brein ${ }^{3}$ have also studied the pathogenic effects of the trypanosome of caderas upon various experimental animals.]

[Jakimoff found that caderas ran a more rapid course in mice and rats than Lignières had found in his experiments already mentioned. Possibly this increased virulence noted by Jakimoff was due to the fact that his trypanosome had been passed through animals a greater number of times. Thus in mice the incubation period was fifteen to twenty hours, and death occurred in one to two days after intraperitoneal inoculation. After subcutaneous injection the incubation period was one to two days, and death occurred in four to five days.]

[Halberstaedter found the blood of mice infective even during the incubation period. Thus, a mouse injected intraperitoneally with rabbit's blood did not show any trypanosomes in the peripheral circulation until thirteen days later, but already on the second day after the intraperitoneal inoculation its blood was infective for another mouse (incubation period nineteen days, death two days later). Halberstaedter also found that the blood of a mouse undergoing treatment with trypanred, although containing active trypanosomes a day or two after the injection of the dye, gave rise to an infection after a very long incubation period-sometimes as long as four weeks-on injection into animals. Ehrlich and Shiga have suggested that the injection of trypanred gives rise to the formation in the body of antiparasitic substances, but an experiment of Halberstaedter's proves that such is not the case.]

[Thomas and Breinl found that white and grey mice became infected somewhat earlier than rats, and that the duration of the disease was slightly shorter. Rats and mice inoculated with blood from animals undergoing treatment with arsenic, and especially during the early stage of such treatment, developed the disease after a prolonged incubation period.]

RATS.-In white or speckled rats the incubation period is three to four days, and the average duration of the disease is seven and a half days after subcutaneous inoculation. After intraperitoneal inoculation the course of the disease is a little more rapid. In rats inoculated intraperitoneally with the blood of a goat or sheep containing very

1 [W. L. Jakimoff, Centralb. f. Bakter, I, Orig., v. 37, p. 668.]

2 [L. Halberstaedter, Centralb. f. Bakter., I, Orig., v. 38, p. 525.]

3 [Thomas and Breinl, Thompson Yates and Johnston Lab. Reports, v. 6, part ii., 1905, p. 35.] 
few parasites, the average duration of the incubation period was eight days and of the total infection sixteen days (maximum twenty-one days, minimum seven and a half days). Lignières states that speckled rats are more resistant than white, and the grey most resistant of all. A sewer rat we injected with caderas died in twelve days. According to Voges, grey rats sometimes recover from the disease; in white and speckled rats it is always fatal. The parasites increase in number in the blood until the time of death, when they are always very abundant. The symptoms preceding death are the same as in the mouse; we have never seen death occur suddenly, as sometimes happens in rats with nagana.

[In white and grey rats Jakimoff found the incubation period to be nineteen to twenty-four hours, and the total duration of the disease three and a half to four and a half days, after intraperitoneal inoculation. After subcutaneous injection the incubation period was two to three days, and death occurred in five to eleven days. Thomas and Breinl obtained similar results in the rats they inoculated, but the incubation period after intraperitoneal injection was rather longer in their animals (thirty-six to sixty hours) than in Jakimoff's.]

Field-Mice (Arvicola arvalis).-A field-mouse showed trypanosomes in its blood thirty-six hours after intraperitoneal inoculation. Two others inoculated subcutaneously showed the parasites on the second and eighth days respectively after inoculation. These animals died so rapidly that it was not possible to study the complete course of the disease in them. The great variability in the length of the incubation period depends probably upon individual variations in the susceptibility of the animals to caderas ( $c f$. nagana in guinea-pigs).

HEDGEHOGs.-The hedgehog (Evinacens europaus) is very susceptible to caderas. Two hedgehogs injected with the blood of an infected guinea-pig died in six and nine days, with very many trypanosomes in the blood.

OTtERs.-Two otters (Nutria sp. ?) inoculated with caderas by Voges died in ten days.

Mulita.-The mulita (armadillo, Tatusia hybrida) is very susceptible, dying in ten days according to Lignières.

Monkeys.-Monkeys are likewise very susceptible to caderas. The disease lasts from seven to fifteen days according to the amount of blood injected. In Nyctipithecus felimus the incubation period was from three to five days, and the disease lasted five to eight days (Elmassian and Migone).

On October 8, I902, we inoculated a monkey (Cercopithecus fuliginosus, or closely allied species) subcutaneously with blood from a mouse with caderas. The monkey's temperature at the time was $38.5^{\circ} \mathrm{C}$. $\left[\right.$ IOI $^{\circ}{ }^{\circ} \mathrm{F}$.]. On October 13 a few trypanosomes were found in the blood and the temperature was raised. From the I 4 th to the I6th the temperature remained at about $39^{\circ}$ to $39^{\circ} 3^{\circ} \mathrm{C}$. $\left[\right.$ IO2 $2^{\circ}$ to 
I02. $\left.8^{\circ} \mathrm{F}.\right]$, and the trypanosomes gradually increased in number. From October $I 7$ the temperature began to fall, and on the 2oth was $35^{\circ} 7^{\circ} \mathrm{C} .\left[96 \cdot 3^{\circ} \mathrm{F}\right.$. $]$, which was much below the normal. On the Igth trypanosomes were fairly numerous in the blood, but on the 2 oth and 2 Ist the blood examination was negative. The monkey died during the night of October 2I-22, the disease having lasted thirteen and a half days.

In monkeys a subnormal temperature before death is usually found in caderas and nagana, as in the infection produced by T. gambiense.

DoGs.-After subcutaneous inoculation trypanosomes appear on the fourth or fifth day. In our experiments the average duration of the disease was thirty-six days. The multiplication of the

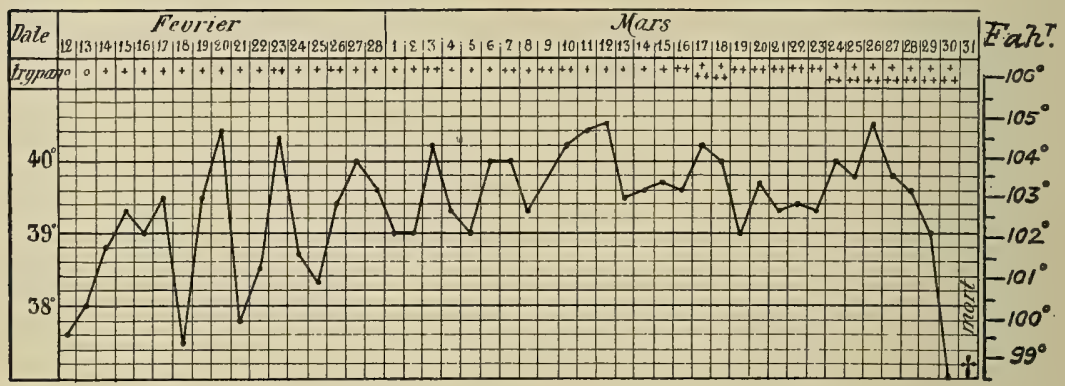

Fig. 40.-Temperature Chart of a Dog inoculated on February i2, I902,

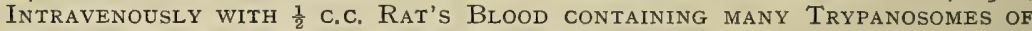
Caderas. Death in Forty-Eight Days. (Lignikeres.)

trypanosomes in the blood of the dog is not so regular as in the foregoing animals. There are exacerbations in the intervals between which the parasites sometimes become very scanty, but these intervals are always short. At the time of death trypanosomes are numerous or very numerous in the blood.

The only constant symptoms are wasting and a variable degree of fever, the latter occurring especially with the appearance of trypanosomes in the blood (see Fig. 40).

When the disease does not progress very rapidly, there is often œdema of the sheath and scrotum, conjunctivitis, iritis-sometimes double, with hypopyon, which may lead to total blindness-and, in the last stages, paresis of the hind-limbs. The disease is always fatal in dogs.

[In Jakimoff's dogs the incubation period, after subcutaneous injection, was two to four days, and death occurred in seven to sixteen days-much more quickly than in Lignières' and Laveran and Mesnil's experiments. The trypanosomes became progressively numerous, and the chief symptoms were somnolence, paresis, and eye troubles.]

RaBBits.-In rabbits inoculated subcutaneously trypanosomes appear in the blood in four or five days. The average duration of 
the disease in the rabbits inoculated by us in Paris was thirty-three days (maximum, forty-six days). Voges gives the duration as from one to three months. Trypanosomes are scanty or very scanty in the blood of rabbits infected with caderas.

The general symptoms are wasting and febrile paroxysms. The commonest local symptoms are: blepharo-conjunctivitis; œedema at the root of the ears, with partial loss of hair ; coryza, with swelling and excoriations of the nostrils; swelling in the region of the anus and vulva; and swelling of the testicles, or even orchitis. The eye lesion is a grave complication if the animals are not well looked after, for the eyelids become stuck together, and pus accumulating beneath them, the corneæ become inflamed. Blindness may result, which prevents the rabbit from feeding itself properly, and so hastens the fatal issue. The eyes should be bathed every morning with boracic lotion; by so doing this complication may be avoided.

Lignières states that the odema fluids in the rabbit often contain more trypanosomes than the blood.

[Jakimoff's rabbits developed a more acute infection than did those of Lignières. After intravenous inoculation the incubation period was less than twenty-four hours, and death occurred in twenty-two days; after intraperitoneal injection the incubation period was two days, and death occurred in fifteen to seventeen days.]

[Thomas and Breinl found the average incubation period four to five days; the total duration was sixteen to fifty-nine days. In a few rabbits the trypanosomes were fairly numerous in the blood-ten or fifteen in a field - but most rabbits showed very few parasites. Fever was irregular; an initial rise to $106^{\circ}$ or $107^{\circ} \mathrm{F}$. ( $4 \mathrm{I}^{\circ}$ to $4 \mathrm{I} \cdot 6^{\circ} \mathrm{C}$.) was noted on the fourth or fifth day in some cases, but, as a rule, the temperature did not exceed $104^{\circ} \mathrm{F}$. $\left(40^{\circ} \mathrm{C}\right.$.). In addition to the symptoms mentioned above, loss of flesh and a somewhat marked anæmia were noted. In animals that were treated with atoxyl, the odema and the urethral, nasal, and ocular discharges soon disappeared, and the hair began to grow again over the bald patches. One rabbit treated with atoxyl was still alive eight months after injection and appeared in perfect health.]

CAPybara (Hydrocharus capybara).-In this large rodent, which is common in Uruguay and in certain parts of the Argentine, and which appears to play some part in the spread of caderas, the incubation period lasts ten days, and the total duration of the disease is from one to two months. During the last stages paralytic symptoms occur.

CoATI (Narica nasua, Lin.).-In two coati inoculated with caderas by Lignières, death occurred in twenty and forty-seven days respectively. Elmassian records a longer duration of the disease in this animal.

The chief symptoms are : wasting, anæmia, swelling of the face, weakness, and, in the last stages, paresis of the hind-limbs. Trypanosomes are present in the blood almost constantly, and often in great numbers (Lignières); they increase with exacerbations. 
Guinea-PIgs.-After subcutaneous inoculation trypanosomes appear in the blood at the end of nine days, on an average. The average duration of the disease was, in our experiments, eighty-four days (maximum 120, minimum 29). The trypanosomes multiply irregularly, and during the exacerbations they may be numerous in the blood, whereas in the intervals they may be so scanty as not to be found on microscopical examination.

At first the guinea-pig shows no symptoms of disease, but eats well and often gains weight. Later on there is wasting, and in some cases localized swellings occur about the head and the region of the anus and vulva. Double blepharo-conjunctivitis, with opacity of both corneæ, was very marked in one of our guinea-pigs, which died on the I36th day of the disease. All the guinea-pigs inoculated by us with caderas have died; but, according to Voges, these animals may sometimes recover.

[In the guinea-pigs inoculated by Jakimoff the incubation period was two to three days (in one case it was only twenty-four hours) after intraperitoneal injection, and three to nine days after subcutaneous injection. Death occurred in eleven to thirty-nine days after the former method of infection, and in twelve to twenty-six days after the latter. Some of the guinea-pigs had to be injected several times before they became infected.]

[Thomas and Breinl found the incubation period after intraperitoneal and subcutaneous inoculation to be 6 to $8 \frac{1}{2}$ and 8 to ro days respectively. The disease lasted from 22 to I 53 days, the average being 75 to 90 days for adults, and 30 to 46 days for young guinea-pigs ( 300 grammes). Most of the animals had trypanosomes in their blood almost constantly, but occasionally the parasites nearly disappeared, especially after a marked exacerbation. There was a slight rise of temperature with the first appearance of trypanosomes in the blood, and afterwards the fever became irregular. Three animals had paresis of the hind-limbs one or more days before death. Of forty-three guinea-pigs infected and untreated not one survived.]

CATs.-According to Lignières and Elmassian and Migone, kittens die in from fifty to eighty days, while full-grown cats live for a long time, although the parasites are often numerous in their blood. Lignières mentions the case of a cat which, eight months after infection, did not show any signs of disease. [In a later paper ${ }^{1}$ he mentions that this cat died two years and seven and a half months after inoculation.]

The chief symptoms in the later stages are wasting and anæmia.

[In full-grown cats Jakimoff noted an incubation period of four days, and a total duration of one month. Thomas and Breinl found the incubation period to be $6 \frac{1}{2}$ to 8 days in kittens and 7 to II days in cats. Kittens died in 30 to 56 days, adult cats in $2 \frac{1}{2}$ to 6 months. With the first appearance of parasites in the blood there was a rise of temperature. In kittens the temperature usually remained high, and trypanosomes were almost constantly found in the blood. In cats the temperature curve was more

1 [Lignières, Report to Path. Section of Eighth Internat. Congress of Vet. Med., Budapest, September, I905; abstract in Bull. Inst. Past. (Mesnil), v. 3, p. 946.] 
irregular - with sharp rises to $104^{\circ}$ or $105^{\circ} \mathrm{F} .\left(40^{\circ}\right.$ to $40^{\circ} 6^{\circ} \mathrm{C}$.) -and try panosomes were rare in the blood, except during the febrile paroxysms. Edema and discharges from the eyes, nose, and genitals were rarely observed.]

Pig, Sheep, Goat, Cattle.-As a rule, these animals do not exhibit any symptoms after inoculation with caderas, so that several observers have looked upon them as refractory to the disease, especially as their blood usually fails to show any trypanosomes on microscopical examination. These animals really contract the disease, but in a very mild form which nearly always ends in recovery. On taking the temperature of inoculated animals, it is found that there are occasional elevations, and on inoculating the blood into very susceptible animals, such as mice and rats, typical infections are produced. Trypanosomes, therefore, are present in the blood, but in such small numbers that they nearly always escape detection by the microscope.

We have inoculated with caderas two sheep, one of which was immunized against nagana; one goat (Goat I.), which for six months had been immunized against nagana, and had since received large doses of blood containing many $T$. brucei; a young goat ( $\delta$ ) weighing I4 kilogrammes; and a kid ( $q$ ) weighing $8 \frac{1}{2}$ kilogrammes (Goat II.).

Only one of these animals died-namely, the sheep which was immunized against nagana. It died sixty-six days after inoculation, and at the time of death its blood was virulent in doses of $\frac{1}{40}$ c.c. The other animals all remained perfectly well. They increased regularly in weight, had no rise of temperature on the days immediately succeeding the inoculation, and never showed any parasites in the blood on microscopical examination. At first the blood was virulent for mice in doses of $\frac{1}{10}$ c.c., with an incubation period of five days. At the end of three months this dose was either not infective or gave rise to a chronic infection in mice after a prolonged incubation period. Later still, towards the end of the infection, as much as 3 c.c. of blood did not infect a rat. We considered animals cured when 8 c.c. of their blood injected into rats failed to infect. The sheep remained infected for four and a half months, the three goats for five to five and a half months.

In the pig the blood is still virulent two months after inoculation with caderas.

In cattle the blood is virulent for several months.

Animals which survive an attack of caderas are immunized against the disease, and they can be injected with very large doses of the virus without becoming reinfected.

[Jakimoff also injected a goat, which never showed trypanosomes in its blood, but this was infective four months after inoculation. The goat died in six months, the only symptom being slight wasting.]

[Frogs and pigeons are immune (Jakimoff); so also are geese and fowls (Mesnil and Martin).] 


\section{Section 3.-Pathological Anatomy.}

Apart from anæmia, which is more or less marked, there is no lesion which is constantly found in the different species of animals susceptible to caderas.

[In addition to the loss of hæmoglobin and the changes in the number of the corpuscles (marked diminution of red, considerable increase of white, especially lymphocytes), Jakimoff ${ }^{1}$ observed a diminution in the alkalinity of the blood. Thus in one dog infected with caderas the alkalinity fell from 0.64 per cent. to 0.24 per cent. two days before death. $\mathrm{He}$ found also that, as in nagana, the cerebro-spinal, pleural, peritoneal, pericardial, and odema fluids and the bile all contained trypanosomes, whereas the urine was always negative. Mayer ${ }^{2}$ noted marked lipæmia in caderas dogs, the blood containing 0.3 to 0.5 per cent. of fat.]

The swelling of the spleen, which is always found in rats, mice, and dogs after infection with caderas, is only slightly marked in guinea-pigs and absent in rabbits.

In mice, average weight 24 grammes, the average weight of the spleen was 0.84 gramme (maxima, I gramme and $\mathrm{I} \frac{1}{4}$ grammes).

In rats, average weight 2I 5 grammes, average weight of spleen was 2 grammes (maximum, 3 grammes).

In a dog weighing $4^{\circ} 7$ kilogrammes the spleen weighed 36 grammes; in two others of $\mathrm{I} 2$ and 25 kilogrammes the spleens weighed respectively Iog and 345 grammes.

In the monkey mentioned above, weighing $\mathrm{I}, 265$ grammes, the spleen, which was large and diffluent, weighed 14 grammes.

In guinea-pigs, weighing about 500 grammes, the average weight of the spleen was 2 grammes (maximum, 5 grammes in a guinea-pig of 670 grammes; minimum, $0{ }^{\circ} 72$ gramme in a guinea-pig of 400 grammes). In one case the spleen weighed I 2 grammes, but it was the seat of a large infarct, which had led to rupture of the organ.

In rabbits of about $\mathrm{I} \frac{1}{2}$ kilogrammes the spleen weighed only I.68 grammes, or less than it does in rats 215 grammes in weight.

In the horse the spleen is congested and increased in size, and the mesenteric glands are also enlarged. Nephritis and interstitial hæmorrhages are common. There is often fluid in the peritoneal, pleural, and pericardial cavities, and a yellowish gelatinous exudation in the spinal canal (Elmassian and Migone).

[In cats Thomas and Breinl found the spleen and lymphatic glands somewhat enlarged. In kittens the gland-juice often contained many trypanosomes.]

[Halberstaedter, who studied the tissue changes and the distribution of the parasites in the tissues and organs of animals experimentally infected with nagana, dourine, and caderas (see Chapter VI.), found that the tissues did not stain nearly so well in caderas as in the other two diseases ]

[Sauerbeck ${ }^{3}$ observed the remains of trypanosomes inside the large mononuclear and polymorphonuclear leucocytes of the bone-marrow of a

3 [Jakimoff, Centralb. f. Bcckter., I, Ref., v. 38, 1906, p. 13 ; ibid., Orig., v. 37, p. 668.]

2 [Mayer, Zeitschr.f. Exp. Path. u. Ther., v. i. 1905.]

3 [E. Sauerbeck, Zeitschr.f. Hy'g. u. Infectionskr., v. 53, 1906, p. 51 2.] 
caderas rat. He made a similar observation in the case of a nagana guinea-pig, and is convinced of the great importance of the macrophages in the engulfment of trypanosomes.]

\section{Section 4.-Pathogenic Agent. ${ }^{1}$}

Voges called the trypanosome of caderas Trypanosoma equina, which should be changed to Trypanosoma equinum, Trypanosoma being neuter. ${ }^{2}$

In fresh blood $T$. equimum very closely resembles $T$. evansi and $T$. brucei; it is only in preparations which are well fixed and stained that one is able to distinguish between them. T. equinum is very active when the blood is drawn, but in ordinary fresh preparations it loses its motility fairly quickly. In animals with many trypanosomes in their blood the movements of the parasites usually become sluggish during the few hours immediately preceding death, probably owing to the asphyxial condition present.

T. equinum is $22 \mu$ to $24 \mu$ long, by about $I^{\circ} 5 \mu$ wide; but parasites undergoing division may be $28 \mu$ to $30 \mu$ long, by $3 \mu$ to $4 \mu$ wide. In the ordinary forms the flagellum measures about $5 \mu$. The parasite has the same length in different species of animals, measurements made in the case of the mouse, rat, monkey, guinea-pig, dog, and horse giving us identical figures.

The most important differential characteristic of $T$. equinum, as compared with the allied species, evansi, brucei, and equiperdum, is the peculiar appearance of the centrosome (see Fig. 4I, I, and Fig. 5, in the coloured plate). Whereas in the trypanosomes of the type evansi the centrosome is very obvious, and stains a deep purple by the ordinary method, in T. equinum it is so insignificant that some observers have denied its existence altogether. ${ }^{3}$ It does exist, however, but it is very small and stains the same colour as the flagellum, which makes it all the more difficult to distinguish. The centrosome, which measures about $\frac{1}{2} \mu$ in $T$. brucei and $T$. evansi, is at the most $\frac{1}{3} \mu$ in $T$. equinum, so that this trypanosome can be readily distinguished from the allied species by the different appearance of the centrosomes in well-stained preparations.

We inoculated both caderas and nagana into a mouse, with the result that the two infections developed simultaneously, nagana preponderating. Owing to the difference in the appearance of the centrosomes, it was easy to distinguish the trypanosomes of caderas from those of nagana in stained specimens of blood.

1 See the references given in Section I ; also A. Bachmann and P. de Elizalde, An. d. circulo medico argentino, March 3r, 1903.

2 Recently Lignières has suggested the name $T$. elmassiani, which cannot, however, be adopted.

${ }^{3}$ Lignières, op. cit. He admits that the beginning of the flagellum is sometimes a little swollen and rounded. It is just this thickening at the origin of the flagellum - which is more constant than he states-that constitutes the rudimentary centrosome of $T$. equinum. 
The nucleus, undulating membrane, and flagellum have almost the same appearance as in $T$. evansi. The chromatic granules in the protoplasm vary considerably in number; in rats and mice they are more numerous in the last stages of the disease than they are at first.

Multiplication is nearly always by equal binary fission. As a rule, the centrosome and the adjacent part of the flagellum are the first to divide (see Fig. 4I, 2, 3). The nucleus divides later and at the same time as the rest of the flagellum. At this stage the parasite, which is twice as wide as a normal trypanosome, shows two nuclei, two centrosomes, two undulating membranes, and two flagella; finally the protoplasm divides. Sometimes parasites with three or four nuclei are seen (Fig. 4I, 4, 5). Evidently in such cases the nucleus and centrosome have divided again, before division of the protoplasm has taken place.
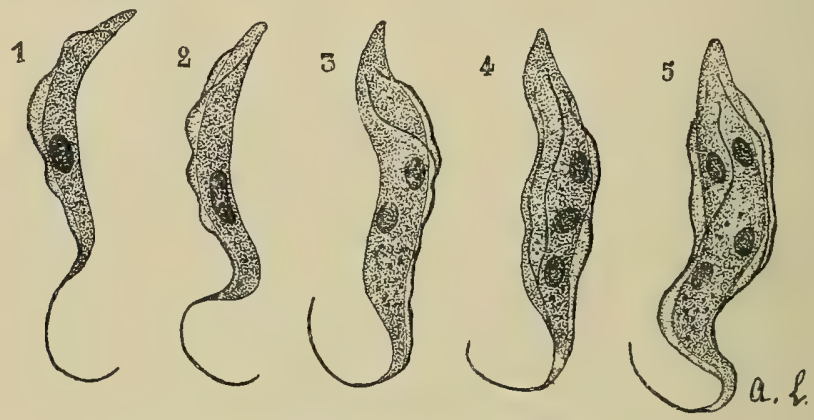

Fig. 4I.-Trypanosome of Caderas,

I. T. equinum, normal form. 2, 3. Ordinary multiplication forms, simple fission ; 2 shows an early stage in division. 4, 5. Less common forms, dividing into three and four parasites. (Magnified about 2,000 diameters.)

According to Lignières $T$. equinum may be kept alive for three days in the ice-chest. At the ordinary laboratory temperature it dies more quickly. The vitality of the trypanosomes is greater in blood mixed with serum than in pure defibrinated blood. The serums of different animals behave rather differently in this respect. Lignières found in his experiments that the mixture with fowl serum gave the best results, the trypanosomes surviving eleven days; next come the serums of the horse and sheep (six days); rat, ox, and frog (five days); pig, cat, and dog (four days); man, guinea-pig, and rabbit (about three days).

Lignières obtained well-marked agglutination of the trypanosomes of caderas with the normal serums of the sheep, pig, rabbit, and horse. Even better results were obtained with the serums of oxen, sheep, pigs, and cats infected with caderas. The normal serums of the ox, man, rat, and cat were only feebly agglutinating, while those of the frog, fowl, guinea-pig, and dog did not agglutinate at all. 
The trypanosomes of caderas agglutinate, like $T$. lewisi and $T$. brucei, by their posterior ends. The rosettes which are thus formed are smaller and looser than those obtained with $T$. lewisi. Agglutination is never complete, but there is always a certain number of unclumped parasites to be seen in the preparations.

Action of Cold and Heat.-This trypanosome is more resistant to cold than to heat. In one experiment of Lignières blood cooled for two hours to $-20^{\circ} \mathrm{C}$. was found to be still virulent. In another experiment blood exposed to $-10^{\circ} \mathrm{C}$. for five hours was no longer virulent. We made several experiments to find out the effect of great cold (temperature of liquid air) upon this trypanosome. A mouse inoculated with $\frac{1}{5}$ c.c. blood which had been exposed for five minutes to $-\mathrm{I} 9 \mathrm{I}^{\circ} \mathrm{C}$. became infected in nine days, and died in sixteen and a half days. Another mouse inoculated with $\frac{1}{3}$ c.c. of the same blood exposed for five minutes, and afterwards for ten minutes, to $-\mathrm{IgI}^{\circ} \mathrm{C}$, , became infected in eight days, and died in twelve and a half days. A control mouse inoculated with infective (uncooled) blood became infected in five days, and died in nine days. This trypanosome is therefore able to withstand for at least several minutes the temperature of liquid air.

T. equimum dies after five hours and forty minutes' exposure to $40^{\circ} \mathrm{C}$., after four hours' exposure to $4 \mathrm{I}^{\circ} \mathrm{C}$., after forty-five minutes to $42^{\circ} \mathrm{C}$., after twenty minutes to $43^{\circ} \mathrm{C}$, after ten minutes to $44^{\circ} \mathrm{C}$., after eight minutes to $45^{\circ} \mathrm{C}$., and after five minutes to $53^{\circ} \mathrm{C}$. (Lignières).

[Jakimoff obtained almost identical results. He also studied the preservation of $T$. equimum and obtained somewhat similar results to those described under T. brucei (see p. I57). The chief differences observed between the two trypanosomes were: (I) T. equinum was less affected by a temperature of $36^{\circ} \mathrm{C}$., defibrinated blood kept for two days at $36^{\circ} \mathrm{C}$. being still capable of infecting mice; and (2) 0.6 per cent. $\mathrm{NaCl}$ solution added to the blood kept at $20^{\circ} \mathrm{C}$. was able to maintain T. equinum alive and infective for five days, whereas $T$. brucei under the same conditions was infective only for two days. On the other hand, blood containing $T$. equimum would not bear such high dilution ( $\mathrm{I}$ in $5 \mathrm{O}, 000$ ) as blood containing T.brucei; caderas blood diluted 5,000 times was still infective for mice, however.]

The involution forms seen in heated blood are similar to those already described under T. brucei-namely, tadpole and spherical forms.

Attempts to cultivate $T$. equinum on blood-agar at the room temperature yielded negative results. Six to eight days after inoculation of the tubes no motile parasites were found, and mice inoculated with the fluid did not become infected.

[Thomas and Breinl made several attempts to cultivate T. equinum on various modifications of Novy and McNeal's medium. One attempt with rabbit's blood trypanosomes inoculated upon a 
rabbit-blood chicken-broth agar medium ${ }^{1}$ yielded a positive result. After twenty-nine days at $22^{\circ} \mathrm{C}$, one tube, out of twelve inoculated, was found to contain a few trypanosomes, many of them being paired, apparently healthy and fairly motile. This culture infected a rat eight and a quarter days after injection. Subcultures upon fresh blood-agar tubes failed to grow.]

[Rabinowitsch and Kempner ${ }^{2}$ state-but without giving any details-that they have cultivated $T$. equinum.]

\section{Section 5.-Caderas is a Distinct Morbid Entity.}

Voges regarded caderas as identical with dourine, while Sivori and Lecler identified it with surra; but since the disease has become better known, these opinions are no longer tenable.

Morphologically the trypanosome of caderas is distinguished from the parasites of nagana, surra, and dourine by the small size of its centrosome. Caderas is not propagated in the same way as dourine, and, moreover, most mammals are susceptible to caderas, whereas the number of species susceptible to dourine is very limited. Finally, animals which have acquired immunity against nagana, surra, or dourine are as susceptible to caderas as normal animals, and vice versa.

During our investigations a goat and a sheep immunized against nagana proved to be as susceptible to caderas as normal animals of the same species, and they contracted an infection lasting the same time. ${ }^{3}$ Lignières made the complementary experiment by showing that an ox, a sheep, and a pig, which had recovered from an infection with caderas, were as susceptible to nagana as normal animals of the same species and contracted an infection lasting the same time. ${ }^{4}$ We found that two goats (I. and II.) which had recovered from an infection with caderas were still susceptible to surra. ${ }^{5}$

Nocard and Lignières showed that dogs immunized against dourine were as susceptible to caderas as the normal animals used as controls. We shall refer to these experiments in the chapter on dourine.

[Ehrlich and Shiga state that mice cured with trypanred possess a temporary immunity - that is to say, they do not become acutely ill after a second injection. This immunity, moreover, is specific. Halberstaedter found that a mouse immunized against caderas after treatment with trypanred promptly became infected on injection

1 [The medium used was chicken-broth (meat I part to water 2 parts), with 0.5 per cent. peptone and 0.25 per cent. sea-salt added, and rendered faintly alkaline, agar 2.5 per cent., and defibrinated rabbit-blood $2: 1$.

2 [Rabinowitsch and Kempner, Centralb. f. Bakter., I, Orig., v. 34, 1903, p. 816.]

3 C. R. Acad. Sciences, v. 135, November 17, 1902, p. 838 .

+ Bol. Agricultura y Ganaderia, third year, No.50, Buenos Aires, February I, 1903 .

Laveran and Mesnil, C. R. Acad. Sciences, June 22, 1903, p. I 529. 
with dourine. After a second injection with trypanred, the mouse again became free from trypanosomes, and was then found to be normally susceptible to nagana.]

\section{Section 6.-Mode of Propagation.}

Caderas can be easily inoculated, it being sufficient to inject very small doses of the virus subcutaneously, or to place traces of it upon the surface of a wound or excoriation. The ingestion of blood or an emulsion of an organ containing trypanosomes is not followed by infection, if there be no recent wound or abrasion of the mucous surfaces. Sexual intercourse does not give rise to infection, as it does in the case of dourine (Lignières).

A priori, one would imagine that caderas was spread by means of biting flies, as in the case of nagana and surra. That opinion has been held by several observers, but it is still denied by some, and it is not in agreement with a number of recorded facts as to the conditions under which caderas is propagated.

Voges has shown that there are many insects which bite horses in the regions where caderas is prevalent. In particular he mentions a Tabamus and a biting fly known in the Argentine as Mosca brava, as being probably concerned in the propagation of this disease.

According to Sivori and Lecler, caderas is propagated by Tabanidæ (sp.?), by Mosca brava, which Dr. Brauer of Vienna says is the Stomoxys nebulosa Fabr., and also by Stomoxys calcitrans. These observers state that they succeeded in infecting horses by allowing them to be bitten by flies which had sucked the blood of sick animals.

Lignières states that St. calcitrans is very widely distributed in the districts where caderas occurs, but he adds that he never met with a case of infection by means of that fly in the infirmaries where infected horses were placed side by side with healthy ones or with horses suffering from other diseases. There were always very many horse-flies and St. calcitrans in these infirmaries.

An epizootic of caderas which occurred on a farm in Paraguay did not spread to a neighbouring farm, which was separated from the former only by a wire-gauze partition (Elmassian and Migone).

The only fact upon which all observers are agreed is that the capybara (Hydrocharus capybara), which is very abundant in Paraguay and in the Argentine portion of the, Chaco, along the banks of the small watercourses running through the cattle-rearing districts, is the source from which the carrier of the disease probably obtains its supply of the virus.

These animals are attacked periodically by an epizootic of an unknown nature. They lie about along the banks of the streams and die there. When the farmers in Paraguay find the dead bodies of the capybara on their farms, they know that caderas will soon break out among the horses. There is a striking analogy between 
this mortality among the capybaras which precedes outbreaks of caderas, and that among rats which precedes epidemics of plague. Elmassian, who recorded these facts, ${ }^{1}$ has hitherto looked in vain for capybaras spontaneously infected with caderas, but that may be because it is very difficult to catch them alive.

[Further observations by Elmassian and Migone ${ }^{2}$ confirm the belief that the capybara is the 'reservoir' of the virus of caderas. They quote instances of an epidemic of caderas breaking out among dogs which, after hunting capybaras, had devoured their still warm bodies. In these cases the virus passed directly from capybara to dog. Later on the infection spread to horses, which could only have got it from dogs or capybaras; but nothing definite is known as to how the infection is conveyed from the latter to the horse, or from one horse to another. Elmassian and Migone state that mosquitoes are the only insects biting at the time of day that capybaras and horses are at the water's edge together.]

[Possibly fleas are concerned in the transmission of the disease. Lignières found that fleas caught on a caderas dog and placed on a healthy dog did not infect the latter, but similar fleas crushed in salt solution infected two rats out of four inoculated.]

\section{Section 7.-Treatment. Prophylaxis. ${ }^{3}$}

Quinine, methylene blue, salicylic acid, permanganate of potash, potassium iodide, intravenous injections of perchloride of mercury, and arrhénal have all been tried unsuccessfully in the treatment of caderas (Voges, Lignières).

As with nagana, arsenious acid has given favourable results in some cases, but the improvement is only temporary. In rats or mice infected with caderas the same results are obtained as with nagana: there is a temporary disappearance of the trypanosomes from the general circulation, and the life of the animals is prolonged, but they are never cured. 0.5 milligramme of arsenious acid is the dose for each Ioo grammes of body weight. A rat thus treated survived for I30 days after inoculation with caderas. ${ }^{4}$ After a time, however, the arsenious acid no longer has any effect upon the trypanosomes, and symptoms of intolerance arise.

Erhlich and Shiga have used with success in the treatment of caderas in mice, a dye of the benzo-purpurine series which they call trypanroth ${ }^{5}$ (trypanred).

On injecting into a mouse simultaneously, but at different parts of the body, the caderas virus and a solution of trypanred, infection does not follow. In mice treated from one to three days after

1 Private communication, December 24, 1903.

2 [Elmassian and Migone, Ann. Inst. Past., v. 18, 1904, p. 589.]

3 [See Chapter XIII. on 'Treatment,' for recent work in this direction.]

4 For details of the arsenic treatment see Chapter VI., p. 170.

5 Ehrlich and Shiga, Berl. klin. Woch., March 28 and April 4, 1904. 
inoculation with caderas the results are remarkable. On injecting 0.3 c.c. of a I per cent. solution of trypanred, the trypanosomes soon disappear from the blood, often permanently, but in a certain number of cases there is a relapse. This is sometimes very long in appearing, in one case coming on only after sixty-five days.

Animals injected with trypanred on the fourth day of the disease lose their trypanosomes, and as a result become immunized to a certain degree against this trypanosome. Inoculated from one to seven days after this apparent or real cure, they only become reinfected after an incubation period of from twelve to sixty days. Even twenty-one days after the disappearance of the parasites the mice are still slightly immune, but after thirty days they are no longer so.

On comparing these results with those of preventive inoculations, it is seen that the production of immunizing substances is associated with the destruction of the trypanosomes by the drug. The curative power of trypanred is also very apparent on giving the dye internally. In mice fed for eight days on biscuit containing trypanred infection with caderas occurs, but it ends in recovery.

With rats the results obtained with trypanred have been much less encouraging than with mice. On injecting into a rat 2 c.c. of a I per cent. solution, the trypanosomes disappear, but only for a time. With guinea-pigs and dogs the results have been still less favourable.

Trypanred has no toxic effect in vitro upon the trypanosomes of caderas. Ehrlich and Shiga think that it acts by provoking in the mouse the formation of a substance inimical to the trypanosomes, which does not persist very long in the blood. Two or three days after they have been injected with trypanred, the mice can be successfully reinoculated with the trypanosome.

We treated four caderas mice by injecting them with trypanred at a time when the parasites were numerous in the blood. The mice received on an average $0^{\circ} 2$ c.c. of a I per cent. solution per Io grammes of animal. The trypanosomes disappeared from the blood in twenty-four to forty-eight hours. In one mouse they reappeared in fifteen days and caused death. In another they reappeared a month later, disappeared again spontaneously, but after ten days reappeared again and remained present till death. The other two mice had no relapse and were still alive two months after the injection of trypanred. ${ }^{1}$

The sheep, goat, ox, and pig which survive an attack of caderas become immunized against the disease, and their serum acquires, during the course of the infection, protective properties, which, unfortunately, are but slightly marked and of short duration.

The serum of a goat which, prior to inoculation with the try-

1 [The authors inform me that these two mice were probably cured when they died by accident, early in I906, nearly two years after the beginning of the experiment recorded above.] 
panosome of caderas, had no action upon the parasite, acquired definite protective properties. Three months after inoculation, I c.c. of this serum mixed with $\frac{1}{10}$ c.c. of trypanosome-containing blood prevented an infection. Later on, towards the end of the infection and after recovery, the serum was no longer protective.

The serum of the sheep immunized against nagana had, prior to inoculation with caderas, no protective power against $T$. equinum. One month afterwards, in doses of 2 c.c., it prevented infection in a mouse, while in doses of I c.c. it prolonged the duration of the disease, but did not prevent it. At the time of death, two months after inoculation with caderas, the serum was protective for a mouse in doses of I c.c. The serums of the other sheep and of the young goat towards the end of their infection, were similarly protective for the mouse, if mixed with infective blood, in doses of $I$ and $\frac{1}{2}$ c.c. respectively.

Attempts to obtain protective or curative serums by other methods have failed hitherto.

A young bull was inoculated by Voges for a year and a half with increasing doses of virulent blood, but at the end of that time the animal's serum had not acquired any curative property.

In other experiments of the same observer the virulence of the trypanosomes was diminished by formalin or by heat. First dead trypanosomes were injected, then trypanosomes of attenuated virulence. Either the trypanosomes remain alive and retain their virulence, or else they die and have no protective action whatever (Voges).

The action of human serum upon caderas is similar to that upon nagana. On injecting $\frac{1}{2}$ to I c.c. of human serum or $0^{*}$ I gramme of powdered serum dissolved in water subcutaneously into a mouse weighing about 20 grammes with a few or even a fair number of caderas trypanosomes in its blood, the trypanosomes disappear in twenty-four to thirty-six hours. The fewer parasites there are in the blood at the time the serum is injected, the more rapidly do they disappear from the blood. When they are very numerous the treatment is often of no avail, death occurring before the serum has time to act.

The trypanosomes disappear for six to eight days, after which they reappear as a rule, so that the treatment must be repeated. Only one out of ten mice treated with human serum was cured by a single injection of the serum.

By means of repeated injections life may be prolonged for a considerable time. In treated mice the average duration of the disease was fifty-seven days, whereas in untreated control mice it was only six to eight days. One mouse which was thus treated lived for II3 days. There arrives a time when the human serum no longer has any effect upon the parasites.

The mouse which recovered had not become immunized against 
the disease, for it became reinfected on injecting a fresh dose of virulent blood.

In rats infected with caderas human serum has the same effect as in mice. For a rat weighing I50 to 200 grammes the dose is 2 c.c. of serum, or $0^{\prime} 25$ to $0^{\circ} 3$ gramme of the powdered serum dissolved in water.

The mode of action of human serum upon the trypanosomes of caderas is the same as upon the trypanosomes of nagana (see p. I76).

By combining the action of human serum with that of arsenious acid, it is possible to prolong life still more: a rat thus treated survived inoculation with caderas for four and a half months.

As in the case of nagana and surra, this method of treatment cannot be used for large mammals.

The impotence of therapeutics makes prophylaxis all the more important. Unfortunately we are still ignorant of the agent which disseminates the disease, so that we cannot formulate with any precision the prophylactic measures to be adopted in preventing the spread of epizootics of caderas.

Caderas occurs chiefly in marshy districts and along watercourses where the capybara abounds. The indications are, therefore, to select dry areas for horse-breeding purposes and to destroy the capybara.

Veterinary surgeons should examine all sick horses in districts where caderas is known to be prevalent, for it is important to diagnose the disease promptly. Animals suffering from the disease should be slaughtered or isolated, and healthy animals should be kept in the stables. Voges recommends having wire-gauze netting fitted to the windows of stables, but we have seen that the rôle of biting flies in the propagation of caderas has not been proved, so that the value of this measure is rather doubtful. 


\section{CHAPTER X}

\section{DOURINE}

\section{(Fr. 'Mal du Coït'; Ger. 'Beschälkrankheit.')}

Pathogenic Agent: Trypanosoma equiperdum, Doflein, Igor.

\section{Section 1.--Historical Survey and Geographical Distribution.}

THIs trypanosome epizootic is also a disease of the Equidæ, but is peculiar in that it is transmitted only by coitus. Only horses used for breeding purposes are affected naturally by the disease; nevertheless, geldings and mules are very susceptible to experimental inoculation.

The disease was first recognised in Europe at the beginning of the nineteenth century. It is the only trypanosome disease known to occur in this part of the world. ${ }^{1}$ Its presence has been recorded throughout a large part of Europe-Spain, Germany, Switzerland, Austria-Hungary, Russia, and Turkey. In France it has made but short incursions in the Pyrenean departments, [but, according to Schneider and Buffard, ${ }^{2}$ dourine appears nearly every year on the Spanish frontier, in the department of Basses-Pyrénées.] As a result of the strict regulations of the sanitary authorities, which practically necessitate the slaughter or castration of every infected stallion, dourine has disappeared from most of the countries just enumerated. At the present time it occurs only in Spain (particularly in Navarre), and, to a less extent, in Hungary and South Russia; also in Turkey, which imports many horses from infected districts.

The disease also exists along the whole south littoral of the Mediterranean Sea, in Morocco, Algeria, ${ }^{3}$ Tunis, Tripoli, Syria, probably throughout Asia Minor, and in Persia.

[In India dourine has probably existed for a long time, but the nature of the disease was only recognised in 1902 by Pease, ${ }^{4}$ in the

1 These details are all taken from Nocard and Leclainche's work, 'Les Maladies microbiennes des animaux,' v. 2, Paris, Masson, I903, pp. 6r5, 616.

2 [Schneider and Buffard, Ann. Inst. Past., v. 19, 1905, p. 715.$]$

3 See Schneider and Buffard's 'La Prophylaxie de la Dourine,' Lyon, I9or. In the year 1902 alone, Billet and Marchal (in Schneider and Buffard, Rec. mid. vétérin., 1902, p. 723) saw sixteen cases of dourine at the remount depôt in Constantine.

4 [Pease, 'Note on Dourine,' also 'Further Note on Dourine,' published by Punjab Government, 1903 ; Vet. Journ., v. 9, 1904, pp. I87, I96 ; v. 10, p. 297 ; v. I2, I905, p. 209.] 
Punjab. It has since been studied in India by Lingard, ${ }^{1}$ Pease, Baldrey, ${ }^{2}$ and others. Baldrey states that the disease is almost certainly present in Beluchistan, in the Bombay Presidency, and in the United Provinces, in the Babugarth Government stud. Mott ${ }^{3}$ has made a careful and minute study of the microscopic changes in the nervous system of cases of chronic dourine which occurred in India.]

In the United States, where it appears to have been introduced recently, dourine continues to make great ravages. Thus, in his General Report for Igor, Salmon, head of the Bureau of Animal Industry, mentions twelve horses (two stallions and ten mares) which were slaughtered on account of dourine. The disease exists in the States of Nebraska, Wyoming, and South Dakota. In spite of all attempts at stamping out the disease, that end has not yet been attained, for dourine exists amongst the half-wild horses of the Indians in Rosebud and Pine Ridge.

'In Chile the disease exists in certain provinces, but was not recognised until recent years' (Monfallet).

It probably occurs in Java. In Igoo a true 'maladie du coït'disease due to coitus-was discovered in the Government studs at Soemedang, and the disease was studied by De Does in Weltevreden. ${ }^{4}$

The trypanosome of dourine appears to have been first seen in I 894 by Rouget in the blood of a sick horse in the remount depôt at Constantine. Rouget made numerous interesting experiments with this trypanosome upon various mammals, the results of which were published in December, I896..$^{5}$ Unfortunately, the virus died out without Rouget having been able to reproduce the disease experimentally in horses.

In I899 Schneider and Buffard, 6 also in Algeria, found a trypanosome in two horses suffering from dourine, and later in a donkey. They were more fortunate than Rouget, inasmuch as they succeeded in reproducing the disease in the horse with a virus which had been passed through the dog. Their results were confirmed by Nocard at Alfort, ${ }^{7}$ and since then the rôle of this trypanosome in the causation of dourine has been generally admitted. At the beginning of July, IgoI, Doflein ${ }^{8}$ gave the name $T$. equiperdum to this parasite.

1 [Lingard, Reports of the Imperial Bacteriologist, 1903-I904, etc. ; Centralb. f. Bakter., I, Orig., v. 37, p. 537.]

2 [Baldrey, Journ. Path. Therap., v. I8, 1905, pp. I-22.]

3 [Mott, Proc. Roy. Soc., Ser. B, v. 78, 1906, pp. I-12.]

4 De Does, 'Boosardige dekziekte in het Soemedangsche,' Veeartsenijkundige Bladen voor Nederl. Indië, v. I3, I 4, I900, I90I (quoted by Nocard and Leclainche, op. cit., v. 2, p. 584 .

5 Rouget, Ann. Inst. Past., v. Io, I896, p. 716.

6 Schneider and Buffard, 'Notes communicated to the Académie de Médecine on July 25, September I9, October 3, and November 21, I899, January, 1900. Archiv. Parasitologie, v. 3, 1900, p. 124. Complete paper in Rec. méd. vétér., I900, pp. 81-105, I 57-169, and 220-234.

7 Nocard, Bull. Acad. Méd., v. 64, meeting of July 31, 1900, pp. 154-163.

8 Doflein, Die Protozoen, etc., Jena, I9or, p, 66. 
A few days later we ${ }^{1}$ called it $T$. rougeti, but Doffein's name has the prior claim.

In 1902 Schneider and Buffard raised doubts as to the true nature of the disease in horses whence Rouget derived his trypanosome, and suggested that he was dealing not with dourine, but with nagana or surra. They came to the conclusion that other trypanosomiases than dourine probably existed in Algeria. That such is really the case has now been demonstrated by the researches of Szewzyck and Rennes and of the Sergents (see p. 2 Io et seq.).

It is highly probable that the observation made by Chauvrat in I892 was upon one of these trypanosomiases different from dourine. As to Rouget's case, we shall continue to look upon it as one of dourine, and later on we shall examine the validity of the arguments brought forward by Schneider and Buffard against the diagnosis of dourine, and of those adduced by Rouget in support of his diagnosis.

\section{Section 2.-Dourine in the Equidæ.}

We quote almost verbatim from the excellent work by Schneider and Buffard the account of the symptoms of the natural affection as it occurs in reproductive Equidæ.

HoRsE.-Dourine in horses may be acute or chronic, the former being much less common than the latter.

Chronic Dourine.-In the chronic form there are three stages, which as a rule are fairly well defined:

Stage I. Presence of Localized Edema.-The first signs of the disease in the stallion are visible from the eleventh to the twentieth day after coitus. The disease nearly always starts with a little œdema at the lower part of the sheath, which may be overlooked, especially if the veterinary officer or the head of the stables has not noted the condition of the sheath before the covering season.

This odema gradually extends along the lower part of the sheath to the scrotum and the inguinal region, and may even reach the abdominal walls. Usually the swelling is cold and painless, but sometimes it is hot and tender to the touch. The end of the penis becomes infiltrated, and the horse frequently gets slight erections. The superficial lymphatic glands in the groin are enlarged, and this enlargement is often unilateral when the initial œdema occurs only on one side.

In the mare the symptoms are less marked than in the stallion. They consist at first of a unilateral or bilateral swelling of the vulva, often extending up to the anus, a bright red colour of the vaginal mucous membrane, and a gradually increasing mucoid or viscid discharge.

The appetite always remains good; the temperature oscillates between $38^{\circ}$ and $385^{\circ} \mathrm{C}$. $\left[1004^{\circ}\right.$ and $\mathrm{IOI}^{\circ} 4^{\circ} \mathrm{F}$. $]$; coitus is still

${ }^{1}$ Laveran and Mesnil, C. R. Acad. Sciences, July I5, 190 I, p. I3r. 
possible, the horse easily getting an erection. Later on, about a month after the appearance of the first symptoms, the initial swellings partially subside and become localized to the genital organs. Sometimes the swelling disappears almost entirely, the end of the penis alone remaining infiltrated. At this time the kidneys are tender on pressure, and the animal almost gives way under the weight of a rider. The horse gets winded after a short trot and already shows signs of wasting.

Stage 2. Presence of Patchy Infiltrations of the Skin (plaques). -These constitute the only pathognomonic sign of the disease, and they usually appear forty to forty-five days, sometimes two months, after the infecting coitus. The plaques are 'salient and

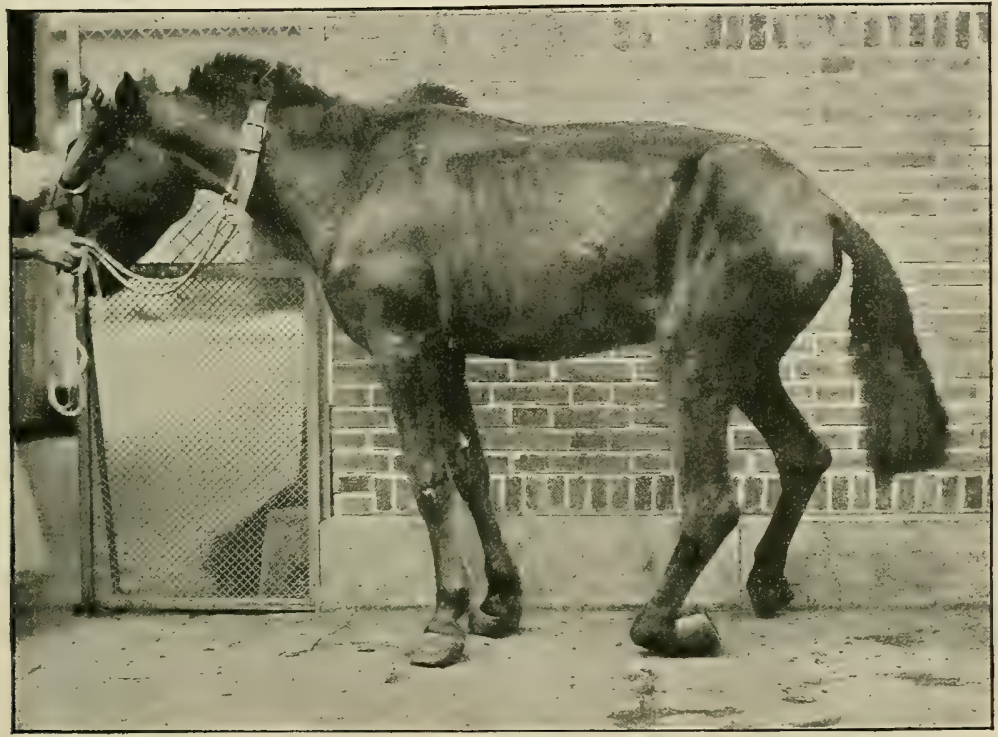

Fig. 42.-Horse suffering from Dourine at the End of the Second Stage.

Note the emaciation, the great feebleness of the hind quarters, and the flexion of the fetlock in left hind-leg. (From a photograph in Nocard's collection, lent by Vallée.)

rounded, and look as though a metal disc had been slipped under the skin' (Pease). They vary in size from a florin to the palm of the hand. The hair over them bristles and the skin is thickened. Sometimes, instead of these well-defined plaques, the skin is slightly swollen in those parts where the plaques usually occur. In some stallions they are at times odematous, and in such cases when they disappear a little fluid exudes, which causes the hair to become matted together. The duration of these patches is very variable: they may appear in the morning and be gone again the same night, or they may persist for five to eight days. They occur most commonly on the sides and hind-quarters, but sometimes upon the neck and shoulders, and on the thighs. 
At this period wasting is very marked (see Fig. 42). The animal constantly lies down, and can only get up with difficulty, as if its hind-limbs were paralyzed. When walking it drags its hind-feet along the ground, and when the weight of the body is supported by either hind-leg there is extreme flexion of the fetlock. Often the synovial sheaths of the joints and tendons of the hind-limbs are swollen. The inguinal glands become enormously enlarged and sometimes ulcerate. The glands of the chest and intermaxillary space are also congested and swollen. The appetite is good, and may even be enormous, the eye is often fixed and staring, and the temperature often rises to $39^{\circ} \mathrm{C}$. $\left[\mathrm{IO}_{2} 2^{\circ} \mathrm{F}\right.$.] in the evening, falling again in the morning to $385^{\circ} \mathrm{C}$. $\left[\operatorname{Ior}^{\circ} 4^{\circ} \mathrm{F}\right.$.]. Connection is practically impossible for stallions, while mares very generally abort.

Stage 3. Presence of Profound Anamia and Paraplegia.-At this stage the mucous surfaces are very pale, wasting is marked, the appetite becomes capricious, and so profound is the weakness that the sick animals cannot move from place to place without propping themselves up against a wall or other support. Often there occur superficial abscesses, which show little tendency to heal ; and sometimes there are eye troubles, such as conjunctivitis and ulcerative keratitis. Micturition is difficult and the urine is thick. The joints of the limbs and spine crepitate on the slightest movement, and fractures frequently occur. Towards the end there is complete paraplegia, and the animal falls down, never to rise again. Its sensibility to pain is so much diminished that the animal may be pricked or even cut without showing the least sign of pain. We shall see that these nervous symptoms are often associated with patches of softening in the spinal cord.

The duration of the disease is variable-usually it is from two to six months, exceptionally it lasts one or two years. Schneider and Buffard $^{1}$ record two cases of cure, but it is possible that these cases relapsed again later.

[Baldrey gives the duration as twelve to eighteen months; Lingard states that certain breeds of horses can retain the materies morbi of dourine in their system for one or four years, just as cattle and camels in India can tolerate the trypanosome of surra, or its 'developmental' forms, in their blood for one to three years. A stallion whose nervous system was studied by Mott died twentyseven and a half months after the infecting coitus.]

[According to Baldrey, dourine is fatal and incurable except in the first stage. Castration performed at this stage of the disease seems to benefit some cases, but if done in the second stage it is useless. Pease states that dourine is an old disease in India, and therefore less deadly than in Europe. He estimates that 70 to 80 per cent. of infected mares die of the disease in India.]

Acute Dourine.-Sometimes dourine runs an acute course, and,

1 Schne:der and Buffard, 'La Prophylaxie de la Dourine,' p. 4. 
after the initial œdema, a sudden acute paralysis or attacks of vertigo may carry off the animal in a few days. In the mare this acute form is more common than in the stallion, and usually paralysis occurs suddenly a few days after the appearance of the plaques.

Experimental Dourine.-Subcutaneous inoculation of the virus reproduces the natural disease in the horse and donkey, the incubation period varying from seven to twenty days, according to the number of trypanosomes injected. There is no difference between the experimentally produced disease and that occurring naturally. The signs and symptoms, as well as the course of the temperature, are the same in the two cases. Sometimes the temperature is very

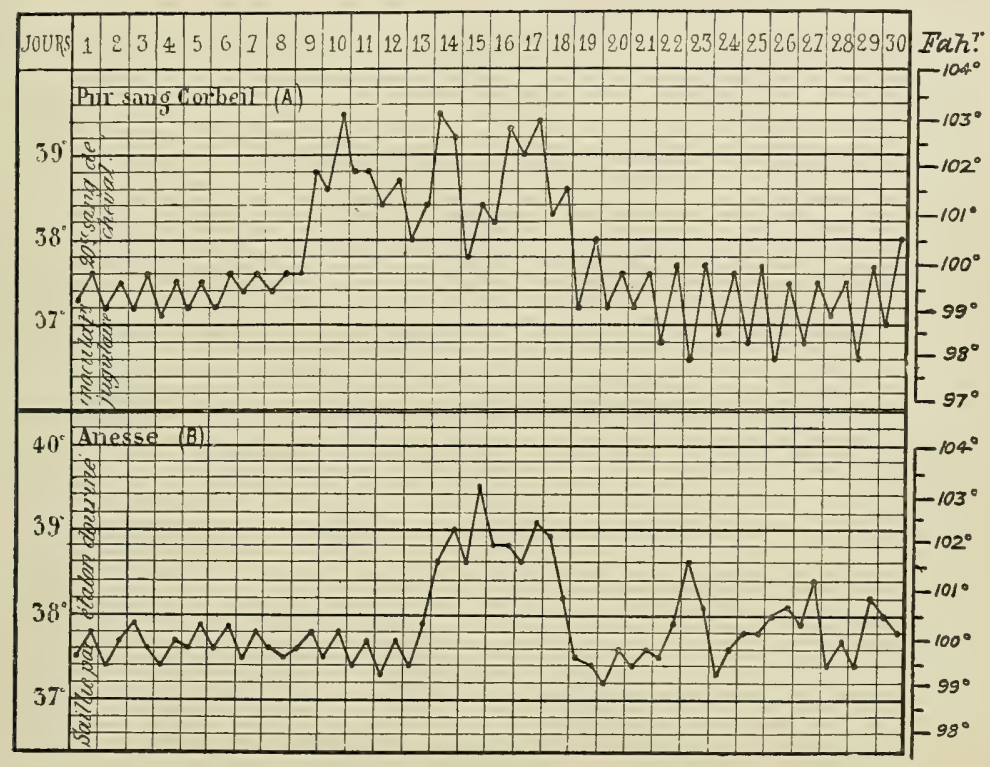

FIG. 43.

A. Temperature Chart of a Horse with Dourine during the First Month AFTER InOCUlation.

This animal, a thoroughbred stallion, was injected intravenously with 20 c.c. blood from a horse.

B. Chart of a Donkey ( $q$ ) During the First Month after the Infecting Coitus. This animal was covered by a diseased stallion.

(Nocard's charts, lent by Vallée.)

high, just as at the onset of nagana and surra. Nocard has recorded several well-marked cases of the kind. 'I have been able,' writes Nocard (Soc. Biologie, May 4, Igor), 'to kill strong healthy horses in four, six, and eight weeks, and the curve of their temperature was identical with that seen in surra and nagana.'

We reproduce (Fig. 43, A) a temperature chart of experimental dourine in which the temperature was above $39^{\circ} 5^{\circ} \mathrm{C}$. $\left[\mathrm{IO}^{\circ} 2^{\circ} \mathrm{F}\right.$.] on several days during the second week after inoculation. Cases of the natural disease have also been recorded in which the temperature rose to $40^{\circ} \mathrm{C}$. [ $\mathrm{IO}^{\circ} . \mathrm{F}$.] at the onset. 
Donkey.-The symptoms of dourine in the donkey, both male and female, are very indefinite, and as a rule a male donkey is recognised as being infected by the victims he makes amongst the females with which he has had connection. The only sign which is invariably present is œdema of the end of the penis; œedema of the sheath appears later, but cutaneous plaques are very rarely presentonly in 2 per cent. of the cases.

In less resistant male donkeys dourine runs a course similar to that in the horse, but such cases are rare. Under those conditions wasting is extreme, the sheath and scrotum become swollen, and finally paralysis supervenes. Some donkeys $(\delta)$ live for more than three years with dourine, and the Arabs state that some animals even recover from the disease, but we know of no authentic case of recovery from dourine.

The experimental disease in the donkey runs a clinical course similar to the natural disease in that animal (see Fig. 43, B).

Distribution of the Trypanosome in the Body.- ' It is difficult to find the parasite in naturally infected animals. It can be found in the blood taken from the region of the odematous swellings and plaques, but we have rarely seen it in the blood obtained from other vessels. The fluid which escapes immediately after puncturing the œdematous swellings or plaques appears not to contain the parasite, but if this fluid be tinged with blood the parasite may be found in it, and the more blood there is present the more numerous are the trypanosomes. Two conclusions may be drawn from these facts: first, that the trypanosome is really a blood parasite; and, secondly, that very probably the swellings and cutaneous plaques are due to embolism caused by masses of the parasites blocking the small bloodvessels.'

'The best time to find the trypanosome in the plaques is on the first appearance of the latter-it is then that the parasite should be looked for, and the success of the examination depends upon this condition being fulfilled. As the cutaneous lesion extends, the parasite becomes more difficult to find in preparations, and some hours after the appearance of the lesions the parasite can no longer be found. Apparently they persist for a longer time in the œdematous swellings. ... .

'At the outset of the disease we have constantly found the trypanosome present. At a later period, when the infiltrated areas in the skin have disappeared, the trypanosome seems to be less freely distributed throughout the body. Blood examined in fresh preparations shows only a few parasites, but this blood is infective on injection' (Schneider and Buffard).

[Lingard ${ }^{1}$ states that the infection does not become generalized - that is to say, the virus does not enter the circulation-until from thirty to forty days from the time of the primary infection. Some-

1 [Lingard, Centralb. f. Bakter., I, Orig., v. 37, p. 537.] 
times it may be as long as ten to twelve or more months before the blood infection occurs in stallions, especially if the swelling of the sheath be detected early and the animal be segregated, given rest and good food, and all connection stopped. When the trypanosome gets into the circulation, plaques may occur at any time after an interval of a few days, and may recur for long periods-more than a year. The blood is not infective on injection during the 'latent' stage of penile swelling. It appears, says Lingard, that the trypanosomes, or their developmental forms, remain shut off for the time being in the affected area.]

[Lingard is of opinion that the plaques are due to a circumscribed angio-neurotic œdema, occasioned by the advent of the trypanosome, or its developmental forms, in the papillary layer of the skin. $\mathrm{He}$ thinks that a toxin elaborated by the trypanosomes produces dilatation of the capillaries and a localized increased secretion of lymph around.]

[Lingard's view of the origin of the plaques is based upon the following observations: In blood drawn from a recent plaque, fully-formed, but small-sized, trypanosomes are present. These might have developed, says Lingard, from the amoboid or plasmodial form, after the latter had been deposited in the plaque. Later on, as long as the oedema persists, trypanosomes or developmental forms are present at some part of the odematous area, the mature trypanosomes diminishing in number-which may be due to the action of the toxin-and the developmental forms increasing. Eventually only developmental forms of the parasite remain in the plaque, and on the disappearance of the latter they return to the general circulation. Similar developmental forms were found in the fluid obtained from the secondary swelling arising at the seat of subcutaneous inoculation with infective blood. In a donkey inoculated subcutaneously with 20 c.c. horse blood, the first immature form was found on the nineteenth day, and the first mature trypanosome only on the thirty-ninth day.]

[Lingard has found that blood or sero-sanguineous fluid taken from a plaque, from which the mature trypanosomes have disappeared, is parasiticidal in vitro, giving rise to loss of motility and granular disintegration of the parasites.]

[Mott does, not agree with the view that the plaques are due to embolism by trypanosomes. He thinks that, by analogy with the origin of herpes zoster, an inflammatory irritation of the posterior spinal ganglia, as these become successively affected by the noxious agent, causes the eruption of the cutaneous plaques. It has, moreover, been shown experimentally by Bayliss that stimulation of the posterior roots produces vaso-dilatation. In the blood or odema fluid produced, as suggested by Mott, by the irritation of the spinal ganglia, the trypanosomes may find suitable conditions for multiplying by fission. According to Mott, therefore, the presence of many trypanosomes in the blood and fluid from a plaque would be the result rather than the cause of this lesion. The eruptions seen in human trypanosomiasis might be similarly accounted for by irritation of the neurotrophic centres in the spinal ganglia.] 
[Trypanosomes are present, though in very small numbers, in the general circulation. Schneider and Buffard ${ }^{1}$ state that, during an epidemic of dourine in 1904 in the department of BassesPyrénées, they were able to find the trypanosome in the peripheral blood of two mares out of four examined. ${ }^{2}$ The trypanosomes were very scanty, and were found only after a long and tedious examination. Marek, in Hungary, also succeeded, after a prolonged search, in finding the trypanosome in the blood of a sick stallion (quoted by Schneider and Buffard).]

[Lingard states that when the plaques are numerous and constantly changing, even small quantities of blood are infective on injection; but when the plaques are very scanty, it may be difficult to infect an animal even with large quantities of blood.]

[Trypanosomes or developmental forms can be found-after staining and careful examination-in the semen of infected stallions. Mares infected by coitus will in most cases sooner or later develop the trypanosome in the vaginal mucus, and it may be observed in the mucus at intervals during the subsequent course of the disease. Sometimes the trypanosome may be present for months in the vaginal mucus without the animal showing any signs of ill-health. Mares inoculated subcutaneously with infective blood-anywhere except in the external genitals-may develop plaques and cerebrospinal symptoms, but the vaginal mucus, when free from blood, may be non-infective in such cases (Lingard).]

[Baldrey also states that the trypanosomes may live in the vaginal mucous membrane and discharges of mares, and in the urethral and seminal fluids of stallions for an indefinite period.]

[In animals succumbing to an acute attack of the disease, accompanied by nervous symptoms, developmental forms of the trypanosome occur in the cerebro-spinal fluid (Lingard). In a very chronic case-lasting twenty-seven and a half months-studied by Mott numerous stained specimens of the fluid were examined for the $T$. equiperdum, but unsuccessfully.]

Pathological Anatomy.-At the autopsy the most definite and characteristic lesions are found in the lymphatic glands and in the spinal cord.

The lymphatic glands are much enlarged, congested, and softened. The first glands to be enlarged are those of the abdomen and hindquarters. Gradually the glands of the anterior part of the body become enlarged, and in chronic cases the lymphatic system throughout the body becomes affected. According to von Thanhoffer, the glands on section show dark grey patches, due to the remains of capillary hæmorrhages.

1 [Schneider and Buffard, Rev. gén. méd. vét., June, 1904; Ann. Inst. Past., v. I9, I905, p. 715. Abstract by Mesnil in B'ull. Inst. Past., v. 4, I905, p. I66.]

${ }_{2}$ [These four mares were suspected of dourine after being covered by a diseased stallion. Three of them died with the classical symptoms of the disease. The stallion had covered thirty-seven mares, but the remaining thirty-three did not become infected. The stallion is said to have recovered eventually.] 
The lesions in the spinal cord are most marked in the lumbar and sacral regions. The cord is in parts transformed into a reddish, diffluent pulp, the softened portions being sometimes as much as 6 to 8 centimetres ( 2 to 3 inches) in extent. No other trypanosome disease gives rise to such a condition, and even in dourine it is found only when the paralysis has been of long duration. On histological examination, Marek, ${ }^{1}$ a Hungarian observer, found degeneration of the fibres of the posterior columns, the other parts of the cord (the grey matter and the rest of the white matter) being healthy. Some fibres, particularly the sensory, of the roots of the spinal nerves are also degenerated. The nerves of the hind-limbs also show degeneration of some of the fibres, those of the fore-limbs being less affected. Owing to these anatomical changes which occur in dourine, Marek calls it polyneuritis infectiosa equorum.

[In an Arab stallion which suffered from chronic dourine and during the course of its illness exhibited ${ }_{5} 6$ cutaneous plaques, together with marked symptoms of paraplegia, Mott ${ }^{2}$ found widespread changes in the spinal cord, posterior spinal ganglia, and nerve roots. The notes of this case sent by Lingard to Mott are as follows: The infective coitus occurred on May 4 to 6, I903; partial paraplegia appeared on February 25, I904; death on August I5, 1905, twenty-seven and a half months after infection. Post-mortem a considerable quantity of gelatinous exudation was found round the lumbar portion of the spinal cord, and a smaller amount around the cervical enlargement, and a certain quantity of cerebro-spinal fluid escaped from within the membranes on removal.]

[The following histological changes were observed by Mott: Intense chronic inflammation of the posterior spinal ganglia-most severe in the lower dorsal and lumbo-sacral regions, but fairly marked also in the upper regions of the cord. Where the inflammation was most intense the ganglion cells were most affected-in some cases being completely destroyed, and their place occupied by inflammatory products. Associated with this extensive neuronic destruction in the lumbar region, there was extensive posterior root destruction and system-degenerative sclerosis of the posterior columns. This lesion, which resembles that of tabes dorsalis, and the comparatively normal appearance of the anterior roots, 'would suggest that this animal suffered with a sensory paralysis of the hind-limbs analogous to tabes dorsalis, rather than a polyneuritis' (Mott).]

[Throughout the grey matter of the spinal cord the ganglion cells showed marked chromolytic changes, and the vessels exhibited

${ }^{1}$ [Marek, Zeitschr.f. Tiermedizin, I900, p. 401 ; 1904, p. 13.]

2 [Mott, Proc. Roy. Soc., Ser. B, v. 78, I905, p. I; Brit. Med. Journ., I906, ii., pp. 300, I775-1777); also Centralb. f. Bakter., I, Ref., v. 39, I906, pp. I-9. In the last two papers Mott states that he has found lesions similar to those described in the text in four other cases of equine dourine, the tissues of which were sent to him by Lingard.] 
evidence of chronic inflammation, with scattered capillary hæmorrhages. The changes were most marked in the lumbo-sacral region, infiltration and thickening of the connective tissue septa, infiltration of the nerve roots and of the vessel walls with lymphocytes and small round cells. Mott states that the condition simulated an acute syphilitic meningitis in many ways, except that in this case there was only occasional evidence of an obliterative arteritis.]

[There was also evidence of a general irritation of the central nervous system, manifested by a subpial and septal proliferation of the neuroglia, a chronic interstitial inflammatory change which, in the spinal cord, was not limited to the posterior columns. The membranes at the base of the brain also seemed thickened, and sections of the peduncles and interpeduncular structures showed a subpial and septal neuroglia proliferation similar to that seen in the lumbar region.]

[These changes in the nervous system in dourine are like those seen in chronic cases of infection with $T$. gambiense-sleeping sickness-and appear to be due to the presence of an irritative agent in the lymphatic system. In dourine this starts "in one seat of primary infection, extends to the inguinal glands, thence presumably by the pelvic lymphatics to the lumbo-sacral plexus and the posterior lumbo-sacral roots to the central nervous system; consequently the lower part of the spinal cord-and especially the posterior columnis first and most affected. In the case of sleeping sickness there may be any number of seats of infection, but the cervical glands are nearly always markedly involved ' (Mott).]

The other lesions are less characteristic and less importantgelatinous exudations under the skin, serous effusions into the pleural and peritoneal cavities, wasting, and pallor of the muscles, which show areas of fatty degeneration and atrophy of the fibres.

[Morax ${ }^{1}$ has studied the eye changes in dogs and goats infected with dourine, and in goats infected with nagana. The most characteristic lesion - which when it occurs in animals is very suggestive of trypanosomiasisis an interstitial keratitis. It is caused by the multiplication of the parasites in the interlamellar spaces of the cornea. The multiplication of the trypanosomes is followed by a leucocytic infiltration, and later on by a formation of new bloodvessels. These changes may lead to complete disorganization of the cornea, or they may disappear and leave only slight traces, especially in resistant animals, such as goats.]

\section{Section 3.-Experimental Dourine in Dogs, Rabbits, Rats, and Mice. Refractory Animals.}

The dog, rabbit, and, in certain cases, the rat and mouse, aie susceptible to the trypanosome of dourine. [It has been shown that buffaloes (Pease) and other bovines, as well as monkeys (Mesnil and Rouget), are likewise susceptible to $T$, equiperdum.]

1 [V. Morax, Ann. Inst. Past., v. 21, 1907, pp. 47-6r.] 
The infection may be transmitted by all the ordinary methods of inoculation. It progresses more rapidly after intraperitoneal or intravenous injection, while intracerebral or intraocular inoculation hastens the appearance of nervous symptoms. Subcutaneous inoculation is best for studying the form of the disease most like the natural.

Rouget was the first to recognise that, contrary to what obtains in the case of the other pathogenic trypanosomes, 'solution of continuity of the integuments is not indispensable, for the trypanosome can pass through healthy mucous membranes. A drop of blood, rich in parasites, placed in the lower conjunctival sac of a rabbit, is sufficient to infect the animal. We have seen a case of probable infection by the vagina. A male rabbit recently infected was intentionally placed in a cage with a healthy female, which contracted the disease.'

This experiment has been repeated many times by Schneider and Buffard. In their paper (pp. 226-228) they mention the following instances: (I) Two dogs became infected after having had connection with a bitch which was experimentally inoculated by introducing into the vagina some blood from a plaque in a diseased stallion; (2) a rabbit ( $q$ ) contracted dourine after coitus with a rabbit experimentally infected by subcutaneous inoculation; (3) a rabbit ( $\delta$ ) became infected after covering another suffering from dourine. A bitch or a rabbit may be infected by carefully placing on the vulva some drops of blood or œdema fluid containing trypanosomes.

[It has already been mentioned (p. 219) that the Sergents infected four animals out of eight, by way of intact mucous membranes -conjunctival and genital-and that six similar experiments with debab were all negative. In this way the Sergents were able to differentiate dourine from other Algerian trypanosomiases.]

'The absorption by the digestive tract,' says Rouget, 'of different substances rich in parasites has never been followed by infection.'

Naturally, the material inoculated will not give rise to dourine unless it contains the specific trypanosome. It is important to bear this in mind when dealing with blood which, as we have seen, always contains few parasites. It explains the contradictory results obtained by the older investigators. It is often necessary to inject 5 , IO, I5, or 20 c.c. of blood to get a positive result.

Nocard found in 1892 that the softened parts of the spinal cord were also infective.

The dog being very susceptible to dourine, the injection into that animal of blood-stained œedema fluid, or, failing that, of blood, affords a valuable means of diagnosis in doubtful cases-as, for example, in donkeys-but it should always be remembered that when one has to inject blood, from ro to 20 c.c. should be inoculated. [But the diagnosis of dourine, even by animal inoculation, is often difficult. Thus Schneider and Buffard's stallion, which served thirty-seven mares and infected four of them (see p. 320, foot- 
note 2), never showed trypanosomes, and its blood was not infective on injection into dogs and rabbits.]

Dogs.- The dog never suffers from the disease naturally, but is very susceptible to experimental inoculation with virulent material from infected Equidæ.

In 1892 Nocard ${ }^{1}$ showed that the dog contracts a disease closely resembling dourine in horses, when inoculated in the anterior chamber of the eye with some softened nerve substance from the spinal cord of a diseased horse.

Rouget (loc. cit.) also studied the disease experimentally in the dog. He drew particular attention to the eye lesions (exophthalmos, keratitis, followed by staphyloma, and hypopyon), to the very marked motor troubles, and to the pronounced œedema of the external genital organs.

Schneider and Buffard gave dourine to the dog by subcutaneous inoculation with the blood or fresh spinal cord of infected animals.

Six to eight days after inoculation the animals have a temperature of $39^{\circ}$ or $39^{\circ} 5^{\circ} \mathrm{C}$. [102. $2^{\circ}$ or $103^{\circ} 2^{\circ} \mathrm{F}$.]. From twelve to twenty days after inoculation, there is extensive œdema of the abdominal wall, usually around the site of inoculation, and also swelling of the genital organs-acute balanitis in the male, acute inflammation of the genitourinary mucous membrane, with an abundant vaginal discharge in the female. There is continued fever $\left(39^{\circ}\right.$ to $39^{\circ} 5^{\circ}$ C. $)$ and the appetite is good, although the animal appears restless and ill. The gait is hesitating; the kidneys are pushed upwards, and are very tender on pressure. The swelling of the genital organs alone persists.

When the disease has established itself, the signs and symptoms are : wasting, in spite of a good appetite; trouble with locomotion, especially with the hind-limbs; localized odemas; and cutaneous plaques. The latter, which are only visible in animals whose hair has been cut short, resemble the plaques seen in horses. If they are punctured soon after they appear, the blood which escapes contains many trypanosomes. Other symptoms are arthritis, with effusion into the joints, and various eye troubles, such as opacity of the cornea and lens, purulent conjunctivitis, and ulcerative keratitis, with hypopyon. Fever is always continued, the temperature being $39^{\circ}$ to $40^{\circ} \mathrm{C}$. $\left[102 \cdot 2^{\circ}\right.$ to $104^{\circ} \mathrm{F}$.]. In very resistant animals the temperature falls to between $38^{\circ} 3^{\circ}$ and $38^{\circ} 9^{\circ} \mathrm{C}$. [ IOI $^{\circ}$ and $102^{\circ} \mathrm{F}$.] at the end of three to four weeks.

There are always periods of improvement, but these remissions are merely temporary, the disease nearly always terminating fatally. Nevertheless Nocard has seen dogs recover after having been extremely ill.

This stage of the disease lasts about a month. Sometimes death occurs rather rapidly after an attack of paralysis. More often 1 Nocard, C. R. Acad. Sciences, v. I I 4, I 892, p. I 88. 
emaciation becomes extreme, so that the animal is a mere skeleton, and finally it refuses food altogether. On the slightest exertion it becomes dyspnœic. Death occurs suddenly, probably from syncope.

$\left[\right.$ Pease $^{1}$ states that, according to Schneider and Buffard, certain breeds of dogs easily contract dourine by subcutaneous inoculation and by coitus, while other breeds are very resistant. Pease found the same thing with pariah dogs in Lahore. Of twelve dogs and bitches inoculated in various ways, only one showed any symptoms whatever, although control animals-other breeds of dogs-were readily affected. These pariah dogs, which were refractory to dourine, as well as the one which showed a slight local infection and then completely recovered, were highly susceptible to surra.]

[Thomas and Breinl ${ }^{2}$ have experimented upon several puppies and one adult dog. The incubation period in the former was from four and a half to eleven days (average seven days), in the latter thirteen days. The total duration of the disease was twenty-two to sixty-four days (average five to six weeks) in the puppies; the adult dog lost flesh and became very anæmic, but recovered in two and a half months. Four months later it was killed, and a pup inoculated with its blood developed the disease. In the puppies the chief symptoms were: profound anæmia, loss of weight, œedema of the genital organs, hind-limbs, and abdomen, purulent discharge from the eyes and nose, and in one case partial paralysis of the hind-limbs. Trypanosomes were at first very scanty in the blood, but increased from about the third week onwards; many dividing forms were then also seen in the blood.]

[Post-mortem the spleen and lymphatic glanos were moderately enlarged; there was often much fluid in the serous cavities, and small petechial hæmorrhages on the surface of the lungs. The serous effusions and odematous patches contained many trypanosomes. There was frequently considerable œedema around the lumbar region of the cord.]

RABBITS.-Rouget clearly recognised the susceptibility of the rabbit to the trypanosome of dourine.

' Fever is irregular and does not, as a rule, appear during the first few days after inoculation. The temperature fluctuates between $39^{\circ} 5^{\circ}$ and $40^{\circ} \mathrm{C}$. $\left[\mathrm{IO}_{3} 2^{\circ}\right.$ and $104^{\circ} \mathrm{F}$.], without falling much in the morning. Then the temperature becomes normal, but from time to time there are sudden rises which cannot be accounted for on examining the animal.

' One of the earliest signs is localized or general œedema of the ears, which hang down, are hot, and pit on pressure. The dilated vessels are seen to be gorged with blood. The fluid which exudes on incision contains the parasite, often in large numbers. The swelling persists for one or more weeks, then further changes take place: the veins become thrombosed, the skin becomes dry and scaly, the hair falls out, and on two occasions we have seen sloughs

1 [Pease, Vet. Journ., v. 9, 1904, p. 187.]

2 [Thomas and Breinl, Thompson Yates and Johnston Lab. Reports, v. 6, part 2. I905, p. 32. Mesnil remarks (Bull. Inst. Past., v. 4, p. I24) that their virus, of which they do not give the source, was of the Schneider-Buffard type.] 
the size of a shilling, which, on removal, left a perforation of the cartilage. ...

' Later on the limbs become swollen and ulcerated, the claws are long and brittle, the skin is covered with scabs, and the hair falls out. At the same time there is weakness of the hind-limbs, which may go on to complete paraplegia and involve the sphincters. The general condition of the animal rapidly gets worse, and in spite of a good appetite, wasting continues, so that the animal often loses more than a third of its original weight.

'There is a muco-purulent conjunctivitis, the parasite being found in the exudate. The eyelids are swollen and irritated by the pus which adheres to them. We have not observed any well-defined lesions of the eyeball, such as are seen in the mouse and dog.

'In some animals there is a discharge from the nose, and the nostrils are covered with thick, adherent crusts, beneath which the tissues are destroyed and the bones exposed.

'The external genitalia are always affected. In the female the vulva and anus are swollen, the congested mucous membrane bleeds easily, and sometimes shows one or two indolent ulcers. In the male there are odema of the sheath and paraphimosis, and the end of the penis thus exposed may necrose. We have seen three cases in which the skin of the scrotum sloughed and exposed the testicle.

'In the rabbit the disease lasts from one to three or four months, varying with the age and weight of the animal. Death occurred in all the animals (twenty-five in number) inoculated by us.'

As in the Equidæ and in dogs, the parasites are comparatively scanty in the blood, and are present irregularly and intermittently; but, says Rouget, 'we have not been able to establish any relation between the febrile paroxysms observed and the presence of the parasite in the blood. In order to discover in which parts of the body the trypanosome may be found, we sacrificed several rabbits which were obviously infected, but whose blood failed to show the parasite on microscopical examination. We found them in the spleen, in the ocular media, on the mucous surfaces, and in the plaques, but never in the marrow of the bones.'

We have ourselves inoculated two rabbits with the oedema fluid of an infected dog, one in the vulva and the other subcutaneously. The former quickly showed the train of symptoms so well described by Rouget, and died in two and a half months. The latter showed no obvious signs until after two and a half months, and died nine months after the inoculation, having almost completely recovered from the cutaneous and ocular lesions. At the seventh month after inoculation its lesions so closely resembled those of two rabbits with nagana which we had under observation at the same time that they were scarcely distinguishable from them; but in the two rabbits with nagana the lesions had appeared much more quickly, and death rapidly supervened. 
'At the autopsy,' says Rouget, 'in addition to the lesions already described, one finds enlargement of the lymphatic glands, exudation into the peritoneal cavity, and congestion of the liver and spleen. The remaining organs appear healthy. The parasite is found everywhere-in the various fluids, in the viscera, in the glands (testicles), and on the mucous surfaces (urethra).

' Rabbits from which the spleen had been removed were inoculated after they had recovered from the operation, and the disease ran the same course as in control animals.'

[Thomas and Breinl have recorded similar results in the rabbits inoculated by them. The incubation period after subcutaneous injection was six to eleven days, and death occurred, as a rule, in twenty-four to Io8 days. Some animals were still alive six months after inoculation.]

RATS AND MICE.-The observations of Rouget on the one hand, and of Schneider and Buffard on the other, differ materially as to the susceptibility of rats and mice. This has led to a good deal of discussion, in which these scientists have taken a prominent part.

Let us consider first the facts as stated by Rouget: 'On subcutaneous injection of small doses of the virus ( $\frac{1}{10}$ c.c. of a mixture of broth and infective blood, in such a proportion that one drop of the mixture shows one or two parasites in each field of the microscope), trypanosomes can be found in the blood taken from the tip of the tail at the end of the third day, and on three occasions they were found in twenty-four hours. After intraperitoneal injection the parasite may be found in the blood after thirty-six or forty-eight hours. The injection of large doses of the virus shortens the incubation period. The parasites increase rapidly, and they go on multiplying until death, which occurs from the fifth to the eleventh day after inoculation. At death the trypanosomes are more numerous than the red corpuscles.

'The mice do not appear ill until just a few hours before death, when they sit quite still and huddled up, the eyes closed, and the hair dry and bristling. They are insensitive to external stimuli, and the corneæ become white and opaque, either partially or completely.

'At the autopsy there is sometimes a blood-stained exudation in the peritoneal cavity, but the most noticeable lesions are hyperæmia of the abdominal parietes and enlargement of the liver and spleenthe latter weighing as much as 2 grammes. The spleen is glistening, swollen, and of a pink colour; the liver is markedly congested, and the bladder is distended with urine. The other organs look normal, and the lungs appear quite healthy. The lymphatic glands near the site of inoculation are enlarged. The parasite is found in the substance of all the internal organs, in the ocular media, and in the testicles, but not in the urine or in the contents of the alimentary canal,

' Grey mice and white rats react in the same manner, but the disease lasts a little longer in the latter (fifteen days). 
'Sewer rats present certain peculiarities. Some are susceptible, others are quite refractory; in others, again, the parasite multiplies in the blood for a time - as can be ascertained by systematic examination of the blood-then permanently disappears, the animal remaining in good health. Of thirty rats caught in the drains of the military hospital at Constantine, seven died of the infection, nine were quite refractory, and fourteen were only slightly susceptible.'

Nocard, who experimented with the trypanosome of Schneider and Buffard, says (loc. cit., p. I62) : 'I have never succeeded in killing a single mouse. At first my mice showed a few parasites in the blood of the tail six to eight days after inoculation, but now, after more than twenty-eight passages through the dog, not only do the mice not die, but they appear absolutely refractory.' Later ${ }^{1}$ he says: 'As regards the rat, which at first appeared as refractory as the mouse, intracerebral inoculation killed a young rat about six weeks old, and during the last few days of its life trypanosomes in appreciable numbers were found in its blood. Since that time the parasite, which in this way had become virulent for the rat, killed-in from six to fifteen days-all the white rats inoculated, even subcutaneously, with a trace of infective blood.' Nocard told us that he lost this virulent strain of the trypanosome during the vacation of Igor, and could not succeed in getting another.

Schneider and Buffard never succeeded in infecting either rats or mice with their trypanosomes, ${ }^{2}$ and they state that Billet and Marchal, in Constantine, similarly failed to infect grey mice. ${ }^{3}$

This difference in their experimental results led Schneider and Buffard to suppose that Rouget's horse was not suffering from dourine. ${ }^{4}$

Rouget ${ }^{5}$ hastened to reply to the criticism, and mentioned the fact that he had inoculated all his rats and mice with a virus which had been passed through the rabbit, which neither Schneider and Buffard nor Nocard had done.

Working on those lines, Nocard tried again to infect mice, and we quote the following remarks upon the subject, which he wrote to us in June, I903:

1 Nocard, C. R. Soc. Biol., May 4, I9or, pp. 464-466.

2 Schneider and Buffard, Rec. méd. vétér., December I 5, 1902, pp. 72I-727.

${ }^{3}$ Dr. Billet has recently confirmed this statement in a letter to us. None of the strains of trypanosome which he found was infective for the mouse.

4 In so far as the diagnosis is concerned, we may remark that it had been made with certainty in 1894 by the veterinary officer Busy. It was only in I 899 that Busy, doubtless owing to the discovery of other trypanosome diseases, expressed slight doubts about his earlier diagnosis in a report which Schneider and Buffard have made public. On reading the two successive reports of Busy (in Schneider and Buffard, loc. cit., pp. 726, 727), one finds that the horse was ill at least three and a half months before death, that it had œdema of the sheath and scrotum, swelling of the mucous membrane of the urethra and of the glans penis; "well-defined, rounded, prominent plaques' on the flanks and hind-quarters (first report; in the second report Busy mentions only slight staring of the coat), and no fever. All these symptoms would point to dourine rather than to mal de li \%usfanu (see p. 2 II), in which there are no odematous swellings.

${ }^{5}$ Rouget, Kec. méd. vétér., February 15, 1903, pp. 81·90. 
'I inoculated three mice with blood from a rabbit with dourine, and on the sixth day two of the mice showed a few trypanosomes in their blood. One of these animals recovered, but the other became very ill, so that on the fourteenth day, seeing that it was dying, I killed it in order to collect some blood. It contained the trypanosome in enormous numbers.

'I had on several previous occasions injected the blood of this mouse into six other mice, without any of them showing the parasite. The day the animal was killed I reinoculated them all, and in addition inoculated four fresh mice. Three days later I found a few trypanosomes in the blood of three of the newly-inoculated mice and of one of those reinoculated, but instead of increasing in number the parasites rapidly disappeared!

'Since then I have inoculated twelve fresh mice-both intraperitoneally and subcutaneously - with large doses (as much as 2 c.c.) of blood taken from rabbits dying of dourine, but without result! This blood was nevertheless infective, for it gave the disease to rabbits and dogs.

' I have, therefore, been the victim of a particular instance of individual susceptibility, and Rouget's hypothesis still remains to be proved.'

In their recent review of the trypanosomes, ${ }^{1}$ Rabinowitsch and Kempner state that, starting with a trypanosome of dourine (obtained from Nocard, who got it from Schneider and Buffard), which was not at all virulent for rats or mice, after more than ten passages they succeeded in infecting white rats. They do not give any other details.

Finally, Rouget has recently published the results of further experiments upon rats and mice with a trypanosome from a stallion in the remount depôt at Blidah, which confirm in every detail the earlier results he obtained in $1896 .^{2}$

To summarize, then, we see that the trypanosomes of Rouget killed all white and grey mice, all white rats, and a certain percentage of sewer rats; that the trypanosome of Schneider and Buffard, in Nocard's hands, killed a series of rats and one mouse, and gave a mild and temporary infection to other mice, but finally was without effect upon numerous rats and mice; while in the hands of Rabinowitsch and Kempner this same trypanosome was rendered virulent for the rat.

As a result, we think that Rouget was dealing with the trypanosome of dourine ${ }^{3}$ and, so far as the susceptibility of rats and mice is

1 Rabinowitsch and Kempner, Centralb. f. Bakter., I, Orig., v. 34, Igo3, p. 8I 5.

2 Rouget, C. R. Soc. Biol., v. 56, May 7, I904, p. 744. According to Rouget, there can be no doubt about the diagnosis of dourine in this Blidah stallion, for it was proved experimentally. A gelding was inoculated with the blood of the stallion, and the progress of the disease thus transmitted was followed step by step by the veterinary officer Chenot. later.]

${ }^{3}$ [This has been confirmed by Mesnil and Rouget's experiments, described 
concerned, we are of opinion that it varies with the strain of trypanosome (for there may be several varieties of dourine), with the species of animals through which the parasite has been passed, and with the rats and mice used.

[Lignières ${ }^{1}$ has made some observations upon rats with the SchneiderBuffard trypanosome of dourine. At first the rats died in about two months, but after a series of passages they died, with some exceptions, in five to six days. This confirms the results of Rabinowitsch and Kempner with a virus obtained from the same source.]

[Mesnil states that this virus, after passage through rats-given him by Rabinowitsch-kills mice in about six days. Lignières' exalted virus killed all the horses and dogs experimented upon; rabbits, however, recovered after a year.]

[Thomas and Breinl inoculated twenty-three rats with large numbers of the parasite; only two showed trypanosomes in their blood, and these rats died in eleven and eighteen days.]

[As in caderas and, to a less extent, in nagana, mice injected with the blood of another mouse undergoing treatment with trypanred become infected after a very long incubation period (Halberstaedter). Thus in one experiment it was found that the incubation period was four weeks, but the course of the infection was not otherwise altered, and the mouse died two days later.]

According to Nocard (Biologie, I9or, p. 465), 'all species of ruminants appear to be absolutely refractory to dourine, as do also the macaque monkeys.' [Subsequent observations have shown, however, that this is not the case. Pease ${ }^{2}$ inoculated two buffaloes with virulent blood containing the trypanosome of Indian dourine, and in both cases observed local swelling and multiplication of the parasite at the site of inoculation. One buffalo was reinoculated three months after the first injection, and trypanosomes were again found at the site of inoculation. A third injection, of Ioo c.c. blood, given a month after the second, produced no effect in three months, so that the buffalo had probably become immunized. The animal was then injected with surra, and $T$. evansi was present in its blood on the twelfth day.]

[Pease also inoculated a sheep and a goat with dourine, but unsuccessfully. Thomas and Breinl likewise failed to infect a goat with this trypanosome.]

[Mesnil and Rouget, ${ }^{3}$ having obtained an exalted virus, repeated Nocard's experiments upon ruminants and monkeys, and found both these species susceptible.]

[A goat was injected subcutaneously with the blood of a mouse infected with Rouget's (1904) trypanosome. On the eighth day and at intervals during the first two months after inoculation a few trypanosomes were seen in blood-films. The goat's blood was still infective (on injection)

1 [Lignières, Report to Pathological Section of Eighth Congress of Veterinury Medicine, Budapest, September, I905; abstract by Mesnil, Bull. Inst. Parst., v. 3, p. 946.]

2 [Pease, Vet. Journ., y. 10, 1904, p. 297.]

3 .'Mesnil and Rouget, Ann. Inst. Past., v. 20, 1905, p. 689]. 
sixteen months after the animal was inoculated. Nineteen months after inoculation the goat was still alive, but then became blind fairly rapidly, and a fatal termination seemed probable. As sometimes happens in equine dourine, there were periods of temporary improvement simulating recovery. One of the mice inoculated with the blood of this goat died in sixty-two and a half days, after passing through a stage of apparent cure. Four dogs out of five inoculated developed blindness.]

[A Breton cow, inoculated at Alfort by Vallée with the same virus, also became infected, the infection lasting several months. The cow became immunized, for a reinoculation made six months after the first failed to reinfect the animal.]

[A young goat ( $\delta$ ) was inoculated with the original Schneider-Buffard virus made virulent for rats by Rabinowitsch. A very mild infection followed, which lasted about six months. The goat recovered and acquired immunity, for a fresh inoculation with the same virus produced no effect. Three months after the second inoculation the goat was injected with the Rouget virus, and was found to be immune against this, as well as against the original Schneider-Buffard trypanosome. This experiment proves the identity of the parasites found in horses having the symptoms of dourine.]

[The monkey inoculated by Mesnil and Rouget was a large Macacus cynomolgus. It was injected with the Rouget virus, and had a severe infection lasting three to four months, with rises of temperature - to $40^{\circ} \mathrm{C}$. ( $\mathrm{IO}^{\circ} \mathrm{F}$.) or above on several occasions, generally coinciding with exacerbations in the numbers of the parasite. The animal died five months after inoculation, but it had apparently recovered from its trypanosome infection.]

In 1896 Rouget stated that 'guinea-pigs were refractory at all ages and under any conditions,' but with a trypanosome derived from another source in I9O4 all his guinea-pigs became infected (personal communication).

[Rabinowitsch and Kempner succeeded in raising the virulence of the trypanosome of Schneider and Buffard, by passage through white rats, so that it became pathogenic for rats and also for guineapigs. Thomas and Breinl inoculated four guinea-pigs and a cat, but with negative results.]

ReFractory Animals. ${ }^{1}$ - 'Birds-fowls, pigeons, and sparrows - and bats are refactory, no matter how many trypanosomes be injected, or what mode of inoculation be employed. Fowls and pigeons, cooled in the ordinary way, were found to be absolutely immune ....'

'Cold-blooded animals - snakes, lizards, frogs-are insusceptible. Frogs placed in the incubator at $37^{\circ} \mathrm{C}$. were inoculated on several occasions in the dorsal lymph sac; but the parasites never multiplied, and no trace of them could be found after thirty-six hours' (Rouget).

1 [Bergeret and Bonin (Lyon médical, v. I04, 1904, p. 622) record a case of dourine in a man who is supposed to have contracted the disease through sexual intercourse with a mare. On reading their account of the case it.is seen that there is no proof that the man really had dourine; the symptoms were urethral discharge, enlarged glands, and general weakness. Trypanosomes were never looked for in the man, nor were they found in the mare.] 


\section{Section 4.-The Trypanosoma equiperdum.}

We certainly have less information concerning the morphology and biology of the trypanosome of dourine than we have of any of the other well-known pathogenic trypanosomes.

Rouget's report of 1896 contains several figures giving a good general idea of the parasite as it appears in the living state; but at that time the methods of staining were insufficient to bring out the distinctive points in the morphology of these parasites.

In their papers published in Igoo Schneider and Buffard give a large number of figures of the trypanosome, accompanied by a long description, but both figures and text are lacking in precision. These authors certainly saw small agglutination rosettes, and possibly, too, forms dividing longitudinally. Since then Schneider and Buffard have not again referred to the morphology of their trypanosome. In their ' Treatise on Micro-biological Technique,' published in I902, Nicolle and Remlinger give, on p. 897, some of Schneider's figures, which are more detailed copies of some of the drawings in his paper of I900. These figures show five stages in the longitudinal division of the parasite, closely resembling those we had given a year previously for the trypanosome of nagana.

Thanks to Nocard and Schneider, we were able, in the year Igoo, to study the trypanosome of dourine in the blood-stained oedema fluid of the horse and dog, and have, on two occasions, given an account of the morphology of this parasite (Biologie, November I 7 , I90o, and March 23, IgoI), showing, among other things, that its mode of longitudinal division is identical with that of $T$. brucei, and that it differs from the latter trypanosome in several details. Fig. 44 is drawn from our preparations made at that time.

In their paper of October, I903, Rabinowitsch and Kempner give four coloured figures of $T$. equiperdum, but none of them shows any dividing forms. Since Igoo we have had the opportunity of studying this trypanosome in the blood of Nocard's mouse (see p. 329), and of other mice obtained from Rouget.

In ordinary fresh preparations the trypanosome of dourine resembles the other pathogenic trypanosomes. Like them, it scarcely moves except in loco, but it is quite easy to make out that it can move from place to place, the flagellum being usually, but by no means always, foremost.

In films made from the blood-stained odema fluid of the dog or horse, and stained by our ordinary method, the parasite has the appearance given in Fig. 44. The centrosome is always very distinct, and is exactly the same as in $T$. brucei and $T$. evansi, while the undulating membrane is almost as much folded as in those trypanosomes. The nucleus is centrally situated, and the free flagellum is about as long as in $T$. brucei. The posterior extremity may vary considerably in form, and sometimes it seems to present two points, 
as seen in Fig. 44, 2, 3. This part of the parasite is undoubtedly very contractile, but apparently a little less so along the edges than in the middle. ${ }^{1}$

The protoplasm stains fairly uniformly, but perhaps a little less deeply than in the case of the other pathogenic trypanosomes. In this respect $T$. equiperdum is rather like $T$. lewisi. The protoplasmic granules seen in $T$. brucei and others are never seen in this trypanosome, which is an important distinguishing feature, for it appears to be a constant one. Schneider has also noticed this point, and as these granules are present in the trypanosomes found in horses suffering from mal de la Zusfana (see p. 2II), he regards those trypanosomes as different from $T$. equiperdum.

Lastly, we should draw attention to the precentrosomic vacuole seen in Fig. 44, 4. This resembles the vacuole, similarly situated,
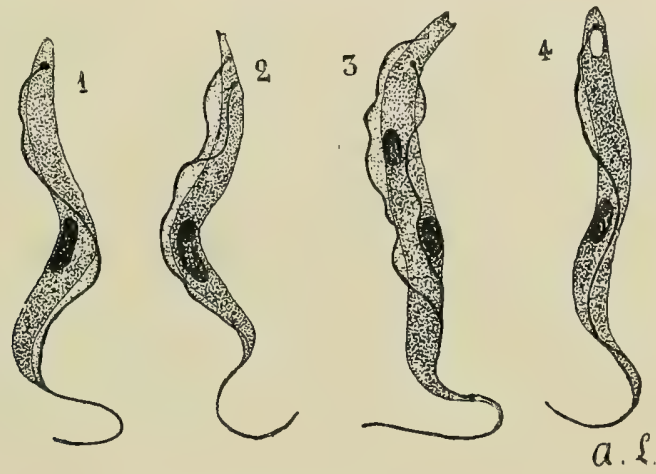

FIG. 44. -T. EQUIPERDUM.

I. Ordinary form, not undergoing division. 2, 3. Stages in binary fission. 4. Form with vacuole.

which is found in $T$. gambiense occurring in the cerebro-spinal fluid in sleeping sickness. Its causation is undoubtedly the same in both cases. It is difficult to fix the trypanosomes perfectly in any of the body fluids other than pure blood. ${ }^{2}$

The parasite is $25 \mu$ to $28 \mu$ long, even in the horse, and is therefore a little shorter than $T$. brucei; it is also slightly narrower.

Fig. 44, 2, 3 shows two stages in longitudinal binary fission, which takes place in the usual way.

As we have already mentioned, the above remarks refer to the trypanosome of Schneider and Buffard, as found in the dog and horse.

In the blood of the mice we have examined the trypanosome is slightly different. For example, in Nocard's mouse the parasites are more stumpy than those we have just described, and the proto-

${ }^{1}$ [Dr. Woodcock suggests that the bifid appearance of the posterior extremity may possibly be due to commencing division, for in both cases figured ( 2 and 3 ) division had begun.]

2 [As previously stated (Chapter III.), I am not entirely in agreement with the authors on this point. In Uganda, trypanosomes in blond-films of huinan beings and of animals infected with $\Gamma$. gambiense often showed a precentrosomic vacuole.-ED.] 
plasm contains a number of large granules. Possibly the trypanosomes showed these characteristics because the mouse was on the point of dying when it was killed. In the blood of Rouget's mice, taken a considerable time before death, these stumpy forms were again met with, and the granules were also present, but they were very scanty. In one specimen we saw a trypanosome with six nuclei, two of which were still dividing. We have recorded similar forms in the case of $T$. brucei and T. equinum. Rabinowitsch and Kempner (loc. cit., p. 8I2) talk of parasites with eight and ten nuclei arranged in the form of a rosette. Possibly, too, one of Schneider and Buffard's figures represents a similar case of multiple division.

[In Lingard's account of the trypanosome of Indian dourine he states that this trypanosome contains fewer and smaller chromatic granules than does $T$. evansi; also that its undulating membrane is less developed. Only one mode of division-longitudinal fissionoccurs. This may begin at either end or in the middle.]

The systematic study of the morphology of $T$. equiperdum in the various susceptible mammals is very necessary.

The few details we possess concerning the biology of this parasite seem to show that it differs hardly at all from that of the other trypanosomes.

'After escaping from the bloodvessels the parasite retains its motility only for a few hours; but we have seen it remain motile for forty-eight hours, in a sealed preparation, kept at a temperature of $36^{\circ} \mathrm{C}$. After twenty-four hours, however, the blood of an infected animal is no longer virulent' (Schneider and Buffard).

So far as we are aware, the action of different serums upon T. equiperdum has not been investigated.

[It has already been mentioned that Lingard found the blood and blood-stained œedema fluid from a plaque microbicidal in vitro, causing loss of motility and granular degeneration of the trypanosomes. We shall also see later (Section 7) that Rabinowitsch and Kempner found human serum, as well as the serum of immunized rats, microbicidal.]

[Jakimoff and Nina Kohl ${ }^{1}$ have made some observations upon the vitality of this trypanosome in corpses, and the former ${ }^{2}$ has, in addition, studied the vitality of $T$. equiperdum under conditions similar to those previously used by him for $T$. brucei and T. equinum (see pp. 157 and 305).]

[Mice that had died of dourine showed motile trypanosomes thirty hours after death when the body was kept at a temperature of $2.5^{\circ}$ to $5^{\circ} \mathrm{C}$. above zero. No living trypanosomes were found after eighteen hours if the corpse was kept at the room temperature.]

[The vitality of $T$. equiperdum was also tested under the following conditions: (I) In defibrinated blood; (2) on adding citrate solution; and (3) on adding horse serum; and in each of these cases the trypano-

1 [Jakimoff and Nina Khol, Arch. des Sc. Biol. (Russes), 1906, Nos. 4, 5.]

2 [Jakimoff, C. R. Soc. Biol., v. 6I, 1906, p. 631.] 
somes were kept at different temperatures: $4^{\circ} \mathrm{C}$. to zero, room temperature $\left(21^{\circ} \mathrm{C}\right.$.), and $32.5^{\circ} \mathrm{C}$. The trypanosome was found to retain its virulence longest (three days) when mixed with the citvate and kept in the vefrigevator. The virulence was retained for two days in the defibrinated blood, and for only one day after the addition of horse serum. In every case the virulence was tested by injecting white mice. The incubation period was always increased ; instead of three to four days, it was eight to thirteen days.]

[On comparing the three trypanosomes, it is seen that $T$. brucei and $T$. equimum are much more resistant than $T$. equiperdum. The first two can survive for six days under certain conditions, and the best media for them are defibrinated blood and horse serum. Nor is the optimum temperature the same; T. brucei and T.equinum survive longest at the room temperature, $T$. equiperdum at, or just below, zero.]

Schneider and Buffard figure a rosette of five parasites united by their posterior ends. Rouget has observed that when the trypanosomes are very numerous in the blood of mice, "they occur in thick clusters,' and we have noticed the same thing.

[Cultivation of T.equiperdum.-Thomas and Breinl have made several attempts to cultivate this trypanosome, with slight success. Two tubes of a modified Novy and McNeal's medium, the same as that used for T. equimum (see footnote, p. 306), out of nineteen inoculated with the blood and odema fluid of a puppy, showed motile trypanosomes on the eleventh day. On the seventeenth day one of the cultures showed many dividing forms which were agglomerated into clumps, but no rosettes were seen. There were also many single parasites, but their motility was much diminished. On this date, the seventeenth day, the culture was still pathogenic.]

\section{Section 5.-The Individuality of Dourine.}

As we have seen, the trypanosome of dourine differs only slightly from the other trypanosomes of the type brucei; but it is particularly scanty in the blood of infected Equidæ.

From the point of view of its clinical course in the Equidæ, dourine resembles the other animal trypanosomiases in some respects, but differs from them in others. The points of resemblance are the following: the anæmia, the fever, the swellings affecting especially the genital organs and the dependent parts of the body, the lesions of the eyeball and eyelids, the wasting-in spite of a normal or even an increased appetite-and the muscular weakness, especially of the hind-limbs. The chief points of difference are: the duration of the disease, the presence of the cutaneous plaques, the symptoms of paralysis of the hind-quarters associated with the presence of patches of softening in the spinal cord, and the long afebrile periods which follow the initial rises of temperature.

The characteristic features of dourine are not constant-the cutaneous plaques, for example, are usually absent in the donkeyand in the horse they may be regarded as having some relation to the very chronic course of the malady, for in the subacute cases they 
do not occur. Nocard (vide supra) was able to kill horses in four to eight weeks, and the temperature in those cases was exactly as in surra or nagana. On the other hand, we have seen that some Togo horses did not succumb for nearly a year to a trypanosomiasis closely allied to nagana.

In the Equidæ dourine behaves, then, like an attenuated form of nagana, and the same may be said of its behaviour in the ordinary laboratory animals-dogs, rabbits, rats, and mice. In rabbits this resemblance is particularly marked. The lesions of the eyes, genital organs, and skin in rabbits suffering from dourine, so closely resemble the lesions produced by nagana or caderas as to be indistinguishable from them. The only difference is that in dourine the progress of the disease is much slower. In nagana the rabbit never lives longer than two months after inoculation, whereas in dourine the animal may live for six months with the characteristic symptoms, and may even recover. ${ }^{1}$

[It has been stated that] many animals susceptible to nagana and to the allied trypanosomiases are refractory to dourine, such as macaque monkeys (according to Nocard), goats, sheep, and cattle. It will be remembered, however, that all these mammals, except perhaps monkeys, are less susceptible to nagana than the ordinary experimental animals. Cattle suffer, as a rule, only slightly in surra, and in mal de caderas are not ill at all, yet we have seen how closely these two epizootics resemble the African diseases which are propagated by tsetse-flies or other biting insects.

[The observations of Pease and of Mesnil and Rouget establishing the susceptibility of ruminants and monkeys to the virus of dourine, bring this trypanosome into line with the other pathogenic mammalian trypanosomes. Injections into these species of animals can, therefore, no longer be relied upon in diagnosing dourine from allied trypanosomiases, as is frequently necessary in Algeria, India, and elsewhere. Pease has shown that the pariah dogs in Lahore are very resistant to dourine, but susceptible to surra, while ordinary dogs are susceptible to both diseases. This might be utilized as a means of diagnosis, and, moreover, tends to show that surra and dourine are distinct diseases.]

So far as the ætiology is concerned, dourine stands alone, for infection occurs by coitus. That may be due simply to the fact that the trypanosome can pass through healthy mucous membranes. The Sergents' comparative experiments on this point with the trypanosomes of dourine and of debab (see p. 2Ig) are interesting, and show that these two Algerian trypanosomiases are distinct morbid entities.

That flies cannot play any part in the propagation of dourine is certain, and may be due to the absence of the appropriate carriers of the infection from countries in which the disease is prevalent, or

1 [Lignières' rabbits recovered after a year. See p. 330.] 
to the fact that very few parasites are present in the blood of infected animals. It would be interesting to know what the ætiology would be in a country in which the tsetse is found.

[Lingard, in his paper on 'Dourine and its Trypanosome,' to which frequent reference has been made, says that flies (sp. ?) can convey the trypanosome of dourine and produce infection in healthy susceptible animals. There is no evidence at present, however, that flies act as an intermediate host.]

The following experiments point clearly to the individuality of dourine. Nocard (Biologie, May 4, Igor, p. 466) inoculated with nagana a control dog and two other dogs highly immunized against dourine. Whereas the control dog lived for fourteen days, the two dogs which were refractory to dourine died in eleven days. Later, Lignières ${ }^{1}$ made an analogous observation by inoculating the parasite of caderas into two dogs immunized against dourine. These dogs succumbed to caderas in about one and a half months, whilst the controls lived about two months.

From these facts it can scarcely be doubted that animals immunized against dourine are susceptible to the other trypanosomiases-to surra, for example. We may therefore conclude that dourine is a definite morbid entity.

[Other, more recent, observations confirming this conclusion as to the speciricity of dourine are those of Pease, who immunized a buffalo against dourine and then found it susceptible to surra, and of Mesnil and Rouget, who immunized a goat against the virus of Schneider and Buffard and then found it insusceptible to the virus of Rouget. The latter result proves the identity of the parasites found in horses suffering from dourine in different parts of the world.]

\section{Section 6.-Mode of Propagation.}

Dourine is transmitted by coitus. [In this respect, as well as in several others, it resembles syphilis. The virus in both cases has the power of penetrating intact mucous membranes. Dourine is due to a specific trypanosome, while syphilis is almost certainly caused by the Treponema pallidum of Schaudinn, and, as was stated in Chapter III., a trypanosome stage in the life history of this parasite has been described by Krzysztalowic $z$ and Siedlecki. Like syphilis in man, dourine may run a very chronic course in horses (two years or more), and the lesion found in dourine in the lumbo-sacral region of the cord to some extent resembles a localized syphilitic meningomyelitis (Mott).]

Before we knew anything about the pathogenic agent of the disease, "the experimental proof of this mode of transmission (by coitus), previously given by Hertwig, was again furnished by Prince

1 Lignières, Riv. Soc. med. argent., v. Io, I902, pp. I I 2-I I 4. 
and Lafosse in I86I-I862. Of fifteen healthy mares which were covered by four diseased stallions, ten became infected-five of them severely-while five remained well. Two healthy stallions which served the infected mares contracted the disease. Trasbot in 1877 and Peuch in 1898 gave the disease to mares by allowing them to be covered by stallions suffering from dourine.

'Numerous observations show that an infected stallion transmits the disease to the majority of the mares covered by it, and during the whole of the breeding season. One may say that two-thirds or three-quarters of the mares exposed to infection become contaminated ' (Nocard and Leclainche, p. 626).

These observations have been confirmed and explained since the discovery of the pathogenic agent, for we have seen that the trypanosome of dourine can pass through healthy mucous membranes, and so give rise to the disease. The disease produced experimentally in horses, dogs, and rabbits by any artificial method, such as subcutaneous inoculation or placing the virus upon a mucous surface, may be transmitted from one sex to the other by coitus. Rouget was the first to show this, and since then Schneider, Buffard, and Nocard have recorded a large number of similar observations.

Rabinowitsch and Kempner (loc. cit., p. 8o8) state that they succeeded in transmitting dourine from rat to rat (of the same sex) by means of fleas.

Is coitus the only natural mode of transmission of dourine in the Equidæ? 'Infection apart from coitus is extremely rare, and doubtless always results from direct infection. The cases observed in geldings or in old mares were due to an infection of the genital mucous membrane through the medium of grooming instruments, such as sponges, or by means of litter' (Nocard and Leclainche, p. 626).

\section{Section 7.--Treatment. Immunity. Prophylaxis.}

Treatment.- 'Treatment with arsenic is alone of any value. Trélut obtained good results with arsenious acid ( 3 to 6 grammes a day), either alone or in conjunction with oil of turpentine or reduced iron ( 6 to 9 grammes). Blaise also recommends arsenious acid or arseniate of soda, together with arseniate of strychnine.

'Arkhangelsky and Novikoff cured stallions of dourine by subcutaneous injections of sodium arsenite or cacodylic acid' (Nocard and Leclainche). Marchal, ${ }^{1}$ veterinary officer at Constantine, states that he cured five stallions of dourine by injecting them subcutaneously with cacodylate of sodium in doses of I gramme in 5 c.c. water on five consecutive days, followed by five days without injections. The duration of the treatment varies 'with the resistance of the animals, and with the extent to which the trypanosomes are distributed throughout the body.'

1 Marchal, Rev. méd. vétér., April 15, I903, p. 230, and April 15, 1904, p. 231. 
[Pease, ${ }^{\mathrm{I}}$ in India, also tried the effect of injections of cacodylate of soda, but did not obtain the successful results Marchal had done. An animal treated by Pease in this way had bouts of fever, œdema, plaques, and trypanosomes, just like an untreated animal. Pease concludes that the successful results quoted by Marchal in his second paper were probably chronic cases which were recovering spontaneously, and in which the arsenic acted as a tonic and alterative rather than as a parasiticide.]

It is naturally interesting to compare these results with those obtained by means of arsenic in the treatment of the other trypanosomiases.

We have tried the effects of trypanred upon mice infected with the trypanosome which we obtained from Rouget. This drug has a very definite effect, but more or less quickly relapses occur, which are, however, amenable to further treatment with the drug.

Rabinowitsch and Kempner (loc. cit., p. 8Io) write as follows: 'According to our observations, not only human serum, but also in some cases the serum of white rats actively immunized against $T$. lewoisi, as well as of passively immunized rats, have a microbicidal action upon the trypanosome of dourine.'

Rouget made certain experiments in serum-therapy. He used the serum of rabbits or dogs in the last stage of the disease when cachexia had begun to develop. This serum injected into miceafter demonstrating the presence of the parasite in the blood by means of the microscope, two or three days after inoculation-did not materially prolong the life of the animals, for they survived the injection only from three to seven days. The therapeutic effect of this serum is therefore insignificant. ${ }^{2}$

Prevention.- Employed as a prophylactic in doses of $\frac{1}{3}$ c.c., the same rabbit serum prevented the excessive multiplication of the trypanosomes in six mice. These mice survived, although on several occasions one was able to demonstrate the presence of the parasite in the blood from the tip of the tail-one or two parasites in each field of the microscope. All the other mice so treated lived only for a short time, varying from seventeen to twenty-three days. The results were the same whether the virus and the serum were injected after mixing in vitro or separately' (Rouget).

These facts quite agree with our own observations upon the serum of goats or sheep suffering from nagana or caderas.

'The serum of animals naturally refractory (pigeon, fowl) does not possess any immunizing properties, even though the animals be previously inoculated with large doses of infective blood' (Rouget, p. 727).

This is again similar to what we found in the case of $T$. brucei.

1 [Pease, Vet. Joum., v. 9, I904, p. I96, and v. I2, I905, p. 209.]

2 [Baldrey states that castration performed in the first stage of the disease seems to benefit some cases. For recent experiments on treatment see Chapter XIII.] 
Immunity.-Animals suffering from dourine (horses or experimental animals) enjoy a certain amount of local immunity, for 'they show no signs of local reaction on reinjecting them with large doses of the virus' (Schneider and Buffard).

'Several dogs recovered after having been extremely ill, and were then quite immune, for on injecting them with enormous doses of infective blood or oedema fluid they did not react in the slightest' (Nocard, Biologie, May 4, Igor, p. 466).

[In the foregoing pages other instances of local and general immunity have been mentioned. Thus, a buffalo inoculated by Pease on three successive occasions showed a local multiplication of trypanosomes after the first two injections, but then appeared immunized against dourine, for the third injection had no effect. The buffalo was normally susceptible to surra, however.]

[Mesnil and Rouget immunized a kid against Schneider and Buffard's virus, and found it also immune against Rouget's virus. Vallée immunized a cow against Rouget's virus.]

PROPHYLAXIS.- The prophylaxis of dourine is a priori a simple matter. There is no advantage in killing infected animals, as in the case of the other animal trypanosomiases; but it is sufficient to make it impossible for them to propagate the disease by the ordinary method. The indication, therefore, is to castrate all stallions suffering from dourine, and in the various countries where the disease occurs this is made compulsory. In the case of mares it is safer to kill them when diseased. In the parts of Algeria under civil administration the mayors or other responsible officials have the right to enforce this slaughter. But in order to apply the laws and regulations concerning dourine, it is necessary to be able to diagnose the disease. Whereas in horses the fully-developed disease is generally easy to diagnose, this is often difficult in the earlier stages, and in the donkey diagnosis is always difficult. The micro-biological method may be of the greatest assistance in the diagnosis. One should examine the adema fluids microscopically for the parasite, and if it is not found, then one should inject into a dog a large dose of œdema fluid or, failing this, of the blood of the suspected animal.

By sanitary inspections alone it has been found possible hitherto to prevent the introduction of dourine into France from Algeria or from Navarre, where the disease is endemic. Suspected animals are effectively prevented from reproduction.

[But, as has already been stated in Section I, Schneider and Buffard maintain that in France dourine appears nearly every year in the department of Basses-Pyrénées, on the Spanish frontier. Mares get infected by the asses or horses by which they are covered. As is also the custom of the Arabs in Algeria, a mare is first covered by an ass, and if not impregnated, she is then served by a stallion. The asses operate on both sides of the frontier, and in this way introduce the disease into France from Spain.]

In Algeria prophylaxis is not such a simple matter. "The male 
donkey propagates the disease by carrying the trypanosome, while the female helps to preserve the parasite. It is the 'roving' male which, by means of clandestine intercourse, is responsible for all the outbreaks of dourine. In the mule-rearing districts he infects the mares covered by him; then, if these mares are not fecundatedwhich one can tell in May-they are allowed to be covered by stallions, which, in their turn, become infected by the mares, who at the time of pairing show very few, if any, symptoms of the disease. In this way is to be explained the periodic appearance of dourine in the Government studs at the end of May and during the first fortnight of June--that is to say, during the last month of the pairing season ' (Schneider and Buffard).

The problem to be solved is a difficult one-namely, to restrict all intercourse to asses officially recognised as healthy.

\section{APPENDIX.}

\section{'Maladie de Soemedang' (Java).}

The following details about this disease are taken from Nocard and Leclainche's treatise (v. 2, pp. 584, 585):

'In February, Igoo, Hubenet discovered in the Government stud at Soemedang a disease among the stallions which was conveyed by coitus to the native mares. This disease was somewhat like surra, which, as we have seen, occurs in other parts of Java, but more like dourine. An infected horse sent to the laboratory at Weltevreden was made the subject of investigation by de Does. ${ }^{1}$ The "maladie de Soemedang" is characterized by swelling of the genital organs, following upon an infecting coitus, accompanied usually by purulent catarrh of the mucous membrane. The swelling extends along the abdominal wall and reaches the chest. White patches occur in the skin, around the genital orifices, and on the perineum. Urticarial swellings occur, and these are followed by progressive atrophy of the muscles and paresis of the hind-quarters. If the disease progresses, complete paralysis occurs, and the animals die. Death is the usual termination with stallions, but mares often recover. The only lesion found, apart from those in the genital region, is softening of the spinal cord, which is surrounded in the lumbar region by a gelatinous deposit, which also infiltrates the sciatic nerve.

'The blood-stained œedema fluid obtained from the swellings of the genital organs contains a few trypanosomes, but the parasites have never been seen in the blood. In appearance they resemble $T$. evansi.

' Rabbits inoculated subcutaneously with $\frac{1}{4}$ to 5 c.c. of the œedema fluid die, much emaciated, in from thirteen to twenty-five days. The only symptom is catarrhal conjunctivitis, and sometimes some paresis before death. Trypanosomes could never be found in the blood, yet the blood was infective for another rabbit when injected in large doses (3 c.c.). The dog inoculated subcutaneously or by scarification did not show any symptoms for four to six weeks. ${ }^{2}$

'Two guinea-pigs remained free from infection after subcutaneous

1 J. de Does, 'Boosaardige deksiekte in het Soemedangsche,' Third Report, Veeartsenijkundige Bladen voor Nederl.-Indië, v. I4, I90I, pp. 20-45.

2 The ten dogs inoculated all died from a severe infection by ankylostomes. 
injection of $\frac{1}{2}$ c.c. blood. De Does thinks that the "maladie de Soemedang" differs from both surra and dourine. So far as the distinction from dourine is concerned-which need alone be discussed-the author points out that the Java disease is by no means always fatal; the lumbar hyperæsthesia and the arching of the back are absent, the swelling of the lymphatic glands is nearly always absent, and the results of animal experiments are different. De Does thinks it may be a variety of dourine which has undergone some evolutionary change. This conclusion, which seems to follow from the facts stated, would have more weight if supported by more extended and more exact experimental investigations.' 


\section{CHAPTER XI}

\section{GALZIEKTE (GALL-SICKNESS)}

Pathogenic Agent: Trypanosoma theileri, Laveran, Bruce, Igo2.

GALZIEKTE or gall-sickness is a disease of Bovidæ endemic throughout a large part of South Africa, due to a trypanosome which, both morphologically and in its pathogenic action, is quite distinct from the parasites we have hitherto studied.

\section{Section 1.-Historical. Geographical Distribution.}

According to Theiler, ${ }^{1}$ this disease was described for the first time by the veterinary surgeon Spreull, and the epizootics described by Kolle under the name of malaria of cattle, ${ }^{2}$ and by Hutcheon, the principal veterinary officer of Cape Colony, under the name of jaundice or biliary fever, ${ }^{3}$ were really gall-sickness.

The disease is generally called gall-sickness or galziekte by the farmers in South Africa, but under the general designation of 'biliary diseases' several different diseases of cattle have been confounded.

Gall-sickness often occurs at the same time as the bovine piroplasmosis or redwater fever of the Transvaal. Theiler draws attention to the fact that the name gall-sickness has been used in the Transvaal since $187 \mathrm{I}$, before the disease redwater was known there, and that even at the present day gall-sickness is met with in parts of South Africa where redwater has never occurred.

The trypanosome which is the causal agent of gall-sickness was discovered by Theiler, and described by one of us and by Bruce almost simultaneously, under the name of $T$. theileri. ${ }^{4}$

Gall-sickness is prevalent over a large part of South Africa. It has been observed among the cattle in Zoutpansberg in North Transvaal, in the valley of the Komati in the east, in the neighbourhood of Standerton in the south, and in Klerksdorp in the west,

1 A. Theiler, 'A New Trypanosoma,' Journ. Comp. Path. and Therap., 1903, v. 16.

2. Kolle, Zeitschr.f. Hyg.u. Infectionskrankh., I898, v. 27, p. 44.

3 Hutcheon, Report for 1897 , quoted by Theiler, $o p$. cit.

4 Laveran, Acad. des Sciences, March 3 and November 3, I902 ; Bruce, Lancet, March 8, I902, p. 664. 
as well as in cattle coming from Cape Colony and the Orange River Colony.

The same trypanosome was also found by Theiler in Pretoria, in the blood of an ox coming from Madagascar; but the animal had been some time in Natal before it arrival in the Transvaal.

Schilling has seen an identical trypanosome in a Togo ox. ${ }^{1}$

Panse $^{2}$ found a very large trypanosome in the blood of a calf on the island of Mafia, German East Africa. This trypanosome was $40 \mu$ to $80 \mu$ long, not including the free flagellum, which itself measured $30 \mu$. The posterior end was very pointed. Panse expressed some doubt about the identity of this trypanosome with T. theileri.

[Koch and Lühe, on the other hand, think that it is certainly T. theileri. Koch has met with the disease in cattle coming from Mafia Island, and also among cattle at the experimental station in Rhodesia. $\left.{ }^{3}\right]$

In the blood of an animal suffering from the 'Jinja' disease (see p. 205) Nabarro found a trypanosome closely resembling, if not identical with, $T$. theileri. This trypanosome was $44 \mu$ long, including free flagellum I8 $\mu$, and had a very pointed posterior end, a single nucleus, and two centrosomes. The other trypanosomes found in this blood-film were either very short and stumpy (length I $3 \mu$, free flagellum 2 to $3 \mu$ ), or fairly long (length $20 \mu$ to $25 \mu$, free flagellum $8 \mu$ to Io $\mu$ ). The animal probably had a double infection, with $T$. dimorphon and $T$. theileri. These observations of Panse, Koch, and Nabarro show that $T$. theileri has a wider distribution in Africa than was formerly supposed. $\left.{ }^{5}\right]$

[Lühe ${ }^{6}$ and Luhs ${ }^{7}$ have also met with this trypanosome in Bovidæ in Transcaucasia. Lühe studied the morphology of the parasite in stained blood-films sent to him by Ziemann, while Luhs was able to make a study of the disease and of its trypanosome on the spot. He observed it in six animals sick with cattle-plaguethat is to say, under the same conditions as Theiler did in South Africa and Holmes in India. He never found it in other cases-for example, in animals with piroplasmosis. The trypanosome was only feebly pathogenic, for the blood of the sick animals could be used without harmful effects, for immunization against cattle-plague.]

1 Schilling, Journ. Trop. Med., I903, p. 47.

2 O. Panse, Zeitschr. f. Hyg., v. 46, I904, p. 376.

${ }^{3}$ [Koch, Lecture before Berlin Medical Society, translation in Brit. Med. Jiurn., I904, II, p. 1446.]

${ }^{4}$ [Nabarro and Greig, Report of the Sleeping Sickness Commission, No. V, 1905, see p. I 8 and Plate I, Fig. I.]

5 [Todd has recently described (Liverpool School Memoirs, 1906, No. XXI, p. 9i) a trypanosome which closely resembles, if it is not identical with, $T$. theileri, found by I)utton and himself in the blood of an antelope killed at Kasongo, in the Congo Free State.]

6 [M. Lühe, in Mense's 'Handbuch der Tropenkrankheiten,' v. 3, I 906, p. I 33.]

i [F. Luhs. Arch. Parcesitologie, v. 10, 1906, p. I7 I ; abstract by Mesnil in Buil. Inst. Past., v. 4, 1906, p. 918.] 
[In India very large trypanosomes have also been observed in the blood of cattle by Lingard and by Holmes. One of these, seen by Lingard in the blood of cattle inoculated with surra, has already been described in Appendix A to the chapter on Surra (p. 286). Lühe states that Blanchard regards these Indian trypanosomes as a distinct species, which he calls $T$. lingardi. Lühe himself is of opinion that they are identical with $T$. theileri, and the evidence we possess at present is certainly in favour of this view.]

[Durrant and Holmes, in their first paper, ${ }^{1}$ state that they found a trypanosome a little shorter than $T$. evansi in spleen smears of a bull postmortem, the animal having died after a fairly acute illness in the laboratory at Muktesar. In a later paper ${ }^{2}$ Holmes describes a very large trypanosome which he found in the blood of 'hill' cattle. The cattle in which this trypanosome was seen were all more or less debilitated, or had been inoculated with 'rinderpest' serum. The 'plains' breed of cattle never showed trypanosomes. This trypanosome was $60 \mu$ to $80 \mu$ long (one measured as much as 9I $\mu$ ), free flagellum I $5 \mu$ to $25 \mu$; the width was $2 \mu$ to $4 \mu$, in one case $6 \mu$. The posterior end was very pointed, and the centrosome was Io $\mu$ to I7 $\mu$ from the tip.]

[Cattle seldom died of the infection unless they were very debilitated. Inoculations into ponies, bulls, and rabbits gave negative results, so that morphologically and in its behaviour in cattle and in experimental animals this trypanosome closely resembles $T$. theileri. Holmes thinks that the parasites previously seen by Durrant and himself in spleen smears were immature forms of this trypanosome, but the figures they give illustrating their paper do not warrant this belief. Many of the parasites depicted look quite mature and normal in appearance, and resemble the surra trypanosome.]

[Lingard ${ }^{3}$ describes a similar trypanosome which he discovered in the blood of ' hill' cattle in I9O2. He calls it T. himalayamm , ${ }^{\text {, but it }}$ probably is the same parasite as that described by Holmes.]

[In the blood of 'plains' cattle, Lingard in I895, during an outbreak of surra, discovered a large trypanosome which he thinks may have been T. theilevi. $\left.{ }^{3}\right]$

\section{Section 2.-Gall-sickness is a Disease Peculiar to the Bovidæ.}

All attempts made by Theiler to infect with gall-sickness animals other than Bovidæ were unsuccessful. His experiments were made upon horses, dogs, sheep, goats, rabbits, guinea-pigs, rats, and mice.

In Bovidæ infection occurs more or less easily, according to the origin of the animals. Theiler states that the Transvaal cattle are the least susceptible, and that cattle coming from Texas and the Argentine Republic become infected in a much larger proportion than do the native cattle.

The disease has been observed in animals of all ages.

1 [Durrant and Holmes, Journ. Comp. Path. and Therap., v. I7, I904, p. 209].

2 [Holmes, Journ. Comp. Path. and Therap., v. 17, I904, p. 317.]

3 [Lingard, Indian Med. Gaz., I904, p. 445.]

4 [Lingard, Journ. Trop. Vet. Sci., v. i, I906.] 


\section{Section 3.-Course of the Disease. Symptoms.}

The disease may run an acute or a subacute course. Three to five days after inoculation there is usually a rise of temperature, lasting several days, after which the temperature falls again to normal. In some cases the presence of the trypanosome appears to have very little effect upon the health of the animals, but as a rule the infection is accompanied by severe anæmia. In one case the red corpuscles were reduced to $2,500,000$ per cubic millimetre. Often basophile granules are seen in the red corpuscles, but they are not characteristic of this disease, as they are very common in bovine piroplasmosis (Theiler), and are usually found in all severe anæmias. As in other grave anæmias, nucleated red corpuscles are frequently met with. The leucocytes are increased in number in most cases.

The trypanosomes are often fairly numerous in the blood, but do not remain present for long. The longest time that they were seen in the blood was thirteen days, and the shortest one day, the average being nine days. According to Theiler's observations, ${ }^{1}$ the number of parasites does not appear to have much influence upon the severity of the disease, and in several cases in which the parasites were numerous the animals apparently enjoyed good health.

When the disease terminates in recovery, the trypanosomes diminish in number, while the red corpuscles increase, and finally the parasites disappear altogether from the blood.

Gall-sickness is often associated with other diseases, and this complicates the study of it. Thus, piroplasms are often found, and spirochætes are sometimes found in the blood of animals infected with $T$. theileri, so that it is impossible to say exactly what part the trypanosome plays in the production of the symptoms noted.

[As has already been stated, the cases of $T$. theileri infection seen by Holmes, Lingard, Luhs, and Nabarro were all complicated by some other infection or occurred in debilitated animals. Holmes' cases were all more or less debilitated, or had been inoculated with rinderpest serum. Lingard's cases were complicated with surra, with filariasis in one case, and with anthrax in another case. Luhs' six animals were all sick with cattle-plague, and Nabarro's case had another trypanosome infection, probably with $T$. dimorphon, to which the animal succumbed.]

Among forty cases which can be looked upon as simple infections by $T$. theileri there were five deaths, giving a mortality of $12 \frac{1}{2}$ per cent. (Theiler).

1 Theiler has counted as many as thirty trypanosomes in one field of the microscope (Zeiss. Obj., No. 6); an average of five is by no means uncommon. In other cases only one parasite may be found in the whole film of blood examined. 


\section{Section 4.--Pathological Anatomy.}

Anæmia and hypertrophy of the spleen are the most noticeable changes seen post-mortem in cattle that have died of gall-sickness.

The blood is watery and all the organs and tissues are pale in colour, but sometimes they have an icteric tint. The subcutaneous tissue of the abdominal walls is often the seat of a serous infiltration, and the pericardium contains a variable quantity of fluid. The heart is flabby and shows subpericardial petechiæ. There is hypostatic œedema of the lungs. The spleen is enlarged and softened, and the mesenteric glands are often swollen.

\section{Section 5.-The Pathogenic Agent.}

$T$. theileri is easily distinguished from the other mammalian trypanosomes by its size. The largest forms measure $60 \mu$ to $70 \mu$

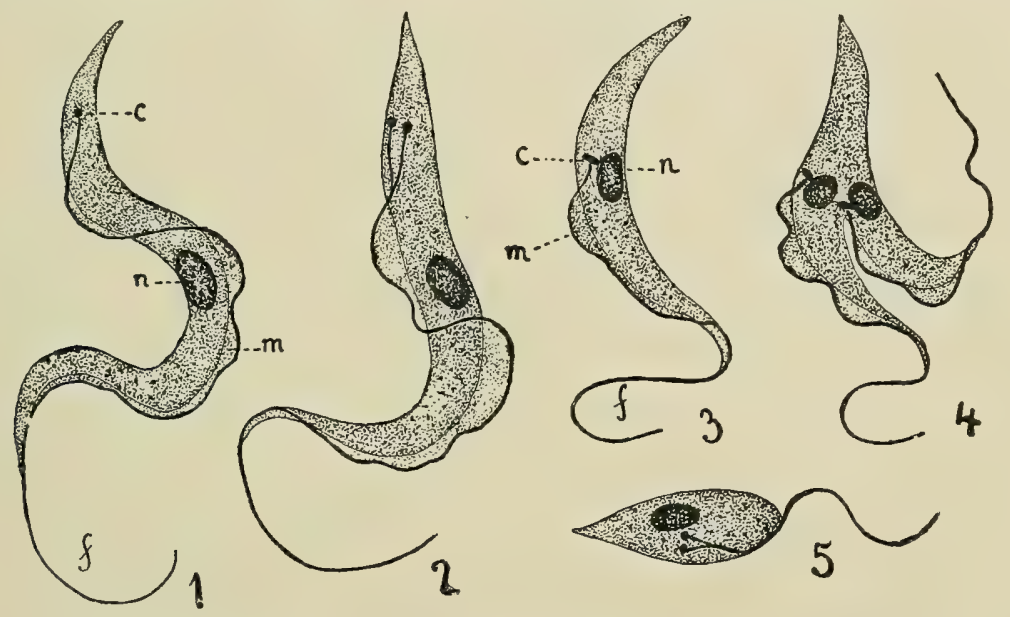

Fig. 45-Trypanosoma theileri and Trypanosoma transvaaliense.

I land 2. T. theileri; Fig. 2 shows a trypanosome undergoing division. 3-5. T. trans. vaaliense; Fig. 4 shows a trypanosome in the last stage of division; Fig. 5, a small form undergoing division. (Magnified about I,700 diameters.)

in length, by $4 \mu$ to $5 \mu$ in breadth. The smallest forms are $25 \mu$ to $30 \mu$ long, by $2 \mu$ or $3 \mu$ wide.

In fresh blood the movements of the parasite are so rapid that one cannot easily make out its shape, but on keeping the preparations for several hours, or even for two or three days, the motility diminishes, and it is then possible to distinguish the chief morphological characters of the trypanosome. This trypanosome moves as a rule with the flagellum foremost, but sometimes the non-flagellated end leads the way. There is a long flagellum and an undulating membrane. The posterior end of the body is usually pointed.

On fixing and staining in the ordinary way, one can distinguish (Fig. $45, I$ ) an oval nucleus $(n)$ situated about the middle of the body, and a round darkly-stained centrosome $(c)$, situated a con- 
siderable distance from the posterior extremity. The free flagellum is about one quarter of the whole length of the parasite. The flagellum itself starts from the centrosome, and runs along the undulating membrane $(\mathrm{m})$, which is fairly wide and shows several folds. The protoplasm stains deeply and contains a large number of chromatic granules.

Multiplication takes place by simple fission. Fig. 45, 2 shows a trypanosome undergoing division; the centrosome has divided, and the root of the flagellum has also begun to divide.

[The trypanosome seen by Lühe in the blood of Transcaucasian cattle appears to differ in some respects from the foregoing. The centrosome is not round, but elongated transversely to the long axis of the body; sometimes it is dumb-bell-shaped-' like a diplosome'-which may possibly indicate commencing division. The nucleus is like that of other trypanosomes; the chromatin is grouped in a number of rounded bodies, one of which-the karyosome-is sometimes more deeply stained than the rest. The relative positions of the nucleus and centrosome may differ. This has led Laveran and Mesnil to distinguish two species of this trypanosome- $T$. theilevi and $T$. transvaaliense-but Lühe thinks they are varieties of the same trypanosome, and Theiler and Luhs are of the same opinion. The form Lühe calls the typical form has a rounded nucleus situated about the middle of the body, a centrosome about midway between the nucleus and the posterior end, and few chromatic granules. The other form-T.transvaaliense of Laveran and Mesnil-has an oval nucleus, which is situated further back than in the typical form, and which may even be posterior to the centrosome. This form has many chromatic granules. Lühe found fewer individuals of the latter variety in his bloodfilms. He also saw transitional forms, and therefore concludes that these two trypanosomes are the same species.]

[In addition to the typical and other forms just described, Lühe figures another (? \&) form of the parasite resembling those he and other observers describe in T. brucei, T. equinum, and some of the other mammalian trypanosomes. This female (?) form is very large, and is broader than the ordinary form. It has a pale-staining alveolar protoplasm; the nucleus consists of an oval ring of chromatic bodies, and lies across the long axis of the body of the parasite.]

[Both Lühe and Luhs describe myonemes, or a sort of fibrillar envelope, like Prowazek found in T. lewrisi.]

[Luhs observed that young forms resulting from longitudinal division always had the centrosome situated close to the nucleus, as in T.transvaaliense. Both he and Lühe regard T. transvaaliense as a developmental stage of $T$. theileri.]

T. theileri can live for seven days in defibrinated blood at the room temperature or in the ice-chest, but not so long in the incubator. In defibrinated blood diluted with horse serum or with physiological saline the trypanosome can live as long as in undiluted blood; but the addition of water or of glycerine and water rapidly kills the parasites. Exposing them to a temperature of $50^{\circ} \mathrm{C}$. kills them in less than twenty-four hours.

[Luhs found the trypanosome sluggishly motile after being kept on ice for nine days, and on warming the preparation the motility 
increased. Kept at $20^{\circ} \mathrm{C}$, the trypanosomes lost their motility in six days, and at $38^{\circ} \mathrm{C}$. in three days.]

Involution forms are often seen in blood-films. The protoplasm stains badly, and the outline of the parasite is indistinct. The centrosome and flagellum are the most resistant parts of the trypanosome, and are often met with amongst the red corpuscles, the rest of the parasite having disappeared.

Agglutination has been seen by Theiler under certain conditions. Occasionally two parasites adhere by their posterior ends. When defibrinated blood is allowed to stand, the trypanosomes come to the surface and adhere together in the form of rosettes. Agglutination is well marked on adding to the blood the serum of a calf which has been inoculated on several occasions with $T$. theileri. In one case in which the trypanosomes were very numerous, agglutination was observed in blood examined fresh twenty-four hours after the animal had received a big dose of immunizing serum. Before this injection the blood had been examined, but no agglutination had been observed.

[Luhs also observed agglomeration of $T$. theileri when the organism was under unfavourable conditions. He found that the addition of salt solution to the blood did not prevent agglomeration.]

In films of blood from a bovine sent to us by Theiler from the Transvaal one of us has seen and described under the name of $T$. transuaaliense a trypanosome which morphologically appears quite distinct from $T$. theileri.

$T$. transvaaliense varies considerably in size. In one and the same film are seen small forms measuring on an average $18 \mu$ in length, including the flagellum; large forms $40 \mu$ and even $5^{\circ} \mu$ long, by $6 \mu$ in width; and intermediate forms, which are the most numerous, $30 \mu$ long, by about $4 \mu$ to $5 \mu$ wide. The posterior extremity is as a rule very pointed.

The nucleus, which is oval in shape, is situated about the middle of the body. The relation of the centrosome to the nucleus is characteristic. In all the trypanosomes described hitherto the centrosome has been situated far from the nucleus, and usually a short distance from the posterior extremity of the body. That, indeed, is an objection which has been raised to the interpretation we have given of this chromatic corpuscule of trypanosomes in which the flagellum takes origin.

In T. transvaaliense the centrosome, which is relatively large, and therefore easily seen, is always situated near the nucleus, and often adjoining it, as in Fig. 45, 3. It is usually elongated in form, and stains more deepiy than the nucleus by our method of staining.

On account of the approximation of the centrosome to the nucleus, the undulating membrane is much less developed in $T$. transvaaliense than in the other trypanosomes. The protoplasm is finely granular, and stains less deeply than that of $T$. theileri.

$T$. transvaaliense multiplies by fission, like $T$. theileri, but the 
division forms are more varied than in the latter species. Fig. 45, 4 shows a parasite of medium size in the last stage of fission, and one can distinguish two nuclei, two centrosomes, two flagella, and two undulating membranes; the protoplasm has also begun to divide. Fig. 5 shows a small parasite undergoing fission, the centrosome and adjacent part of the flagellum having already divided. In the process of fission the flagellum divides throughout its whole length. Some films were made with blood which had been kept for twentyfour hours, and many of the trypanosomes were agglutinated into more or less well-formed rosettes. Agglutination occurs by the posterior extremities, as with T. lereisi and T. brucei. The protoplasm of these agglutinated trypanosomes was already showing signs of degeneration, and contained large chromatic granules.

The coloured plate at the end of the book shows a T. theileri (Fig. 8) and a $T$. transvaaliense (Fig. 9).

From Theiler's researches it would appear that $T$. transvaaliense is not a distinct species, but a variety of $T$. theileri, for on injecting blood containing T.transvaaliense into cattle Theiler succeeded in producing ordinary gall-sickness.

[We have already seen that on morphological and developmental grounds Lühe and Luhs also regard these two trypanosomes as varieties of one species.]

\section{Section 6.-Mode of Propagation.}

Gall-sickness is easily inoculable from one bovine into another by the subcutaneous or intravenous injection of virulent blood. The

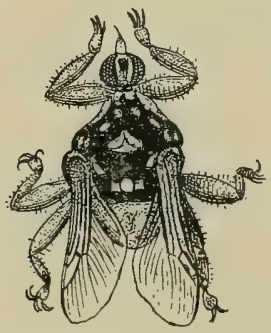

Fig. 46.-Hippobosca rufipes. $\times 2$ (about). (After Theiler.)

The small, round, white area, mesially situated at the posterior part of the thorax, is in nature red in colour, and on either side there is a small grey area.

incubation period is from four to six days when a large dose of blood containing many trypanosomes is injected, but may be as long as eighteen or twenty days if the blood injected contains very few parasites. Blood may still be virulent, even though it show no parasites on microscopical examination. According to Theiler, the inoculations of defibrinated blood for cattle-plague in the Transvaal have contributed to the spread of gall-sickness.

By analogy with what happens in nagana, one would suppose 
that gall-sickness was propagated by biting flies. Theiler's investigations show that the greatest suspicion falls upon Hippobosca rufipes, which is very common in South Africa.

Several specimens of Hippobosca, after having been starved, were placed upon an animal infected with gall-sickness, and immediately afterwards upon a healthy animal. In two cases out of four the healthy animals so experimented upon became infected. An hour after feeding the blood in the stomach of the flies shows trypanosomes as active as in fresh blood.

Theiler has sent us some specimens of Hippobosca from the Transvaal, which have been identified by Dr. Speiser of Bischofsburg. According to Speiser, these flies belong to two species: $H$. rufipes, v. Olfers, and $H$. maculata, Leach. The latter is very rare in South Africa, where it appears to have been introduced at the time of the Boer War with cavalry horses coming from India. ${ }^{1}$

\section{Section 7.-Treatment. Prophylaxis.}

At present we know of no treatment for gall-sickness. Cattle which survive an attack are immune. We have seen that the native cattle in the Transvaal are less susceptible to the disease than imported cattle; therefore foreign animals should not be introduced into areas where the disease is prevalent.

Sick animals should be isolated. Killing them would be the most radical and efficacious measure, but it is difficult to enforce this in the case of a disease the gravity of which is not yet known with certainty, and which, moreover, often ends in recovery.

Healthy cattle should be protected as much as possible from biting flies by the methods suggested in previous chapters.

The inoculations for cattle-plague appear to have spread gallsickness in the Transvaal; therefore, if such inoculations should still be necessary, it should be ascertained beforehand that the animals from which the blood is taken are free from gall-sickness.

1 Laveran, 'Concerning Two Species of Hippobosca from the Transvaal,' Soc. de Biol., February 21, 1903. 


\section{CHAPTER XII}

\section{HUMAN TRYPANOSOMIASIS, INCLUDING SLEEPING SICKNESS}

Pathogenic Agent: Trypanosoma gambiense, Dutton, I902.

\section{Section 1.-Historical.}

AT the beginning of last century the English observer Winterbottom ${ }^{1}$ recorded the occurrence among the negroes on the West Coast of Africa of a curious disease characterized particularly by a tendency to sleep. Since that time the disease has been described by a large number of observers, and under the name of sleeping sickness it has found a place in all treatises upon tropical diseases.

In I840 Clarke $^{2}$ of Sierra Leone gave a short account of the disease. From I86I to I900 French naval surgeons published a series of very interesting papers upon the clinical and pathologicoanatomical study of sleeping sickness. The most important of these are by Dangaix, ${ }^{3}$ Nicolas, ${ }^{4}$ Griffon du Bellay, ${ }^{5}$ Chassaniol, ${ }^{6}$ Santelli, ${ }^{7}$ Guérin, ${ }^{8}$ Le Roy de Méricourt, ${ }^{9}$ Corre ${ }^{10}$ Mahé, ${ }^{11}$ Nielly, ${ }^{12}$ and Le Dantec. ${ }^{13}$

Guérin's excellent thesis deserves special mention. He studied the disease in Martinique in negroes coming from the West Coast of Africa. In the course of a dozen years he observed as many as I 48 cases.

Hirsch has given a good description of the disease, and in his excellent work on the 'Historical and Geographical Pathology of

1 Winterbottom, 'An Account of Native Africans in the Neighbourhood of Sierra Leone,' 1803.

2 Clarke, London Med. Gaz., September, 1840; Edinburgh Monthly Journ. Med. Science, 1842 , p. 320.

3 Dangaix, Moniteur des Hôp., I86r, p. Ioo.

4 Nicolas, Gaz. hebdom., I86I, p. 670.

5 Griffon du Bellay, Arch. de méd., 1864, I ${ }^{\mathrm{e}}$ sem., p. 73.

6 Chassaniol, Arch. de méd. nav., I865.

7 Santelli, ibid., 1868.

8 Guérin. Thèse, Paris, 1869.

9 Le Roy de Méricourt, article 'Sleeping Sickness' in the Dict. cncycl. des sc. méd., I 87 I.

10 Corre, Arch. de med. nav., I877.

i1 Mahé, 'Syllabus for the Study of Tropical Diseases,' 1888.

12 Nielly, 'Tilém. de pathol. exotique,' I 881, p. 503.

13 Le Dantec, 'Pathol. exotique,' 1900, p. 759. 
Diseases' gives a bibliography of all the papers dealing with sleeping sickness. ${ }^{1}$

The conquests of bacteriology were destined to receive, and have received, their set-back in the history of sleeping sickness. A. de Figueiredo, ${ }^{2}$ Cagigal and Lepierre, ${ }^{3}$ Marchoux, ${ }^{4}$ Bettencourt, ${ }^{5}$ Broden, ${ }^{6}$ and Castellani, ${ }^{7}$ have attributed to different Bacteria (bacilli, pneumococci, streptococci, etc.) the rôle of pathogenic agent in sleeping sickness. The variety of the microbes isolated by these observers tends to prove that the causes of secondary infection, so common in this disease, have been looked upon as the actual causal agent of the disease.

Several observers have attributed the disease to bad or insufficient feeding. Ziemann has held this opinion, and, according to him, the disease is due not to an infection, but to an intestinal intoxication. This intoxication is said to be produced by the root of the manioc eaten raw or only slightly cooked, as is often done by natives. ${ }^{8}$ A large number of facts tell against this opinion. In Casamance, where little or no manioc is eaten, sleeping sickness is very prevalent, whilst in Dahomey, where this root enters largely into the native dietary, the disease occurs but rarely. ${ }^{9}$ It has been shown, moreover, that the disease may occur in well-nourished people, who for several years have left those districts where manioc is eaten. The incrimination of certain mud fish in Casamance is also unjustified.

In I9oo Manson gave a very full account of two patients from the Congo who died in Charing Cross Hospital, and the pathological anatomy of these two cases was very carefully worked out by Mott. ${ }^{10}$

Manson has attributed sleeping sickness to an infection by a filaria which he has described under the name of Filaria perstans. It is true this filaria is often met with in the blood of negroes suffering from sleeping sickness, but it is also found in districts where the disease does not occur. ${ }^{11}$ Low has recorded the existence of $F$.perstans in British Guiana, where sleeping sickness has never occurred. We

1 Hirsch, 'Handbuch der hist. geogr. Patholog.,' 1862 ; second edition, 18801882. [Translation published by New Sydenham Society, see vol. iii., pp. 595602.]

${ }^{2}$ Figueiredo, 'Inaugural Dissertation,' Lisbon, I89I.

3 Cagigal and Lepierre, Médecine moderne, January 26, I898.

4 Marchoux, Ann. d'hyg. et de méd. colon., 1899 , p. I3.

5 Bettencourt, 'Doença do Somno,' Lisbon, Igor.

6 Kuborn's Report upon Broden's work, Acad. de méd. de Belgique, October 26, I90I.

7 Castellani, Brit. Med. Journ., March I4, I903.

8 Ziemann, Centralb. f. Bakter., I, Orig., v. 32, I903, p. 413.

9 Kermorgant, Acad. de méd., December 29, 1903.

10 Manson, 'Tropical Diseases,' third edition, I903, p. 335. [Also 'African Lethargy, or the Sleeping Sickness,' P. Manson and F. W. Mott, Trans. Path Soc., v. 5I, 1900.]

i1 Low, Brit. Med. Journ., March 28, I903. Christy, Annual Meeting of British Medical Association at Swansea, July, 1903; Journ. Trop. Med., 1903. Low, Royal Society Reports of the Sleeping Sickness Commission, November, 1903. Christy, ibid., November, I903. 
may add that in many cases of sleeping sickness the filaria cannot be found. This hypothesis of Manson's must, therefore, be given up. ${ }^{1}$

The researches of Dutton and Todd, of Castellani, and of Bruce, Nabarro, and Greig upon human trypanosomiasis mark the latest phase in the elucidation of the rtiology of sleeping sickness. It would seem that the careful investigations of these observers have at last discovered the true cause of this disease.

On May I0, I90I, Forde received under his care at the hospital in Bathurst (Gambia) a European, aged forty-two, the captain of a steamer on the River Gambia. This man, who had navigated the river for six years, had suffered from several attacks of fever which were looked upon as malarial. The examination of his blood revealed the presence not of the malarial parasite, but of small wormlike bodies, concerning the nature of which Forde was undecided.

On December 18, Igor, Dutton, in conjunction with Forde, examined this patient, whose condition had become more serious, and recognised that these worm-like bodies seen by Forde were trypanosomes. Dutton has given an excellent description of this parasite, which he has called Trypanosoma gambiense. ${ }^{2}$

In Igo2 Dutton and Todd came across further cases of human trypanosomiasis. Of $\mathrm{I}, 000$ persons examined in Gambia, ${ }^{3}$ six natives and one quadroon showed trypanosomes in their blood. The cases observed amongst the natives were as follows: A woman of thirtyfive, a boy of nine, a girl of sixteen, a boy of twelve, a man of thirtyfive, and another of twenty-two. The first three belonged to the same village-Lamin.

Manson has recorded two cases of trypanosomiasis in Europeans contracted on the Congo. ${ }^{4}$ In one of these cases the trypanosomes

${ }^{1}$ [For a full account of the earlier hypotheses as to the ætiology of sleeping sickness, see Centralb. $f$. Bakter., I, Orig., v. 35, I903, p, 45, 'Ueber die Ætiologie der Schlafkrankheit,' by Bettencourt, Kopke, de Rezende, and Mendes ; also p. 62 for a paper by Castellani.]

2 Forde, Journ. Trop. Med., September I, I902; Dutton, ibid., December I, 1902 ; Dutton, Thompson Yates Lab. Report, I902, v. 4, part ii., p. 455. The patient who formed the subject of these observations by Dutton and Forde died in England on January 1, 1903. See a note by Annett, Brit. Med. Journ., February 7, I903.

Previously, Nepveu had recorded the existence of trypanosomes in the blood of several patients coming from Algeria, but his descriptions and figures are so lacking in precision that there is considerable doubt about the correctness of this diagnosis. Although in recent years the blood of a very large number of patients in Algeria has been examined, trypanosomes have never been seen again in them (Nepveu, Soc. Biol., I891, Memoirs, p. 49; and Soc. Biol., December 24, 1898).In 1894 Barron recorded the case of a woman of thirty-nine with a uterine fibroid, in whose blood a large number of flagellated Protozoa were found. The retrospective diagnosis of trypanosomes may be made in this case. The patient had not lived in Africa, so far as can be judged from the short note by Barron (Trans. of the Liverpool Med. Institution, December 6, 1804). In I898 Brault brought forward the hypothesis that possibly the trypanosome was the causal agent in sleeping sickness (Janus, July to August, I 898, p. $4 \mathrm{r}$ ).

${ }_{3}$ Dutton and Todd, First Report of the Trypanosomiusis Expedition to Senegambia, I002, Liverpool, 1903.

4 Manson, Journ. Trop. Med., November I, 1902, and March 16, 1903. Manson and Daniels, Brit. Med. Journ., May 30, 1903. 
were discovered by Broden, Director of the Bacteriological Laboratory at Leopoldville, Congo Free State. ${ }^{1}$

Brumpt has recorded the existence of $T$. gambiense at Boumba, situated at the junction of the Ruby and the Congo, in a ship's steward, who suffered for several months from irregular fever, which did not respond to quinine. ${ }^{2}$ Lastly, Baker has seen three cases of trypanosomiasis in Entebbe, Uganda. The parasites were numerous in one case, scanty in the other two. ${ }^{3}$

The relation between human trypanosomiasis and sleeping sickness was not suspected ${ }^{4}$ when Castellani, examining the cerebrospinal fluid of negroes in Uganda suffering from sleeping sickness, discovered trypanosomes which were at first thought to be a different species, and were described under the names of $T$. ugandense (Castellani) and T. castellanii (Kruse). ${ }^{5}$

This important discovery was immediately confirmed by Bruce and Nabarro, who found trypanosomes in each of thirty-eight cases in the cerebro-spinal fluid obtained by lumbar puncture from sleeping sickness patients in Uganda, and twelve out of thirteen times in the blood. The cerebro-spinal fluid of 'control' individuals, not suffering from sleeping sickness, never showed trypanosomes.

We shall frequently have occasion throughout this chapter to quote from the interesting reports by Bruce, Nabarro, and Greig, ${ }^{6}$ upon sleeping sickness in Uganda.

In the Congo State, Brumpt found trypanosomes twelve out of fifteen times in patients suffering from sleeping sickness. ${ }^{7}$

The Portuguese Commission for the study of sleeping sickness re-examined the blood-films of twelve of their patients which had been used during the course of their investigations, and found trypanosomes in four of them. ${ }^{8}$

1 Broden, Bull. de la Soc. d'études coloniales, Brussels, I903, No. 4.

2 Brumpt, Acad. de méd., March I7, 1903 ; Bulletin, v. 49, p. 372.

3 Baker, Brit. Med. Journ., May 30, 1903, p. 1254.

4 [Before the publication of Castellani's discovery of trypanosomes in the cerebro-spinal fluid of sleeping sickness patients, Maxwell-Adams had suggested the relationship between trypanosomiasis and sleeping sickness on the ground that certain features (puffiness of the face and lower eyelids, irritability and apathy, and characteristic alteration in the voice) were common to both diseases. He also made the rather improbable suggestion that the disease is conveyed by the bite of rats, or by a rat-flea (see Brit. Med. Journ., March 28, I903, and April I6, I904, p. 889).]

5 Castellani, letter dated Entebbe, Uganda, April 5, I903, addressed to the Royal Society, Brit. Med. Journ., May 23, I903, and June 20, I903; also Journ. Trop. Med., June I, Iço3. Kruse, 'Sitzungsbericht der niederrhein. Gesell. f. natur. Heilk,' 1903. Castellani, Proc. Roy. Soc., v. 71, I903, p. 501, and Keports of the Sleeping Sickness Commission, No I, August, I903.

${ }^{6}$ Royal Society, Reports of the Sleeping Sickness Commission: No. I, by Bruce and Nabarro, Progress Report on Sleeping Sickness in Uganda, London, August, 1903; No. 4, Further Report on Sleeping Sickness in Uganda, by Bruce, Nabarro, and Greig, November, I903. Résumé in Brit. Med. Journ., November 21, I903.

7 Brumpt, Congrès d'Hygiène de Bruxelles, 1903.

8 A medicina contemporanea, June 28, 1903; also Report on Sleeping Sickness presented to the Minister of Marine and of the Colonies by the Commission presided over by Bettencourt, Lisbon, 1903 . 
In the Congo Free State, Dutton, Todd and Christy, ${ }^{1}$ and Broden ${ }^{2}$ also found trypanosomes in the cerebro-spinal fluid of a large number of negroes suffering from sleeping sickness.

[Subsequent investigations in all parts of Africa in which sleeping sickness occurs have confirmed the original observations of Castellani, Bruce, and Nabarro. If carefully looked for, trypanosomes are practically always found in the cerebro-spinal fluid, blood, and gland-juice of sleeping sickness patients. Moreover, clinical and experimental evidence is being gradually accumulated, which goes to prove that a trypanosome is the cause, and probably the sole cause, of sleeping sickness. Patients who had acquired trypanosomiasis in Africa have died of sleeping sickness as long as three years after returning to Europe, and a patient of Thiroux's with sleeping sickness had left the endemic area eight years. ${ }^{3}$ In a monkey experimentally infected with $T$. gambiense, in which the infection ran a chronic course (like trypanosomiasis ending as sleeping sickness does in the human subject), the macroscopic and microscopic lesions in the central nervous system typical of human sleeping sickness have been observed. ${ }^{4}$ Lastly, we have seen that in uncomplicated chronic dourine-another trypanosome infection-microscopical changes very similiar to those occurring in sleeping sickness have been described by Mott.]

It still remained to be seen if $T$. gambiense and $T$. ugandense were different species, or if the parasite found in sleeping sickness patients was really the $T$. gambiense.

Bruce, Nabarro, and Greig showed that twenty-three out of eighty natives from parts of Uganda where sleeping sickness is endemic had trypanosomiasis in their blood, ${ }^{5}$ whilst in $\operatorname{II} 7$ natives from uninfected areas the blood examination was negative in every case.

The same observers have shown that, contrary to the statements of Castellani, there is no appreciable morphological difference between $T$. gambiense and T. ugandense.

Dutton, Todd, and Christy, in their 'Report upon Trypanosomiasis on the Congo, ${ }^{\prime 6}$ arrived at the same conclusion. They state that the parasites seen in the blood of individuals, whether showing signs of sleeping sickness or not, are identical, and there is no reason to suppose that the trypanosome observed on the Congo differs from T. gambiense.

The pathogenic action of the two trypanosomes upon different species of mammals is the same. Thomas and Linton have made a comparative study of the human trypanosomes derived from

1 Brit. Med. Journ., January 23, 1904, p. 186.

2 Broden, Bull. de la Soc. d'études colon, Brussels, February, 1904.

3 [Thiroux, C. R. Soc. Biol., v. 6o, 1906, p. 778.]

4 [Harvey, R.A.M.C. Journ., v. 4, I905, p. $62 \mathrm{I}$.]

5 [For the after-history of these twenty-three infected individuals, see later (Section 10).]

${ }_{6}^{6}$ Brit. Med. Journ., January 23, I904; [also Thompson Yates and Johnston Lab. Reports, v. 6, part i., I905, pp. I-45.] 
different sources: (I) Trypanosomes brought from Gambia by Dutton and Todd; (2) trypanosome of Bruce and Nabarro from Uganda ; and (3) trypanosomes of Dutton, Todd, and Christy from the Congo (from the cerebro-spinal fluid of sleeping sickness patients, and from the blood of individuals showing no sign of this disease). Thomas and Linton inoculated the trypanosomes from these various sources into.a large number of animals, and they found that the pathogenicity was almost the same in all cases. ${ }^{1}$ Our researches upon T. gambiense and T. ugandense have yielded the same results. ${ }^{2}$

[Laveran ${ }^{3}$ has since studied the pathogenic action upon animals (guinea-pigs, rats, and mice) of three different strains of the human trypanosome: (I) The Gambian trypanosome from a patient in the first stage of trypanosomiasis; (2) the Uganda parasite from a case of sleeping sickness; and (3) a trypanosome from the blood of a missionary in Paris, who contracted the disease in the Ubangi district (French Congo). The virulence of all three strains was about the same.]

[Plimmer ${ }^{4}$ concludes, from certain experiments he has made upon rats, that $T$. gambiense and $T$. ugandense are 'quite distinct and separate.' Rats inoculated with the 'Gambia fever' trypanosome all showed trypanosomes in blood-films, and died on an average two months and twelve days after inoculation. Paralysis and nervous symptoms were absent, but shortly before death the animals became heavy and apathetic. Post-mortem, the spleen was very enlarged and congested, the lymphatic glands were swollen, and the liver was congested and showed cloudy swelling. The cerebral capillaries contained many organisms. On the other hand, rats inoculated with the sleeping sickness trypanosome never showed the parasites in their blood, even on centrifuging post-mortem. All three animals inoculated with this trypanosome became paralyzed two to four weeks before death, and the total duration of the infection was six to nine months. Post-mortem, the spleen was not enlarged in any of the rats, nor could any lesions be found in the other organs. Trypanosomes were found only in the mashed spinal cord, and extracts of the mashed cord produced paraplegia on injection into other rats, but no trypanosomes could be found in these animals.]

[The fact that cases of 'trypanosome fever' frequently appear to terminate in sleeping sickness, Plimmer thinks may be explained by a double infection with these two trypanosomes. $\left.{ }^{5}\right]$

[Thomas and Brein $1,{ }^{6}$ who experimented on a large number of rats with several strains of the human trypanosome, including the same two strains used by Plimmer, obtained results confirming those of Thomas and Linton and of Laveran, and opposed to those of Plimmer.]

1 Thomas and Linton, Lancet, May I4, I904, pp. I337-I340.

2 Owing to the kindness of Dutton, Todd, Annett, and Bruce, I have been able to study the trypanosomes of Gambia and Uganda at the Pasteur Institute. I take this opportunity of thanking them all for their kindness.-A. LAVERAN.

${ }^{3}$ [Laveran, C. R. Acad. Sciences, v. I42, 1906, p. 1065.]

4 [Plimmer, Proc. Roy. Soc., v. 74, 1905, p. 388.]

5 [In a later paper (Proc. R'oy. Soc., Ser. B., v. 79, I907, p. 95) Plimmer gives up the idea that the two strains of human trypanosome are distinct species.]

6 [Thomas and Breinl, Thompson Yates and Johnston Lab. Keports, v. 6, part ii., I905, pp. 93, 94.] 
[Gray and Tulloch ${ }^{1}$ have also studied the effects of different strains of the trypanosome upon white rats. Their results do not confirm those of Plimmer, for they found that the same strain of trypanosome produced the two different clinical types of the disease mentioned by Plimmer.]

[It is now almost, if not quite, universally believed that $T$. gambiense and $T$. ugandense are the same species.]

The objection might still have been raised that the symptoms produced by $T$. gambiense were not those of sleeping sickness. The case recorded by Manson shows that symptoms of sleeping sickness may supervene in a patient who at first has exhibited only the symptoms due to $T$. gambiense. It was one of those cases originally recorded as an instance of typical T. gambiense infection ${ }^{2}$ (p. 354). The patient, who died on November 26, 1903, presented characteristic symptoms of sleeping sickness during the last few months of her illness, and the lesions found at the autopsy were like those met with in sleeping sickness. ${ }^{3}$ This observation is of great importance. It is a very strong argument in favour of the identity of $T$. gambiense and T. ugandense, and, moreover, it shows that, contrary to what was formerly believed, Europeans are not immune against sleeping sickness.

Finally, it appears to follow from observations of Nabarro, ${ }^{4}$ as well as from our own, ${ }^{5}$ that monkeys which have acquired immunity against $T$. gambiense are also immune against $T$. ugandense, and vice versa.

[Subsequent investigations have shown, however, that this acquired immunity is apparent rather than real. Trypanosomes may disappear from the blood of monkeys and remain absent for more than a year, as in the case recorded by Harvey, reappearing shortly before death. Laveran ${ }^{6}$ and Thomas and Breinl ${ }^{7}$ have recently expressed the opinion that no immunity is attainable with the trypanosome of sleeping sickness.]

According to the rules of priority in nomenclature, the name T. ugandense (Castellani) should disappear, and the older name T. gambiense (Dutton) should be retained.

Further, the name sleeping sickness, which aptly describes the ultimate symptoms produced by $T$. gambiense, ${ }^{8}$ is not a good one to

1 [Gray and Tulloch, Sleeping Sickness Reports, No. 8, 1907, pp. 53-63.]

2 Manson, Brit. Med. Journ., May 30, 1903, and December 5, I903, p. I46I.

3 [Low and Mott, Brit. Med. Journ., April 30, 1904, p. 1000.]

4 Nabarro, 'Epidemiological Society,' Lancet, January 23, 1904.

5 Laveran, C. R. Acad. Sciences, April 5, 1904.

${ }^{6}$ [Laveran, Report presented to the Fifteenth International Medical Congress, Lisbon, 1906. Abstract in Brit. Med. Journ., June 2, 1906, p. 1287.]

7 [Thomas and Breinl, op. cit., p. 24.]

8 Bruce and Nabarro found that many of their cases of sleeping sickness in Uganda did not really sleep excessively. Dutton, Todd, and Christy also draw attention to the minor importance of sleep in their cases of Congo trypanosomiasis. Corre had previously made the same observation, for in 1877 he wrote: 'Somnolence, exceptionally pushed as far as coma, rarely continues, and is not absolutely constant. All the patients with sleeping sickness do not sleep. Many remain 
give to the early stage of the infection, which is characterized solely by the presence of a few trypanosomes in the blood and by irregular rises of temperature. The name Human Trypanosomiasis is therefore to be preferred to the older names Sleeping Sickness and Negro Lethargy.

\section{Section 2.-Geographical Distribution.}

Trypanosomiasis is endemic only in certain parts of Equatorial Africa. It has sometimes been seen in the Antilles, but only in negroes coming from the West Coast of Africa. That explains how Guérin was able to study the disease in Martinique.

The chief foci of the disease are found along the coast of West Africa, extending from Senegal to St. Paul de Loanda (see map).

[Wellman ${ }^{1}$ has recently described cases of human trypanosomiasis in South Angola-considerably to the south of St. Paul de Loanda -and the natives state that sleeping sickness is advancing south through the province.]

In Senegambia the disease is common in Casamance and in Sine and Saloum. Cases have been recorded along the banks of the River Senegal in the neighbourhood of Bakel, Kayes, and Bafoulabe. ${ }^{2}$

In Gambia trypanosomiasis is by no means rare; and, as we have seen above, it was in Gambia that trypanosomes were first discovered in human blood. According to the researches of Dutton and Todd, only a small proportion of the natives of Gambia are infected ; but it is probable that, by using other methods of diagnosis, a larger proportion of infected individuals would be found. Cases of true sleeping sickness are met with, as well as the earlier stages of trypanosome infection which were studied by Dutton and Todd.

Trypanosomiasis is prevalent from the mouth of the Gambia to a distance of 250 miles up the river. In French territory, at Maka, about thirty miles from the banks of the River Gambia, Dutton and Todd examined a hundred natives, but did not find trypanosomes in any of them.

Amongst the most infected areas may be mentioned: Upper Guinea, the hinterland of Sierra Leone, of Liberia, and of the Ivory Coast, Lobi, or the country of the Bobos, and Yatenga.

On several occasions the disease has become epidemic at Roba, in Yatenga. In 1850 there were more than 180 victims in this district. From 1886 to $I 889$, in four other villages in the same district, 300 natives died of sleeping sickness.

Secondary foci have been recorded at several points along the

lying down, the eyelids closed, half closed, or quite open, but profoundly indifferent to all going on around them ' (quoted from Hirsch's 'Geographical Pathology').]

i [F. C. Wellman, Journ. Hyg., v. 6, 1906, p. 237.]

2 Kermorgant, 'Distribution of Sleeping Sickness in the French West African Colonies,' Acad, de méd., December 29, 1903. 


\section{0 \\ TRYPANOSOMES AND THE TRYPANOSOMIASES}

Upper Niger, [but Laveran ${ }^{1}$ has recently stated that human trypanosomiasis is rare in this district].

[Renner ${ }^{2}$ has described sporadic or imported cases of the disease in Sierra Leone; but later investigations by Grattan and Cochrane ${ }^{3}$
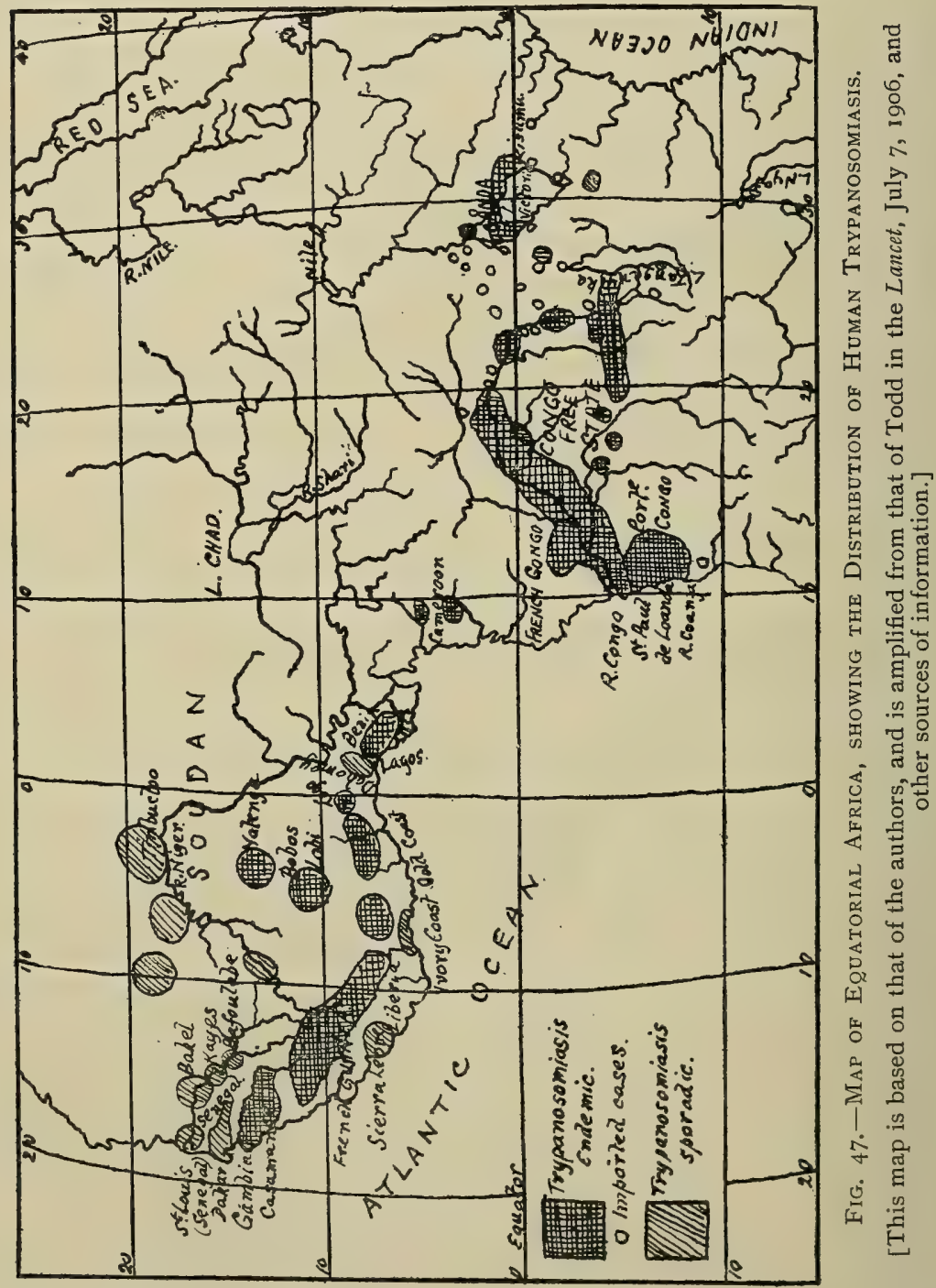

show that trypanosomiasis is much commoner in Sierra Leone than is usually supposed.]

[Between July and December, I905, Grattan ${ }^{4}$ saw eighteen cases-all confirmed by the microscope-and states that probably several more were

1 [Laveran, C. R. Acad. Sciences, v. I43, July 9, 1906, p. 94.]]

2 Renner, Journ. Trop. Med., v. 7, November 15, 1904.]

3 Grattan and Cochrane, R.A.M.C. Journ., v. 6, 1906, p. 524.]

4 [Grattan, R.A.M.C. Journ., v. 7, 1906, p. 485.] 
not diagnosed. Gland palpation and puncture were used in the diagnosis, and found to be much easier than the examination of the blood, either in films or by centrifuging. The disease has been contracted in Sierra Leone by natives who have never been out of the colony or protectorate, so that some of the fly belts are now infected.]

[Gl. palpalis is common in Sierra Leone; Grattan caught specimens at Maferri, near Port Lokkoh, Melanki, Robarri, Ronietta, Yonnibana, Moyamba, Makali, Medina, and Magbele. Some of the flies caught at Warima and Robarri were Gl. fusca. Trypanosomiasis was found to occur at Freetown, Regent, Yonnibana, Kissi Wellington, Port Lokkoh, Waterloo, Tikonko, and Bonthe, at all of which (except the last three) Gl. palpalis is present.]

[An imported case of sleeping sickness in a native of Lagos has been described by Taylor and Currie. ${ }^{1}$ According to G. Martin, ${ }^{2}$ sleeping sickness is probably present throughout French Guinea. G1. palpalis is also very widely distributed, but only a small proportion of the natives are affected by trypanosomiasis.]

[Krueger ${ }^{3}$ has described several cases of sleeping sickness in Togo, and states that the disease is endemic there, II5 deaths having occurred from this disease in recent years. Gl.palpalis is also very prevalent.]

According to Ziemann, sleeping sickness is also prevalent along the coast at Monravia and Accra, and in several districts of Cameroon, notably at Etun-beka-ha and at Etun-bekani. ${ }^{4}$

[Gl.palpalis is found in several of the coast districts in Cameroon, such as Victoria, Buea, Barombi, so that all the conditions for the spread of sleeping sickness are present (Ziemann). $]^{5}$

The islands of Princes, St. Thomas, and Fernando Po are also infected.

In Portuguese Congo (Angola) the disease occurs principally in the region of Quissama and along the banks of the River Coanza. ${ }^{6}$ In the town of Dondo and its environs there were, in I 900,98 deaths from sleeping sickness amongst 2,000 negroes or half-castes. At Tombo, near the mouth of the Coanza, practically the whole population has been carried off by the disease. At Benguella the disease is less prevalent than in Loanda, and still further south, particularly in the province of Mossamedes, it disappears entirely.

[Wellman ${ }^{7}$ states that the River Coanza seems to mark the south limit of the zones of endemicity of sleeping sickness, but recently

1 [Taylor and Currie, Brit. Med. Journ., February 4, I905, p. 248.]

2 [G. Martin, Ann. Hyg. et Méd. colon., v. 9, 1906, p. 304; Les Trypanosomiases de la Guinée française, Paris, 1906, chapter x. 1

3 [Krueger, Arch. f. Schiffs in Tropen Hyg., v. 8, 1904, p. 479; abstract in Bull. Inst. Past., v. 3, 1905, p. 47.]

4 Deutsche med. Wochenschr., April 2, I903. For the distribution of human trypanosomiasis in Togo, see K. Hintze, Deutsche med. Wochenschr., May I9 and 26, 1904, pp. 776, 81 2 .

5 [Ziemann, Centralb. f. Bakter., I, Orig., v. 38, 1905, p. 307.]

6 Keport of the Portuguese Commission, Lisbon, Igo3.

7 [F. C. Wellman, 'Notes from Angola' in Journ. Trop. Med., v. 8, pp. 319, 320, and 327,328 ; also Journ. Hyg., v. 6, 1906, p. 237.] 
three cases of trypanosomiasis were seen by Wellman and Fay in natives who had never left their district (South Angola). According to native accounts, sleeping sickness is advancing south through this province. To the north of the Coanza typical Gl.palpalis are found, but to the south one finds a variety-Gl.palpalis wellmani, Austen.]

[Kopke $^{1}$ has studied fifty-two cases of sleeping sickness from various Portuguese West African colonies. He used gland puncture in the diagnosis and found T. gambiense present in every case. Lumbar puncture was performed in forty cases, and in every caseeven in the absence of nervous symptoms - trypanosomes were found in the cerebro-spinal fluid.]

Sleeping sickness is endemic in French Congo and in the Congo Free State, where for several years it has been extending rapidly and causing great ravages. Sporadic cases are also met with at l'Ouellé, Basako, Bangala, Leopoldville, and Boma. The disease is endemic in the neighbourhood of the falls, especially at Banza Manteka. In the training college at Berghe-Ste.-Marie, at the junction of the Kasai and the Congo, cases of sleeping sickness have multiplied rapidly in recent years. The mortality, which in I8g6 was I3 per cent. and in I897 I9 per cent., rose in the following years to 39 and 22 per cent. respectively, and in the first quarter of 1900 to 73 per cent. The majority of the children died of the disease. At the time van Campenhout ${ }^{2}$ visited the colony eighty-two patients with sleeping sickness came to see him, and there were many others who did not come.

The whole population of the left bank of the Congo, from the mouth of the Kasai to Bolobo, higher up the river, has fallen a prey to the scourge which has decimated the villages of the Botanguis.

[Kermorgant, ${ }^{3}$ in a series of papers, gives details of the frequency of sleeping sickness along the French banks of the Congo and of its tributary the Ubangi, as far as Bangi, and in Loango. The disease is rare at Libreville, and in the valleys of the Ogoué and Upper Sanga.]

[In an interesting paper on 'A Means of Checking the Spread of Sleeping Sickness,' Todd ${ }^{4}$ shows that the enormous spread and great increase of sleeping sickness in the Congo basin have been due in great measure to the increase in travel following the opening up of the country. Before Europeans entered the Congo basin (I884) the

1 [A. Kopke, 'Fifteenth International Congress of Medicine,' Lisbon, 1906: also Arch. de Hyg. et Path. exot., v. I, 1905; abstract by Mesnil in Bull. Inst. Past., v. 4, I906, p. 671.]

2 Van Campenhout and Dryepondt, Rapport sur les travaux du laboratoire médicale de Léopoldville en I899-I900, Brussels, I90I, and Paris International Congress, 1900 (section on Colonial Medicine).

${ }_{3}$ [Kermorgant, Ann. d'Hyg. et de Méd. colon, 1903-1906; ibid., v. 9, 1906, p. I26; abstract by Mesnil in Bull. Inst. Past., v. 4, I906, p. 53I.]

${ }_{4}^{4}$ [J. L. Todd, Transactions of the Epidemiological Socicty, 1906; also Lancet, July 7, I906, pp. 6-9, with three maps; and Memoir I8 of the Liverpool School of Tropical Medicine, 1906, pp. 23-38, with four maps.] 
disease seems to have been confined to the region of the Lower Congo and to the banks of the main river as high up as Bumba. With the advent of white men new steamer and caravan routes were opened up, with the result that the infected areas have increased rapidly in number and extent. Todd's maps show that 'imported' cases of the disease have occurred at many points along the eastern boundary of the Congo State, in close proximity to the British and German East African possessions, and that cases have already occurred at Ujiji, on the eastern shore of Lake Tanganyika, in German territory. $\left.{ }^{1}\right]$ [Unless effective quarantine measures - such as those outlined by Todd-dependent upon cervical gland palpation, be speedily introduced and enforced, there is very grave danger of the disease being carried into Nyassaland and other parts of British Central Africa, as well as into areas of Uganda and German East Africa at present unaffected. The danger is not only great but imminent, and prompt and energetic action on the part of the Governments concerned is imperative.]

For some years trypanosomiasis has made great ravages in Uganda. ${ }^{2}$ [The disease appears to have broken out first in Busoga in I896. ${ }^{3}$ Dr. Moffat, then Principal Medical Officer, Uganda and East African Protectorates, explains its first appearance in Busoga by the fact that a large number of Emin Pasha's Soudanese, with their wives and followers, were brought into and settled in Busoga. They came from the Congo Free State, where sleeping sickness has been endemic for many years. Finding the necessary conditions present, the disease rapidly spread, and in a few years became endemic around the northern shores of the Victoria Nyanza-from Buddu on the west to south of Kavirondo Bay on the east-in Busoga and Chagwe, around Entebbe, and on the islands in the lake. The disease was first definitely diagnosed by the Drs. Cook, ${ }^{4}$ of the C.M.S. Hospital, Mengo, in IgOI, and so merciless has been its onslaught that it has claimed probably more than Ioo,00o victims in Uganda alone during the past six years (I90I-I906).]

[The distribution of the disease in Uganda is peculiar. Except in the district of Busoga, sleeping sickness has not become endemic far from the shore of the lake or from the banks of rivers. The villages along the lake shore and on the islands of the lake have been most severely visited, many of them having lost two-thirds or more of their inhabitants. The extent to which the disease spreads inland from the lake differs in different parts and varies from ten up to thirty or forty miles.]

${ }^{1}$ [In a later paper Kinghorn and Todd (Lancet, February 2, 1907, p. 282) state that the disease now extends much further south. It is now endemic along the eastern shores of Lake Tanganyika, between about $4^{\circ}$ and $7^{\circ}$ S. Lat. Imported cases have been reported from places even further south.]

2 Royal Society, Reports of the Sleeping Sickness Commission. See reports by Bruce, Nabarro, and Greig, by Low and Castellani, and by Christy.

3 [Hodges, Lancet, July 30, I904, p. 290.]

4 [J. H. Cook, Journ. Trop. Med., July 15, 1901.] 
[Bruce, Nabarro, and Greig have shown that this peculiar distribution of the disease almost coincides with, and is probably dependent upon, the distribution of a tsetse-fly-the Gl. palpalis. This fly is found only near large water-courses where there is dense undergrowth and shade, as from large trees; it is not found in papyrus swamps or in large open plains. For some reason at present unexplained, the tsetse-fly has been found under other conditions in various parts of Busoga, which no doubt is the cause of the greater area of endemicity of sleeping sickness in that district than in other parts of Uganda. Of course, there may be-and investigations by Nabarro and Grieg concerning the shores of the Albert Nyanza and the banks of the Nile to the north of this lake show that there are-uninfected fly areas where sleeping sickness does not occur. An imported case of the disease may be the means of making such areas endemic foci of trypanosomiasis.]

Christy gives the following dates for the appearance of the disease in different parts of Uganda: Several parts of Busoga or the island of Buvuma, I896 (Hodges); Kampala, February, Igor; South Kavirondo, October, I9or ; Sesse Islands, December, Igor; Kasagunga, January, I902; Lusinga Islands and Kasachonga, March, I902; Kisengere, May, I902 ; and German East Africa, September, I902.

According to these dates the disease is manifestly spreading, and it is to be feared that the Uganda Railway, which runs from the East Coast to Lake Victoria, will facilitate its spread, for Kisumu, the terminus of the railway on the lake, is already infected. It is possible, too, that the disease may spread to Egypt along the valley of the Nile.

[In $1903^{1} \mathrm{Mr}$. Wyndham, of the Uganda Civil Service, found that Gl. palpalis was prevalent on both shores (Uganda and Congo Free State) of the Albert Nyanza, and since then the fly has been found between Fajao, on the Victoria Nile, and the north end of the Albert Nyanza, as well as at various places down the Nile, including the neighbourhood of Wadelai and Nimule. The furthest point north at which Gl.palpalis was met with by Hodges $^{2}$ was near a small stream about thirty-five miles south of Gondokoro, between Kanda and Shindiro.]

[Balfour ${ }^{3}$ states that $\mathrm{Gl}$. palpalis has been found in the Lado Enclave at Wandi, and also in the southern part of the Bahr-el-Ghazal province. Another species of Glossina, Gl. morsitans, occurs in many parts of this province-sometimes in very large numbers-and has been found by Morant as far north as S. Kordofan.]

[When Gl. palpalis was first discovered in the region of the Victoria Nile and around the Albert Nyanza in I9o3, sleeping sickness was unknown there. By the end of 1904 , however, this fly area had certainly become infected, for cases of sleeping sickness were seen by Greig ${ }^{4}$ at Fajao and at other places near the northern extremity of the Albert Nyanza, and along the Nile as far north as Wadelai. Subsequently

1 [Nabarro and Greig, Report of Slecping Sickness Commission, No. 5, pp. 46, 47.]

2 [Hodges, Report of Sleeping Sickness Commission, No. 8, pp. 86-99.]

3 [Balfour, Second Report of the Welliome Research Laboratory, Khartoum, I 906 ; see p. 29 and map.]

${ }^{4}$ [Greig and Gray, Report of Sleeping Sickness Commission, No. 6, p. 276.] 
(1905) Hodges found that the disease had spread in this part of Uganda (Unyoro) and in the Nile Valley; but the conditions favourable to the occurrence of a great epidemic such as that on Victoria Nyanza ${ }^{1}$ are not present in these parts of Uganda and the Nile Valley, except, perhaps, on the shores of Lake Albert.]

[Sleeping sickness will probably make its way down the Nile as far as Gl.palpalis extends, but the danger of its spreading to Egypt is not great, because this tsetse has not been found along the Nile north of Gondokoro ; nor is it likely to occur here, for the character of the country is unfavourable to the fly (Greig). We know, however, that several other species of tsetse-fly occur in the Anglo-Egyptian Sudan, in British and German East Africa, in British Central Africa, and as far south as Natal and the Transvaal. If, as appears highly probable from the experiments of Nabarro, Greig, and Wiggins in British East Africa, other species of Glossina than Gl.palpalis can act as carriers of $T$. gambiense, there is grave danger of sleeping sickness spreading east to the coast, north into the Sudan, and south through British Central Africa even as far as the Transvaal. The most stringent precautions and energetic measures should be taken to prevent the infection of this enormous fly-belt by natives from the endemic foci in Uganda and the Congo State.]

[In November, I903, Nabarro saw a case of sleeping sickness, which had been imported from Uganda, in the hospital at Nairobi, British East Africa, but hitherto the disease is unknown amongst the Nandi and Masai tribes of British East Africa.]

[In September, I904, Dr. Ahlbory, ${ }^{2}$ in writing from Muanza, German East Africa, states that sleeping sickness and Gl.palpalis occur on the Gori River ; also that an imported case of sleeping sickness from Uganda had been observed in Muanza. He states further that trypanosomiasis had been discovered in a child of two born in Tabora, which had never left the districts of Unyamwezi and Ussukuma. ${ }^{3}$ ]

[A European who contracted the disease in German East Africa recently died in Hamburg. ${ }^{4}$ All these facts show that trypanosomiasis is obtaining a footing in this German colony, which has

1 [Hodges gives the following as being the conditions necessary for the occurrence of a great epidemic: (I) The presence of Gl.palpalis in large numbers over a considerable area; (2) a thickly-gathered, numerous population; (3) free and frequent intercommunication, much of it within the fly range; (4) a considerable part of the population either living or daily employed within the fly range; (5) a coast or banks much broken by inlets, estuaries, and rivers, and with adjacent islands. These conditions rarely coexist in such fatal completeness as they do round the northern shores of Victoria Nyanza (Hodges).]

2 [See R.A.M.C.Journ., v. 4, 1905, p. I24.]

3 [For the latest information concerning the progress of sleeping sickness in Uganda and German East Africa, consult the Reports of H.M. Commissioner for the Uganda Protectorate, also Koch in the Supplement to No. 5 I of the Deutsch. med. Wochenschr., December, I go6.]

${ }^{4}$ [Nocht and Mayer, in Kolle and Wassermann's 'Handbuch der Pathogenen Micro-organismen,' Appendix, p. 56.] 
probably become infected both from Uganda and from the Congo. $\left.{ }^{1}\right]$

We may summarize the information we already possess about the geographical distribution of this disease by saying that it extends along the valleys of the Senegal, Niger, Congo, and Upper Nile, and occurs also in the valleys of less important rivers situated between them (Manson). Hitherto the disease has not been recorded either in North or South Africa.

\section{Section 3.-Predisposing Causes. The Influence of Age, Sex, Occupation, Race, etc.}

Age appears to have no marked influence upon the disease. Christy has seen symptoms of sleeping sickness in many infants of eighteen months to two years, and these children had become infected a considerable time previously. ${ }^{2-}$ [By gland puncture Dutton and Todd, on the Congo, found trypanosomes in an infant less than nine months old.] The two sexes are attacked in the same proportion. The influence of occupation and social position is very marked. The majority of cases are seen amongst the agricultural workers and amongst the poorest classes. The native chiefs and persons belonging to the upper classes living in the villages are attacked in a much smaller proportion than the poorer negroes who work in the fields all day.

For a long time it was believed that the disease attacked only negroes. In 1859 Chassaniol reported a case of the disease in a mulatto at Gorée. In Portuguese Congo half-castes are often attacked (Bettencourt). The cases recorded by Dutton and Todd, Manson, Broden, Brumpt, and others, show that trypanosomiasis does

1 [Kudicke (Centrxlb.f. Bakter., I, Orig., v. 4I, 1906, p. 72) has described a case of human trypanosomiasis in a native of German East Africa, apparently not due to $T$. gambiense. Clinically the patient was in the first stage of trypanosomiasis: febrile attacks not responding to quinire, enlarged lymphatic glands, pulse-rate increased, and spleen slightly enlarged. Occasionally there were slight fibrillary tremors of the tongue and difficulty with speech; once there was an urticarial eruption, which did not irritate, and soon disappeared. Trypanosomes could not be found in blood-films, but a Cercopithecus injected with 9 c.c. blood showed very few trypanosomes in its blood seventeen and twenty-one days after injection. The monkey died of sepsis a few days later. Rats, monkeys, and a cow injected with the monkey's blood all failed to become infected. The trypanosome was considerably larger and broader than $T$. gambiense; the posterior end was very pointed, and the oval nucleus was situated at the junction of the middle and posterior thirds of the body. The parasites were all alike, and resembled $T$. theileri, but were rather smaller, and the undulating membrane was better developed. The patient was treated with arsenic for a time, and the febrile attacks ceased. The after-history of the case is unknown.]

[Todd and Tobey (Liverpool School of Tropical Medicine, Mem. 21, Sep. tember, 1906, pp. 93, 94) describe a very similar trypanosome which was observed in the fresh blood of two monkeys (Cercopithecus schmidti) at Kasongo, Congo State. The trypanosomes were not found in stained specimens. Todd and Tobey think it is a hitherto undescribed parasite of the monkey, and that it is the same as that seen by Kudicke.]

" Christy, 'The Epidemiology and Etiology of Sleeping Sickness.' Royal Society, Report of the Sleeping Sickness Commission, November, I903. 
not spare Europeans. We have already seen that in a case of Sir Patrick Manson's the characteristic symptoms of sleeping sickness were observed in a white woman.

In IgO4 Dupont, ${ }^{1}$ a practitioner from the Congo Free State, recorded three cases of sleeping sickness in Europeans who had lived on the Congo. Trypanosomes were found in the cerebro-spinal fluid of only one of these patients, but in the other two cases the clinical symptoms pointed clearly to the existence of sleeping sickness.

[During the last few years several more cases of trypanosomiasis and sleeping sickness have been recorded in Europeans. Nearly all the European countries with African colonies have contributed to the ever-increasing list of victims to this disease. A recent writer ${ }^{2}$ in the British Medical Journal states that hitherto seven cases of trypanosomiasis are known to have occurred amongst English people. Four of these have died; of the three still alive, one is said to be quite well and the parasite has disappeared, while the other two have been free from active symptoms for several months. The writer of the article-and Sir Patrick Manson is of the same opinion-thinks that there may be a small percentage of recoveries from trypanosomiasis in man, and that the terminal and fatal condition-sleeping sicknessis not inevitable.]

[Two of these cases are of more than ordinary interest. One was that of a European who was under the care of Dr. Moffat in Entebbe, Uganda, in March, I903, as already recorded by Bruce and Nabarro in their 'Progress Report on Sleeping Sickness in Uganda,' p. I7. This patient was curator of the Botanical Gardens, which are situated on the lake shore, and are full of tsetse-flies and rank vegetation. The disease started with irregular fever and delirium; no malarial parasites were seen at any time, but trypanosomes were discovered in the blood on April 2, I903. The patient was invalided to England on April 6, and died exactly three years later-on April 6, I 906-of typical sleeping sickness.]

[While in England the patient had periodical febrile attacks. He first became drowsy in November, 1904, having previously suffered from delusions. Latterly he became more drowsy and apathetic, and was admitted to hospital for the third time in October, I905. ${ }^{3}$ He was then very weak and had difficulty in swallowing his food. While in hospital he was sometimes very lethargic, not speaking for days, while at other times he brightened up and took his food well. On several occasions his temperature rose to $103^{\circ}$ or $104^{\circ} \mathrm{F}$., accompanied by a rigor or fit, epileptic in character. Neither trypanosomes nor malarial parasites were found in the blood on these occasions. On March 28, I906, the temperature fell to $92^{\circ} \mathrm{F}$., but rose again to $98^{\circ} \mathrm{F}$. a few days later. It then steadily and progressively fell, and on the day of death-April 6 , I906-was $84^{\circ} 2^{\circ} \mathrm{F}$. On April 4 he was very drowsy, but still sensible. In the evening he had a fit, which lasted about ten minutes. The next day he was almost unconscious, but winced when lumbar puncture was performed. Trypanosomes

1 Dupont, Le Caducée, April 16, I904.

${ }^{2}$ [Brit. Med.Journ., May 5, 1906, p. 1062.]

3 [I am indebted to Dr. Rose Bradford, under whose care the patient was while in University College Hospital, for permission to use the notes of the case, and to make the post-mortem bacteriological investigation.] 
were easily found in the cerebro-spinal fluid. The next day the patient died, and post-mortem the brain showed the typical macroscopic appearance of sleeping sickness, and there were broncho-pneumonic patches in both lungs. The heart blood and cerebro-spinal fluid were found to be sterile.]

[Dr. Mott, who examined the brain and other organs microscopically, found the typical lesions of sleeping sickness present.]

[The other case was that of a young scientist, ${ }^{1}$ a member of the Royal Society's Sleeping Sickness Commission in Uganda, who cut himself during the dissection of an infected rat. Trypanosomes soon appeared in his blood, and after an unusually rapid course, the infection ended fatally in June, I906, having lasted only a few months.]

[Records of other recent cases of trypanosomiasis in white people have been summarized by Mesnil. ${ }^{2}$ These cases have been reported by Carlos França, ${ }^{3}$ Dias de Sa, ${ }^{4}$ Martin and Girard, ${ }^{5}$ Sicard and Moutier, ${ }^{6}$ and Willems. ${ }^{7}$ In all but Dias de Sa's case, in which hypnotic symptoms were absent throughout, these were all cases of sleeping sickness. Willems mentions four cases which came under his observation. There seems to be no doubt about the diagnosis, as the symptoms were very definite, but the diagnosis was not confirmed microscopically. In all the other cases trypanosomes were found in the body fluids of the patients or in the blood of injected animals.]

[Other cases have been recorded by Broden ${ }^{8}$ in a missionary from the Congo, and by Günther and $W^{2}$ ber $^{9}$ in a patient who probably contracted the infection in Cameroon in I902. Reference has already been made to the case of a European who became infected in German East Africa and died recently in Hamburg.]

[Nabarro and Greig ${ }^{10}$ have recorded the case of a Persian-the first reported instance of the disease in an Asiatic-who, having been in Uganda on and off for eighteen years, had come into close contact with the natives and contracted sleeping sickness, of which he died towards the end of I903 (see Fig. 5I). Two cases of trypanosomiasis have recently been observed by Gray in Indian soldiers in Uganda.]

The seasons appear to exert no influence upon the course of the disease, but on account of the long period of incubation, or rather of latency, which, as a rule, precedes the appearance of nervous symptoms, it is difficult to be sure about this.

We have already mentioned that the disease has been wrongly

1 [I refer to the late Lieutenant Forbes Tulloch, R.A.M.C., whose untimely fall on the field of battle against disease is deplored by all.]

${ }^{2}$ [Mesnil, Bull. Inst. Past., v. 4, 1906, p. 126, from which the following five references are taken.]

${ }^{3}$ [Carlos França, quoted from Le Semaine médicale, 1905, p. 48.]

4 [Dias de Sa, Porto medico, $190 \%$, No. 2.]

5 [Martin and Girard, Bull. médic., April 29, I905 ; Lancet, May 20, 1905, p. I385; also L. Martin, Ann. Inst. Past., v. 21, 1907, pp. I61-193.]

${ }^{6}$ [Sicard and Moutier, Bull. Soc. mét. des Hôpitaux, June 30 and December 8, 1905.

7 [Willems, 'La Maladie du Sommeil,' Brussels, 1905.]

8 [Broden, Publications de la Soc. 'd' Etudes colon. de Belg., 1905.]

9 [Günther and Weber, Mïnch. med. Woch., June, 1904, pp. I044-1047.]

10 [Nabarro and Greig, Sleeping Sickness Reports, No. 5, 1905, p. 33.] 
attributed to manioc-eating. Fish, or at least certain kinds of fish, has often been mentioned amongst the causes of the disease, on account of the frequency of its occurrence along the banks of certain watercourses and, in Uganda, in the villages bordering on Lake Victoria. But in certain districts where the natives eat as much, and the same kinds of, fish as in the infected districts the disease is not endemic.

Wars and famine have favoured the spread of sleeping sickness in Africa. This is the usual effect of these scourges upon epidemic diseases, and sleeping sickness, naturally endemic, may assume an epidemic form.

The Soudanese soldiers disbanded after the relief of Emin Pasha by Stanley in I 888 contributed, according to Christy, ${ }^{1}$ to the rapid spread of the disease throughout Uganda. In I90I and I902, according to the same observer, the mortality from this disease was increased by famine in the islands in Lake Victoria.

Along the Ivory Coast, before its invasion by bands of Samori, sleeping sickness was much less common than it is at the present day (Kermorgant).

The chief foci of the disease are met with along the rivers in wellwooded districts. This is easily explained. We shall see later on that sleeping sickness is spread by a biting fly, Gl.palpalis, and that this fly lives along the watercourses in districts where vegetation is abundant. When patients suffering from trypanosomiasis arrive in those areas where Gl. palpalis abounds, the disease is spread; on the other hand, in districts where the fly is absent the introduction of infected individuals is followed by no serious result. It is just the same as with malaria, yellow fever, nagana, and, in short, with all diseases which are propagated by a special insect: the disease is infectious only where the insects capable of propagating it occur.

\section{Section 4.-Description of the Disease.}

All authors are agreed that the onset of the disease is insidious. We know that trypanosomes may be present for a long time in the blood before getting into the cerebro-spinal fluid, and thus giving rise to the symptoms of sleeping sickness, so that two distinct stages. can be recognised during the course of the disease.

In the first stage trypanosomes occur in the blood, but usually only in small numbers. Generally they give rise to no symptoms in negroes, but in white people there is irregular fever. In the second stage pain in the back, tremors, and, later, drowsiness occur, and the temperature is of a hectic type. The drowsiness goes on to lethargy, and the patient finally falls into a comatose condition. The temperature becomes subnormal before death. Trypanosomes are practically always present in the cerebro-spinal fluid.

${ }^{1}$ [Christy obtained this infurmation from Dr. Moffat; see p. 363.] 
Hitherto the descriptions of sleeping sickness have referred only to this second phase of the disease.

First Stage [Trypanosome Fever, so called.]-In negroes the diagnosis could formerly only be made by the examination of the blood. The parasites are very scanty, and therefore often difficult to discover in the blood. [Mott was the first to suggest that possibly the trypanosomes might be more easily found in the enlarged lymphatic glands than in the blood. Greig and Gray, in Uganda, practically tested Mott's suggestion, and found that trypanosomes were often discernible in the fluid obtained by gland puncture when they could not be seen in the blood. Gland puncture is therefore the best means of making the diagnosis of early trypanosomiasis, but in the absence of the necessary apparatus and skill, gland palpation may be relied upon almost as much. Dutton and Todd have found that cervical gland enlargement, without obvious cause, in a native who has been exposed to the risk of infection, is almost certainly due to trypanosomiasis, and should be regarded as such until the contrary is proved.]

This latent period of the infection varies very much in length: sometimes it is very short ; at other times it may extend over several years. [In a case of trypanosomiasis in a European contracted on the Congo, Dutton, Todd, and Christy ${ }^{1}$ found 'that the incubation period between the time of infection with $T$. gambiense and the appearance of the symptoms associated with trypanosomiasis may be as short as four weeks.']

According to Corre, the inhabitants of the island of Gorée (Senegal) who had lived in Casamance did not consider themselves safe from the disease until at least seven years after they had left the infected area. Guérin, in the Antilles, has recorded cases in negroes who had quitted Africa five to eight years previously, and there is no doubt that these negroes had brought the germ of the disease from Africa. The disease, however, never spread in the Antilles. Manson writes: 'The disease may appear seven years after having left an endemic area.'

The observations of Dutton and Todd in Gambia, and of Bruce, Nabarro, and Greig in Uganda, show that in negroes this first stage is accompanied, as a rule, by no obvious signs of disease [except the glandular enlargement above referred to]; but this is not the case with mulattos and whites. Fever is the chief sign of the infection. The patients suffer from an irregular intermittent fever, the temperature being raised for two to four days, then falling to normal or below normal for four or five days. In other cases the fever is of the hectic type, the temperature being normal in the morning, but rising in the evening to $38.5^{\circ}$ or $39^{\circ} \mathrm{C}$, and rarely to $40^{\circ} \mathrm{C}$. [104 $4^{\circ} \mathrm{F}$. . The rises of temperature are not preceded by rigors, and the sweat-

1 [Dutton, Todd, and Christy, Thompson Vates and Johnston Lab. Reports, v. 6, part i., 1905, section 6.] 
ing which occurs at the end of the rise is, as a rule, only slightly marked. The respiration and pulse rates are increased quite apart from the febrile attacks; the respiration rate is from 25 to 30 a minute, and the pulse rarely falls below 9o. Cardiac excitability is, according to Broden, a constant symptom, the pulse rate. often reaching $I 40$ a minute.

Localized œedemas and erythemas are often present, such as puffiness of the face, œdema of the eyelids and ankles, and evanescent congested or erythematous patches on the face, trunk, or limbs. Anæmia, general weakness, and wasting are slightly marked at first, but gradually increase, and headache is present in many cases.

The spleen is often enlarged, but this is not always the case,

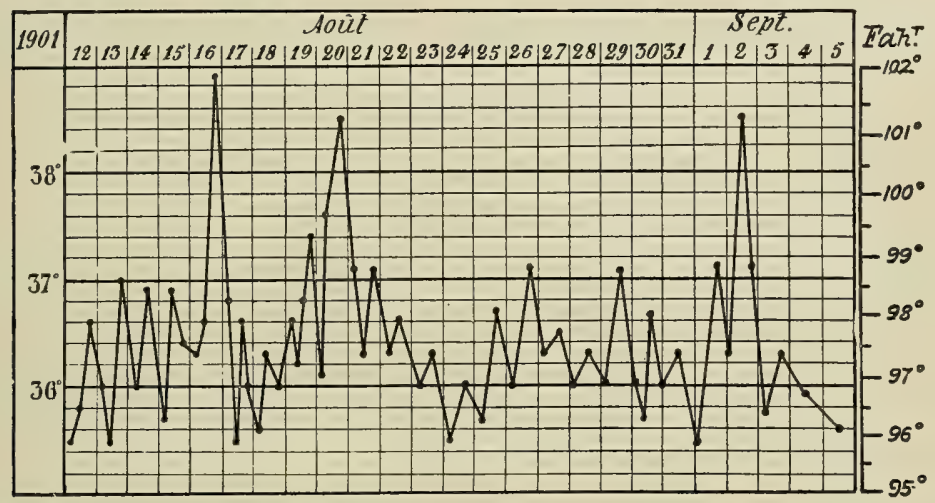

Fig. 48. - Human Trypanosomiasis. Part of the Temperature Chart of Dutton's First Patient during his Stay in the Liverpool Hospital.

especially at the outset. Baker observed splenic enlargement in only one of his three cases. Broden also thinks that the spleen is only slightly enlarged in the first stage of the disease. Moreover, the prevalence of malaria in those districts where trypanosomiasis occurs may itself give rise to enlargement of the spleen. The liver dulness is increased in some cases. In the absence of complications, the other organs show nothing abnormal.

In Dutton's first case the duration of the disease was at least nineteen months before it ended fatally. Dutton and Todd's case in a quadroon also ended fatally after one year and a half.

In certain cases trypanosomiasis may terminate fatally without giving rise to the characteristic symptoms of sleeping sicknesssymptoms which may occur in Europeans and Asiatics as well as in blacks, as is shown by the very interesting case published by Manson in $I 903$ [and by the later cases], to which we have already referred.

Second Stage [Sleeping Sickness, so called.]-The chief signs are fever and symptoms associated with the nervous system.

The temperature, which in the first stage of the disease shows considerable elevations, with fairly long intervals of apyrexia, is in the second stage of a hectic type. As a rule, considerable oscillations 
are observed, the temperature in the evening often rising to $39^{\circ} \mathrm{C}$. $\left[\mathrm{IO}^{\circ} 2^{\circ} \mathrm{F}\right.$.], and falling in the morning to $37^{\circ} \mathrm{C}$. [98.6 $\mathrm{F}$.]. It is important to bear in mind this type of fever, for the diagnosis has often to be made between trypanosomiasis and malaria. In malaria we know that, as a rule, the highest temperatures are observed in the morning, whereas in trypanosomiasis and in 'hectic' fever the reverse is the case.

The rise of temperature is, as a rule, unaccompanied by rigors or sweating. Patients will sometimes attend to their daily occupation with a temperature of $39^{\circ}$ to $39^{\circ} 5^{\circ} \mathrm{C}$. [ $\left[\mathrm{IO}^{\circ}\right.$ to $\mathrm{IO}^{\circ} \mathrm{F}$.] , and it is therefore necessary to use a thermometer in order to ascertain whether the temperature is raised, and not to rely upon the statements of patients.

The pulse-rate is increased, and, moreover, may vary considerably in frequency during the day, increasing, perhaps, from 90 to I 30 per minute, without a corresponding rise in the temperature. The respiration rate is also increased to 20 or 30 per minute. There are often irregularities in the temperature chart due to complications, and particularly to malarial attacks. For several weeks before death the temperature falls below normal, and this subnormal temperature, when it occurs in the evening as well as in the morning, is always of fatal import. Fig. 49 shows a typical temperature chart of a sleeping sickness patient. [During the last seven to fourteen days the rectal temperature often falls to below $95^{\circ} \mathrm{F} .\left(35^{\circ} \mathrm{C}\right.$.), and for several days may be as low as $92^{\circ} \mathrm{F} .\left(33^{\circ} 3^{\circ} \mathrm{C}\right.$.) in the morning. In the case of the European mentioned on p. 367 , the temperature steadily fell from $95^{\circ} \mathrm{F}$. during the last five days of life, and shortly before death reached the very unusual figure of $84^{\circ} 2^{\circ} \mathrm{F} .\left(29^{\circ} \mathrm{C}\right.$.), taken in the rectum.] With this fall of temperature the frequency of the pulse and respiration is usually diminished.

Headache and a change in the mental condition or temperament of patients are constant symptoms at the commencement of the second stage of the disease. Headache occurs in the supra-orbital regions; some patients complain of a sense of constriction in the temples (Guérin). The headache is often accompanied by pain in the back and in the upper part of the chest. ${ }^{1}$ Patients who were active and intelligent become lazy and dull. Apathy is one of the chief features of the disease; the face loses its ordinary expression, and the patient is drowsy, and when asked questions replies only after a considerable interval. [Figs. 50 and 5 I show very well the characteristic expression and facial aspect in sleeping sickness.]

Tremor of the tongue and hands is nearly always present. In the tongue it is usually of a fibrillary character. The tremor of the hands and arms which is present during rest often becomes increased on voluntary movement, such as raising a glass of water to the lips,

${ }^{1}$. [In two cases recorded by L. Martin pain in the feet was a very marked and persistent symptom.] 
etc. In certain cases tremors also affect the lower limbs and trunk, and may even shake the bed on which the patient is lying.

Towards the end rigidity of the neck muscles and marked flexion of the lower limbs are frequently observed. Epileptiform
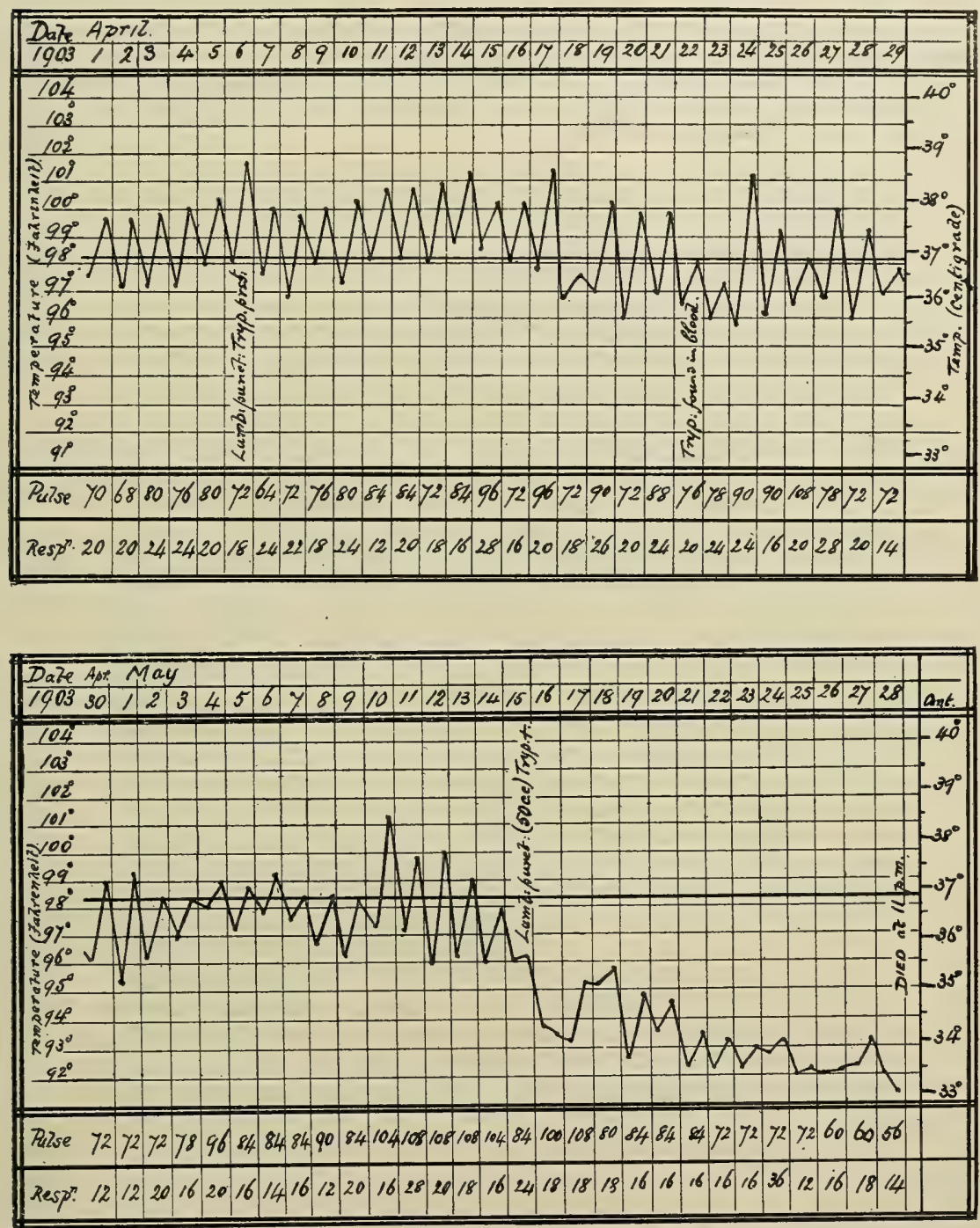

Fig. 49. - The Temperature Chart of a Sleeping Sickness Patient. ${ }^{1}$ (Case 42. Bruce and Nabarro's Report, No. I, p. 85.)

convulsions, either generalized or localized to certain groups of muscles, occasionally occur. Paralyses are very rarely seen, but

1 [This chart is inserted in place of that given in the original, as being more complete and probably more correct in details. The temperatures given by Low and Castellani, whose chart Laveran and Mesnil copied, were taken in the axilla, and no records were made below $95^{\circ} \mathrm{C}$. The temperatures in all our cases were taken in the rectum.-ED.] 
there is general weakness or asthenia, which gradually becomes more pronounced, and is accompanied by progressive emaciation.

In the last stages there is incontinence of urine and fæces.

Beyond the pains already referred to there is little or no sensory disturbance. There is no anæsthesia, but zones of hyperæsthesia are sometimes found. The superficial reflexes are usually normal; the pupils are equal and generally dilated, and there are no ocular paralyses or changes in the fundus oculi.

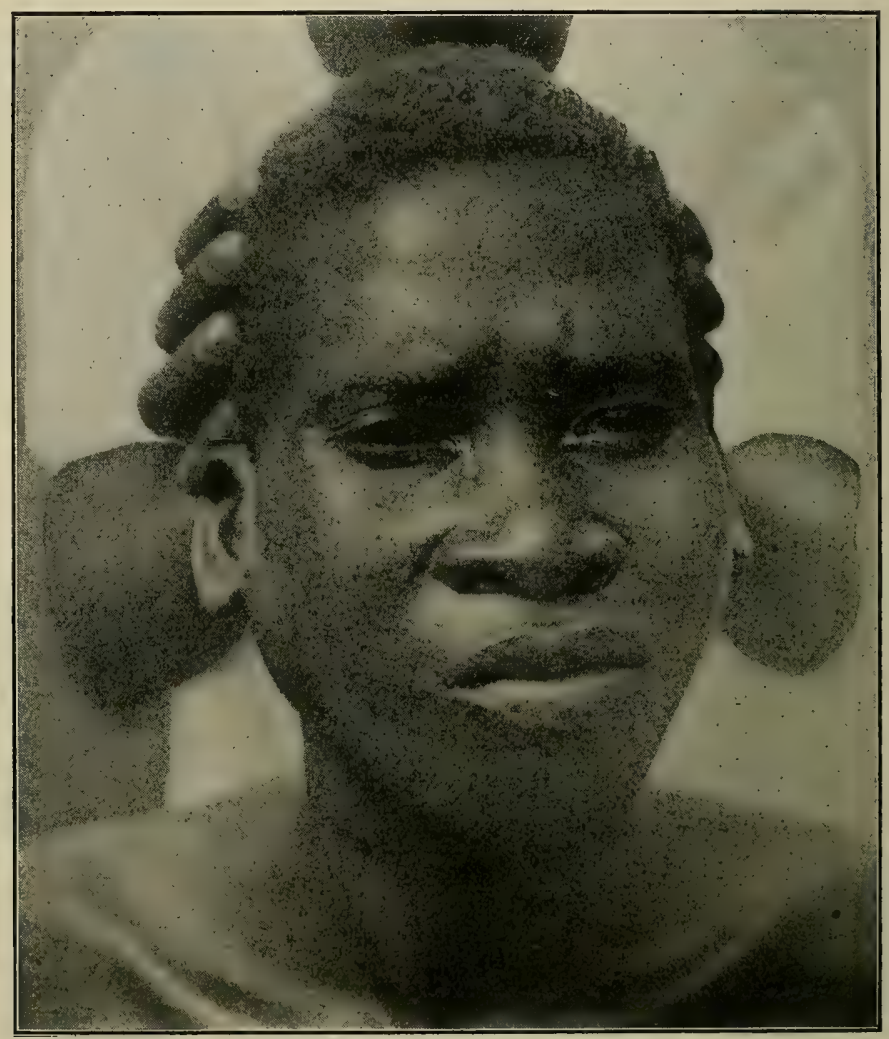

Fig. 50.-Photograph of an Advanced Case of Sleeping Sickness in a Native OF UGANDA.

An exposure of $\frac{1}{100}$ second with a 'focal-plane' shutter was used, and even then it was found necessary to hold the head on account of the fine tremor present. Photographed by Mr. R. J. Stordy, Principal Veterinary Officer, Uganda and East Africa Protectorates.

The intellectual faculties gradually become impaired, the patient has a certain amount of difficulty in understanding what is said to him, and becomes emotional, often crying for no reason whatever. Delirium is usually absent.

The drowsiness increases, and the patient's attitude becomes characteristic. The head falls forward on the chest and the eyelids 
are closed. At first the patient is easily aroused from this drowsy condition, but soon he reaches that stage in which he falls sound asleep, almost in any attitude and under any conditions, especially after meals. These periods of sleep, which become gradually longer and more profound, lead eventually to a comatose condition, from which the patient can be aroused only with the greatest difficulty.

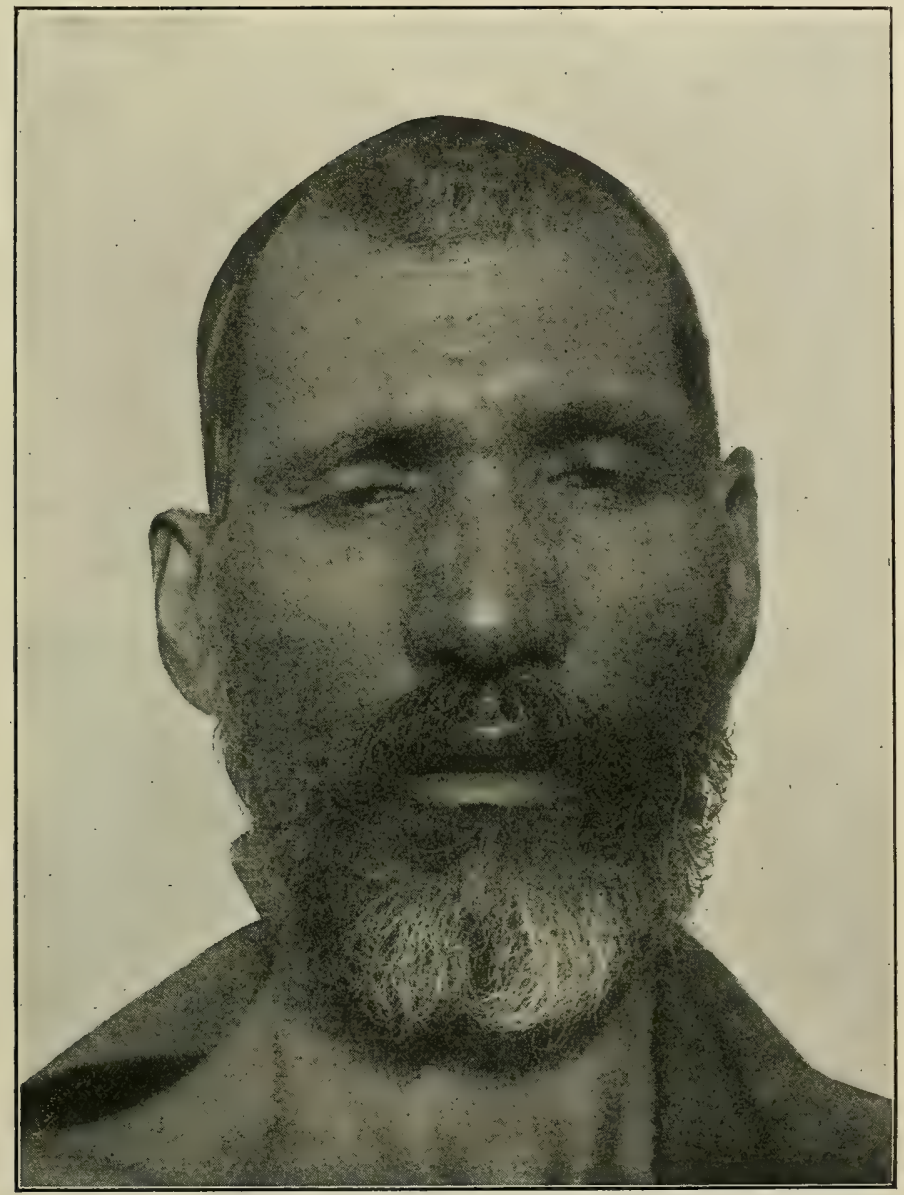

Fig. 5I.-Photograph of a Persian suffering from Sleeping Sickness.

This photograph was also taken by Mr. Stordy, with the same exposure as Fig. 50. This and the preceding photographs show well the characteristic lethargic appearance, which may be correlated with the advanced chronic interstitial meningeal and perivascular inflammation of the lymphatic system of the brain found in these two cases by Dr. Mott.

It is at this stage that the temperature becomes subnormal and death occurs.

[This condition of drowsiness and sleep, leading eventually to coma, is by no means invariably present. In the early part of the 'sleeping sickness' stage patients often sleep more than usual, but later on they do not sleep excessively. They become lethargic or apathetic (see Figs. 50 
and 5 I), and indifferent to their surroundings, and often lie with their eyes closed. When spoken to, they hear and understand what is said to them, and after a longer or shorter interval give'a very brief reply. Many years ago Corre had drawn attention to this fact; Bruce and Nabarro noticed the same thing in Uganda, and Dutton and Todd in the Congo State. Mott states that there is a parallelism between the intensity of the lethargy or apathy, the chronicity of the disease, and the characteristic histological changes in the central nervous system.]

[Lorand ${ }^{1}$ has suggested that the drowsiness of late trypanosomiasis is due to the same cause as the sleepiness of myxœedema-namely, atrophy of the thyroid gland. He thinks that sleeping sickness is due to thyroid degeneration brought about by the action of the toxins of the trypanosomes on the thyroid gland. Sleeping sickness, he says, presents not only all the clinical symptoms of myxœdema, but also the same anatomicopathological changes in the nervous system. Acting upon this hypothesis, Lorand treated a case of sleeping sickness with thyroid tablets with good results, especially in the symptoms referable to the nervous system.]

Anæmia is nearly always present, but it is often difficult to determine how much of this is due to the trypanosomiasis and how much to other diseases from which the patient may be suffering, such as malaria, ankylostomiasis, etc. In the patients observed by Low and Castellani the average number of red corpuscles was $3,500,000$ per cubic millimetre. Occasionally they number only $2,000,000$, and in one case there were $6,200,000$ red corpuscles present per cubic millimetre at the time of death.

[In one Uganda patient whose blood was examined a few days before death I found over 8,000,000 red corpuscles per cubic millimetre. The next day the number had fallen to $7,400,000$. On neither occasion was cyanosis present.]

The hæmoglobin is diminished in proportion to the diminution in the number of red corpuscles. In the majority of patients the large mononuclears are increased in number, and in young subjects the eosinophiles are also increased. [This eosinophilia probably bears no relation to the trypanosomiasis, but is due to helminthiasis. Practically every patient shows, post-mortem, an infection with one or more species of worms.]

[From seventeen observations on fifteen patients in the later stages of sleeping sickness, I have obtained the following differential leucocyte count : polymorphonuclears, 50.6 per cent.; lymphocytes, $27^{\circ} 3$ per cent. ; and large mononuclears, $15^{\circ} 3$ per cent. The eosinophiles varied from o to 20 per cent., and the basophiles from 0.2 to I'2 per cent.]

[An analysis of Greig and Gray's blood-counts²-IO4 observations on fifty-four patients in all stages of sleeping sickness-gives the following results: polymorphonuclears, 40 per cent.; lymphocytes, $3^{8} 7$ per cent. ; and large mononuclears, $14^{\circ} 7$ per cent. The higher percentage of polymorphonuclear leucocytes found by Nabarro is

1 [Lorand, Monthly Cyclopadia of Practical Medicine, v. 9, 1906, p. 145; also Lancet, May I 3 1905, p. 1292, and June 23, 1906, p. 1775.]

${ }^{2}$ [Greig and Gray, op. cit., 1905, pp. 18-24.] 
probably due to the fact that in nearly every case the blood-films were taken from patients within a few weeks of death. Comparative blood examinations, made several weeks before death and again a few weeks later, reveal the fact that the mononuclear leucocytosis which is usually present in the first stage of trypanosomiasis and in the early stage of sleeping sickness proper often gives place to a polymorphonuclear leucocytosis.]

[This is well shown by the leucocyte counts of the following two cases :

Patient I.--On June 29, 1903, the polymorphonuclears were 46.4 per cent., the lymphocytes 34 per cent., and the large mononuclears 16 per cent. On July 2I, I903, the numbers were $8 \mathrm{r}^{\circ} 4,8{ }^{\circ}$, and $\mathrm{IO}^{\circ} 4$ per cent. respectively. The patient died on July 23 .

Patient II.-On April 30, I903, the polymorphonuclears were 57 per cent., the lymphocytes 29 per cent., and the large mononuclears 9 per cent. On June 29,1903 , the numbers were $74^{\cdot} 8,7^{\circ} 4$, and 13.6 per cent. respectively. The patient died on July 7.$]$

[Several of Low and Castellani's ${ }^{1}$ differential leucocyte counts made several weeks, and again shortly before death, show the same thing. This terminal polymorphonuclear increase is no doubt due to the secondary or terminal bacterial infection which is so often found post-mortem in sleeping sickness patients.]

[Dutton and Todd and others describe an auto-agglutination of the red corpuscles which has been regarded as pathognomonic of trypanosome infections. 'It occurs in man and in infected animals as long as the trypanosome infection lasts.]

The trypanosomes are always very scanty in the blood, but in a small percentage of cases they may be found in ordinary bloodfilms. We shall mention later on the methods which have to be employed when the trypanosomes are very scanty.

[There is sometimes an apparent periodicity, and in a few cases examined from this point of view Greig and Gray found the trypanosomes more numerous in the blood at night than by day. Todd and Tobey, ${ }^{2}$ from a number of observations made on sleeping sickness patients in the Congo State, conclude that 'no marked qualitative or quantitative change, corresponding to day and night, occurred in the peripheral blood of the cases' examined from this point of view. Thomas and Breinl (op. cit., p. Io) found a marked periodicity in the presence of the parasite from day to day. In one case in which the intervals of absence were longest the trypanosomes were present on twenty-two days and absent on seventy-eight days; the largest number of successive days on which they were present was three, while the longest period of absence was twentyfive days.]

Malarial parasites and pigmented leucocytes are often found in the blood of patients, pointing to the presence of a malarial complication. In certain districts embryo filariæ are also frequently met with in the blood, and the very frequent coincidence of sleeping

${ }^{1}$ [Low and Castellani, Reports of the Sleeping Sickness Commission, No. 2, 1903, pp. 43-61.] p. 62.]

"[ Todd and Tobey, Liverpool School of Tropical Medicine, Mem. 21, 1906, 
sickness with filariasis led to the idea that these two diseases were related in some way (Manson).

Edematous swellings have been noted by some observers, and van Campenhout and Dryepondt speak of well-defined hard swellings, particularly at the upper part of the tibia. Roughness of the skin, which has been looked upon by some authors as one of the symptoms of the disease, is far from constant. According to Low and Castellani, the skin remains perfectly normal in many cases right up to the end. We have seen that in the first stage of the disease various skin eruptions may occur in white people. In negroes in the second stage it is not uncommon to find papulo-pustular eruptions, pruritus, and lesions due to scratching. But these skin conditions are also frequently met with in negroes who are not attacked by trypanosomiasis (Low and Castellani). In several cases of trypanosomiasis in Europeans, Dupont noted very extensive and pruriginous papular or vesicular eruptions. ${ }^{1}$

[Nattan-Larrier and Tanon, ${ }^{2}$ in a case of trypanosomiasis in a European, saw two types of skin eruption. (I) A vesiculo-papular eruption - as described by Broden, Dupont, and Martin-localized to the arms and lower part of the chest. This form of eruption causes much irritation. (2) The more characteristic circinate erythema-as described by Forde, Manson, Günther, and others-localized to the shoulder and arms and to the hypochondriac, epigastric, infra-axillary, lumbar, and interscapular regions. This form of eruption consists of round or oval rings, purplish red in colour, 4 to 12 millimetres ( $\frac{1}{6}$ to $\frac{1}{2}$ inch) wide, enclosing areas of skin 2 to I 2 centimetres (I to 5 inches) in diameter. Erythematous patches looking somewhat like urticaria are thus produced, but they never irritate. These patches arise in simultaneous crops, and run their course in ten to fifteen days.]

[As in the case of the plaques seen in dourine, superficial scarifications of these erythematous eruptions showed several trypanosomes in smears, while a simultaneous or previous examination of blood from the finger was negative. These characteristic erythemas are important, therefore, from both the clinical and micro-biological points of view. The presence of trypanosomes in the superficial capillaries of the skin may have an important bearing upon the transmission of the infection by biting flies.]

[Mott thinks that the various skin eruptions seen in T. gambiense infections may be accounted for by irritation of the neurotrophic centres in the spinal ganglia, as has already been stated in discussing the origin of the plaques in dourine (p. 3I9).]

The lymphatic glands in the neck, groin, and axillæ are as a rule enlarged in patients suffering from sleeping sickness. 'We always found enlargement of the cervical glands,' writes Bettencourt. ${ }^{3}$ 'Cervical adenitis is, therefore, a constant symptom.' General lymphatic enlargement was observed by the Portuguese Commissioners in 74 per cent. of the cases.

1 Le Caducée, April 16, 1904.

${ }^{2}$ [Nattan-Larrier and Tanon, C. R. Soc. Biol., v. 60, 1906, p. 1065; abstract by Mesnil, Bull. Inst Past., v. 4, 1906, p. 670.]

3 The Report of the Portuguese Commission already referred to. 
According to Low and Castellani, the frequency of skin affections, tuberculosis, and syphilis accounts for the glandular enlargement, which, they say, should not be looked upon as a special symptom of trypanosomiasis. The glands, which vary in size from that of a bean to that of a hazel-nut, are hard and not adherent to the skin, and only rarely suppurate.

[The subsequent Uganda Commissioners, as well as Dutton and his co-workers in the Congo State, frequently found suppuration of the lymphatic glands; and Mott, ${ }^{1}$ who has recently examined the deep paravertebral glands of the neck and other glands from many sleeping sickness cases, finds that there is nearly always evidence of suppuration. The glands are purulent, or show minute necrotic areas or points of suppuration, or, on staining, contain numerous foci of Gram-positive diplococci or diplostreptococci.]

The following observations, however, leave no doubt as to the relation between trypanosomiasis and enlargement of the lymphatic glands. Greig and Gray ${ }^{2}$ punctured the cervical lymphatic glands in fifteen cases of sleeping sickness, and found trypanosomes present in the fluid withdrawn, always in greater numbers than in the blood or cerebro-spinal fluid. The parasites were also found, but in smaller numbers, in other glands. The glands of five natives suffering from trypanosomiasis in its first stage also contained trypanosomes. ${ }^{3}$.

[Greig and Gray, ${ }^{4}$ Dutton and Todd, ${ }^{5}$ and many subsequent observers, have since confirmed this observation, so that at the present time cervical gland puncture is regarded as the most reliable means of making the diagnosis of trypanosomiasis. Moreover, Todd states that from the examination of over 6,000 apparently healthy negroes in the Congo Free State, he and Dutton found, by using gland palpation and puncture, that every negro whose neck glands are enlarged without obvious cause must be regarded as the subject of trypanosomiasis until the contrary is proved.]

The spleen as a rule shows some enlargement, but this is often due to malaria, as can be proved at the autopsy. We have seen that enlargement of the spleen is a very common symptom of the trypanosomiases, and that it is observed in the majority of animals inoculated with $T$. gambiense. It is therefore quite likely that enlargement of the spleen may occur in man as a result of uncomplicated trypanosomiasis.

The respiration rate is increased especially at night, and is

1 [Mott, 'Histological Observations on Sleeping Sickness and other Trypanosome Infections,' Sleeping Sickness Reporis of the Royal Society, No. 7, 1906.]

2 [It was Dr. Mott, a member of the Tropical Diseases Committee of the Royal Society, who conceived the idea that trypanosomes might easily be found in the enlarged lymphatic glands, and at his suggestion Greig and Gray were asked to examine the glands for living trypanosomes.]

${ }^{3}$ [Greig and Gray, Brit. Med.Journ., May 28, 1904, p. I252.]

4 [Greig and Gray, Report of Slecping Sickness Commission, No. 6, I905, pp. 7-18.]

${ }_{5}$ [Dutton and Todd, Liverpool School of Tropical Medicine, Mem. I6, I905, pp, 97-I02; and Mem. I8, I906, pp. I-2I.] 
usually in proportion to the acceleration of the pulse. It may be from 20 to 30 per minute. Cheyne-Stokes breathing is not uncommon during the last days of the illness. Physical examination towards the end of the disease shows signs of hypostatic congestion and œdema of the lungs.

The pulse rate is rarely normal, but is increased to 90 to 130 beats per minute, great variations taking place during the course of the day. The relation of the pulse rate to the temperature is not constant, and great frequency of the pulse may be found together with a low temperature. During the later stages, however, the pulse rate becomes diminished coincidently with the fall of temperature (see Fig. 49).

The pulse is regular and of very low tension. When the temperature is much raised the pulse may be dicrotic. For twentyfour to forty-eight hours before death it may become thready or imperceptible.

There are no symptoms in connection with the heart, neither palpitation nor signs of endocarditis, but occasionally a hæmic murmur may be present. ${ }^{\mathrm{I}}$

The appetite is normal, or even increased, at the onset of the disease. There is no vomiting, and constipation is usually present. The fæces, which are hard, usually contain a variable number of the ova of worms: Ankylostomum duodenale, Ascaris lumbricoides, and Trichocephalus. The liver is often enlarged, but less so than the spleen.

The quantity of urine passed in the twenty-four hours is at first normal, but is often increased in the later stages of the disease. The urine is clear, pale, of low specific gravity, and its reaction is nearly always alkaline, on account of the vegetarian diet of the patients. On analysis an excess of carbonates and of earthy phosphates is found. Albumin is absent as a rule, but there may be a trace when the temperature is high. Sugar and bile pigments are absent. In the last stages there is incontinence of urine. Low and Castellani never met with a case of retention.

[When studying the disease in Uganda, we found it convenient to recognise three stages in sleeping sickness proper. Although these are not sharply defined, but pass gradually the one into the other, we nevertheless found them useful in the clinical description of the malady.]

[I. Stage of Fever.-In this stage it is often impossible for a European to recognise that the patient is ill at all, but his friends have already recognised the subtle change in intelligence or demeanour which they have learnt to associate with the onset of sleeping sickness. On examination, the temperature is usually found raised and of the 'hectic' type (see Fig. 49); the pulse is accelerated, small, and of low tension. Trypanosomes are nearly always found by lumbar puncture.]

[2. Stage of Tremors.-After a variable time-usually one to two months-the dull, heavy expression which appears during the first

${ }^{1}$ [ In a case of the disease in a European recorded by Martin. the patient suffered from dyspnoa and palpitation at an early stage of the disease, and clinically resembled a patient with cardiac disease (Ann. Inst. Past., v. 21, 1907, pp. I6I-193). ] 
stage deepens, and the face may become puffy. The gait becomes shuffling and uncertain. The speech is slow, thick, and mumbling, and the voice is often high-pitched. There is a marked tremor of the tongue and hands, and often of the lips and legs. The patient becomes more listless and apathetic, and is still able to get about, though with increasing difficulty. The temperature continues to oscillate, but not so much as in the first stage, and is rarely so high as at first. This stage lasts as a rule two to four weeks, and passes gradually into]

[3. Stage of Subnormal Temperature and Profound Lethargy.-The patient is now quite lethargic and helpless. He takes his food with the greatest difficulty, and often has to be forcibly fed. He passes urine and fæces under him, or there may be obstinate constipation. The temperature is subnormal, often registering only $92^{\circ}$ to $95^{\circ} \mathrm{F}$. (see Fig. 49). The pulse is almost imperceptible, and the patient dies, often conscious almost to the end, but absolutely lethargic and indifferent to his surroundings.]

[Dutton, Todd, and Christy ${ }^{1}$ adopt a somewhat similar classification in their description of the disease :

Type A.-Cases with no definite symptoms of illness.

Type B.-Cases with few symptoms.

Type C.-Fatal cases with well-marked symptoms, the most notable being fever, lassitude, weakness, and wasting. Cases under Type $C$ they further subdivide into (I) those showing no sleep symptoms; and (2) those showing sleep symptoms.]

Complications.-Amongst the most common complications are laryngitis, bronchitis, broncho-pneumonia, and odema of the lungs. Bed-sores are common, especially if the patients lie on hard, coarse beds and are not properly attended to when they pass urine and fæces under them.

[In Uganda we often found secondary bacterial complications post-mortem, such as purulent meningitis, pneumonia, and generalized suppuration of the lymphatic glands. Christy, Dutton, and Todd on the Congo also found that secondary bacterial infection seemed to determine the fatal issue in many cases of sleeping sickness.]

DuRation.-We have already seen that the first stage of the disease may last for several years. The second stage lasts from four to eight months. Sometimes there are periods of remission, but relapses invariably occur. It is quite exceptional for the disease to be prolonged for more than a year from the time that the nervous symptoms become manifest.

[Sometimes, however, the disease may last even longer than a year. The European (see p. 367 ), who contracted trypanosomiasis in Uganda early in I903, had delusions and became drowsy before November, Ig04, but did not die of sleeping sickness until April, Igo6-more than eighteen months from the onset of nervous symptoms.]

1 [Dutton, Todd, and Christy, Thompson l'ates and Johnston Lab. Reports, v. 6, part i., p. I4. See also Stephens, Nature, v. 69, February I I, I904.] 


\section{Section 5.-The Pathogenicity of Trypanosoma gambiense for Different Species of Animals.}

T. gambiense is pathogenic for a very large number of mammals : monkeys (at least certain species), lemurs, the dog, [jackal], cat, rabbit, guinea-pig, rat, mouse, [jerboa], hedgehog, marmot, horse, donkey, [cow], goat, and sheep.

MONKEYS AND LEMURS.-The disease produced in monkeys by inoculation with blood or cerebro-spinal fluid of sleeping sickness patients is very interesting, inasmuch as it is closely analogous to human trypanosomiasis. [Different species of monkeys show great variations in their susceptibility to infection with $T$. gambiense, and until recently baboons were thought to be refractory.] Dutton and Todd inoculated without success several baboons (Cynocephalus $\operatorname{sphin} x$ ), and we also failed to infect a baboon which was injected on several occasions with infective blood from a rat or dog. [Thomas and Linton also found the Cynocephalus sphinx refractory; but in later experiments Thomas and Breinl ${ }^{1}$ successfully inoculated four baboons-one Cynocephalus sphinx and three C. babuin.] On the other hand, the Macacus is easily infected. Bruce, Nabarro, and Greig experimented upon $M$. rhesus and Cercopithecus (blackfaced variety). All the macaques we inoculated ( $M$. rhesus, $M$. cynomolgus) became infected. In addition to the two species of Macacus already mentioned, Brumpt and Wurt $z$ have infected the following: Cercopithecus ruber and C. callitrichus, the marmoset (Hapale penicillatus), Cebus capucinus, Lemur rubriventer, and L. mongoz. A Cercopithicus fuliginosus was refractory. ${ }^{2}$ Thomas and Linton (op. cit.) obtained the following results by the inoculation of $T$. gambiense derived from various sources into different species of monkeys. All the Macacus rhesus and Cercopithecus callitrichus inoculated became infected, but the Cynocephalus sphinx was absolutely refractory. A chimpanzee inoculated with a trypanosome from the Gambia had a mild infection, and died of broncho-pneumonia seven months after inoculation.

[A chimpanzee injected subcutaneously by Gray and Tulloch in Uganda showed trypanosomes in blood-films on the seventeenth day. Four months after injection enlarged glands were felt in the axilla, and actively motile trypanosomes were found in the gland juice. The animal died about a month later, probably from the effects of captivity. Trypanosomes were not found in the blood during the last two months of life, but they were present in the gland juice until one week before death. There were no characteristic lesions found in the brain or other organs postmortem.]

[As already mentioned, Thomas and Breinl succeeded in infecting baboons, but they state that the baboon is certainly the most resistant

1 [Thomas and Breinl, Thompson Yates and Johnston Lab. Reports, v. 6, part ii., 1905, pp. I3-15.]

2 Brumpt and Wurtz, 'Experimental Sleeping Sickness,' three notes, Soc. Biol., March 26, 1904. 
animal with which they have experimented. The other species of monkeys used by them-Macacus rhesus, Cercopithecus callitrichus and C. nuber, Pithecia satanas (Jew and Sooty monkeys)-were almost equally susceptible, the Sooty monkey apparently rather less so than the others.]

Inoculations into the vertebral canal or brain of monkeys give the same results as subcutaneous inoculations. Moreover, the same results are produced when the blood of a patient in the first stage of trypanosomiasis or the cerebro-spinal fluid of a patient suffering from sleeping sickness is used for inoculation.

[The average incubation period in seventeen monkeys (Macacus rhesus and Cercopithecus sp.) inoculated with blood or cerebro-spinal fluid by the various Uganda Commissioners was eighteen daysminimum nine days, maximum forty days. The average duration in thirteen of these monkeys was seven and a half months--minimum two months, maximum eighteen months. Three of the monkeys survived inoculation more than a year. In the monkey (a Macacus) which survived the longest (eighteen months) Harvey found the typical lesions, meningo-encephalo-myelitis, of chronic sleeping sickness.]

As a rule, there is no marked febrile reaction when the trypanosomes first appear in the blood, but rises of temperature have been seen in some cases.

We may briefly refer to two experiments mentioned in the Reports by Bruce, Nabarro, and Greig. A Macacus rhesus inoculated subcutaneously on March 23, I903, with cerebro-spinal fluid from a patient who had just died of sleeping sickness, ${ }^{1}$ died on July I2, with trypanosomes in its blood. During the last four weeks ${ }^{2}$ of its life the monkey was in a lethargic condition resembling that seen in patients suffering from sleeping sickness.

A Macacus rhesus was inoculated in the vertebral canal with I c.c. of cerebro-spinal fluid from a patient with sleeping sickness. Nineteen days later trypanosomes appeared in the blood. The temperature was raised on several occasions; four months after inoculation the monkey showed signs of sleeping sickness and died [about three weeks later. Post-mortem, there was no meningo-encephalitis or perivascular infiltration. The capillaries of the brain showed endothelial proliferation, and there was an increase of glia tissue. Marked chromatolysis of the cells of the cord, medulla, and, to a less degree, of the cortex was present (Mott). This gliosis of the brain is in all probability the earliest stage of the changes in the nervous system found in the human subject.]

At the Pasteur Institute we inoculated two Macacus cynomolgus; one died at the end of fifty days, the other recovered from its

1 [The monkey did not become infected as a result of this inoculation, probably because the patient had been dead too long, and the cerebro-spinal fluid contained many micrococci. The animal was re-injected with cerebro-spinal fluid on May I I, I903, and became infected seventeen days later.]

2 [This is erroneously given in the original of Laveran and Mesnil's book as ten days.-ED.] 
trypanosomiasis. Two Macacus rhesus died in thirty-three and sixtythree days.

The chief symptoms of the infection in these animals were wasting, ${ }^{1}$ anæmia, and, in the last stages, a lowering of temperature with drowsiness. Trypanosomes were always scanty, or even very scanty, in the blood, except in the last stages of the disease, when they were fairly numerous. In one of the monkeys the temperature went up from $38.5^{\circ} \mathrm{C}$. [102.2 ${ }^{\circ} \mathrm{F}$.] the normal temperature of monkeys, to $40^{\circ} 2 \mathrm{C}$. [104.4 $4^{\circ} \mathrm{F}$.], when the parasites first appeared in the blood, but the temperature remained normal after this initial rise.

During the last days of the illness the temperature falls progressively to $37^{\circ}, 35^{\circ}$, and $33^{\circ} \mathrm{C}$. $\left[98^{\circ} 6^{\circ}, 95^{\circ}\right.$, and $91^{\circ} 4^{\circ} \mathrm{F}$. , and sometimes on the day of death the rectal temperature may be as low as $28^{\circ}$ or $27^{\circ} \mathrm{C}$. $\left[82^{\circ} 4^{\circ}\right.$ or $80^{\circ} 6^{\circ} \mathrm{F}$. $]$. With the lowering of temperature there is a tendency to drowsiness, and the liveliest monkeys remain quiet and sleep almost constantly, assuming the ordinary attitude of sleep-namely, the sitting posture, with the head bent down between the legs. The monkeys inoculated by Thomas and Linton with $T$. gambiense never showed any signs of drowsiness. We have already seen that in the other trypanosomiases monkeys usually have a subnormal temperature for some time before death. In the case of other infections in monkeys this tendency to drowsiness and lowering of temperature may also be present. ${ }^{2}$ When these symptoms occur in the very last stage of the disease in monkeys inoculated with $T$. gambiense, they cannot be looked up as characteristic of this infection; but when the stage of lethargy occurs some weeks ${ }^{3}$ before death, as it did in one of the experiments recorded by Bruce, Nabarro, and Greig, the resemblance to human sleeping sickness is marked.

In the marmoset, the Cebus capucinus, Lemur rubriventer, and L. mongoz, the course of the disease is more rapid than in the Macacus. According to Brumpt and Wurtz, the average duration in the marmoset is twelve days, in C. capucinus eight days. The disease may end in recovery, but monkeys that have recovered are not always rendered immune (Thomas and Linton, $o p$. cit.).

[It is perhaps doubtful whether monkeys ever recover from $T$. gambiense infection. The trypanosomes may disappear from the blood after the first few months and may remain absent for a whole year, as in Harvey's case. This monkey remained apparently well for seventeen months, yet it died a month later with typical sleeping sickness symptoms, and a trypanosome was found in a blood-film three days before death.]

[Baboons show the ordinary symptoms of the disease in monkeys - fever, anæmia, and loss of weight-but trypanosomes are usually

1 One of the monkeys, which weighed I,240 grammes at the beginning of the experiment, weighed only 805 grammes the day it died.

2 Brumpt arid Wurtz, Soc. de Biol., March 26, 1904.

3 Vide footnote 2 to p. 38.3 . 
scanty in the blood. Three Cynocephalus babuin died in forty-one, fifty, and seventy-five days after inoculation. The trypanosome obtained from one of these baboons, after being passed through a rabbit, was found to be very virulent, and gave rise to a much shorter incubation period, and to a more acute disease than the ordinary strain of T. gambiense (Thomas and Breinl, op. cit., p. I4). Microscopically many small hæmorrhages were found in the grey matter of the brain and spinal cord in two of the baboons.]

DoGs.-Dogs are easily infected, the parasites appearing in the blood at the end of ten to fifteen days, but as a rule they are scanty, and the disease takes a slow course.

[In Uganda we found the incubation period to vary from two to five weeks in adult dogs. After being present for some time in the blood, the trypanosomes diminished in number, and in some cases disappeared, but the animals all died from anæmia due to ankylostomiasis. Four puppies inoculated by us failed to show trypanosomes in the peripheral blood at any time. Two of them died quickly of ankylostomiasis, but the other two lived for three and a half and four and a half months respectively, and succumbed to canine piroplasmosis. One puppy had received two injections of infective blood, the other four injections of cerebro-spinal fluid, yet neither animal showed trypanosomes in its blood. The blood was nevertheless infective, for on injection into a monkey the latter developed trypanosomiasis in fifteen days, as well as piroplasmosis on the eleventh day. $\left.{ }^{1}\right]$

According to Brumpt and Wurtz, the incubation period in the dog is seventeen days, and the disease lasts sixty-six days. The temperature is subnormal before death. Thomas and Linton state that dogs die in five to six weeks.

[In a later paper Thomas and Breinl (op. cit.) give three weeks to nine months as the limits of the duration of the disease in their dogs. A bitch inoculated by Dutton and Todd and brought to England recovered from the infection. and was then found to be immune. Its serum was not protective. The offspring of this bitch were quite as susceptible as normal animals to the various strains of T. gambiense. One dog, which lived for more than nine months, had 7,820,00o red corpuscles per cubic millimetre of blood on the sixty-ninth day of the disease. Puppies were found to be more susceptible than adult dogs.]

False membranes which form in the anterior chamber of the eye may lead to loss of sight in one or both eyes. In dogs the temperature is raised, as is seen in Fig. 52, which shows the first part of the temperature chart of a dog inoculated by us on November I4, I903, with blood containing $T$. gambiense. From November, I903, to April, 1904, the dog showed a series of rises of temperature with apyrexial intervals of eight to ten days. When the blood was examined during the pyrexial attacks, the parasites were, as a rule,

${ }^{1}$ [Nabarro and Greig, Sleeping Sickness Commission lieport, No. 5, p. 36.] 
scanty, or even very scanty, in the blood, while in the apyrexial intervals the blood examination was usually negative. [This dog was still infected five and a half months after inoculation (April 30, I904), but appeared to have recovered at the end of six and a half months. Reinoculated a month later, it contracted a fresh infection, which lasted only a very short time. A third inoculation was followed by a similar result, but the dog succumbed to the fourth injection. ${ }^{1}$.

[In Uganda the dogs at Bugangu, at the north-east end of Lake Albert, were found by Gray and Tulloch ${ }^{2}$ to be naturally infected with T.gambiense. It is possible, therefore, that dogs may help to spread human trypanosomiasis, especially as the parasites are fairly numerous in their blood.]

[JACKAL. - A jackal inoculated by us in Uganda became infected in eleven days, and trypanosomes were found in its blood for about six weeks. During the next six weeks they could not be found in

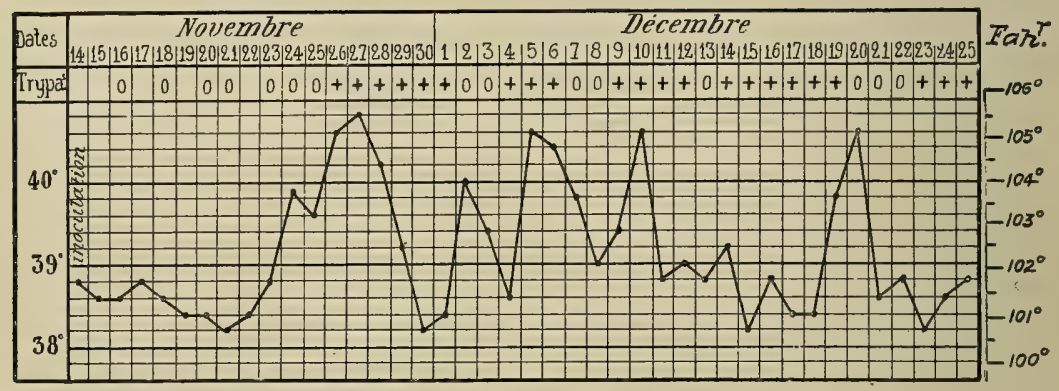

Fig. 52. - The Beginning of the Temperature Chart of a Dog inoculated with T. gambiense.

blood-films. The jackal then devoured a monkey infected with the Uganda mule trypanosome (see p. 208). Thirty-six hours later this trypanosome was found in its blood, and the jackal died a fortnight afterwards.]

CATS.-Cats react in almost the same way as dogs (Thomas and Linton).

[Nabarro and Greig inoculated a cat on September 23, I903, with Io c.c. of blood from a case of early trypanosomiasis. As the cat had not become infected, it was reinjected on November II, I903, with 8 c.c. of blood. Trypanosomes first appeared in its blood sixty-nine days after the second inoculation, and were present irregularly until May IO, I904. After that date the blood examination was always negative until the animal died-from the effects of traumatism-on September 3. A kitten inoculated by Greig and Gray on two occasions showed trypanosomes in its blood fourteen days after the second injection, and was still alive and well nearly four months after inoculation.]

[Thomas and Breinl give the duration of the disease in cats as three to ten weeks, but exceptionally it may be considerably longer-up to six and a quarter months. Kittens died in from three to seven weeks. Anæmia and fever were present in most cases.]

1 [Information kindly furnished by the authors.]

2 [Gray and 'Tulloch, Sleeping Sickness Commission Report, No. 8, 1907, p. 64.] 
Guinea-PIGs. - T gambiense gives rise to a very chronic infection in guinea-pigs (Dutton and Todd). Brumpt and Wurtz (op. cit.) state that they never succeeded in finding trypanosomes in the blood of inoculated guinea-pigs. On the other hand, we have found a fair number of trypanosomes in the blood in the later stages of the infection in nearly all the guinea-pigs inoculated by us with $T$. gambiense. Guinea-pigs inoculated on December II, I903, were still alive on May 30, I904, and showed no signs of disease, although the parasites were fairly numerous in the blood. [They died, however, during the following month. ${ }^{1}$ ] About half the guinea-pigs inoculated by Thomas and Linton remained uninfected, and, according to these observers, the disease may terminate either by death or by recovery.

[In Uganda we found that our guinea-pigs were very resistant to injections of blood or cerebro-spinal fluid containing $T$. gambiense. One guinea-pig was injected with cerebro-spinal fluid on May 5, I903, September I, 1903, and August 18, I904 (5 c.c., 2 c.c., and 3 c.c. respectively), and trypanosomes were first observed in its blood on October 4, 1904-forty-seven days after the third injection. Another animal received three injections of blood-on May I, Igo3, September 2 I, I903, and June 23, I904. Trypanosomes were first found in its blood on August 2, forty days after the last injection.]

[In a recent investigation upon the relative virulence of three strains of T. gambiense (from Gambia, Uganda, and Ubangi), Laveran? found that all his guinea-pigs injected intraperitoneally became infected and died. The average duration was Ioo days. Trypanosomes were present irregularly, and were usually numerous near the time of death. The spleen was found enlarged, and had ruptured in some cases. Thomas and Breinl also found the spleen acutely swollen and ruptured in four of their guinea-pigs.]

RABBITS,-In rabbits the disease runs a chronic course, as in guinea-pigs, and the trypanosomes are scanty in the blood. Brumpt and Wurt $z$ have observed (op. cit.) lesions resembling those seen in rabbits inoculated with dourine. In one of the rabbits inoculated by us the head became swollen and altered in appearance, and there was double blepharo-conjunctivitis with discharge of muco-pus. Thomas and Linton also noted these symptoms, which appear to be common to the different trypanosomiases in the rabbit. [The average duration of the disease was 50 to I 28 days in Thomas and Breinl's experiments, but animals suffering from a mild chronic infection lived as long as 150 and 273 days.]

White RATs.-White rats are easily infected, but less constantly than with $T$. brucei, $T$. evansi, and $T$. equinum. Intraperitoneal inoculations are more successful in rats (as they are also in guinea-pigs and rabbits) than subcutaneous inoculations. One of our rats was not infected by a subcutaneous inoculation, but became infected after inoculation into the peritoneum. Even intraperitoneal

1 [Information furnished by the authors.]

2 [Laveran, C. R. Acad. Sciences, v. I42, I906, p. 1065; abstract by Mesnil in Bull. Inst. Past., v. 4, I906, p. 585.] 
inoculations do not always succeed in rats. Trypanosomes appear in the blood on an average fifteen days after inoculation (minimum six days, maximum thirty-six days). During the earlier stages of the disease the parasites are very scanty and do not multiply progressively, but in the later stages they are always numerous in the blood. Rats suffering from any skin affection were found less resistant than normal rats. The average duration of the disease was three months; but in animals treated with arsenious acid the disease lasted a longer time, one rat living for 208 days after inoculation.

$T$. gambiense does not always produce such a serious infection in the rat, but sometimes there is a very mild infection, the parasites occurring in the blood only for a few days and then disappearing. Some of the rats which contract this abortive form of the disease are immunized by it; others become reinfected on reinoculation with $T$. gambiense, and they may then suffer from a severe form of the disease. Thomas and Linton state that rats which have recovered are by no means always immune.

[Laveran, in the investigation already referred to, found that the majority of the rats inoculated intraperitoneally became infected, some, however, only after the second or third injection; a few resisted even three injections. The average duration of the disease was sixty-two, seventy-seven, and eighty-one days with the Gambia, Uganda, and Ubangi trypanosomes respectively. Rats infected with the Gambia strain all died; in the case of the other two strains there were some spontaneous cures. Only one of the rats-inoculated with the Ubangi virus-had paresis. Young rats were usually more susceptible than adults.]

[In Uganda, Bruce and Nabarro found the incubation period in grey wild rats to be eleven days.]

It is seen, therefore, that this trypanosomiasis runs a much longer and more irregular course in rats than do the other trypanosomiases which we have already studied.

Mice.-In mice the infection is milder than in rats. Trypanosomes appear in the blood about the seventh day after intraperitoneal inoculation, but, as a rule, only in very small numbers, and they very rapidly disappear. Mice which survive the infection are immune, and they may be reinoculated with large doses of infective blood without causing a reappearance of the trypanosomes in their blood.

Under certain conditions at present not well understood, T. gambiense may acquire a greater virulence for mice. ${ }^{1}$ Of ten mice inoculated by us only two became infected. [The infection was moderately severe, but the mice did not appear ill, yet they succumbed to the disease, and post-mortem the spleen was found enlarged..$^{2}$ ] Thomas and Linton obtained variable results with mice, according to the source of the particular strain of $T$. gambiense used for inoculation.

1 See especially Brumpt and Wurtz, Soc. de Biol., March 26, 1904, p. 567.

${ }^{2}$ [Information kindly furnished by the authors.] 
[With the three strains of trypanosomes already mentioned, Laveran found mice more resistant than rats. A considerable percentage of the animals did not become infected at all-two out of eleven with the Gambia strain, six out of eight with the Ubangi strain. Many of the infected mice recovered--three out of the nine which were susceptible to the Gambia virus, and two out of four infected with the Uganda virus. The disease was sometimes very chronic, lasting as long as a year.]

[JERBOA.-A jerboa inoculated by Laveran ${ }^{1}$ with the Gambian trypanosome developed a fairly severe infection, and died in fortynine days. It was thus more susceptible than the rat to the same virus.]

MARMOT.-Parasites appeared in the blood seven days after inoculation, and the disease lasted twenty-nine days. Death occurred after a subnormal temperature and drowsiness.

HEDGEHOG.-A hedgehog died with a subnormal temperature forty-one days after inoculation. ${ }^{2}$

GoATs.-According to Dutton and Todd, and Thomas and Linton, T. gambiense is pathogenic for the goat, but the infection produced is a mild one and practically without symptoms. A nanny goat and a billy goat inoculated by us subcutaneously became infected. They had no rises of temperature, but the nanny goat showed very few parasites in the blood on several occasions, whereas in the billy goat parasites could never be found in blood-films, and it was necessary to inject a rat to show that the blood was infective. The blood of the nanny goat was still virulent in doses of $2 \frac{1}{2}$ c.c. four months after inoculation. [Both goats had recovered from their infection about five months after inoculation. $\left.{ }^{3}\right]$

[A native Uganda goat was inoculated on five occasions between May II, I903, and August I8, I904, with infective blood or cerebrospinal fluid, before trypanosomes could be found in the peripheral circulation - thirteen days after the last injection.]

[Thomas and Breinl's goats inoculated with various strains of T. gambicnse all died. The animal inoculated with the Uganda virus died in fifty-eight days. The symptoms were: fever, anæmia, and loss of weight. Parasites were fairly constantly present in small numbers.]

SHEEP.-Two sheep and a lamb inoculated by us became infected. In one of the sheep there were two slight rises of temperature $\left[\mathrm{IO}_{4} .6^{\circ}\right.$ and $\mathrm{IO}_{4}{ }^{\circ} \mathrm{F}$.] at the commencement of the disease, but the other sheep had no fever. In one case trypanosomes could be detected in very small numbers by the microscope, but in the other two cases (the lamb and one sheep) it was necessary to inoculate rats in order to show the presence of the infection. The blood of

1 [Laveran, C. R. Soc. Biol., v. 59, I905, p. 250.]

2 Brumpt and Wurtz, ibid., loc. cit.

${ }^{3}$ [Information furnished by the authors.] 
one of the sheep was no longer virulent four months after inoculation : this animal, therefore, recovered from its trypanosomiasis.

(Thomas and Breinl had a similar case of recovery in a sheep which became infected ten days after inoculation and remained infected for five months.]

[A native Uganda sheep received eight injections of cerebro-spinal fluid (62 c.c. in all) between May I I, I903, and August I8, I904, yet the weekly blood examinations never revealed the presence of trypanosomes in the peripheral circulation. Another sheep inoculated on two occasions with infective blood likewise failed to become infected.]

PIG.-A pig three or four months old inoculated on several occasions ' never showed any parasites,' say Brumpt and Wurtz. It is important to know if the blood of this pig was inoculated into rats, or whether a simple microscopical examination was made, for the latter alone is not sufficient.

Horses. - In the horse T. gambiense produces a chronic infection. The horse inoculated by Dutton and Todd on February I4, 1903, was still alive on September 28, I905 (Thomas and Breinl). One month after inoculation trypanosomes appeared in the blood, but were always present only in very small numbers. In August, I903, the microscopical examination of the blood was negative, yet the blood was still virulent for rats; in October, however, this was no longer the case. The horse showed several symptoms, such as rises of temperature, wasting, and dejection after inoculation, but improved soon after its arrival in England. [On September 28, 1905, the temperature still showed occasional rises to $100^{\circ}$ or IOI $^{\circ} \mathrm{F}$.; the horse was in good condition, however, and had increased in weight. Trypanosomes were never seen in the blood, nor was the latter infective for rats in doses of I to 2 c.c. (Thomas and Breinl)].

A horse inoculated at Alfort by Vallée ${ }^{1}$ became infected, and showed slight odema of the genital organs and limbs. [It succumbed to the infection on September 4, I904..$^{2}$ ]

In Gambia horses are sometimes naturally infected with trypanosomes. The question suggested itself to Dutton and Todd whether this trypanosome of the horse in Gambia was not the same as the parasite found in man. This question has now been answered, for it has been shown that they are two distinct species. We have already described this trypanosome, $T$. dimorphon, and we need not here repeat the morphological and other characteristics which enable us to distinguish it from T. gambiense.

In the donkey $T$. gambiense produces a mild infection (Thomas and Linton).

[Trypanosomes are always scanty in the blood, and the animals present few signs of ill-health. There may be slight anæmia, fever, loss of weight, and glandular enlargement. The blood was found to

\footnotetext{
1 Letter from Dr. Vallée, dated May 8, 1904.

${ }^{2}$ [Information furnished by the authors.]
} 
be still infective for rats eighteen months after injection (Thomas and Breinl).]

[Three donkeys were injected in Uganda with blood or cerebro-spinal fluid containing T. gambiense, but trypanosomes could never be found in their blood. The donkeys remained well until they were injected with the various ' animal' trypanosomes found in Uganda, to which they all succumbed.]

Cattle.-According to Bruce, Nabarro, and Greig, cattle are refractory. [Two oxen inoculated, the one on six occasions with cerebro-spinal fluid, the other on three occasions with blood containing T. gambiense, failed to show the parasite on microscopical examination. It appears to follow from Bruce, Nabarro, and Greig's observations in Uganda, that the native cattle, sheep, and goats are very resistant to $T$. gambiense, much more so than the European species of these animals.]

[In Chapter VI. an account was given of the various trypanosomiases that were found to occur naturally in Uganda cattle. None of these trypanosomes appears to be identical with the human species.]

At our request, Vallée inoculated a cow with the blood of a rat heavily infected with $T$. gambiense. The cow became infected, but there was no febrile reaction, nor were trypanosomes seen on microscopical examination of the blood. The cow's blood inoculated into rats proved infective. Thomas and Linton also succeeded in infecting an ox.

[A cow inoculated by Thomas and Breinl had a rise of temperature on the eighth day to $103^{\circ} 6^{\circ} \mathrm{F}$. No parasites were visible in blood-films, but a rat inoculated with the blood became infected. Trypanosomes were first found in the cow's blood on the nineteenth day after inoculation. At the outset the cow lost weight and was out of condition, but it soon regained its normal appearance. More than a year later the blood was still infective for rats, though only in larger quantities than at first.]

[The milk of this cow was peculiar: about two months after infection it was noticed to be watery, and was found to be very poor in fat. Dogs, cats, and human beings fed on the milk, even after it had been sterilized, developed diarrhœa. The milk was proved to be free from pyogenic bacteria and trypanosomes.]

\section{Section 6.-Pathological Anatomy.}

Long ago the existence of meningeal lesions and an increase of cerebro-spinal fluid had been noted in patients who had died of sleeping sickness.

In 1840 Clarke recorded the presence of cerebro-spinal meningitis at five autopsies. In I86I Dangaix found injection of the meninges and an increase in the cerebro-spinal fluid at a post-mortem on a patient in Gaboon. Griffon du Bellay, in I863, noted injection of the meninges and brain in negroes who had died of sleeping sickness in Gaboon. Similarly, a patient who died in Guadeloupe had 
inflammation of the meninges and increase of the cerebro-spinal fluid (Gaigneron and Lherminier). ${ }^{1}$ Guérin, in Martinique (I869), observed that meningeal congestion and great increase of the cerebrospinal fluid were the lesions most constantly found in sleeping sickness. Nielly, summarizing our knowledge of the pathological anatomy of sleeping sickness in I880, writes: 'Meningeal lesions predominate, but they are not invariably present.'

Recent researches carried out with the aid of more perfect methods than were at the disposal of previous observers have shown that the meningeal lesions are always present, and have enabled us to make out that, even in cases where there are no macroscopic lesions, the inflammatory changes typical of cerebro-spinal meningitis are seen on microscopical examination. The injection and inflammation of the meninges vary very much in intensity. Sometimes the meningitis is well marked, the membranes being injected and thickened, adherent to the brain, which is torn when they are removed, and showing much perivascular exudation. Sometimes there is only slight hyperæmia on macroscopic examination. The subarachnoid fluid is usually increased in the skull as well as in the spinal canal. It is usually clear, but sometimes cloudy or even purulent, especially in the sulci of the brain and along the bloodvessels. In the centrifuged cerebro-spinal fluid a large number of mononuclear leucocytes and a variable number of trypanosomes are found, but the latter are usually scanty. ${ }^{2}$

[The increase in amount and the turbidity of the subarachnoid fluid give to the typical sleeping sickness brain rather a characteristic appearance. The convolutions are somewhat flattened, and the sulci are filled with a turbid exudate having a 'ground-glass' appearance. The vessels of the meninges are usually injected. A well-marked meningitis-and especially a purulent meningitis - is to be regarded as a complication, due to a terminal infection with pyogenic micro-organisms (such as pneumococci, diplo-streptococci), rather than as a typical sleeping sickness lesion.]

On cutting into the brain the only morbid conditions present as a rule are excess of fluid and dilatation of the lateral ventricles. The presence of meningo-encephalitis and meningo-myelitis is shown under the microscope by an infiltration with mononuclear leucocytes over the whole convex surface of the brain, in the sulci, and along the vessels. Often the exudation is so marked that the vessels come to lie in grooves on the surface of the brain, pons, bulb, and spinal cord. The histological changes have been well described by Mott. ${ }^{3}$

1 Dutroulau, 'Traité des Maladies des Européens dans les Pays chauds,' second edition, Paris, 1868 , p. 160.

2 As Bruce, Nabarro, and Greig state in their report, to which frequent reference has been made in this chapter, the scarcity of trypanosomes in the cerebro-spinal fluid is no argument against the pathogenic action of these parasites. In certain animal species the trypanosomes of nagana are always scanty in the blond, and one might also mention the case of dourine, in which the trypanosomes are equally scanty in spite of the gravity of the infection.

3 Manson, 'Tropical Diseases,' third edition, 1933, p. 340 ; [also Trans. of the Path. Soc., v. 51, part ii., 1900]. 
[During the last few years the minute anatomy of the central nervous system and other organs of sleeping sickness patients has been carefully studied by Mott, ${ }^{1}$ Low, Breinl, ${ }^{2}$ and others. ${ }^{3}$ It is especially to Mott that we are indebted for our present knowledge of the extensive microscopic changes which are found in sleeping sićkness.]

[The lymphatic gland enlargement, which is considerable in all early cases of trypanosomiasis, is almost certainly induced by the trypanosomes, for in the early stages of the disease the glands are practically always sterile as regards bacteria. The change in the glands is inflammatory in nature, and terminates in fibrosis. Trypanosomes are always very scanty in sections of glands and other tissues, so that the chronic irritation which they set up in the lymphatic glands generally, and later in the lymphatics of the brain and spinal cord, is probably not due to their mere mechanical presence, but to a chemical toxin. There is, however, very little, if any, further evidence at present to hand of the existence of such a toxin.]

[The trypanosomes probably infect the lymphatic glands and the cerebro-spinal fluid by escaping from ruptured capillaries, but it is possible that in certain instances they may, by their 'boring' movement, force their way through the walls of minute vessels into the perivascular spaces.]

[The posterior spinal ganglia always show chronic inflammatory changes, which may be due to the absorption of toxins from the neighbouring infected paravertebral glands (Mott).]

[We have seen that the cervical glands are enlarged in practically all cases of trypanosomiasis and sleeping sickness, and, according to Mott, the most chronic change is found about the base of the brain. $\mathrm{He}$ argues from this that probably 'the chronic inflammation of the lymphatics spreads along the nerves, spinal ganglia, and roots to the central nervous system, and especially along the lymphatics of the nerves and vessels entering the base of the skull.']

[The changes observed by Mott in the lymphatic glands in early trypanosomiasis and in sleeping sickness were as follows: Increased vascularity and lymphocytes in all stages up to the formation of

1 [F. W. Mott, 'The Cerebro-spinal Fluid in Relation to Disease of the Nervous System,' Brit. Med. Journ., December 10, I904, pp. I559, I 560; 'The Post-mortem Findings in a European Case of Sleeping Sickness,' Low and Mott, Brit. Med. Journ., April 30, 1904, p. 1000 ; 'Observations on the Brains of Men and Animals infected with Various Forms of Trypanosomes,' Proc. Roy. Soc., Ser. B, v. 76, 1905, pp. 235-242 ; ibid., v. 78, 1906; Brit. Med. Journ., December 22, 1906, pp. I772-1777; and especially Report of the Sleeping Sickness Commission of the Royal Society, No. 7, igo6.]

2 [A. Breinl, Proc. Roy. Soc., Ser. B, v. 77, I9o6, pp. 233-236 ; Thompson Yates and Johnston Lab. Rep., v. 6, part ii., 1905, pp. 66-92.]

3 [Warrington, Brit. Med. Journ., 1902, No. 2, p. 929 ; Bettencourt and coworkers, 'La Maladie du Sommeil,' Lisbon, 1903; Sicard and Moutier, La Presse méd., December, I905; Kopke, 'Investigaeses sobre a Doenca Somno,'Arch. de Hyg. e Path. exot., v. I, fasc. I; Mole, 'The Lesions in the Lymphatic Glands in Human Trypanosomiasis,' Liverpool School Tropical Med., Mem. 21, I906, pp. 69-83.] 
large plasma cells of Marscholko, and large numbers of degenerated swollen plasma cells, like those seen occasionally in the perivascular lymph spaces of the brain in sleeping sickness. Proliferated endothelial cells, some of which had taken on a phagocytic function, and had engulfed lymphocytes and chromatin particles, were also seen. There was also hyperplasia of the trabeculæ and walls of the lymph sinuses and vessels, leading eventually to fibrosis of the whole gland.]

[In sleeping sickness the majority of the glands removed after death, and a certain proportion of those removed during life, showed

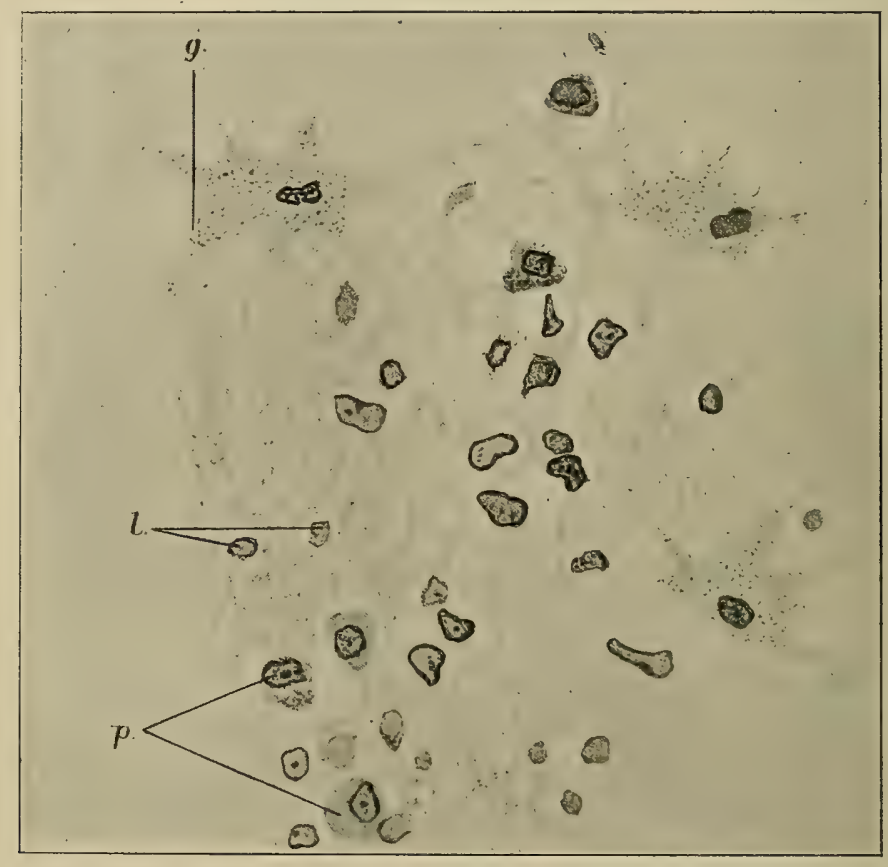

Fig. 53.-Changes seen in the Brain in Sleeping Sickness.

Three large glia cells $(g)$, their branches ending in a network around and upon a small vessel; lymphocytes $(l)$ and plasma cells $(p)$ are seen scattered about. Mag. 500 (From Mott.)

points of suppuration in their interior due to an infection with diplostreptococci, whereas the glands removed during life from natives in the early stages of trypanosomiasis were free from micrococci.]

[The changes in the nervous system are thus summarized by Mott: 'The chronic inflammatory change of the nervous system is manifested by a proliferation and overgrowth of the neuroglia cells, especially of those which are related to the subarachnoid space and perivascular lymph spaces, with accumulation of lymphocytes in the meshwork (see Figs. 53 and 55). In chronic cases plasma cells of Marscholko, similar to those in the lymphatic glands, may be found. Various other cells are met with in less numbers, some being the result of degenerative changes and others of endothelial origin, 
and possessing a phagocytic activity (see Figs. 53 and 54). The characteristic morbid change affects the soft membranes and the vessels.']

[There is a chronic leptomeningitis of the whole of the central nervous system, most marked where the cerebro-spinal fluid is most abundant. The subpial and septal neuroglia cells are increased in size and number, and their processes are also increased in number and thickness. The irritative processes which give rise to this overgrowth of glia tissue also produce proliferation of the endothelial cell nuclei and an infiltration of the pia arachnoid with lymphocytes, which may become transformed into plasma cells.]
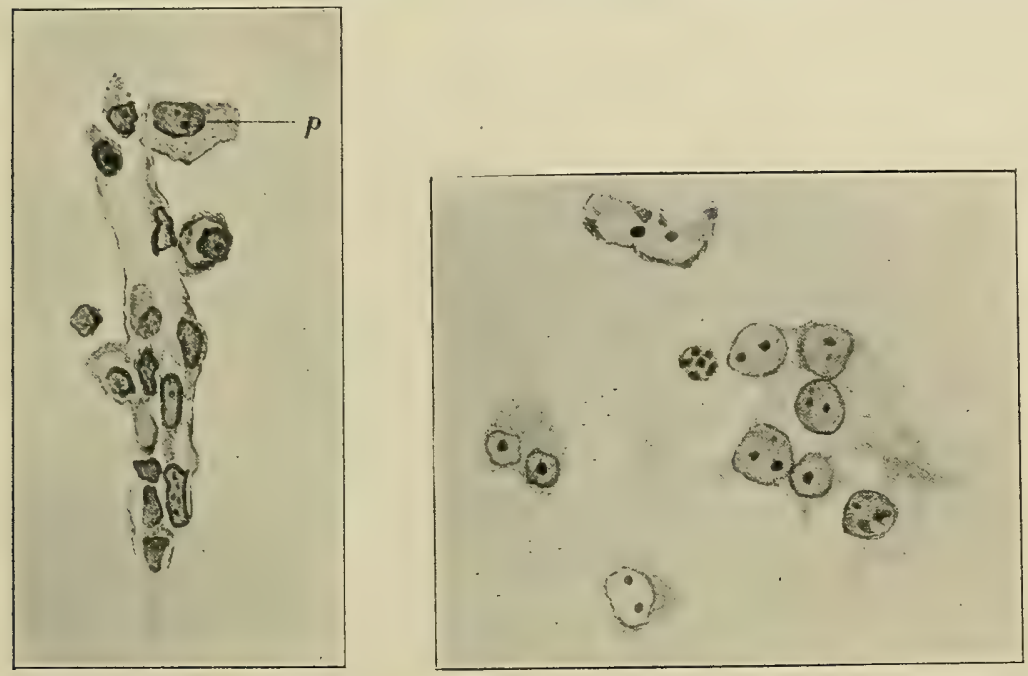

Figs. 54 and 55.-Changes seen in the Brain in Sleeeping Sickness.

Fig. 54.- Small vessel, showing endothelial nuclei proliferated, and three plasma cells. Fig. 55.-Active proliferating young glia cells found in great numbers in sleeping sickness tissues. The pale nucleus, with distinct nuclear membrane, contains chromatin granules, with an arrangement indicating mitosis. Surrounding the nucleus is the cytoplasm, staining pink with eosin, with a tendency to form starlike processes. Mag. 500. (From Mott.)

[The anatomical lesion distinguishing this disease from all other chronic nervous affections (Mott) is 'the universal perivascular cellinfiltration of the central nervous system' (see Fig. 56). This infiltration is most marked, and appears earliest in regions where the cerebro-spinal fluid is most abundant-namely, medulla, pons, cerebellum, and at the base of the brain. It does not consist entirely of lymphocytes, as was formerly supposed, but, as has been shown by Eisath's differential method of staining, ${ }^{1}$ of lymphocytes, proliferated nuclei of the glia cells, and endothelial nuclei in varying proportions, all entangled in a meshwork of greatly proliferated and enlarged glia cells.]

[Some degree of perivascular neuroglia proliferation was found in, two cases of experimental sleeping sickness in monkeys where there

${ }^{1}$ [G. Eisath, Monatschrift für Psychiatrie u. Neurologie, v. 20.] 
was no obvious lymphocyte infiltration around the vessels. Mott regards this neuroglia proliferation as being probably the first stage of the changes seen in the nervous system in human sleeping sickness.]

[When the trypanosomes get into the cerebro-spinal fluid they themselves-or possibly a toxin which they elaborate-slowly give rise to chronic inflammatory changes in the lymphatics of the nervous system. This inflammation, together with the perivascular cell-infiltration, gradually interferes with the circulation of the lymph and cerebro-spinal fluid, and sets up increased intracranial pressure. ${ }^{1}$ This increased pressure "interferes with the circulation of the blood in the small vessels, and the characteristic symptoms of the disease

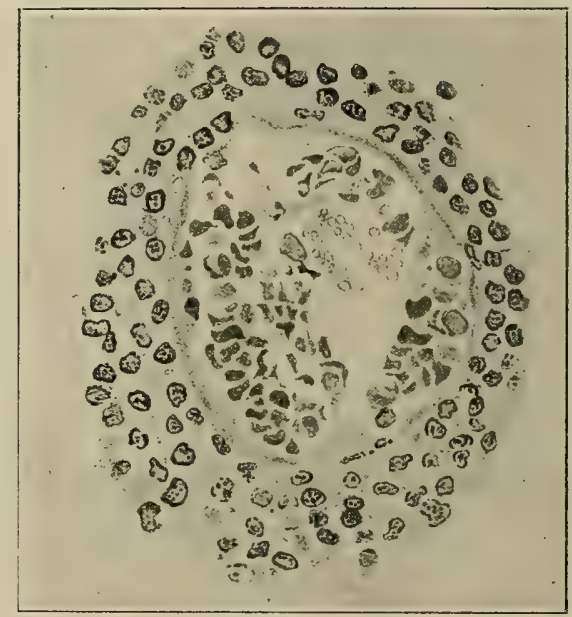

Fig. 56.-Section of a Vessel in a very Chronic Case of Sleeping Sickness, Showing Marked Perivascular Infiltration.

Mag. 250. (From Mott.)

- viz., lethargy, tremors, and muscular weakness-may be explained by the functional depression of the nerve cells from a deficient nutrition and interference with oxidation processes, brought about by mechanical and biochemical interferences with the activities of the nerve cells, and not by neural destruction. This is shown by the patients retaining comprehension of their surroundings, and by their intelligent response to questions when aroused from their lethargy-a totally different picture to general paralysis (also a meningo-encephalitis), in which there is a profound parenchymatous change, whereas sleeping sickness is a primary interstitial process, although later on in the disease, especially when it is chronic and of

1 [The intracranial pressure cannot be much increased-for optic neuritis is never seen in sleeping sickness patients-nor does the increased pressure in the subdural space of itself give rise to the symptoms of the disease, for I have obtained as much as 50 c.c. of cerebro-spinal fuid without producing any amelioration in the patient's condition. The anæemia of the brain is probably due to the pressure of the perivascular cell-infiltration, and, to some extent, to the endothelial proliferation.-ED.] 
long standing, marked chromolytic cell changes and a certain amount of destructive degeneration of the neurons occur' (Mott).]

[Other parts of the nervous system show the effects of the chronic irritation. (I) In chronic cases the central canal of the spinal cord is filled up owing to a proliferation of the cells of the ependyma. (2) The spinal nerve roots and the posterior spinal ganglia show more or less marked inflammatory changes in all cases. There is marked perivascular infiltration, and the capsules of the cells and the lymphatic sheaths of the nerves are at times crammed with lymphocytes. (3) Sections of the spinal cord may show septal glia proliferation, these changes in the spinal cord and ganglia being similar to, but less intense than, those found and described by Mott in dourine. $]^{1}$

[As with other trypanosomiases, capillary hæmorrhages may occur in the central nervous system.]

[On the whole, Mott thinks that the changes found in the nervous system in sleeping sickness very closely resemble those found in syphilis, except that the proliferative endarteritis leading to thrombosis, so common in the latter disease, is absent in the former.]

[The other tissues-e.g., the heart, pericardium, liver, alimentary canal, and testicles-show, though usually in far less degree, an infiltration and accumulation of lymphocytes in the lymphatics, suggesting a defensive reaction (Mott). Trypanosomes, even when numerous in the blood or in smears of tissues, such as the lymphatic glands during life, are nearly always very scanty in sections of organs and tissues. This may be due to the fact that the parasites undergo morphological changes which are not easily recognisable in sections, or to the fact that the terminal microbic infection, which so often carries off sleeping sickness patients, leads to a rapid destruction of the trypanosomes.]

In addition to the cerebro-spinal lesions, enlargement of the spleen, liver, and lymphatic glands is frequently seen in patients who have died of sleeping sickness. As trypanosomiasis is often complicated by malaria, it is difficult to say what share each of these diseases has in the splenic enlargement and congestion of the liver. The marked pigmentation of the viscera seen in many cases shows that malaria often plays an important part.

[The enlarged glands in sleeping sickness, as well as in animals infected with T.gambiense, may sometimes be hæmorrhagic (Dutton, Todd and Christy, Thomas and Breinl). Greig and Gray describe small superficial ulcers in the stomach, resulting from necrosis of the mucous membrane into which petechial hæmorrhages had occurred. These ulcers are not always present, for I failed to find them in the stomach of a European who recently died of chronic sleeping sickness at University College Hospital.]

1 [The above account has been taken largely, and in some parts almost verbatim, from the interesting paper in the Reports of the Sleeping Sickness Commission of the Royal Society, No. 7, 'Histological Observations in Sleeping Sickness and other 'Trypanosome Infectiuns,' by Dr. Mott.] 
Death often occurs as the result of pulmonary complications, and at the autopsy signs of congestion or odema of the lungs or of bronchopneumonia are frequently found. The heart is usually pale and flabby; the kidneys are normal. The intestines are often congested, and in Uganda they nearly always harbour the Ankylostoma duodenale and Ascaris lumbricoides. In addition one often finds Bilharzia hamatobia, Trichocephalus dispar, and sometimes, in the mesentery, adult forms of Filaria perstans (Low and Castellani).

In animals dying after experimental inoculation the spleen is as a rule greatly enlarged. This is most marked in rats ; thus, in a rat weighing 175 grammes the spleen weighed Io grammes, the average weight in a normal rat being 4 to 5 grammes. In a monkey weighing 805 grammes the spleen weighed 8 giammes, and in another monkey of I,297 grammes it weighed I9 grammes. In a rabbit weighing 3,070 grammes the spleen weighed I3 grammes.

Thomas and Linton never found trypanosomes in the cerebrospinal fluid of animals inoculated with $T$. gambiense, not even monkeys.

[Greig and Gray, however, on several occasions found trypanosomes in the cerebro-spinal fluid of monkeys, obtained during life by lumbar puncture and after death.]

\section{Section 7.-The Pathogenic Agent.}

As a rule the trypanosomes are scanty, or very scanty, in the blood of man, and the examination of the blood, made in the ordinary way, is not sufficient to show the presence of the parasite. Dutton and Todd have counted as many as twenty-three trypanosomes in a blood-film, but this is quite exceptional.

Bruce and Nabarro recommend the following method of examining the blood when the ordinary method fails to show the parasites. Ten c.c. of blood are taken from a vein into a tube containing a little citrate of potash solution, and centrifuged for ten minutes. At the end of that time the supernatant plasma [together with some of the middle layer (which contains the leucocytes and most of the trypanosomes) and a little of the red corpuscle layer] is pipetted off and centrifuged again for ten minutes. The supernatant liquid is again pipetted off and centrifuged for ten minutes, and the process repeated once again if necessary. [A drop of the deposit obtained on pipetting off or pouring away the supernatant liquid should be examined as an ordinary moist film with a magnification of 200 or 300 diameters, for some minutes after each centrifuging, because when the parasites are more numerous in the blood than usual, they may thus be discovered after one, two, or possibly three centrifugings. If not discovered previously, actively motile trypanosomes are practically always found in the sediment obtained after the fourth centrifuging.] 
[Dutton and Todd ${ }^{1}$ centrifuged small quantities of citrated blood in a capillary pipette and examined the leucocyte layer under the microscope. Possibly the smaller percentage of positive results obtained by these investigators, as compared with those obtained by us in Uganda, was due to the fact that they used much smaller volumes of blood than we did.]

The Cerebro-spinal Fluid.-In order to find the trypanosomes in the cerebro-spinal fluid during life, lumbar puncture must be resorted to. Ten or I $_{5}$ c.c. of this fluid are obtained and centrifuged for fifteen to twenty minutes. All the supernatant liquid is then poured away, and a platinum loopful of the deposit examined fresh with a magnification of about 200 diameters. ${ }^{2}$

In practising lumbar puncture, ${ }^{3}$ it is advisable to attend to the following details which we have taken from the excellent work of Tuffier on 'L'Analgésie chirurgicale par voie rachidienne,' Paris, Igor.

Use a platinum-iridium $n \in$ edle (which can be sterilized easily), 8 centi-metres [about $3 \frac{1}{2}$ inches] long, fitted to a hypodermic syringe. The external diameter of the needle should be I millimetre, and the internal diameter $0^{*} 6$ millimetre; the point should be bevelled off very little.

It is unnecessary to use a local anæsthetic. The patient should sit up with the arms held out in front. The lumbar region is thoroughly washed and rendered aseptic. The iliac crests are used as a guide, the transverse line joining their highest points crossing the spine at the level of the fourth lumbar vertebra (spinous process).

The left index-finger being placed on this spinous process, the patient is told to bend the head well forward. This serves to separate the laminæ of the vertebræ between which the needle has to pass. At the moment of inserting the needle the patient must be warned that he is going to be pricked, and that he must remain perfectly still and on no account straighten the back by sitting upright. The needle of the syringe, properly sterilized, is introduced at a point I centimetre [about $\frac{1}{2}$ an inch] from the middle line close up against the edge of the indexfinger, which is kept on the spinous process. The point of the needle is directed slightly towards the middle line and upwards, and when it enters the subdural space it is distinctly felt that there is no longer any resistance. A clear liquid immediately wells up in the needle and can be collected in a tube, or a syringe can be fitted to the needle.

[In sleeping sickness, when the pressure in the spinal canal is often much increased, the cerebro-spinal fluid may rush out in a steady stream directly the needle enters the subdural space, so that as much as 50 c.c. may be collected in less than a minute. Sometimes the fluid refuses to flow at first; this may be due to a fragment of skin or other tissue blocking the end of the needle. Slight aspiration with the syringe will overcome the difficulty in such cases, and on removing the syringe from the needle, the fluid flows out as usual. It is not safe to draw off a large quantity of cerebro-spinal fluid by forcible aspiration with the syringe, as damage may thereby be done to the bloodvessels of the spinal cord.]

1 [Dutton and Todd, Thompson Yates and Johnston Lab. Reports, v. 6, part ii., I905, pp. 97-IOI.]

2 [A $\frac{2}{3}$-inch ( 16 millimetres) objective with a 12 or 18 compensating eye-piece (Zeiss), or a $\frac{1}{6}$-inch ( 2 millimetres) objective with a 3 or 4 eye-piece, shows the parasites quite well.]

3 [In the original this description of the method of performing lumbar puncture occurs as a footnote in a later part of the chapter.] 
[Christy recommends local anæsthesia, produced by injecting cocaine both subcutaneously and deep into the muscles over the spot to be punctured. The patient is placed on his right side.]

[In Uganda we found it more satisfactory to use chloroform and to perform the operation with the patient lying on his side, the back brought right up to the edge of the bed or table, and the thighs and body in a position of extreme flexion. By anæsthetizing the patient there is no danger of a sensitive individual suddenly jerking backwards at the critical moment-as I saw happen on several occasions when the operation was performed without an anæsthetic-and possibly breaking the needle in his back, which I once witnessed.]

[The subsequent steps in the process were carried out as already described, and our patients usually got up and walked away ten or fifteen minutes after the operation, none the worse for the anæsthetic or the puncture.]

The trypanosomes are never very numerous, [and sometimes it is necessary to examine several drops of the deposit before one trypanosome is found; but occasionally they are comparatively numerous, and one or two parasites may be seen in each field of the microscope. In rare cases the trypanosomes have been so numerous in the cerebro-spinal fluid as to be seen in films of the uncentrifuged fluid. On the other hand, we met with a few cases of undoubted sleeping sickness in Uganda, and similar cases have been described by other observers, in which the trypanosome was not found in the cerebro-spinal fluid until after two, three, or even four lumbar punctures. I think, therefore, that trypanosomes are present in the cerebro-spinal fluid of every case of sleeping sickness. The fact stated above that the trypanosomes are occasionally absent at a particular examination is possibly due to a certain periodicity ${ }^{1}$ which they may exhibit in the cerebro-spinal fluid as well as in the blood].

The cerebro-spinal fluid is rarely as limpid as normal, but is usually very pale yellow in colour, or slightly turbid from the presence of a variable number of red blood corpuscles or of mononuclear leucocytes.

[Greig and Gray found that there is a progressive increase in the number of lymphocytes in the cerebro-spinal fluid as the disease advances. The average numbers found were 23 per cubic millimetre in the early stage of trypanosomiasis, and 257,355 , and 730 per cubic millimetre respectively in the different stages of sleeping sickness.]

On making dried and stained films of the deposit obtained on centrifuging the cerebro-spinal fluid, the trypanosomes, as well as the leucocytes, are badly fixed, and as a rule not well stained. On the other hand, the parasites occurring in the blood fix and stain well. It is therefore in the blood that $T$. gambiense must be studied; but as the trypanosomes are as a rule too scanty in the blood of man to

[C. Christy, I04 lumbar punctures. Thompson Yates and Johnston Lab. Reporls, v. 6, part i., 1905,pp. 57-71 ; Brit.Med.Journ. August 2, 1 SO4, pp. 372-378.] 
allow of this study, it is necessary to inoculate the blood or cerebrospinal fluid of a patient into susceptible animals in whose blood the $T$. gambiense multiplies.

Greig and Gray (op. cit.) recommend puncturing an enlarged lymphatic gland with an ordinary hypodermic syringe, and looking for the parasites in the drop of gland-juice thus obtained.

[Dutton and Todd have confirmed the observations of Greig and Gray by a large series of gland punctures, and have concluded that 'gland puncture is by far the most efficient method of demonstrating the presence of trypanosomes in cases of trypanosomiasis.' Gland puncture is of greater value in early cases of trypanosomiasis than in late cases, because (I) in early trypanosomiasis the trypanosomes are often very scanty in the blood and absent from the cerebro-spinal fluid, whereas they are particularly numerous in the lymphatic glands; and (2) in late trypanosomiasis (sleeping sickness) the trypanosomes are practically always present in the cerebro-spinal fluid-and therefore easily found on centrifuging this fluidwhereas they tend to diminish in number, and, in a small percentage of cases, even to disappear from the lymphatic glands. This is possibly to be correlated with the fibrosis and atrophy of the glands which are often observed in advanced sleeping sickness.]

[The posterior cervical glands gave the largest percentage of successful results ( 95 per cent.); then come the axillary ( 87 per cent.), the femoral ( 72 per cent.), and the epitrochlear ( $54^{* 2}$ per cent.).]

[Gland puncture is quite a simple operation, but it is necessary to attend to a few details of technique. The syringe must be boiled before use, then washed with sterile salt solution, every trace of which should be got rid of before using the syringe. After obtaining the drop of gland juice, the piston should be allowed to return almost as far as it will, and then fixed with the finger, otherwise much of the fluid aspirated would be lost in the body of the syringe. The drop of fluid in the syringe must be forced out by one sharp push of the piston rather than by two or three movements, so as to avoid getting air bubbles in the preparation. The drop is quickly covered with a cover-glass (care being taken to avoid the minute piece of skin punched out by the needle), ringed with vaseline, and examined at once (Todd).]

[Dutton, Todd, and Christy found trypanosomes in centrifuged hydrocele fluid when they were absent from the blood and cerebro-spinal fluid. Living trypanosomes were seen in the centrifuged pericardial exudate as long as twenty and a half hours after death. Pleural and peritoneal fluids may also show trypanosomes post-mortem.]

We have seen in Section I, dealing with the history of our knowledge of this disease, that at the beginning of the investigations on human trypanosomiasis, $T$. gambiense, the parasite of the irregular fevers of Gambia, and T. ugandense, the parasite of sleeping sickness, were thought to be distinct species. We have also seen that the most recent investigations have led to the conclusion that these are one and the same disease caused by $T$. gambiense. The most careful and acute observers have failed to confirm the characteristic differences pointed out by Castellani between $T$. gambiense and $T$. ugandense.

Trypanosoma gambiense.-T. gambiense (see Figs. 57 and 58, and coloured plate, Figs. 6 and 7 ) measures $17 \mu$ to $28 \mu$ long, by $\mathrm{I}^{\circ} 4 \mu$ to $2 \mu$ wide. Forms undergoing division are a little longer and 
considerably wider $\left(2{ }^{\cdot} 5 \mu\right.$ to $\left.3 \mu\right)$ than the ordinary forms. The free flagellum is often one-third or a quarter of the total length ; but sometimes the protoplasm of the body is continued along the whole length of the flagellum. Such forms without free flagellum were fairly numerous in the blood of a Macacus rhesus which died of trypanosomiasis. The undulating membrane is narrow; the nucleus, oval in shape, is situated about the middle of the body, and the centrosome is well seen in stained specimens. The posterior end of the body varies in shape: sometimes it is pointed, as in Fig. $57, I$; sometimes rounded, as in 3 , and in the latter case the trypanosome is nearly always shorter than in the former. These variations in the appearance of the posterior extremity are undoubtedly dependent upon the changes
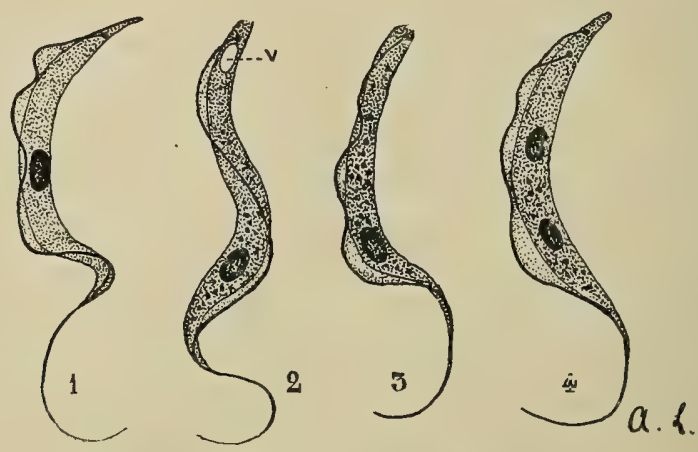

Fig. 57.-Trypanosoma gambiense.

I. T. gambiense well fixed in the blood. 2. Trypanosome in a smear of blood-stained œdema fluid. 3. Trypanosome with a rounded posterior end, and showing many chromatic granules in the protoplasm. 4. Form undergoing division. (Magnified about 2,000 diameters.)

in shape which the parasite undergoes during its migrations. The trypanosome contracts and elongates alternately, so that rapid drying of the blood fixes the parasite in one or other of these phases.

When the posterior end is drawn out and pointed the centrosome is further removed from the tip than when this is contracted and rounded off, so that the distance of the centrosome from the tip is a variable quantity, and cannot be used as an aid to diagnosis.

Close to or around the centrosome there is often seen a clear space or vacuole (Fig. 57, 2 v), to which Castellani attributed great importance. According to him, in T. ugandense the centrosome, which is very near the posterior end, is situated outside the vacuole which is found at this end of the parasite, whereas in T. gambiense the centrosome is further removed from the end and is in the interior of the vacuole. These distinctions are, however, artificial. When the trypanosomes have been well fixed in blood-films, vacuoles are not seen. They are best marked in badly fixed preparations made 
from the cerebro-spinal fluid or from the blood of very anæmic individuals. ${ }^{1}$

The protoplasm often contains chromatic granules which are sometimes remarkable on account of their size and number $(\mathrm{Fig} .57,3)$.

[As has already been mentioned, the trypanosomes seen in a blood-film may differ morphologically in appearance. Some are long and slender, stain well, and have an elongated nucleus and a long flagellum; others are broader and shorter, with clear, palestaining cytoplasm containing many granules, with short flagellum, and well developed undulating membrane. These two types of trypanosomes are regarded by Prowazek, Lühe, Nocht and Mayer, and othèrs as being in all probability sexual forms, the slender parasites being looked upon as the male and the broad as the female. In
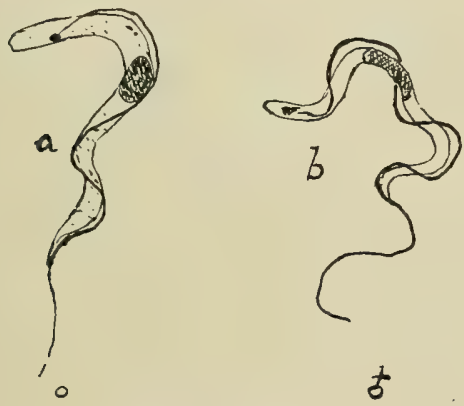

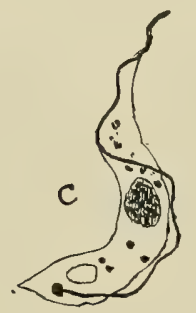

\&

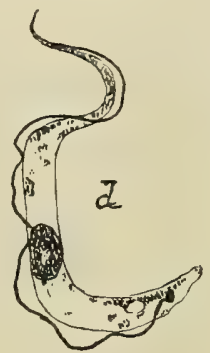

o

Fig. 58.-Sexual and Indifferent Forms of $T$. gambiense.

a. Indifferent form. b. Male form. c. Female form. $d$. Indifferent form, which in the fly becomes female: note the short flagellum. $a$ and $d$. From the gut of the tsetsefly forty-eight and twenty-four hours respectively after $a$ feed. $b$ and $c$. From the blood of an infected monkey. Magnified 2,000 diameters. (After Minchin, Gray, and Tulloch.)

addition to these, there are the ordinary or 'indifferent' forms (see Fig. 58). The short ( $q$ ) form may predominate in the blood of infected animals shortly before death (Nocht and Mayer).]

[It has been pointed out in a previous chapter (see p. ${ }_{5} 6$ ) that Koch has described similar morphologically distinct forms (which he regards as sexual forms of $T$. gambiense) in the digestive tube of tsetse-flies, but in all probability the flagellates seen by Koch were not $T$. gambiense, but ' wild' tsetse-fly trypanosomes. The same remark applies to the so-called multiplication forms of $T$. gambiense described by Gray and Tulloch ${ }^{2}$ as occurring in the stomach of $\mathrm{Gl}$. palpalis.]

As with the majority of trypanosomes, multiplication is by binary division. The centrosome and nucleus divide, then the flagellum divides longitudinally (see Fig. 57, 4), and finally the protoplasm

1 [I do not agree with the authors that vacuoles are not seen in well fixed bloodfilms. In Uganda we frequently found that a vacuole was present, just in front of the centrosome, in blood-films taken from human beings and from monkeys infected with $T$. ganbiense, and immediately fixed and stained by Leishman's method (see Plates 1.-III. in Sleeping Sickness Commission Report, No. I, by Bruce and Nabarro).]

2 [Gray and Tulloch, Sleeping Sickness Commission Report, No. 6, 1905, pp. 282-287.] 
shows signs of division. Castellani has described small amœeboid bodies, which he regards as another mode of reproduction of the parasite in the cerebro-spinal fluid. ${ }^{1}$ These forms have, however, never been seen by other observers. [But in sections of lymphatic gland and brain, in which ordinary trypanosomes are rarely seen, one may often find small rounded bodies, I $\mu$ to $2 \mu$ in diameter, with a large and small chromatin corpuscule, closely resembling the Leishman body of kala-azar. These are possibly degenerated trypanosomes, and have been described by Mott as occurring in trypanosomiases other than the human.]

[In a recent paper Salvin-Moore and Brein ${ }^{2}$ describe certain forms met with in rats infected with $T$. gambiense, which they regard as spore-like resistant phases of the trypanosome. The first stage is the development of a thick stainable band between the centrosome and the nucleus. This is seen in from 5 to 20 per cent. of the parasites present in the blood at the height of an exacerbation. The second stage-seen in the lungs, spleen, and bone marrow when the trypanosomes are rapidly diminishing in the blood-is the disintegration of the parasites and the formation of small rounded bodies. These consist of the nucleus surrounded by a hyaline layer. Moore and Breinl regard these round bodies as 'resistant forms,' and state that they are probably identical with Plimmer and Bradford's 'nuclei of a plasmodium,' and with Holmes' 'free nuclei' (see p. 274). Small trypanosomes are said to develop directly from these 'spore-like resistant bodies,' and thus the life cycle begins over again.]

[The formation of the stainable band and the ensuing union of the substance of the nucleus and centrosome may, according to Moore and Breinl, correspond to a sexual act; but before we can accept their interpretation of these phenomena, it is necessary that the observations themselves be confirmed. It may be added that, in order to demonstrate the appearances above described, the blood, or 'organ smears,' must be fixed, while still wet, in a reagent, such as Flemming's fluid.]

The trypanosomes often occur in pairs, with their posterior ends overlapping one another to a variable extent. Sometimes this overlapping is so marked that the nuclei of the parasites come to lie side by side, and this gives rise to the appearance of a trypanosome undergoing division. The presence of the flagella at the two extremities prevents one from falling into this error if the film is well stained.

When blood containing $T$. gambiense is mixed with salt solution or horse serum, the trypanosomes remain alive for five or six days at the temperature of the laboratory. They live much longer in tubes of rabbit's blood and agar. Tubes inoculated on January II, I904, with the blood of a rat severely infected with $T$. gambiense, and kept at $22^{\circ} \mathrm{C}$., showed a fair number of actively motile trypanosomes on January 30. A certain number of these parasites were unusually large, as much as $35 \mu$ to $40 \mu$ long; many dividing forms were also

1 Castellani, Centralb. f. Bukter., I, Orig., 1903, v. 35, p. 62, and Slecping Sickness Commission Report, No. 2, November, Iç03.

2 [J. E. S. Moore and Breinl, Lancet, May 4, I907, p. 1219.] 
seen, but no rosettes. Subcultures were unsuccessful, so we cannot say that there was any growth in vitro in this experiment.

Two rats inoculated on January I 8 with the contents of one of these tubes, seven days after insemination, showed no sign of infection.

[Thomas and Breinl ${ }^{1}$ have also made several attempts to cultivate $T$. gambiense, but with very slight success. They found veal and chickenmeat infusion more suitable than beef infusion; I to I' 5 per cent. peptone, $\frac{1}{4}$ to $\frac{1}{2}$ per cent. sea-salt, and 2.5 to 3.5 per cent. agar were added. The best proportion of this medium to blood (rabbit's best, then sheep or goat) was 2 to I or 3 to 2 . In this medium living trypanosomes were seen as late as the twenty-fifth to the fortieth day, and in one experiment on the forty-seventh day. On renewing the medium, living trypanosomes were seen as late as the fifty sixth day. There was no evidence of actual multiplication in any of the tubes, nor were the 'cultures' virulent for animals.]

[Gray and Tulloch's ${ }^{2}$ experiments in this direction were attended by one partial success. The medium used was Novy and McNeal's agar, to which three times its volume of undefibrinated dog's blood was added. The water of condensation was inoculated with a drop of blood from a white rat very rich in trypanosomes. On the eighth and tenth days no trypanosomes were seen in a drop of the 'culture' fluid, but on the fifteenth day several active ones were found. They were single and in groups of three or four; dividing forms were also seen. These trypanosomes were distinctly larger than those originally inoculated, some of them measuring as much as $54 \mu$. Besides being longer and broader than the original trypanosomes in the blood of the rat, the centrosome was situated nearer to the nucleus than in the trypanosomes inoculated. These cultural trypanosomes closely resembled certain forms found in the gut of tsetseflies twenty-four hours after the latter had fed on an infected monkey. After the twentieth day the trypanosomes were killed by contaminating cocci. Subcultures from the original tube before contamination with cocci failed to grow. No mention is made of the pathogenicity of the culture for animals.]

In a certain number of cases Castellani found streptococci in the blood or cerebro-spinal fluid of sleeping sickness patients; these were evidently associated with secondary infections. Streptococc1 are found as a rule only in the last stage of the illness or in the cadaver (Castellani).

[The Portuguese Commissioners had previously found that a very large percentage of sleeping sickness patients showed diplostreptococci post-mortem, so that they-not unnaturally-regarded this organism as being causally connected with the disease. Castellani himself held this view at first, as the result of his earlier investigations in Uganda. These micrococci may be found not only in the heart-blood and cerebro-spinal fluid, but also in the lymphatic glands, in the brain, spinal ganglia, and other parts of the nervous system, and in other organs (Mott). This generalized infection is undoubtedly a secondary or terminal phenomenon in sleeping sickness, due to the greatly diminished resisting power of the patient

1 [Thomas and Breinl, Thompson Yates and Johnston Lab. Reports, v. 6, part ii., I905, p. 45.]

2 [Gray and Tulloch, Sleeping Sickness Commission Report, No. 8, 1907, p. I33.] 
resulting from the chronic trypanosome infection. It is not to be regarded as an essential factor in the rtiology or pathology of the disease-though it undoubtedly hastens the fatal issue-for in some cases of undoubted sleeping sickness the blood and cerebro-spinal fluid are sterile. I found this to be the case in the European who contracted the disease in Uganda, and died three years later in London. Martin and Girard, and Sicard and Moutier also obtained negative bacteriological results in their cases of sleeping sickness in Europeans.]

\section{Section 8.-Mode of Propagation.}

All the West African tribes regard sleeping sickness as contagious, and they very generally isolate sufferers from the disease in special huts. The irresistible spread of trypanosomiasis in Portuguese Congo and in Uganda quite bears out the idea that the disease is transmissible, at least under certain conditions.

In Jamus of July 15, I903, we wrote as follows: 'Several authors have concluded that sleeping sickness is not contagious, because in many countries, notably in the Antilles, it has often been introduced without becoming endemic or epidemic. This is the case, however, with all hæmatozoan diseases which are propagated by insects-e.g., malaria, nagana, etc. ... These diseases are only contagious in districts where the necessary insects exist to propagate the pathogenic agents; thus nagana is propagated in Africa only where the tsetse-fly occurs.'

The natives of French Guinea attribute to flies the power of propagating the disease, and this native belief was thought by all observers to be probably correct, as soon as it was shown that sleeping sickness was a trypanosomiasis like nagana and surra, of which the propagation by means of biting flies had already been proved.

Dutton and Todd studied the biting flies of Gambia capable of propagating human trypanosomiasis. Specimens of flies were caught on the boats plying up and down the River Gambia. Two of these flies bite man and the lower animals-namely, Tabamus dorsovitta, Welka, and Gl.palpalis, Rob. Desvoidy. The latter is very common in West Africa, and abounds in the mangroves which occur in profusion along the banks of the watercourses, and during the hottest months gives rise to great annoyance. The attempts made in Gambia to convey trypanosomiasis to animals by means of these flies were unsuccessful.

Sambon ${ }^{1}$ and Brumpt ${ }^{2}$ suggested that tsetse-flies might propagate sleeping sickness. The distribution of sleeping sickness is not the same as that of Gl.morsitans, contrary to what had at first been stated by Brumpt; but, as we shall soon see, it corresponds closely with that of another species of Glossina.

1 Sambon, Journ. Trop. Med., July I, 1903.

2 Brumpt, Soc. de Biol., January 27 and November 28, 1903. 
It was Bruce and Nabarro who first showed by actual experiment that it is the Gl. palpalis ${ }^{1}$ which is certainly the most important, if not the only, agent concerned in the spread of this trypanosomiasis.

In their first report (August, I903) Bruce and Nabarro state that a monkey-a black-faced Cercopithecus - was submitted to the bites of numerous tsetse-flies caught in Entebbe, Uganda, and that trypanosomes appeared in the blood of this monkey. The experiment was begun on May I3, I903, and on the 27th of that month trypanosomes were first found in the blood.

The experiments recorded in the second report of Bruce, Nabarro, and Greig (November, I903) are of considerable interest. Tsetseflies (Gl.palpalis) were allowed to suck blood from negroes affected with sleeping sickness, and were afterwards fed on five monkeys (Cercopithecus). At the end of about two months trypanosomes appeared in the blood of the monkeys. Positive results were obtained even when the flies had fed on the patients twenty-four and forty-eight hours before being fed on the monkeys. We have just seen that positive results were sometimes obtained with freshly caught flies which had not been intentionally fed on patients. The flies in Uganda have numerous opportunities of becoming infected with trypanosomes by biting human beings, and perhaps also lower animals, affected with trypanosomiasis. We have already seen that $T$. gambiense is pathogenic for a number of mammals, and gives rise to a chronic infection which is well suited for the maintenance and propagation of the pathogenic agent.

Bruce, Nabarro, and Greig give a map of Uganda showing the distribution of sleeping sickness, and another showing that of $\mathrm{Gl}$. palpalis. On glancing at these two maps, which agree in almost every detail, one is struck by the close relation existing between the trypanosomiasis and Gl.palpalis, and one cannot fail to be convinced that $G l$. palpalis is the chief disseminator of the disease.

[Of course, there may be-and observations show that there aredistricts in which Gl.palpalis occurs, and where sleeping sickness is unknown. This means that the 'fly belt' has fortunately' not yet become infected by imported cases of trypanosomiasis. It is extremely important that such fly belts should be accurately mapped out, and suspected cases of trypanosomiasis rigidly excluded. The importance of this precaution is seen from the following instance: In I903 we ascertained that Gl. palpalis was prevalent all round the shores of the Albert Nyanza, but this fly belt was not infected, and sleeping sickness was unknown there. Owing to intercourse with infected natives from Busoga and other parts of Uganda around the Victoria Nyanza, in I904 sleeping sickness had appeared in this fly belt, and is now travelling down the Nile as far as the distribution of the tsetse-fly will carry it.]

1 The characters of Gl. palpalis are given in a note upon the tsetse-flies (see Chapter XVIII.). 
One of us recently received specimens of tsetse-flies from the following districts in the Congo Free State: Swata, Kwamouth, Bokala, and Yumbi. In every case they were Gl. palpalis, and we know that sleeping sickness is prevalent in all those districts, particularly at Berghe Ste. Marie, situated opposite Kwamouth, where it has made great ravages for several years past.

We have likewise found $\mathrm{Gl}$. palpalis amongst flies sent us by Dr. Tautain from a district of Bas Rio-Nunez, where sleeping sickness occurs; and two tsetse-flies coming from the neighbourhood of Rufisque, Senegal, given to us by Dr. Kermorgant, belonged to the same species.

[New habitats of Gl. palpalis are being constantly found. In Igo3 we ascertained that it occurred all round the Albert Nyanza, and Greig found it down the Nile at Wadelai, Nimuli, and at a spot about midway between the latter and Gondokoro. In Cameroon, Ziemann found it to occur at several places along the coastVictoria, Buea, and Barombi. It also occurs in Sierra Leone, and doubtless careful investigation will show that the whole West Coast of Africa from the Gambia to Angola is infested by $\mathrm{Gl}$. palpalis. During a journey of some 2,000 miles through the Congo Free State, Dutton and Todd found this fly practically everywhere along their route.]

[In South Angola, where sleeping sickness seems to be spreading, Wellman has found a variety of this Glossina, which Austen has named Gl. palpalis wellmani. This variety of $G l$. palpalis has also been found recently at several places in the Upper Congo district near Portuguese territory. Massey, ${ }^{1}$ who reports this observation, states that isolated cases of sleeping sickness occur, but trustworthy evidence as to where the disease was contracted is wanting.]

Can tsetse-flies, other than Gl. palpalis, propagate human trypanosomiasis?

[Experiments which we carried out ${ }^{2}$ in conjunction with Wiggins in East Africa seem to show that the East African tsetse-flies (Gl.pallidipes, Gl. fusca, and Gl. longipennis) can convey T. gambiense from infected to healthy animals by feeding on them. We also found that $\mathrm{Gl}$. palpalis is able to convey in a similar manner from diseased to healthy animals the various animal trypanosomes that occur in Uganda. It is, therefore, highly probable that species of Glossina other than palpalis may propagate human trypanosomiasis, and the possible results of the infection of the enormous fly areas of Africa by $T$. gambiense are too appalling to contemplate. Moreover, the disease may then not be confined to Africa, for recently the tsetse-fly has been found in Arabia. $\left.{ }^{3}\right]$

${ }^{1}$ [Yale Massey, Lancet, August 4, 1906, p. 296. For further information see Chapter XVIII.]

2 [Nabarro and Greig, Sleeping Sickness Commission Report, No. 5, 1905, p. 45.]

${ }^{3}$ [Carter, Brit. Med. Journ., November 17, 1906, p. I393.] 


\section{Section 9.-Diagnosis.}

It was formerly difficult to diagnose the disease in the first stage. The rises of temperature which occur may easily be mistaken for those of malaria or filariasis, which are often endemic in the same districts as trypanosomiasis.

It is necessary to examine the blood microscopically in order to distinguish these different diseases. If it be a case of malaria, the characteristic hæmatozoa will be found at the time of the febrile paroxysms; if it be filariasis, embryo filariæ will be found on examining the blood at the proper time-thus in the case of Filaria nocturna the blood should be examined at night.

We have already seen that trypanosomes are nearly always very scanty in human blood; therefore, if they are not found on microscopical examination of films it is necessary to use the special method recommended by Bruce and Nabarro (see p. 398), or to inoculate susceptible animals with several cubic centimetres of the patient's blood.

If enlarged glands are present, these may be punctured and the fluid thus obtained examined for trypanosomes.

[We have already seen that gland puncture-especially of the cervical glands-is of most value in early trypanosomiasis, when the diagnosis by means of the rather tedious process of centrifuging the blood is much more difficult and uncertain. Dutton and Todd think that cervical gland enlargement, in the absence of any obvious cause, in negroes should be regarded as evidence of trypanosomiasis.]

Malaria responds to treatment by quinine, which is without action on the fever of filariasis or trypanosomiasis. Trypanosome fever reaches its maximum at night, whilst in malaria the highest temperatures are usually observed during the morning. Tachycardia and rapid changes in the frequency of the pulse, occurring independently of variations in temperature, are mentioned by all observers as important signs of the first stage of trypanosomiasis.

In the second stage, when tremor of the tongue and hands, headache, and drowsiness are present, the diagnosis is much easier, especially as one is guided, as a rule, by the endemicity of the disease in the district in which the patient is seen or from which he comes.

Trypanosomiasis in the second stage may be confounded with general paralysis, certain cerebral affections, such as syphilis and tumour, tabes, beri-beri, and uræmia.

In general paralysis and tabes there is no hectic fever, as there is in trypanosomiasis ; in general paralysis the cerebral symptoms are more marked, as a rule; and in tabes inco-ordination of movements is the dominant symptom.

Beri-beri is characterized by peripheral neuritis, which is absent in sleeping sickness; on the other hand, tremors, fever, and lethargy are not seen in beri-beri.

In chronic nephritis with uræmia the urine is albuminous, and œdema is usually present [to a greater extent than in trypanosomiasis]. 
The district from which the patient comes often furnishes a valuable clue to the diagnosis. In doubtful cases it is always necessary to examine the blood, [glands], or cerebro-spinal fluid. We have already mentioned how this examination should be made to find the trypanosomes directly; in addition to this, one should inoculate rats or dogs-the former intraperitoneally, the latter subcutaneouslywith blood and with the remainder of the cerebro-spinal fluid which was not used for microscopical examination.

\section{Section 10.-Prognosis.}

Authors are agreed that sleeping sickness is always fatal. By careful nursing, so that bedsores are prevented and the patients properly fed, one may prolong the life of patients, without, however, being able to prevent the fatal issue.

Whether the prognosis is as grave in the first stage of the disease, when the trypanosomes are not found in the cerebro-spinal fluid, is at present undecided.

Different species of animals, notably Macacus monkeys, inoculated with $T$. gambiense may recover from the infection, from which it may be supposed, as Bruce, Nabarro, and Greig say in their report, that a certain number of cases of blood infection in man may recover, without going on to the second stage of the diseasenamely, sleeping sickness.

[But, as has already been stated, in monkeys infected with $T$. gambiense the trypanosomes may apparently disappear from the peripheral blood for months, so that the animals have come to be regarded as cured. More prolonged observation, however, shows that the infected animals eventually succumb to the trypanosomiasis, as was the case, for example, in one of our Uganda monkeys, which died eighteen months after infection, with nervous symptoms and the characteristic anatomical lesions post-mortem. Until the last month the monkey, though somewhat thin, appeared well, and for more than a year trypanosomes could not be found in the peripheral blood (Harvey).]

[As the course of the disease is so very chronic, extending as it often does over several years, it is impossible to say at present whether any individual in whom the trypanosome has been found has definitely recovered from the infection. Even if recovery is possible, it can only occur in a very small percentage of cases.]

[In March to June, Igo3, we found trypanosomes in the blood of twenty-three individuals in Uganda who showed no signs of sleeping sickness, and who, with the exception of the European already referred to, were apparently in good health. Three years later (April, I go6) twelve of these individuals had died-ten of sleeping sickness and two of pneumonia-and one was in an early stage of sleeping sickness; all traces of the remaining ten had been lost. These observations tend to show that until a specific remedy is found for the disease the prognosis in human trypanosomiasis is very grave.] 


\section{Section 11.-Treatment. Prophylaxis.}

Authors recommend tonic treatment; iron, quinine, and arsenic in combination give fairly good results, especially in cases complicated by malaria (Low and Castellani).

In animals infected with $T$. gambiense arsenious acid causes the trypanosomes to disappear from the general circulation, at least temporarily, and it may hasten recovery from the infection. ${ }^{1}$ The dose required for rats is $O^{\circ} I$ milligramme of arsenious acid per 20 grammes of body-weight, or I milligramme for a rat weighing 200 grammes; smaller doses are useless, or produce only very slight results.

Brumpt and Wurt $z$ have shown that in the marmoset arsenious acid, in doses of 2.8 milligrammes per kilogramme of body-weight, is very toxic for the trypanosomes; they state that, unfortunately, this drug is also very toxic for monkeys, even in doses of I milligramme per 600 grammes of body-weight. ${ }^{2}$

Animal experiments show that better results would probably be obtained in the treatment of human trypanosomiasis by giving occasional large doses of arsenious acid than by prescribing frequent small doses, as is usually done. Moreover, treatment should be started early-as soon as trypanosomes have been found in the blood [or glands] -without waiting for symptoms of sleeping sickness to appear.

Broden, ${ }^{3}$ who used injections of Fowler's solution in the treatment of trypanosomiasis in its early stages, noted that the injections caused the trypanosomes to disappear from the blood, and yet he seems to have used only small doses of the drug.

[Greig and Gray (op. cit., p. 67) have also tried the effect of arsenic, in the form of sodium arsenite, in five cases of early trypanosomiasis. The drug was beneficial to some extent, but its action was only temporary. The trypanosomes disappeared first from the blood and later from the lymphatic glands. After a variable time they reappeared temporarily and intermittently in the blood, but did not reappear in the glands. Greig and Gray think that the arsenic has (I) a direct destructive effect upon the trypanosomes, and (2) a secondary effect which comes into play later-namely, the production of active immunity by the destroyed trypanosomes. The after-history of these patients is not given in the report.]

[Gray and Tulloch (op. cit., p. 30) treated ten further cases of trypanosomiasis with arsenite of sodium, but with disappointing results. In most of the cases trypanosomes continued to be present in, or disappeared only temporarily from, the lymphatic glands, but in one case the glands remained free from trypanosomes for as long as ten months.]

1 Laveran, Acad. des Sciences, February 22, 1904. The same solution was used as in the treatment of nagana (see Chapter VI.).

2 Soc. de Biol., May 7, 1904.

3 Broden, Bull. de la Soc. d'études colon., February, 1904. 
We have tried the effect of trypanred upon rats infected with $T$. gambiense, but with unsatisfactory results.

One would imagine a priori that $T$. gambiense, which multiplies in the blood of man as well as in that of most other mammals, would be unaffected by human serum, unlike the trypanosomes of nagana, surra, and caderas, against which man enjoys a natural immunity. Our own observations show this to be actually the case. Dried and powdered human serum injected in doses of $0^{*} 2$ to $0^{*} 3$ gramme into rats weighing I70 to 200 grammes infected with $T$. gambiense was quite inactive.

Rats infected with $T$. gambiense at first show very few parasites in the blood, and sometimes a negative result may be recorded after the parasites have once been present; but after a month or a month and a half the trypanosomes are permanently established in the blood, and they are, as a rule, sufficiently numerous to enable one to study the effect of different drugs upon them. That is the time to study the action of serums and other drugs.

The serum of normal guinea-pigs, sheep, and horses, like human serum, has no action upon $T$. gambiense. This was to be expected, seeing that these animals are susceptible to $T$. gambiense.

Manson treated a patient suffering from trypanosomiasis with injections of horse serum, but unsuccessfully. ${ }^{1}$ This, again, was to be foreseen, as the horse is not refractory to $T$. gambiense.

Unfortunately, this trypanosome develops in the blood of most mammals, but we must add that the serum of a Cynocephalus, which is very resistant to $T$. gambiense, proved just as inactive as the serum of animals markedly susceptible to this trypanosome.

Experiments should be made with the serum of animals highly immunized against $T$. gambiense, but the results of previous experiments made in this direction with other pathogenic trypanosomes, and of researches upon the curative power of the serum of animals enjoying acquired immunity against $T$. gambiense itself, leave little hope as to the ultimate result of such experiments.

[Thiroux ${ }^{2}$ has found that the serum of sleeping sickness patients possesses some preventive action. Mice injected with a mixture of this serum and citrated blood containing T. gambiense became infected after a longer incubation period (a delay of from nine to thirty-two days over the control mice) than did control mice injected with citrated blood alone. The serum had no curative action on animals already infected with the trypanosome.]

Good hygienic conditions and a liberal diet are important factors in the treatment of trypanosomiasis. In Africa sleeping sickness makes the greatest ravages amongst the poor, overworked, and badly-fed negroes. ${ }^{3}$ Similarly, it is found that animals which are

1 Manson and Daniels, frit. Med. Journ., May 30, 1903.

2 [Thiroux, C. R. Soc. Biol., v. 6o, 1906, p. 778.]

3 Christy, Sleeping Sickness Commission Report, November, Igo3. 
enfeebled become more severely infected than those which are in good condition and well fed.

The application of the cautery to the spine is indicated when the symptoms of spinal meningitis are well marked. ${ }^{1}$ We may add in passing that the treatment of sleeping sickness most used by the natives of Africa is removal of the enlarged lymphatic glands. ${ }^{2}$

ProphylaXis. - Trypanosomiasis is propagated by tsetse-fliesGl. palpalis in particular-therefore the most important prophylactic measure consists in the protection of the person from the bites of these flies.

The site for a house should be chosen far from damp and marshy áreas, which the tsetse-flies infest, and also far from the native huts in which cases of sleeping sickness occur. The house itself should be fitted with wire-netting in the doors and windows, so that the flies cannot enter.

When one has to pass through a 'fly belt,' it is advisable to use a veil of fine mosquito netting round the head, similar to that which has been suggested for the protection of the head and neck against mosquitoes.

It would be as well to study the means of destroying the Gl. palpalis - at least, in the immediate vicinity of towns and villages. It is possible that in this case, as with nagana, the destruction of the big game and its disappearance may give satisfactory results.

[In Uganda, and probably also in some other parts of Africa, Gl. palpalis is not dependent on big game for its existence. Moreover, the destruction or extermination of the fly will be extremely difficult, if not impossible, for it has recently been shown by Bagshawe, ${ }^{3}$ in Uganda, that the natural habitat of the pupa of Gl.palpalis is the loose, crumbling soil around the roots of the banana-trees along the lake-shores, and not inland. As bananas form the staple food of the natives, it would be impossible to destroy the plantations without creating a famine. Minchin ${ }^{4}$ suggests that to keep down the fly the natives should be encouraged or constrained to keep fowls in banana plantations where the fly is abundant, and that in forest districts it might even be advisable to introduce the Indian junglefowl. He thinks these birds would probably scratch up and eat many of the pupæ of the fly.]

[By snipping off ' a piece of leg for the purposes of identification, Bagshawe found that individual flies can travel at least a mile. That is the reason, he thinks, why the breeding-places have eluded search so long.]

It is important, in districts where trypanosomiasis is endemic, to obey carefully all the laws of hygiene, for disregard of those laws may favour infection.

1 [The value of this mode of treatment is very problematical.-ED.]

2 For the later experiments and observations on treatment see next chapter.]

3 [Bagshawe, in Minchin's letter to Nature, October 25, 1906.]

4 [Minchin, Nature, November 8, 1906.] 
[Dutton and Todd point out that, 'while sleeping sickness may be quickly carried from place to place, it spreads but slowly from an infected centre.' This, no doubt, explains the enormous extension of the disease in recent years in Uganda, the Congo, and other parts of Africa, brought about by the introduction of large numbers of infected porters, labourers, and soldiers into uninfected fly-infested districts.]

[In gland palpation we have a simple means of diagnosing trypanosomiasis, which an intelligent layman can apply accurately. The following measures based on this fact have been recommended by Todd, ${ }^{1}$ and are already being applied in the Congo State: (I) 'The establishment of posts of inspection along the main roads to prevent the entrance of infected persons into uninfected districts, and (2) the removal of infected persons from posts in uninfected districts to places already infected.']

['The application of these measures will be obtained by informing every agent of the State of the significance of enlarged cervical glands. Each chief of a post will be directed to examine his staff and to send suspected persons towards infected regions to the nearest doctor, where, if it is necessary for diagnosis, the patient will be watched. Each white man will be made to understand the importance of preventing persons with enlarged glands from proceeding towards uninfected districts, and will be given authority to prevent their progress.']

$\left[\right.$ Hodges $^{2}$ has recently studied the epidemiology of the disease in Unyoro and the Nile Valley (Uganda), and is of opinion that quarantine or isolation of infected persons is not feasible. $\mathrm{He}$ suggests that the sick in each community should be removed from direct communication with the neighbouring fly belt, and that they should on no account be allowed to approach any water where the fly is. Vigorous measures along these lines are now being taken in Uganda, and the patients are being placed in a number of specially organized settlements inland, away from any fly belt. Special arrangements are being made to remove all sources of danger from the European settlements at Entebbe and Jinja, by the removal of all vegetation which is capable of harbouring the tsetse-flies. $\left.{ }^{3}\right]$

[Probably over half a million natives of Africa have died of sleeping sickness during the past ten years. In many of the villages visited by Dutton and Todd 30 to 50 per cent. of the population were infected with trypanosomes, and a similar condition, no doubt, obtains in other parts of Africa. The large majority of these infected persons will die of their trypanosomiasis. We should, therefore, do our utmost to protect uninfected areas from a similar fate.]

1 [Dutton and Todd, Liverpool School of Tropical Medicine, Mem. 13, 1906, pp. $25-38$.

2 [Hodges, Slceping Sickness Commission Report, No. 8, 1907, pp. 86-99.]

${ }^{3}$ [Brit. Med. Journ., January 26, 1907, p. 223.] 


\section{CHAPTER XIII}

\section{THE TREATMENT OF THE TRYPANOSOMIASES ${ }^{1}$}

\section{Section 1.-Historical Review of the Treatment of the Trypano- somiases in General.}

[IN the foregoing chapters reference has been made to the large number of drugs that have been tried in the treatment of the various trypanosomiases. As has been pointed out, many of these drugs have a microbicidal action upon the trypanosomes in vitro, but very few of them are of real value in the treatment of trypanosome infections.]

[A good résumé of the development of the chemical therapeutics of the trypanosomiases is given by Mesnil and Nicolle ${ }^{2}$ in their interesting paper on 'The Treatment of the Trypanosomiases by Benzidine Dyes,' which will be considered in detail later. They say: 'Lingard, and after him Bruce, were the first to demonstrate the undoubted value of arsenical treatment in Equidæ suffering from surra and from nagana. They even succeeded in curing some of their animals. Laveran and Mesnil thereupon initiated the study of arsenical compounds in the treatment of experimental nagana. They showed that by repeated injections the life of infected dogs and rats could be materially prolonged, but that a permanent cure could not be obtained with these drugs. On the other hand, they succeeded in curing, with human serum, several mice infected with nagana. Laveran obtained analogous results in mice infected with caderas or surra. These observations are very interesting from a theoretical point of view,' but, unfortunately, cannot be applied in practice, owing to the large doses of serum that would be required for animals such as cattle and horses.]

['Ehrlich and Shiga's discovery of trypanred inaugurated a great forward movement in the treatment of the trypanosomiases. By means of this dye they were able to cure a considerable proportion of mice infected with caderas. This result has since been confirmed by Laveran and Mesnil, Halberstaedter, ${ }^{3}$ Franke, and Thomas. Unfortunately, as Ehrlich and Shiga's work showed, trypanred is

1 ['The whole of this chapter has been added.-ED.]

2 [Mesnil and Nicolle, Ann. Inst. Past., v. 20, I906, pp. 417-448, and 513-538.]

3 [Halberstaedter, Centralb. f. Bakter., I, Orig., v. 38, I905, pp. 525-532.] 
less efficacious in the treatment of other animals infected with caderas, as well as in the treatment of the other trypanosome infections. All observers are agreed at the present time that trypanred is unable to cure mice infected with nagana. It is equally valueless in mice infected with the virus of Mauritian surra-unless the treatment be repeated (Laveran) - as well as in rats and other animals infected with $T$. gambiense (Laveran, Thomas). On the other hand, trypanred is able to cure mice infected with mbori (Laveran, Franke) or with dourine (Halberstaedter).']

['Methyl or ethyl green (malachite green, "brilliant" green), recommended by Wendelstadt and Fellmer, ${ }^{1}$ never cured a single animal when the dye was used alone. The most that these dyes could do was to prolong for some time the life of rats infected with nagana.']

['An important step in advance was made by Thomas, who first used, in the treatment of the trypanosomiases, atoxyl, an arsenical compound remarkable for its slight toxicity. By repeated administration of this drug, he apparently cured a certain proportion of animals suffering from various trypanosome infections. On theoretical grounds we (Mesnil and Nicolle) had already begun to experiment with atoxyl before the publication of Thomas's work.']

['We may briefly refer to the experiments of Balfour and Neave with chrysoidin, which appears to be valueless in treatment.']

['The impossibility of obtaining a certain cure by the exclusive use of any one of the above-mentioned drugs suggested the employment of two or more of the most valuable of them in combination. Shortly after the discovery of trypanred, Laveran treated animals on these lines by injecting the dye the day after an injection of arsenic, and repeating these alternate injections more or less frequently. In this way he was able to cure rats, mice, and dogs infected with mbori and with Mauritian surra; rats, dogs, and two macaques (Macacus rhesus) infected with $T$. gambiense; and two dogs infected with experimental dourine.']

['By means of a similar combined treatment Franke ${ }^{2}$ cured rabbits and a monkey (Cercopithecus) infected with mal de caderas. Thomas $^{3}$ also recommends the combined treatment with sodium arseniate and trypanred, but, having found that atoxyl is more valuable than sodium arseniate, he used the combination of atoxyl and trypanred in his later experiments on treatment. Unfortunately, he gives very few details of the results obtained in this way. He draws attention to the toxicity of trypanred, and in his first report upon the subject he says that if further efforts be made to produce

1 [Wendelstadt, Deutsche med. Wochenschr., I904, No. 47.]

2 Franke, 'Therapeutische versuche bei Trypanosome-erkrankung,' 'Veterin.mediz. Dissert.,' Giessen, G. Fischer, Jéna, I905; abstract by Mesnil in Bull. Inst. Past., v. 4, 1906, pp. 138, 139.]

${ }_{3}$ [Thomas, Brit. Med. Journ., May 27, 1905, pp. II 40-1143.] 
a substance like trypanred, but less irritating in action, the combination (with atoxyl) ought to be of service in the treatment of human trypanosomiasis.']

['Wendelstadt and Fellmer ${ }^{1}$ have attempted to improve upon their method of treatment by the alternate administration of "brilliant green " and arsenious acid. In this way they cured at least one rat and a Macacus rhesus infected with $T$. brucei. The latter even became highly immunized, which is rarely the case in animals which recover from trypanosome infections as the result of treatment.' About seven months after the treatment was begun the monkey was permanently cured, and it was then refractory to two successive inoculations of the trypanosome. The serum of this monkey, in doses of $\frac{1}{2}$ c.c., brought about the immediate disappearance of the trypano. somes from the blood of heavily infected rats ; in vitro it agglutinated the trypanosomes, whereas the serum of normal monkeys has no microbicidal or agglutinating action upon T. brucei.]

[Mesnil and Nicolle conclude their résumé of the chemical therapeutics of the trypanosomiases by alluding to the failures of Brumpt and Wurt $z^{2}$ and of de Magalhaes ${ }^{3}$ to cure, by means of arsenic and trypanred, monkeys and rats infected with $T$. gambiense.]

[Mesnil and Nicolle's researches, undertaken with the view of studying the chemical factors which determine the activity of the 'benzidine' dyes, as well as of finding, if possible, therapeutic agents better than trypanred for the treatment of the various trypanosomiases, have led to the discovery of synthetic preparations of very great value in both the prophylaxis and treatment of several trypano. some infections.]

[In addition to these experiments upon the treatment of the trypanosomiases by chemical agents, which appear to give the greatest promise of ultimate success, other experiments have been carried out along different lines, and with very little, if any, success in most cases. Amongst these may be mentioned: (I) The treatment with serums and extracts of spleen; (2) the injections of cultures of organisms; and (3) the exposure to $\mathrm{X}$ rays and other rays.]

\section{Section 2.-Experiments on Treatment with Agents other than Chemical Substances : Serums and Spleen Extracts; Cultures of Organisms ; X Rays and other Rays ; Light.}

[The Action of Serums.-In previous chapters it has been stated that human serum injected into animals (rats and mice)

1 [Wendelstadt and Fellmer, Zeitschr. f. Hyg., v. 52, 1906, pp. 263-28I ; Sitzungsber. d. Niederrhein. Ges.f. $\Lambda$ at. u. Heilkunde zu Bonn, January 22, 1906 ; Verhandl. d. deut. colon. Congresses, I905, pp. 287-29I ; abstract by Mesnil, in Bull. Inst. Past., v. 4, I906, pp. 378.380.]

2 [Brumpt and Wurtz, C. R. Soc. Biol., 1904, No. 16, v. 59, 1905, p. 6I.]

3 [De Magalhaes, Arch. Inst. roy. de Bact. Camera Pestana, v. I, I906, pp. $17 \mathrm{I}-176$. $]$ 
infected with various trypanosomes (nagana, surra, caderas, and $T$. dimorphon) causes a rapid disappearance of the trypanosomes from the blood. In most cases this disappearance is only temporary, but occasionally in nagana and caderas infections the animals so treated have been permanently cured. Laveran ${ }^{1}$ has since found that baboon's serum has a similar effect upon the trypanosome of nagana, surra, and caderas, but that it is distinctly less potent than human serum. Unlike the latter, however, baboon's serum had an effect upon $T$. gambiense in the blood of the mouse, in a dose of $0^{\circ} 2$ gramme of dried serum for a mouse weighing 18 grammes.]

[On p. I99 mention was made of the specific serum obtained by Kleine and Moellers from donkeys immunized against $T$. brucei. This serum completely protected mice from infection with $T$. brucei, and under certain conditions was able to cure nagana-infected mice. This action of the serum was specific, for this serum had little or no effect upon mice infected with $T$. gambiense.]

[The Action of Spleen Extract.-Acting upon Rodet and Vallet's observation that an extensive destruction of trypanosomes occurs in the spleen of an infected animal (see p. I45), Roux and Lacomme $^{2}$ have endeavoured to treat nagana-infected dogs by injecting extract of ox spleen (pounded spleen to which three times its volume of saline is added) in 20 c.c. doses. Three dogs were injected (two subcutaneously, one intravenously), and in each case the trypanosomes disappeared from the blood. In the two dogs injected subcutaneously a staphylococcal abscess developed at the site of inoculation, and this may have had something to do with the disappearance of the trypanosomes. In one of these two dogs the trypanosomes reappeared in seven days; in the case of the other two the observations had not been made sufficiently long to say whether the disappearance of the trypanosomes was permanent or temporary.]

[The Action of Bacteria upon Trypanosome Infections. $-\mathrm{Nissle}^{3}$ treated rats infected with $T$. brucei with intraperitoneal injections of $\frac{1}{20}$ loopful of a potato culture of $B$. prodigiosus. Some of the rats died in half an hour to three hours after the injection, but in others the trypanosomes had almost completely disappeared from the blood in twenty-four hours.]

[Massaglia found that the trypanosomes rapidly disappeared from the peripheral circulation when the host became infected with streptococci. Thomas and Breinl ${ }^{4}$ injected cultures of various bacteria into trypanosome-infected animals, in most cases without permanent benefit.]

1 [Laveran, C. R. Acad. Sciences, v. 139, I904, p. 177.]

2 [G. Roux and L. Lacomme, C. R. Acad. Sciences, v. I43, I906, p. I35.]

3 [Nissle, Hyg. Rundschau, 1904, No. 21, p. 1039.]

4 [Thomas and Breinl, Thompson Yites and Johnston Lab. Reports, v. 6, part 2, I905, P. 6I.] 
[The Action of X Rays.-Mention has already been made of the observations of Russ upon T. lewisi (p. 76) and of Ross, De Nobele, and Goebel upon T. brucei (p. I59), that $\mathrm{X}$ rays have no effect upon these trypanosomes in vitro. Ross has also found that Finsen rays and radium emanations are equally inactive against T. brucei.]

[Ed. and Et. Sergent, ${ }^{1}$ working with an Algerian trypanosome (see p. 2I4), found that the $X$ rays had a slight favourable influence when applied to the entive body of rats when the curve of the trypanosomes was at its highest. On the other hand, when the rays were applied during the incubation period, they hastened the fatal issue.]

[De Nobele and Goebel ${ }^{2}$ tried to find out if radio-therapy had any effect upon the course of experimental nagana in the guinea-pig, mouse, or rabbit. The guinea-pigs and mice were placed in cages at a distance of Io centimetres (4 inches) from the tube. In rabbits the rays were applied only to the testicles-organs for which, as Van Durme has shown, the trypanosomes have a special predilection. The results were absolutely negative, whatever the period and duration of the treatments, and in spite of the fact that the doses of $\mathrm{X}$ rays absorbed by the animals were always above the maximum compatible with the integrity of the skin, and even with life in some cases. De Nobele and Goebel add : 'It is illusory, it seems, to hope for favourable results in sleeping sickness from radio-therapeutic treatment, as Mense ${ }^{3}$ has thought possible.']

[The Action of Light after Injection of Colouring AGENTS.-Busck and Tappeiner ${ }^{4}$ injected certain dyes into animals and then tested the effect of their serum upon trypanosomes. Methylene blue serum rapidly lost its colour, and was found to be inactive in the light on animals tested. Thionin, methylene violet, bengal rose, and other dyes gave fair results. Eosin and erythrosin alone gave serums which were coloured and active for a long time. Trypanosomes (in saline suspensions) were killed in from one to three hours in the light, whereas they survived at least twenty-four hours in the dark.]

[Mice, rats, and rabbits infected with $T$. brucei were tested for the action of light after injections of eosin or erythrosin. The animals were depilated on the back by means of calcium sulphhydrate, and exposed in a wire cage to the sun's rays filtered through a solution of ferrous sulphate, which arrests nearly all the infra-red rays. It is necessary to have a good current of air circulating

${ }^{1}$ [Ed. and Et. Sergent, Ann. Inst. Past., v. 20, 1906. See also Salomonsen and Dreyer, C. R. Acad. Sciences, June 13, 1904, p. I 543.]

2 [De Nobele and Goebel, Ann. Soc. de Méd. de Gand., v. 86, I906, pp. 52-63; abstract by Mesnil, in Bull. Inst. Past., v. 4, 1906, p. 672.]

${ }^{3}$ [Mense, Arch. f. Schiffs. u. Tropenhyg., July, 1905.]

${ }^{4}$ [Busck and Tappeiner, Deutsche Arch. f. Klin. Medizin, v. 87, 1906, pp. 98-IIo; abstract by Moutin, in Bull. Inst. Past., v. 4, I906, p. 672.] 
around the animal, otherwise its temperature may become dangerously high.]

[In rabbits $0^{\circ} \mathrm{I}$ gramme eosin proved dangerous to life, producing insolation or subsequent necrosis; $0^{\circ} 05$ gramme eosin was well tolerated, but had no effect upon the trypanosomes.]

[In rats and mice better results were obtained. An infection was prevented by treating the animals, immediately after the inoculation of trypanosomes into the back, with a subcutaneous injection of the dye in the abdomen, and then exposing the back to light for six or seven hours. This exposure to light after the injection of the dye killed the trypanosomes inoculated, for control animals kept in the dark became infected in the ordinary way. The dose of the dye was $0^{\circ}$ I c.c. of a 2 per cent. solution per 30 grammes of mouse. If treatment was delayed for twenty-four hours no beneficial result followed, so that this mode of treatment will be of no use practically.]

\section{Section 3.--Recent Experiments on Treatment with Arsenic Compounds, including Atoxyl, and with Trypanred.}

[We have already seen that the first successful attempts to treat trypanosome infections were those of Lingard, and later of Bruce, who succeeded in prolonging the life of animals spontaneously infected with surra or nagana by the administration of arsenic. Occasionally a permanent cure was thus effected, but in most cases the animals died-either of the disease, if the treatment was stopped on account of intolerance, or of arsenical poisoning, or necrosis at the seat of inoculation, if the treatment was persisted in. Laveran and Mesnil subsequently used arsenic in the treatment of experimental nagana in dogs and rats; but a cure was never effected, although the progress of the infection was checked for a time. Moore and Chichester ${ }^{1}$ state that they had good results from arsenic in the treatment of trypanosome-infected cattle in Southern Nigeria (see p. I70). Broden and others have treated cases of human trypanosomiasis with arsenic, usually in the form of Fowler's solution, with apparent success (see p. 4II). On the other hand, Nabarro, Greig, Gray, and Tulloch ${ }^{2}$ in Uganda treated sleeping sickness patients, as well as cases of early trypanosomiasis, with injections of arsenic, but on the whole with disappointing results. The trypanosomes disappeared from the blood and lymphatic glands, but reappeared in every case except one, in which the glands remained free for as long as ten months. Gray and Tulloch also found that arsenic was of very little value as a prophylactic or curative agent in the case of monkeys infected with $T$. gambiense.]

[Arsenic being evidently of considerable value in the treatment of these diseases, the indication was to find, if possible, a less toxic form of the drug than the ordinary inorganic salts of arsenious

\footnotetext{
1 [Chichester, Journ. Trop. Med., v. 8, 1904, p. 195.]

${ }^{2}$ [Gray and Tulloch, Sleeping Sickness Commission Report, No. 8, 1907, pp. 30-53.]
} 
and arsenic acids. This induced Thomas $^{1}$ to try atoxyl, ${ }^{2}$ which, as we have seen in Section I, was being experimented with by Mesnil and Nicolle before the publication of Thomas's paper. By means of atoxyl alone, but more especially in conjunction with trvpanred, introduced by Ehrlich and Shiga early in I904, encouraging results have been obtained by Thomas in some cases.]

[The following are the details of some of Thomas and Breinl's experiments with atoxyl and trypanred :]

[ATOXYL.-TRYPANOSOMA GAMBIENSE.-Rabbit (weight, 2,oio grammes) infected with the 'ordinary' strain of T.gambiense. Many trypanosomes were present in the blood at the time treatment was started. The animal was given I c.c. of a 5 per cent. solution of atoxyl three times a week, the dose being gradually increased to I c.c. of a Io per cent. solution. After three and a quarter months of treatment the rabbit became very ill and was killed. The whole of its blood injected into a monkey failed to infect.]

[Guinea-pigs, infected with the same strain of $T$. gambiense, were treated with an initial dose of $0^{\circ} 3$ c.c. of a ro per cent. solution of atoxyl, followed by $0^{\circ}$ I to $0^{\circ} 3$ c.c. of a 5 per cent. solution three times a week for two months. The animals so treated all increased in weight. One died sixtytwo days after treatment was discontinued; its blood was not infective for three rats. Two other guinea-pigs were killed 80 and 100 days respectively after discontinuing the treatment, and their blood was found to be non-virulent.]

[A monkey (Macacus vhesus) inoculated with a very virulent strain of T. gambiense improved greatly, and gained in weight during treatment. The dose administered was I c.c. of a Io per cent. solution twice a week. Unfortunately the monkey developed acute dysentery, to which it succumbed. Its blood was non-infective in doses of I c.c., but was infective in the dose of I $_{5}$ c.c. $]$

[Many rabbits infected with this same highly-virulent strain of T. gantbiense were treated with atoxyl. Whereas the untreated rabbits died in fourteen to thirty-six days, several of the treated animals survived inoculation for eight months. Guinea-pigs infected with this strain did not react so well to the treatment. Rats and mice reacted well if the treatment was started early and large doses of atoxyl were administered.]

[TRYPANOSOMA BRUCEI.-Successful results were obtained in the case of guinea-pigs infected with T. brucei. Atoxyl injections produced a rapid and permanent disappearance of the trypanosomes in several of the inoculated animals, and the whole of the blood was found to be non-infective on injection into rats. One guinea-pig had lived for nine and three-quarter months after becoming infected, or five and a quarter months after the treatment was stopped. The initial dose was $0^{\circ} 4$ c.c. of a 5 per cent. solution of atoxyl, and the subsequent dose was O.I c.c. injected two or three times a week.]

[In rats the treatment was begun when the animals were apparently. moribund and getting into the semi-comatose condition. At this stage the parasites often numbered 200 or more to a field. Rats weighing about I 25 grammes were given an initial dose of $0^{\circ} 5$ c.c. of a 5 per cent.

1 [Thomas, Brit. Med. Journ., May 27, I905, pp. I I40-1143; Thompson Yates and Johnston Lab. Reports, v. 6, part 2, I905, pp. 49-63.]

2 [Atoxyl, or meta-arsenic-anilide, has the formula $\mathrm{C}_{6} \mathrm{H}_{5} \cdot \mathrm{NH} . \mathrm{AsO}_{2}$. It contains about $3 ; 7$ per cent. of arsenic, and is said to be forty times less toxic than Fowler's solution. It is especially suitable for either subcutaneous or intravenous administration. It produces no necrosis or pain, and very much higher doses of arsenic can be given without producing toxic results (Thomas). It is soluble in about $I$ in 6 of water.] 
solution, and in nineteen hours trypanosomes were no longer visible in the blood from the tail. The condition of the rats was greatly improved by the atoxyl injections; some lived I 26 days after inoculation, and fiftyseven days after treatment was stopped. Subinoculated animals did not become infected. When only one injection of atoxyl was given, a relapse occurred in from six to eleven days, and the disease then took its natural course. $\left.{ }^{1}\right]$

[With $T$. evansi the results obtained in the small laboratory animals were about the same as with T. brucei. Guinea-pigs and rabbits infected with $T$.equinum also responded well to atoxyl treatment. Animals infected with $T$. equiperdum or $T$. dimorphon did not respond nearly so well.]

[TRYPANRED.- The best results with this dye were obtained in the case of animals infected with T. equinum. Ehrlich and Shiga, and Laveran and Mesnil had previously obtained similar results in animals infected with this trypanosome. Thomas and Breinl found that some rats lived as long as 107 days, and in some cases the blood was found non-infective post-mortem. In other animals infected with $T$. equinum, such as rabbits, guinea-pigs, cats, and dogs, and in the case of animals infected with the other trypanosomes, less favourable results were obtained. The improvement in the animals' condition was only temporary, and no definitive cures resulted.]

[The combination atoxyl-trypanred proved of benefit. Animals infected with $T$. dimorphon reacted better to the combined treatment than to either drug used separately; but the treatment had to be pushed in order to keep the animals free from trypanosomes. As a result many of the animals died from the toxic effects, especially from nephritis brought about by the trypanred.]

[Laveran has published a series of papers ${ }^{2}$ upon the treatment of animals experimentally infected with various trypanosomes. He used arsenic and trypanred, either separately or in combination, the best results being obtained when the drugs were administered in combination. The following are the details of some of Laveran's experiments on treatment: $\left.:^{3}\right]$

[TRYPANOSOMA EVANSI.--In rats and mice infected with $T$. evansi an injection of trypanred caused the trypanosomes to disappear from the general circulation in about forty-eight hours, but the disappearance was only temporary. Much better results were obtained by using trypanred with arsenious acid, as the following experiment shows: A white rat, weighing 80 grammes, infected with $T$. evansi, was injected subcutaneously with 2 c.c. of a I per cent. solution of trypanred. Two days later the trypanosomes had disappeared from the blood; the parasites were absent for a few days, but then reappeared. Six days after the original injection of trypanred, the trypanosomes having reappeared, 0.45 milligramme of arsenious acid was injected subcutaneously, and twenty-four hours later 2 c.c. of a I per cent. solution of trypanred. Two days after the last injection the trypanosomes had disappeared from the blood, and were not seen again subsequently. Two and a half months later the rat's weight had increased to I 34 grammes.]

1 [Moore, Nierenstein, and Todd (Annals of Trop. Med. and Parasit., v. I, No. I, February, I907, p. I6I) state that, in nagana-infected rats, the best combination is atoxyl, followed by small doses of perchloride of mercury after the trypanosomes have left the peripheral blood. It is suggested that this treatment should be tried in human trypanosomiasis.]

${ }_{2}^{2}$ [Laveran, C. R. Acad. Sciences, v. I39, 1904, p. I9; v. Ifo, 1905, pp. 287 and I081; V. I 41,1905, p. 91.$]$

${ }^{3}$ [The details of these experiments are taken almost verbatim from Laveran's interesting papers.] 
[In mice trypanred used alone allowed $T$. evansi to reappear in the blood, whereas permanent cures were obtained by the combined use of trypanred and arsenious acid. Some of the rats and mice treated in this way by Laveran remained free from trypanosomes for seven, eight, and nine months.]

[Animals infected with mbori, which, as has been pointed out, is a variety of surra less virulent than the Indian and Mauritian strains, were cured more easily by these drugs. In mice a cure may be effected by a single injection of trypanred $\left(\mathrm{O}_{3} 3\right.$ c.c. of a I per cent. solution for a mouse of 15 to 20 grammes). In rats the dye used alone produces only a temporary disappearance of the trypanosomes. Much better results, including several permanent cures, were obtained by the alternate injection of trypanred and arsenious acid at intervals of twenty-four hours. Some of these rats and mice remained free from trypanosomes for seven, eight, and nine months, and the cure was complete, because the blood was non-infective even when the whole of the blood of a rat was injected intraperitoneally into a guinea-pig.]

[A bitch, weighing $12 \cdot 5$ kilogrammes, inoculated with mbori on September 7 , I904, was alive and well on January 30, I905, having been cured by the combined treatment.]

[Animals cured by treatment in this way are not immunized, as are animals, such as bovines, goats, and sheep, which recover spontaneously from an attack of mbori or surra. 'Cured' mice and rats were often reinoculated with mbori, with the result that the disease always progressed as in control animals, and invariably endel fatally.]

[TRYPANOSOMA GAMBIENSE.-Cures were obtained in the case of rats and dogs infected with this trypanosome. The guinea-pig, which is much more susceptible than the rat to arsenious acid and to trypanred, responds much less readily to treatment. ${ }^{1}$ When animals infected with $T$. gambiense are treated with arsenious acid, and two days later with trypanred, and the trypanosomes are allowed to reappear before any further injections of the drugs are given, the results are much less favourable than if the double injections are repeated without waiting for the reappearance of the trypanosomes. The same thing has been observed in malaria, which is also a hæmatozoan infection.]

[In rats three double injections at intervals of a week were usually sufficient to effect a cure. Each 'treatment' consisted of an injection of arsenious acid ( $0^{\circ} \mathrm{I}$ milligramme per 20 grammes of body-weight), followed in forty-eight hours by an injection of trypanred (2 milligrammes per 20 grammes of body-weight). This treatment produces a temporary loss of weight in the rats; the skin is stained a deep red, and the urine is pink and often contains albumin. The injections made with sterile solutions into the muscles of the shoulder do not give rise to any local irritation in the rat. Of three rats treated in this way, two remained free from trypanosomes for three months, and one for six months.]

1 [A rat weighing 200 grammes can tolerate I milligramme of arsenious acid and 2 centigrammes of trypanred, whereas a guinea-pig weighing 400 to 500 grammes will not tolerate more than I milligramme of arsenious acid or 1.5 centigrammes of trypanred. Several guinea-pigs treated with trypanred died of nephritis or of renal congestion.] 
[Dogs withstand injections of arsenious acid given intravenously, but local necrosis is produced if the injections are made under the skin or into the muscles. It is advisable not to exceed $I^{*} 5$ milligrammes of arsenious acid per kilogramme of body-weight; and in dogs weighing Io to I 2 kilogrammes a dose of I 4 to I6 milligrammes of arsenic usually causes the trypanosomes to disappear. $\left.{ }^{1}\right]$

[Dogs weighing ro to I2 kilogrammes, as a rule, tolerated 30 to 40 centigrammes of trypanred. Two of the animals died, however, while undergoing treatment; one of them had a chronic interstitial nephritis, which caused death through failure to eliminate the drugs, while the other had a parenchymatous nephritis.]

[Laveran gives details of two dogs infected with $T$. gambiense which were cured, the one after three double injections, the other after four. He adds that 'spontaneous recovery from infections with $T$. gambiense being quite exceptional, the above observations upon rats and dogs seem to show that these infections are curableat least, in certain species of animals. The best results are obtained by the successive administration of arsenious acid and trypanred, but it is desirable to find a drug which is as efficacious as, but less toxic than, the latter. The doses required being almost toxic ones, and the toxicity varying in different species of animals, the dose for each species must be carefully ascertained, and this is somewhat difficult in the case of man.']

[Two monkeys (Macacus sinrcus) infected with the Uganda strain of $T$. gambiense were treated with arsenious acid and trypanred in combination. The dose injected was I milligramme of arsenic, ${ }^{2}$ or about 2 centigrammes of trypanred (used in a 2 per cent. solution) per kilogramme of body-weight. Both drugs were injected subcutaneously. The arsenic injections gave rise to no bad results; the trypanred produced a marked coloration of the skin and urine, but did not give rise to necrosis. One monkey was treated four times, the other three times, with the double injection (arsenious acid, followed in two or three days by trypanred), an interval of eight to ten days being allowed between the successive courses of treatment. In each case trypanosomes were found in the blood ten days after inoculation, but not after the treatment was begun. Both animals gained a little in weight, and were well about three months (eighty-nine and eighty-three days) after inoculation. Laveran states that they were apparently cured; but, as we saw in the last chapter, monkeys may become free from trypanosomes without the intervention of any

${ }^{1}$ [For these intravenous injections Laveran used a solution of the following composition : Chemically pure $\mathrm{NaCl}, \mathrm{I}^{\circ} 7$ grammes; chemically pure sodium arsenite, 1.63 grammes; distilled water, 250 grammes. The solution is made distinctly alkaline by the addition of a drop of caustic soda, and is then sterilized in the autoclave. One c.c. of this solution is equivalent to 4 milligramines of arsenious acid.]

2 [The arsenical solution used for these injections had the following composition: Arsenious acid, 0.5 gramme; sodium bicarbonate, 0.5 gramme; distilled water, 250 grammes. The strength of arsenious acid was, therefore, 2 per $1,000$. 
treatment, and remain apparently in good health for many months, yet ultimately the trypanosomes reappear and the animals die, often after exhibiting symptoms of sleeping sickness.]

TRYPANOSOMA EQUIPERDUM (dourine).-Although this trypanosome always gives rise to a fatal infection in dogs that are untreated, Laveran succeeded in curing two dogs by means of the combined treatment. One dog was cured after three double injections ( 16 milligrammes arsenious acid, followed in a few days by 30 centigrammes trypanred); the other dog required six double injections before it recovered from its infection. This animal weighed ro kilogrammes, and received in the space of forty-seven days 72 milligrammes arsenious acid and I*2 grammes trypanred without ill-effects. It gained 2 kilogrammes in weight during the experiment.]

[Mode of Action of Trypanred,-Laveran writes: 'This dye is only feebly toxic. A mouse of 15 to 20 grammes may safely be given a dose of $0^{\circ} 3$ c.c. of a I per cent. solution. ... Its mode of action is still obscure. Ehrlich and Shiga have shown that in vitro it has only a slight microbicidal effect upon trypanosomes; but it is admittedly difficult to study in vitro slowly acting microbicides, because the trypanosomes lose their motility fairly quickly without the addition of any microbicide. This microbicidal action may occur, but it is slow. In injected animals the trypanosomes do not entirely disappear from the general circulation until the end of forty-eight hours. Trypanred is, therefore, much slower in its action than is arsenious acid.']

['Thomas and Breinl have made similar observations. They noted, moreover, that injections of arsenic or of trypanred were followed by a marked polymorphonuclear leucocytosis. This leucocytosis no doubt plays a part in the destruction of the trypanosomes, but the injection of agents, such as nuclein, colchicin, etc., which cause a leucocytosis, after the administration of arsenic or trypanred, or both, did not give better results than were obtained with the arsenic or trypanred alone.']

[The Value of Therapeutic Measures in the TrypanoSOMIASES. - 'There is no doubt,' says Laveran, 'that by means of the combined treatment with arsenic and trypanred complete and permanent cures can be obtained in the different trypanosomiases. I have in my possession animals which have been cured for over a year of trypanosomiases, which in untreated animals of the same species always run an acute and invariably fatal course. The blood of these cured animals is no longer infective, even though the whole of it is injected into another animal.']

['Animals that have recovered from a trypanosomiasis after being treated with arsenious acid and trypanred, or with the latter alone, are not immunized against the disease. When they are reinoculated with the virus they develop a fresh infection, and the disease runs 
the same course as in a control or normal animal. I had previously made similar observations in mice which had recovered from nagana, surra, or caderas after injections of human serum. It seems to follow, therefore, that we cannot hope to do much good by therapeutic means in combating the trypanosomiases of domestic animals in regions where these diseases are enzootic or epizootic; for the animals when cured would again be exposed to the infection-except in the case of dourine, in which it is possible to take effective precautions against reinfection. It must be our aim, therefore, to prevent these diseases rather than to cure them. Therapeutic attempts, however, are all-important in human trypanosomiasis, and especially in the case of Europeans who are able to leave the country in which the disease is endemic.']

\section{Section 4.-Treatment by Means of Benzidine Dyes (Mesnil and Nicolle).}

[The important investigations of Mesnil and Nicolle ${ }^{1}$ upon the action of various derivatives of benzidine in trypanosome infections were suggested by the fundamental work of Ehrlich and Shiga on trypanred. Mesnil and Nicolle's experiments were carried out upon nagana, surra, and caderas in mice, and upon T. gambiense infections in young rats and macaque monkeys. Wenyon studied the action of these dyes upon $T$. dimorphon infections, and, more recently, Mesnil, Nicolle, and Aubert ${ }^{2}$ have made fuller investigations upon their action in $T$. gambiense infections.]

[Nagana, surra, and caderas run such a uniform course in mice that a prolongation of less than twenty-four hours attributable to the drug can be recognized. It is, therefore, possible to classify the dyes experimented with by the differences in the prolongations to which they give rise. In some cases a permanent cure was obtained with one injection of a dye.]

In order to enable the reader to understand the technical terms used in the following description of these experiments, it will be advisable to make a brief reference to the constitution of some of the more important chemical substances in question. 'The benzidine dyes, in their simplest form (diazo compounds), consist of one molecule of a diazotized base (benzidine or one of its homologues), serving as a nucleus, united, it may be, to two molecules (identical or otherwise) of a phenol or aromatic amine, or to one molecule of a phenol and one molecule of an aromatic amine-lateral chains of the diazo compound, so to speak. There exist, then, symmetrical derivatives and asymmetrical derivatives. Certain compounds of benzidine, which contain one or two molecules of amine, are able, after having been diazotized, to give rise, in their turn, by union with phenols and amines, to triazo and tetrazo compounds.']

1 [Mesnil and Nicolle, Ann. Inst. Past., v. 20, 1906, pp. 417-448, and 514-538; abstracts in Full. Inst. Past., v. 4, 1906, pp. $817-821$, and in Brit. Med. Journ., December 22, I906, p. 1777, from which the account given above is largely taken.]

${ }_{2}^{2}$ [Mesnil, Nicolle, and Aubert, Ann. Inst. Past., v. 21, January, 1907.] 
[Benzidine, naphthalene, etc., belong to the group of organic substances known as the carbocyclic or ring compounds, the basis of which is benzene, $\mathrm{C}_{6} \mathrm{H}_{6}$ or<smiles></smiles>

It is usual to number the hydrogen atoms I to 6, thus-<smiles>C1CCCCC1</smilesall these atoms being of equal value. When two of the hydrogen atoms are replaced by other atoms or groups, the diderivatives or disubstitution products $-\mathrm{C}_{6} \mathrm{H}_{4} \mathrm{X}_{2}$-resulting from the replacement of the hydrogen atoms can exist in three distinct modifications. These are known as ortho-, $m e t a-$, and para-derivatives (usually written as $0_{-}, m_{-}$, and $p_{-}$). In the orthocompounds two adjacent hydrogen atoms of benzene have been replaced. Taking any one of the $\mathrm{H}$ atoms arbitrarily as $\mathrm{I}$, it is seen that two orthopositions are possible-namely, I, 2 and I, 6 . Meta-compounds are produced when the $H$ atoms I, 3 or I, 5 are replaced; two meta-positions are, therefore, possible. Para-compounds are produced when the $\mathrm{H}$ atoms replaced are in the position $I, 4$, at opposite angles of the hexagon; there is, therefore, only one para-position.]

[Benzidine is p-diamino-diphenyl, the two benzene hexagons being joined directly by one angle, and the

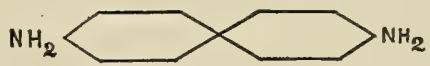

amino-groups being at the opposite angles of the hexagons-the paraposition. Naphthalene consists of two benzene hexagons united by one side :

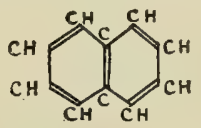

The following symbols represent the constitution of some of the derivatives of benzidine and naphthalene shortly to be mentioned:

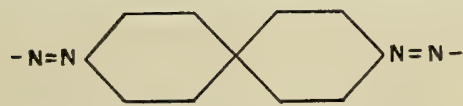

Diazotized benzidine radical.

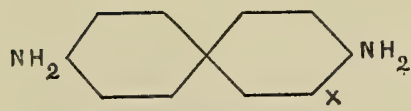

o-nitro benzidine $\left(\mathrm{NO}_{2}\right)$;

o-benzidine sulphonic acid $\left(\mathrm{SO}_{3} \mathrm{H}\right)$, etc.<smiles>NC1CCCC2CCCCC12</smiles>

$a$-naphthylamine (amino-naph thalene).<smiles>NC1CCC(S(=O)(=O)O)CC1C(=O)O</smiles>

$\alpha$-naphthylamine4,6 , disulphonic acid.

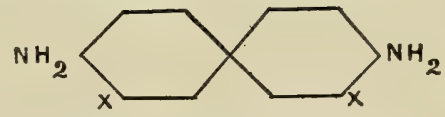

o-dimethyl benzidine (tolidine); o-dimethoxy benzidine (dianisidine) ; o-dinitro benzidine ;

o-benzidine disulphonic acid, etc.

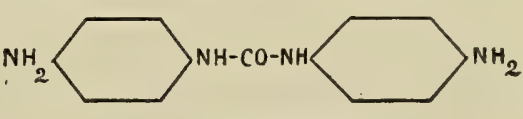

p-diamino-diphenylurea.<smiles>NCC1CCCC2CC(S)CCC1C2O</smiles>

$\beta$-naphthylamine3,6 , disulphonic acid (acid R).

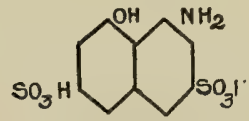

I, 8, amino-naphthol3,6 , disulphonic acid (acid $H$ ).<smiles>OCCCCC1CCCCC1O</smiles>

oxynaphthalene or a-naph thol. 
[Treatment of Experimental Nagana in Mice.-Mesnil and Nicolle studied a very large number of synthetic compounds, ${ }^{1}$ with the view of ascertaining which were the best bases and substitution products, and which the best lateral chains. Reviewing first the symmetrical diazo compounds, let us enquire what is the respective influence of the lateral chains and of the diazos on the curative power.]

[Experiments show that the benzenic chains (even combined with the best bases) always give rise to inactive derivatives. So also do the naphthalenic chains which do not contain the $\mathrm{NH}_{2}$ group, or which, containing it, do not present at least two $\mathrm{SO}_{3} \mathrm{H}$ groups. On the contrary, it is amongst the naphthylamine-disulpho compounds, the disulphonic amino-naphthols, the disulphonic naphthylene diamines, and the trisulphonic naphthylamines that active chains are found. Amongst the disulphonic $a$-naphthylamines the best type is $I, 5,7$; amongst the disulphonic $\beta$-naphthylamines the best types are I, 5,7 , and especially $2,3,6$, the lateral chain of trypanred. Amongst the disulphonic amino-naphthols the best types are $I, 8,4,6$ (acid $K$ ), and especially $I, 8,3,6$ (acid $H$ ). Amongst the disulphonic naphthalene diamines the best type is $2,7,3,6$. The $a$ - and $\beta$-trisulphonic naphthylamines are very inferior to the corresponding disulphonic derivatives; the addition of a third sulpho-group is, therefore, harmful rather than beneficial.]

[Each of the good lateral chains was studied in combination with a number of diazos (as many as twenty-seven for acid $\mathrm{H}$ ), and it was found that, according to the base used, the activity of the resulting derivatives varied from o to $\left.\infty .^{2}\right]$

[Acid H, like all the disulphonic amino-naphthols, can be united with the diazos in an acid as well as in an alkaline medium. In an acid medium the mode of insertion of the azo group is completely inverted, and the therapeutic power is much diminished. It is easy to unite two molecules of acid $\mathrm{H}$ successively in an acid and alkaline medium; the asymmetrical colours thus formed manifest an efficacy varying with the base selected.]

[The conditions affecting the activity of the lateral chains were also studied in detail; it was found that the best of these chains were those which contain the sulpho-groups 5,$7 ; 4,6$; and especially 3,6 .]

[What part do the diazos play in the efficacy of these colours? Mesnil and Nicolle found that the benzidinic bases are the only ones of any value; but their activity depends upon a number of conditions. (I) On the position of the diazotizable $\mathrm{NH}_{2}$ group ; the meta-position (in relation to the benzidinic link) is bad; the ortho-

1 [The authors express their indebtedness to several dye works, and especially to the Farbenfabriken at Elberfeld, for supplying the dyes.]

2 [By $\infty$ is meant that a permanent disappearance of the trypanosomes followed a single injection of the dye.] 
position, on the other hand, is good. (2) On the general structure of the diazo; the benzidinic nuclei, strictly speaking, always appear better than the bases in which the two hexagons, instead of being directly linked, are joined by a bivalent group or atom (as in p-diamino-diphenylurea; see p. 427). Benzidine is, on the whole, a good nucleus. In its derivatives the activity varies with the position and nature of the substituting groups or atoms. Thus, to mention two of the many facts ascertained by the authors, it was found (I) that the ortho-position (in relation to the benzidinic link) was always bad; and (2) that the substituting groups $\mathrm{CH}_{3}$ and $\mathrm{OCH}_{3}$, as a rule, exerted a favourable influence.]

[To determine with any degree of completeness the influence of the lateral chains on the bases, it would have been necessary to experiment with thousands of compounds. With the resources at their disposal Mesnil and Nicolle were able to arrive at at least one of the laws which regulate this influence. This is the law: On classing the three most commonly used bases in the following order, benzidine, dianisidine, tolidine $(\mathrm{B}, \mathrm{D}, \mathrm{T})$, it is found that when a lateral chain possesses a sulpho-group in position 6 , the activity of the compound resulting from the union of this chain with one of the three bases increases from $\mathrm{B}$ to $\mathrm{T}$; when the lateral chain possesses a sulpho-group in position 7 , the activity of the compound diminishes from B to T. This law of the sulpho-groups 6 and 7 also applies to other bases than benzidine, dianisidine, and tolidine.]

[No doubt there are other laws governing the formation of active compounds, because it is not sufficient to combine any good chain with any good base in order to produce an active compound.]

[Theoretical considerations, which are given at length in Mesnil and Nicolle's paper, led these authors to conclude that the auxochrome $\mathrm{NH}_{2}$ constitutes the essential element of the lateral chains. The group $\mathrm{NH}_{2}$ is also present in other colours used by certain investigators (notably methyl and ethyl green), as well as in normal serums.]

[The derivatives, which are active in vivo against nagana, are solutions having a blue, violet, rose, or red colour; they are transparent or opaque, and colour the mice more or less permanently; they are quite harmless in the therapeutic dose $\left(0^{\circ} 5\right.$ to I centigramme), and often in larger doses. The best of these derivatives is 'dichlorobenzidine +acid $\mathrm{H}$-alkaline, alkaline' (called $\mathrm{Cl}$ for short-bluish violet in solution). Then come the compounds 'tolidine + acid $\mathrm{H}$ - alkaline, alkaline' (called $\mathrm{A}^{1}$ - violet solution), and

1 [Bouffard (Ann. Inst. Past., v. 20, 1906, pp. 539-546) has studied the distribution of this dye in the organs and tissues of healthy animals injected with it in vivo. He finds the dye in the convoluted tubules of the kidney, in the cells of Kuppfer of the liver, and in the interstitial cells of various organs and tissues. The cerebro-spinal fluid remains uncoloured. This seems to show that the dye, and probably, therefore, other remedial agents, are unable to penetrate the spinal canal, at least in certain cases. It suggests an intraspinal method of treatment for sleeping sickness, in addition to subcutaneous or intramuscular medication.] 
'tolidine + acid $\mathrm{H}$-acid, alkaline' (called A'-violet). These three dyes can, in many cases, bring about the permanent disappearance of the trypanosomes after a single injection. Such results are only rarely obtained with the compound 'benzidine $+2,7$ diaminonaphthylene $-3,6$ disulphonic acid' (called $a$ for short-dark cherry-red solution), and quite exceptionally with trypanred-'benzidine o-monosulphonic acid + acid R' (clear cherry-red solution), and with the compound ' $\mathrm{p}$-diamino-diphenylurea + acid H' (called $\mathrm{Ph}$-violet).]

[The treatment of relapses is only successful with the compound $\mathrm{Ph}$. This dye is able to prevent the reappearance of the parasites when a full initial dose is injected, followed in a week by a smaller dose. Finally, it was found that the dyes $\mathrm{Cl}$ and $\mathrm{A}$ possessed a remarkable preventive power against the infection (nagana).]

[Mesnil and Nicolle state that the asymmetrical diazo compounds offer no advantage over the symmetrical, but rather the reverse; and that triazo and polyazo compounds never manifest any curative power, even when they contain good lateral chains.]

[In the second part of their paper ${ }^{1}$ Mesnil and Nicolle give a detailed account of the therapeutic action of the six best dyes, and also compare their action with that of some arsenic compounds. About one-third of the mice treated with $\mathrm{Cl}, \mathrm{A}$, and $\mathrm{A}^{\prime}$ were permanently cured by one injection (dose I centigramme for a mouse of I5 to 20 grammes); so also were one-quarter of those treated with $a$ (dose 0.75 centigramme); one out of thirteen treated with trypanred (dose $0^{\circ} 5$ centigramme) ; and one out of fifteen treated with $\mathrm{Ph}$ (dose I centigramme). The dyes were always dissolved in distilled water (I per cent. solution), and injected hypodermically.]

[In the cases which were not permanently cured a relapse occurred after a variable time (five to twenty days, average twelve days). A relapse, if untreated, ran the same course as an original infection. Few dyes were found of use in the treatment of relapses; as has been mentioned, $\mathrm{Ph}$ alone gave good results, and then only if the relapses did not occur very soon after the treatment.]

[Of the arsenic derivatives used, a certain number (arsenite and arseniate of soda, arrhénal, and atoxyl), given in suitable doses, caused the trypanosomes to disappear : but relapses always occurred rapidly, except with atoxyl, which behaved like the dye a (two mice out of eight cured by one injection). Atoxyl was also preventive, almost as much as $\mathrm{Cl}$.]

[The Disappearance of the Trypanosomes.-The parasites disappear from the blood in sixteen to seventy-two hours, according to the nature and dose of the dye used, and the number of parasites present when the injection is given. The trypanosomes in the circulating blood show involution forms; they become granular, stumpy, and increasingly sluggish ; their remains are found in the leucocytes. In vitro these dyes do not exhibit any appreciable trypanosomicidal activity.]

${ }^{1}$ [Mesnil and Nicolle, Ann. Inst. Past., v. 22, July, 1906, pp. 513-538.] 
[The investigations made to try to find out what becomes of the trypanosomes from the time that they disappear from the blood to the time the relapse occurs were carried out on mice treated with $\mathrm{A}$ and $\mathrm{Ph}$. It was found that, even on inoculating a number of mice with the blood, spleen, liver, kidneys, and brain of mice killed some days after the disappearance of the trypanosomes, the parasites were not always found in the inoculated animals, although, as has been pointed out above, a relapse would almost certainly have occurred in the mice treated with the dye. This failure to find the trypanosomes in the subinoculated mice is due to their small numbers, or to their physiological condition, or to some unknown cause. In one interesting case the brain alone was infective.]

[The Treatment of Experimental Caderas in Mice.The comparative study of the therapeutics of nagana and caderas shows at once that the order of activity of the dyes is subject to important variations on passing from one trypanosomiasis to another. Mesnil and Nicolle's observations, corroborated by those of Wenyon for $T$. dimorphon, show that this variation depends on the structure of the lateral chains, and especially of the diazos.]

[In caderas, as in nagana, the dye $\mathrm{Cl}$ causes the complete disappearance of the trypancsomes in many cases (about 50 per cent.) after a single injection. Trypanred also gives good results, as Ehrlich and Shiga had originally shown (almost 50 per cent.). The dyes $\mathrm{A}, \mathrm{A}^{\prime}$, and $a$ cure only exceptionally, and $\mathrm{Ph}$ never.]

[The interval between the treatment and the relapse (when this occurs) is about the same as with nagana, except in the case of trypanred, when, according to Ehrlich and Shiga, it may be as long as two months. The treatment of relapses is successful with $\mathrm{Cl}$, trypanred, and, above all, with $a$.]

Treatment of Experimental Surra in Mice.-Here again the best colour is $\mathrm{Cl}$, which cures nearly all the animals after one injection. After this comes trypanred. The curative power of A and $\mathrm{A}^{\prime}$ is doubtful, that of $a$ and $\mathrm{Ph}$ inefficient. The derivative $\mathrm{Cl}$ has a prophylactic action which is quite remarkable.]

[To summarize the foregoing observations, we may say that $\mathrm{Cl}$ (dichlorobenzidine + acid $\mathrm{H}$ ) constitutes, at the present time, the best chemical agent to oppose the three animal trypanosomiases, nagana, caderas, and surra; but for the relapses $\mathrm{Ph}$ is to be recommended in nagana, $a$ in caderas, and perhaps atoxyl in surra.]

[TREATMENT OF EXPERIMENTAL TRYPANOSOMA DIMORPHON INFECTIONS IN MICE.-In the treatment of the three above-mentioned trypanosomiases the best lateral chain is acid $\mathrm{H}$, and the best diazo is dichlorobenzidine. The blue dyes are therefore, as a rule, better than the red dyes. In $T$. dimorphon infections, however, Wenyon found that the opposite condition holds. The only derivative capable of curing mice after a single injection was the compound $a$. Trypanred, administered even in repeated doses, was only exceptionally able to prevent the reappearance of the parasites. With the other 
derivatives relapses always occurred, sometimes, however, after a long interval.]

[TREATMEnT OF EXPERIMENTAL Infections With TRYPanOSOMA GAMBIENSE IN RATS AND MONKEYS.-In these cases it is impossible to obtain a permanent cure after a single injection of the dye. The relative value of the drugs used is estimated by the interval which elapses between the treatment and the relapse. The most efficacious dye was found to be $\mathrm{Ph}$; after this come the compounds ' $\mathrm{p}$-diamino-

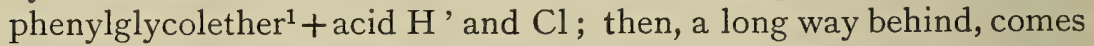
trypanred, and further still the compound $a$. We see, therefore, that in $T$. gambiense infections, as in nagana, caderas, and surra, the best lateral chain is acid $\mathrm{H}$; but, whereas in the last three diseases the best diazo is dichlorobenzidine, for $T$. gambiense it is p-diaminodiphenyl urea. As has already been mentioned, the best drug in T. dimorphon infections is a-a red dye. ' It is permissible to hope,' say Mesnil and Nicolle, 'that we shall one day arrive at a means of distinguishing the various pathogenic trypanosomes by the chromotherapeutic test.']

[Parallel experiments were made with atoxyl and sodium arsenite. ${ }^{2}$ With the former permanent cures were occasionally obtained after a single injection in the monkey and rat. As in the case of the other trypanosomiases, sodium arsenite is far inferior to atoxyl in treatment.]

[Having ascertained that the two best drugs were the dye $\mathrm{Ph}$ and atoxyl, Mesnil, Nicolle, and Aubert used them in different ways in order to find out how the best ultimate results could be obtained. The alternate use of the dye $\mathrm{Ph}$ and of atoxyl was found of more value that either drug used singly; in some cases the relapses were treated only as they occurred, in others preventive injections were given, so as to try and ward off a relapse.]

[A rat which received four injections of $\mathrm{Ph}$ (each time on the appearance of trypanosomes in the blood) lived for I43 days; a control rat died in forty-seven days. Another rat which received seven injections was still alive after eight months; trypanosomes were absent from the blood for three and a half months after the last injection of $\mathrm{Ph}$. A rat treated with atoxyl had a relapse after twenty-two days; a fresh injection of atoxyl cured the animal, for no relapse had occurred when the rat died, I 47 days afterwards. Another rat injected with atoxyl had a relapse after I I 5 days. Two rats which were injected only once never showed trypanosomes, although they survived I2I and I7I days.]

The preventive treatment of relapses gave even better results, especially with $\mathrm{Ph}$. It is interesting to note, however, that in some cases relapses occurred after a very long interval-as much as six months. These delayed relapses in rats show that under the influence

1 [This is perhaps more correctly called p-aminophenyl-diether of glycol. For its formula see p. 427.]

2 [The dyes were used in I per cent. solution in distilled water; the atoxyl in 2 per cent. solution; and the sodium arsenite 1 per 500.] 
of treatment a subacute disease may be converted into a chronic one, and also that one must be very careful in speaking of cures where T. gambiense infections are concerned.]

[In monkeys the best results were obtained by using atoxyl alone, or the combination Ph-atoxyl alternately, and without waiting for a relapse to occur before injecting a further dose of the drug. Animals treated with $\mathrm{Ph}$ alone were never permanently cured; but by means of atoxyl, or of atoxyl and $\mathrm{Ph}$ used alternately, the trypanosomes disappeared from the blood and remained absent for five or six months (experiments still in progress). Mesnil, Nicolle, and Aubert think that some of these monkeys were really cured, for the following reasons: (I) For five months or more after the last treatment trypanosomes had not reappeared in the blood; (2) the blood of two of the monkeys was not infective for rats in 8 c.c. doses; (3) the whole of the blood and the extracts of organs of another monkey failed to infect a dog; and (4) the bloods of all the monkeys lost the power of agglutinating their own red corpuscles. Time will show whether these monkeys are permanently cured of their trypanosomiasis.]

[These drugs, in therapeutic doses, are well borne by monkeys, and no doubt the same applies to human beings. As we shall see in the next section, atoxyl is the only drug which is at present receiving the serious attention of investigators in the treatment of human trypanosomiasis. In the case of negroes the alternate administration of atoxyl and the dye $\mathrm{Ph}$ may prove superior to atoxyl alone; but in whites the violet coloration of the integuments produced by the dye will be a bar to its general use.]

\section{Section 5. - Treatment of Human Trypanosomiasis (Sleeping Sickness) and of Experimental Infections with Trypano- soma gambiense in Animals. ${ }^{1}$}

[Before it had been discovered that $T$. gambiense was the cause of sleeping sickness, Low and Castellani treated their cases in Uganda with iron, quinine, and arsenic. This produced a distinct but temporary improvement, especially in cases complicated with malaria.]

[When the probable rôle of the trypanosome in the causation of the disease was demonstrated by Castellani, Bruce, and Nabarro, treatment by injections of arsenic and other drugs, which had proved of some value in nagana and surra, suggested itself. Nabarro and Greig, in I903, treated a patient by intravenous and intraspinal injections ( 8 c.c. dose in each case) of a I per cent. solution of methylene blue. No permanent benefit resulted from this treatment. Two other patients in an early stage of sleeping sickness were

1 [Laveran has recently given a good résumé of the various attempts that have been made to treat human trypanosomiasis. See Bull. de l'Acad. de Méd., February 26, 1907.] 
treated by intramuscular injections of arsenious acid (arsenious acid I gramme, sodium carbonate I gramme, water 500 c.c.). The injections were given every two or three days. The initial dose was I5 minims (about I c.c.), and the dose was rapidly increased to 40 minims (about 2.5 c.c.). There was a distinct improvement in the patients' condition, but it was only temporary, and the patients eventually succumbed to the disease.]

[Greig and Gray ${ }^{1}$ followed up this line of treatment, but with equally unsatisfactory ultimate results. Dutton, Todd, and Christy ${ }^{2}$ and Broden ${ }^{3}$ likewise obtained temporary beneficial effects by means of arsenic. Gray and Tulloch ${ }^{4}$ found that safe doses of arsenic had little or no power to protect monkeys against an infection with T. gambiense.]

[Attempts were next made to cure $T$. gambiense infections in animals by the combined use of arsenic and trypanred. The experiments of Laveran and of Thomas and Breinl on these lines have already been alluded to (Section 3). A certain percentage of recoveries resulted from this mode of treatment.]

[Gray and Tulloch tried the effect of 'tragarot' in well-marked cases of sleeping sickness, but although no ill effects were produced (as often result from the administration of trypanred), no beneficial effects could be observed in the patients so treated.]

[Other drugs or forms of treatment which have been suggested or tried, but without permanent benefit, are chrysoidin (Balfour and Neave ${ }^{5}$ ), 'brilliant green' (Wendelstadt and Fellmer ${ }^{6}$ ), thyroid tabloids (Lorand ${ }^{7}$ ), and $\mathrm{X}$ rays $\left(\right.$ Mense $\left.^{8}\right)$.]

[ATOXYL.-The drug which has hitherto proved most useful in the treatment of sleeping sickness in man and of $T$. gambiense infections in animals is atoxyl. As has been mentioned in Section I, it was Wolferstan Thomas who first used atoxyl in the treatment of the trypanosomiases, though Mesnil and Nicolle had independently experimented with this drug before Thomas published his paper in 1905.$]$

[Thomas warmly advocates the use of atoxyl in human trypanosomiasis, and the encouraging results obtained with it in experimentally infected animals by Thomas and Breinl, Laveran, Mesnil,

${ }^{1}$ [Greig and Gray, Sleeping Sickness Commission Report, No. 6, I905, pp. 5 o-69.]

2 [Dutton, Todd, and Christy, Liverpool School of Tropical Medicine. Memoir xiii.]

${ }^{3}$ [Broden, 'La Trypanosomiase chez l'Européen,' Brussels, I905.]

${ }^{4}$ [Gray and Tulloch, Sleeping Sickness Commission Report, No, 8, 1907. pp. 30-53.]

${ }_{5}$ [Balfour and Neave, Lancet, June 17, 1905 ; Second Report of the Wellcome Research Laboratories, Khartoum, 1906, pp. 15.3-170.]

${ }_{6}^{6}$ [Wendelstadt and Fellmer. See references given on p. 4I7.]

7 [Lorand, German Congress of International Medicine, Deutsche med. Wochenschr., v. I, I905.]

8 [Mense, Arch.f. Schiffs und Trop. Hyg., July, 1905.] 
and others (see Sections 3 and 4 ), and by Ayres Kopke, ${ }^{1}$ Broden, ${ }^{2}$ Van Campenhout, Todd, ${ }^{3}$ Koch, ${ }^{4}$ and others, in human trypanosomiasis show that it is a valuable remedy for this disease. Koch, who has recently used it on a large scale in Uganda, speaks most enthusiastically of it, and apparently regards it as a specific cure for trypanosomiasis. Unfortunately, other observers have been unable to corroborate Koch's statements. Kopke, Broden and Rodhain, and others, have found that the administration of atoxyl causes a great and rapid amelioration in the condition of the patients, but that death eventually occurs in spite of the treatment. Kopke quotes one case in which the treatment was continued for fifteen months, the patient receiving in all thirty-three injections of atoxyl (in doses of $\mathrm{I}$ to $\mathrm{I}^{\circ} 5$ grammes). Trypanosomes, nevertheless, continued present in the cerebro-spinal fluid, and the patient died after a series of epileptiform fits.]

[Van Campenhout advocates the combined administration of atoxyl and large doses of strychnine sulphate. He recommends giving the latter in small pills, each containing I milligramme ( $\frac{1}{60}$ grain). Three of these pills are given on the first day, and the dose is increased by one pill a day until ten pills are taken per diem; then give eleven pills each day for a week, and gradually increase the daily dose by one pill each week until fifteen ( $\frac{1}{4}$ grain) are taken every day. After this the dose is gradually diminished. This treatment gave very good results even when the patients (natives) had reached the paralytic stage of the disease.]

[Thiroux and d'Anfreville ${ }^{5}$ treated a native of Portuguese Guinea, aged about twelve to fourteen years, with three subcutaneous injections of atoxyl ( $0^{\circ} I_{5}$ gramme each) and one intraspinal injection ( $0^{\circ} 02$ gramme); strychnine was also given (dose from 3 increasing to 7 milligrammes). About a fortnight after the treatment was started, and again a month later, trypanosomes could no longer be found in the centrifuged cerebro-spinal fluid, and this fluid, as well as the blood, was not infective on inoculation into monkeys. Two and a half months after all treatment was stopped, the patient was apparently well. It is remarkable that the patient improved so rapidly after the small quantity of atoxyl injected. Possibly, as Laveran suggests, trypanosomiasis is more easily curable in children (this patient was only twelve or fourteen years old) than in adults.]

[All authorities are agreed that atoxyl is of considerable value in the treatment of human trypanosomiasis. It is possible, moreover,

1 [Ayres Kopke, 'Traitement de la Maladie du Sommeil,' Medicina contem. poranea, Lisbon, 1907.]

2 [Broden and Rodhain, Arch. f. Schiffs u. Trop. Hyg., v. Io, I906, p. 693.]

3 [Todd and Breinl, Brit. Med Journ., January 19, I907; Todd and Kinghorn, Lancet, February 2, 1907. A full bibliography is given in these two papers.]

${ }^{4}$ [Koch, Deutsche med. Wochenschr. Supplement to No. 5I, I9o6; abstract in Brit. Med. Journ., January 19, 1907 ; also January 10, 1907, p. 49.]

5 [Thiroux and d'Anfreville. See in Laveran, Bull. Acad. Méd., February 26, 1907.] 
that permanent cures may occasionally be effected by its administration, especially in the early stages of the disease. Nevertheless, we must be very careful not to be misled by the immediate improvement which usually follows its use, and to regard the improvement as being evidence of a permanent cure. Atoxyl is undoubtedly an advance on the older methods of treatment, but it is not a specific. Finally, the very chronic course that human trypanosomiasis runs (lasting often for several years) makes it necessary for us to follow up the cases for years after treatment before we can confidently talk of a permanent cure.]

[The Mode of Administration of Atoxyl in Human TRYPANOSOMIASIS.-Atoxyl is usually given in subcutaneous injection, but it may also be administered by intramuscular, intravenous, or intraspinal injections, or by the mouth. Broden and Rodhain state that the drug is well borne by the stomach and intestines after oral administration; but most observers agree that atoxyl is decomposed by the acid gastric contents, and that signs of arsenical poisoning are thus more easily produced.]

[In early trypanosomiasis, before the trypanosomes are found in the cerebro-spinal fluid, intramuscular or subcutaneous injections are sufficient; but when signs of sleeping sickness have developed, and trypanosomes are present in the spinal fluid, it is probably advisable to inject the atoxyl into the spinal canal as well as into the muscles or subcutaneously. ${ }^{1}$ Before injecting the drug, Io to $I_{5}$ or more c.c. of cerebro-spinal fluid should be withdrawn by lumbar puncture. Correa Mendes ${ }^{2}$ in Loanda, injected intraspinally Io c.c. of a I per I,00o solution of atoxyl. Kopke, and Thiroux and d'Anfreville have also used intraspinal injections.]

[The usual strength of the atoxyl solutions used for intramuscular or subcutaneous injections is to per cent. Van Campenhout, however, prefers 5 per cent. solutions. Breinl and Todd recommend a 20 per cent. solution in sterile normal saline. The atoxyl separates out on cooling; but if the solution is warmed to blood-heat just before use the drug completely redissolves, and there is no pain at the site of injection.]

[The doses of atoxyl recommended by different authorities vary considerably. Ayres Kopke has injected subcutaneously as much as $I$ and $I \cdot 5$ grammes of atoxyl into adult negroes without producing any ill effects. Broden and Rodhain never gave more than 0.8 gramme for a dose. In two patients doses of $0^{\circ} 6$ and $0^{\circ} 8$ gramme were followed by toxic symptoms. Van Campenhout commences with a

${ }^{1}$ [In 1903 Nabarro and Greig injected a patient intraspinally and intravenously with a solution of methylene blue, with the result that the trypanosomes disappeared from the cerebro-spinal fluid. For intraspinal injections drugs must be used in small doses ; otherwise there is a danger that they may combine with the nerve elements and produce serious results.]

2 [Quoted by Kopke in communication made to the Society for Medical Studies, Lisbon, December 29, 1906: Medicina contemporanea, Lisbon, 1907.] 
dose of 0.2 to 0.25 gramme of atoxyl, and increases it daily by 0.05 gramme until a dose of 0.8 gramme or more is reached. If there are then no signs of poisoning, the daily dose remains at this point for a fortnight or three weeks. It is then gradually diminished by $0^{\circ} 05$ gramme daily until the initial dose $\left(0^{\circ} 2\right.$ gramme) is reached, and the treatment is then interrupted for one or two months. Three or more such courses of treatment are given as required. $\left.{ }^{1}\right]$

[Breinl and Todd recommend the following dosage: $0^{\circ} 6$ c.c. of a 20 per cent. solution of atoxyl (warmed) subcutaneously daily for four days. On each of the four succeeding days 0.8 c.c. is given, and the dose is then raised to I c.c. each day. This dose is given daily for a week, then on every other day for a fortnight, and afterwards twice a week until all symptoms have disappeared and the patient's blood is non-infective when inoculated into susceptible animals. Afterwards I c.c. should be given weekly for as long a period as possible. ${ }^{2}$ If toxic symptoms occur the same doses should be given, but less frequently.]

[Certain precautions are necessary in the administration of atoxyl by injections. Van Campenhout points out that the syringes and needles used for the injections should be sterilized by boiling, since carbolic acid decomposes atoxyl. It has also been found that solutions of atoxyl decompose (with the liberation of free aniline) and become yellowish if exposed to light. All solutions should, therefore, be kept in dark (brown) bottles, and made up fresh every week.]

[As has already been mentioned, the strong solution (20 per cent.) of atoxyl recommended by Breinl and Todd should be warmed to blood-heat before use. Atoxyl solutions must, however, not be overheated. Prolonged heating in the autoclave causes the solution to decompose with liberation of aniline (Lanzenberg). Laveran has found that solutions of atoxyl sterilized in the autoclave are at first more toxic, and also more active against the trypanosomiases, than solutions prepared in the cold. The initial increase of activity of solutions sterilized in the autoclave rapidly diminishes, however, and on this account the solutions used should always be recently prepared and sterilized.]

[Toxic Symptoms.-When the initial dose is small and the subsequent doses are gradually increased, there may be no toxic symptoms. L. Martin states that a rise of temperature may often follow the earlier injections of atoxyl. Schild, ${ }^{3}$ who first used atoxyl

1 [Quoted from Breinl and Todd's paper in Brit. Med. Journ., January I9, 1907.

2 [The disappearance of the auto-agglutination of the red corpuscles may be of considerable help in deciding when it is safe to discontinue the atoxyl treatment.]

${ }^{3}$ [W. Schild, Berl. klin. Wochenschr., No. 13, 1902; Dermatolog. Zeitschr., v. Io, Heft I.] 
therapeutically, describes the symptoms of poisoning as 'feelings of chilliness, vertigo, headache, and tickling in the throat.' The administration of atoxyl is never followed by albuminuria.]

[Broden and Rodhain observed severe abdominal cramps radiating to the hypochondria, slowing of the heart, and marked coldness of the extremities. Intestinal colic and diarrhœa were absent in their patients.]

[Daniels states that 'burning on micturition, general dryness of the mucous membranes and skin, with the formation of pruriginous vesicles,' followed the administration of 0.2 gramme of atoxyl twice weekly for fourteen months in one case. In another case vomiting and diarrhœa occurred early in the course of treatment. When these symptoms occurred the dose was reduced, and subsequently very gradually increased.] ${ }^{1}$

[The action of atoxyl upon the trypanosomes is similar to that of arsenious acid (Laveran, Thomas and Breinl). When a sufficiently large dose of atoxyl is injected into an animal showing many trypanosomes in its blood, the parasites are obviously diminished in number at the end of four or five hours. Of those still present many are involuted and degenerating: some are deformed, others spherical, movement is diminished and the protoplasm granular. At the end of eighteen to twenty-four hours the trypanosomes have entirely disappeared.]

[Finally, we may again refer to the opinion of Mesnil, Nicolle, and Aubert that the alternate administration of the dye $\mathrm{Ph}$ and of atoxyl may prove to be more valuable than atoxyl alone in the treatment of human trypanosomiasis.]

${ }^{1}$ [Quoted by Breinl and Todd in the article previously referred to.] 


\section{CHAPTER XIV}

\section{TRYPANOSOMES OF BIRDS}

\section{Section 1.--Historical Review and Geographical Distribution.}

UNTIL quite recently all that was known about the trypanosomes of birds was contained in a paper by Danilewsky, published in Russian in 1888 , and summarized in French in $1889 .{ }^{1}$ This paper contains numerous details about the appearance of the trypanosomes in fresh blood, the changes they undergo, and their cultivation (?) in vitro.

Chalachnikov, in his ' Recherches sur les parasites du sang chez les animaux à sang froid et à sang chaud' (Charkov, I888), devotes a chapter to the trypanosomes of birds; but he scarcely does more than reproduce the descriptions given by his teacher, Danilewskydescriptions truly remarkable, seeing that they are based on observations made almost exclusively upon fresh blood.

In I903 our knowledge was considerably increased by the researches of Laveran ${ }^{2}$ upon the Trypanosoma avium of an owl (Syrnium aluco), of Dutton and Todd ${ }^{3}$ upon the trypanosomes of birds of the genera Crithagra and Estrelda of Gambia, and of Hanna ${ }^{4}$ upon the trypanosomes of the pigeon and raven of India. These papers give detailed accounts of the structure of the adult parasites, but say nothing about their mode of reproduction.

According to Danilewsky, Wedl ${ }^{5}$ was the first to see a trypanosome in bird's blood, but his description is vague. The parasite was $75 \mu$ to I50 $\mu$ long, by $5 \mu$ to $6 \mu$ wide; with one end pointed, the other blunt. It had a corkscrew-like movement. The host was Loxia coccothraustes. Possibly this parasite was a Filaria.

In I845 Gros, ${ }^{6}$ after having discovered the flagellated parasites of the blood of field-mice and moles, stated that he had found in the blood of goat-suckers and cranes similar parasites, which measured

1 Danilewsky, Biol. Centralb., 1885; Arch. slaves de Biol., 1886-1887; and 'Researches upon the Parasites of the Blood of Birds,' in Russian, Charkov, I888; French edition, Charkov, I889.

2 Laveran, C. R. Soc. Biol., v. 55, May 2. 1903, p. 328.

3 Dutton and Todd, First Report of the Expedition to Senegambia, 1902, Liverpool, 1903, pp. 55, 56, pl. ii., figs. I, 2 .

${ }^{4}$ Hanna, Quart. Journ. Micr. Sc., v. 47, December, 1903, pp. 437, 438, pl. 32.

5 Wedl, Denkschr. Akad. Wien., v. I, I850.

6 Gros, Bull. Soc. Natur., Moscow, v. 18, 1845, p. 423. 
Io $\mu$ to I5 $\mu$ in length and were very thin. His hæmatozoon of the raven, I00 $\mu$ to I $30 \mu$ long and thinner than the small diameter of the red corpuscles, he calls a trypanosome, but it is very doubtful if this is the correct interpretation.

It appears quite certain that the $T$. eberthi Kent seen by Eberth in the digestive tube of the fowl was not a trypanosome, but a Trichomonas, as was first suggested by Stein, and later by Leuckart.

The trypanosomes of Danilewsky have been found in several Russian birds-the owl, the roller-bird (Coracias garula), and others. Danilewsky saw trypanosomes in the blood of young roller-birds only three or four days old. The parasites were numerous in the blood in summer, but absent during the winter.

Ziemann, in Heligoland, found trypanosomes in the chaffinch (Fringilla colebs), ${ }^{1}$ but he did not describe the parasites. The same observer states that he found, in a small white owl in Cameroon, trypanosomes which showed male and female forms (?). ${ }^{2}$ [In a later paper $^{3}$ Ziemann mentions the occurrence of trypanosomes in the kingfisher (Alcyon) in Cameroon.]

The owl in which a trypanosome was found by one of us was bought in a Paris market. We have sought in vain for trypanosomes in a large number of other French birds.

Of twenty-five birds examined at Bathurst, Gambia, belonging for the most part to the genera Estrelda and Crithagra, Dutton and Todd found only one (Estrelda estrelda) infected with the species of trypanosome they call $T$. johnstoni. The other trypanosome described by the same observers was common in the blood of the species of Crithagra and Estrelda found at St. Louis on the Senegal (seven birds infected out of fifteen examined) and at Bathurst.

Hanna, in India, found a small proportion of the domestic pigeons infected with trypanosomes. He studied at the same time a trypanosome of the Indian raven (sp. ?) in blood-films made by Ross in I896. Donovan informs us that he has found trypanosomes in the blood of A thene brama from the neighbourhood of Madras.

The brothers Sergent, ${ }^{4}$ who examined the blood of a large number of Algerian birds particularly in the environs of Algiers, found trypanosomes in only three species: in one goldfinch (Fringilla carduelis) out of forty-six examined; in two black-cap warblers (Sylvia atricapilla) out of five examined; and in three swallows out of ten examined. The parasites were always so scanty that these observers were never able to find them again in stained preparations.

Schaudinn, ${ }^{5}$ in his inquiries into the alternation of generations and the change of host of Trypanosoma and Spirochata, used the

1 Ziemann, 'Ueber Malaria u. andere Blut parasiten,' Jena, I898, p. 106.

2 Ziemann, Arch. f. Schiffs u. Trop. Hyg., v. 6, 1902, p. 389 . Probably he saw sexual forms of Hemamaba ziemanni.

3 [Ziemann, Centralb. f. Bakter., I, Orig., v. 38, 1905, p. 308.]

${ }_{4}$ Ed, and Et. Sergent, C. R. Soc. Biol., v. 56, 1904, p. 132.

5 Schaudinn, Arb. a. d. kaiserl. Gesund., v. 20, 1904, p. 387. 
Athene noctua of Rovigno, Istria, which contained trypanosomes in their blood (see Chapter III.).

[Since Igo3 our knowledge of the trypanosomes of birds has been considerably increased by the investigations of Novy and McNeal, Petrie, Thiroux, and others.]

[Thiroux ${ }^{1}$ has made an extended morphological and experimental study of the trypanosome of the Java sparrow (padda, or rice-bird, Padda oryzivora), discovered by Levaditi in I904, and named by Laveran and Mesnil T. padda. As will be mentioned later, Thiroux succeeded in growing the trypanosome artificially on a modification of Novy and McNeal's medium.]

[Novy and $\mathrm{McNeal}^{2}$ have published a long paper upon the trypanosomes of birds. These observers examined $43 \mathrm{I}$ birds, representing forty species. It is a remarkable fact that the microscope showed trypanosomes in only twenty-four out of $43 \mathrm{I}$ birds, whereas the cultural method applied in only fifty-three cases gave twenty-nirie positive results ( 55 per cent.). By the two methods of examination (microscopical and cultural), trypanosomes were found in thirty-eight birds, representing fifteen species, as follows: Red-winged blackbird (Agelaius phoniceus), rusty blackbird (Scolephagus carolimus), bluebird (Sialia sialis), blue jay (Cyanocitta cristata), mourning dove (Zenaidura macroura), American goldfinch (Spimus tristis), flicker (Colaptus auratus), red-shouldered hawk (Buteo lineatus), Baltimore oriole (Icterus galbula), robin (Merula migratoria), English sparrow (Passer domesticus), song sparrow (Melospiza fasciata), brown thrasher (Harporhynchus rufus), hairy woodpecker (Dryobates villosus), and house wren (Troglodytes adon).]

[Of the thirty-eight cases in which trypanosomes were discovered, either microscopically or culturally, twenty-three showed an infection with other parasites, such as Filaria, Hamoproteus majoris, $H$.danileroskyi, $H$. ziemanni, etc. Novy and McNeal state that the study of the trypanosomes found in the blood, and also of those obtained by cultivation, shows that there are several distinct species which exhibit no constant association with a given cytozoon. Thus, the commonest species of trypanosome (T. avium) found in the birds examined was associated with five different species of intracorpuscular parasites, or with Filaria, in addition to its very frequent single occurrence. These observers add that 'under these circumstances it would indeed be difficult to estabish a relationship with any of the intracellular organisms mentioned.']

[In addition to $T$. avium, which was found in twenty-five out of the thirty-eight cases, several other species of trypanosome were encountered. In four cases a variety (?) of $T$. avium was found; in each of two other cases trypanosomes which Novy and McNeal

1 [Thiroux, Ann. Inst. Past., v. 19, 1905, pp. 65-83.]

2 [Novy and McNeal, American Medicine, v. 8, November 26, 1904, pp. 932934 ; Journ. Infect. Dis., v. 2, March I, 1905, pp. 256-308. There are many excellent photo-micrographs illustrating the latter paper.] 
regard as distinct species, to which they give the names $T$. laverani and $T$. mesnili; and in the remaining seven cases trypanosomes of other species not yet sufficiently differentiated.]

[In April, I905, Vassal ${ }^{1}$ discovered a trypanosome in the blood of a pheasant (Polyplectrum germani), caught near Nhatrang, in Annam. The bird was also heavily infected with intracorpuscular parasites. Vassal suggests the provisional name $T$. polyplectri for this trypanosome.]

[Petrie, ${ }^{2}$ at Elstree, in Hertfordshire, found trypanosomes in several species of birds-house-martin (Chelidon urbica), song-thrush (Turdus musicus), blackbird (Merula merula), swallow (Hirundo rustica), chaffinch (Fringilla ccelebs), and yellow-hammer (Emberiza citrinella). In every case the blood examination was negative, but the trypanosomes were found in the bone marrow. In one case, that of the house-martin, spirochrtes were present in the blood. All the house-sparrows, starlings, crows, and jackdaws examined were negative as regards trypanosomes.]

[In the Anglo-Egyptian Sudan, Sheffield Neave ${ }^{3}$ found trypanosomes in the common vulture of Egypt (Neophron percnopterus) and the red-breasted shrike (Laniarius cruentus). These trypanosomes were very scanty in the birds' blood.]

[Wellman ${ }^{4}$ has found a trypanosome in the blood of an African dove (Treron calva) in Portuguese Angola.]

[Sjöbring ${ }^{5}$ states that trypanosome infection of birds is widespread in the neighbourhood of Säftstraholm in Södermannland, Sweden. This observer found the parasite in nearly all passerine birds examined by him, with the exception of Corvus and Pica. The infection was apparently local, for it was not met with in other districts. No details of morphology are given, but it is simply stated that the forms observed resembled those of Danilewsky.]

[Donovan ${ }^{6}$ has recently described a trypanosome in the blood of the Indian kite (Milvus govinda).]

[Lastly, trypanosomes have been found in several species of South American birds. Aragao discovered them in Nicticorax gardenia in Brazil (thirty-seven birds infected out of forty-seven examined). Cerqueira ${ }^{7}$ found the same trypanosome in egrets; it appears to be identical with the $T$. avium minus of Danilewsky. In one specimen of Tachyphormus ornata a different species of trypanosome was found.]

1 [Vassal, C. R. Sor. Biol., June I7, I905, v. 58, p. Ior4.]

2 [Petrie, Journ. Hyg., v. 5, I905, pp. 191-200.]

3 [Neave, Second Report of the Wellcome Research Laboratory, Khartoum, I906, p. I98.]

4 [Wellman, Journ. Trop. Med., v. 8, I905, p. 285.]

5 [Quoted from the paper by Novy and McNeal previously mentioned.]

6 [See in Thiroux's paper, Ann. Inst. Past., v. 20, 1905, p. 79.]

7 [Cerqueira, These de Rio de Janeiro, I906; abstract by Marchoux in Bull. Inst. Past., v. 4, p. 104t.] 


\section{Section 2.-Morphology and Cultivation of the Trypanosomes of Birds.}

The trypanosomes of the owl and roller-bird, studied by Danilewsky, ${ }^{1}$ appear as fusiform parasites (Fig. 59), with a tapering or even sharply-pointed posterior extremity which varies in length. The anterior extremity gradually tapers off, and is prolonged into a wavy flagellum of variable length, which gradually diminishes in thickness, so that at last it becomes a scarcely visible filament. The flagellum is closely connected with the undulating membrane, which appears in the form of a hyaline, unstained border, more or less narrow, extending from the flagellum to the posterior extremity. The intimate relation between the flagellum and undulating membrane

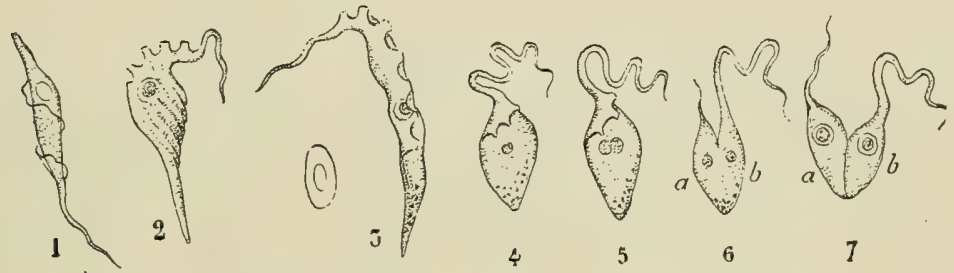

Fig. 59.-Trypanosomes of Birds. (After Danilewsky.)

I-3. Different adult forms. Between 2 and 3 is a red corpuscle of the bird, to give an idea of the relative sizes of the trypanosomes and red corpuscles. 4-7. Stages in the binary division of a trypanosome.

is plainly seen on watching the movements of the parasite, and Danilewsky draws particular attention to this point.

Danilewsky figures the vesicular nucleus, which he says he saw very distinctly in the living state. The forms showing striations (Fig. 59, 2), which resemble the parasites seen in the Batrachia, are rare. From the point of view of size the trypanosomes seen in the blood may be divided into two groups: one consisting of stumpy forms with a long flagellum, measuring I $8 \mu$ to $22 \mu$ in length, not including the flagellum; the other of long forms $45 \mu$ to $60 \mu$ in length; but transition forms also occur. In all the parasites the width is from $7 \mu$ to $8 \mu$.

Even in young roller-birds three or four days old the parasites are specially numerous in the red marrow of the bones, where many different forms are seen. 'In many cases,' says Danilewsky, 'when I found only one or two trypanosomes after a long search in the heart blood, I found many in the red marrow of the bones.' There are cases, however, in which the blood contains many more parasites than the bone marrow.

1 Danilewsky has confused in the same description, and under one name$T$. avium - the trypanosomes of the owl and roller-bird, although they were probably different species. 
Danilewsky describes in detail the longitudinal division of the parasite. According to his figures, which are reproduced here (Fig. 59, 4 to 7 ), the process is analogous to the unequal division of T. lereisi; one of the two trypanosomes retains the original undulating membrane and flagellum, while a new flagellum develops in the other parasite. Often before the two daughter trypanosomes separate, the one with the new flagellum starts dividing again.

All these changes were studied by Danilewsky under the microscope, either in hanging-drop or in ordinary fresh preparations. The division of a trypanosome into two usually takes from twenty to thirty minutes, or at the most an hour. It is, however, still a little doubtful whether this is the normal mode of reproduction of the trypanosome in circulating blood.

Danilewsky also describes reproduction by a process of segmentation, which he observed in blood kept for six to eight days at $22^{\circ} \mathrm{C}$. in sterilized pipettes. A large spherical form, without trace of a flagellum, divides into two, then into four, and so on. A 'morular' mass is thus produced, containing thirty-two elements, which elongate, then become fusiform, and finally become transformed into an equal number of trypanosomes, with a fairly long flagellum, but no undulating membrane. It is to these forms that Danilewsky gave the name Trypanomonas. Knowing what we do now about the cultivation of trypanosomes, it is probable that these masses of Trypanomonas were really cultivation forms. It is much more difficult to admit that they were derived from the 'morular' masses, as Danilewsky thinks.

TRYPANOSOMA AVIUM, Danilewsky, Laveran emend.-One of us (loc. cit.) has given the following description of the trypanosome of Syrnium aluco:

'In fresh blood the trypanosome of the owl appears as a very active worm-like body, with an undulating membrane and an anterior flagellum.

'In blood dried, fixed, and stained by my ordinary method (silver oxide-blue, eosin, and tannin), I obtained excellent preparations of the trypanosomes (see Fig. 60 and Fig. Io in the coloured plate). The trypanosome is fusiform; the protoplasm stains so darkly that the nucleus and centrosome are often scarcely visible; the anterior extremity tapers off and terminates in a flagellum; the posterior extremity is drawn out to a variable extent. About the middle of the parasite is an oval nucleus $(I, n)$. The centrosome is spherical and fairly large $(I, C)$, and when the posterior end is very pointed, the centrosome is situated a considerable distance from the tip. The undulating membrane is well developed and bordered by the flagellum, which joins the centrosome. Below the wavy flagellum a parallel line or striation is often seen, and this appears to be due to a fold in the membrane. These lines are seen in Fig. 60, I, at the prominent parts of the undulating membrane. 
'The trypanosome is from $33 \mu$ to $45 \mu$ in length, including the flagellum.

'In one case (Fig. 6o, 2) the centrosome had divided, which may certainly be looked upon as the beginning of a dividing stage. This is in agreement with the observations of Danilewsky, who saw only one mode of multiplication-namely, longitudinal division-in the trypanosomes of birds.

"The trypanosomes were scanty in the blood of the owl, and they were not more numerous in the kidneys than in the peripheral blood.

'From the above description of the parasite it is evident that the trypanosome of the owl should be included in the genus Trypanosoma. It is highly probable that it is the same parasite as that seen by Danilewsky in an owl, and called by him Trypanosoma avium. I think that this name should be adopted for the trypanosome of the

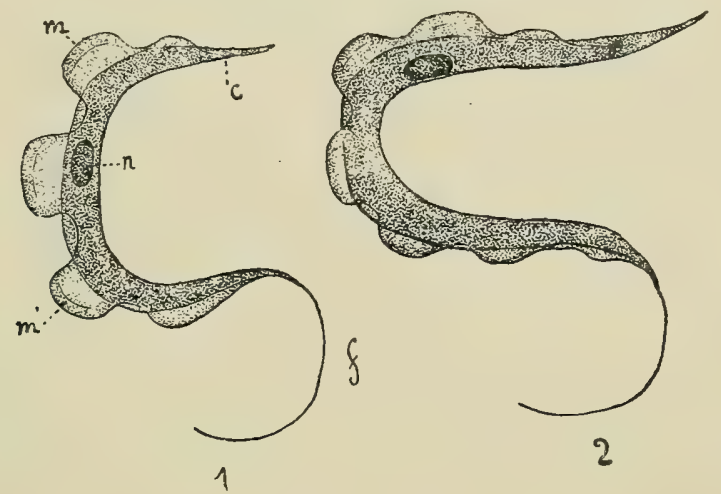

Fig. 6o.-Trypanosoma avium.

$n$. Nucleus. $c$. Centrosome. $m, m^{\prime}$. Undulating membrane. $f$. Flagellum. In 2 the centrosome has divided.

owl, and that it be left an open question whether the trypanosome of the roller-bird belongs to the same species or not.'

It is doubtless an allied species which Schaudinn observed in A thene noctua (see Chapter III.).

TRYPANOSOMA fOHNSTONI, Dutton and Todd, I903.-Found in the blood of Estrelda estrelda in Bathurst.

The following description of the parasite and Fig. 6I, $I$, are taken from the authors' paper :

' Only two to four parasites were seen in a coverslip preparation. In fresh preparations the parasite appears as a very actively moving spirillum-like body; so striking, indeed, is this resemblance, that at first sight it was thought to be a true spirillum. The undulating membrane is scarcely recognisable, and the parasite has no free flagellum. When its movements have become slower, the organism is seen to possess a long straight body, pointed at both ends. At a point about one-third of its length distant from the posterior end of 
the parasite is seen a refractile spot, the centrosome. A little further on a slightly refractile area indicates the position of the nucleus....

'In stained preparations (see Fig. 6r, $I$ ) the above details are better seen. Along the free border of the undulating membrane runs the flagellum, stopping abruptly at the anterior end of the organism in a small red dot. The nucleus is elongated, granular, and does not quite extend across the short axis of the parasite. The centrosome is a small dot or oval spot of chromatin surrounded by a small halo. The protoplasm takes on a uniform blue colour.

' The length of the parasite is from $36 \mu$ to $38 \mu$, and its width at the nucleus $\mathrm{I}^{4} 4 \mu$ to $\mathrm{I}^{\circ} 6 \mu$. The distance from the centrosome to the

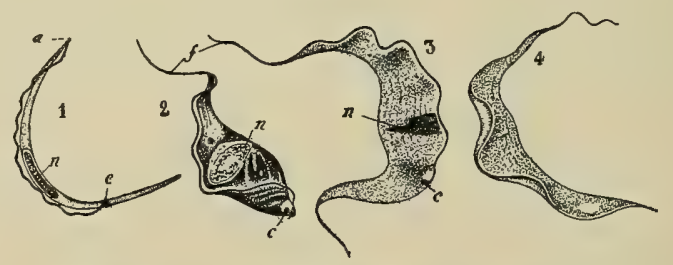

Fig. 61.-Other Bird Trypanosomes.

I. T. johnstoni. 2. Another Gambian trypanosome. 3. Trypanosome of the Indian pigeon. 4. Trypanosome of the Indian raven. n. Nucleus. c. Centrosome $a$. Anterior chromatic granule. $f$. Flagellum, Magnif. I,000 diams. (I and 2 after Dutton and Todd; 3 and 4 after Hanna.)

posterior end is $\mathrm{IO}^{\circ} 4 \mu$. Distance from the centrosome to the centre of the nucleus is $9 \mu$ to Io $\mu$.

' No developmental or dividing forms were seen in any preparation. Two larks were inoculated, each with 0.5 c.c. blood from this bird, but unsuccessfully.'

Another Gambian Trypanosome - Trypanosome of the Pigeon and Raven of India.- These trypanosomes, instead of being very thin and elongated, like $T$. johnstoni, are short and stumpy, and have a free flagellum (see Fig. 6I, 2 to 4 ).

[The table on the opposite page, ${ }^{1}$ based upon those of the authors and of Thiroux, gives the most important dimensions of these three trypanosomes, as well as of several of the other bird trypanosomes hitherto described.

1 [It must not be inferred that all the trypanosomes mentioned in this table are regarded as distinct species.] 


\begin{tabular}{|c|c|c|c|c|c|c|c|c|c|c|c|c|c|c|c|}
\hline & 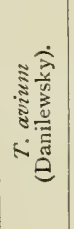 & 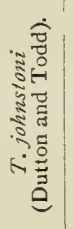 & 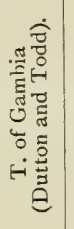 & 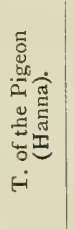 & 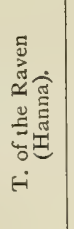 & 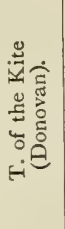 & 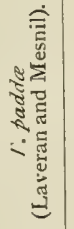 & 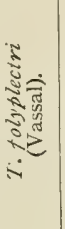 & 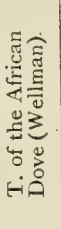 & 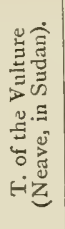 & 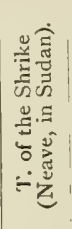 & 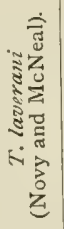 & 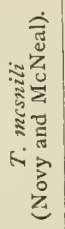 & 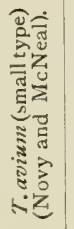 & 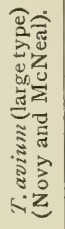 \\
\hline Total length & 33 to & 36 to & $32.5 \mu$ & 45 to & 40 to & $50 \mu$ & 30 to & $4^{6} \mu$ & $28 \mu$ & 58 to & $28 \mu$ & $20 \mu^{*}$ & $50 \mu^{*}$ & 18 to & 5 to \\
\hline $\begin{array}{l}\text { Width at } \\
\text { level of } \\
\text { nucleus }\end{array}$ & $\left|\begin{array}{c}45 \mu \\
3 \mu \\
(\mathrm{abt} .)\end{array}\right|$ & $\begin{array}{r}30 \mu \\
\mathbf{1} 4 \text { to } \\
\mathbf{r} 6 \mu\end{array}$ & $8 \mu$ & $\begin{array}{l}6 \mu \\
8 \mu\end{array}$ & $\begin{array}{c}50 \mu \\
3 \text { to } \\
4^{-8} \mu\end{array}$ & $\begin{array}{r}3 \text { to } \\
3.5 \mu\end{array}$ & $\begin{array}{l}40 \mu \\
5 \text { to } \\
7 \mu\end{array}$ & $5, u$ & ¿ $\mu$ & $\begin{array}{l}4 \mu \\
4 \text { to } \\
5 \mu\end{array}$ & $3 \mu$ & $6 \mu$ & $8 \mu$ & $\begin{array}{c}45 \\
4 \mu \\
6 \mu\end{array}$ & $\begin{array}{l}4 \text { to } \\
5 \cdot 5 \mu\end{array}$ \\
\hline $\begin{array}{l}\text { Distance of } \\
\text { centrosome } \\
\text { from pos- } \\
\text { terior end }\end{array}$ & $\begin{array}{l}3 \text { to } \\
5 \mu\end{array}$ & $10^{\circ} 4 \mu$ & $\begin{array}{c}2 \mu \\
(\text { abt. })\end{array}$ & $\begin{array}{l}\text { Ig to } \\
22 \mu\end{array}$ & $\begin{array}{c}8 \text { to } \\
9.5 \mu\end{array}$ & $5 \mu$ & $10 \mu$ & го $\mu$ & $4 \mu$ & $7 \mu$ & $4 \mu$ & I $\mu$ & $7 \mu$ & $\begin{array}{c}0.5 \text { to } \\
6 \mu\end{array}$ & $\begin{array}{l}13 \text { to } \\
25 \mu\end{array}$ \\
\hline $\begin{array}{l}\text { Distance of } \\
\text { centrosome } \\
\text { from centre } \\
\text { of nucleus }\end{array}$ & II $\mu$ & $\begin{array}{l}9 \text { to } \\
\text { Io } \mu\end{array}$ & $9^{6} 6 \mu$ & $\begin{array}{c}4 \text { to } \\
6.5 \mu\end{array}$ & - & $12 \mu$ & $\begin{array}{l}9 \text { to } \\
\text { Io } \mu\end{array}$ & $12 \mu$ & - & - & - & - & Io $\mu$ & $\begin{array}{c}6.5 \text { to } \\
\text { I2 } \mu\end{array}$ & $\begin{array}{l}6.5 \text { to } \\
\text { Io } \mu\end{array}$ \\
\hline $\begin{array}{l}\text { Distance of } \\
\text { centrosome } \\
\text { from an- } \\
\text { terior end }\end{array}$ & $\begin{array}{l}\text { I7 to } \\
20 \mu\end{array}$ & $26 \mu$ & $20 \mu$ & $\begin{array}{l}29 \text { to } \\
36 \cdot 5 \mu\end{array}$ & $\begin{array}{c}32 \text { to } \\
46^{\circ} 5 \mu\end{array}$ & $27 \mu$ & $23 \mu$ & $24 \mu$ & -- & - & - & - & - & $\begin{array}{l}\text { r3 to } \\
23 \mu\end{array}$ & $\begin{array}{l}25 \text { to } \\
45 \mu\end{array}$ \\
\hline $\begin{array}{l}\text { Free flagel- } \\
\text { lum }\end{array}$ & $\begin{array}{c}\text { II } \mu \\
(\text { abt. })\end{array}$ & $\circ$ & $\begin{array}{l}\text { Io to } \\
\text { I2 } \mu\end{array}$ & $6.8 \mu$ & $6.8 \mu$ & $16 \mu$ & $\begin{array}{c}\text { I.5 to } \\
6 \mu\end{array}$ & $I 2 \mu$ & $8 \mu$ & Io $\mu$ & Io $\mu$ & (?) & (?) & $\begin{array}{l}6 \text { to } \\
13 \mu\end{array}$ & $\begin{array}{l}\text { Io to } \\
20 \mu\end{array}$ \\
\hline
\end{tabular}

* These dimensions are the length of the body without free flagellum. In the case of $T$. laverani and $T$. mesnili the free flagellum was not stained, but Novy and McNeal think one was present.

In the two Indian species, especially that of the pigeon, the posterior end is drawn out to a very fine point. Although these trypanosomes are of about the same length, they differ markedly in two respects : (I) the parasite of the raven is half as wide as that of the pigeon, and (2) the centrosome of the former is only half as far from the posterior end as it is in the latter. A more detailed comparison between these two species is not possible, since Ross's preparations of the raven's blood in which Hanna studied the parasite have faded.

The trypanosome of the pigeon (Fig. 6I, 3) has a very small centrosome, situated in the centre of a clear vacuole; a well-defined undulating membrane, with a short free flagellum; and a thin ribbonlike nucleus, extending across the width of the body. In the homogeneous or longitudinally striated cytoplasm there are several chromatic granules, which are particularly numerous near the posterior end.

The parasites were always relatively scanty in the blood of the pigeons, and Hanna did not observe any dividing forms.

The parasite of the small birds (gen. Estrelda and Crithalda) of St. Louis and Bathurst (Fig. 6I, 2) differs from the foregoing in its very blunt posterior end and in the terminal position of the centrosome. The body stains a deep blue, and shows an appearance of longitudinal striation. The nucleus is large and elongated transversely, and its chromatin stains diffusely. The relatively large centrosome is in the centre of an unstained vacuole. The free flagellum is much longer than in the foregoing species. Dutton and Todd did not see any developmental or dividing forms. Two pigeons and two larks were inoculated without success. 
TRYPANOSOMA PADDE, Laveran and Mesnil. - In May, Igo4, Dr. Levaditi discovered, in the blood of a Java sparrow (Padda oryzivora) bought in a shop in Paris, a trypanosome for which we propose the name $T$. padda. Dr. Levaditi kindly gave us the infected bird, which enabled us to study this new trypanosome.

The Padda oryzivora is very often infected with an intracorpuscular hæmatozoon, Hamamceba danilewskyi, ${ }^{1}$ but the occurrence of trypanosomes in the blood of these birds must be very rare. One of us had examined a large number of them, but had never before seen trypanosomes in their blood.

$T$. padda is $30 \mu$ to $40 \mu$ long, by $5 \mu$ to $7 \mu$ wide. These measurements vary a little, because the parasite is constantly contracting and elongating.

The body of the parasite is fusiform, bulging more or less towards the middle. The anterior end, which is always very attenuated, accompanies the flagellum almost to its tip. The posterior end, which varies a little in shape, is usually very sharply pointed. ${ }^{2}$ There are fine longitudinal striations on the surface of the body. In the protoplasm fine granules are seen, in addition to the nucleus and centrosome. In front of and behind the nucleus clear spaces, which are quite as large as the nucleus itself, can be made out in lightly stained preparations. The nucleus is situated about the centre of the body, and stains only moderately well by our ordinary method. The centrosome is large and rounded, and stains a deep violet. It is situated a variable distance from the posterior end, according to the state of contraction or elongation of this extremity; but it is sometimes nearer the nucleus, especially in forms about to divide. The undulating membrane is narrow and much indented, and is so thin that it remains uncoloured, even in the most carefully stained preparations. The free and attached parts of the flagellum are thin.

[Thiroux, from whose paper ${ }^{3}$ the following additional facts concerning $T$. paddce are taken, states that the nucleus, flagellum, and undulating membrane stain feebly by the ordinary methods, but that better results are obtained by staining at a temperature of $53^{\circ}$ to $54^{\circ} \mathrm{C}$.]

[Multiplication forms are seen only in birds that are heavily infected. Equal binary longitudinal fission is the usual mode of reproduction, but sometimes unequal fission is observed. At first the centrosome enlarges and becomes fusiform, and simultaneously the adjacent base of the flagellum broadens out. Then the flagellum begins to split, and at the same time the centrosome and nucleus segment, and lastly the protoplasm divides.]

1 Laveran, Soc. de Biol., April 30, 1899, and January 13, 1900.

2 The posterior end is sometimes as pointed as the anterior, so that in badly stained preparations in which the flagellum is not visible, one night think that there was a flagellum at each end of the parasite.

${ }^{3}$ [Thiroux, Ann. Inst. Past., v. 19, February, 1905, pp. 65-83.] 
[Agglutination of Trypanosoma padda.-Agglutination rosettes, similar to those seen in the case of $T$. lewisi, may be produced by adding a drop of blood from a bird which has recovered from the infection, or in which the infection is of long standing, to the blood of a bird rich in parasites. The serum of normal birds does not agglutinate. As with $T$. lewisi, the trypanosomes are joined by their posterior ends, the flagella remaining free and active at the periphery of the rosettes. Auto-agglutination is often seen in birds whose blood contains very many parasites, and it appears that in such cases the parasites diminish in number more quickly than when auto-agglutination is absent.]

[T. padda dies in thirty hours in blood mixed with I per cent. citrate at $0^{\circ}, 22^{\circ}$, or $35^{\circ} \mathrm{C}$., but remains alive in cultures for a long time.]

[Cultivation of Trypanosoma padda.-Thiroux succeeded in growing this trypanosome artificially on a modification of Novy and McNeal's medium.]

[The medium was blood-agar containing 30 grammes of agar per litre, and an equal volume, or one and a half times its volume, of defibrinated goose blood. Pigeon blood was found not to answer. The water of condensation was inoculated with the heart blood of a padda containing trypanosomes. At $37^{\circ} \mathrm{C}$. the trypanosomes begin to multiply in the water of condensation in eight or nine days, and reach their maximum development in twelve to fifteen days. Involution forms then appear in the culture, but the trypanosomes remain motile for more than forty days. Successive subcultures on blood agar were also made, and these showed very active reproduction of the trypanosomes.]

[In young cultures the trypanosomes are fusiform, more or less granular parasites, with a flagellum at the anterior end, but without any trace of an undulating membrane. They vary in length from $12 \mu$ to $30 \mu$ and in width from ${ }^{\circ} 5 \mu$ to $5 \mu$. The larger forms often have two flagella. In cultures fifteen or more days old numerous involution forms are seen in addition to the Herpetomonas-like parasites. These involution forms assume all stages from a fusiform to a spherical shape. In very old cultures the spherical, and occasionally also the fusiform, parasites present a morular appearance, due to the segmentation of the protoplasm. Thiroux suggests that this may represent the beginning of an encysted condition and the appearance of 'resistant spores.' This idea is apparently confirmed by the fact that the Herpetomonas, which are closely allied to the trypanosomes, particularly in their culture forms, have analogous resisting forms. ${ }^{1}$ ]

[The parasites are not easily stained in liquid cultures, but satisfactory results can be obtained by fixing thin films in absolute alcohol and staining by the eosin-Borrel-blue tannin method. The fusiform parasites have the nucleus near the anterior end of the body, while the centrosome, with the attached flagellum, is between the nucleus and the anterior end. This gives the parasites an appearance like that of Herpetomonas rather than of trypanosomes. These cultural forms are similar to those of the LeishmanDonovan bodies. The amoboid involution forms seen in very old cultures are often devoid of a nucleus, but contain large chromatic granules.]

[Agglutination may be produced in cultures of $T$. padda by adding a drop of blood from a bird that has recovered from the infection or that

1 [Prowazek, Arb. a. d. kaiserl. Gesund., v. 20, part iii., I904, p. 440.] 
has been infected for a considerable time and has very few parasites in its blood. The blood of healthy birds has no such effect. Rosettes form in from five to fifteen minutes, and all the parasites have their flagella directed towards the periphery.]

[Auto-agglutination is seen in cultures, as it is in the blood of birds that are severely infected.]

[INOCUlation ExPERIMENTs.-This was the first trypanosome of birds to be successfully inoculated into animals (I904). Since Thiroux's experiments, Novy and McNeal have succeeded in inoculating a canary with $T$. avium.]

[Paddas. - Thiroux was able to transmit the infection from one padda to another by means of subcutaneous, intramuscular, intravenous, or intraperitoneal injections of infective blood. Intraperitoneal inoculation was the most reliable method, the incubation period varying from twelve hours to eighteen days. After subcutaneous injection the incubation period was never less than twelve days. The infection produced varied considerably in severity. In some cases it was very slight, and only an occasional parasite was found in blood-films; in other cases the parasites were very numerous-sometimes even equalling the red blood-corpuscles in numbers-and in such cases the birds usually died of the infection. In an average case, of moderate severity, after a variable incubation period, which, however, was usually short, the parasites increased progressively in number for nine to fifteen days and then diminished, the infection remaining stationary sometimes for more than forty days. Ultimately the parasites very gradually disappeared, and the infection sometimes remained latent for two or three months, trypanosomes being only very rarely seen in the blood. The long duration of this latent phase makes it impossible to study the immunity produced by an attack, for one can never be certain that a bird is really cured.]

[After a severe infection of long duration the spleen is often enlarged considerably. Many parasites are present in the spleen and bone marrow, but apparently not more than were present in the blood during life.]

[Thiroux found all paddas more or less susceptible to intraperitoneal inoculation with this trypanosome.]

[Intraperitoneal injections of cultures of $T$. padd $a$ also gave rise to infection. The incubation period varied from fourteen to eighteen days, and the infection was always mild. Very few parasites were found in the blood, and they did not differ in appearance from those seen in paddas which were infected directly with blood.]

[Other Birds.-The following species of birds were also found susceptible on intraperitoneal inoculation: Serinus meridionalis, S. canarius, Lagonosticta minima, Mariposa phonicatis, and Estrelda cinerea. The following birds were found insusceptible: Geese, pigeons, Fringilla calebs, Passer domesticus, Emberiza citrinella, and 
Pytelia subflava. It is interesting to note that, although the goose is refractory to inoculation, $T$. padda is able to grow artificially on media containing goose blood.]

[It follows from Thiroux's observations that one species of trypanosome can infect several species of birds; also that one species of bird may be susceptible to different trypanosomes. Thus, the canary, which is susceptible to $T$. padda, is, according to Novy and $\mathrm{McNeal}$, equally susceptible to $T$. avium. Also T. padda may be inoculated into birds of the genus Esirelda, which Dutton and Todd found to be naturally infected with $T$. johnstoni.]

[The rat, mouse, and frog were found to be refractory on inoculation.]

[Levaditi ${ }^{1}$ and Sevin ${ }^{2}$ have studied the action of normal serums on $T$. padda. They found that the serum of a normal guinea-pig (which is refractory to $T$. padda) agglutinates only slightly, but that the same serum heated to $56^{\circ} \mathrm{C}$. agglutinates well. The explanation seems to be that normal guinea-pig serum contains a thermo-labile anti-agglutinin. If a trace of fresh guinea-pig serum is added to the heated serum agglutination is prevented.]

[The serum of the rat, which is also refractory to $T$. padda, can immobilize and disintegrate this trypanosome. Under these conditions the trypanosome becomes spherical and vacuolated, and shows only a vestige of flagellum and undulating membrane. This trypanolytic property of rat serum disappears when the serum is heated to $56^{\circ} \mathrm{C}$. It cannot be restored by adding the fresh serum of another species of animal. Sevin has found that this heated serum favours the trypanolytic action of the same serum unheated.]

[According to Levaditi and Sevin, animals which are normally resistant to $T$. padda may be divided into two groups: (I) Those whose serum is not agglutinating or paralyzing, as, for instance, mice ; and (2) those whose serum has a trypanolytic action, as, for example, white rats. In group (I) the trypanosomes injected intraperitoneally at first show signs of multiplication by fission, and later on become, while still living, the prey of the phagocytes. In group (2) the trypanosomes injected are rapidly destroyed and show no signs of multiplication.]

[Novy and McNeal's Observations on the Trypanosomes of BIRDS.-As has already been stated, these investigators have found that trypanosome infections of birds are very common and widespread. The methods they used included the direct detection of trypanosomes in the blood, cultivation, and the inoculation of birds with pure cultures. The detection of trypanosomes in fresh bloodfilms was not easy, owing to the extreme scarcity of the parasites; yet this method was, as a rule, more delicate than the examination of stained films. Nocht's modification of Romanowsky's stain was used. It was found, however, that these bird trypanosomes did not stain so well as $T$. lereisi or $T$. brucei, for it was the exception to find

1 [Levaditi and Sevin, C. R. Soc. Biol., v. 58, 1905, pp. 694-697.]

2 [Sevin, ibid., v. 59, 1905, pp. 122, I23; abstracts in Bull. Inst. Past., v. 3, pp. 578,804 .] 
specimens with well-stained nucleus and flagellum. Even when no free flagella could be seen in stained specimens, they could be seen in fresh blood-films.]

[The cultivation method proved to be far superior to direct examination, inasmuch as it rendered possible the isolation of the trypanosomes when apparently none could be detected by the microscope. In cultivating the trypanosomes it is a mistake to have the meat - extract too concentrated. This is particularly true of the initial or first generation.]

[The best formula is the following: The extractives of 125 grammes of rabbit or beef meat in I litre of distilled water, 2 per cent. Witte's peptone, $0^{\circ} 5$ per cent. salt, 2 per cent. agar, and Io c.c. of normal sodium carbonate solution. The agar thus prepared is filled into tubes, and sterilized in the autoclave at $110^{\circ} \mathrm{C}$. for thirty minutes. When cooled to about $50^{\circ} \mathrm{C} ., 2$ volumes of defibrinated rabbit's blood are added, and the mixture is then allowed to solidify in an inclined position. When firmly set it is placed upright for a few minutes until a few drops of water of condensation appear. This liquid is then inoculated with a drop of blood taken from the heart of the bird by means of a fine pipette.]

[The cultures of the bird trypanosomes usually develop very quickly at $25^{\circ} \mathrm{C}$. As a rule, growing trypanosomes can be recognised on the third day. On the sixth or seventh day they are usually extremely abundant and very actively motile. After this they diminish in number, and involution forms appear. Sometimes colonies are formed on the agar above the water of condensation. Subcultures succeed well as a rule. By means of the cultural characteristics it is possible to differentiate the bird trypanosomes from one another, as well as from the mammalian trypanosomes (T. lewisi, T. brucei, and T. evansi).]

[Novy and McNeal consider that they have investigated at least four distinct species. The one most frequently met with (in twentyfive out of thirty-eight birds infected) they identify with Danilewsky's $T$. avium. Two types of this trypanosome were found in the blood, corresponding closely to the majus and minus of Danilewsky. These two types belong to the same species, and always give rise to the same. cultural forms. Novy and McNeal regard the large form as a multiplication, or possibly sexual, type of the smaller and more common form.]

[The large form varies considerably in length: body $35 \mu$ to $65 \mu$, free flagellum I $5 \mu$ to $20 \mu$. The width is $5 \mu$ to $7 \mu$. The centrosome lies close to the nucleus and is in a large clear space. The nucleus stains badly. Six or eight well-marked striæ or myonemes are present along the entire length of the body. The posterior end is long and tapering. In the living condition the contortions of the parasite are very active, but there is little tendency to travel out of the field of the microscope.]

[The small form is the most common one met with in the blood 
of birds. The total length, flagellum included, is about $25 \mu$ to $30 \mu$, the body proper being about $20 \mu$ long. The width ranges from $3.5 \mu$ to $5 \mu$. In some parasites the centrosome appears to be at the very tip, in others it is $4 \mu$ or $5 \mu$ distant. Novy and McNeal suggest that the larger of the small forms, with its centrosome at a distance from the tip, constitutes a transition to the large form, which has its centrosome close to the nucleus.]

[This smaller variety is thought to be identical with Laveran's T. avium found in the owl (see p. 444), and also with the short, broad trypanosome found by Dutton and Todd in Senegambia. Hanna's Indian bird trypanosomes Novy and McNeal identify with their large form of T. avium. T. johnstoni (Dutton and Todd) they regard as a distinct species; and $T$. paddce they regard as being culturally distinct from $T$. avium, and as closely resembling $T$. laverani.]

[Cultural Characteristics of Trypanosoma avium.-Two types of parasite are seen in cultures: (I) Round, oval, or spindleshaped cells, with the centrosome by the side of, or anterior to, the nucleus. These are arranged in rosettes-simple or primary and multiple rosettes-in which the flagella are directed centrally, as in the culture or ' multiplication 'rosettes of $T$. lewisi; and (2) extremely slender, long, wavy, darting forms-the spirochætiform parasites of Schaudinn. These 'spirochætes' have a well developed undulating membrane, the centrosome lying in the posterior part of the body. They are often joined by their posterior ends, the flagella being directed peripherally ('agglutination rosettes'). It might be supposed, say Novy and $\mathrm{McNeal}$, that the rosette and spirochæte forms represent two distinct species; but on various grounds (the two forms were met with in every one of the eighteen strains examined, etc.) they are of opinion that the two forms belong to one species.]

[Injections of these cultures into birds were not very satisfactory. Only one canary out of many birds (canaries, robins, sparrows, etc.) injected became infected.].

[TRYPANOSOMA MESNILI, Novy and McNeal, I905.-This trypanosome was met with in the blood of a hawk (Buteo lineatus). It differs morphologically from $T$. avium, and an even greater difference can be seen in cultures. This trypanosome is characterized by its large size, and by a wide, rounded posterior extremity. Its dimensions are given in the table on p. 447. The cytoplasm stains deeply and is coarsely granular.]

[Cultures grow very rapidly and show two types of cells, resembling in a general way those met with in $T$. lewisi and $T$. avium: (I) Small cells, Io $\mu$ to I $2 \mu$, even I $5 \mu$, long, by $4 \mu$ to $6 \mu$ or $7 \mu$ wide. The rapidly growing cells are much smaller, and have a very short free flagellum; the fully developed cells have a free flagellum about as long as the body. These cells form the multiplication rosettes. (2) Large cells, $20 \mu$ to $25 \mu$ long, by $4 \mu$ to $6 \mu$ wide; free flagellum, 
I7 $\mu$ to $20 \mu$. These correspond to the spirochæte stage of $T$. avium, but they are shorter and wider, and have a very long free flagellum. These cells form the typical agglomeration rosettes, with the flagella directed peripherally.]

[Injections of these cultures were made into an owl and three chickens, but unsuccessfully.]

[TRYPANOSOMA LAVERANI, Novy and McNeal, I905.-This trypanosome was met with in the blood of a goldfinch (Spinus tristis). In size it agrees with the smaller variety of $T$. avium (see table on p. 447). The posterior end is very pointed; the posterior part of the cell is coarsely granular.]

[In artificial culture growth is very slow and scanty. The rosettes are smaller and less numerous than in $T$. avium and T. mesnili. The characteristic feature of this trypanosome is the presence of a terminal rod, which usually lies against the wall at the posterior end of the cell. This rod is best seen when division is about to occur. Many free forms are seen in cultures. They are either typical spindle-shaped cells, I4 $\mu$ to $20 \mu$ long, by $4 \mu$ to $5 \mu$ in greatest width, with a long free flagellum; or slender cells, $20 \mu$ to $25 \mu$ long, by $2.5 \mu$ to $3 \mu$ wide, and with a shorter free flagellum.]

[TRYPANOSOMA SP. (?).-This trypanosome was obtained twice from blue jays and once from a rusty blackbird. It was got only by cultivation, and although Novy and McNeal feel sure it is a distinct species, they have not yet named it. Growth is rapid; rosettes are very scarce, the free forms predominating. These are wide, actively motile cells, about I5 $\mu$ long, by $3 \mu$ or less in width.]

[Relation of Trypanosomes to other Blood Parasites.-Many of the birds inoculated by Thiroux with $T$. paddce had a double infectionwith the trypanosome and Halteridium. From his experiments upon paddas and canaries Thiroux has come to the conclusion that $T$. padda and $H$. danilewskyi have no relation to one another, that their respective life cycles are quite distinct, and that the birds are really suffering from a double infection. Novy and McNeal have come to the same conclusion with regard to $T$. avium, and the various intracorpuscular hæmatozoa met with by them in birds. Reference has already been made (see p. 45) to their interpretation of Schaudinn's observations. They maintain that $T$. noctua and Spirochata ziemanni of Schaudinn probably represent trypanosomes which have multiplied in the mosquito; they are not to be considered as stages in the life-history of cytozoa. The facts that the same species of bird may be infected by several species of trypanosomes; that the trypanosomes may or may not be associated with intracorpuscular parasites; that no constancy can be shown to exist between a given trypanosome and a given cytozoon; and, lastly, that birds infected only with cytozoa do not give rise to cultures of trypanosomes, even when many mature gametes are present, have led Novy and McNeal 
to conclude that the simultaneous presence of trypanosomes and other blood parasites is to be regarded as an accidental coincident infection, and that there is no genetic relation between the parasites.]

[TRYPANOSOMA POLYPLECTRI, Vassal, I905.-The dimensions of this trypanosome are given in the table on p. 447. In the fresh condition it is very active, but does not move much from place to place. It has a long, pointed posterior extremity, and sometimes there are granules between the centrosome and the posterior end of the body. Multiplication takes place by simple binary fission. As has already been mentioned ( $\mathrm{p}$. 442), this trypanosome was found by Vassal near Nhatrang, Annam, in a pheasant, which was severely infected with intracorpuscular hæmatozoa. Vassal has given it the provisional name $T$. polyplectri, but he adds that in the light of Novy and McNeal's observations it is wise to keep an open mind upon its specific individuality until a more complete study of it has been made.]

[Trypanosomes of Birds in the Anglo-Egyptian Sudan. -Neave found trypanosomes in several vultures and in a redbreasted shrike. The dimensions of these parasites are given in the table on p. 447. The trypanosomes found in the blood of vultures were about as large as the large form of $T$. avium described by Novy and McNeal. The posterior end was very pointed, and was apparently prolonged into a flagellum $4 \mu$ to $6 \mu$ long.]

[The trypanosome of the shrike was considerably shorter than that of the vulture (see p. 447). Its centrosome was rod-shaped, and Neave states that he is unable to identify this trypanosome with any previously described species.]

[Trypanosome of the African Dove (Wellman).--This trypanosome is short and stumpy. The nucleus lies with its long axis (longest diameter about $6 \mu$ ) across the width of the body. Faint longitudinal striæ or myonemes are present. The cytoplasm contains fine granules. The dimensions of the parasite are given on p. 447.]

[TRYPAnosome of Nicticorax.-This trypanosome, discovered by Aragao in Nicticorax gardenia in Brazil, has been further studied by Cerqueira. It appears to be identical with $T$. avium mimus of Danilewsky, and belongs to the type lewisi. Cerqueira found that it grew well in Novy and McNeal's medium in three days. Three types of multiplication forms are described: (I) ordinary trypanosome forms; (2) Spirocheta-like forms; and (3) large and small spherical forms.]

[Attempts at artificial infection gave negative results.]

To sum up, the trypanosomes of birds known up to the present belong to three different types:

I. Trypanosomes of the type lewisi of rats ; 
2. Trypanosomes of the type rotatorium of the frog; and

3. Long, thin trypanosomes without free flagellum, of a distinctive type.

Concerning the natural mode of spread of the infection we have only Schaudinn's work, mentioned in Chapter III. ; he regards Culex pipiens as the carrier of the infection. [We have seen, however, that this is doubted by several authorities.] 


\section{CHAPTER XV}

\section{TRYPANOSOMES OF REPTILES}

UNTIL I902 we knew nothing definite about the trypanosomes of reptiles. It is true authors mentioned that the tortoise served as a host to trypanosomes, but they gave no exact details. From the existence of flagellates with undulating membrane in the digestive tract of Ixodes testudinis, an acarine ectoparasite of tortoises, Leydig ${ }^{1}$ concluded, without positive proof, that they existed in the blood of the tortoises.

Later, Kunstler ${ }^{2}$ writes: 'In the blood of the mud tortoise there occurs a parasite which, I think, is allied to Trypanosoma.'

In I902 we found on two occasions out of four in an Asiatic tortoise, Damonia reevesii, a trypanosome which we have described under the name $T$. damonia. ${ }^{3}$ We reproduce here our description, with diagram (Fig. 62, and Fig. II in the coloured plate):

'In the two infected tortoises $T$. damonice was very scanty in the blood. It measures $32 \mu$ in length (including the flagellum), by $4 \mu$ in width. In stained preparations it is easy to see that $T$. damonice has the typical structure of the genus Trypanosoma. The body of the parasite is usually curved, and the anterior extremity ends in a flagellum $(f)$. Towards the middle of the body is an oval nucleus $(n)$, in which the chromatin is in the form of granules of variable size. Near the posterior end is the centrosome (c), which is clearly visible, while the convex border of the parasite is furnished with a festooned undulating membrane $(m)$. The flagellum starts from the centrosome, and borders the undulating membrane. The protoplasm is finely granular with some larger granules, especially in the posterior part.

'We have not seen any multiplication forms. We may add that $T$. damonice is relatively stumpy, compared with the species found in fishes and mammals. It is intermediate between these and the T. votatorium of frogs.'

In their paper on the trypanosomes of Gambia, Dutton and Todd state that they saw trypanosomes in snakes and tortoises. They give no details-neither of the snakes infected nor of their parasites.

1 Leydig, Lehrbuch der Histologie, 1857, p. 346.

2 Kunstler, C. R. Acad Sciences, v. 97, I883, p. 755.

3 Laveran and Mesnil, C. R. Acad. Sciences, v. 135, October 20, 1902, p. 609. 
Upon the subject of the trypanosomes of the tortoise they write as follows :

' Two tortoises obtained from the marshes at Cape St. Mary, out of several examined, contained trypanosomes in their blood. Their blood was constantly examined during a period of three months, but only occasionally were parasites seen, and then only in small numbers. Two varieties were observed. One was a

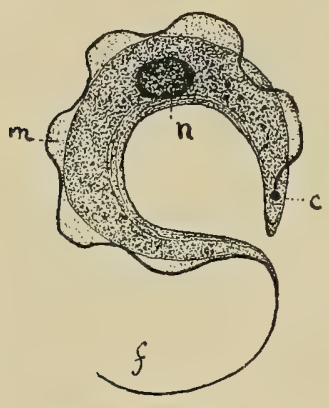

Fig. 62.-TRYPANOSOMA DAMONIÆ,

$n$, Nucleus. c. Centrosome $m$. Undulating membrane.

f. Flagellum. long thick form; the other, short and slender, possessed a comparatively broad undulating membrane, which extended the whole length of the organism.

'The few parasites seen in stained films of blood from these tortoises have not been sufficiently perfect to permit their detailed description.'

Dr. Simond has shown us a drawing of a trypanosome which he observed in the blood of a tortoise at Agra, in India, probably Emys or Kachuga tectum.

Gehrke ${ }^{1}$ has recorded the occurrence of a trypanosome in a gecko.

[Minchin, Gray, and Tulloch, ${ }^{2}$ while looking for a possible vertebrate host of the trypanosomes of tsetse-flies (see Chapter XVIII.) in Uganda, on one occasion found a trypanosome in a film of crocodile's blood. Beyond its large size and general resemblance to other reptilian trypanosomes, it was not possible to make out any details of structure in this parasite, as the blood-film was not very well preserved. These observers found that a hæmogregarine was quite common in the blood of crocodiles.]

[TRYPanosome OF THE LizARd.-Quite recently G. Martin ${ }^{3}$ has described a trypanosome which he found in a lizard, Mabuia raddonii, in French Guinea.]

[This trypanosome differs from $T$. damonice in appearance, and resembles the flat form (without ridges) of $T$. rotatorium of the frog. Examined fresh, the nucleus and centrosome are not easily made out; the flagellum never extends beyond the limits of the body. When stained, the protoplasmic body of the parasite measures $40 \mu$ in length, by almost as much in width. The nucleus is long and thin, and curved or arched, as in T: borreli of the frog (see next chapter). It stains uniformly pale violet, except for one or two darker spots. As in T. borreli, the centrosome stains deeply, and is

1 Gehrke, Deutsche med. Wochenschr., I903, ver.-beil., p. 402.

2 [Minchin, Gray, and Tulloch, Sleeping Sickness Commission Reports, No. 8, I907, p. 130.]

${ }_{3}^{3}$ [G. Martin, C. R. Soc. Biol., v. 62, 1907, pp. 594-596.] 
in contact with one end of the nucleus. The undulating membrane begins in the centrosome, and in its general direction describes an arc of a circle; it presents few folds.]

[Martin has given the name $T$. bouteti to this trypanosome.]

[Robertson ${ }^{1}$ has described, under the name $T$. pythonis, an endocorpuscular parasite in a python from Gambia. It has all the characters of a hæmogregarine, but possesses, in addition to the nucleus, a chromatic dot like the centrosome of a trypanosome, which is joined to the nucleus by a filament. Mesnil thinks it is premature to call this parasite a trypanosome.]

We know nothing about the mode of propagation of the trypanosomes of reptiles. It is very probable that it takes place by means of ectoparasites, acarine or blood-sucking, which are not rare upon the bodies of reptiles.

: [Robertson, Proc. Roy. Phys. Socy. Edin., v. 16, I906, pp. 232-247.] 


\section{CHAPTER XVI}

\section{TRYPANOSOMES OF BATRACHIANS}

\section{Section 1.-Historical and Geographical Distribution.}

ONE year after the discovery of the first trypanosome-that of the trout-by Valentin, Gluge, ${ }^{1}$ in $I 842$, recorded the presence, in the blood of frogs, of another of these parasites. He described it as a fusiform microscopic organism with pointed ends, having on its side three projections-probably the undulating membrane-which were endowed with very active mobility when the parasite moved from place to place. The body was transparent, and showed no trace of structure.

The following year the same parasite was studied by Mayer and Gruby. In July, I843, Mayer ${ }^{2}$ described it under two names: Amoeba rotatoria, a pointed parasite with two extremities; and Paramocium loricatum or costatum, ${ }^{3}$ an ovoid form without free flagellum (the drawing is quite definite upon this point). In November, I 843 , Gruby ${ }^{4}$ gave a fairly complete description of the same trypanosome, and we reproduce it here in part: 'Its elongated body is flattened, transparent, and twisted like a wimble; its head end terminates in long slender filaments, as also does the tail end. The parasite is from $40 \mu$ to $80 \mu$ long, and $5 \mu$ to Io $\mu$ wide. The filamentous, pointed head end is very actively motile, the length of this filament being Io $\mu$ to I2 $\mu$. The body is elongated, flattened, and serrated like the blade of a saw along one of its borders. As I mentioned above, it is smooth and twisted two or three times around its long axis like a wimble or a corkscrew, and on this account I propose to call this hæmatozoon Trypanosoma.'

The description is fairly precise, but the figures are not so accurate as those of Mayer. Gruby called this species T. sanguinis.

In I850 Chaussat, ${ }^{5}$ repeating the observations of his predecessors,

' Gluge, Miiller's Archiù, v. 9, I842, p. 148.

2 Mayer, 'De Organo Electrico et de Hæmatozois,' Bonn, July, I843.

3 In the text the name loricatum occurs; in the description of the plates, that of costatum.

${ }^{4}$ Gruby, C. R. Acad. Sciences, v. I7, I843, p. 1134 . This note is reproduced in Ann. Sc. natur. Zool., 3rd series, v. I, 1844, p. 104, where it is illustrated by six figures, which prove that the author saw long thin forms and short stumpy forms.

5 Chaussat, 'Thesis for the Faculty of Medicine,' Paris, 1850, No. 192, 51 pp. and 2 plates. 
described and figured the trypanosome of frogs, his figures being distinctly better than those of Mayer. He has accurately drawn and correctly interpreted the change of shape into a spherical form with loss of flagellum.

In the same year, I850, Wedl ${ }^{1}$ again recorded the existence of the trypanosome of Rana esculenta, and described for the first time a trypanosome identical with the preceding in the blood of the green frog (Hyla viridis). His paper gives good figures of the various forms of these trypanosomes.

In I870 Lieberkühn ${ }^{2}$ figured and described under the name Monas rotatoria a parasite in the blood of the frog which was evidently a trypanosome.

In I87 I Ray Lankester ${ }^{3}$ rediscovered the same species in $R$. esculenta, and gave it the name Undulina ranarum.

In I875 Rättig ${ }^{4}$ gave a further account of the parasite, and studied the action of the following reagents upon it : alkalies, distilled water, acetic and hydrochloric acids (which kill it almost immediately), and salt solution, which in a strength of $\frac{1}{4}$ or $\frac{1}{2}$ per cent. acts upon it more slowly.

Gaule ${ }^{5}$ devoted himself especially to establishing the non-parasitic nature of trypanosomes, a suggestion which had already been brought forward by Milne-Edwards, Remak, von Siebold, and Stein. He endeavoured to establish a relation between these organisms and the normal elements of frog's blood. Only the figures which illustrate this paper are of any value, and two of them (iii. and v., in Fig. 2) are good.

In I88I-I883 Grassi ${ }^{6}$ recorded the presence of $T$. sanguinis, Gruby in Hyla viridis, Bufo vulgaris, and $R$. esculenta. He created in addition the genus Paramocioides for the representatives of the family of Trypanosomata (S. Kent) with undulating membrane but without trace of flagellum, and he recorded a species of this genus (Paramcecioïdes costatus, n. sp.) in the blood of $R$. esculenta. From this author's description and figures-a cell with a narrow and undulating lateral expansion extending from the anterior extremity to about the middle of the body-he was evidently dealing with the 'ribbed' form of $T$. sanguinis, which had assumed a spherical shape. The globular form of Amceba rotatoria of Mayer resembled Paramocium costatum.

Danilewsky, first in 1885 and later in I888, gave numerous

1 Wedl, Denkschr. Akad. Wien., v. I, I850, I plate.

2 Lieberkühn, Ueber Bevegungserscheinungen der Zellen, Marburg and Leipzig, I870, Table II., Fig. I7 (quoted by succeeding writers).

${ }^{3}$ Ray Lankester, Quart. Journ. Micr. Sc., v. II, I87 I, p. 387.

4 Rättig, Inaugural Dissertation, Berlin, 1875 (quoted by succeeding writers).

5 J. Gaule, of Leipzig, Arch.f. Anat. u. Physiol. (Physiolog. Abth.), I88o, p. 375, I plate.

6 Grassi, Arch. ital. Biologie, v. 2, p. 426, and v. 3, p. 23, I883; also Atti Soc. ital. d. sc. Nat. Milano, v. 24, I881, p. I35.

7 Danilewsky, Biol. Centralb., v. 5, November I, I885, p. 529 ; also 'Nouvelles Recherches sur les parasites du sang des Oiseaux,' Charkov, 1889. 
details of the $T$. sanguinis of Gruby which he observed in the blood of $R$. esculenta, $R$. temporaria, Hyla arborea, and of tadpoles. In the frog he distinguished at least four varieties. We shall return to this again when speaking about the morphology of the parasite.

Chalachnikov, ${ }^{1}$ who has written a long account of the trypanosomes of frogs, accompanied by numerous drawings, also groups them into two main classes, comprising five varieties.

The work of Ziemann, published in $1898,{ }^{2}$ in which there is a figure of a frog trypanosome, marks the first application of Romanowsky's method of staining (modified by the author) to trypanosomes. As we have already pointed out, it is from the time that these methods of staining were applied that our exact knowledge of the morphology of the trypanosomes dates. Ziemann's results, it is true, were not entirely satisfactory-he stained two chromatic masses, a large and a small, evidently the nucleus and centrosome, but he did not attempt to give any interpretation of them, nor did he succeed in staining the undulating membrane or flagellum.

We took up the study of the trypanosome of $R$. esculenta in Igor, ${ }^{3}$ and were able to give for the first time all the details of its structure. In addition to its intrinsic interest, that study enabled us, as we have seen in Chapter III., to settle the question of the generic names which should be used in designating the various trypanosomes.

In the numerous accounts to which we have just referred, it has always been the trypanosome seen by Gluge, Mayer, and Gruby which has been the object of study.

In November, I903, Dutton and Todd ${ }^{4}$ described two new species of trypanosome which they found in frogs in Gambia, in addition to $T$. rotatorium or sanguinis, but it is quite likely that these were simply special forms of the species of Mayer-Gruby.

Early in 1904 Ed. and Et. Sergent ${ }^{5}$ recorded an undoubtedly new species of trypanosome in $R$. esculenta which they found in very large numbers in the blood of a frog at Kabylie. They have called this new trypanosome $T$. inopinatum.

[Continuing their researches upon the trypanosomes of frogs in Algeria, the Sergents ${ }^{6}$ examined eighty-two specimens of $R$. esculenta, with the result that $T$. inopinatum was found once and $T$. rotatorium eighteen times. In eight of the latter cases the trypanosomes were of the ordinary large size ( $40 \mu$ to $60 \mu$ ), while in the remaining ten cases the parasites were similar in form, but much smaller in size (average length only $22 \mu$ ). The name $T$. rotatorium var. nana is suggested by the discoverers for this smaller form.]

1 Chalachnikov, 'Recherches sur les parasites du sang,' etc., Charkov, 1888 , in Russian.

${ }_{2}$ Ziemann, Centralb. f. Bakter., I, v. 24, I 898.

3 Laveran and Mesnil, C. R. Soc. Biol., June 22, 1901, p. 678.

4 Dutton and Todd, First Report of the Expedition to Senescambia, 1902, Liverpool, November, IŞO3.

${ }_{5}^{5}$ Ed. and Et. Sergent, C. R. Soc. Biol., January 23, I904, p. 123.

${ }^{6}$ [Ed. and Et. Sergent, C. R. Soc. Biol., v. 58, 1905, pp. 670-672.] 
[Fifty-eight of the frogs were examined in summer and autumn: forty-one of these harboured various parasites-trypanosomes, Filaria, and Drepanidium. The twenty-four frogs examined in winter showed no parasites.]

[França and Athias ${ }^{1}$ found trypanosomes in six frogs caught in Lisbon. The parasites present were $T$. votatorium and $T$. inopinatum (Sergent). These authors think that the species rotatorinm should be split up into-(I) T. lovicatum or costatum (Mayer), in which the body is ovoid, generally fairly wide, striated or not, and with the centrosome situated near the nucleus; and (2) T. votatorium (Mayer), in which the body is more slender, the centrosome is near the posterior extremity, and the undulating membrane is well developed, and extends along the whole length of the body. Mesnil thinks there is no justification for this subdivision of the species, and that intermediate forms occur. He likewise doubts the validity of two new species created by França and Athias: (I) $T$. undulans, which is said to be $30 \mu$ long, by $6 \mu$ to $9 \mu$ wide, with a narrow undulating membrane, and without free flagellum; and (2) $T$. elegans, which is also $30 \mu$ long, but only $3 \mu$ wide.]

[Broden ${ }^{2}$ examined twenty frogs (sp. incert.; probably two) at Lusambo, Congo Free State, and found eighteen of them infected with $T$. rotatorium. Six of the frogs also showed forms corresponding to $T$. mega of Dutton and Todd; this was probably only a variety of $T$. rotatorium.]

[Lewis and Williams, in I904, examined 140 frogs from the River Niagara. Fourteen (Io per cent.) were infected with $T$. rotatorium; five with Drepanidium ranarum; one with Filaria. In one case Trypanosoma and Drepanidium occurred in the same blood. The infections with trypanosomes were distributed as follows: In July, of fifteen frogs examined, two were found infected; in August, ten out of twenty-six; in September, two out of fourteen; and from October to December, of eighty-five frogs examined, none showed trypanosomes.]

[These observers examined many other animals for the presence of hæmatozoa, but with negative results: normal cats, dogs, rabbits, and guinea-pigs; fifty-one English sparrows (half in winter, half in spring); twenty-seven mud-puppies (Necturus maculatus) in March, and forty toads in the summer.]

[In I904 Laveran ${ }^{3}$ described a trypanosome which had been discovered by Theiler in two Transvaal frogs $(R$. angolensis, Bocage, and $R$. theileri, Mocquart). This trypanosome appears to be a distinct species, to which Laveran has given the name $T$. nelspruitense.]

[Nabarro and Stevenson have found trypanosomes in the blood of a frog from Hong-Kong. They were very scanty in the blood,

1 [França and Athias, Arch. inst. roy. de bacter. Camara Pèstana, v. I, May 1906, pp. I 27-165; abstract by Mesnil, Bull. Inst. Past., v. 4, I906, p. 756.]

2 [Broden, Arch.f. Sch. u. Trop. Hyg., v. 9, 1905, p. 18.]

3 [Laveran, C. R. Soc. Biol., v. 57, 1904, p. I 58.] 
and the frog was also infected with a large hæmogregarine (sp. ?). The species of frog in which these parasites occurred has not yet been determined. Dr. Bell of Hong-Kong, to whom we are indebted for sending us the blood-films, has told us that the infected frog was a small brown frog, closely allied to, if not identical with, $R$. temporaria.]

[Early in 1907 Marchoux and Salimbeni ${ }^{1}$ described a new trypanosome in a Hyla (allied to H. lateristriga, Spix and Agassiz) in Brazil. This trypanosome is distinct from $T$. rotatorium, and its discoverers have given it the name $T$. borreli.]

[During the past few years interesting observations have been made upon the cultivation of T. rotatorium by Lewis and Wiiliams ${ }^{2}$ and by Bouet; ${ }^{3}$ upon the development of $T$. inopinatum in leeches and its transmission by them (Billet, ${ }^{4} \mathrm{Brumpt}^{5}$ ); and upon the possible relation between the trypanosomes and hæmogregarines of the frog. Details of these observations will be given later on.]

One of us ${ }^{6}$ has observed, in specimens of the blood of a toad (sp. ?) at Imi, Ogaden, which had been sent to him by Brumpt, 'several trypanosomes having the structure of the ordinary trypanosomes of the frog, but much smaller in size.'

[Brumpt ${ }^{7}$ has since given a short description of this trypanosome of the African (Somaliland) toad, Bufo reticulatus. He regards it as a new species, to which he has given the name $T$. somalense.]

[In Igo6 Tobey $^{8}$ recorded the occurrence of trypanosomes in the blood of newts (Diemyctulus viridescens). The trypanosomes were constantly present in the blood and in large numbers. This is the first time that newts have been found infected with these parasites.]

The $T$. rotatorium of frogs appears to occur in all parts of the world, and in this respect it resembles the T. lewisi of rats. We may mention that about half the frogs, $R$. esculenta, caught in the neighbourhood of Paris contain trypanosomes in the blood, but sometimes in very small numbers.

The older observers have noted that trypanosomes were seen in the blood of frogs much more frequently in summer than in winter. [It has already been mentioned that the Sergents in Algeria, and Lewis and Williams in America, found the same seasonable prevalence of trypanosome infections in frogs.] This is the case with all hæmatozoa, but recently Koninski of Wieliczka has stated that the

1 [Marchoux and Salimbeni, C. R. Soc. Biol., v. 62, 1907, pp. 592-594.]

2 [J. Lewis and H. V. Williams, Proc. Soc. for Exper. Biol. and Med., v. 2, I905, p. 44, in Amer. Med., v. 9, March 25, 1905 ; abstract by Mesnil, Bull. Inst. Past., v. 3, 1905, p 4I3.]

3 [G. Bouet, Ann. Inst. Past., v. 20, July, I906, pp. 564-577.]

4 [Billet, C: R. Acad. Sciences, v. 139, 1904, pp. 574-576.]

5 [Brumpt, C. R. Soc. Biol., v. 6I, I906, pp. 167-169.]

${ }^{6}$ Laveran, C. R. Soc. Biol., February 27, 1904, p. 332.

7 [Brumpt, C. R. Soc. Biol., v. 6o, 1906, p. 164.]

8 [Tobey, Journ. Med. Resecurch, Boston, New Series, v. 10, 1906, pp. I47, 148.] 
seasonal influence is $n i l .{ }^{1}$ He gives statistics of the frequency with which trypanosomes occur in the various Batrachians, but obviously these numbers apply only to the district in which he made his observations. He found trypanosomes in the three following species: R. esculenta, $32 \cdot 8$ per cent.; R. temporaria, $\mathrm{I} 3^{\circ} 2$ per cent.; and Bufo viridis, I2 $\mathrm{I}$ per cent. On the other hand, trypanosomes were never found in the blood of Hyla arborea, Bufo cinereus, and B. calamita (very few specimens of these three species were examined), Pelobates fuscus (twenty-eight examined), Bombinator igneus (fifty-two examined, of which twenty-five were larvæ), and Triton vulgaris (twenty examined).

The proportion of infected animals of a given species increases with age. Koninski found $R$. esculenta infected immediately after their metamorphosis. On the other hand, he never found tadpoles infected as Danilewsky (loc. cit., p. 79) and Kruse ${ }^{2}$ had done. The males of $R$. esculenta contained twice as many parasites as the females. Gruby had noticed that the proportion of infected males was smaller than that of females.

\section{Section 2.-Trypanosoma rotatorium (Mayer). ${ }^{3}$}

On studying the blood of frogs ( $R$. esculenta) infected with trypanosomes, one is struck with the great variety of forms presented by the parasite. All observers have drawn attention to this pleomorphism. Danilewsky, for example, distinguishes at least four varieties:

I. 'The simplest form, membranous,' with a flat, transparent body, passing insensibly into the undulating membrane, very active, and with a long, wavy flagellum.

2. 'The flat form rolled on itself,' or the 'funnel-shaped' form.

3. 'The plane spiral form,' with pointed posterior extremity. The undulating membrane extends only along the anterior half of the body.

4. 'The pectinated spiral form,' with the variety in the form of a cornucopia.

Chalachnikov, who has made a special study of the trypanosomes of Batrachians, distinguishes:

I. The group of flattened forms, comprising $(a)$ the simple flat form, $(b)$ the folded form, and (c) the transitional form-plane spiral.

2. The group of pectinated forms, comprising $(a)$ the pectinated spiral form, and (b) the spiral and tubular pectinated forms (cornucopia).

These five varieties, of which the author gives good descriptions

1 Karl Koninski, Biol. Centrabl., v. 2 I, I90 I, p. 40.

2 Kruse, Virchow's Archiv, v. I20, 1890, p. 557.

3 The synonyms of this species are as follows: Paramcecium loricatum or costatum + Amoba rotatoric $[$ Mayer, July, 1843$]=T$. sanguinis [Gruby, November, $1843]=$ Monas rotatoria [Lieberkühn, 1870] =Undulina ranarum [Ray Lankester, $\mathrm{I} 87 \mathrm{I}]=T$. sanguinis (Gruby) + Paramoecioides costatus $\mathrm{n}$. $\mathrm{sp}$. [Grassi, I882]. 
and figures, occur in the blood of $R$. esculenta, R. temporaria, and Hyla arborea.

Chalachnikov has seen these various forms become spherical by the retraction of the flagellum. He gives numerous figures showing this change in vitro, and the reproduction of the parasite by segmentation, which leads eventually to the formation of extremely small spherical bodies. In the present state of our knowledge it is impossible to say what is the significance of these bodies. [They may possibly bear some relation to the 'morular' masses of small spherical bodies described by Thiroux in cultures of $T$. padda, and by Bouet in those of $T$. rotatorium itself.]

In ordinary fresh preparations the trypanosomes usually move about in loco, so that one is able to study the very complex movements of the undulating membrane, and the amoboid movements which result in the ever-varying shape of the body.

By using our ordinary method of staining we have studied the cytological details of the structure of $T$. rotatorimn.(Fig. 63 , and Fig. I5 in the coloured plate).

The trypanosomes show a very much folded undulating membrane, with a thickened border, which is prolonged anteriorly into a free flagellum. These structures all stain reddish-violet by our method. The posterior end of the flagellum (or thickened edge of the undulating membrane) is situated at a variable point in the posterior half of the body, ${ }^{1}$ and comes into relation with a vacuole, in the centre of which is the centrosome, a fairly large granule staining deep violet.

The nucleus is oval in shape and stains pale reddish-violet. It takes the stain uniformly, except for two or three distinct dots in it. The nucleus is situated anteriorly to the centrosome, as in other trypanosomes, but in this species the nucleus and centrosome are nearly always very close together. The protoplasm stains a very deep blue, and sometimes a number of dark blue granules are visible in it. In addition to these, numerous unstained dots are seen, but only in parasites that have been damaged. We have figured the two chief varieties of $T$. rotatorium - the one with its surface covered with numerous ridges or ribs (Fig. $63, I$ and 4 ), the other flattened and with a smooth surface (Fig. 63, 2 and 5). Both forms have the same chromatic structure. It will be observed that in $I$ and 4 the centrosome is quite close to the nucleus, and, consequently, the undulating membrane is found only along the anterior part of the body. In 2 the centrosome is about midway between the nucleus and the posterior extremity, while in 5 it is very near the posterior end of the body.

The anterior end usually tapers off and ends in a relatively short flagellum. The posterior end varies much in shape. It may be

1 The undulating membrane never extends as far as the posterior end of the body, as Senn states in his differential diagnosis, doubtless on the authority of Gaule's inexact figures, which he reproduces. 
rounded off (5), or in the form of a blunt cone (2), or it may have a short point (4) or a very long point $(I)$. There is scarcely any difference between this last form and those which Dutton and Todd figure (see Fig. 64) in illustration of their provisionally new species, T. mega $a^{1}$ and $T$. karyozeukton. ${ }^{2}$

In $R$. esculenta in France, Laveran and Mesnil have seen parasites with the posterior extremity quite as elongated as those figured by Dutton and Todd, and occasionally this extremity has been
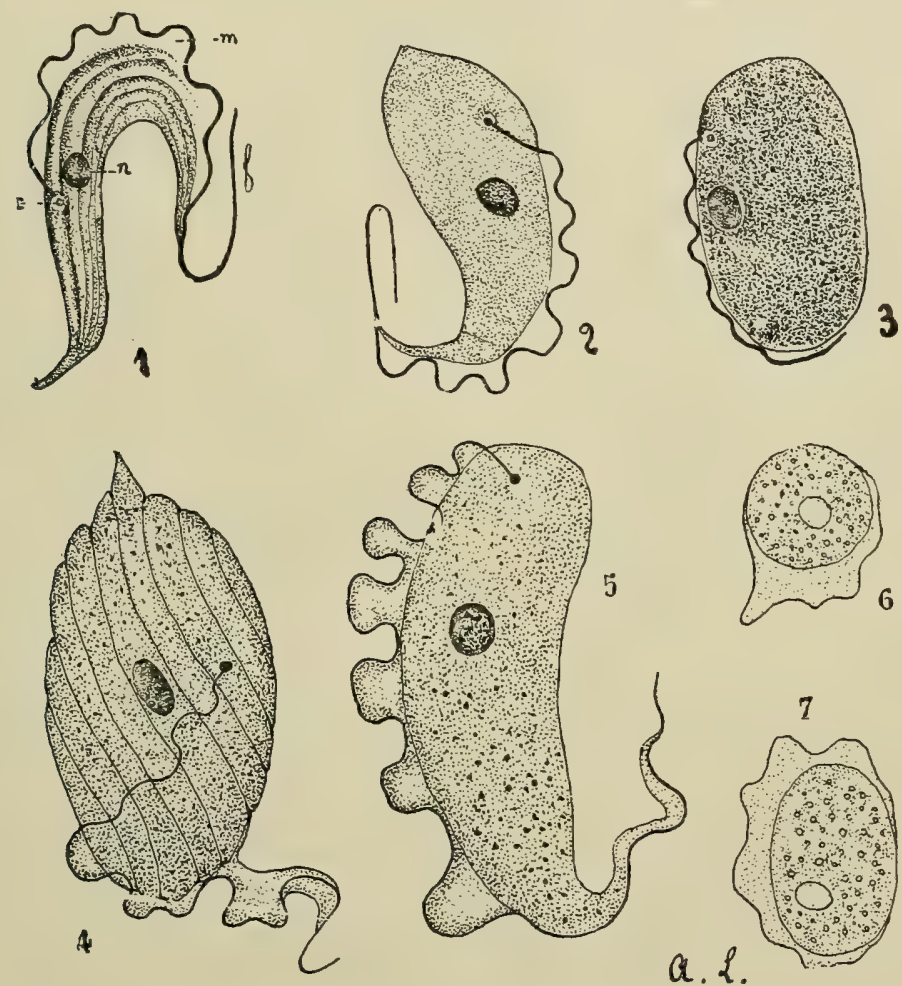

Fig. 63.-TRYPanosomes of Rana esculenta.

I and 4. Folded forms, thin (I), stumpy (4). 2 and 5. Flattened forms. 3. Contracted form. 6 and 7. Young forms examined fresh. (Magnified about I,400 diameters.) $n$. Nucleus, c. Centrosome. $m$. Undulating membrane. $f$. Flagellum.

1 Dutton and Todd found this form in a small frog (sp. ?) on McCarthy Island. The figure reproduced here (Fig. $64, I$ ) gives an idea of the well-developed undulating membrane with its numerous folds, of the longitudinal striation of the body, of the depth of staining of the protoplasm, and of the faintly-staining nucleus, all of them characteristic of $T$. rotatorium. The body proper is $72 \mu$ long, and the flagellum Io $\mu$ to $15 \mu$; the width of the body at the level of the nucleus is $8 \mu$.

${ }_{2}$ Dutton and Todd saw a single specimen of this variety in a frog (sp. ?) at Cape St. Mary, which harboured $T$. rotatorium in addition. Length of body: $67^{\circ} 2 \mu$; flagellum, I $5^{\circ} 2 \mu$; width at level of nucleus, $6.4 \mu$. The chief characteristic of this parasite appears to be the presence of a chain of chromatic granules between the nucleus and centrosome (see $k$ in figure). The authors, however, did not see this chain of granules in the trypanosomes of two other frogs in the same district, although the parasites had the same general appearance as the first form, but were smaller in size. 
so thin as to give the impression that it was prolonged into a short flagellum. Fig. 64, 2, shows this to be almost the case with T. karyozeukton. There is, therefore, considerable doubt as to the validity of these new species of Dutton and Todd, the more so as these observers recognise the existence of $T$. sanguinis $^{1}$ in frogs in Gambia.

To sum up, then, we may say that the trypanosomes of frogs vary very much in shape as well as in size. Some of them are relatively thin and short, others are very large and broad, and many intermediate forms occur. The length varies from $40 \mu$ to $60 \mu$, and only exceeds this figure in forms in which the two ends are attenuated and drawn out. The width, on the other hand, may vary from $5 \mu$ up to $40 \mu$. In some frogs there are found only ovoid forms with rounded ends, a very short free flagellum, the undulating membrane extending only half-way along the body, and which measure in films of fixed blood $50 \mu$ to $60 \mu$ in length, by $30 \mu$ to $40 \mu$ in width.
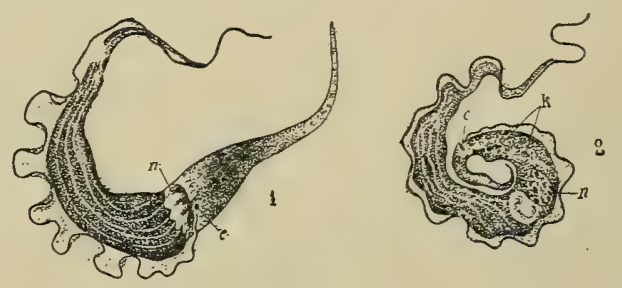

Fig. 64.-Trypanosomes of Frogs in Gambia.

I. T. mega (Dutton and Todd). 2. T. karyozeukton (Dutton and Todd). n. Nucleus. $c$. Centrosome. $k$. Chain of granules extending from nucleus to centrosome. (Magnified about $\mathbf{r}, 000$ diameters.)

Fig. 63,3 , represents a form which is not uncommon, and has often puzzled observers. Some, such as Mayer and Grassi, have looked upon it as a new species, and even as a new genus (Paramcecioides, Grassi). It is nothing more than an ordinary trypanosome which has become spherical, probably owing to the abnormal conditions met with outside the bloodvessels. Both varieties, the pectinated and the smooth, may exhibit this change of shape into spherical form, and we have on several occasions seen the change take place. The undulating membrane is retracted, and in the fresh condition the flagellum seems to have disappeared. The examination of stained specimens does not show any difference in structure between this form and the normal forms. The forms represented in Fig. 63, 6 and 7 , seen in a drop of fresh blood, appear to us to be very young forms of trypanosomes.

All the foregoing remarks apply to the parasites seen in $R$. esculenta. Observers are agreed in regarding as very closely allied, if not identical, the parasites of $R$. temporaric, of Bufo, and of Hyla. IVe

1 They found this parasite in fourteen out of thirty-five frogs examined ( $R$. trinodis (?) and other species). 
have had the opportunity of studying a number of trypanosomes in the blood of a Hyla arborea, bought at a shop in Paris. Fig. 65 represents a trypanosome of the Hyla. It is $75 \mu \mathrm{long}$, flagellum included, by $7 \mu$ in width, and in general appearance it closely resembles a young $T$. rotatorium of Rana esculenta.

We have sought in vain for reproduction forms of $T$. rotatorium in our frogs.

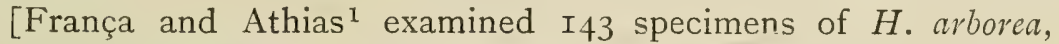
var. meridionalis, six of which were found to be infected with $T$. rotatorium. The trypanosomes were very numerous in the blood of these frogs, much more so than is usually the case in $R$. esculenta.]

[In stained films, in addition to the ordinary forms of trypanosome (resembling that seen in Fig. 65, except that the nucleus was fusiform, elongated, and fairly narrow), there were others which appeared to be forms undergoing division by segmentation. In blood-films fixed immediately after they were made only the initial phases of division were seen-the first division of the centrosome, and the conversion of the nucleus into a pale, ill-defined area bounded by a ring of small chromatic masses.]

[In blood fixed five hours after leaving the bloodvessels, and kept between slide and coverslip, more advanced stages of division were observed. Areas of cytoplasm became more or less (but

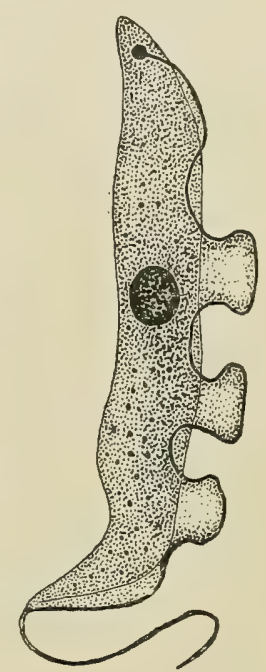

a.

Fig. 65. - TrypanoSOME OF Hyla arborea. never completely) divided off, and in them two nuclei and two centrosomes were seen. There were also appearances almost suggesting mitotic division, and França and Athias think that 'in the division of trypanosomes by segmentation the blepharoplast possibly plays a rôle like that of the centrosome during mitotic division.']

[Marchoux and Salimbeni ${ }^{2}$ have found a new trypanosome in a H. lateristriga (?) from Brazil. In the fresh condition the young forms are seen to differ considerably from the adult forms of the parasite. The young parasites are morphologically like $T$. rotatorium. The undulating membrane is clear and transparent, and is quite distinct from the granular cytoplasm of the body. In the adult forms the protoplasmic portion is very expansive and rolled on itself, so that the undulating membrane comes to lie inside a cigar-shaped body which is open at both ends. When pressure is applied to the film containing the trypanosomes the body becomes flattened out. There is no free flagellum extending beyond the limits of the body.]

1 [França and Athias, C. R. Soc. Biol., v. 6o, I906, p. I I08.]

2 [Marchoux and Salimbeni, C. R. Soc. Biol., v. 62, April I9, I907, pp. 592-594.] 
[When stained-and Billet's ${ }^{1}$ method gave the best results-the protoplasm and centrosome were found to take the stain readily; the nucleus and chromatic filament, on the other hand, stained badly. The nucleus has a curious shape; it is a long, narrow, arched body, with a rod-like prolongation at one end. The total length, including the terminal rod, which measures $5 \mu$ to $6 \mu$, is $25 \mu$ to $30 \mu$. The centrosome is situated at the anterior extremity of the nucleus. This arrangement of the nucleus and centrosome is similar to that previously described by França and Athias ${ }^{2}$ in a trypanosome from another species of Hyla.]

[The total length of this trypanosome is from $20 \mu$ to $80 \mu$. Marchoux and Salimbeni regard this trypanosome of $H$. lateristriga (?) as a new species, to which they have given the name $T$. borreli.]

[Cultivation of T. ROTATORIUM.-Lewis and Williams (op.cit.) were the first to achieve any success in this direction. They used nutrient agar, to the water of condensation of which two or three drops of frog's or toad's blood were added. Cultures made from two infected frogs showed, after two weeks, growths of flagellated organisms. These were of a very long oval form, the largest measuring $\mathrm{I} 8 \mu$ by $2 \mu$. There was one long flagellum attached to a centrosome situated at the anterior end of the body. The largest parasites showed a rudiment of an undulating membrane. Motility was not very marked. Numerous small forms were seen, evidently representing various developmental stages.]

[The growth was never luxuriant, and rosette formation was not observed. Only one generation of subcultures was successful, and all the cultures soon died. A single attempt to inoculate a normal frog gave a negative result.]

[Attempts to cultivate Drepanidium did not succeed, although the parasite remained alive for a long time in the medium. In one of these experiments trypanosomes were found in the tubes after ten days, and it appeared as though trypanosomes had developed from Drepanidium. This was not so, however; the trypanosomes had evidently been introduced with the blood of the frog which served for the preparation of the medium, for an uninoculated tube of the batch also showed trypanosomes. This

1 [Billet, C. R. Soc. Biol., v. 6r, 1906, pp. 753,754. Billet's method is a modification of Giemsa's method, which I have found to give very good results with trypanosomes, malarial and other chromatin-containing parasites, as well as with ordinary blood.]

[It is prepared as follows: Add 0.3 per cent. of sodium carbonate to a I per cent. solution of Grübler's medicinal methylene blue, and heat to $50^{\circ} \mathrm{C}$. for three hours. This is called the 'carbonated blue.' To stain films, put Io c.c. distilled water plus ro drops of commercial Giemsa's solution in a graduated cylinder or test-tube, add 2 or 3 drops of the 'carbonated blue'; shake and pour the mixture into a dish (see p. 9) containing the film, which is previously fixed for a few seconds in absolute alcohol, or in a mixture of alcohol and ether. Keep in the paraffin-chamber at $45^{\circ}$ to $50^{\circ} \mathrm{C}$, and at the end of ten to fifteen minutes the film will be well stained. Wash in a large quantity of water, and if too dark decolourize rapidly with a few drops of Unna's solution of tannin-orange.] fasc. 2 .] 
is comparable with Novy and McNeal's observations and culture experiments upon the avian trypanosomes.]

[Bouet ${ }^{1}$ has been more successful in his culture experiments than have Lewis and Williams. He obtained excellent results with Novy and McNeal's blood-agar medium (original formula, see p. 452), the best proportion being one of agar to two of blood. In this medium cultures grew in four or five days at the room temperature. At the time Bouet's paper was written (July, I906) the organism was in the tenth generation of subculture. In subcultures the trypanosomes appeared at the end of a very variable time-from three days up to twenty-five or even thirty-four days. The average time was from six to ten days on Novy and McNeal's type medium. The trypanosomes were most numerous from the eighteenth to the twenty-fifth days.]

[Both forms of the trypanosome, the stumpy and the thin forms, seen in the original frog's blood, gave positive results on cultivation. The majority of the frogs whose blood was cultivated harboured other parasites (filariæ and hæmogregarines), in addition to $T$. votatorium. A frog which had only trypanosomes in its blood gave positive results. On the other hand, frog's blood in which hæmogregarines were alone present gave negative results.]

[In young cultures the young trypanosomes were sometimes agglutinated into rosettes, but it was especially towards the end of the first month that auto-agglutination was observed in moist coverslip preparations of the culture. As has been observed by McNeal in cultures of $T$. lewisi, by Novy and McNeal in cultures of avian trypanosomes, and by Thiroux in cultures of $T$. duttoni, colonies may appear upon the agar medium above the water of condensation, especially in old cultures.]

[The vitality of cultures is very great. In one case living trypanosomes were found after five months in a culture-tube of the third generation.]

[Morphology of $T$. rotatorium in Cultures (see Fig. 66). - In the fresh condition the majority of the parasites are fusiform or pearshaped, like Herpetomonas or Crithidia. The protoplasm is finely granular, and the nucleus and centrosome are hardly discernible. It is difficult to make out an undulating membrane. There is a very long, actively motile flagellum. Movement takes place with the flagellar end foremost, and in agglutination (which is usually only temporary) the flagella are, as a rule, directed towards the centre of the rosette.]

[In addition to the above forms, which are by far the most numerous, all intermediate stages between them and the forms seen in the blood of frogs may be met with, especially in the earliest generations of cultures. In old cultures spherical forms are common,

1 [Bouet, Ann. Inst. Past., v. 20, 1906, pp. 564-577.] 
and these often contain very refractile, round, non-staining granules, consisting possibly of reserve material.]

[Multiplication is by longitudinal fission. In the colonies which grow on the agar itself the trypanosomes occur in 'morular' form. The flagellum is very short and hardly motile, and the protoplasm contains small, very refractile granules. These are possibly early stages of a resistant form of the parasite, as has been suggested when speaking of the cultivation of $T$. paddee (p. 449).]

[The trypanosomes seen in cultures are much smaller than those seen in the blood of frogs. The long forms are about $25 \mu$, flagellum included, by $2 \mu$; the spherical forms are $5 \mu$ in diameter.]

[Stained Preparations. - The best results were obtained by Gray and Tulloch's method (see p. I2), and then staining with Giemsa for
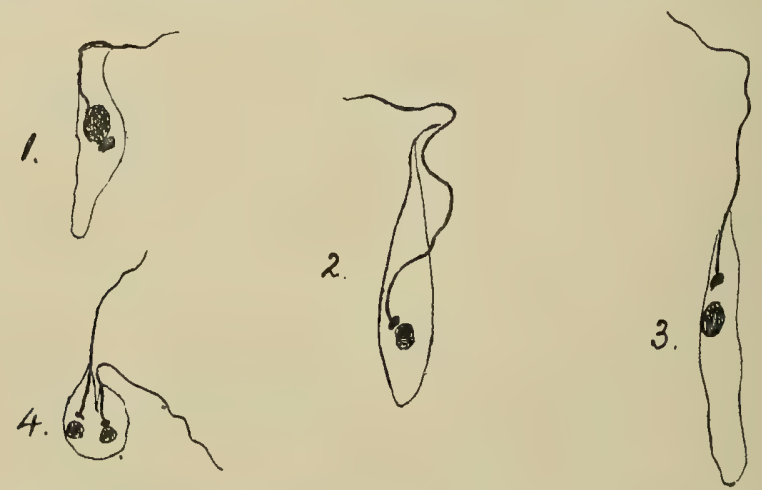

Fig. 66.-Culture forms of T. Rotatorium. (After Bouet.)

In I the centrosome is posterior to the nucleus. In 2 the centrosome is anterior to the nucleus, and a well-marked undulating membrane is present. 3 is a young form, in which the undulating membrane has not yet developed. 4, a very young spherical form undergoing division. (Magnified about $\mathrm{x}, 800$ diameters.)

three-quarters of an hour to one hour. The film is finally cleared with clove oil for about a minute.]

[The nucleus is usually situated near the anterior end of the body, especially in the Herpetomonas-forms; but it may be centrally or even posteriorly situated (Fig. 66, $I$ and 2). The centrosome is always near the nucleus, between it and the anterior end of the body; sometimes it actually touches the nucleus. The flagellum is attached to the centrosome. An undulating membrane is present in the adult culture forms (Fig. 66, 2), but not in the young forms (3).]

[Spherical forms may occur in very young or very old cultures. In the former they are to be regarded as very young trypanosomes, already possessing the power of division (Fig. 66, 4), in the latter case as involution forms, which will eventually give rise to the 'morular' stage and the stage of 'encystment.']

[As has already been stated, auto-agglutination may occur in cultures. Usually the flagella are directed towards the centre of the rosette, but sometimes they may be directed peripherally.] 
[The serum of an infected frog was found to possess agglutinating properties; that of a normal frog, or of another species of animal, had no agglutinating properties.]

[Frogs injected intraperitoneally with cultures did not become infected. Negative results were also obtained on inoculating specimens of Bufo and Pelobates.]

In conclusion, we may add that the type $T$. rotatorium is the largest of the trypanosomes hitherto known. There are several species, particularly amongst the parasites of fishes, which are as long as, and even longer than, this trypanosome, but in none of them does the width exceed $I_{5} \mu$. On account of its usually stumpy appearance, its very short flagellum-which in some cases appears even to be wanting-and its amobiform changes of shape, T. rotatorium stands alone in the genus Trypanosoma, and it is easy to understand why an attempt was made, when our cytological knowledge of the trypanosomes was still in its infancy, to create a new genus for the thin and slender trypanosomes of mammals. Now we are acquainted with a whole series of species intermediate between the very large and stumpy $T$. rotatorium and the trypanosomes of the type lewisi; and, moreover, in the species rotatorium itself all kinds of intermediate forms are to be found.

\section{Section 3.-Trypanosoma inopinatum, Sergent.}

We cannot do better than reproduce the authors' own figure and description of this parasite:

'When examining the blood of a green frog ( $R$. esculenta) caught at Dra-el-Mizan, Kabylie, we found a trypanosome which does not resemble any of the hitherto described trypanosomes of frogs and Batrachians generally.

'The parasite is about $25 \mu$ to $30 \mu$ long-flagellum included-by $3 \mu$ wide. It closely resembles $T$. lewisi of rats, but differs from it in being more stumpy and less drawn out, especially in the post-centrosomic portion, and its nucleus is situated near the middle of the body, whereas in T. lewisi it is in the anterior part of the body. The centrosome is well developed, as in $T$. leveisi, and often it appears as a transversely elongated body occupying the whole width of the parasite. The undulating membrane is usually unfolded, and appears even more rigid than that of $T$. lewisi. Although the blood preparation may show as many trypanosomes as red blood corpuscles, all the parasites seen are of about the same size, and, indeed, we have only once seen a dividing form of the parasite (Fig. 67, A) which showed two centrosomes and commencing division of the undulating membrane adjacent to them. The two centrosomes were situated close to the nucleus instead of occupying the normal position of the centrosome. In several other parasites we have also seen the centro- 
some close to the nucleus, which, in all probability, means that these parasites were about to divide.

'This trypanosome of green frogs is undoubtedly different from $T$. votatorium, Mayer ( $=T$. sanguinis, Gruby), which is the only trypanosome hitherto recorded in this species of frog. It resembles not only $T$. lewisi and the other mammalian trypanosomes, but also the trypanosomes of fishes, especially T. remaki of the pike, described in detail by Laveran and Mesnil. We shall call it Trypanosoma inopinatum.

' Other frogs in the same locality were not infected, and frogs from other parts of Algeria showed the ordinary frog trypanosome, T. rotatorium.'

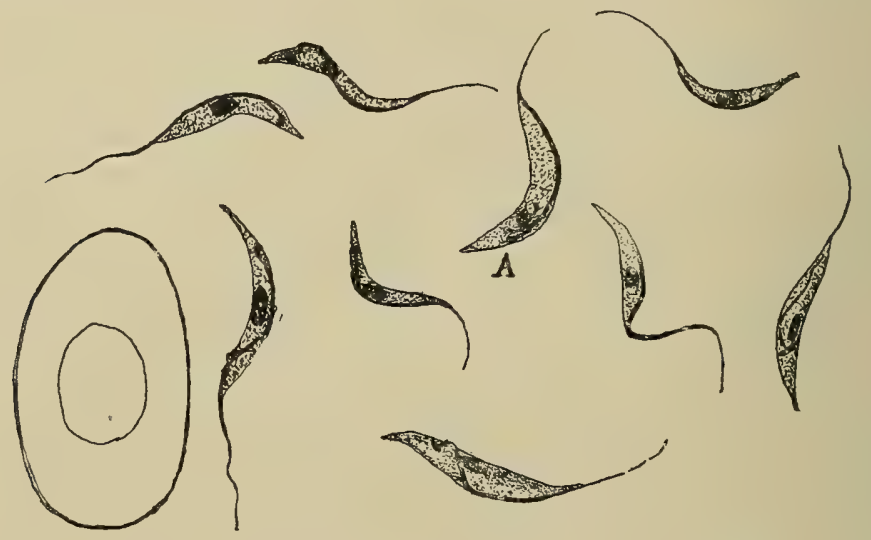

FIG. 67.-TRYPANOSOMA INOPINATUM OF R. ESCULENTA.

A. Commencing division. A red corpuscle of the frog is shown for the purpose of comparison. (Magnified about I,000 diameters.)

[During the past few years Billet and Brumpt have made several interesting observations upon T. inopinatum, particularly with regard to its development in leeches and its possible relation to Drepanidium.

[In his first paper $^{1}$ upon the subject Billet states that he saw the trypanosomes ( $T$. inopinatum) penetrate the red blood-corpuscles of the frog, at the same time losing the flagellum. In addition to these trypanosomes, the frog's blood contained (I) numerous free gregarinoid forms (Billet says that the analogy between some of these forms and the trypanosome-forms minus flagellum is striking); (2) endoglobular, elongated forms, undergoing longitudinal division; and (3) rounded forms, giving rise to all stages of schizogony, characteristic of the Hamogregarina splendens (a Lankestevella).]

[In his recent paper ${ }^{2}$ Billet brings forward evidence to show that the alternate or invertebrate host of $T$. inopinatum is a leech, Helobdella algiva.

1 [Billet, C. R. Soc. Biol., v. 57, ISO4, pp. 16I-I64; abstract by Mesnil in Bull. Inst. Past., v. 2, 1904, p. 724.]

2 [Billett, C. S. Acad. Sciences, v. I 39, I904. pp. 574-576; abstract in Bull. Inst. Past., v. 2, 1904, p. 989. See also Woodcock's article in Oucurt. Journ. Micr. Sc., v. 50,1906, p. 251 .] 
Trypanosomes were frequently found in the intestine of the leeches which had been ectoparasitic on frogs, harbouring either hæmogregarines plus trypanosomes or hæmogregarines alone. The T. inopinatum found in the leeches showed a variety of forms which were never met with in the frogs' blood. The leech, therefore, appears to be the normal host of this trypanosome.]

[Twenty-four or more hours after the infection of the leeches, their intestine contains forms, with a distinct nucleus and centrosome, which seem to be stages intermediate between a hæmogregarine and a trypanosome. After the third day only $T$. inopinatum are present. Lastly, frogs apparently free from all hæmatozoa, infected by the bites of leeches containing only trypanosomes, contracted an infection exclusively hæmogregarine. From these observations Billet concludes that $T$. inopinatum is ontogenetically related to the hæmogregarine parasite in $R$. esculenta in Algeria.]

[Brumpt ${ }^{1}$ found in the digestive tract of leeches (Placobdella catenigeva), which had sucked the blood of an African tortoise, Emys leprosa (probably containing the Hamogregarina bagensis), forms (? ookinetes) with a nucleus and a smaller chromatic body, resembling the centrosome of a trypanosome.]

[In a later paper ${ }^{2}$ Brumpt states that he was able to infect frogs with $T$. inopinatum by allowing infected leeches to bite them. In one frog the trypanosomes appeared in the blood on the tenth day after the bite of the leech. They multiplied rapidly, and on the thirtieth day there were fifty trypanosomes to one red corpuscle. The frog became very anæmic, and died on the thirty-fifth day. Similar results were obtained with other frogs, thus showing that $T$. inopinatum may be pathogenic.]

[Brumpt showed, moreover, that $T$. inopinatum is inoculable. Frogs inoculated in the dorsal lymph sac with the heart blood of frog (I) mentioned in the preceding paragraph became infected in forty-eight hours, and died in twelve to sixteen days. Post-mortem there were found œdemas, hydropericardium, ascites, and hæmorrhages. Heart puncture furnished a 'purulent, chylous fluid,' containing many agglutinated trypanosomes.]

[The trypanosomes in the leech rapidly gave rise to Herpetomonasforms varying in length from $6 \mu$ to I $4 \mu$ for the body and $3 \mu$ to I $4 \mu$ for the flagellum.]

[Brumpt is of opinion that there is no relation between the trypanosomes and the hæmogregarines of the frog. Leeches fed on frogs containing many hæmogregarines did not develop flagellates in a month. We have already seen that Lewis and Williams, Novy and McNeal, and others are of the same opinion.]

\section{Section 4.-0ther Trypanosomes of Batrachians.}

TRYPANOSOMA NELSPRUITENSE, Laveran, IgO4. ${ }^{3}$-This trypanosome from Nelspruit, Transvaal, was discovered by Theiler in two frogs. There appear to be at least two species of frogs in the Transvaal: $R$. angolensis, Bocage, and a new species to which Mocquart has given the name $R$. theileri.]

[T. nelspruitense varies in size. Its length may be as much as $70 \mu$, of which the free flagellum may occupy $35 \mu$; the width is $3 \mu$.

1 [Brumpt, C. R. Soc. Biol., v. 57, 1904, pp. 165-167; abstract by Mesnil (reproduced above) in Bull. Inst. Past., v. 2, 1904, p. 724.]

2 [Brumpt, C. R. Soc. Biol., v. 6r, 1906, pp. 167-169.]

3 [Laveran, C. R. Soc. Biol., v. 57, 1904, p. 158.] 
The body is slender and vermiform in appearance, much like T. granulosum of the eel. The anterior two-thirds of the body stains much more intensely than the posterior third, and anteriorly there are many deeply staining granules. The nucleus is round or oval, and stains less deeply than the centrosome. The latter is situated at a variable distance from the posterior end. The undulating membrane shows several folds.]

[Trypanosome of Frogs in Hong-Kong (Fig. 68). - This trypanosome was found by Nabarro and Stevenson in blood-films of a frog sent to them by Dr. J. S. Bell, of Hong-Kong. The trypanosomes were extremely scanty, only two being found in several large blood-films. The frog was more obviously infected with a large hæmogregarine, ${ }^{1}$ and it is conceivable that if this frog's blood had been cultivated and the trypanosomes had developed, it might
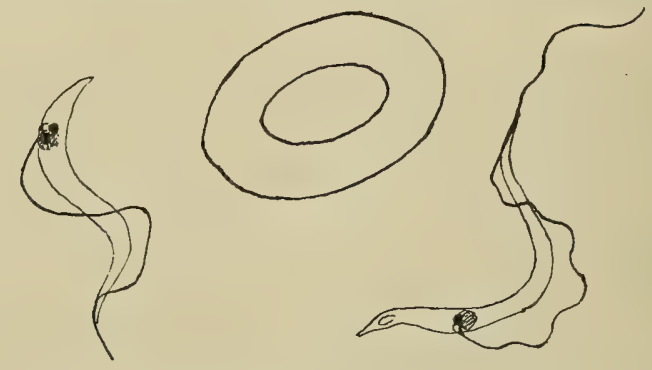

Fig. 68.-Trypanosome of Frogs in Hong-Kong.

Note the great width of the undulating membrane, and the unusual position of the nucleus, to which the centrosome is closely applied. A red corpuscle is also represented to show the relative sizes. (Magnified about 1,500 diameters.)

have been thought that the hæmogregarines had given rise to the trypanosomes.]

[Morphology.-The larger of the two trypanosomes seen was 4I $\mu$ long, including the free flagellum, which measured $16 \mu$. The body itself was long and slender, its greatest width being only about $\mathrm{I}^{\circ} 4 \mu$. The nucleus was small and spherical, and about $I^{\prime} 3 \mu$ in diameter. Its posterior edge was partly overlaid by the centrosome, which was about $7{ }^{\circ} 5 \mu$ from the posterior end of the body. There was a large vacuole I $\mu$ from the posterior extremity, and another smaller one close to the centrosome. Stained by Giemsa's method the cytoplasm (blue) was seen to be fairly homogeneous, no striations or chromatic granules being visible in it. The nucleus was stained a reddish-violet and the centrosome a deep violet. The undulating membrane was distinctly visible (pale reddish-violet); it was very wide $\left(2{ }^{\circ} \mu\right.$ in the widest part); the edge was wavy, but the membrane itself showed few folds.]

1 [Professor Laveran, to whom I sent the films, informs me that this hæmogregarine resembles that which he had observed in a frog from the Transvaal.] 
[The other trypanosome was $25^{\circ} 6 \mu$ long, including the flagellum, $3 \mu$. The total width of the parasite was $3.8 \mu$, that of the body itself being $I^{\bullet} 9 \mu_{0}$. The nucleus was spherical, $I^{*} 3 \mu$ in diameter, and more irregularly stained than in the first trypanosome. The centrosome was $4^{\circ} 6 \mu$ from the posterior end of the body, and in close contact with the nucleus. There was a small vacuole just behind the nucleus and centrosome. The posterior half of the body was much more deeply stained than the anterior.]

[This trypanosome appears to be different from the hitherto described species of frog trypanosomes. I think it is a new species, for which I propose the name Trypanosoma belli.]

[Trypanosome of THE Toad (Trypanosoma somalense, Brumpt, ${ }^{1}$ I906).-This trypanosome was found by Brumpt in the blood of a Somaliland toad, Bufo reticulatus. The body is arched as it is in some forms of $T$. rotatorium. T. somalense differs from the latter in its small size, the body measuring $30 \mu$ and the free flagellum $7 \mu$. The centrosome is $4 \mu$ from the posterior end. The nucleus is situated in the widest part of the body, a little anterior to the centrosome.]

[Trypanosome of the Newt.-This trypanosome was found by Tobey ${ }^{2}$ in American newts (Diemyctulus viridescens). The infected newts appeared to differ from healthy newts in the following respects: they were darker in colour, the spots on the side were a brighter red, and the shoulder and pelvic girdles were very prominent owing to the wasting of the muscles. They seemed as active as healthy newts, but some of them died soon after they came under observation.]

[In the fresh condition the trypanosome was very active, and moved with a spiral motion. The whole body was contractile.]

[In stained specimens three different forms were seen: 'wide, long, and short.' The first two seemed to be but different phases of a contractile state. The average length was $45 \mu$ to $50 \mu$, the width $2 \mu$ to $5 \mu$, the free flagellum $24 \mu$. The nucleus was situated slightly behind the middle of the body. The centrosome, which was small and often difficult to see, was situated in the centre of an unstained vacuole a short distance from the posterior extremity of the body. The undulating membrane was well developed and showed several folds.]

[Inoculations into healthy newts were unsuccessful, as were inoculations into mud puppies (Necturus maculatus). Several attempts were made to infect frogs, but in only one case was there an apparent infection.]

1 [Brumpt, C. R. Soc. Biol., v. 6o, 1906, p. 164.]. 148.]

2 [E. N. Tobey, Journ. Med. Research, new series, v. Io, No. I, 1906, pp. I47, 


\section{Section 5.-Modes of Infection.}

We are quite ignorant of the mode of infection of frogs and the other Batrachians. It is quite probable that it occurs through the agency of some ectoparasite, such as the leech or tick. The same remarks apply to the hæmogregarines of the Batrachians, and it is to be hoped, now that attention has been drawn to these questions, that some definite information will soon be forthcoming.

[We have seen, however, that the recent investigations of Billet and Brumpt (see Section 3) have furnished a reply to these questions. The $T$. inopinatum of frogs can undoubtedly multiply in the intestine of the leech, and infected leeches are able to transmit the infection to healthy frogs by biting them.]

We have tried to infect green frogs experimentally with the blood of other frogs containing trypanosomes. The infective blood was injected into the dorsal lymph sac or into the peritoneum. We experimented upon seven or eight frogs, with only one successful result. $^{1}$ One frog inoculated on June 30 , 1902, showed a few parasites in its blood from the 4 th to the 8 th of July.

Why were these experiments unsuccessful? Perhaps on account of the mode of inoculation, but it is nearly always successful with the trypanosomes of fishes and rats. Possibly it was because the frogs experimented upon had acquired immunity as the result of a previous infection, for, as a matter of fact, they were got from districts where about half the frogs were infected.

1 [It has already been mentioned (see p. 475) that Brumpt succeeded in transmitting $T$. inopinatum to several green frogs by inoculation into the dorsal lymph sac.] 


\section{CHAPTER XVII}

\section{TRYPANOSOMES OF FISHES}

\section{Section 1.-Historical. Species known to be Infected.}

IN I84I Valentin saw in the blood of a trout (Salmo fario) a parasite which he classed with the Amœbæ of Ehrenberg, ${ }^{1}$ but which, from the short description and figures he gives of it, should be grouped with the hæmatozoa to which Gruby in I843 gave the name trypanosomes.

Remak observed in the blood of the pike (Esox lucius) and of several other fresh-water fishes very motile hæmatozoa, possessing a transparent membranous part and tooth-like projections, which disappeared when the parasites were at rest. ${ }^{2}$ In all probability these were trypanosomes, for in fresh preparations the undulating membrane gives one the impression of tooth-like projections, as described by Remak.

Gros $^{3}$ recorded the existence of vermicules in the blood of a number of fishes-gudgeon, rockling, perch, sterlet, lote, tench, etc. The parasite of the rockling was $45 \mu$ long, by I $\mu$ wide, and was very active. It was pleomorphic, but most frequently presented the appearance of a ribbon folded and twisted into various shapes. From this description one cannot fail to recognise these parasites as trypanosomes.

Berg and Creplin have described the trypanosome of the pike, which was seen by Berg in four cases out of five. He states that the length of the parasites is from one and a half to three times the large diameter of the red corpuscles. ${ }^{4}$

The hæmatozoa found by Wedl in the gudgeon and in a tench appear to belong rather to the hæmogregarines than to the trypanosomes. ${ }^{5}$

Chaussat found in the blood of the barbel a hæmatozoon resembling the trypanosome of the frog. ${ }^{6}$

1 Valentin, 'Archiv. de J. Müller,' I84I, p. 435.

2 Remak, Canstatt's Jahresbericht, I842, p. Io.

3 Gros, Bull. de la Soc. imp. des Natur. de Moscow, I845, v. 18, part i., p. 423.

4 Berg, 'Hämatozoën des Hechtes,' Archiv skand. Beiträge zur Natur. geschichte, I845, v. I, p. 308.-Creplin, Observations upon Berg's comniunication.

5 Wedl, Denk. der Wiener Akad. der Wissen., I850, part ii., p. I 5.

${ }^{6}$ Chaussat, 'Thèse,' Paris, 1850. 
In 1883 Mitrophanov $^{1}$ gave a good description of two species of fish trypanosomes under the names of Hamatomonas cobitis and H. carassii. From his descriptions and figures it is obvious that these parasites belong to the genus Trypanosoma.

$T$. cobitis was found in the blood of a loach (Cobitis fossilis). Its length is given as $30 \mu$ to $40 \mu$, and its breadth as I $\mu$ to I.5 $\mu_{*}{ }^{2}$ The body is elongated and worm-like, and has a spirally-arranged undulating membrane. The two ends are pointed, and the one which is foremost when the parasite moves ends in a flagellum.

$T$. carassii was found in the blood of the Prussian carp (Carassius vulgaris). It is much larger and more flattened than the preceding, which, however, it otherwise closely resembles. These trypanosomes are very nearly related to the trypanosome of the pike, which is described later on.

Danilewsky found trypanosomes in Cyprimus carpio, Tinca tinca, Cobitis fossilis, and C. barbatula, Esox lucius, Perca fluviatilis, and Carassius vulgaris. ${ }^{3}$ According to Danilewsky, two kinds of trypanosome are to be distinguished in fishes-a thin, ribbon-like form and a spindle-shaped form, each of them with an undulating membrane and a flagellum. They multiply by unequal binary division.

Chalachnikov ${ }^{4}$ found trypanosomes in the blood of a large number of fishes caught in the rivers and streams in the district of Cherson, Russia, notably in the Cyprinus carpio, Esox lucius, Carassius vulgaris, and Acerina vulgaris. He recognises two kinds of trypanosome in fishes: (I) A flat form very closely resembling the flat trypanosome of the frog. A variety of this trypanosome is said to have two flagella-a long anterior and a short, thin posterior one. (2) A fusiform trypanosome with undulating membrane arranged spirally. There would appear to be three varieties of this form, but they are not sharply marked off from one another.

The young trypanosomes of fishes may, according to Chalachnikov, multiply by longitudinal division. He states that he also saw in the blood of Cyprinus carpio and Esox lucius, kept for some days in vitro, protoplasmic masses undergoing division, as well as young trypanosomes.

Kruse $^{5}$ says that he has often seen herpetomonads (trypanosomes) in the blood of fishes from the Mediterranean Sea.

According to Lingard, ${ }^{6}$ the fresh-water fish of India often harbour trypanosomes which sometimes are very numerous. In form these trypanosomes appear to be allied to the species de. scribed by Mitrophanov. Fishes which live in the mud are more

1 Mitrophanov, Biol. Centralbl., March 15, 1883, vol. 3, p. 35.

2 [These are the dimensions given by Mitrophanov. In the original of Laveran and Mesnil's book the width is incorrectly given as $\frac{1}{2} \mu_{\text {. }}$ ]

${ }^{3}$ Danilewsky, Biol. Centralbl., November I, 1885 ; and 'Rech. sur les parasites du sang des Oiseaux,' Charkov, i889.

4 Chalachnikov, 'Recherches sur les parasites du sang,' Charkov, 1888.

5 Kruse, in 'Flügge,' v. 2, 1896, p. 627.

${ }^{6}$ Lingard, 'Report on Surra,' etc., v. 2, part i., I899, p. 155. 
often infected than other kinds. Lingard found trypanosomes in the following species: Trichogaster fasciatus, Ophiocephalus striatus, Macrones seenghala, and $M$. tengara (Siluridæ). It is during the months of May and June that trypanosomes are seen in largest numbers in the blood of these fishes.

[Lingard ${ }^{1}$ has since made a further study of the fish trypanosomes in India. He has found trypanosomes in the following species from the river at Poona: Barbus carnaticus, Ophiocephalus striatus, and Rhynchobdella aculeata. The trypanosomes were obtained by gently scraping the gills without drawing blood. In fish from the River Jumna, Lingard found two species of trypanosome-a large form in Ophiocephalus striatus and a small one in Trichogaster faciatus and Macrones seenghala or M. tengara.]

Sabrazès and Muratet have described the trypanosome of the eel. ${ }^{2}$

We were the first to describe trypanosomes in salt-water fishsole, ray, and dogfish-and we also discovered a trypanosome peculiar to the red-eye, for which we have created a new genus, Trypanoplasma. ${ }^{3}$ We give below a list of the fresh-water and saltwater fishes that we have examined for trypanosomes. The number or word in brackets after the specific name of each fish refers to the number of specimens examined.

\section{Fresh-WATER Fishes.}

A. Fishes in which Trypanosomes were found. - Pike, Esox lucius (6) ; carp, Cyprinus carpio (9); tench, Tinca tinca (I0); red-eye, Scardinius erythrophthalmus (many); bream, Abramis brama (4) ; eel, Anguilla vulgaris (many).

B. Fishes in which Trypanosomes Were not Found. Gudgeon, Gobio gobio (many); loach, Cobitis barbatula (many); stickleback, Gasterosteus aculeatus (many); roach, Leuciscus rutilus (3); chub, Squalius (Leuciscus) cephalus (several); trout, Trutta fario (4).

\section{Salt-Water Fishes. ${ }^{4}$}

A. Fishes in which Trypanosomes were found.-Cartilaginous fishes: Ray, Raja punctata (II), Raja mosaica (3), Raja clavata (I), Raja macrorynchus (I); small dogfish, Scyllium canicula (38); large dogfish, Scyllium stellare (3). Bony fishes: Sole, Solea vulgaris $(2 \mathrm{I})$.

B. Fishes in which Trypanosomes Were not found. Cartilaginous fishes: Mustelus canis (5); Galeus galeus (2); Acanthias

1 [Lingard, Ind. Med. Gaz., December, 1904, pp. 445-447; abstract by Mesnil in Bull. Inst. Past., v. 3, p. 414.]

2 Sabrazès and Muratet, 'Trypanosome of the Eel' (résumé of communications made to the Société linnéenne de Bordeaux, in Decernber, 1901, March, 1902, and July 2, I902).

${ }_{3}$ Laveran and Mesnil, C. R. Acad. Sciences, October 28, I9or, and October 13, 1902; also Arch. f. Protistenkunde, I 902, v. I.

${ }_{4}^{4}$ These fishes were obtained from the waters of Roscoff, or from those of L'anse St. Martin (St. Martin's Bay), near Cap de la Hague (Manche). 
acanthias (II); Squatina angelus (4-2 large and 2 small); Torpedo torpedo (2); Raja alba (3); Raja microcellata (2); Raja mirelatus (I). Bony fishes: Syngnathus sp. (many); Nerophis lumbricoides (several); Orthagoriscus mola (2); Blennius pholis (many); Blennius montagui (many); Callionymus dracunculus (2); Gunnelus vulgaris (5); Lophius piscatorius (5); Gobius sp. (many); Mullus surmuletus (4); Trigla (4); Scomber scomber (I); Trachurus trachurus (2); Cottus scorpius (several); Cottus bubalis (I8); Zeus faber (2); Pagellus centrodontus (2); Pagellus erythrinus (7); Cantharus griseus (I); Chrysophrys aurata (I); Labrus sp. (many); Crenilabrus melops (6); Spinachia vulgaris (4); Ammodytes tobianus (7); Ammodytes lanceolatus (7); Gadus pollachius (Io); Gadus luscus (4); Lota molva (2); Motella tricirrata (8); Motella mustela (4); Rhombus maximus (2); Platessa vulgaris (II); Platessa microcephala (5); other Pleuronectes, sp. var. (several); Lepadogaster gouanii (many); Conger conger (IO).

From these lists it is seen that trypanosomes are rare in the bony salt-water fishes, and that the cartilaginous fishes are more often infected.

[The investigations of the past few years have considerably extended the list of fishes in which trypanosomes are known to occur. They have also made known some interesting facts concerning the evolution of the fish trypanosomes in the body of particular species of leech.]

[Several new species of Trypanoplasma have been described in the blood of fishes, and, in addition, Léger ${ }^{1}$ has described one, which he calls Trypanoplasma intestinatis, in the osophagus and anterior part of the stomach of a salt-water fish, Box boöps.]

[Of the recent observations upon the trypanosomes of fishes the following may be mentioned: Petrie ${ }^{2}$ found a number of goldfish (Cavassius auratus) infected at Elstree, Hertfordshire. This trypanosome closely resembles $T$. danileweskyi of the carp, with which Petrie thinks it is identical.]

[Robertson ${ }^{3}$ has found trypanosomes in Pleuvonectes flesus, P. platessa, and Raja micvocellata caught at Millport, Scotland.]

[Montel ${ }^{4}$ found a trypanosome resernbling that of the eel in the blood of a fish of the genus Clavias (Silurus clarias) in Cochin China. He has given it the provisional name $T$. clavia. $]$

[Neave ${ }^{5}$ has found trypanosomes in several species of Nile fish, the noke (Mugil), the dabib (Polypterns), the bagara (Bageus bayard), and the gargur (Lynodontis schal).]

[Castellani and Willey ${ }^{6}$ describe trypanosomes in several fish caught in a lake in Colombo, Ceylon. To one of them, found in a member of the

1 [Léger, C. R. Soc. Biol., v. 58, 1905, pp. 5 I I-513.]

2 [Petrie, Journ. of Hyg., v. 5, April, 1905, p. 197.]

3 [Robertson, Proc. Roy. Phys. Soc. Edin., v. I6, 1906, pp. 232-247.]

4 [Montel, C. R. Soc. Biol., v. 58, June 17, 1905, p. 1014.]

5 [Neave, Second Report of the Wellcome Research Laboratory, Khartoum, 1906, p. 197.$]$

${ }_{6}^{6}$ [Castellani and Willey, Quart. Journ. Micro. Sc., v. 49, 1905, pp. 383-402.] 
Siluridæ, Saccobranchus fossilis, they have given the name $T$. saccobranchi. Trypanosomes were also found in Macrones cavasius and Gobius ginvis.]

[The trypanosome of the eel, first described by Sabrazès and Muratet in IgOI, has been found by $\mathrm{Manca}^{1}$ in eels (eight out of seventeen examined) from different parts of Oristano, Sardinia.]

[Dr. Bell has informed me that in Hong-Kong he found a large trypanosome (? T. granulosum) in eels.]

[Brumpt and Lebailly ${ }^{2}$ have briefly described a number of new fish trypanosomes, many of which were associated in the same host (marine Teleostians) with new hæmogregarines. The following species of fish were found to be infected: Pleuronectes platessa (Platessa vulgavis), plaice; Pl. flesus (Flesus vulgaris), flounder; Limanda platessoides; Platoplivys laterna; Bothus vhombus (Rhombus lavis), brill ; Blennius pholis ; Gobius nigev; Callionymus dracunculus; and Cottus bubalis.]

[In addition to the above, Brumpt ${ }^{3}$ has described several new species of trypanosome and of trypanoplasm in fresh-water fishes, as well as their mode of evolution in particular species of leech. The new trypanosomes were found in Bavbus fluviatilis, barbel; Perca fuviatilis, perch; Acevina cermua; Cottus gobio, river bull-head; Scavdinius evythrophthalmus, rudd or red-eye; various species of Leuciscus, roaches; Gobio fuviatilis, gudgeon; and Squalius (Leuciscus) cephalus, chub. A trypanosome found in the minnow (Phoxinus lavis) is regarded by Brumpt as a new species, but Laveran ${ }^{4}$ thinks it has all the characteristics of $T$. danilewskyi of the carp.]

[The new trypanoplasms described and named by Brumpt were found in Cottus gobio; Barbus fuviatilis ; Abramis brama, bream; and Salmo fario, trout.]

[Léger, ${ }^{5}$ in 1904 , described a new trypanosome as well as a new trypanoplasm in Cobitis barbatula, loach.]

[Lastly, Keysselitz ${ }^{6}$ states that he has found both trypanosomes and trypanoplasms in the following fishes obtained from various German waters: Perca fluviatilis, Acevina cermua, Lota vulgavis, Bavbus fluviatilis, Cyprimus carpio, Cavassius vulgavis, Tinca vulgavis, Abramis brama, Lenciscus idus, L. cephalus, L. evythrophthalmus, L. rutilus, Esox lucius, and Cobitiș barbatula. Trypanosomes (Trypanosoma) were found alone in Anguilla vulgavis and Silurus glanis.]

[The trypanosomes of fishes belong, therefore, to two genera - Trypanosoma and Trypanoplasma. The latter genus comprises several species, in addition to that originally described by Laveran and Mesnil in the red-eye and minnow ( $T p l$. borreli), and that described shortly after by Miss Plehn ${ }^{7}$ in the carp ( $T p l$. cyprini). Trypanosomes of both genera are now known to occur in several species of fish-carp, loach, river bull-head, red-eye, barbel, bream, minnow, and others.]

1 [Manca, C. R. Soc. Biol., v. 6o, I906, p. 494.]

2 [Brumpt and Lebailly, C. R. Acad. Sciences, v. 139, I904, p. 613 ; Lebailly, ibid., p. 576.]

3 [Brumpt, Rev. Scientif., September, 1905, pp. 32I-332; abstract by Mesnil, Bull. Inst. Past., v. 3, p. 920 . Also C. R. Soc. Biol., v. 57, 1904, pp. 161-164; and v. 60, I906, pp. I60.162, and 162-164.]

4 [Laveran, C. R. Soc. Biol., v. 57 , 1904, pp. 250, 251.]

5 [Léger, C. R. Soc. Biol., v. 57, Iyo4, pp. 344, 345.]

6 [Keysselitz, Arch.f. Protisten., v. 7, 1906, pp. I-74.]

7 [Plehn, Arch.f. Protisten., I903, v. 3, p. I7.5.] 


\section{Section 2.-Technique. Preservation of the Trypanosomes of Fishes.}

Examination of Living Parasites.-Several drops of blood can be easily obtained from a fish by incising two or three rays at the base of the caudal fin. An ordinary fresh preparation is made and at once examined for trypanosomes. If it be desired to preserve the blood in hanging-drop preparation or for subsequent inoculations, the addition of a little citrated salt solution will prevent coagulation, but leave the motility of the trypanosomes unimpaired.

The trypanosomes of fishes can live for several days in vitro. Berg (loc. cit.) kept the trypanosomes of the pike alive for six days at $12^{\circ} \mathrm{C}$. in an ordinary blood preparation. Mitrophanov succeeded in keeping them alive for three or four days in blood mixed with salt solution. He states that a fairly low temperature is favourable to their preservation (loc. cit., p. 39), which agrees with our own observations upon $T$. lewisi.

Chalachnikov (loc. cit.) states that he saw in the blood of Cyprinus carpio and Esox lucius, kept for several days in vitro, protoplasmic masses undergoing division, which he looked upon as multiplication forms of the trypanosomes. Possibly the trypanosomes of fishes may become agglutinated in vitro, as other trypanosomes do, which would explain certain of the forms described by this observer.

We have kept the trypanosomes of the pike alive for several days in pure blood and in blood mixed with salt solution, but we have not observed the division forms described by Chalachnikov, or any agglutination of the parasites. In order to see agglutination easily, it is obviously necessary that the trypanosomes should be fairly numerous in the blood, which we have never found to be the case with the parasites of fishes. Possibly the fishes examined by Chalachnikov were more severely infected than our own, and consequently agglutination was able to occur.

Sabrazès and Muratet ${ }^{1}$ kept the trypanosomes of the eel alive in vitro for eight days at a temperature of $10^{\circ}$ to $19^{\circ} \mathrm{C}$.

[Lebailly ${ }^{2}$ succeeded in keeping trypanosomes of the eel alive for nine days in ordinary slide and coverslip preparations, ringed with vaseline and kept at $24^{\circ} \mathrm{C}$. For the first four to five days there was a definite multiplication of the parasites.]

The method of staining fixed specimens of blood is the same as that previously given, but in order to obtain good results certain special precautions are necessary. The fishes must be opened while still living and blood taken from the heart with a pipette. The blood is then spread in a thin layer on slides, rapidly dried over the flame of a spirit-lamp, and fixed in absolute alcohol or in alcohol and ether. The atmosphere is saturated with water-vapour at the seaside, and consequently blood-films dry so slowly in the ordinary way

1 Sabrazès and Muratet, Soc. de Biol., January 16 and 30, 1904.

2 [Lebailly, Thèse Fac. Med., Paris, 1906; also Arch. parasitologie, v. 10, 1906.] 
that the corpuscles and parasites have time to become altered in appearance. In the blood of dead fishes the blood-corpuscles and trypanosomes undergo rapid changes.

\section{Section 3.-Description of the Trypanosomes of Fishes belonging to the Genus Trypanosoma.}

We shall consider first the trypanosomes of fresh-water fishes, and later those of salt-water fishes.

TRYPANOSOMA REMAKI, Laveran and Mesnil, Igor.-We have given the name $T$. remaki to the trypanosome of the pike, after Remak who first saw the parasite. ${ }^{1}$

This trypanosome appears to have a wide geographical distribution-Remak, Berg, Danilewsky, Chalachnikov, and we ourselves have seen it in the pike in various parts of Europe. The infection
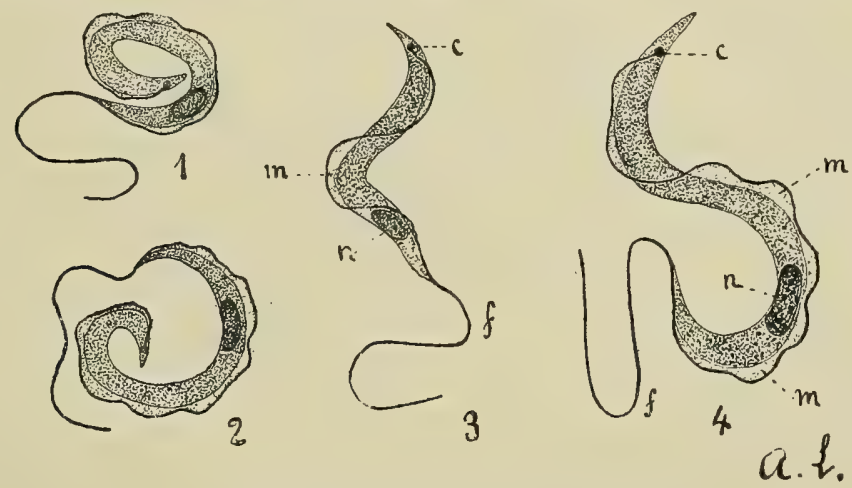

Fig. 69.-Trypanosomes of the Pike.

51, 2, 3. T. vemaki var. pavva. 4. T. vemaki var. magna. n. Nucleus. c. Centrosome. $m$. Undulating membrane. $f$. Flagellum. (Magnified about 2,00o diameters.)

is also a fairly frequent one; thus, in Paris, as in Lorraine, we found parasites in three out of four pike weighing 500 grammes or more. The trypanosomes are never very numerous, and they are sometimes so scanty that a prolonged examination is necessary in order to find a single parasite.

In fresh blood $T$. remaki looks like a minute worm endowed with very active movements, with an undulating membrane along one side. It wriggles and twists about more than $T$. lewisi, and often becomes rolled on itself. Its structure can only be satisfactorily made out in stained specimens.

Most infected pike show parasites differing much in length, so that we have described two varieties of $T$. remaki (var. parva and magna).

T. remaki var. parva measures on an average $28 \mu$ to $30 \mu$ in length, including the flagellum, the body itself being $\mathrm{I}_{5} \mu$ to $20 \mu$

1 Laveran and Mesnil, Acad. des Sciences, October 29, I90I. 
long. Some specimens we have measured were as much as $42 \mu$ in length (body $25 \mu$, flagellum I7 $\mu$ ), whilst others were only $20 \mu$ long (body and flagellum each Io $\mu$ ). This variation in length appears to be independent of the process of division, for we have seen it when there were no dividing forms. The width of the parasites is about I. $4 \mu$.

Fig. 69, $I, 2$, and 3 , gives a good idea of this variety of $T$. remaki. The protoplasm of the body stains pale blue, is fairly homogeneous, and no definite granules can be seen in it. The nucleus $(n)$ and the centrosome $(c)$ are stained deep violet. The nucleus, usually oval in shape, is situated at the junction of the anterior and middle thirds of the body. It is composed of fine chromatic granules closely packed together and surrounding a central vacuole; in which there is often seen a granule larger than the rest. The spherical centrosome is small compared with those of the other species of piscine trypanosomes. The flagellum borders the undulating membrane and joins the centrosome. The undulating membrane is not much folded, showing at the most five or six folds, and is very like that of $T$. lewisi. The part of the body posterior to the centrosome is very short and bluntly conical.

T. remaki var. magna (Fig. 69,4 ) is at least $45 \mu$ long (body $26 \mu$ to $28 \mu$ ) by $2 \mu$ to $2{ }^{\circ} 5 \mu$ wide. Some specimens may be as long as $57 \mu$.

Besides the difference in size, this variety differs from the variety parva by the darker staining of its protoplasm, which may be partly due to the greater thickness. As in the case of parva, the protoplasm stains evenly, no granules being visible. In other respects the two varieties are practically identical-the nucleus has the same structure in both, the centrosome is close to the posterior end, and the undulating membrane is slightly folded.

These large trypanosomes are not forms undergoing division, for we have never seen any specimens of $T$. remaki showing signs of division.

In naturally infected pike we have never seen undoubted dividing forms, but have occasionally met with parasites of the variety parva with two nuclei. We have only met with dividing forms in the two pike experimentally inoculated. The pike from which these two were inoculated contained only the small variety of $T$. remaki, and all the parasites seen in these two experimentally inoculated pike likewise presented the appearances of the variety parva. The trypanosomes showed the same variations in length as did those of the naturally infected pike, and during the ten to fifteen days that the parasites were most numerous in the blood dividing forms were not infrequently met with.

Trypanosomes about to undergo division increase a little in size, and especially in width. The length of such parasites varies from $28 \mu$ to $35 \mu$. The nucleus may divide first, as in Fig. 70, 3, but more often the centrosome is the first to divide ( 2 and 4 ). 
The centrosome enlarges $(I)$ and then divides into two small spherical masses. These are at first quite close together, but soon separate, remaining joined for a time by a small connecting portion, thus giving rise to the appearance of a dumb-bell. At the same time the root of the flagellum divides ( 2 and 4 ), and afterwards the flagellum divides throughout its whole length.

The nucleus when about to divide becomes elongated in the long axis of the parasite ( $I$ and 4 ). The nuclear vacuole and its chromatic granule also elongate and divide, so that the chromatin becomes concentrated at the two extremities of the nucleus. Finally, there are two nuclei, one behind the other (Fig. 70, 3 and 5), each containing a vacuole with a chromatic granule. The nuclear division
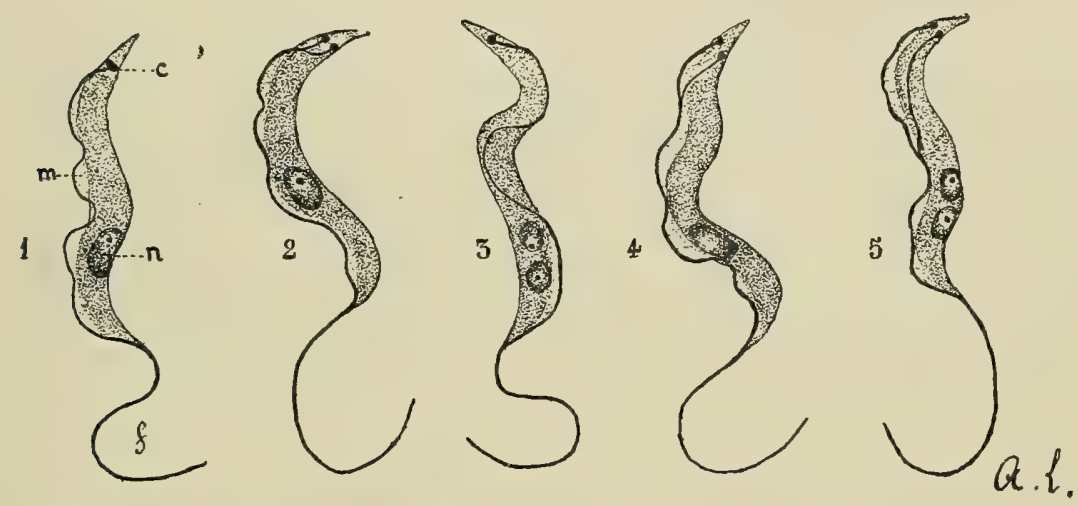

Fig. 70.-Different Stages in the Longitudinal Division of $T$. remaki.

(Magnified about 2,00o diameters.)

is of the direct type. At a given moment the trypanosome shows two nuclei, two centrosomes, two undulating membranes, and two flagella; the protoplasm then quickly divides. The division is equal or subequal, so that the newly formed trypanosomes are not easily distinguished from the older ones. This mode of division is identical with that of $T$. brucei.

Do the large and the small forms which we have described constitute distinct species?

In the pike which contained the two varieties parva and magna we did not find intermediate forms of the parasite. The large trypanosomes do not always coexist with the small ones. Finally, inoculations made with the small variety reproduced only small forms of the trypanosome. These facts are in favour of a specific difference, but they are not conclusive. On the other hand, the great resemblance between the small and the large trypanosomes, except for the size, is in favour of their being a single species. Possibly the trypanosomes of the variety magna only appear in pike which have been infected for a long time with the variety parva. 
We hope that this question will be answered by the experimental method we have initiated.

TRYPANOSOMA DANILEWSKYI, Laveran and Mesnil, I904.-Danilewsky was the first to record the existence of trypanosomes in the carp (Cyprinus carpio), and for this reason we have given. his name to this species. We found $T$. danilereskyi in one out of four carp from Garches (Seine et Oise), and in two out of three carp, $I_{4}$ to I5 centimetres [about 6 inches] long, bought in Paris. These observations were made in March, Ig03. In September and October, I90I, the blood of two carp bought in Paris had given negative results. It is an interesting fact that we have often found small leeches fixed between the scales on the bodies of the carp, especially those coming from Garches. The trypanosomes were always very scanty in the infected earp.
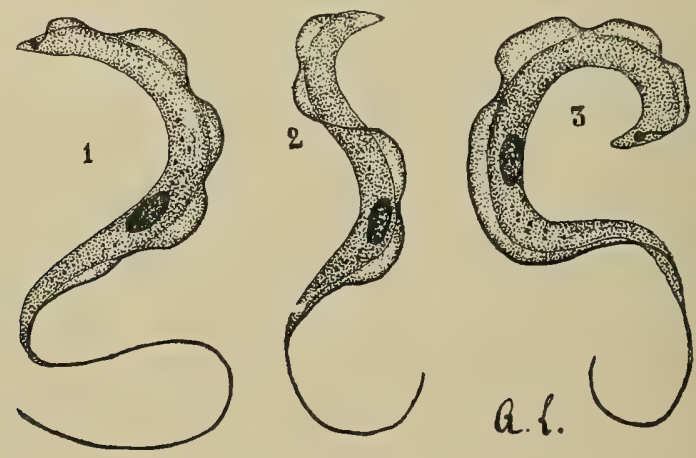

Fig. 7x.-Trypanosomes of the Carp and of the Tench.

I. T. danilewskyi. 2 and 3. T. tinca. (Magnified about 2,000 diameters.)

T. danilereskyi (Fig. $7 \mathrm{I}, I$ ) is $35 \mu$ to $45 \mu$ long, by about $3 \mu$ wide, the free flagellum measuring I5 $\mu$ to I7 $\mu$. The undulating membrane is broad and has many folds. The centrosome is fairly large and is close to the posterior extremity. The nucleus is elongated, and is situated towards the middle of the body, but usually rather nearer the anterior end. The protoplasm contains chromatic granules varying in size and number. We have not seen any multiplication forms.

We have already mentioned that another trypanosome, Trypanoplasma cyprini, Plehn, has been found in the carp. This hæmatozoon, which is quite distinct from $T$. danilereskyi, will be described later.

[The trypanosomes found by Petrie in nineteen goldfish (Carassius auratus) examined at Elstree apparently belonged to this species. The parasites were scanty in every case. In the fresh condition they were very active and showed many contortions. The length of three individuals was $32 \mu, 38 \cdot 4 \mu$, and $48 \mu$ respectively; the width was $2 \mu$ to $3 \mu$.] 
[Attempts to cultivate this trypanosome on rabbit-blood agar were unsuccessful. Short 'tadpole' forms, not very active, were seen in the culture tube in ten days, and on the eleventh and twelfth days small groups of four or five parasites were present. Petrie regards these flagellates seen in culture as degeneration forms.]

[A minnow (Phoximus lavis) examined by Laveran harboured a trypanosome having all the characteristics of $T$. danilereskyi of the carp. In addition to this trypanosome, a trypanoplasm, apparently identical with that of the red-eye (Tpl. borreli), has been found in the minnow by Léger and by Laveran.]

TRYPANOSOMA TINC.E, Laveran and Mesnil, Ig04.-We found trypanosomes in three out of six tench (Tinca tinca) bought alive in Paris in March, I903. Tench from 20 to 25 centimetres [8 to Io inches] long were more often and more severely infected than younger ones from I2 to I5 centimetres long. We had previously examined this fish on several occasions (in Lorraine, August, IgoI ; in Paris, September and October, Igor), but with negative results.

The trypanosomes were scanty or very scanty in the infected tench, but in one the parasites were fairly numerous in the blood, and particularly so in the kidneys.

Doflein had previously recorded the occurrence of trypanosomes in the tench. The fish found infected by him were obviously ill, and died in large numbers. ${ }^{1}$

In fresh blood the trypanosome is very motile, and is nearly always rolled on itself, so that one is unable to make out its shape and structure. It is necessary to study stained specimens in order to ascertain the details of structure.

The parasite is about $35 \mu$ long, by $2.5 \mu$ to $3 \mu$ wide. Its posterior end is in the form of a short, blunt cone (Fig. 7I, 2, 3), and the centrosome, which is fairly large, is not far from the posterior end. The nucleus is situated towards the middle of the parasite; the undulating membrane is wide and shows several folds. The free flagellum is fairly long.

In several parasites the centrosome and root of the flagellum had divided, so there is little doubt that in this case, as in that of T. remaki, multiplication takes place by equal division or bipartition, although we did not succeed in finding more advanced stages of multiplication.

TRYPANOSOMA ABRAMIS, Laveran and Mesnil, I904.- In July, I902, we found trypanosomes in the blood of a bream (A bramis brama) caught in the River Sarthe, between Sablé and Avoise. Unfortunately the fish died, and the blood on its arrival in Paris was already too decomposed to allow of a study of the trypanosome.

The blood of three bream bought in Paris did not contain trypanosomes.

1 Doflein, 'Die Protozoen,' etc., Jena, I90I, p. 7 I. 
[We shall see later that Brumpt has described a new trypanoplasm, which he calls $T p l$.abramidis, in the bream.]

TRYPANOSOMA GRANULOSUM, Laveran and Mesnil, Ig02.-Sabrazès and Muratet, of Bordeaux, were the first to give a description of the trypanosome of the eel (Anguilla vulgaris), which we have called T. granulosum. The infected eels were caught in the Garonne at Portets, and were from 25 to 30 centimetres [Io to 12 inches] in length. Eels of about the same size caught at various other places in the West of France did not harbour trypanosomes.

Six eels, caught in the River Sarthe, near Sablé, in July, were examined by us, and this trypanosome was found in every case. The parasites were scanty in the blood. Of nine eels examined at Roscoff in August, I902, only one showed trypanosomes in the blood, and they were very few in number. We have looked in vain for trypanosomes in the blood of eels from the ponds at Garches, as well as of those bought in shops in Paris.

[We have already mentioned the fact that trypanosomes have been found in eels in Sardinia (Manca), in Hong-Kong (Bell), and in Germany (Keysselitz).]

In fresh specimens we have only been able to make out the great contortions of the body in many cases, and the large size the parasites may attain.

In stained preparations trypanosomes of all sizes are found. The largest are $80 \mu$ long-body $55 \mu$, flagellum $25 \mu$-by $2{ }^{*} 5 \mu$ to $3 \mu$ wide. We measured two others which were smaller: one was $70 \mu$ long, the flagellum being $30 \mu$; the other was $44 \mu$ long, of which the flagellum measured $I_{3} \mu . \quad T$. gramulosum is shown in Fig. 72, 2, and in Fig. 13 of the coloured plate.

[In eels caught in the sea Lebailly found a trypanosome which is smaller than that of eels caught in rivers. He calls this marine eel trypanosome $T$. granulosum var. parva.]

The posterior extremity beyond the centrosome is very short and rather pointed, while the anterior end is very pointed. The centrosome is spherical and relatively large. There is a well-developed undulating membrane, bordered by a flagellum which stains particularly well. The protoplasm contains large granules scattered throughout the length of the body. These granules stain deep violet, and often appear upon an almost unstained background. They are sometimes grouped around the nucleus, which is then seen with difficulty. The nucleus stains reddish-violet, and is composed of a mass of chromatic granules. Sometimes it occupies the whole width of the body; at other times, when it is narrower, it lies close to the concave side.

Sabrazès and Muratet found that the trypanosomes of the eel can live for more than a week in blood kept in vitro at a temperature of $10^{\circ}$ to $19^{\circ} \mathrm{C} .{ }^{1}$ Under these conditions the authors observed multi-

1 Sabrazès and Muratet, oc. Sde Biol, January 16 and 30, 1904. [It has already been mentioned that Lebailly kept them alive for nine days at $2+^{\circ} \mathrm{C}$.] 
plication of the trypanosomes, and young forms as well as dividing forms became quite common. Sabrazès and Muratet also succeeded in cultivating this trypanosome according to the method of McNeal and Novy. Attempts should be made to get a series of subcultures of $T$. granulosum, as has been done with $T$. lewisi.

TRYPANOSOMA SOLEE, Laveran and Mesnil, Igor.-We found this parasite in only one of four soles (Solea vulgaris) caught in St. Martin's Bay, near Cap de la Hague, in the province of Manche. The trypanosome was very scanty in the infected sole, which harboured in addition the Hamogregarina simondi. ${ }^{1}$

At Roscoff we also found trypanosomes in the sole, but in even a smaller proportion than among the fish caught in St. Martin's Bay.

In fresh blood $T$. solea looks like other trypanosomes; its move-

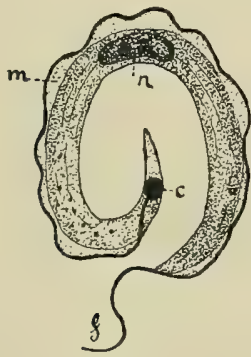

1

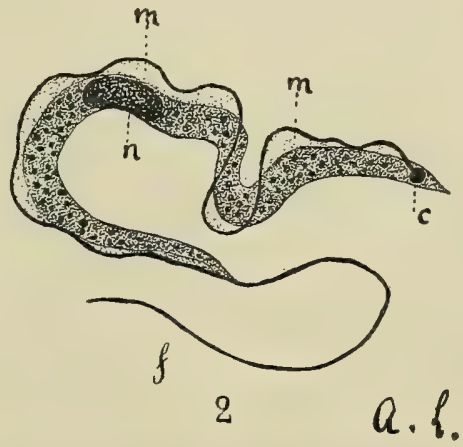

Fig. 72.-Trypanosomes of the Sole and of the Eel.

I. Trypanosome of the sole. 2. Trypanosome of the eel. (Magnified about 2,000 diameters.)

ments are very active, and the undulating membrane, with the free flagellum at the anterior end, can be distinguished.

In stained specimens the following details can be made out (see Fig. $72, I$, and Fig. 12 in the coloured plate): The parasite is $40 \mu$ long, the flagellum, which is very short, measuring only $8 \mu$. The anterior extremity is often less pointed than the posterior. The oval nucleus, containing large chromatic granules, is situated about the middle of the body. The spherical centrosome is near the posterior extremity, and is considerably bigger than in T.remaki. The undulating membrane is well developed and the flagellum is well seen in stained specimens. The protoplasm contains several small chromatic granules in the posterior part of the body, and also shows some fine longitudinal striations.

TRYPANOSOMA SCYLLII, Laveran and Mesnil, I902.-We found this trypanosome at Roscoff in sixteen out of thirty-eight small dogfish

1 Laveran and Mesnil, C. R. Acad. Sciences, v. , 133, October 14, I90I. 
(Scyllium canicula), and in one out of three large dogfish (Scyllium stellare), examined. ${ }^{1}$

The trypanosome is nearly always rolled on itself, so as to form in fresh blood and even in stained specimens an almost complete circle bordered by the undulating membrane. The extremities, which are often folded under and hidden by the body of the parasite, are not easily examined. Fig. $73, I$, shows a $T$. scyllii in a blood-film stained in the usual way. Its length is from $70 \mu$ to $75 \mu$, the flagellum measuring about I $4 \mu$, and its width is $5 \mu$ to $6 \mu$. The posterior extremity is conical and not very pointed. The protoplasm, which is finely granular, stains a deep blue by our usual method, and is easily distinguished from the undulating membrane, which is pale blue. The round or oval nucleus is situated at the junction of the anterior and middle thirds of the body. The centrosome, situated near the posterior end, is smaller than in $T$. solea. The flagellum joins the centrosome and borders the undulating
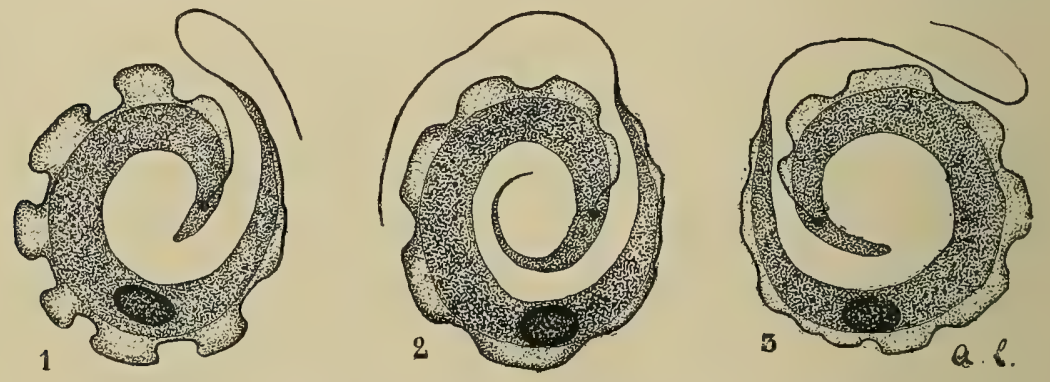

Fig. 73.-Trypanosomes of the Dogfish and of the Ray.

I. T. scyllii. 2 and 3. T. raja. (Magnified about $\mathrm{I}, 600$ diameters.)

membrane, which is broad, and presents several well-marked folds. We did not find any multiplication forms. The parasites were always scanty in the blood of the dogfish examined.

TRYPANOSOMA RAfE, Laveran and Mesnil, I902.-We found this trypanosome at Roscoff in Raja punctata in all of four medium-sized or large fish examined. The blood of seven small rays gave negative results. It was found also in one $R$. macrorynchus examined, in one $R$. mosaica out of three examined, and in the one $R$. clavata examined (from St. Martin's Bay). The blood of $R$. alba, $R$. microcellata, and $R$. mirelatus gave negative results.

We think that the trypanosomes found in $R$. punctata, $R$. mosaica, $R$. clavata, and $R$. macrorynchus belong to the same species, but we are not sure of this. The description which follows applies to the trypanosomes of $R$. punctata and of $R$. mosaica, studied in fresh blood and in stained films.

${ }^{1}$ In our earlier papers we followed E. Moreau ("Manuel d'Ichthyologie française,' 1902, p. 6) in giving to the small dogfish the name Sc. stellare (catulus), and to the large the name Sc. comicula. There is no doubt that in Moreau's work the names of the two dogfish were transposed by mistake. 
T. raje is $75 \mu$ to $80 \mu$ long, by about $6 \mu$ wide, the flagellum measuring about $20 \mu$. The posterior extremity is usually very pointed, so much so that it sometimes seems to end in a flagellum. The various intermediate forms of the posterior end and its staining reactions show, however, that this is not a flagellum. Fig 73, 2, shows a trypanosome of the ray with such a pointed posterior end. Fig. 73, 3, shows a trypanosome of the same species with a conical posterior end, and looking quite different from the parasite seen in Fig. 73, 2. The trypanosome with the blunt end bears a close resemblance to $T$. scyllii.

The protoplasm stains a dark blue and contains fine chromatic granules. The round or oval nucleus is situated at the junction of the anterior and middle thirds of the body. The centrosome, small and round, stains deeply, and is situated a considerable distance from the posterior end, especially when this is very pointed. The flagellum joins the centrosome and is very thin, both where it borders the undulating membrane and in its free part. Fig. I 4 in the coloured plate shows a T. raje as it appears in films stained by our method.

We have never seen any multiplication forms. These trypanosomes, which are always scanty in the blood, appear to have no pathogenic action.

[The following is a brief description of the more recently described trypanosomes of fishes:

Trypanosoma clarice (Montel, I905).- This trypanosome was found in a Clavias (Silurus clarias) in Cochin China. In fresh preparations it is fairly active, but less so than $T$. leveisi. When stained its length is about $60 \mu$, and its breadth about $4 \mu$; there is a short free flagellum. The posterior end is thick and truncated; sometimes it appears bifid. The anterior end tapers off towards the free flagellum. The protoplasm stains a deep blue, but in the anterior half of the body there are clear areas, which stain badly, and about the middle of the body in the region of the nucleus there are some longitudinal striæ. The nucleus is large and feebly staining; it contains chromatic granules, one of which is larger than the rest. The centrosome is large, and stains very deeply; it is situated near the posterior end of the body. There is a well-developed undulating membrane with numerous folds. The free flagellum is short, and does not stain very well.]

$[T$. claria resembles $T$. scyllii, $T$. vaja, and especially $T$.gvanulosum. It is thinner than the first two and a little broader than the last.]

[Trypanosoma platessa (Lebailly, I904).- Total length, $52 \mu$; width, $3 \mu$ to $3.5 \mu$; free flagellum, I2 $\mu$. There are numerous granules in the cytoplasm; the nucleus is at the junction of the anterior and middle thirds of the body. The posterior extremity is very pointed. The host is a plaice, Pleuronectes platessa (Platessa vulgavis), which is found to harbour also a new hæmogregarine, Hamogregarina platessa. About one in six of the fish examined was infected with the trypanosome.]

[Trypanosoma flesi (Lebailly, I 904).--Total length, $55 \mu$; width, $5 \mu$; free flagellum, Io $\mu$. The cytoplasm contains numerous granules; the nucleus is situated about the middle of the body. The posterior end of the body is pointed, and the centrosome is rather nearer the tip than in T. platesse. The host is Pleuronectes flesus (Flesus vulgavis), flounder, which 
harbours in addition a new hæmogregarine, $H$. flesi. About 25 per cent. of the fish examined were found to be infected.]

[Typanosoma laterne (Lebailly, r904).- Total length, $65 \mu$; width, 5. $\mu$ to $6 \mu$; free flagellum, $8 \mu$. The cytoplasm contains large and numerous granules. The host is Platophrys laternce, in which a new hæmogregarine, $H$. laterne, is also found. Only one in twenty of the fish examined was infected.]

[Trypanosoma limanda (Brumpt and Lebailly, I904).-Total length, $45 \mu$; width, $2 \mu$ to $2.5 \mu$; free flagellum, $20 \mu$. The posterior end of the body is very pointed. The host of this trypanosome is Limanda platessoides, one in six of which was found to be infected.]

[Trypanosoma delagei (Brumpt and Lebailly, I904). - This trypanosome is thin and fusiform, like the foregoing, but is rather shorter. Total length, $33 \mu$; width, $2.5 \mu$; free flagellum, I2 $\mu_{0}$ The posterior extremity is pointed; the undulating membrane is almost as wide as the body itself. As in $T$. limanda, the nucleus is nearer the centrosome than the free flagellum. The host is Blennius pholis, which harbours in addition the Hamogregarina bigemina (Laveran and Mesnil). About 4 per cent. of the fish were found infected with this trypanosome.]

[Trypanosoma gobii (Brumpt and Lebailly, I904).-.Total length, $66 \mu$; width, $5 \mu$.to $5.5 \mu$; free flagellum, Io $\mu$. The posterior extremity of the body is generally blunt or rounded off. The host is Gobius nigev, in which two new hæmogregarines are found, $H$. blanchardi and $H$. gobii. About half the fish were found infected.]

[Trypanosoma callionymi (Brumpt and Lebailly, I904).--Total length, $70 \mu$; width, $5 \mu$; free flagellum, only $5 \mu$. The cytoplasm sometimes contains large granules. The posterior extremity of the body is long and tapering, and as a result the centrosome is situated some distance from the tip. The host is Callionymus dracunculus (about 20 per cent. infected); two new hæmogregarines, $H$. callionymi and $H$. quadrigemina, are associated with this trypanosome.].

[Trypanosoma cotti (Brumpt and Lebailly, I904).-Total length, $53 \mu$; width, $5 \mu$; free flagellum, $8 \mu$. The posterior end is short and rounded. The host is Cottus bubalis (three out of four found infected). This species of Cottus harbours also a new hæmogregarine, $H$. cotti.]

[These new trypanosomes of Brumpt and Lebailly belong to two types: (I) The type of $T$. raja of Laveran and Mesnil, in which the body is long and fairly wide $(45 \mu$ to $65 \mu$ in length, by $5 \mu$ to $7 \mu$ in width), and the free flagellum short $(5 \mu$ to Io $\mu)$. To this type belong $T$. flesi, $T$. gobii, $T$. callionymi, $T$. cotti, and $T$. laterna. (2) The type of $T$. lewisi, in which the body is short and thin $(20 \mu$ to $25 \mu$ long, by $2 \mu$ to $2.5 \mu$ wide), and the free flagellum is fairly long: (I $2 \mu$ to $20 \mu$ ). To this type belong $T$.limanda and T.delagei. The $T$. platess $a$ is intermediate between these two types.]

[The hæmogregarines found in these fishes are also of two types. $H$. quadrigemina and $H$. gobii are I $7 \mu$ long, by $\mathrm{I} \cdot 8 \mu$ broad, and resemble $H$. bigemina and $H$. simondi of Laveran and Mesnil. All the others are shorter and wider (9 $\mu$ to I $2 \mu$ long, by $2 \mu$ to $3 \mu$ wide). The hæmogregarines and trypanosomes frequently coexisted in the same fish, and the trypanosomes were always much scarcer in a given species than the hæmogregarines. ${ }^{1}$ ]

1. [From Mesnil's abstract in Bull. Inst. Past., v. 2, 1904, p. 990]. 
[Typanosoma bothi (Lebailly, ${ }^{1}$ I905). - Total length, $42 \mu$; width, $3 \mu$; free flagellum, I $3 \mu$. The posterior end is thin and tapering. The nucleus is situated in the anterior half of the body. The host is Bothus vhombus (Rhombus lavis), brill, which harbours also a new hæmogregarine, H. bothi.]

[Trypanosoma barbi (Brumpt, ${ }^{2}$ I 906).- Total length, 5x $\mu$; width, $3 \mu$; free flagellum, I6 $\mu$. The centrosome is $I^{\circ} 5 \mu$ from the posterior end. The nucleus is $14 \mu$ from the origin of the free flagellum, and I $8 \mu$ from the posterior tip. The host is Barbus fuviatilis, barbel, in which a trypanoplasm also occurs.]

[Trypanosoma perce (Brumpt, I906). - Total length, $57 \mu$; width, $3 \mu$; free flagellum, I6 $\mu$. The centrosome is I $5 \mu$ from the posterior end; the nucleus is equidistant from the anterior and posterior ends of the body. The host is Pevca fuviatilis, perch.]

[Trypanosoma acerine (Brumpt, I906).--Total length, $47 \mu$; width, $3 \mu$; free fragellum, I7 $\mu$. The centrosome is $I \cdot 5 \mu$ from the posterior end; the nucleus $8 \mu$ from the origin of the free flagellum, and I9 $\mu$ from the posterior extremity of the body. The host is Acerina cernua.]

[Trypanosoma phoxini (Brumpt, I906).-In the adult forms the dimensions are as follows: Total length, $46 \mu$; width, $5 \mu$; free flagellum, I2 $\mu$. The centrosome is I.5 $\mu$ from the posterior end; the nucleus is equidistant from the anterior and posterior ends of the body. In young forms the absolute and relative dimensions are a little different. The host is Phoximus lavis, minnow, which harbours also the Trypanoplasma borveli.]

[It has already been mentioned that Laveran regards this trypanosome of the minnow as identical with $T$. danilereskyi of the carp.]

[Trypanosoma langeroni (Brumpt, Igo6).- Total length, $50 \mu$; width, $3 \mu$; free flagellum, I $3 \mu$. In old forms the width may be $6 \mu$ to $9 \mu$, and the free flagellum Io $\mu$. The centrosome is $\mathrm{I} 5 \mu$ from the posterior end; the nucleus is $16 \mu$ from the origin of the free flagellum, and I $8 \mu$ from the posterior end. The host is Cottus gobio, which harbours also a trypanoplasm.]

[Trypanosoma scardinii (Brumpt, I906).-Total length, $54 \mu$; width, $4 \mu$; free flagellum, I $8 \mu$. The centrosome is $2 \mu$ from the tip, the nucleus I $3 \mu$ from the origin of the flagellum, and I9 $\mu$ from the posterior end. The host is Scardinius erythrophthalmus, red-eye, in which a trypanoplasm also occurs.]

[Trypanosoma leucisci (Brumpt, Igo6).--Total length, $48 \mu$; width, $3 \mu$; free flagellum, I $8 \mu$. Centrosome, I $5 \mu$ from tip; nucleus, $9 \mu$ from origin of flagellum, and I $8 \mu$ from the posterior end. This trypanosome is parasitic in various roaches, Lenciscus genus.]

[Trypanosoma elegans (Brumpt, I906).- - Total length, 5 ${ }^{\circ} 5 \mu$; width, $4.5 \mu$; free flagellum, I5 $\mu$. Centrosome, $2 \mu$ from tip; nucleus, ${ }_{5} \mu$ from origin of flagellum, I7 $\mu$ from posterior end. The host is Gobio fluviatilis, gudgeon.]

[Trypanosoma squalii (Brumpt, I906).- - This was seen only in the fresh condition in the blood of Squalius (Leuciscus) cephalus, chub. In size and general appearance it resembled the trypanosome of the gudgeon.]

These various trypanosomes, which wẹre found by Brumpt in fresh-water fishes, can be divided into several groups according to their mode of evolution in the bodies of leeches, Hemiclepsis (see Section 6).

1 [Lebailly, C. R. Soc. Biol., 59, 1905, p. 304.]

2 [Brumpt, C. R. Soc. Biol., v. 60, 1906, pp. 160-162.] 
[Trypanosoma barbatule (Léger, ${ }^{1}$ I904).-This trypanosome is rather short and relatively stumpy. Its total length is $30 \mu$ to $40 \mu$; width, $4 \mu$ to $6 \mu$; free flagellum, i I $\mu$ to I2 $\mu$. The undulating membrane is broad, and presents deep folds. The host is Cobitis bavbatula, loach, which harbours also a new trypanoplasm.]

[Trypanosoma saccobranchi (Castellani and Willey, ${ }^{2}$ I905).-This trypanosome was discovered in a member of the Siluridæ, Saccobranchus fossilis, in Colombo. The discoverers give few details of morphology; they merely state that the centrosome was very near the posterior end. The degree of infection in these fish varied very much. Endo-corpuscular parasites were not found in association with the trypanosomes.]

[Trypanosomes in Nile Fish.-Neave ${ }^{3}$ found trypanosomes in several species of Nile fish. The parasite of the noke (Mugil) had the following dimensions: Length, $50 \mu$; width, $4 \mu$; free flagellum, I2 $\mu$; diameter of nucleus, $4 \mu$; distance of centrosome from tip, $5 \mu$. Neave states that this fish is a species of grey mullet, and has a gizzard.]

[The trypanosome of the bagara (Bageus bayard) had the following dimensions: Average length, 5 I $\mu$ to $5^{8} \mu$; width, $5 \mu$; free flagellum, $8 \mu$; diameter of nucleus, $3 \mu$; distance of centrosome from end, $0^{\circ} 2 \mu_{\text {. }}$ ]

[The trypanosome of the gargur (Lynodontis schal) measured as follows: Length, $24 \mu$ to $43 \mu$; width, $2 \cdot 5 \mu$ to $4 \mu$; free flagellum, $8 \mu$ to Io $\mu$; nucleus, $2 \mu \times 3 \mu$; distance of centrosome from tip, $0.5 \mu$. From Neave's figures it appears that this is a more slender-looking parasite than the first two.]

[Trypanosomes were also found in the dabib (Polypterus) when the blood was examined fresh on three occasions, but Neave was unable to secure a stained specimen of the trypanosome.]

\section{Section 4.-Description of the Trypanosomes of Fishes belonging to the Genus Trypanoplasma.}

We have already pointed out the characteristics of this genus (p. 26), so shall not refer to them again here. ${ }^{4}$

[Recent observations show that many species of fish harbour trypanoplasms. Brumpt is of opinion that the trypanoplasms found by him in several fresh-water fish are distinct species. Keysselitz, on the other hand, thinks that all the trypanoplasms seen by him in the fishes mentioned on p. 483 belong to one species, Tpl. borreli.]

TRYPANOSOMA BURRELI, Laveran and Mesnil, IgOI.-This parasite was found in the blood of half the number of red-eye (Scardinius [Leuciscus] erythrophthalmus) caught in the ponds in Garches. Young fish are less frequently infected than those $I_{5}$ to $I_{7}$ centimetres long. In all the red-eye we examined, whether naturally or artificially infected, the trypanoplasm was scanty in the blood, a prolonged examination often being necessary to find the parasite.

In fresh blood Tpl. borreli is very active, so that it is impossible

1 [Léger, C. R. Soc. Biol., v. 57, 1904, pp. 344-347].

2 [Castellani and Willey, Quart. Journ. Micr. Science, v. 49, I905, p. 383-402.]

3 [Neave, Second Report of the Wellcome Research Laboratory, Khartoum, 1906, p. 197.$]$

${ }_{4}$ We have pointed out previously (p. 22, note I) how we were induced to modify our earlier descriptions of the structure of the trypanoplasms. 
to see any details of its structure. One can only see that it often changes its shape; sometimes it takes the form of the letter $\mathrm{C}$, with the undulating membrane along the outer side of the curve; at other times it becomes straightened out like an amœba, when the body becomes as transparent as the undulating membrane. When the parasite moves, it travels with the larger end foremost. Neither nucleus nor granules of any kind are to be seen in the protoplasm.

Stained specimens show that the structure is different from that of the trypanosomes. The body of Tpl. borreli is flattened and often curved, as shown in Fig. 74, $I$, and Fig. I6 of the coloured plate. The inner (concave) side of the body is thicker than the outer (convex) side, which merges into the undulating membrane without any sharp line of demarcation. The outer side of the body stains less deeply than the inner, but the protoplasm stains uniformly,

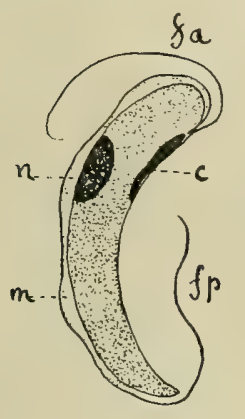

1

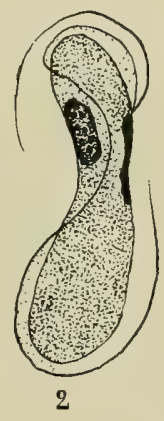

2

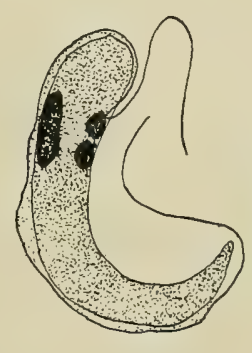

3
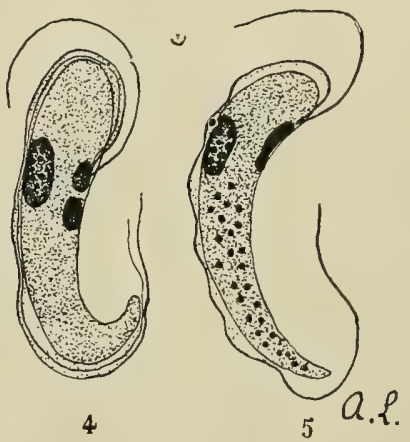

FIG. 74. - TRYPANOPLASMA BORRELI.

I and 2. Trypanoplasm of the red-eye. 3 and 4 . Dividing forms. 5. Trypanoplasm of the minnow. (Magnified about $\mathbf{I}, 800$ diameters.)

except for some dark granules which are occasionally present. In some parasites the posterior end stains a dark blue, while the anterior end is very pale. The posterior extremity may terminate abruptly, or it may taper off, extending for some distance along the flagellum.

The body of the parasite undergoes frequent changes of shape as the result of amœboid movements, so that the form shown in Fig. 74, $I$, although the most common, is by no means always seen. The form shown in Fig. 74, 2, is fairly common, and sometimes the body of the parasite is even straighter, so that it comes to be more or less regularly oval. The length of the body, flagella excluded, is usually about $20 \mu$, while the width varies from $3 \mu$ to $4 \mu$ or more.

In well-stained preparations there are seen, at the junction of the anterior and middle thirds of the body, two masses of chromatin, which are usually elongated with their long axes in the direction of the long axis of the body. Of these two chromatic masses, the one situated as a rule on the convex or outer side of the parasite is the 
nucleus (Fig. 74, $I, n$ ). It is larger and more oval in form, and stains less deeply than the other. The smaller of these two masses, which is usually situated in the concavity of the body, is more elongated and thinner than the other, and stains a deeper purple than the nucleus; it represents the centrosome (Fig. 74, I, c).

The centrosome gives rise to two flagella ${ }^{1}$ : an anterior flagellum, which at once becomes free $(f a)$; and a posterior flagellum $(f p)$, which winds round the anterior extremity of the body, borders the whole length of the undulating membrane, and finally becomes free at the posterior end of the body. Sometimes it may become detached from the body before reaching the posterior end, as is shown in Fig. 74,3 and 5 . The free part of each flagellum measures about $15 \mu$.

We have seen some dividing forms in the blood of red-eye experimentally infected; the centrosome divides first, then the flagella divide (Fig. 74, 3 and 4).

Léger has often seen trypanoplasms very closely resembling Tpl. borreli in the blood of minnows (Phoxinus lavis, Agass.) in Dauphiné. In some minnows the parasites are very numerous, and give rise to profound anæmia. The infected fish remains motionless, refuses to feed, and finally dies. ${ }^{2}$

In form and size the trypanoplasm described by Léger is closely allied to $T p l$. borreli of the red-eye, but there are certain differences between them. In the minnow the parasites are more regular in shape than in the red-eye ; secondly, the former parasites often show large chromatic granules (Fig. 74,5 ), and even pigment granules (Léger), which are not found in Tpl. borreli; and, lastly, the trypanoplasm of the red-eye appears to be less pathogenic than that of the minnow.

[As the result of inoculation experiments, Laveran ${ }^{3}$ agrees with Léger's view that the trypanoplasm of the minnow is the same species as Tpl. borreli of the red-eye. Of four minnows injected intraperitoneally with the trypanoplasm of the red-eye, two became infected, and the trypanoplasm of the minnow similarly infected a red-eye.]

[Our knowledge of the trypanoplasms of fishes has been considerably extended by the very thorough observations of Keysselitz published early in I906. 4 The following facts have been taken from the author's long paper on the subject.]

[The infection in the carp, tench, and bream often presents a certain periodicity, especially a seasonal one, the infection being

${ }^{1}$ [Both Léger and Keysselitz state, however, that the flagella arise from a wellmarked granule - the diplosome - which lies very close to the centrosome.]

2 Léger, Acad. des Sciences, March 28. and April 4, 1904.

3 [Laveran, C. R. Soc. Biol., v. 57, 1904, pp. 250, 251.]

4 [Keysselitz, Arch. f. Protisten., v. 7, 1906, pp. I-74; abstract by Mesnil in Bull. Inst. Past., v. 4 , I906, pp. 297-300, from which the account given in the text is largely taken.] 
more intense in the hot weather. ${ }^{1}$ It is found also that a latent or mild infection may develop into a severe one after the fish has laid its eggs. Keysselitz goes fully into the question of relapses and their causation. He succeeded only once in transmitting the infection from one fish to another by injection, and he consequently regards the so-called successful inoculation results of other investigators as instances of a relapse rather than a fresh infection.]

[As previously described by Léger, Keysselitz found a certain percentage of the infected fish succumb to the infection. The most important symptom is anæmia, which is evidenced by the pallor of the gills. Post-mortem one finds serous fluid in the peritoneum, hydropericardium, and œdema of the organs.]

[Keysselitz gives a detailed account of the morphology of the trypanoplasm, and of its evolution in the intestine of a leech, Piscicola geometra (this will be referred to again in Section 6). According to this author, the life cycle and evolution of Trypanoplasma are very complex, and analogous to those of $T$. noctuce and Hamamoeba or Spirochata ziemanni of the owl (see pp. 42-44), as originally described by Schaudinn.]

[The blepharoplast (centrosome) contains both true chromatin and plastin or nuclear substance-a fact which is in favour of its nuclear origin. In successfully stained specimens eight peripheral chromosomes could be seen grouped around a central body.]

[The nucleus, says Keysselitz, consists of a central karyosome and of eight small chromatic granules, which are often joined to the central body (karyosome) by very slender filaments. In certain cases, as in heavily infected fishes which are very anæmic, the granules of nuclear material are more or less scattered throughout the protoplasm.]

[The nucleus divides by 'pseudo-mitosis.' The karyosome elongates and becomes dumb-bell-shaped, a fine thread uniting the two resulting karyosomes; each chromosome divides into two, and eventually two nuclei are formed.]

[In addition to the ordinary or 'indifferent' forms of the parasite, Keysselitz describes two kinds of large formis, which he regards as sexual elements and calls gametes. The most characteristic difference between these two sexual forms is the presence of a welldeveloped blepharoplast (centrosome) and a relatively small nucleus in the male gametes, and a relatively large nucleus and small centrosome in the female gametes. When blood containing these sexual forms is swallowed by the appropriate leech, conjugation, followed by further evolution, occurs (see Section 6).]

TRYPANOPLASMA CYPRINI, M. Plehn, Igo3. ${ }^{2}$ - Miss Flehn found

1 [Brumpt thinks that this is due, at least under natural conditions, to fresh bites of leeches, in which the digestion of the blood and the evolution of the flagellates occur very quickly in hot weather.]

2 M. Plehn, Arch. f. Protisten., v. 3, 1903, p. 175.-Hofer, 'Handbuch der Fischkrankheiten,' and Allgemeine Fischerei Zeitung, 1904, No. 3, p. 48.- 
this parasite in the blood of carp obtained from various sources, at the piscicultural station in Munich. In some cases the parasites were very numerous, and the infected fish could be recognised by the pallor of their gills.

According to Hofer, this disease was prevalent in certain ponds in Germany in which carp-breeding was practised. The sick fish would remain for weeks on their side, the body bent in the form of an arc. When touched they would swim a few strokes and then resume their original attitude. Hofer's assertions as to the cause of the epizootics of the carp (I900-I9O2) must be accepted with some reserve, since $T p l$. cyprini was only described in Igo3.

The parasite is fairly resistant, for it remains motile for several days in preparations of blood mixed with salt solution. When its movements slow down the flagella and undulating membrane can be distinguished. The latter begins at the anterior end, and does not quite reach the posterior end of the body. Contrary to what is found in Tpl. borreli, the flagella are of unequal length; the anterior is more than half as long as the body, while the posterior is only a quarter the length of the body. The posterior flagellum is slender and very difficult to stain.

The body, which is flattened and lamelliform, twists and folds itself into all shapes, much like a piece of cloth that is shaken about in water. The protoplasm contains only a few fine granules. The body measures from $20 \mu$ to $25 \mu$, but may be as little as Io $\mu$ or as much as $30 \mu$ long.

Miss Plehn found that intra vitam staining with methylene blue stained the flagella well, but left the nucieus unstained. Dried films stained by the Romanowsky-Ziemann method show two chromatic masses near the anterior end of each trypanoplasm. One of these masses, the nucleus, is irregularly spherical, stains red, and contains a number of granules which stain more intensely than the rest of the nucleus. The other chromatic mass, which is rod-shaped and close to the edge of the parasite, stains a deep purple, and gives rise to the two flagella. ${ }^{1}$ Miss Plehn questions the centrosomic nature of this mass of chromatin, but does not give any definite interpretation of it. By Romanowsky's method the posterior flagellum does not stain.

In blood-films treated for two hours with gold chloride, and then exposed to the sun for four hours in formic acid (Apathy's method), the chromatic mass from which the flagella arise-and which we regard as the centrosome-is alone stained black. With other trypanosomes the same staining reaction is obtained in the corresponding chromatic masses (personal communication from Miss Plehn).

Chalachnikov had previously seen this trypanoplasm; some of the figures given by this author (op. cit.) leave no doubt upon the point.

1 We would here thank Miss Plehn for a letter which she sent to one of us on March 26, I904, correcting her earlier description of Tpl. cyprini. 
Multiplication forms are rarely seen. In a film which Miss Plehn kindly sent us we found several parasites evidently undergoing division, as they showed two nuclei and two centrosomes.

[The recently described trypanoplasms include the following:

Trypanoplasma varium (Léger, ${ }^{1}$ I904).-Length of body about $25 \mu$; of free flagella, $I \& \mu$ to $20 \mu$. This trypanoplasm is allied to $T p l$. borreli, but its flagella are a little longer, and it does not possess the well-marked cytoplasmic granules seen in the latter form. Unlike Tpl. bovreli, it presents giant forms, which are amœboid or vermiform in appearance. Moreover, Léger found that in certain streams containing both loach and minnows only the former were infected with this parasite. From this fact he concludes that Tpl.varium and Tpl.bovreliare distinct species. The host of this trypanoplasm is Cobitis barbatula, loach, which harbours also a trypanosome, T. barbatule.

[Trypanoplasma guevnei (Brumpt, ${ }^{2}$ I 905).- - Total length, $54 \mu$; anterior flagellum, $\mathrm{x} 6 \mu$; posterior flagellum, $4 \mu$. The centrosome is $9 \mu$ long, the nucleus $7 \mu$. The protoplasm always contains black pigment. The host is Cottus gobio.]

[Trypanoplasma barbi (Brumpt, I905). - In the fresh condition the body alone measures $26 \mu$. In stained specimens the parasite assumes the irregular forms described by Léger in Tpl. varium. The anterior flagellum measures I $8 \mu$, the posterior $9 \mu$. The centrosome is I I $\mu$ long, the nucleus ro $\mu$. The cytoplasm never contains pigment granules. This parasite occurs in the barbel, Bavbus fuviatilis.]

[Trypanoplasma abramidis (Brumpt, I905).--This trypanoplasm, parasitic in the bream, was seen only in the fresh condition. Its body measures $30 \mu$, the anterior flagellum about I $5 \mu$, the posterior $5 \mu$ to $6 \mu$.]

[Trypanoplasma trutte (Brumpt, I905). - Like the foregoing, this trypanoplasm was seen only in the fresh state. It is a small species, the body measuring only $20 \mu$, the anterior flagellum I $2 \mu$, and the posterior flagellum about $4 \mu$. The host is Salmo fario, trout.]

[Trypanoplasma intestinalis (Léger, ${ }^{3}$ I905).-This trypanoplasm, the first to be described as occurring outside the blood, was found by Léger in the œesophagus and anterior part of the stomach of a salt-water fish (Box boöps). The body is I4 $\mu$ long, the anterior and posterior flagella each I6 $\mu$. Léger describes, in addition to these typical forms, globular forms with three anterior flagella and a rudimentary undulating membrane. These, he thinks, are female forms, for he witnessed all stages of their penetration by typical forms (males).]

[Trypanoplasma ventriculi (Keysselitz, ${ }^{4}$ I906).-In the paper on $T p l$. borreli already referred to, Keysselitz incidentally mentions, and gives figures of, this new trypanoplasm, which he found in the stomach and adjacent part of the intestine of Cyclopterus lumpus, from Bergen, Norway. In appearance this parasite closely resembles the blood trypanoplasms; it has two well-developed flagella, each of

1 [Léger, C. R. Soc. Biol., v. 57, I904, pp. 344-347.]

2 [Brumpt, Rev. Scientrf., September 9, I905, pp. 32I-332 ; abstract by Mesnil, Bull. Inst. Past., v. 3, 1905, p. 920 ; also C.R. Soc. Biol., v. 60, 1906, pp. 162-164.]

3 [Léger, C. R. Soc. Biol., v. 58, I905, pp. 5 II-5I3; abstract by Mesnil, Bull. Inst. Past., 3, rgo5, p. 448.]

${ }^{4}$ [Keysselitz, Arch.f. Protisten., v. 7, 1906, p. 37.] 
which takes origin in a distinct granule (diplosome) situated in front of the blepharoplast. The latter was frequently divided into two.]

\section{Section 5.-Mode of Multiplication of the Trypanosomes of Fishes.}

There is very little information to be obtained upon this point in the works and papers hitherto published. ${ }^{1}$ Danilewsky states that the trypanosomes of fishes multiply by unequal binary division, the young forms looking like monads. ${ }^{2}$ According to Chalachnikov, the young trypanosomes of fishes multiply by longitudinal fission. $\mathrm{He}$ also states that he saw in the blood of Cyprimus carpio and of Esox lucius, kept for several days in vitro, protoplasmic masses undergoing division, which he regarded as multiplication forms of the trypanosomes. ${ }^{3}$

The trypanosomes are, as a rule, very scanty in the blood of fishes, and this partly accounts for the difficulty experienced in finding dividing forms. It may also be due in part to the fact that these forms are only found at the beginning of the infection, as in the case of $T$. lewisi. In order, therefore, to obtain fish under favourable conditions for examining multiplication forms, we inoculated fishes experimentally by means of intraperitoneal injections of infective blood from fish of the same species. These experiments, which we made with the pike, eel, and red-eye, enabled us to study the most important phases in the multiplication of $T$. remaki and Tpl. borreli.

In our experiments we found that the trypanosomes multiply by equal or subequal binary fission, like $T$. brucei, for example. ${ }^{4} \mathrm{We}$ have already mentioned the various stages met with in the reproduction of $T$. remaki and Tpl. borreli, so there is no occasion to refer to them again.

\section{Section 6.-Wodes of Infection.}

We have seen in previous chapters that the mammalian trypanosomes are, as a rule, conveyed by biting flies. It is probable that the trypanosomes of fishes are conveyed by ectoparasites, which attach themselves either to the gills of the fish or to the surface of the body between the scales. We have often found small leeches on the bodies of soles, rays, and red-eye-fish which are frequently infected with trypanosomes or other hæmatozoa. ${ }^{5}$

${ }^{1}$ [Keysselitz's paper on Tpl. borreli gives many details of the multiplication of this parasite, both in infected fish and in the stomach of the leech. Léger and Brumpt also describe the evolutionary changes of trypanosomes and trypanoplasms in various species of leech.]

${ }_{2}$ Biol. Centralbl., v. 5 , November I, 1885.

3 'Rech. sur les parasites du sang,' Charkov, 1888.

4 Laveran and Mesnil, 'Sur le mode de multiplication des trypanosomes des poissons,' Acad. des Sciences, June 16, 1902, and Arch.f. Protisten., v. 1, 1902.

5 We have described hremogregarines in the sole and ray (Laveran and Mesnil, Acad. des Sciences, October 14, I901, and October 13, 1902). 
Leydig long ago found trypanosomes in the ingested blood of leeches caught on fish. From this it follows that leeches are probably concerned in the transmission of these parasites.

Keysselitz, in Munich, succeeded in infecting various fishes by means of leeches taken from fishes of the same species (tench, carp, and pike) infected with trypanosomes. ${ }^{1}$

Van Beneden and Hesse have described, under the name of Hemibdella solea, ${ }^{2}$ the leech which is frequently found on the bodies of soles, particularly at Roscoff.

According to Léger, leeches serve to propagate the trypanosome of the minnow (private communication).

The following experiments show that it is easy to inoculate the trypanosomes of a fish into another fish of the same species by injecting it intraperitoneally with a little infective blood.

EXPERIMENT I.-On April I 5, I902, a pike weighing about 500 grammes was killed, and its blood, which contained trypanosomes in very small numbers, was mixed with citrated salt solution and injected, in doses of $\frac{1}{2}$ c.c. of the mixture, into the peritoneal cavity of two young pike ( 15 and I 2 centimetres long). These pike had been kept under observation for several months prior to injection, but trypanosomes had never been found in their blood. The results of the injection were as follows :

Pike A (15 centimetres long): April 23, eight days after inoculation, trypanosomes absent ; May 3, trypanosomes present, but scanty ; May I I, trypanosomes more numerous. From May 20 onwards the trypanosomes diminished in number, and on June 4 only one was found after a long search. The fish survived, and trypanosomes were still present in July, 1902. Pike $B$ ( 12 centimetres long): May 2, seventeen days after inoculation, very few trypanosomes seen in blood; May 7, trypanosomes more numerous, as many as five being seen in one field with a magnification of 480 diameters; May I3, fish killed; fewer trypanosomes present. The trypanosomes were not more numerous in the vessels of the kidneys or spleen than in the blood taken from the heart or from the periphery.

EXPERIMENT II.-An eel, which had never shown any trypanosomes, was injected intraperitoneally with infective blood from another eel. Twelve days later trypanosomes were found in the blood of the injected eel.

EXPERIMENT III.--On May 8, IgO2, the blood of a red-eye containing a few $T p l$. borveli was inoculated intraperitoneally into five other red-eye (two medium-sized and three small). In each case the dose injected was about $\frac{1}{2}$ c.c. blood considerably diluted with citrated salt solution. The five red-eye were carefully examined for trypanosomes before the inoculation, but with negative results. On May I6 the blood of two fishes was examined; trypanoplasms absent. From May 21 to 26 , three of the fishes showed trypanoplasms in small numbers; negative in the case of the other two fishes. On May 29 two of the fishes-those in which the blood examination was negative-were found dead (one small and one medium-sized). The trypanoplasms were scanty in the other three redeye. The two small ones were killed; the parasites were scanty in the spleen and kidneys, as well as in the peripheral blood. In the case of the medium-sized red-eye which survived, the blood was examined during the early days of June, and showed very few parasites.

1 Hofer, op. cit.

2 'Rech. sur les Bdellodes,' Mém. Acad. Sciences Belgique, v. 34, p. 4r. 
This experiment with the trypanoplasm of the red-eye has been repeated several times, with similar results, the parasites appearing in the blood of inoculated fishes at the end of fifteen to twenty days, increasing in number for the next ten or twelve days, and then diminishing more or less rapidly. In no case has the blood of the inoculated fishes shown very many parasites, nor has death ever resulted from the direct effects of the presence of these hæmatozoa.

Miss Plehn has succeeded in producing similar artificial infections in the carp. Of seven carp inoculated into the heart with blood con taining trypanoplasms, five were infected at the end of two to three weeks, but in each case the infection was a mild one.

Inoculations from one species of fish into another have hitherto given uniformly negative results. This fact justifies the description of different species of trypanosome for the different species of fish, although the parasites themselves do not present any marked morphological differences.

[The recent interesting observations of Léger, of Brumpt, and of Keysselitz prove that various species of leech act as the alternate host of the trypanosomes and trypanoplasms of fishes, and that in all probability leeches play an important, if not the sole, part in the transmission of the infection from one fish to another.]

[Léger ${ }^{1}$ examined the intestinal contents of leeches (Piscicola sp.) which had sucked the blood of loaches infected with $T$. barbatule. Eighteen hours after the meal of blood he found pyriform bodies without flagellum and with one nucleus, or with two unequal nuclei, which arose from the first by heteropolar division. Later on there were trypanosomes resembling the 'male,' 'female,' and 'indifferent' types of Schaudinn. The last multiplied actively by equal binary fission; the others, which were larger, multiplied by very unequal binary fission, and finally the intestine of the leech contained many very small trypanosomes.]

[Leeches (Hemiclepsis marginata) which had fed upon the blood of loaches infected only with trypanoplasms (Tpl. varium) contained at the end of several days numerous small elongated trypanoplasms, some of which lacked the anterior flagellum.]

[Brumpt ${ }^{2}$ states that the trypanosomes of fresh-water fishes undergo their evolution in leeches of the genus Hemiclepsis. That of the trypanoplasms is said to occur, according to the species of trypanoplasm, in Hemiclepsis or in Piscicola. For instance, the trypanoplasms of the loach and of the barbel, which are morphologically very closely allied, undergo their evolutionary cycle, the first exclusively in Hemiclepsis, the second exclusively in Piscicola. 7

${ }^{1}$ [Léger, C. R. Soc. Biol., v. 57, 1904, p. 344 ; abstract by Mesnil, Bull. Inst. Past., v. 3, 1905, p. 18.]

2 [Brumpt, C. R. Soc. Biol., v. 57, 1904, pp. 165-167; ibid., v. 60, 1906, pp. 160162; and v. 61, 1906, pp. 77-79 ; abstract by Mesnil, Bull. Inst. Past., vols. 2 and 4.$]$ 
[According to their mode of evolution in Hemiclepsis, the fish trypanosomes may, according to Brumpt, be split up into several groups: (I) Those which undergo evolution solely in the stomach of the leech, and never pass into the intestine or into the sheath of the proboscis. Such are $T$. abramis, T. remaki, T. barbi, T. perce, $T$. squalii, and T.acerina. (2) Those in which development starts in the stomach and goes on in the intestine; finally the trypanosomes get into the sheath of the proboscis. This is the mode of evolution of T. granulosum. (3) Those in which development occurs in the stomach, and after a certain time the trypanosomes reach the sheath of the proboscis, as with $T$. danilereskyi and $T$. phoxini. In the case of $T$. barbatula, $T$. langeroni, $T$. scardinii, and $T$. leucisci, the complete evolutionary cycle was not studied owing to lack of material.]

[Brumpt describes fully the evolution of $T$. gramulosum of the eel as it occurs in Hemiclepsis. Some hours after ingestion the trypanosomes in the leech's stomach become pyriform, and have the centrosome near, or in front of, the nucleus, as in Crithidia. They multiply actively in the stomach, and at the end of forty-eight hours have nearly all passed out into the intestine. Here they become very elongated and assume Herpetomonas-forms, which they retain for several months. These give rise to trypanosome forms, which reach the sheath of the proboscis as early as the fifth day after the meal of infective blood. It is these forms which are inoculated into the eels, and by simple elongation assume the typical form of $T$. gramulosum. The complete evolutionary cycle as just described occurs only in Hemiclepsis; in other leeches, such as Callobdella punctata, Hirudo troctina, and Piscicola geometra, the parasites. undergo certain evolutionary changes and then die off after a variable time. From Brumpt's observations it appears that the infection is never conveyed to the embryo leeches, and Keysselitz has come to the same conclusion.]

[Brumpt succeeded in infecting healthy fish by allowing them to be pricked by infected leeches. $]^{1}$

[Two young carp and two river bull-heads, free from parasites, were infected simultaneously with trypanosomes and trypanoplasms by the bites of embryo Hemiclepsis and Piscicola, themselves heavily infected. The incubation period was ten to seventeen days.]

[Of eleven uninfected eels which were allowed to be pricked by embryos of Hemiclepsis, ten showed trypanosomes in the blood in less than six days. The eleventh eel did not become infected, owing to the removal of the part pricked, the pectoral fin.]

[One Cottus bubalis, pricked by Callobdella punctata, also became infected.]

[The evolution of the trypanosomes of salt-water fishes is a little peculiar. In the leech's stomach the parasites lose their flagella and

1 [Brumpt, C. R. Soc. Biol., v. 6I, 1906, pp. 77-79; abstract by Mesnil, Bull. Inst. Past., v. 4, I905.] 
divide a certain number of times in this condition; after some days flagellated forms again develop. $T$. solece and $T$. cotti develop exclusively in the stomach of Callobdella punctata, and never pass into the sheath of the proboscis. T. scyllii and T. raja develop at first in the stomach of Pontobdella, and then pass into the intestine, where they have flagella and divide a great number of times.]

[The different species of Trypanoplasma develop, according to Brumpt, in different leeches: Tpl. guernei, like Tpl. borreli of the red-eye and minnow, evolutes in Piscicola; so also does Tpl. barbi. On the other hand, Tpl. abramidis undergoes its development exclusively in Hemiclepsis, and never passes into the sheath of the proboscis. Tpl. trutta does not develop in Hemiclepsis or Hirudo; it probably does in Piscicola.]

[The most detailed account we possess of the development of the trypanoplasms in leeches is that of Keysselitz in the paper on $T$. borreli previously mentioned. According to this author, the trypanoplasms of the various fishes studied by him all undergo their evolution in one species of leech, Piscicola geometra.]

[When blood containing the gametes described on p. 499 is swallowed by this leech, the sexual forms conjugate. There is fusion of the two cytoplasms, followed by that of the respective nuclei, the true nuclei uniting after at least one reduction process. There results from this fusion an ovoid mass, without flagellum, resembling the ookinetes of the endocorpuscular hæmatozoa or of T. lewisi (Prowazek; see p. 89). Two chromatic masses can be distinguished in this oval parasite : a compact mass which arises from the union of the two blepharoplasts, and a granular one which arises from the fusion of the two reduced nuclei.]

[These ookinetes, or 'copulas,' soon give rise to typical trypanoplasms, of which there are three kinds. On the analogy of those described by Schaudinn in T. noctua, these three kinds are called 'male,' 'female,' and 'indifferent.' As in the case of the gametes (see p. 499), the chief difference is in the size of the nuclei.]

[From this time onward there is an abundant multiplication of the parasite in the intestinal contents of the leech, and different forms are seen. Some are very thin (Keysselitz calls them spirochætiform), yet they have all the characteristics of trypanoplasms; others are short and stumpy. The latter are to be correlated with the thick consistency of the contents of the leech's intestine, for as soon as these contents become thinner, as after a fresh meal, the parasites assume the normal shape, and it is in this form that they are inoculated into fishes.]

[The multiplication of the stumpy forms was followed with great care-it is by equal or unequal binary longitudinal fission. In certain favourable cases a system of longitudinal fibrils or myonemes, like those described by Schaudinn in T. noctua, was seen. Lastly, Keysselitz observed in certain trypanoplasms in the leech some- 
what complicated nuclear changes, which he regards as evidence of parthenogenesis.]

[The infected leeches may present morbid phenomena which coincide at times with the period of intense multiplication of the trypanoplasm. The most obvious sign is marked swelling of the body from the clitellar region backwards; in addition to this, there is an alteration in the colour of the body, the animal becomes less and less active, and finally dies.]

[Unlike other investigators, Keysselitz failed to produce experimental infection in fishes by means of infected leeches.]

[Keysselitz thinks that other leeches besides Piscicola can subserve the development of Trypanoplasma. In experiments, which he had not concluded at the time of writing the paper, he observed the initial stages of evolution in Hirudo medicinalis.]

[Finally, it may be added that Keysselitz is of opinion that all the hitherto described trypanoplasms parasitic in the blood of fishes belong to one species, which should be known as Trypanoplasma borreli, Laveran and Mesnil.] 


\section{CHAPTER XVIII}

\section{THE TSETSE-FLIES AND THEIR TRYPANOSOMES}

\section{Part 1.-The Trypanosomes of Tsetse-Flies.}

[FlAGELLATES (trypanosomes) have been found in the gut of 'native' or freshly caught tsetse-flies. This is an important fact in view of the statements of Gray and Tulloch, and of Koch, that ingested mammalian trypanosomes (T. gambiense and $T$. brucei) undergo developmental (? sexual) changes in tsetse-flies.]

$\left[\right.$ Novy $^{1}$ has studied these fly trypanosomes in the intestinal contents of specimens of Glossina palpalis sent to him from Uganda. From a review of the recorded observations of Koch, ${ }^{2}$ and of Gray and Tulloch, ${ }^{3}$ as well as from his own. cultivation and other experiments, Novy concludes that the trypanosomes met with in the tsetse-flies are harmless non-parasitic flagellates corresponding to the equally harmless Herpetomonas and Crithidia observed by him in mosquitoes. ${ }^{4}$ He bases this view upon the following facts: (I) The large size of the tsetse-fly parasites compared with the blood-forms ; (2) their presence in flies which had not been fed experimentally on infected animals; (3) the failure to obtain any development of the

1 [Novy, Journ. Infect. Dis., v. 3, I906, pp. 394-4II ; abstract by Mesnil in Bull. Inst. Past., v. 4, I906, p. 606.]

${ }_{2}$ [Koch, Deutsche med. Wochenschr., November 28, I905, pp. I865-1869.]

${ }^{3}$ [Gray and Tulloch, Sleeping Sickness Commission Report, No. 6, 1905, pp. $282-287$.

${ }^{4}$ [In a recent paper upon the 'Trypanosomes of Mosquitoes and other Insects' (Journ. Inf. Dis., v. 4, No. 2, April, I907, pp. 223-276), Novy, McNeal, and Torrey maintain their earlier view (see p. 38) that "the mosquito flagellates are not stages of intracellular organisms, but are probably parasites peculiar to the insects.' These observers have given the name Trypanosoma culicis to a flagellate found in several specimens of Culex, and they state that the parasite found by the Sergents in the gut of a larva of Anopheles maculipennis (see p. 37) closely resembled their flagellate. They state, further, that the flagellates of mosquitoes belong to two easily differentiated types, Crithidia and Herpetomonas; and that these two types are common in other insects, and should be placed under the trypanosomes, instead of being classed as distinct genera.]

[They give the name $T$. christophersi to a trypanosome found by Christophers in the gut of a dog-tick. Rhipicepildus sanguincus, in Madras. The flagellates seen were typical cultural forms ; total length, $30 \mu$ to $45 \mu$, including frce flagrellum $8 \mu$ to $12 \mu$. There is a prominent undulating membrane, extending over about half the length of the body. The nucleus is situated about the middle of the body; the centrosome is close to, and on one side of, the nucleus, but sometimes it lies immediately in front of it. This trypanosome is not a developmental phase of Pircplasma canis, and we have no proof at present that piroplasms pass through a trypanosome stage in their life-cycle.] 
trypanosomes ingested by flies fed on infected animals ; (4) the failure to infect susceptible animals with the flagellates of the tsetse-fly; and (5) analogy with the flagellates (trypanosomes) of mosquitoes.]

Several varieties of trypanosome were present in these flies, and Novy thinks there may be several species. He gives the name Trypanosoma grayi to the numerous forms seen in one of the flies. These very varied forms come under two types, just as Novy had found in the case of T. avium of birds. Type I (see Fig. $75, a$ ) is thin and very elongated (body $I 8 \mu$ to $29 \mu$ long, by $0^{\circ} 6 \mu$ to I $\mu$ wide), with a long free flagellum, so that the total length may be $36 \mu$ to $48 \mu$. The nucleus is rod-shaped, very deeply stained, and compact; the centrosome is large, deeply stained, quite round, and immediately anterior to the nucleus. In this type, as well as in the other types
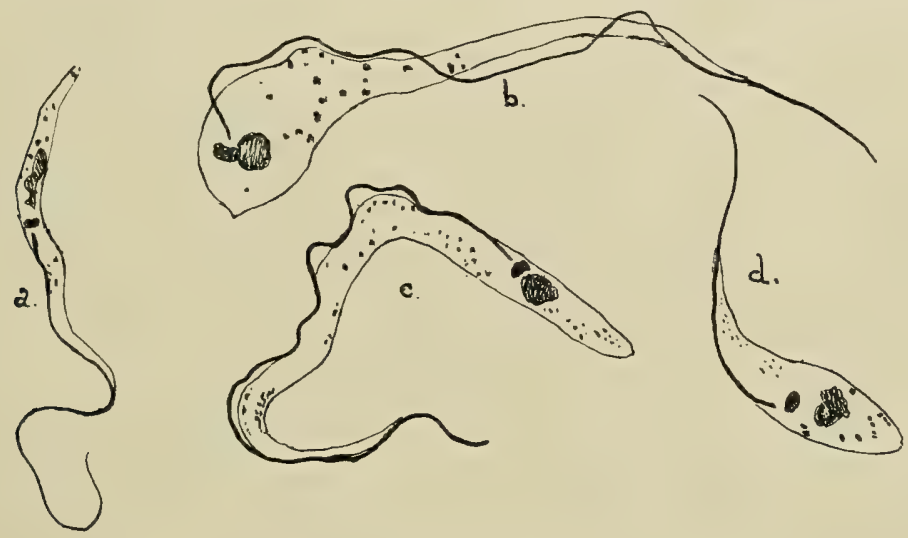

Fig. 75.-TRypanosomes OF THE TSETSE-FLy ( $T$. grayi).

$a$. Male form with compressed nucleus and long free flagellum. $b$ and $c$. Female forms, posterior extremity thickened, short free flagellum. In a later publication (Nature, November 15, I906), Minchin calls $c$ an 'indifferent' form. $d$. Young form resulting from the unequal division of a large female form. $a, b$, and $c$ are from the gut of the tsetse-fly (Gl. palpalis) dissected ten days after having fed on an uninfected monkey. $d$ is from the gut of a fly twenty-four hours after its first feed of blood. (After Minchin, Gray, and Tulloch. Magnified about 2,00o diameters.)

of tsetse-fly trypanosomes, there is, says Novy, a more or less marked diplosome situated near the nucleus, usually between it and the centrosome. It may be mentioned that a diplosome has also been described in some of the Herpetomonas.]

[Type 2 (see Fig. $75, b, c$ ) is a wide and long ribbon-like form, with a short free flagellum and a well-developed undulating membrane. The nucleus is rounded (about $2 \mu$ in diameter), and the centrosome is usually just in front of the nucleus, but may be lateral or even posterior to it. Novy compares these two types to the 'male' and 'female' types respectively of $T$. gambiense described by Koch in Gl.palpalis.]

[As Gray and Tulloch had shown, division is very unequal (see Fig. $75, d)$ in these trypanosomes, and usually takes place in the ordinary way, starting in the centrosome.] 
[The preparations from the other tsetse-flies showed forms differing from the preceding and from one another, although evidently of the same generic group. Novy distinguishes three types, one of which he says might possibly be regarded as having some relation to T. gambiense (see Fig. 76).]

[Minchin, Gray, and Tulloch ${ }^{1}$ have studied, in Uganda, the Gl. palpalis in its relation to $T$. gambiense and other trypanosomes. They confirm Novy's observations upon these trypanosomes of the tsetse-fly, and Minchin has given the name Trypanosoma tullochii ${ }^{2}$ to the type which Novy says is most like the human trypanosome. T. tullochi (Fig. $76, a, b, c)$ is distinguished by its more rounded nucleus, situated near the middle of the body, and by its small, usually circular, centrosome, situated well behind the nucleus.]
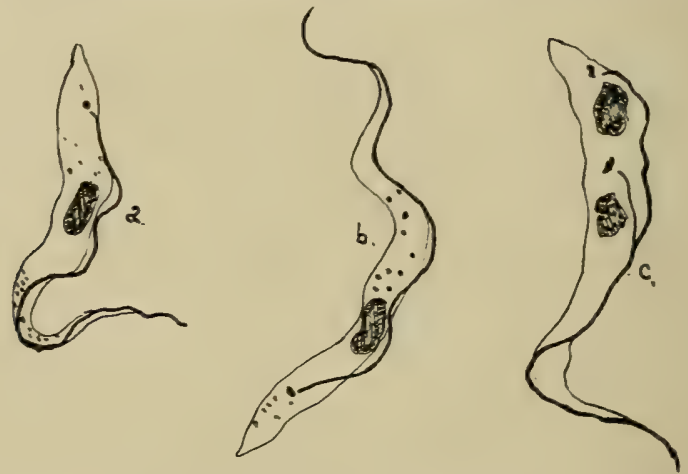

Fig. 76.-Trypanosomes of the Tsetse-Fly ( $T$. tullochi).

$a$. Large form, with minute circular centrosome, from the gut of a fly. $b$. Large form, from the proventriculus of the same fly. c. Dividing form, common type, from the gut of the same fly. (After Minchin, Gray, and Tulloch. Magnified, 2,000 diameters.)

[Tsetse-flies known to contain T. grayi or T. tullochi, or both, were fed on various animals-fifteen monkeys, two guinea-pigs, one white rat, and two hens-but in no case did these animals become infected by the bites of the fly. As we have seen, however, in the chapter on Human Trypanosomiasis, Bruce, Nabarro, and Greig succeeded in infecting monkeys with $T$. gambiense by allowing freshly caught tsetseflies to feed upon them.]

[An interesting observation of Minchin, Gray, and Tulloch seems to show that goat serum has a remarkable effect upon $T$. grayi, and not upon T. gambiense. A large number of freshly caught tsetse-flies were fed on a native goat, and on dissecting these flies (some 500 in number) not a single one was found to contain trypanosomes. Other flies, caught at the same time and place, fed on the other experimental animals (monkeys,

1 [Minchin, Gray, and Tulloch, Proc. Roy. Soc, Series B, v. 78, 1906, pp. 242258 (with 3 plates); reprinted in Report of Slecping Sickness Commission of the Royal Society, No. 8, 1907, pp. 122-136.]

2 [In conformity with the rules of nomenclature followed by Laveran and Mesnil and adopted throughout this volume, I propose to amend the name given by Minchin, and to call this trypanosome Trypanosoma tullochi.-ED.] 
etc.), were found to contain trypanosomes in the usual proportion. Preparations were then made of goat serum and $T$. grayi, and of the same goat's serum and $T$. gambiense. It was found that in the preparation of T. grayi the trypanosomes were rapidly immobilized and died off, while the $T$. gambiense remained active. Similar preparations made with human serum instead of with goat serum showed that the trypanosomes were unaffected in either case. This result seems to furnish an additional means of distinguishing between $T$. gambiense and T. grayi.]

[Minchin ${ }^{1}$ has recently described a stage of encystment of $T$. grayi in the proctodæum of $G l$. palpalis. The undulating membrane disappears, so that the parasite comes to resemble the genera Herpetomonas and Crithidia; the flagellum becomes gradually shorter, and appears to become retracted into a pink-staining vacuole (see Fig. 9), reminding one of the flagellar vacuole described by Leishman during the formation of the flagellum in cultures of the Leishman body. A pink-staining cap, which is formed at the hinder pole, gradually extends round the parasite, and in this way an irregularly-circular cyst, containing a darkly stained centrosome and a somewhat fragmentary nucleus, is produced. ]

[The other parts of the gut of this fly contained normal trypanosomesvery few differentiated sexual forms, but many indifferent forms, which gave rise to young forms and ultimately to the small Herpetomonas-like forms found in the proctodæum. Owing to lack of nutriment here these forms, it is suggested by Minchin, became encysted.]

[These cysts closely resemble the 'schleim-cysten' described by Prowazek $^{2}$ in Herpetomonas musca-domestica, and, as in the case of the house-fly, they are no doubt destined to pass out of the fly's gut with its dejecta. The house-fly, being a foul feeder, becomes infected with the Herpetomonas by swallowing these cysts with its food. Not so, however, the tsetse-fly, which is very particular about its food. Minchin suggests that these cysts are destined to be swallowed accidentally by some vertebrate, the-as yet unknown-host of $T$. grayi, in order to germinate in its digestive tract, to pass thence into the blood, and to be taken up again with the blood by the tsetse-fly. Minchin proposes to call this type of infection-which has been proved by Schaudinn to occur in the case of Amaba coli infection-the contaminative type. In the other mode of infection, which Minchin calls the inoculative, the parasite, after going through developmental changes in the insect, passes back again into a second vertebrate host through the proboscis, as in the case of malaria transmitted by a mosquito.]

\section{Part 2.-The Tsetse-Flies.}

The study of the African trypanosomiases is intimately connected with that of the tsetse-flies. In the chapter on Nagana and Human Trypanosomiasis we have quoted the experiments of Bruce, Nabarro, and Greig, showing the rôle of the tsetse-fly in the propagation of these diseases. A volume like the present one, dealing with the trypanosomiases, ought, therefore, to include an account of the morphology, biology, and taxonomy of the genus Glossina. We have taken these from Austen's monograph, ${ }^{3}$ which gives a comprehensive account of the tsetse-flies, as well as a full bibliography.

1 [Minchin, Proc. Roy. Soc., Series B, v. 79, I906; reprinted in Sleeping Sickness Commission Report, No. 8, 1907, p. 137-142.]

2 [Prowazek, Arb. a. d. kaiserl. Gesund., v. 20. 1904, p. 446].

3 E. E. Austen, 'A Monograph of the Tsetse-Flies (genus Glossina, Westwood), based on the Collection in the British Museum, with a Chapter on the MouthParts, by H. J. Hansen, London, I903. 
For a long time the name tsetse-fly was synonymous with one well-known species, Glossina morsitans, Westwood. It was this tsetse-fly which was suspected of playing the principal part in the propagation of nagana, and the attention of explorers was naturally drawn to this particular fly on account of the personal annoyance it produced, and loss of animals to which it gave rise. Later on, however, other species of the same genus, with habits similar to those of Gl. morsitans, were discovered, and writers have not always discriminated between these species of tsetse-fly. Thus, the name 'tsetse-fly' is now used to include all the species (Austen recognises eight) ${ }^{1}$ of the genus Glossina.

We shall first consider the genus Glossina as a whole, for it is highly probable that $G l$. morsitans is not the only species concerned

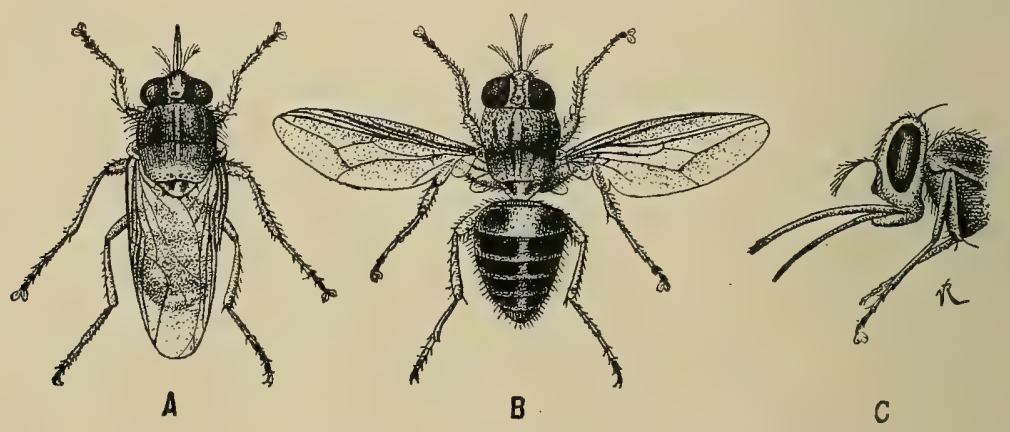

Fig. 77.-Glossina morsitans. (After. Bruce.)

A. Resting condition, with the wings completely overlapping. B. Fly with the wings extended $\left(\times 2 \frac{1}{2}\right)$. C. Anterior part of fly seen in profile.

in the propagation of nagana and the other African animal trypanosomiases. Moreover, it has been proved that it is another speciesGl. palpalis-which conveys the parasite of human trypanosomiasis.

The tsetse-flies are not very large in size, being a little larger than the ordinary house-fly (Musca domestica), and decidedly larger than Stomoxys calcitrans. Their appearance does not bear out their terrible reputation.

A tsetse-fly, when at rest, can be at once recognised by the wings, which almost completely overlap like the blades of a pair of scissors (see Fig. 77, A). In other flies which resemble the tsetse more or less closely (Stomoxys, Hamatopota) and, like it, suck blood greedily, the wings are always separated a certain distance when the flies are at rest. The proboscis of the tsetse projects horizontally in a line with the axis of the body (Fig. 77, c). This is also the case in Stomoxys, but in the latter the palpi do not ensheath the proboscis, as they do in Glossina, so that the proboscis appears thinner. The male tsetse is easily distinguished from the female by the external

${ }^{1}$ [The original gives the number as seven, but, as has been stated on p. 120, footnote 3 , Austen now recognises Gl. tachinoides as a distinct species.] 
genital organ, which forms a well-marked protuberance (hypopygium) on the ventral surface of the abdomen near the distal end. The females have no hypopygium.

The tsetse-fly is usually found in low-lying, hot, moist regions, such as the banks of rivers and lakes, and never occurs far from water. It is found in the vicinity of dense undergrowth or in forestsnever in open plains. It appears to have a predilection for the shade of certain trees or shrubs, such as the mimosa, as noted by Chapman in South Africa, and by Morel on the Shari. Occasionally it is met with on hills, as, for example, in North Transvaal. In North Rhodesia the tsetse has been found 4, IIo feet above the level of the sea. [In Uganda, which is about 4,00o feet above sea-level, tsetseflies abound along the shores of the Victoria Nyanza and on all the islands in the lake. The tsetse-fly, Gl.palpalis, has also been found recently on both shores (Congo Free State and Uganda) of the Albert Nyanza, about 2,400 feet above the sea.]

In the so-called 'fly belts' the tsetse is not necessarily universal.

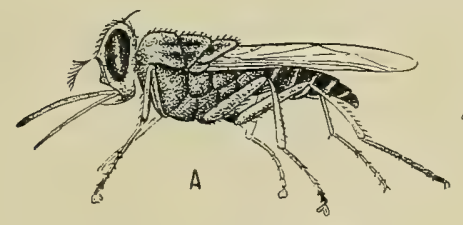

Fig. 78.-Glossina morsitans. (After Bruce.)

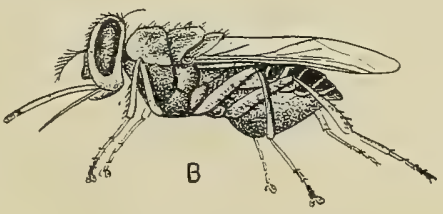

A. Fasting fly. B. Fly with its abdomen distended with blood after a feed. $\left(\times 2 \frac{1}{2}\right.$.)

It is often strictly localized to small areas which are thickly wooded, or where there is dense undergrowth, the intervening areas being quite free. Or a region may be infested with the tsetse except in glades or clear spaces, where it is never met with. It is, therefore, possible to travel with animals through such an infested region by travelling at night and spending the day in these glades. Sometimes the fly is found on one bank of a river and not on the other, as Livingstone described in the case of the River Chobe.

The fly bites most furiously during the day, less so in the evening, and only very rarely at night, when the moon is shining brightly. Both sexes are blood-sucking.

'On entering "fly country,", says Bruce, 'one is not long left in ignorance of the presence of the tsetse. The natives may be seen slapping their naked legs, the dogs bite round, and the horses kick.' In places covered with dense undergrowth one may be attacked by thirty or forty flies in the space of a few minutes.

The fasting tsetse 'has a direct flight, flopping, if I may use the term, suddenly on the animal attacked ' (Bruce), and, as the fly likes shade and hides itself under leaves, amongst the hairs of animals, and in the folds of the skin, it is not easily seen, but the effects of its bite quickly reveal its presence. 
' The fly makes a loud buzzing sound when flying, but after its feed and at rest it emits a peculiar sharp, shrill note, probably caused by an action of the wings' (Bruce).

The fly alights on the skin so gently that one does not feel it. According to Foà, the insertion of the proboscis is painless; Bruce says it is less painful that in the case of the mosquito; while others compare it to the sting of the bee or wasp. Occasionally, in as short a time as twenty or thirty seconds, the fly fills itself with blood (see Fig. 78, A and B), and the abdomen becomes first pink and then dark red. When the fly withdraws its proboscis after feeding, slight irritation at the place punctured is usually experienced.

A tsetse gorged with blood is lethargic and can be easily caught. As a rule, the fly quickly goes back to the undergrowth, where it hides and digests its meal in peace.

That the tsetse follows the big game is the unanimous opinion of explorers, travellers, and sportsmen who have been in Central and South Africa. There may be game without tsetse, says Theiler, but there is no tsetse without big game. The latter appears, however, not to be true in the case of British East Africa and Uganda, where tsetse-flies are found in the absence of big game ; but before attempting to explain this fact further observations upon this point are necessary to show conclusively that such is really the case. If true, it may be explained, for example, by the survival of the flies during a dry season which has caused all the big game to migrate from its ordinary haunts; or by the fact that the British East African species, to which the observation refers, are different from those found in South and Central Africa-Gl. morsitans, etc.

Unlike most of its allies, the tsetse avoids the excretions of animals, so that animals whose bodies are covered with a layer of excrement are thereby protected from the bites of the fly (Livingstone). As soon as one begins to disembowel an antelope covered with tsetse-flies the latter disappear (Foà).

Mode of Reproduction.-We are indebted to Bruce for a detailed account of the mode of reproduction of the tsetse. The tsetseflies-or at least some of them-belong to 'the group of parasitic flies which, on account of their peculiar mode of reproduction, have been termed Pupipara' (Austen). ${ }^{1}$ They extrude a yellowish larva, which is almost as large as the abdomen of the mother. The larva in the species studied by Bruce (according to Austen, this was not Glossina morsitans, but the closely allied species Gl. pallidipes, or possibly both) is about $6 \frac{1}{2}$ millimetres long, by about $3 \frac{1}{2}$ millimetres wide. It is annulated and consists of twelve segments. "Immediately on being born the larva creeps about actively, evidently searching for some cover or hole in which to hide. Having found a resting-place, it

1 [Of these the best known are the mammal and bird parasites belonging to the family Hippoboscida (Austen, op. cit., p. 24).] 
immediately begins to change colour, and after a few hours has turned into a jet-black hard pupa.' (For details of structure, see Fig. 79.)

'If these pupal cases are kept in a perfectly dry place, the perfect insect hatches out in about six weeks. ... It has often been surmised that this fly is bred in buffalo-dung, but from a consideration of the foregoing facts it is evident that nothing is wanted except any moderately dry place' (Bruce).

[It has been mentioned (see p. 4I3) that in Uganda Bagshawe has found that the natural habitat of the pupa of Gl.palpalis is the loose soil around the roots of banana-trees along the lake-shores.]

It is very probable that all the species of Glossina are pupiparous,
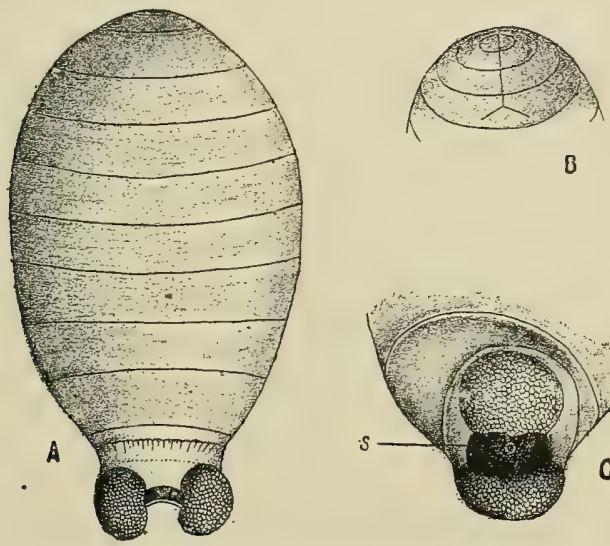

$B$

Fig. 79.-Pupa of Zululand Tsetse-Fly. (After Austen.)

A. Dorsal aspect $(x 8)$. B. Anterior extremity, showing bifurcated longitudinal seam which opens to permit the escape of the imago $\left(\times 5 \frac{1}{2}\right)$. C. Posterior extremity, showing pit and right larval stigma $s$ ( $X I 6)$.

but this has not yet been definitely established in the case of some species.

[The pupa of $\mathrm{Gl}$. palpalis varies in length from $5 \frac{1}{4}$ millimetres to $6 \frac{1}{2}$ millimetres, and in greatest width from 3 to $3 \frac{1}{3}$ millimetres. In general appearance it closely resembles that of the $Z$ ululand tsetse (see Fig. 79), but on closer examination slight differences are discernible. "The tumid lips on the last segment are much closer together in the pupa of Gl.palpalis than in the Zululand tsetse, the space between them being reduced by quite one half, while the lips themselves are somewhat larger, and covered with sparser, and therefore more conspicuous, granules' (Austen). Other minor points of difference are present, and Austen expresses the opinion that, did we but know them, all the species of tsetse-flies might be distinguished in the pupal stage by the characters of the last segment.]

[Amongst the recent publications upon the habits, anatomy, etc., of the tsetse-fly may be mentioned those of Austen, '

${ }^{1}$ [Austen, Brit. Med. Journ., September 17, 1904.] 
Notes on the Tsetse-Flies,' from which the above account of the pupa of Gl.palpalis is taken ; of Giles," 'Anatomy of Stomoxys and Glossina'; of Stephens and Newstead," "The Anatomy of the Proboscis of Biting Flies '; of Minchin, 'The Anatomy of the Tsetse-Fly (Gl.palpalis) '; of Sander, 'Die Tsetsen'; of Koch, ${ }^{5}$ and of Dutton, Todd, and Newstead. $]^{6}$

The tsetse-fly appears to be limited to tropical Africa. ${ }^{7}$ It is found between $13^{\circ}$ N. Lat.-in Senegambia, on the Shari, and possibly, if James Bruce really saw the tsetse, on the confines of the Sudan and Abyssinia-and $26^{\circ} \mathrm{S}$. Lat. Austen's map shows it to be particularly prevalent in the river-basins of East Africa, but that is because the fly is better known in these regions, to which the attention of explorers and sportsmen has been particularly drawn, especially the basin of the Zambesi. Nevertheless, recent accounts appear to show that the fly is no less prevalent in the river-basins of West Africa.

[Many of the recent observations on the distribution of the tsetseflies in various parts of Africa have already been referred to (see especially Chapters VI. and XII.). Amongst others, the following publications may be mentioned: that of Laveran, ${ }^{8}$ on 'Sleeping Sickness' and 'The Distribution of the Tsetse-Flies in Various Parts of French West Africa and the Congo Free State'-species of Glossina were obtained from Sénégal, French Guinea, French Sudan, French Congo, and the Congo Free State; of Major Smith, ${ }^{9}$ on the occurrence of Gl.palpalis in Sierra Leone; of Balfour and Neave, ${ }^{10}$ on the distribution of Gl. morsitans and Gl. palpalis in the Anglo-Egyptian Sudan, and the occurrence of the former as far north as $12^{\circ} \mathrm{N}$. Lat., in South Kordofan ; of Nabarro, ${ }^{11}$ Greig, and the later members of the Sleeping Sickness Commission, on the distribution of tsetse-flies, especially $G l$. palpalis, in Uganda and

1 [G. M. Giles, Journ. Trop. Med., v. 9, 1906.]

2 [Stephens and Newstead, Liverpool School of Tropical Medicine, Mem. I8, I906, pp. 53-74.]

${ }^{3}$ [Minchin, Proc. Roy. Soc., Series B, v. 76, 1905, pp. 53I-547; reprinted in Sleeping Sickness Commission Report, No. 8, 1907, p. 106.]

4 [Sander, Arch.f. Schiffs u. Tropenhyg., v. 9, I905, pp. I93, 254, 309, 355.]

5 [Koch, Deutsche med. Wochenschr., No. 47, November 23, 1905.]

6 [Dutton, Todd, and Newstead, in the Annals of Trop. Med. and Parasitology, Liverpool, v. I, No. I, I937, pp. 54-75.]

7 [Captain Carter (Brit. Lied. Journ., November 17, 1906, p. 1393) records the capture of tsetse-flies in South Arabia on October 10, 1903. The fly has been identified by Newstead as Gl. tachinoides, and Carter states that it 'does not depend for its existence on the big game, for, excepting gazelle, nothing else frequents the belts of bush which it haunts.]

[Carter's observation is very interesting, because it disproves the belief that the tsetse-fly does not occur beyond the African continent.]

8 [Laveran, C. R. Soc. Biol., v. 59, 1905, p. 332; C. R. Acad. Sciences, v. I39, 1904, p. 658 ; v. I40, 1905, p. 75 ; v. I4I, 1905, p. 929.]

[Smith, R.A.M.C. Journ., v. 5, 1905, p. 692.]

10 [Balfour and Neave, Second Report of the Wellcome Resiarth Labor., Khartoum, 1906, pp. 29-32, with a map; see also Brit. Med. Journ., June 17, 1905.]

11 [Nabarro, Greig, etc., Slecping Sickness Commission Ricports, Nos. 5, 6, and 8, 1905-1907.] 
the East Africa Protectorate; of Austen, ${ }^{1}$ on 'The Distribution of the Tsetse-Flies'; of Dutton, Todd, and Newstead, ${ }^{2}$ on the wide distribution of tsetse-flies in the Congo Free State, and the description of a supposed new species, which Newstead calls $G l$. maculata; of Carlos França, ${ }^{3}$ on a 'New Species of Glossina from

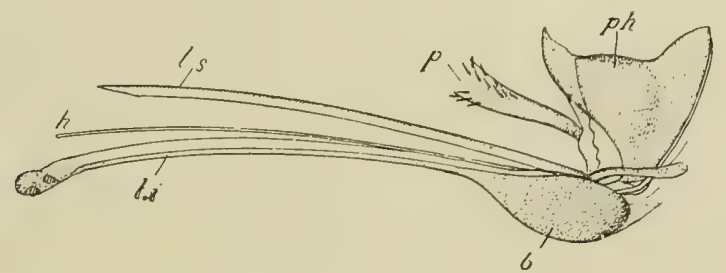

Fig. 80. - External Mouth Parts and Pharynx of Glossina morsitans. (After Hansen, in AUSTEn.)

l.i. Labium (inferior). h. Hypopharynx. l.s. Labium superior, or labrum. p. Basal part of left maxillary palpus. $\quad b$. First segment of the labium (bulb of the proboscis). p.h. Pharynx.

Cazengo, Angola, to the North of the River Coanza '; of Wellman, ${ }^{4}$ on 'Trypanosomiasis in Angola,' and the description of a new subspecies of Gl. palpalis; and of Yale Massey, ${ }^{5}$ on the occurrence

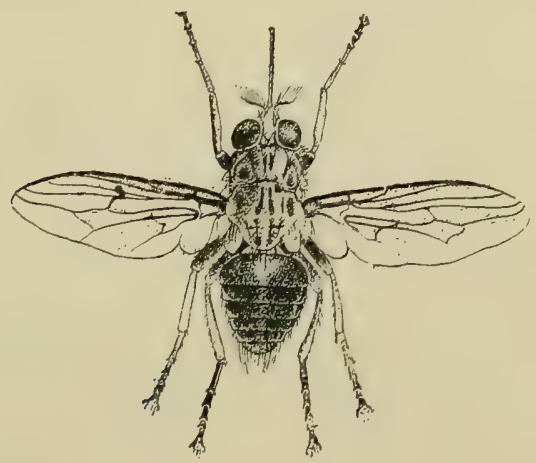

Fig. 8I.-GLossina PALpalis. (Afrer Austen.)

of sleeping sickness and of Gl. palpalis on the Lualaba River, Central Africa.]

[Lastly, reference has already been made to the interesting fact that tsetse-flies ( $G l$. tachinoides) have been found in South Arabia.]

The genus Glossina was created in 1830 by Wiedemann for his species Gl. longipalpis. It belongs to the order Diptera, or twomap.

1 [Austen, Sleeping Sickness Commission Report, No. 6, 1905, pp. 278-282, with

2 [Dutton, Todd, and Newstead, op. cit., 1907, pp. 54-75, with map.]

3 [C. França, Jornal de Sciencias Math., Phys., e Naturaes, 2nd Series, v. 7, No. 27, Lisbon, 1905, pp. 134-1 36.]

${ }_{4}^{4}$ [Wellman, Journ. of Hyg., v. 6, part iii., 1906, pp. 237-245; and Annals and Magazine of Natural History, September, 1906, pp. 242-244.]

[Yale Massey, Lancet, August 4, 1906, p. 296, and March 30, 1907, p. 908.] 
winged flies, and is a member of the family Muscida (used in the narrower sense), which comprises the genera Beccarimyia, Stomoxys, Hamatobia, Lyperosia, and Glossina, all of which are blood-sucking insects. By the venation of its wings, the ramification of the hairs of the arista ${ }^{1}$ the bulb at the root of the proboscis, the conformation of the male genital apparatus, and, lastly, by its pupiparity, the genus Glossina occupies a unique position among this group of insects.

Austen gives (op. cit., p. 60) the following diagnosis of the genus:

Glossina, Wiedemann, I830 (=Nemorhina, Robineau-Desvoidy, I830).-Narrow - bodied, elongate, dark greyish - brown or yellowishbrown, dull-coloured fies, ranging in size from $7 \frac{1}{3}$ millimetres $^{2}$ in the case of a small specimen of G1. morsitans, Westw., to I2 millimetres in that of a large female of G1. fusca, Walk.; recognisable when alive and at rest by the wings being closed flat one over the other above the abdomen (beyond which they project considerably), instead of divaricate (as in the case of Stomoxys), or tectiform (as in Hæmatopota), and by the proboscis (i.e., proboscis ensheathed in the palpi), which in length is equal to the thorax without the scutellum, projecting horizontally in front of the head; palpi, as seen in the natural position, extending slightly beyond the proboscis, their imner sides grooved so as to form a sheath for the latter, to which in life they are applied so closely as entively to conceal it; base of proboscis suddenly expanded beneath into a large onion-shaped bulb. (For details of the proboscis, see Fig. 8o.)

The dichotomous table ${ }^{3}$ (see p. 52I), borrowed from Austen, enables one to distinguish easily between the eight species of the genus, except perhaps between Gl. longipalpis and Gl. morsitans."

[The recently described new species (?) and subspecies are the following: Gl. decorsei (Brumpt), Gl. bocagei (França), Gl. maculata (Newstead), and Gl.palpalis wellmani (Austen).]

Glossina decorsei.-In Igo4 Brumpt $^{5}$ described, under the name of Gl. decorsei, a species of tsetse caught by Decorse in the Shari basin and on the shores of Lake Tchad. It is a small fly, the males measuring 7.33 millimetres and the females 8.27 millimetres in length. The colour of the abdomen is like that of Gl. morsitans : the median dorsal longitudinal band is well marked, dull yellow in colour, broad on the second segment, but getting gradually narrower on the third, fourth, and fifth segments, and only a narrow line on the sixth segment. The transverse interrupted stripes are very dark in colour and occupy the anterior four-fifths of each

1 The appendix to the terminal segment of the antennæ. This is the only visible part of the antennæ in the different figures of Glossina reproduced here.

${ }^{2}$ [Length measured from the face to the end of the abdomen, excluding the proboscis and wings (Austen).]

${ }_{3}$ [The table given in the original has been amplified so as to include the eighth species (Gl. tachinoides), now recognised by Austen, as well as a few minor details which he gives in his ' Revised Synopsis of the Species of Glossina' in the 'Supplementary Notes on the Tsetse-Flies' (Brit. Med. Joum., September 17 , I904), previously quoted.]

4 Austen says that the most characteristic distinguishing features between these two species are furnished by the hypopygium in the male. (See table on p. 52I.)

5 Brumpt, C. R. Soc. Biol., April 16, 1904, p. 628. 
segment, so that the extreme hind margin of each segment is pale or cinereous, and crosses the median longitudinal band at right angles. There are circular black patches on the second abdominal segment. The hind tarsi are black, which makes this new species allied to Gl.palpalis. Indeed, specimens caught by Decorse and presenting the above char. acteristics were examined by Austen, who stated that these flies were undoubtedly $G l$. palpalis var. tachinoides, Westwood. (Letters of April 8 and I3, I904.)

[Since then, however, Austen has had the opportunity of examining many more specimens of this fly, and has come to the conclusion that Gl. tachinoides, Westwood, is a distinct species. Moreover, 'an examination of some of Decorse's specimens shows,' says Austen, 'that the supposed new species ( $G l$. decorsei) is in reality none other, than Gl. tachinoides, Westwood.' $]$

[Glossina bocagei.-The following is the description given by França ${ }^{1}$ of this supposed new species, which came from Cazengo, Angola. Length of body, 8.5 millimetres; of specimens filled with blood, I I millimetres. Length of wing, 8 millimetres; wing-expanse, I 9 millimetres. Thorax, grey, the anterior segment lighter, with brown spots. Abdomen brown, with a longitudinal yellowish line well marked in the second, third, and fourth segments; less marked in the fifth, and hardly visible in the sixth and seventh segments. The posterior borders of the abdominal segments are not pale. The legs are amber-coloured, except the hind tarsi and the last two joints of the middle pair. There are no dark patches on the anterior tarsus. The third joint of the antennæ is uniformly yellowish; the second joint is brown.]

[Austen has examined the typical example of Gl. bocagei, França, and, after comparing it with the type of his Gl. palpalis wellmani, he has come to the conclusion that Gl.bocagei is merely a synonym of $G l$. palpalis wellmani. (Letter of September 25, I906.)]

[Glossina maculata (Newstead). ${ }^{2}$-This is the name given by Newstead to a fly (a somewhat imperfect specimen of a female) caught at Ghumbiri, on the Congo River. His original description is here reproduced.]

['Geneval Appeavance.-Very dark brown; posterior surface of head cinereous, spotted with black; thorax dark brown and faintly cinereous in places, with elongated transverse black spots; pleuræ, coxæ, and femora cinereous, with conspicuous black spots; first and second segments of hind tarsi black. (The remaining segments wanting.)]

['Female.-Head with the frontal stripe rich ochreous brown; frontal margins and ocellar spot bright yellowish-white to pale ochreous; ocelli black; posterior surface of head cinereous, with lavge well-defined irvegular black spots. Antennce dull red-brown in front, sides with a cinereous surface; arista dull reddish-brown. Palpi blackish above, paler beneath. Bulb of proboscis dark castaneous, shining. Thorax dark brown and cinereous, with numerous irregular elongated black spots placed transversely; anterior angles pale brown. The scutellum spotted like the thorax, margin pale brown; pleuræ cinereous and pale brown, with numerous irregular and more or less confluent black spots. Abdomen very dark brown; the narrow basal segment cinereous, with numerous black spots; the broad second segment, with the large median area, the anterior angle, and broad hind margin, cinereous with black spots; the remaining segments, with the exception of the last, with a narrow welldefined median stripe, and hind margins narrowly cinereous; lateral margins of segments three to five, with a cinereous triangular patch ; the hind margins with an occasional more or less obscure black spot, with the exception of the sixth, which has a regular series of ten or eleven on

1 [França, see footnote ${ }^{3}$, p. 517.]

2 [Newstead, Annals of Trop. Med. and Parasit., Liverpool, v. I, I907, p. 73.] 
the grey cinereous band; last segment darker than the rest, with a lateral greyish spot, in the centre of which is a small black one. Legs: All the coxæ cinereous and pale reddish-brown, with numerous more or less confluent black spots; hind femora, with the basal third and apex, pale redbrown, the rest faintly cinereous, with a few large, faint, dull brown blotches; anterior and hind tibiæ pale brown, mid tibiæ slightly darker, with, in some lights, faint indications of dark spots; anterior tarsi and first segment of the mid pale brown; first and second segment of the hind tarsi dark brown. (The remaining segments of the hind and mid tarsi are wanting.) Wings uniformly brown.]

['Length, exclusive of proboscis and with the abdomen somewhat curved, 9 millimetres ; length of wing, 9 millimetres.']

[Newstead states that 'this species may be readily recognised from the other species of the genus by the curious spotted or mottled appearance of the thorax, pleuræ, and femora. To the naked eye it looks like a dark specimen of $G l$. palpalis, but a pocket lens immediately reveals the peculiar markings.']

[It is possible that this supposed species, of which only a single specimen has hitherto been obtained, may eventually prove to be merely a variety of $G l$. palpalis, or, at the most, to be entitled to subspecific rank.]

[Gl. palpalis wellmani, Austen. ${ }^{1}$-This subspecies of Gl. palpalis was found by Wellman at Esupua, on the lower reaches of the River Katumbela, Angola. It has also been found by Massey on the Upper Congo, and we have already seen that Gl. bocagei, França, from the River Coanza, Angola, is regarded by Austen as identical with this subspecies of $G l$. palpalis.]

['The new subspecies may be characterized shortly as follows by indicating the points in which it differs from the typical form :

['Gl.palpalis wellmani (subsp. n., $\delta$ o ).--Frontal stripe pale ochraceous ; thoracic markings much reduced, so that the thorax in a wellpreserved specimen appears spotted, the antero-lateral markings taking the form of spots or blotches; the spot immediately behind the inner extremity of the humeral callus on each side small, ovoid, or nearly circular, and especially conspicuous when the insect is viewed from above and slightly from behind; femora pale, the dark blotches much reduced' (Austen).]

[Austen adds that the specimens caught by Wellman 'are of especial interest as being the first recorded examples of any form of Gl. palpalis from Portuguese West Africa, and as showing that the range of the species in question, which has recently been stated Ly Laveran 2 to occur at Sengaleam (about thirty miles from Cape Verde), extends at least as far south as $12^{\circ} 30^{\prime} \mathrm{S}$. Lat. In all probability $\mathrm{Gl}$. palpalis wellmani will eventually be proved to exist right down to the Cunene River, the southern boundary of Angola, if not further; and it is to be hoped that evidence to decide the southern limit of $G l$. palpalis will shortly be forthcoming.'. $]^{3}$

1 [Wellman, Journ. Hyg., v. 6, 1906, pp. 237-245; also Annals and Magazine of Natural History, September, 1906, pp. 242-24t. Also Austen, ibid., Series 7, v. I5, April, 1905, p. 390.]

2 [Laveran, C. R. Acad. Sciences, v. 139, 1904, p. 659.]

3 [Austen, Annals and Magazine of Natural History, v. I 5, April, 1905.] 


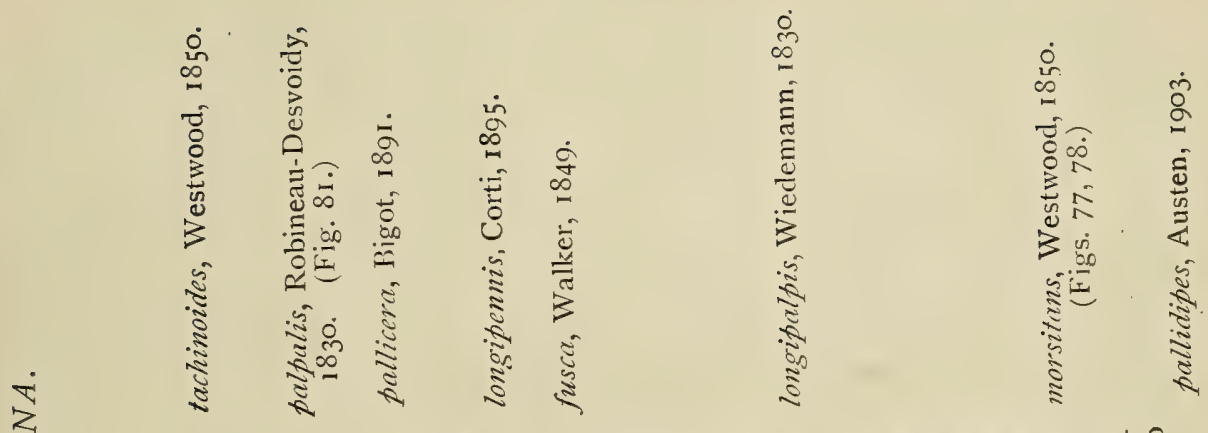

5

10

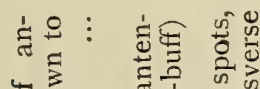

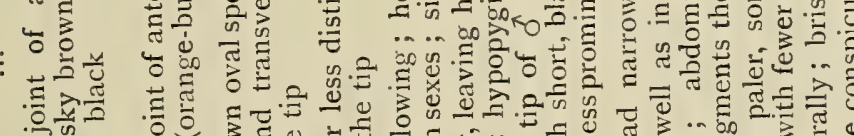

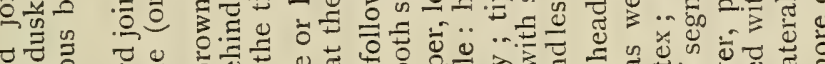

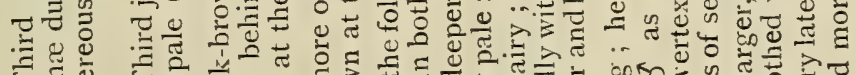

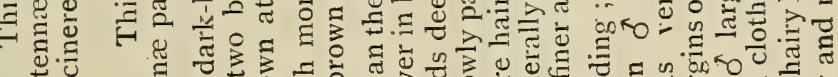

(I)

西

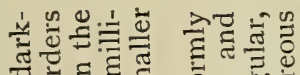

tris

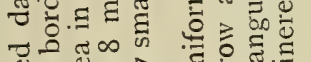
记

is

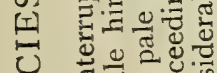

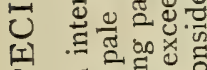

in

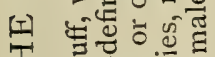

密 sicis

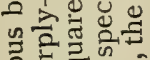

5 ○ क्ष

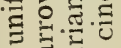
$+5$

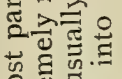
政 E

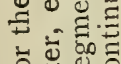

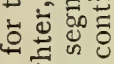
b.

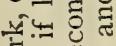

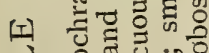
픈 年

至

4

$\rightarrow$ 政

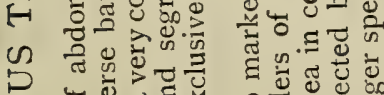

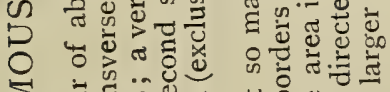

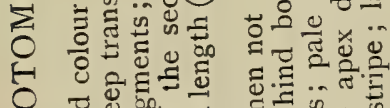

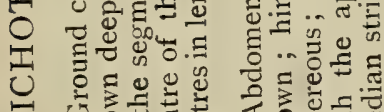

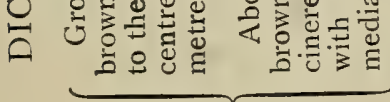

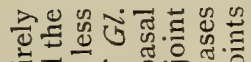

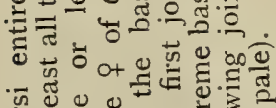

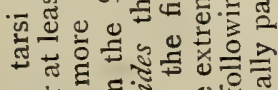

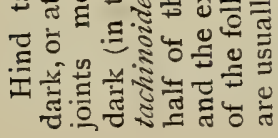

(n)

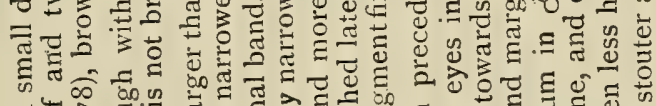

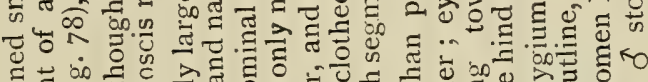

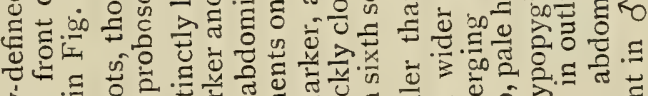
竞:

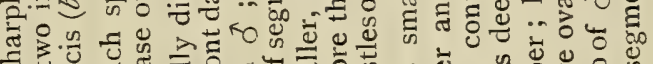

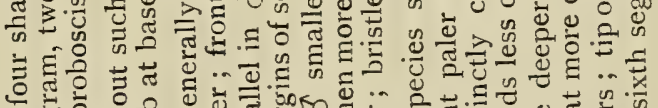

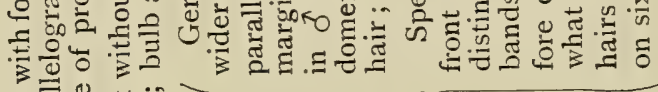

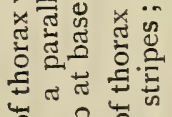

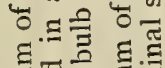
諘定

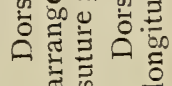
云市

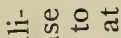
..를 g

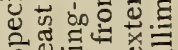
के 更

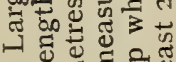

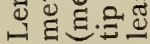

童竞: (5) ह 空言

을 政: 言. $3 \cdot \overline{\text { ज }}$ ज䎸

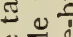
을 $\frac{d}{\sigma}$ 政 t. 줄 毫童产

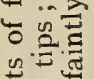

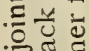
글

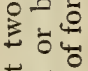
苛言总

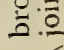

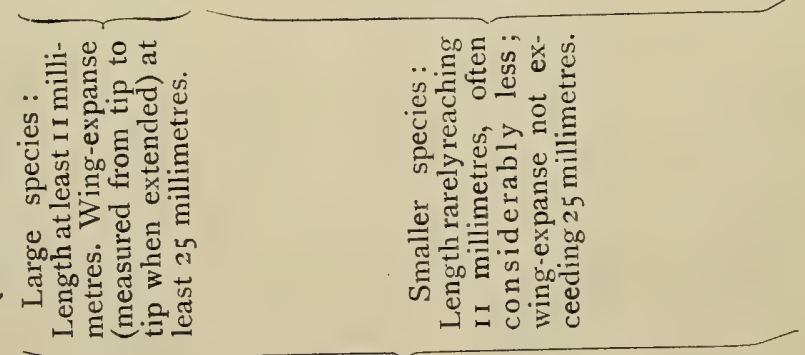

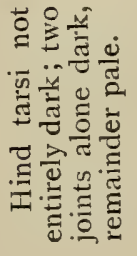




\section{N DEX}

Abbreviations : T. for Trypanosoma ; Tpl, for Trypanoplasma ; T-sis for Trypanosomiasis ; T-ses for Trypanosomiases.

Abramis brama, T. in, $48 \mathrm{I}, 483,489$

Tpl. in, 483,490

Abyssinia, T-ses of, 202

Abyssinian frontier, T-sis of, 207

Acanthias acanthias, $48 \mathrm{I}$

Acerina cernua, T. in, 483, 495

Tpl. in, 483

vulgaris, $T$. in, 480

Action of heat and cold on $T$. brucei, I $57-9$
$T$. dimorphon, 243
T. equinum, 305
T. equiperdum, 334-5
T. evansi, 275
T. lezvisi, 75-6
T. theileri, 348-9

Active immunity, production of, 90

Agelaius phoniceus, T. in, 44I

Agglomerated trypanosomes, virulence of, 84 vitality of, 84

Agglomeration of dead trypanosomes, 82 of $T$. levisisi, 80

of $T$, brucei, $16 \mathbf{I}$

Agglutinates, formation of, $8 \mathrm{I}$ morphology of, 8I

Agglutinating power, 87

Agglutination of trypanosomes, 30

Agglutinins, 8I

specific, 85

in different normal serums, 86

Aino of the Somalis, II 5,201

Alcyon, T. in, 440

Algeria, $\mathrm{T}$-ses in, 210

Ammodytes lanceolatus, 482 tobianus, 482

Amoba binucleata, 56 rotatoria, 3I, 460, 46I

Amomum melesueta, I85

Ancyromonas, 36

Anguilla vulgaris, T. in, 48I, 483, 490

Annam, T-sis of horses in, 286

Anopheles maculipennis, T. in, 37, 508

Antidorcas euchore, nagana in, I39

Arsenic compounds in treatment, 420. $V$. also Sodium arsenite

in dourine, 338

in human T-sis, $4 \mathrm{II}$

in surra, 282

Arsenious acid in caderas, 300

Arvicola arvalis, inoculations, 67, 126

Ascitic fluid, I74

Ass, Togo virus in, I97

Cameroon, $\mathrm{T}$-ses of, $20 \mathrm{I}$

dourine in, 318

Entebbe, $T$-sis of, $2 \mathrm{co}$

Masai, T-sis of Uganda, 206

nagana in, I5I

treatment of, $I 69,170$

Togoland, 193

T-sis of, $5,2 \mathrm{I} 3$

$\mathrm{T}$-sis of Abyssinian frontier, 207
Ass, $T$. gambiense, 382,390

Athene brama, $\mathrm{T}$. in, $44^{\circ}$ noctua, T. in, $43,440,445$

Atoxyl, I84

in treatment, $4 \mathrm{r} 6,420,434$

mode of administration, 436

toxic symptoms, 437

Atylotus nemoralis, $2 \mathbf{I 9}$

tomentosus, 2Ig

\section{Baacy-poy, 294}

Baboon, ' $\Gamma$-sis of Entebbe, 209

Bacteria, action of, upon T. infections, 418 isolation from, $\mathbf{r}_{4}$

Badger, $T$. of, IIo

Bagara (fish), T. in, 482,496

Bageus bayard, T, in, 482,496

Baleri, 226

Bandicoot, Indian, T. in, 67, I02

Barbel, Tpl. in, 495, 501 T. in, 479,483

Barbus fluviatilis, Tpl. in, 483, 501

T. in, 483,495

Bat and dourine, $33 \mathbf{I}$

and surra, 109, 266

T. of, IO7

Batrachia, mode of infection, 478

T. in, $I, 460$

Benzidine, formulæ, 427

dyes, treatment by, 4I5, 426

Beschälkrankheit, 312

Biliary fever (cattle), 343

Billet's staining method, 470

Biology of trypanosomes, 27

Birds, protozoon in, 2

T. in, $\mathbf{I}, 3,7, \mathbf{r}_{3}, 439$

cultivation of, $449,45^{2}$

geographical distribution, 439

morphology of, 443

Sudan, 455

table of dimensions, 447

T. of, historical review, 439

Blackbird, $T$. in, 442

red-winged, $\mathrm{T}$. in, 44I

rusty, $T$. in, $44 \mathrm{I}, 454$

Blackcap warbler, ' $T$. in, 440

Blennius montagui, 482 pholis, 482

T. in, 483,494

Blepharoplast, 2I, 52

Blood-counts in sleeping sickness, 376

Bluebird, $T$. in, $44 \mathrm{~T}$

Bodo musce-domestice, 32

Bodonace, 36

Bodonida, 37

Bombinator, 39 igneus, 465

Bombrix mori, 38

Borrel blue, 9

Bothus rhombus, T. in, 483, 495 
Bouet, cultivation of $T$., I3

Bouton d'Alep, 48

Bovidæ, galziekte in, 343 nagana in, 136 surra in, $257,28 \mathrm{I}$

Box boöps, T. in, 482 Tpl, in, 50I

Bradford and Plimmer's staining method, I2

Bream, T. in, $48 \mathrm{I}, 489$ Tpl. in, 483,501

Brill, T. in, $483,+95$

Bruce and Nabarro's method for blood examination, 398, 409

Buffalo, African, nagana in, $\mathrm{I}_{4} \mathrm{O}$ dourine in, 322 nagana in, 168 surra in, $248,250,258,282$ Togo virus, 197

Bufo calamita, 465 cinereus, 465 reticulatus, T. in, 464,477 viridis, $T$. in, 465 vulgaris, T. in, $46 \mathbf{I}$

Burra dhang (fly), 296

Bushbuck, naganz in, I40, I68

Buteo lineatus, T. in, $44^{\mathrm{I}}, 45^{8}$

Cachexial fever, $4^{8}$

Caderas, 292

animals susceptible to, 293

armadillo, 297

Ar vicola arvalis, 297

birds, 293

capybara, 293, 299, 308, 3II

cats, 204, 293, 300, 302

cattle, 292, 301, 307, 310

Cercopithecus fuliginusus, 297

coati, 292, 299

dog, 293, 298, 302, 304, 306, 308

donkey, 293

ducks, 293

Equidæ, 293, 302

fleas, 308

Erinaceus europeus, 297

fowl, 293,304

frog, 304

geographical distribution, 292 goat, 293, 295, 301, 306, 309 guinea-pig, 293, 300, 302, 304 hedgehogs, 293, 297

historical, 292

horses, 292, 293, 295, 302, 304, 307, 3II

Hydrochcerus capybara, 299

individuality of, 306 mice, 293, 295, 296, 297, 302, 303, $305,306,309,3^{10}, 43^{\mathrm{x}}$

mode of propagation, 307

monkey, 293, 297, 302

mule, 293

mulita, 297

Narica nasua, 299

Nyctipithecus felinus, 297

otter, 293, 297

$0 \mathrm{x}, 293,304,306,309$

pathogenic agent, 303

pathological anatomy, 303 pigs, 293, 301, 304, 306, 309

prophylaxis, 308

rabbit, $293,298,302,304$

rat, $293,296,302,304,306,308,309$, 3 II

sheep, 293, 296, 301, 304, 306, 309, 310

symptoms, 292

Tatusia hybrida, 297

treatment, $308,415-6,422,43 \mathrm{I}$ turkey, 293

Calentura, 251
Callionymus dracunculus, 482

T. in, 483,494

Callobdella punctata, T. in, 505

Camelidæ, surra in, 247, 26I, 28I

$\mathrm{T}$. in, 3, II 5

Somaliland, $\mathrm{T}$-sis, $20 \mathrm{r}$

Cameroon, T-ses of, 199

Canary and $T$. avium, $45^{\circ}$

Cantharus griseus, 482

Capybara, caderas in, 299. $\quad V$, also Caderas epidemic among, 307 and nagana, 169

Carassius auratus, 482 T. in, 488

vulgaris, T. in, 480,483

Tpl. in, 483

Carbocyclic compounds, formulæ, 427

Carp, experiments on, 504

Tpl. cyprini in, 500

Prussian, T. in, 480

Cat, caderas in, 300,302

nagana in, 128, I49, I69

surra in, 268

Togo virus in, 197

T-sis of Gambia in, 237

T. gambiense in, $3^{82}, 3^{87}$

Catoblepas gnu, nagana in, I40, I68

Cattle, Abyssinia, T-ses of, 202

caderas in, 301

nagana in, II2, II4, II7

serum in nagana, 180

surra in, 259,284

Togoland, I93

Togo virus, 197

T-ses of Cameroon, 200

T-ses of Sudan, 203

T-ses of Uganda, 204

T-sis in, 4, 5, 28, 29

'T-sis of Gambia in, 238

T. gambiense, 39r

ข. Bovidæ, etc.

Cebus capucinus and $T$. gambiense, $3^{82}$

Centrifuging blood, 7,398

Centrosome, I6, 2I, 5 I

Cercocebus fuliginosus, T. in, I29

Cercomonadince, 36,37

Cercomonas muscce-domestica, 32 muscarum, 32

Cercopithecus and T. gambiense, $3^{82}$

$\mathrm{T}$, in, 129

callitrichus, 228, 236

and $T$. gambiense, $3^{82}$

fuliginosus, 297 and $T$. gambiense, 382

ruber and $T$. gambiense, $3^{82}$

sabceus, 201, 203

schmidti, 366

Chaffinch, T. in, 440,442

Chelidon urbica, ' $\Gamma$. in, 442

Chimpanzee, $T$. gambiense, $3^{82}$

Choanoflagellata, 37

Chromatic body, posterior, 5 I

Chromo-therapeutic reactions, I9o, 426

Chrysoidin, in treatment, 204, 416, 434

Chrysophrys aurata, 482

Chrysops, 290

Chub, 48I

T. in, 483,495

Classification, Doflein's, 36

Koch's, of $T$. diseases, 187

Minchin's, 37

Coati, caderas in, 299

Cobitis barbatula, T. in, 48o, 48I, 483, 496 Tpl. in, $483,496,5$ or

fossilis, T. in, 480

Cabra venom, 159

Codonceca, 36

Colaptus auratus, T. in, 44I 
Conger conger, 482

Congo State, measures against $T$-sis, $4 \mathbf{r}_{4}$

Coracias garula, T. in, 440

Cottus bubalis, T. in, 483,494 gobio, T. in, 483,495

Tpl. in, 483,495 , 5 이

scorpius, 482

Cow, human T-sis, 366 surra in, $253,275,278$

T. in, 210

T. gambiense, 382

Crane, T. in, 439

Crenilabrus melaps, 482

Creolin in nagana, 185

Cricetus frumentarius, $T$. in, 105

Crithagra, T. in, 439

Crithidia, 37, 508

fasciculata, $37.3^{8}, 5^{1}$

minuta, 39

Crocodile, hæmogregarine in, $45^{8}$ T. in, $45^{8}$

Cross-immunization experiments, 187

Crow, 442

Culex fatigans, 37 pipiens, $37,45^{6}$

Cultivation of Drepanidium, 470 of $T$. duttoni, 13,100

of $T$. padda, $45^{2}$

of $T$. rotatorium, 470

Cultural characteristics, I90

Cultures of T., I3. 30

of $T$. brucei,

of $T$. danilewskyi, 489

of $T$. equiperdum, 335

of $T$. evansi, 275

of $T$. gambiense, 405

of $T$. granulosum, 49ז

of $T$. levisi, 13,76

of $T$. paddce, 449

of $T$. rotatorium, 470-3

Culture medium, 13, I6I, 306, 452

Cyanocitta cristata, $\mathrm{T}$, in, $44 \mathrm{I}$

Cynocephalus babuin and T. gambiense, $3^{82}$ sphinx, 236 and T. gambiense, 382

Cyclopterus lumpus, Tpl. in, 5 or

Cyprinus carpio, multiplication lorms in, 502 T. in, $480,483,488$ Tpl. in, 483

Dabib (fish), T. in, 482

Damonia reevesii, T. in, 457

Debab, el, in dromedaries, 186,218

Delhi boil, 48

Diemyctulus viridescens, T. in, 464, 477

Diplosome, 24, 498

Diplostreptococci in human T-sis, 405

Disagglomeration, 84

Dofleın's classification, 36

Dog, arsenious acid in, 424

caderas, 298, 302, 306, 308

dourine, 322-4I

galziekte, 345

mbori, 220

nagana, III, I27, I39, I49, I5 I, I83

serum treatment, I74

Soemedang, maladie de, $34 \mathrm{I}$

surra, $254,258,261,262.4,272,279,280$

Togoland, 193

Togo virus, 197

treatment of nagana, 170

T-sis, 8, 29, 67, 212, 216 of Gambia, 236-7

T-ses of Abyssinia, 202

of Abyssinian frontier, 207

of Cameroon, 200

of Entebbe, 209

of Sudan, 203

of Uganda, 204
Dog, $T$, equiperdum, 332-4I, 425

T. gambiense, 382, 424

tsetse-fly, 165

Dogfish, T. in, $48 \mathrm{r}, 492$

Dog-tick, T. in, 508

Donkey, dourine in, 3 I $3,317-8,335,340$

in Togoland, I93

Masai, T-sis of Uganda, 206

nagana, I5I, I69, I70

surra, 256,279

$\mathrm{T}$, in, $5,2 \mathrm{r} 3$

T. gambiense, 382,390

T-sis of Abyssinian frontier, 207 of Entebbe, 209

Dormouse, $\mathrm{T}$. in, I07, I32

Dourine, $5,15,48,312$

acute, 316

animals susceptible to, 312,322

buffalo, $330,33 \mathrm{I}, 34^{\circ}$

cow, 33I

dog, 322, 330-6, 339, 340, 34I, 4I6, 425 donkey, 3I3, 3I7, 3I8, 335, 340

Equidæ, 314, 320. $\quad V$. also Donkey and Horse

goat, $330,331,336,339,340$

guinea-pig, 331

horse, I5, 3I2-7, 320, 323, 328, 330, $332-3,336-8,340,34 \mathrm{r}$

mouse, $322,327 \cdot 30,332-6,339$

monkey, 330, 33r, 336

rabbit, $322,325,326-7,329,336$, $33^{8-9}, 34 \mathrm{I}$

rat, $322,327-30,336$

ruminants. 330,336

sheep, 330, 336, 339

chronic, $3^{\text {I } 4}$

experimental, 317,322 . $V$. also under

Treatment

flies, 336,337

geographical distribution, 312

historical, $312-4$

immunity, 338,340

individuality of, 335

man, supposed case of, in, 331

mode of propagation, 337

pathogenic agents, 332-5

pathological anatomy, $320-2$

plaques in, 315

prevention, 339

prophylaxis, $33^{8}, 34^{\circ}$

refractory animals, $33 \mathrm{I}$

bats, $33 \mathrm{I}$

birds, $33 \mathrm{r}$

cat, 33I

cold-blooded animals, 331

fowls, 331, 339

frogs, $33 \mathrm{I}$

lizard, $33 \mathrm{I}$

pigeon, 33I, 339

snakes, $33 \mathrm{I}$

sparrows, 33 I

serum-therapy, 339

symptoms, 3I 4

treatment, $338,416,422,425$

Dove, Arrican, T. in, 442

mourning, $T$. in, 44I

Drepanidium, 45

cultivation of, 470

ranarum, 463

Dromedary, T-sis of, $5,214,219$

Dryobates villosus, T. in, $44^{1}$

Dum-dum fever, $4^{8}$

Ectoplasm, 20

Eel, experiments on, $50_{3}$

T. in $48 \mathrm{I}, 483,490$

Egret, T. in, 442

El debab, 2 I 4

Elephant, surra in, 26r 
Eliomys quercinus, $\mathbf{2} 23 \mathbf{I}_{32}$

Emberiza citrinella, T. in, 442 and $T$. avium, 450

Emys leprosu, 475 $T$. in, 458

Entebbe, T-sis in, 208

Equidæe, caderas in, 293, 302 surra in, 254

T. in, $2,3,58, \mathbf{r}_{32}$. V. also Ass, Donkey, Horse

Erythrea, T-ses of, 202

Esox lucius, T. in, 479,480 multiplication forms in, 502 Tpl. in, 483

Estrelda estrelda, T. in, $440, \cdot 445$ cinerea, 450

Etymology, I

Examination of $T$. in living state, 7

Experiments on carp, 504, 505

on eel, 503

on pike, 503

on red-eye, 503

on river bull-head, 505

Euglenoidina, 37

Field-mouse, T. in, $1,58,67, \mathrm{I}_{32}$

Filaria, 44I, 463

nocturna, 409

perstans, 353,398

Finsen rays, $4 \mathrm{rg}$

Fishes, technique of examination of, 484

Tpl. of, description, 496

T. of, I, 2, 3, 7, I8, 25-8, 33, 479

description, 485

modes of infection, 502

preservation of, 484

Flagellates, position of T. amongst, 35

Flagellosis of Equidæe, 292

Flagellum, I6, 22

Fleas, $T$. lewisi in, 88

Flesus vulgaris, T. in, 483, 493

Flicker (bird), T. in, 44I

Flounder, T. in, 483, 493

'Fly belt,' 407

Fly, dourine, 336, 337 experiments, 190

Formulæ, benzidene, 427

Fowls and dourine, 33I nagana, I40

Fox, nagana in, 130

French Guinea, T-ses of, 226 Sudan, T-ses of, 219

Fringilla carduelis, T. in, $44^{\circ}$ coelebs, $440,442,45^{\circ}$

Frog, dourine, 331 hæmogregarines of, 475 mode of infection, 478

serum, 473

green, T. of, $46 \mathrm{I}$

Hong Kong, T. of, 463,476

$\mathrm{T}$. of, $\mathrm{I}_{3}, 3,8, \mathrm{I}_{3}$

T. inopinatum, 462,473

T. nelspruitense, 463,475

$T$. rotatorium, 20,2 I, 28,3 I, 457,465

Gadus luscus, 482 pollachius, 482

Galeus galeus, $48 \mathbf{I}$

Gall-sickness, 343

Galziekte, 5,343

course of, 346

and $\mathrm{dog}, 345$

geographical distribution, 343

goar, 345

guinea-pig, 345

historical, 343

horses, 345

mice, 345

mode of propagation, $35^{\circ}$
Galziekte, pathogenic agent, 347 pathological anatomy, 347 peculiar to Bovidæ, 344 prophylaxis, 35I

rabbits, 345

rats, 345

symptoms; 346

treatment, 35

Gambia, T-sis of horses in, 229

course of the disease, 230-40

in birds (insusceptible), 239

in cats, 237

in cattle, 238

in dogs, 236

in goats, 238,244

in guinea-pigs, 235

in horses, 230

in mice, $234,43 \mathrm{I}$

in monkeys, 236

in rabbits, 235

in rats, 2.33

in sheep, 239

geographical distribution, 229

individuality of, 243

mode of propagation, 244

pathogenic agent ( $T$. dimorphon $), 240$

pathological anatomy, 240

treatment, $245,43 \mathrm{I}$

Game, big, destruction of, 185

Gargur (tish), T, in, 482,496

Gasterosteus aculeatus, 48I

Gecko, T. in, $45^{8}$

Geese, T. in, I40

Togo virus, 197

Geisselwurzel, 52

Geographical distribution, caderas, $2 \varsigma 2$ dourine, 312

human T-sis, 359

nagana, I $\mathbf{I} 3$

surra, 246

T-ses, I

T. dimorphon, 229

$T$. lewisi, 59

Gerbil and T-sis of Sudan, 204

Gerbillus pygargus, 204

Giaàn in Erythrea, 202

Giemsa's solution, ro, 13

method, modified, 470

Gland puncture in human T-sis, 370, $40 \mathrm{I}$

Glossina, 186,518

bocagei, description, 5 r 9

decorsei, $\mathbf{1 2 0}$

description, 518

diagnosis.of, 518,521

dichotomous table of species, 521

fusca, 156, 167, 192, 208, 228, 408, 521

longipalpis, II6, I99, 228, 521

longipennis, II5, I66, I67, 20I, 408, 521

maculata, 5I7

description, 5 I 9

morsitans, $116, \quad \mathbf{5} 6, \quad \mathbf{6 6 - 7}, \mathbf{1 9 2}, \mathbf{1 9 9}$ $228,364,406,52$ I

description, $5 \mathrm{I} 2$

pallicera, $5^{21}$

pallidipes, 167, 192, 205, 408, 514, 521

palpalis, 204, 208-9, 228, 244, 36r, 369, $406,508,5 \mathrm{I} 2-3,5 \mathrm{I} 5,5 \mathrm{I} 7,521$

in human $\mathrm{T}$-sis, 407

pupa of, $5^{15}$

wellmani, $362,408,519$

description, 520

tachinoides, I17, 120, I99, 228, 517, 521

Goat, caderas, 301,306

dourine, 217,322

galziekte, 345

mbori, 221

nagana, I38, I5I, 177

serum, $\mathbf{I} 77$ 
Goat, surra, 269, 270, 275, 277

Togo virus, 197

$T$-sis in, $67,238,244$

of Abyssinia, 202

of Abyssinian frontier, 207

of Cameroon, 200

of Entebbe, 209

of French Guinea, 227-8

of Uganda, 206

$T$. dimorphon, 238,244

T. gambiense, 382,389

Goat-sucker, T. in, 439

Gobio fluviatilis, T. in, 483, 495 gobio, $48 \mathbf{I}$

Gobius, 482 giuris, $\mathrm{T}$. in, 483

niger, $\mathrm{T}$. in, 483,494

Goebel on osmosis, 27

Goldfinch, T. in, 440, 454

American, $T$. in, 44I

T. laverani in, 454

Goose and $T$. avium, $45^{\circ}$

Togo virus, 194

nagana in, $140,18 \mathrm{I}$

Gray and Tulloch's method, 12, 472

Green, brilliant, in treatment, 434

Gudgeon, 48I

T. in, $479,483,495$

Guinea, French, T-ses of, 226

Guinea-pig, atoxyl, treatment with, 42 I

caderas, 300,302

dourine, $34 \mathrm{I}$

el debab, 216

galziekte, 345

immunity, 9I

mbori, 220

nagana, I3I, I49, I5I, 42I

Soemedang, maladie de, 34I

surra, 266-7, 27I, 275-6, 280, 422

Togo virus, 197

T-sis of, 105,216

T. dimorphon, 235

T. gambiense, $382,387,421$

T. lezvisi, 20, 66, 72, 86, 9 I

T-sis, Abyssinia, 202

Aby $=$ sinian frontier, 207

Entebbe, 209

Uganda, 206

Gunnelus vulgaris, 482

Hamameba danilewskyi, $44^{8}$

ziemanni, 43

Hamatobia, 2 I9

Hamatomonas, 33

Hematomonas carassii, 480

cobitis, 480

Hamatopinus spinulosus, T. in, 88

Hematopota, 290

Hæmatozoa, intracorpuscular, of reptiles, 2

Hæmocytozor, 42

Hæmoflagellates, I9

Hamogregarina bagensis, 475

balfouri, 38

bigemina, 494

blanchardi, 494

bothi, 495

callionymi, 494

cotti, 494

flesi, 494

gobii, 494

laterne, 494

platessce, 493

quadrigemina, 484

simondi, 49I

splendens, 474

Hæmogregarine in crocodile, $45^{8}$

in frog, 475

Hamoproteus danilewskyi, 44I majoris, $44 x$
Hamoproteus siemanni, 44I

Halberstaedter's method, 13

Halteridium, 43, 45, 454

noctuce, 53

Hamster, T. in, I05

Hapale pencillatus, T. gambiense in, 382

Harporhynchus rufus, T. in, 44I

Hawk, T. mesnili in, $44 \mathrm{r}$ red-shouldered, $T$. in, 44 I

Heat and cold, action of, on trypanosomes, $V$. Action of heat and cold

Hedgehog, caderas in, 297 nagana in, 126 T. gambiense, 382,389

Heidenhain's stain, II

Helobdella algira, T. in, 474

Hemiclepsis, 495. 505 marginata, 504

Herpetomonas, $25,32,36,37,508$

algeriense, 37

tombycis, $3^{8}$

biitschlii, 99

jaculum, 37, 39

lezvisi, $5^{8}$

musce-domestica, 38, 5II

subulata, 38

Herpetosoma, 34

Hippobosca, 202, 225, 228, 290 maculata, 35I

rufipes, 350

Hirudo meducinalis, Tpl, in, 507 trociina, T. in, 505

Hirunas rustica, T. in, 442

Historical account of T., I survey of the genera, $3^{1}$

Horse, caderas, 292-3II dourine, 48,3 I 2

galziekte, 345

mbori, 222

nagana, 28, III, I5I, I70

serum, 177

surra, $247^{-85}$

Togoland, 193

Togo virus, 197

$T$-sis in Algeria, 2 Io

Horses, T-sis in Abyssinia, 202

in Abyssinian frontier, 207

in Annam, 286

in Gambia, 229

in Somaliland, 20

T. gambiense, 382,390

and tsetse-fly, 165

House-martin, T. in, 442

House-sparrow, 442

Human serum in nagana, I77

Human trypanosomiasis, 352

blood-counts in, 376

cerebro-spinal fluid, 399

complications, $38 \mathbf{r}$

Congo State measures, 4I 4

description of, 369

diagnosis, 409

duration, $38 \mathrm{I}$

Glossina palpalis, 407

fish-eating in, 369

geographical distribution, 359

gland puncture in, 370,401

historical, 352

influence of age, occupation, race, sex 366

lumbar puncture in, 399

mode of propagation, 406

pathogenic agent, 398

pathological anatony, 391

predisposing causes, 366

prognosis, 4 ro

prophylaxis, 4II, 4I3

skin eruptions in, $37^{8}$

treatment, 4II, 433 
Hyæna and nagana, 168

Hydrocherus capybara, 299, 307

Hyla artorea. T. in, 462,469 lateristriga, T. in, 464,469 viridis, $\mathrm{T}$. in, 46I

Icterus galbula, T. in, $44 \mathrm{I}$

Immunity, active, mode of production, 90 if inherited, 9.o

passive, 93,96

Immunization, results of passive, 97

Immunizing serum, nagana, 178

Incubation period, nagana, 15

Indian squirrel, T. in, 98

Infection, mode of, in Batrachia, $47^{8}$

in caderas, 307

in dourine, 337

in fishes, 502

in human trypanosomiasis, 406

in nagana, 165

in surra, 278

natural in rats, 88

Infectivity of trypanosomes, 28

Inoculation experiments, $\mathbf{1}_{5}, \mathbf{1} 87$

Isolation of trypanosomes, I4

Ixodes testudinis, T. of, 457

Jackal and nagana, 169

and $T$. gambiense, 382,386

T-sis of Entebbe, 209

Jackdaw, 442

Jaculus gordoni, and T-sis of Sudan, 204 orientalis, 266

Jaundice of cattle, 343

Java sparrow, T. of, 44I

Jay, blue, T. in, 44I, 454

Jenner's stain, Io

Jerboa, surra, 266 T. gambiense, 382,389

Jinja disease, 205,344

Kachuga tectum, T. in, $45^{8}$

Kala-azar, 48, 5I

Karyosome, 5I

Kinetonucleus, 2 I

Kingfisher, T. in, 440

Kite, Indian, T. in, 442

Koch's classification of $T$. diseases, 187

Koodoo, nagana in, 140 , I68

\section{Labrus, 482}

Lagonosticta minima, $45^{\circ}$

Lark and T., 446

Laniarizes cruentus, $\mathrm{T}$. in, 442

Laveran's staining method, 9

Leech, morbid phenomena, 507

Tpl. in, 499, 504

$\mathrm{T}$. in, 474,504

Leishman-Donovan body, Io, 48 development of, 49 staining method, IO, I2

Leishmania donovani, $5 \mathbf{I}$ tropica, 5 I

Lemur mongoz and $T$.gambiense, $3^{82}, 3^{8} 3$ rubriventer and $T$. gambiense, 382

Lepadogaster gouanii, 482

Leptomonas, 36

Lepus cuniculus, T. in, IO4

Leuciscus, T. in, 483, 495 cephalus, $48 \mathrm{I}$

T. in, 483,495

erythrophthalmus, T. in, 483

Tpl. in, 483

idus, T. in, 483

Tpl. in, 483

rutilus, $48 \mathrm{I}$

T. in, 483

Tpl, in, 483

Leucocytozoon ziemanni, 43
Lice, $T$. lewrisi in, 88

Light, action of, after injection of colouring agents, $4 \mathrm{IO}$

Limanda platessoides, T. in, 483, 494

Lingard on measurements, I7

Lissoflagellata, 37

Lizards and dourine, 33I

T. in, $45^{8}$

Loach, T. in, $480,48 \mathrm{I}, 483,496$

Tpl. in, 483,501

Lophius piscatorius, 482

Lota molva, 482

vulgaris, T. and Tpl. in, 483

Lote, T. in, 479

Loxia coccothraustes, 439

Lumbar puncture, 399

Lynodontis schal, 482,496

Mabuia raddonii, T. in, $45^{\circ}$

Macacus, inoculations, 68 cynomolgus, inoculation, $33 \mathrm{I}$ and $T$. gambiense, $382,3^{8} 3$ rhesus, 129, 236, 382, 383, 417 sinicus, 424

Macrones cavasius, T. in, 483 seenghala, T. in, 48I tengara, T. in, $48 \mathrm{I}$

Macronucleus, 20

Mal de caderas, 5, 292

Mal du coït, 5,312

Mal de la Zusfana, 2 I I

Maladie de Soemedang, 34I

Malaria of cattle, 343

Mammals, small, trypanosomes of, 98

Manioc root, 353

Marino's stain, II

Mariposa phonicatis, 450

Marmoset and T.gambiense, 382

Marmot and $T$. gambiense, 382,389 and nagana, I26

Masai donkey, immunity of, 192

Mathis' medium, I4

Mbori, 202, 219

dog, 220

dromedary, $\mathbf{x} 86$

guinea-pig, 220

horse, 222

rabbit, 220

rat, 220

sheep, 221

treatment, 222, 4I6, 423

McNeal's method, cultivations, I3

Measurements, Lingard on, I7

Meles taxus, T. of, Iто

Melophagus ovinus, $3^{8}$

Melospiza fasciata, T. in, 44 I

Membrane, undulating, 22

Merula merula, T. in, 442 migratoria, $T$. in, $44 \mathrm{I}$

Method for staining, etc., 7

Billet's, 470

Bradford and Plimmer's, I2

Bruce and Nabarro's, 398, 400

Giemsa's solution, I0, I3 modified, 470

Gray and Tulloch's, I2, 472

Halberstaedter's, I3

Heidenhain's, II

Jenner's, Io

Laveran's, 9

Leishman's, I0, I2

McNeal and Novy's, $r_{3}$

Marino's stain; II

Mathis' medium, I4

Ross's, I2

Ruge's, I2

Tulloch's, I2

Methylene blue in treatment, 433

Micronucleus, 5I 
Milvus govinda, T. in, 442

Mimosa polyacantha, 120

Minchin's classification, 37

Miniopterus schreibersii, T. in, I07

Minnow, T. in, 483, 495

Tpl. in, 495,496

Mole, T. in, I, 58, Iog, Iro

Monadidea, 37

Monas rotatoria, frog, $46 \mathrm{t}$

Monkey, arsenic treatment, 434

atoxyl treatment, 421

caderas, 297, 302

dourine, $322,33 \mathrm{I}$

experimental infection, treatment of, 432

Gambia, 236

human $T$-sis in, 366

nतgana, $\mathbf{2} 29$

serum, I77

surra, 266

treatment, 432

T-sis of Abyssinian frontier, 207 of Entebbe, 209

of Sudan, 203

of Uganda, 206

$T$. gambiense, $35^{8}, 3^{82}, 421$

Morphology of trypanosomes, of agglutinates, 81

of birds, 443

comparative, I6

general, $\mathbf{6} 6$

T. brucei, $\mathbf{1} 50$

$T$. duttoni, Ioo

T. of frog, Hong Kong, 476

$T$. rotatorium. $47 \mathrm{I}$

$\mathrm{T}$. in French Sudan, 225

T. in tsetse-fly, ${ }_{5} 6$

T. vivax, 200

'Morular' mass, 444

Mosca brava, $3 \circ 7$

Mosquito, T. in, 43,508

Motella mustela, 482

tricirrata, 482

Mouse, benzidene dyes in, 426

caderas in, 295, 297, 302-3, 305, 309, $43 I$

colouring agents, 4I9

dourine, 322,327

experimental T-sis, 428, $43 \mathrm{I}$

galziekte, 345

mbori, 220, 222

nagana, I23, I25, I32, I 5I, I73, 428

serum treatment, ${ }^{7} 74$

surra, $265,275,431$

Togo virus, 197

T-sis, 99, 211, 21 5,228

$T$. dimorphon, 234

T. gambiense, 382,388

Movement of trypanosomes, 27

Mugil, T. in, 482,496

Mukebi, 204, 205

Mule, surra in, $247,252,279,280$

T-sis in Entebbe, 208

T-ses of Uganda, 204

Mulita, caderas in, $\approx 97$

Mullus surmuletus, 432

Mus decumanus, 58, 60, 85, I25

giganteus, I02

minutus, IOI

musculus, 60, 99

niveiventer, 60

raitus, $5^{8}$

rufescens, 58, 60

spicatus, IO2

sylvaticus, 58, 67, 123, 132

Musca domestica, 512

Mustelus canis, $48 \mathbf{I}$

Myocyte layer, 27

Myonemes, 24, 27

Myotis murinus, 109, 217
Myoxus avellanarius, T. of, Io7 glis, 107

Nagana, 2, 4, 5. II I

ætiology, I64

animals susceptible to, I2I

Bovidæ, 136

cat, I28, I49, I69 cattle, II2, II4. II 7

diseases allied to, 185 dog, III, I27, I5I, I 83

donkey, I5I

Equidæ, I32

fox, 130

geographical distribution, II3

goat, I38

goose, I8I

guinea-pig, I3I

hedgehog, 126

historical, III

horse, 28, III, II8, I5I

human serum, I76

inmunizing serum, I $^{8} 8$

incubation period, 15

koodoo, I40, I68

monkey, I29

mouse, I23, I25, I32, I5I experimental, 428-3I

pathological anatomy, 140 pig, I5, I35, I42, I59

prophylaxis, 178

rabbit, 130

rat, I23, I25, I4I, I5I

serum-therapy, I74

sheep, II2, I38, I5I

squirrel, I30

treatment, I69, 4I7-9, 42I-2, 428-3I

Narica nasua, 299

Necturus maculatus, 463, 477

Negro lethargy, 359

Neophron percnopterus, 442

Nepa cinerea, 39

Nerophis lumbricoides, 482

Nesokia providens, 67, 102 giganteus, 102

Newt, T. in, 464,477

Nicticorax gardenia, T. in, 442,455

Nile fish, T. in, 482

Noke (fish), T. in, 482,496

Novy and McNeal's method for cultures, I3

Nucleolus, $5 \mathbf{I}$

Nucleus, 16, 20

Nutria, 297

Nutrition of trypanosomes, 27

Nyctipithecus felinus, 297

Oicomonadacece, 36

Oicomonas, 36

Ookinete, 42

Opiocephalus striatus, T. in, $48 \mathbf{x}$

Oriole, Baltimore, 'T, in, $44 \mathrm{I}$

Orthagoriscus mola, 482

Osmosis, Goebel on, 27

Osmotic properties of trypanosomes, 27

Otter, caderas in, 297

Owl, T. in, $439,440,443$

$T$. noctuce, 24

Ox, T-sis of Abyssinian frontier, 207 of Entebbe, 209 of Uganda, 206

Padda oryzivora, 448 T. in, $44 \mathrm{I}$

Pagellus centrodontus, 482 erythrinus, 482

Pantastomina, 37

Paradoxurus, 288

Paramaba eilhardi, 56 
Paramecioïdes, 33, 46r costatus, $46 \mathbf{I}$

Paramacium costatum, 3I, 460 loricatum. 3I, 460

Passer domesticus, T. in, 44I and $T$. avium, $45^{\circ}$

Passive immunity, 93 mode of production of, 96 results of, 97

Pelobates fuscus, 465

Perca fluviatilis, 480, 483, 495

Perch, T. in, $479,483,495$

Periplast, 20

Peste de cadeiras, 292

Petrie, cultivations, I3, IO4

Pheasant, T. in, $44^{2}$

Phoxinus levis, T. in, 483, 495, 496 Tpl. in, 489

Phyllomonas, 36

Phytoflagellata, 37

Pig, caderas in, 301 nagana in, $I_{5}, I_{35}, I_{42}, I_{59}$

Togo virus, I97

T-sis of, 213

T. gambiense, 390

Pigeon, dourine, 33I, 339

T. in, 440

and $T$. avium, $45^{\circ}$

Pike, experiments on, 503

T. in, $479,48 \mathrm{r}, 485$

T. remaki, in, $2 I$

Pipistrellus pipistrellus, T, in, 108

Piroplasma canis, 508 aonovani, $5 \mathbf{I}$

Piroplasmosis, bovine, 343

Piscicola geometra, 499, 504, 505

Pithecia satanas and 1. gambiense, 383

Placobdella ca'enigera, 475

Plaice, T. in, 493

Platessa microcephala, 482 vulgaris, $482,483,493$

Platophrys laterna, T. in, 483, 494

Platytheca, 36

Pleuronectes, 482 Resus, 482, 483, 493

P'immer's method, I 2

Polymastigina, 37

Polyneuritis infectiosa equorum, 321

Polyplectrum germani, T. in, 442

Polypterus, T. in, 482, 496

Position of the irypanosomes, 35

Posterior chromatic body, 5I

'Precipitin' reaction, Igo

Precipitinogen, I50

Preservation of trypanosomes, 29 of birds, 444 of fishes, 484 of $T$. brucei, 156

of $T$. equinum, 304

of $T$. equiperdum, 334

of $T$. evansi, 275

of $T$. gambiense, 404

of $T$. lewisi, 74

of $T$. paddce, 449

of $T$. theileri, 348

Protomastigina, 36, 37

Protomonadina, 36

Protoplasm of trypanosomes, I9

Pteropus medius, T. of, I08, Io9

Pytelia subflava, 45I

Python, T. in, 459

Rabbit, atoxyl treatment, $42 \mathrm{I}$ caderas, 292, 302 colouring agents, 419 dourine, $322,329,336,338-9$ galziekte, 345

mbori, 220

nagana, I3o, I49, I5I, I69
Rabbit, Soemedang, maladiẹ de, $34 \mathrm{I}$ surra, 255, 266, 267, 27I-2, 279

Togo virus, I9?

T-sis in, 67, 74, I03, 213, 215, 287 of Entebbe, 209 of Somaliland, 201 of Uganda, 206

$T$. dimorphon, 235

$T$. evansi, 422

T. gambiense, $382,387,421$

Radium, action of, 76, $4 \mathrm{rg}$

Raja alba, 482, 492

clavata, $48 \mathrm{I}, 492$

macrorynchus, $48 \mathrm{r}, 492$

microcellata, 482,492

mirelatus, 482, 492

mosaica, 48I, 492

punctata, 48I, 492

Rana angolense, 463,475

esculenta, 46r, 465, 467, 473

temporaria, 462

theileri, 463,475

trinodis, 468

Rat, caderas, 296, 302, 306, 308

colouring agents, $4 \mathrm{r} 9$

dourine, 322,327

experimental infection of, $6 I$ treatment of, $43^{2}$

galziekte, 345

human T-sis, 366

mbori, 220

nagana, I23, I25, I4I, I49, I 5 I, I7 I

natural modes of infection in, 88

parasite peculiar to, $5^{8}$. $\quad V$. also $T$. lezvisi surra, 266, 27I, 276, 280, 28I

Togo virus, 197

T-sis in, $2,3,7,8, \pi 4,25,58-9,60,6 \mathrm{I}-2$ 65,2 II, 215,287

of Abyssinian frontier, 207

of Cameroon; rs9

of Entebbe, 209

of Somaliland, 201

of Uganda, 206

T. brucei, $42 \mathrm{I}$

T. dimorphon, 233

T. lewisi, I5, 21, 28, 30, 6r, 68, 77, 88, 94

T. gambiense, $357,3^{82}, 3^{87}, 423$ treatment, 432

T. ugandense, 357

Rat-louse, T. in, 88

Raven, Indian, $T$. in, 440

Ray, T. in, $48 \mathbf{r}, 492$

Red-eye (fish), T. in, $48 \mathrm{r}, 483,495,496$ experiments on, 503

Redwater fever, 343

Reptiles, T. of, 3,457

Rhipicephalus sanguineus, T. in, 508

Rhizomastigina, 36

Rhombus lavis, 483

maximus, 482

River bull-heads, experiments on, 505 T. in, 483

Roach, 48r, 483, 495

Robin, T. in, $44 \mathrm{I}$

Rockling, T. in, 479

Roller-bird, T. in, 440, 443

Ross's method, I2

Rudd. $V$. Red-eye

Ruge's method, I2

Ruminants, dourine in, 330,336

Saccobranchus fossilis, T. in, 483,496

Salmo fario, 'T. in, 479

Tpl, in, $483,50 \mathrm{I}$

Scardinius erythrophthalmus, 48I, 483, 495, 496 Tpl. in, 495

Schleim-cysten, 5 I I

Sciurus griseimanus, T. in, I30 palmarum, T. of, 103, I06 
Sciurus vulgaris, T. in, $\mathbf{1} 30$

Scolephagus carolinus, T. in, 44I

Scomber scomber, 482

Scyllizm caniculi. T. in, $48 \mathrm{r}, 492$ stellare, T. in, 48r, 492

Sections, staining of trypanosomes in, 12

Serinus canarius, 450 meridionalis, 450

Serum, aninal, in nagana, $\mathbf{1 7 7}$

chimpanzee, I77

diagnosis, 189

fowl, 162,177

frog, 473

goat. I62, 177

in surra, 283

on T. grayi, 5 IO

goose, 162,177

horse, 162, 177

human, in nagana, 177

in surra, 283

in treatment, 95, 245

and $T$. gambiense, 4I2

of immunized rats, 93

of monkey, I77

of pig, $162, \mathbf{I} 77$

of rat, 162

of sheep, 162, 177

Serums, action of, 417 on T. padd $, 4,45$

Sexual forms of T., 42-44, 88, I56, 348, 403, 509

of Tpl., 499, 506

Sheep, caderas in, $30 x, 306$

dourine, 336

galiziekte, 345

mbori, 22 I

nagana, II2, 138, I 51

surra, 269

Togo virus, 197

T-ses of Cameroon, I99

Entebbe, 209

Uganda, 206

$T$. dimorphon, 239

T. gambiense, 382,389

Shrike, red-breasted, T. in, 442

Sialia sialis, T. in, 44 $\mathrm{T}$

Sigui tégué (flies), 226

Silurus clarias, T. in, 482, 493 glanis, T. in, 483

Sleeping sickness, $35^{2}, 37 \mathrm{I}$

Snake, dourine, $33 \mathrm{I}$

Sodium arsenite, $95,169,245 . \quad V$. also Arsenic

Soemedang, maladie de, $34 \mathrm{I}$

Sole (Solea vulgaris), T. in, 48I, $49 \mathrm{I}$

Somaliland, $\mathrm{T}$-sis of, $20 \mathrm{I}$

Sorex caruleus, roz

Souma, II5, 186, 202, 205, 223

Soumaya. Same as Souma

Sparrow and dourine, $33 \mathrm{I}$

English, T. in, 44T

song, $T$. in, $44 \mathrm{I}$

Specific agglutinıns, 85

Spermophile, $T$. of, I06

Spermophilus guttatus, T. of, I06

musivus, T. of, I06

Spinachia vulgaris, 482

Spinus tristis, $\boldsymbol{T}$. in, $44 \mathrm{I}$

$T$. laverani in, 454

Spirillum duttoni, 46 obermeieri, 46

Spirochata, 37, 45, 442

evansi, 247

obermeieri, 46

pallida, 47

plicatilis, 47

refringens, 46, 47

ziemanni, $44,+54$

Spleen extract in treatment, $4 \mathrm{I} 8$

Splenomegaly, tropical, 48
Springbok, nagana in, 139

Squalius cephalus, 48r, 483, 495

Squatina angelus, 482

Squirrel, Indian, T. in, 98, 103, 106

nagana in, 130

Staining methods, 8. $V$. Method for staining

Starling, 442

Stegomyia fasciata, 37, 107

Sterlet, T. in, 479

Stickleback, $48 \mathrm{r}$

Stomoxys, 38, 186, 200, 202, 210, 219, 225 calcitrans, 279, 307, 512

geniculatus, 253, 280

nebulosa, 307

nigra, 279

Strepsiceros capensis, nagana in, $\mathbf{I} 40, \mathbf{I} 68$

Strychnine sulphate in sleeping sickness, 435

Sudan, Anglo-Egyptian, T-ses of, 203 French, T-ses of, 219

Surra, 2, 3, 246

ætiology, 278

animals susceptible to, 254

bat, 109,266

Bovidæ, 257, 28I

buffalo, 248, 25I, 257-8, 28I-2

camel, 247, 26I, 28 I

cat, 268

cattle, 247,279

dog, $254,258,26 \mathrm{r}-4,274,279,280$

donkey, 256, 279

elephant, 26r

Equidæ, 246, 257

geographical distribution, 246

goat, $269,270,275,277$

guinea-pig, 266-7, 27I, 275-6, 280

horse, $247,249,250-2,254-5,275,279$. 280-I

jerboa, 266

mode of infection, 278

monkey, 266

mouse, $265-6,275$

experimental treatment in, 422-3, $43 \mathrm{I}$

mule, $247,252,279,280$

pathogenic agent, 272

pathological anatomy, 27 I

prophylaxis, 282

rabbit, 255, 266-7, 27I-2, 279

rat, 266, 27r, 276, 280, 28r

sheep, 269,270

treatment, $282,422 \cdot 3,431$

Sutoko, 205

Swallow, T. in, 440,442

Sylvia atricapilla, 440

Syngnathus, 482

Synkaryon, 53

Syphilis, organism of, 47

Syrnium aluco, T. in, 439, 444

Tabanidæ, 186, 200, 202, 219, 307

Tabanus, 225, 228

dorsovitta, 406

glaucopis, 38

lineola, 278

nemoralis, 219

tergestinus, $3^{8}, 39$

tomentosus, 219

tropicus, 278

Tachyphormus ornata, T. in, 442

Tadpole, T. in, 462

Taher (horse disease), 218

Talpa europea, T. of, IIo

Talusia hybrida, 297

Technique, 7

Tench, T. in, 479, $48 \mathrm{I}$

Tesiudo elongata, 289

Therapeutic measures, value of, 425 reactions (chromo), Iģo

Theropithecus gelada, 201

Thiroux's cultivation, 13,100 
Thrasher, brown, $\mathrm{T}$. in, $44 \mathrm{I}$

Thrush, song, T. in, 442

Thyroid tabloids in treatment, 434

Tibarsa, 246

Tinca tinca, T. in, $480-\mathbf{T}, 489$ vulgaris, $T$. in, 483 Tpl, in, 483

Tmerdjin (horse disease), 218

Toad, T. in, 464,477

Togo virus, 196, 197

Togoland, T-ses of, 193

Torpedo torpedo, 482

Tortoise, Asiatic, T. in, 457 mud, $T$. in, 457

Trachurus trachurus, 482

Tragarot in treatment, 434

Tragelaphus scriptus sylvaticus, I40, I68

Treatment, $415-3^{8}$

arsenic compounds, 420

arsenious acid, 434

atoxyl, 4I6, 420, 434-8

attempts at, of $T$. lewisi infection, 95

benzidene dyes, 415,426

brilliant green, 434

caderas, $308,415-6,422,43 \mathrm{r}$

chrysuidin in, 4I6

cultures of organisms, $4 \mathrm{I} 8$

dourine, $33^{8}, 416,422,425$

Finsen rays, 419

galziekte, $35 \mathrm{I}$

Gambian T-sis, 245 experimental, $43 I$

human T-sis, 4II, $433,434.8$

light, 419

mbori, 222, 416, 423

methylene blue, 433

nagana, 169

experimental, 4I7-9, 42I-2, 428-3I

radium emanations, 419

serum-therapy, 339

serums, 417 . $V$. Serum

sleeping sickness. $V$. Human T-sis

spleen extract, 4 18

strychnine sulphate, 435

surra, 282-3

$$
\text { experimental, 422-3, 43r }
$$

$T$. gambiense infections, experimental, $4 \times 6$, 421, 423-5, 432-4

thyroid, 434

tragarot, 434

trypanred, $415,420,434$

$\mathrm{X}$ rays, $76,159,419,434$

Treponema pallidum, 46, 337

Treron calva, $\mathrm{T}$. in, 442

Trichogaster fasciatus, $T$. in, $48 \mathrm{r}$

Trichomonas (Donné), 33

in fowl, 440

intestinalis, $\mathrm{r} 7$

Trigla, 482

Trilobus gracilis, 99

Triton vulgaris, 465

Troglodytes adon, T. in, 44I

Trophonucleus, 20

Trout, T. in, $I, 48 \mathrm{I}$

Tpl. in, 501

Trutta fario, $48 \mathrm{I}$

Trypanomonas, sub-genus, 34

Trypanomorpha, of Woodcock, 26

Trypanophis, 24, 26

Trypanoplasma, 16, 24, 481

abramidis, 490,501

harbel, in, 495, 50I

barbi, 501

Barbus fluviatilis, in, 483 , 5 OI

borreli, 18, 24, 483, 496, 506-7 multiplication forms in, 502, 506

Box boöps, in, 501

bream, in, 501

carp, in, 499
Trypanoplasma, Cobitis barbatula, in, 501

Cottus gobio, in, 483, 501

Cyclopterus lumpus, in, $50 \mathrm{I}$

cyprini, $483,488,499$

development of, 506

description, 26

evolution of, 499

fishes, of, 496

guernei, description of, 5or

intestinalis, 482, 50I

leech, in, 499, 504

loach, in, 501

minnow, in, 489, 498

new genus, 35

Phoxinus lavis, in, 496

Piscicola geometra, in, 499

Salmo fario, in, $49_{3}, 501$

trout, in, $50 \mathrm{t}$

trutte, 5 or

varium, 501

ventruculi, 5 о

Trypanosoma abramis, 489

Abramis brama, in, 48I

Acerina cernua, in, 483, 495

vulgaris, in, 480

acerine, description, 495

African dove, in, 442 description, 455

Agelaius phoniceus, in, 44I

American goldfinch, in, $44 \mathrm{I}$

Anguilla vulgaris, in, 481, 490

Athene brama, in, 440 noctua, in, 42, 445

avium, 23, 439, 44I canary, in, 450

cultural characteristics, 453

description of, 444

minus, 442

badger, in, 1 ro

bagara (fish), in, 482, 496

Bageus bayard, in, 482,496

balbianii, 34

Baltimore oriole, in, 44

bandicoot, in, 67,102

bandicotti, IO2

barbatule, description, 496

barbel, in, $479,483,495$

barbi, description, 495

Barbus fluviatilis, in, 483,495

bat, in, 107

Batrachia, in, $x, 460$

belli (n. sp.), description, 477

birds, of, I, 2, 3, 7, 439

cultivation of, 443

morphology of, 443

relation of, to other blood parasites, 454

Sudan, 455

table of dimensions, 447

blackbird, in, 442

red-winged, in, 44I

rusty, in, $44 \mathrm{I}$

blackcap warbler, in, $44^{\circ}$

blanchardi, description, I07

Blennius pholis, in, 483, 494

bluebird, in, $44 \mathrm{r}$

borreli, 458, 464, 469-470

bothi, description, 495

Bothus rhombus, in, 483, 495

boueti, description, $45^{8}$

bream, in, $48 \mathrm{r}, 489$

brill, in, 483,495

brown thrasher, in, $44 \mathrm{x}$

brucei, 14, 17, 18, 19, 20, 24, 25, 38, 74 , II2, I21, I47, 167, I99. $V$. also under Nagana

agglomeration, $x 6 \mathrm{x}$

atoxyl, 42 r

attenuation of virus, 18 


\section{TRYPANOSOMES AND THE TRYPANOSOMIASES}

Trypanosoma brucei, compared with $T$. evansi, 273

cultures, I59

involution torms, 163

morphology of, 150

multiplication forms, $\mathbf{I} 53$

nucleus of, 20, 2 I

preservation of, 156

staining of, 152

Bufo reticulatus, in, 1464,477 viridis in, 465

bull-head, river, in, 483

Buteo lineatus, in, $44 \mathrm{I}$

callionymi, description, 494

Callionymus dracunculus, in, 483,494

camels, in, 2, 3 , II $5,201,219,247,26 \mathrm{I}$, $28 \mathrm{I}$

carassii, 480

Carassius auratus, in, 488

vulgaris, in, 480,483

castellanii, 355

cat, in. $V$. Cat

cazalboui, II8, 225, 227

chaiffinch, in, $440,44^{2}$

characters of, 25

Chelidon urbica, in, 442

christopher si, 508

chub, in, 483,495

clarice, 482

description of, 493

cobitis, 480

Cobitis barbatula, in, 480,496

fossilis, in, 480

Colaptus auratus, in, 44I

comparative study of, I6

congolense, $\mathbf{1} 6$

costatum, 463

cotti, description, 494

Cottus bubalis, in, 483,495 gobio, in, 483, 495

crane, in, 439

crocodile, in, 459

culicis, 508

cuniculi, IO3

Cyanocitta cristata, in, $44 \mathrm{I}$

Cyprinus carpio, in, 48C-I, 483,488

dabib (fish), in, 482,496

damonia, description, 457

danilezuskyi, 482 description, 488

delagei, description, 494

Diemyctulus viridescens, in, 464,477

dimorphon, 18, 19, 23, 116, 118, 203, 226-7 229, 240. $V$. Gambia, 'T-sis of horses in

individuality of, 243

dog, in. $V$. dog

dogfish, in, 48I, 492

dogtick, in, 508

donkey, in. $V$. Ass and Donkey

dormouse, in, $\mathrm{IO} 7$

dove, mourning, in, 44I

dromedary, in, 5, 21 4,219

Dryobates villosus, in, $4+\mathrm{I}$

duttoni, 20, 99

eberthi, 34,440

eel, in, $48 \mathrm{r}, 490$

egret, in, 442

elegans, of frog, $46_{3}$ of gudgeon, description, 495

Emberisa citrinella, in, 442

English sparrow, in, 44I

Equidæ, in, 2, 3, 58, I32, 292

equinum, 19, 21, 292, 303

equiperdum, 15,152

cultivation of, 335

in dourine, $3^{12}, 33^{2}$

Esox lucius, in, $479,480-1$

Estrelda estrelda, in, 445
Trypanosoma evansi, 18, 19, 74, 117, 223, 246, 272

compared with $T$. brucei, 273

treatment, 422

field-mouse, in, $x, 58$

fishes, in, $r, 2,3,7,25,27,28,33,479$

grouping of, 505

mode of multiplication of, 502

flesi, description, 493

Flesus vulgaris, in, 483,493

flicker (bird), in, 44I

flounder, in, 483, 493

Fringilla caelebs, in, 442

frog. $\quad V$. frog and Rana

$T$. rotatorium of, $20,2 \mathrm{I}, 28,3 \mathrm{x}, 465$ gambiense, I9, 23, 29, 38, 243, 352, 40r.

$V$, also Human T-sis

and atoxyl, 421

cat, $3^{82}$

cattle, $39 \mathbf{r}$

Cebus capucinus, $3^{82}$

Cercopithecus fuliginosus, $3^{82}$

chimpanzee, 382

cow, $3^{82}$

Cynocephalus sphinx, 382

description of, 4 or

$\operatorname{dog}, 3^{82}$

donkey, $3^{82}, 390$

experimental infections, treatment of, $4 I 6,421,423-5,432$

goat, $3^{89}$

guinea-pig, 382

Hapale pencillatus, $3^{82}$

hedgehog, $382,3^{89}$

horse, 382,390

jackal, 382

jerboa, $3^{82}, 3^{89}$

Lemur, $3^{82}$

mongoz, 382

rubriventer, 382

Macacus cynomolgus, $3^{8} 3$ rhesus, $3^{8} 3$

marmoset, 382

marmot, $3^{82}, 3^{89}$

monkey, 382

mouse, 382,388

pathogenicity of, $3^{82}$

pig, 390

Pithecia satanas, $3^{8} 3$

rabbit, 382

rat, $3^{82}$

sheep, $3^{82}, 3^{89}$

treatment. $V$. Treatment

gargur (fish), in, 482,496

gecko, in, $45^{8}$

genus, 36

giant, of Lingard, 286

goat, $67,238,244$. $\quad V$. Goat

goat-sucker, in, 439

gobii, description, 494

Gobio fluviatilis, in, 483, 495

Gobius giuris, in, 483

niger, in, 483,494

goldfinch, in, $44^{\circ}$

granulosum, 23,483

description of, 490

var. parva, 490

grayi, 509

green frog, in, 46I

grobbeni, 26

gudgeon, in, 478, 483, 495

guinea-pig, in, 105. $V$. Guinea-pig

hamster, in, Io5

hairy woodpecker, in, 44I

Harporynchus rufus, in, 44I

hawk, red-shouldered, in, 44I

Helobdella algira, in, 474

himalayanum, 345

Hirundo rustica, in, 442 
Trypanosoma, in horse. $V$. Horse

house-martin, in, 442 -wren, in, 44I

human, $35^{2}$

Hyla arborea, in, 469 lateristriga, in, 469 viridis, in, 46r

Icterus galbula, in, 44I

Indian kite, in, 442 raven, in, 440

inopinatum, 462, 475, 478 description, 473

Java sparrow, in, 44I

jay, blue, in, 44I

jay, sp. (?), in, 454

johnstoni, 18,23

description, 445

in estrelda, 440

karyozeukton, 467,468

kingfisher, in, $44^{\circ}$

langeroni, description, 495

Laniarius cruentus, in, $44^{2}$

lark, and, 446

laterna, description, 494

laverani, 442

description, 454

in goldfinch, Spinus tristis, 454

leech, in, 474, 504

leucisci, description, 495

Leuciscus, 483, 495 cephalus, in, 495

lewisi, $8,14,15,17,19,20,24,25,5^{8}$ agglomeration of, 80

cultivation of, 13, 76

differential chasacters of, 73

flea, in, 88

guinea-pig, 20, 66, 72, 86, 9 I

lice, 88

nucleus of, 20

preservation of, 7

radium, action of, 76

rat, in, I5, 2I, $28,30,61,68,77,88$, 94

study of, 68

$\mathrm{X}$ rays on, 76

Limanda platessoides, in, 483, 494

limandae, description, 494

lingardi, 345

lizard, in, $45^{\circ}$

loach, in, $480,481,496$

longocaudense, 20, 60

loricatum, 463

Lota vulgaris, in, 483

lote, in, $47^{8}$

luis, 47

Lynodontis schal, in, 482,496

Mabuia raddonii, in, $45^{8}$

Macrones cavasius, in, 483 seenghala, in, 481

tengara, in, $48 \mathrm{I}$

measurements of, 16

$m e g a, 463,467$

Meles taxus, of, iro

Melospiza fasciata, in, $44 \mathrm{I}$

Merula merula, in, $44^{2}$ migratoria, in, $44^{\mathrm{I}}$

mesnili, 44 I

description, 453

in Buteo lineatus, 453

Milvus govinda, in, 442

minnow, in, 483,495

mole, in, $\mathrm{I}, 58$, rog. I ro

monkey. V. Monkey

mosquito, in, 43,508

mouse. $V$. Mouse

mugil, in, 482,496

mule. $V$. Mule

musculi, ro

myoxi, 107
Trypanosoma, nanum, 203

nelspruiterse, 463

description, 475

Neophron percnopterus, in, 442

newt, in, 464, 477

nicolleorum, 108

Nicticorax gardenia, in, 442

Nile fish, in, 482,496

noctuce, 20, 2I, 24, 4I, 89, I67

noke, in, 482,496

Ophiocephalus, in, $48 \mathrm{I}$

owl, in, 24, 440, 443

padde, $44 \mathrm{I}$

action of serums on, $45 \mathrm{I}$

agglutination of, 449

cultivation of, 449

description of, $44^{8}$

inoculation experiments, 450,453

Passer domesticus, in, 44I

pecaudi, 226

Perca fluviatilis, in, 480, 483, 495

perce, description, 495

perch, in, $479,483,495$

pestanai, I Io

pheasant, in, 442

phoxini, description of, 495

Phoxinus lavis, in, 483, 495

Phyllostoma, in, 107

pig. $\quad V$. Pig

pigeons, in, $44^{\circ}$

pike, in, $479,48 \mathrm{r}, 485$

plaice, in, 483,493

platessce, description, 493

Platessa vulgaris, in, 493

Platophrys laterna, in, 483, 494

Pleuronectes flesus, in, 482-3, 493 platessa, in, 482-3, 493

polyplectri, 442 description, 455

Polyplectrum germani, in, 442

Polypterus, in, 482, 496

preservation of, 29

Prussian carp, in, 480

pythonis, 459 description, 459

python, in, 459

rabinowitschi, 105

rabbit. $V$. Rabbit

Raja clavata, in, $48 \mathrm{r}, 492$ macrorynchus, 48I, 492

microcellata, 482

mosaica, $48 \mathbf{I}, 492$

punctata, 48 r, 492

raja, description of, $492 \cdot 3$

Rana angolensis, in, 463, 475

esculenta, in, 46r-2, 465, 459

temporaria, in, $462,464,465,468$

theileri, in, 463,475

trinodis, in, 468

rat. $V$. Rat

ray, in, $48 \mathrm{I}$

red-eye, in, $48 \mathrm{I}, 483,495^{-6}$

remaki, $\mathbf{1} 8,2 \mathbf{I}$

description of, 485

magna, 485

parva, 485

multiplication forms of, 502

reptiles, in, 3, 7, 457

Rhipicephalus sanguineus, in, 508

Rhombus lavis, in, 483,495

river bull-head, in, 483

roach, in, 483,495

robin, in, 44I

rockling, in, 479

roller-bird, in, 440,443

rotatorium, $15,17,20,23,27,457,462-4$, 466,469

cultivation of, 470

description of, 465 


\section{TRYPANOSOMES AND THE TRYPANOSOMIASES}

Trypanosoma rotatorium, morphology of, $47 \mathrm{I}$ synonyms of, 465 var. nana, 462

rougeti, $3 \mathbf{I} 4$

rudd, in. $V$. T. in red-eye

saccobranchi, 483

description of, 496

Saccobranchus fossilis, in, 483, 496

sanguinis, 34, 90, 46I

description of, 460

Scardinius erythrophthalmus, in, $48 \mathrm{r}, 483$, 495,456

Scolephagus carolinus, in, $44 \mathrm{I}$

scardiniz, description of, 495

scyllii, description of, 492

Scyilizum caniculi, in, $48 \pi, 492$

stellare, in, $48 \mathrm{I}, 492$

sexual forms of. $V$. Sexual forms of $\mathrm{T}$.

Sialia sialis, in, 44I

Siluridæ, in, 496

Silurus clarias, in, 482, 493

sole, in, 48I, $49 I$

solece, $18,2 \mathrm{I}$

description of, 491

Solea vulgaris, in, $48 \mathrm{I}$, $49 \mathrm{I}$

somalense, description of, 464,477

song-sparrow, in, 44I

song-thrush, in, 442

spermophile, in, 106

Spinus tristis, in, 441

squatii, description, 495

Squalius cephalus, in, 483, 495

squirrel, in, 106

sterlet, in, 479

sub-genus, 34

suis, II4

swallow, in, 440,442

Syrnium aluco, in, 439, 444

Tachyphormus ornata, in, 442

tadpole, in, 462

Talpa europaca, in, IIO

talpe, n. sp., 1 ro

tench, in, $478,48 \mathbf{I}$

theileri, 20, 205, 343 description of, 347

Tinca tinca, in, $480-1,489$

vulgaris, in, 483

tince, description of, 489

toad, in, 464,477

transvaaliense, $21,347,348-9$

Treron salva, in, 442

Trichogaster fasciatus, in, $48 \mathbf{I}$

Troglodytes cedon, in, $44 \mathrm{I}$

tsetse-flies, and, $2,4,26,38,156,167$, 403,508

tullochi, description, of, 5 Io

Turdus musicus, in, 442

ugandense, 355, 402

undulans, 463

vespertilionis, 107, 108

vivax, 28, 73, II7, I99

infection with, 200

vulture, Egypt, in, 442

yellow-hammer, in, 442

Zenaidura macroura, in, 44I

ziemanni, 43

Trypanosomatida, 37

Trypanosome fever (so-called), 370

Trypanosomes, agglutination of, 30

biology of, 27

comparative study of, I6

cultivation of. $V$. Cultures

fishes, grouping of, 505

mode of multiplication of, 502

infectivity of, 28

mammals, of small, 98

movement of, 27

nutrition of, 27
Trypanosomes, pathogenic, methods of identifying, 187

preservation of, $\mathbf{1 3}, 29$

virulence of, 28

Trypanosomiases of Abyssinia, 202 of Abvssinian frontier, 207 of Algeria, 2 ro of Anglo-Egyptian Sudan, 203 of Annam, 286

of Cameroon, 199

of Entebbe, 208

of Erythrea, 202

of French Guinea, 226

Sudan, 219

of Gambia, 2I9

of German East Africa, Igo

of horses in Annam, 286 Gambia, 229

of Somaliland, $20 \mathrm{r}$

of Togoland, I93

of Uganda, 204

treatment of, $415 . V$. Treatment

Trypanosomiasis, human, $35^{2}$

Trypanosomide, 36

Trypanosomosis, $\mathrm{J}$

Trypanozoon, 26, 33

criceti, 105

Trypanred, 6, 95, I73

in caderas, 308

in dourine, 339

in surra, 282

in treatment, $420,422-5$

inode of action, 425

and $T$.gambiense, 412

Trypanrot, I73, 308. $\quad V$. Trypanred

Tumby-a, 292

Tumby-baba, 292

Turdus musicus, T. in, 442

Turtur humilis, 289

Tsetse-flies, the, $5 \mathrm{I}$

and their trypanosomes, $2,4,26,38$, I $56,167,403,508$

and $\mathrm{dog}, \mathrm{I} 55$

and horse, I6 5

mode of reproduction, $5 \mathrm{I} 4$

rôle of, 2

Uganda, T-ses of, 204

Undulating membrane, 16,22

Undulina ranarum, 32, 46I

Vacuole, 19

Value of therapeutic measures in the $T$-ses, 425

Vespertilio kuhli, I08, I10, 217

nattereri, $T$. of, I08

noctula, 108

Vesperugo pipistrellus, T. of, 108 serotinus, $T$. of, 108

Virulence of trypanosomes, 28

Vulture, Egypt, T. in, 442

Wildebeest, nagana in, 140,168

Woodpecker, hairy, T. in, 44I

Wren, house, $T$. in, 44I

$\mathrm{X}$ rays, action of, $4^{\mathrm{I}} \mathrm{g}$

in treatment, 434

on $T$. lewisi, 76

Yellow-hammer, T. in, 442

Zebra and T-sis, 192

and Togo virus, 197

Zebus, soumaya of. V. Souma

Zenaidura macroura, $\mathbf{T}$, in, $44 \mathbf{I}$

Zeus faber, 482

Zusfana, mal de la, r86, 2II, 33 


\section{AUTHORITIES}

Adami, II5, I86, 202

Agatarchides, I I 5

Ahlbory, 365

Alvares, C. D., 60

Annett, 29, 23I, 24I

Aragoa, 442, 455

Arkhangelsky, 338

Athias, 463

Aubert, I90, 426

Austen, II2, I66, 208, 244, 5II, 5I 5, 5I7, 5I 8 , 520

\section{Bagshawe, 4I3, 5I5}

Baker, 355

Balbiani, 32, 247

Baldwin, I42, I45

Baldry, 313, 316, 320, 339

Balfour, A., 38, II5, I86, 203,364

and Neave, 4I6, 434, 5 I6

G. W., 169

Barron, 354

Battaglia, I08

Bayliss, 3 I9

Bell, 262, 464, 490

Bellay, Griffon du, 352, 39r

Beneden, Van, 503

Berg, I, 479, 484 and Creplin, 479

Bergeret and Bonin, 33I

Bettencourt, 353, 354, 366, 378, 393 and Franca, 104, I08, I09, IIO

Billet, $44, .464,474$ and Marchal, 3I2, 328

Birt, 5I

Blaise, 328

Blanchard, R., I26, I74, 248, 345

Blandfurd, 7, 29, 66, 90, III, I22, I25-9, I30, $I_{34}, I_{49}, I_{52}, I_{54}$

Blin, 248, 249, 288

Blochmann, 36

Bodin, 249

Bonin, $33^{I}$

Bosc, IO4

Bouin, P., 54

Bouffard, G., 225, 429

Bouet, 464, 466, 47I

Bradford, 8, 5I, 367 and Plimmer, $\mathbf{1 2 5}, \mathbf{1 3} \mathbf{I}, \mathbf{I} 38-9, \mathbf{I}+5$

Braid, James, 169

Brau, 249, 287

Brauer, 307

Brault, 354

Breinl, A., 46, 240, 393, 404 and Todd, 436,437

Broden, 23, II $3, \operatorname{I16}, 353,356,366,368,378$, $4 \mathrm{II}, 420,434,463$

Broden and Rodhain, $436,43^{8}$

Brornan, 54

Bruce, David, 2, 5, 6, 29, IIr, II4, I27, I36, I $38-9, \mathbf{1 5}$ I $164-5,170, \mathbf{I} 86,204,354-8$, $364,370,376,433,513$

James, II 5,516

and Nabarro, 6I, 398

and Greig, 392, 407

Brumpt, I, 29, 6I, I05, I07, II2, II 5, I20, I86, $201-2,355,366,382,384-5,406$, $464,474-8,483,495,499,5$ or, 504, 506, 518

and Lebailly, 483,494

Brumpt and Wurtz, 387, 389, 4 II

Buard, 60

Buffard. V. Schneider and Buffard

Busck and Tappeiner, 4I9

Busy, 328

Butschli, 32, 33, 36

Byloff, 62
Cagigal, 353

Calmette, 60

Campbell, W: G. 248]

Campenhout, Van, $362,378,435,43^{5}$

Carougeau, 249, 266, 272

Carré, 278

Carter, 408, 516

Carter, Vandyke, 59, 247

Castellani, 6, 353-4-5-6, 373, 402, 404, 4II, 433 and Willey, 482,496

Cazalbou, II8, I20, I86, 205, 219, 224, 225, 229 282

Cerqueira, 442,455

Certes, 34

Clarke, 352, 39I

Clegg, 25 $\mathrm{I}$

Cochrane, 360

Cook, J. H., 363

Corre, 352,376

Coutts, J. M., 253, 26r, 264, 266, 268

Chalachnikov, $2,32,35,59,60,77$, 105-6, 439, $462,465-6,480,484,500,502$

Chantemesse, I28

Chapman, James, Ir4, 5ז3

Chaptol, 249

Charmoy, de, 280

Chassaniol, 352,366

Chatterjee, 37,49

Chaussat, I, 58, 90, 460,479

Chauvrat, I2I, 2I0, 3 I4

Chenot, 329

Chichester, II 7,420

Christophers, $37,48,49$

Christy, $186,353,356,364,366,369,370,381$, $397,400,412$

Creplin, 479

Crookshank, 2, 33, 59, 247

Cumming, Gordon, II 4

Currie, 36r

Curry, 25I

Dangaix, 352, 39I

Dangeard, 54

Daniels, $354,43^{8}$

Danilewsky, $2,32,33,36,40,59,60,70,439$, $443,46 \mathrm{r}, 465,480,502$

Dantec, Le, 352

Daruty, 279

D'Anfreville, 435

Decorse, 120, 230, 519

Deixonne, M., 25I-2, 258, 279, 283-4

Demoor, A., I42-3

Diesing, II7, I78, I80, I85-6, I99, 201

Dionisi, Io7

Does, De, 3r3, 34r

Doflein, $33,36,40,3{ }^{1} 3,489$

Donovan, 48, 51, 74, 106, 248, 440

Dryepondt, 352,378

Drysdale, J. J., 165

Dupont, 367,378

Dupuy, II9

Durbam, 7, 29, 66, 90, I07, III, I22, I25-6, I28-9, I3O, I34, I4I, I49, I 52-3-4 Miss Florence, II 2

Durme, Van, $142,143,419$

Durrant and Holmes, 345

Dutroulau, 392

Dutton, 5, 6, 26, 29, 344, 354, $35^{6}$ and Todd, 6o, 99, II $3,119,354,359,366$, $370,376,379,3^{81-2}, 3^{8} 7,397-8,414,439$ $440,462,445,467$ and Christy, $40 \mathrm{r}, 434$ and Newstead, 5 I6

Eberth, 32, 440

Edington, A., 6r, 252, 26r, 264, 266, 268, 283 


\section{TRYPANOSOMES AND THE TRYPANOSOMIASES}

Eisath, G., 395

Ehrlich and Shiga, 6, I73, 296, 306, 308-9, $4 \mathrm{I} 5,425$

Elmassian, 5, 292, 294, 299, 300, 302, 307, 308

Evans, G. H., 246, 261

Fay, 362

Fellmer, 4I6, 434

Figueiredo, A. de, 353

Foà, 114, 121, 185, 514

Forde, $6,354,378$

França and Athias, 463, 469, 470 Carlos, $368,517,519$

Francis, 8, 59, 65, 74, 80, 81, 87, 88, 90, 94

Franke, 4I5

Gaigneron, 392

Galli-Valerio, 107

Gaule, J., $46 \mathrm{I}$

Gehrke, $45^{8}$

Ghika, N. D., II5

Gibson, 248, $25^{8}$

Giemsa, ro

Giles, G. M., 5 16

Girard, 368

Gluge, $3 I, I, 460,462$

Goebel, O., 27, 122, I40, I43, I48, 159, 419

Gouzien, 248

Gowers, W. F., II7

Grassi, 33, 60, 46r

Grattan, 360

Gray, 38, 186, 202, 204, 210, 364, 368, 376, 379 and Tulloch, 206, 358, 386, 403,405, 4I r, $420,434,508$

Greffuhle, I86

Greig, 29, II4, I44, I69, I86, I90, 202, 204, $210,354-5,364,368,370,376,379,3^{8} 5$ 420,433 and Gray, 206, 386, 397, 400, 40I, 4II, 434

Griffiths, 203

Gros, I, 58, 439, 479

Grothusen, I33, I92

Gruby, I, 25, 3I, 460, 462, 465

Guérin, 352, 359, 372, 392

Guignard, 53

Gunn, 247

Günther, 378 and Weber, 368

Haig, 248

Halban, I74

Halberstaedter, I3, I42, I44, I46, 296, 302, $306,4 \mathbf{I} 5$

Hallen, II 5,202

Hanna, 439

Hare, 78

Harris, W. C., II 4

Harvey, 356, 4 I0

Head, A. S., II5, 186, 203

Heidenhain, II

Heider, 54

Henneguy, 53

Hertwig, 56, 337

Hesse, 503

Hewby, II7, I86

Hirsch, 352

Hodges, $363,364,414$

Hofer, 500

Hoffmann, 47

Holmes, 102, 274, 345, 346

Hubenet, 34I

Hultgen, 66

Hutcheon, 343

\section{Ishikawa, 54}

Jakimoff, W. L., I22, 126, J30-I, I4I-5, I49, $157,296,299,301,305,334$

Jenner, Io
Jolyet, F., I03

Jürgens, $59,74,77,80,90$

Kanthack, 7, 29, 66, 90, III, I22, 125, 126.9, I30, I34, I4I, I49, I 52-4

Kempner, 3, 8, 5I, 59, 60

Kendall, A. 1., 6r, ror

Kent, Saville, 32, 40, $5^{8}$

Kermorgant, 249, 359

Kerr, 48

Keysselitz, 24, 20́, 483,490, 498, 50r, 503, 506

Kinghorn, 46 and Todd, 363

Kinyoun, 25I

Kisskalt, 108

Kitasato, 60

Kleine, $\mathbf{1 7 8}$, 179 and Moellers, $189,199,4$ r8

Knapp, 46

Koch, $29,3^{8}, 74,112,118,156,167,183,186$, $190,344,365,435,508$

Kohl, Nina, 334

Kolle, 343

Koninski, Karl, 464

Kopke, Ayres, 354, 352, 393, 435, 436

Korff, von, 53

Korschelt, 54

Krueger, 361

Kruse, $355,465,480$

Krzysztalowicz, 46, 337

Kuborn, 353

Kudike, 366

Kummer, 192

Kunstler, 35, 105, 457

Labbé, 33, 35

Laboulbène, 116

Lacerda, 292

Lacomme, L., 129, 169, 418

Lafosse, $33^{8}$

Landsteiner, $\mathbf{1 7 4}$

Lankester, Ray, 3r, 46I

Lanzenberg, 437

Lascaux, II9

Laveran, A., 2, 5, 32, 59, I09, II8-9, 174, 186 , 203, 219, 225-6, 229, 245, 247, 262, 266. $283,343,35 \mathrm{I}, 357-8,360,387,389,4 \mathrm{II}$, $425,433,437,439,448,463-4,475,483$, $498,516,520$

and Martin, 230

and Mesnil, 25, 5I, 59, 6I, 69, 75, 87, Ir2, I16, I18, 122, 123, 141, 154, 174, 188-9, 23I, $233,24 I, 264-8,272,277,286,289$, $290-1,314,348,420,457,462,48 \mathbf{r}, 485$, $488-9,490,492,496,502$

Lebailly, $483-4,490,493,495$

Lecler. V. Sivori and Lecler

L.edoux-Lebard, 87

Lees, Kay, 278

Leger, 22, 24, 26, 32, 37, 40, 4r, 55, 483, 489, $496,498,501,503$

Leidy, 32

Leicester, 248, 250

1.eishman, 48

Lemaire, 116

Lepierre, 353

Lepinte, 249

I. esur, Aimé, 252 Alfred, 25I-2, 256-7

Leuckart, 32,440

Levaditi, $3^{8}, 44 \mathrm{I}, 44^{8}$ and Sevin, 45

I.ewis, $x, 2,32,90$ T., 58

J., and Williams, H. V., $463,470,475$

Leydig, 457, 503

Lherminier, 392

Lieberkubn, 46r 
Lignières, I36, I39, I69, I88, 292, 295, 299, $300,304,306-7,330,336-7$

Lingard, 5, I7, 58, 67, 90, 102, I70, 247-8, 254 , $256-7,26 \mathbf{r}, 266,278,280,28 \mathbf{r}, 282,285,3 \mathbf{r}$, $316,318-9,320,337,345-6,480$

Linton, 356

Livingstone, II4, I2I, I64, I69, 513

Lorand, 376,434

Low, 353. 373, 377, 379, 393, 4II, 433 and Castellani, 433

Lühe, 26, 33, 40, I05, 344, 345, 348, 403

Luhs, F., 344, 346

Lustrac, 34

Mahé, 352

Maillard, 54

Manca, I04, 483,490

Manders, N., 253, 280

Manson, P., 29, 353-4, 358, 366, 378, 392 and Daniels, $4 \mathrm{I} 2$

Marchal, 312, $33^{8}$

Marchoux, 353 and Salimbeni, 464,469

Marek, 320

Markl, I4I, I42, I68

Martin, C. J., 264 G. , II9, I $86,205,227,24 I, 36 I, 458$ L.., $372,378,380,437$ and Girard, 368, 406

Martini, E., 59, 73, I I3, I22, I33, I35, I4I, I55, I78-9, I83, 186, 192, I93, 196-8

Martoglio, II5, 186, 202

Massaglia, A., I42, I45, I46, 27I, 4I8

Massey, Yale, 408, 517

Mathis, $14,77,130$

Maus, 25I

Maxwell-Adams, 355

Mayer, I, 3I, I35, I42.3, I50, 365, 460, 462

McNeal, 3, 25, 38, 44, 67, 70, 88, 92, I49, I55, I6I and Novy, 59, 6I, 77, 78,87

Megnin, I65

Meinmo, II 5, 186, 202, 205

Mendes, 354,436

Mense, 4I9, 434

Méricourt, Le Roy de, 352

Mesnil, 6, 32, 40, 45, 48, 52, 89, I04, I08, II6, I $44,184,194,201,208,212,322,325,330$, 368

Mesnil and Martin, 122, I40, I48, I77, I8I, 301 and Nicolle, I90, 4I 5-6, 426, 428 and Aubert, $426,432-3,438$ and Rouget, $213,217,330,336-7,340$

Meves, 53, 54

Migone, 292, 300, 302, 307, 308

Milne-Edwards, 46 I

Minchin, 37, 38, 167, 413, 511, 516 Gray and Tulloch, 458,5 ro

Mitchell, W., Iт2

Mitrophanov, 2, 27, 33, 480, 484

Möbius, 34

Moffat, $363,367,369$

Mole, 393

Mollereau, 248

Möllers, $178, \mathbf{1 7 9}$

Monfallet, $3 I_{3}$

Montel, 249, 482, 493

Moore, I02, II7, 170, I86 and Chichester, 420

B., 422

J. E. S., I 52

J. E. S. and Breinl, 404

Moreau, E., 492

Morel, I20, $5 \mathbf{I}_{3}$

Morax, V, 322

Mott, F. W., 48, 206, 209, 272, 313, 316, 319, $320-I, 322,353,368,370,379,393-6,405$

Moutier, 368

Musgrave, 186
Musgrave and Clegg, 60, 65. 74, 251, 259, 266, $277,280,285$

and Williamson, 25I, 266

Muratet, $48 \mathbf{I}$

Mutin-Boudet, 249, 287

Nabarro, 6, 29, II4, I22, I4I, I44, I69, I90, $202,204,210,344,346,354-6,35^{8}, 364$ $368,370,376-7,420,433,463$

and Greig, 230, 385-6, 408, 436, 516 and Stevenson, 476

Nabias, B. de, I03

Nattan-I,arrier, 378

Neave, $48,442,455,482,496$

Neporojny, $\mathbf{1} 43-5$

Nepveu, 354

Newstead, 5 I 6

Nicolas, 352

Nicolle, 6, 48, 104, 184, 217 and Comie, I08, 110 and Remlinger, 332

Nielly, 352, 392

Nierenstein, 422

Nissle, I42, I45, $4 \mathrm{I} 8$

Nobele, de, I59, 4 I9

Nocard, I12, I27, 129, I37-8, I65, 180, 188, 506, $3^{1} 3,3^{1} 7,324,328,33^{\circ}, 33^{2}, 336,337^{-8}$, 340 and Leclainche, 3 r2, 338, $34 \mathrm{I}$ and Vallée, 259, 278

Nocht, 8,135 and Mayer, 365,403

Novikoff, $33^{8}$

Novy, $3,3^{8}, 44,46,1_{5} 6,167,508$ and McNeal, II2, I59, I60, 44I, 45 I, $453-4,475$

and Hare, $247,273,276,278$

and Torrey, 508

Nuttall, C. E., 247

Ochmann, II4, I86, I93

Osler, 247

Panisset, 221, 247, 269

Panse, I9r, 344

Patton, 50

Pease, 246, 255, 258, 26I, 264, 282, 3I $2-3,3 \mathrm{I} 5$, $322,325,330,336,339,34^{\circ}$

Pécaud, I18, 186, 219, 224, 225, 229

Penning, 60, 250, 254, 266, 280

Perrin, 34

Petrie, I3, 59, 7I, 74, I04, I08, 44I, 442, 482, 488

Peuch, $33^{8}$

Pfeiffer, $3^{8}$

Phillips, 48

Pierre, II9

Pitchford, 185

Plehn, Miss, 22, 35, 483, 488, 499, 500

Plimmer, 8, I2, 29, 51, 357 and Bradford, III-2, I2I, I4I, I5I, I54, 242

Poche, 26

Prenant, 54

Pricolo, A., IOI-2, 274

Prince, 337

Prowazek, 20, 2I, 24, 32, 38, 4I, 59, 68, 79 , $88-9, \mathrm{I}_{43-4}, \mathrm{I}_{52}, \mathrm{I}_{55}, \mathrm{I} 67,4 \mathrm{O}, 449,5$ II

Rabinowitsch, $3,8,5 \mathrm{I}, 59,60,33 \mathrm{I}$ and Kempner, $6 \mathrm{I}, 64,68,70,85,88,90$, IO5, I5I, 306, 329, 33I-2, 339

Raillet, $60,165,214$

Rättig, I, 46I

Ray Lankester, I, 3I, 46I

Remak, I, 46I, 479

Remlinger, $48,33^{2}$

Renner, 360

Rennes, 120, I86, 21 I, 212, 3I4

Rezende, de, 354 


\section{TRYPANOSOMES AND THE TRYPANOSOMIASES}

Richard, II9

Robertson, 459, 482

Rodet, A., and Vallet, G., I43, I45, 4I8

Rodhain, 435

Roger, I86

$$
\text { and Greffulhe, 213 }
$$

Rogers, 48, 49, 74, 257, 279

Romanowsky, 8

Ross, I2, 44, I52, I59, I67, 419

Rost, E. R., 283

Rouget, 5, 6x, 210, 313-4, 322-4, 326, 328, 33 $\mathbf{r}_{\text {, }}$ 338-9

Roux, G., 4I8

Ruge, I2

Russ, 76, 419

Sa, Dias de, 368

Sabrazès and Muratet, 6o, 48r, 484, 490

Saint-Sernin, 249, 287

Salimbeni, 464

Salmon, 313 and Stiles, $25 \mathrm{r}$

Sambon, 40, 406

Sander, I9I, 5 I6

Santelli, $35^{2}$

Sauerbeck, I4I-2, I44, 302

Saville Kent, 32

Savouré, II5, 186, 202

Sawtchenko, 93

Schat, 250, 254, 259, 279

Schaudinn, 2O, 2I, 23, 24, 39, 4I, 42, 45, 47 $52,56,89,167,440,445$

Schild, W., 437

Schilling, 29, II2, II8, I22, 135, I40, I48, I55, I78, 180, 186, 193, I94-6, 344

Schneider, 332, 333 and Buffard, 5, 210, 3I2, 31 $3.4,316,318$ $320,324,328,33^{2}-3,335,337,33^{8}, 340-1$

Schmidt, I66, I9I

Schoch, I65

Schoo, 6o

Séguin, 249

Senn, 3, 8, 2I, 32, 33, 36, 40, 52, 55, 59, 466

Sergent, Ed. and Et., 37, 74, I08, I20, 2 II, 214 $218,314,336,419,440,462,464$

Sevin, $45 \mathrm{I}$

Shiga, 6, I73, 296, 306, 308-9, 415, 425

sicard and Moutier, 368, 393, 406

Siebold, Von, $46 \mathrm{I}$

Siedlecki, 46, 337

Simond, $45^{\circ}$

Sivori and Lecler, 6I, 74, 88, 292, 294, 306-7

Sjöbring, 442

Slee, $25 \mathrm{I}$

Smedley, 59, 80, I6I

Smith, Allen, $25 \mathbf{I}$ Donaldson, 115

Major, II8, I86, 230, 516

Speiser, 35I

Spreull, 343

Stähelin, I94

Statham, 48, 50

Steel, J. H., 247, 256, 269, 280

Stein, 32, 440, 46r

Stephens, 37, 38r and Newstead, 516

Stevenson, 122, 141, 463

Stordy, R. J., 6I, II4, I86, 207, 374

Strasburger, 53

Stuhlmann, $166,190,192$

Szewczyk, I20, I86, 2II, 3 I $4_{4}$
Tanon, $37^{8}$

Tappeiner, 4I9

Tartakovsky, 60

Tautain, II9, 226, 408

Taylor and Currie, $36 \mathrm{r}$

Teppaz, 242, 245

Terry, B. T., 6I, 65

Theiler, 5, 6I, II2-3, II5, I34, I85-7, 20I, $343-4,346,5$ I4

Thiroux, 44, 6r, 74, 99, 356, 412, 44I, 448.9. 466 and D'Anfreville, $435^{-6}$ and Teppaz, 242, 245

Thomas, 6, 6I, I84, 4I5, 42I, 434 and Breinl, 228, 23I, 233, 24I, 255, 264-8, $272,276,296,299,302,305,325,327$, $33 \mathrm{C}-\mathrm{I}, 357^{-8}, 377,3^{82}, 3^{86}, 3^{89}, 39 \mathrm{I}$, $397,405,418,425,434$ and Linton, $356-7,382,384-5,387,390$

Thomson, J. D., IIO

Tobey, $366,464,477$

Todd, $5,26,29,344,356,362,401,422,435$ and Tobey, 366, 377

Torrey, $3^{8}$

Trasbot, $33^{\circ}$

Trélut, $33^{8}$

Tulloch, $3^{8}, 35^{8}, 368$

Valentin, I, 3I, 479

Vallée, 247, 278, 33I, 390 and Carré, 188, 278 and Panisset, $188,222,260,278$

Vallon, 2I4

Van Beneden, 503

Van Campenhout, $362,378,435,436$

Vassal, 6I, 249, 25I, 253, 286, 289, 442, 455

Vles, 34

Voges, 140, 292, 306, 310, 3II

Von Siebold, $46 \mathrm{r}$

Vrijburg, 250, 257

Warrington, 393

Wasielewski, 3, 8, 2I, 32, 52, 55, 59 and Senn, 70

Wassermann, 365

Weber, 368

Wedl, I, 439, 46r, 479

Wellman, $359,442,455,517,520$ and Fay, 362

Wendelstadt, 416 and Fellmer, 434

Wenyon, rgo, 43I

Westwood, II5

Wiggins, 365,408

Willey, 482

Willems, 368

Williams, H. V., 464

Williamson, 25I

Wilson, E. B., 54

Winterbottom, 352

Wvodcock, 19, 26, 52, 108, 333, 474

Wright, 48,51

Wurtz, 29, $3^{82}, 3^{84}, 3^{85}$

Wyndham, 364

Yersin, 249

Zabala, 292

Ziemann. $3,8,24,28,61,66,73, \mathbf{I 1 2}, \mathbf{1 1 6}, \mathbf{1 1 8}$, 129, $186,193,195-6,200,353,361,440$, 462

ERRATUM. -Page $3^{8} 4$, line 8 from top, for $\left[\mathrm{IO}^{\prime} 2^{\circ} \mathrm{F}\right.$. $]$ read $\left[\right.$ Ior $^{\prime} 3^{\circ} \mathrm{F}$.] 



\section{DESCRIPTION OF PLATE.}

I. T. lewisi.

2. Multiplication form of $T$. lewisi.

3. T. lewisi, small form resulting from the disintegration of a rosette.

4. T. brucei.

5. T. equinum.

6. T. gambiense.

7. The Same, undergoing Division.
8. T. theileri.

9. T. transvaaliense.

Io. T. avium.

II. T. damonia.

12. $T$. solece.

13. T. granulosum.

14. $T$. raja.

I5. $T$. rotatorium.

16. Tpl. borreli.

All these figures have been drawn from preparations stained by the eosinBorrel-blue-tannin method described in the text, under a magnification of about 2,000 diameters, with the exception of Fig. I5, which was drawn under a magnification of about 1,400 diameters. 

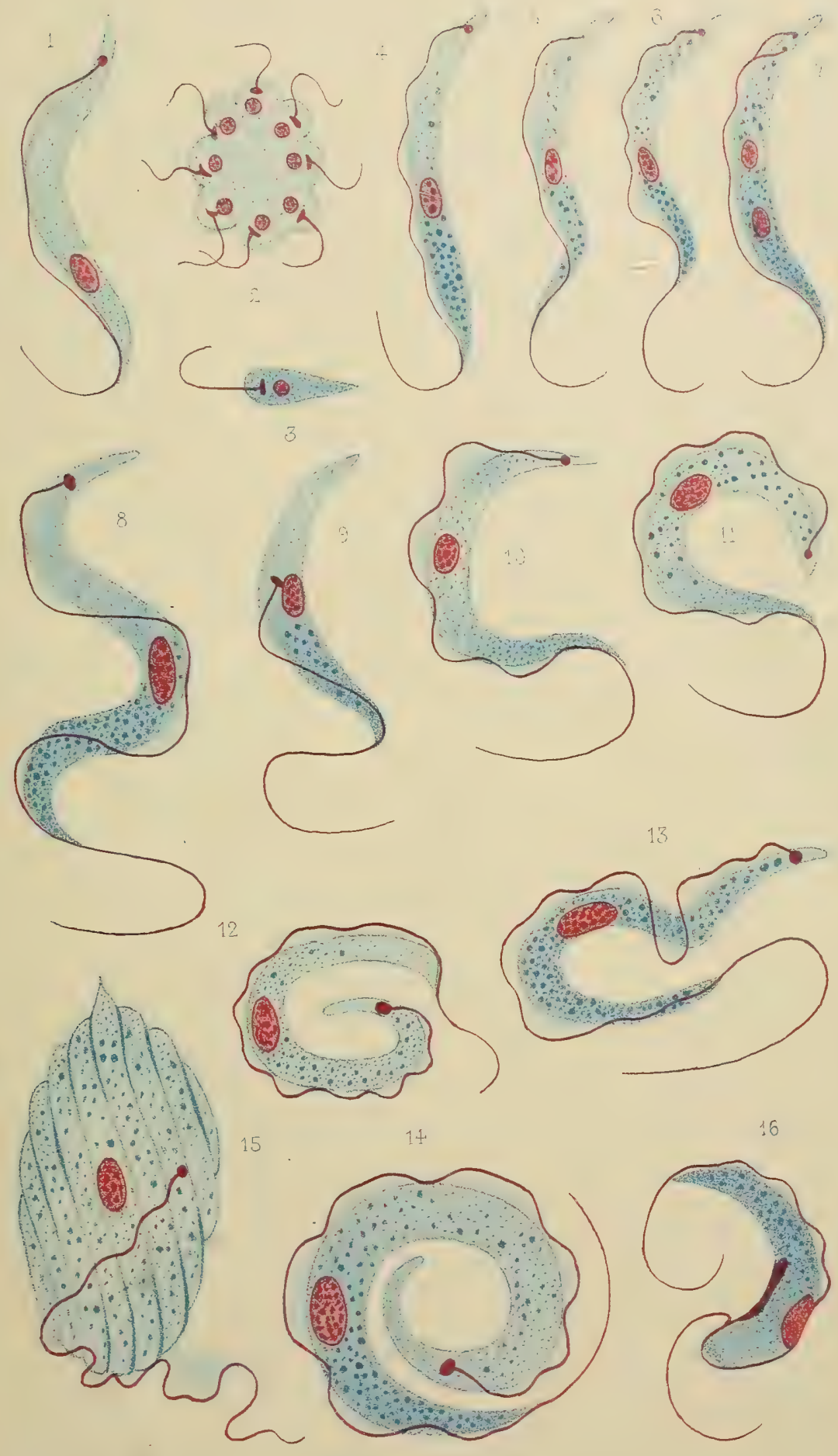



